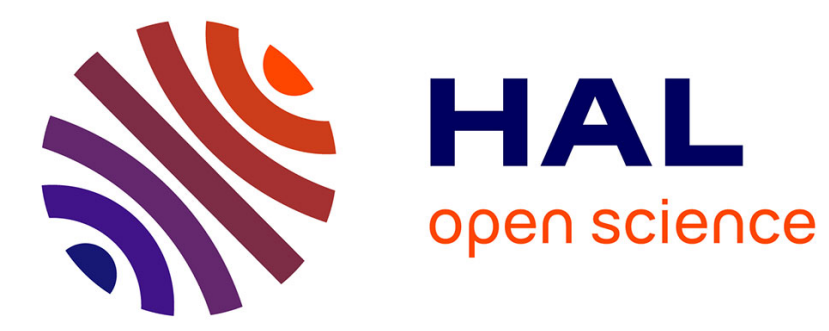

\title{
Revisiting carbenium chemistry on amorphous silica-alumina: Unraveling their milder acidity as compared to zeolites
}

Fabien Leydier, Céline Chizallet, Dominique Costa, Pascal Raybaud

\section{To cite this version:}

Fabien Leydier, Céline Chizallet, Dominique Costa, Pascal Raybaud. Revisiting carbenium chemistry on amorphous silica-alumina: Unraveling their milder acidity as compared to zeolites. Journal of Catalysis, 2015, 325, pp.35-47. 10.10.1016/j.jcat.2015.02.012 . hal-01176479

\section{HAL Id: hal-01176479 \\ https://hal.science/hal-01176479}

Submitted on 15 Jul 2015

HAL is a multi-disciplinary open access archive for the deposit and dissemination of scientific research documents, whether they are published or not. The documents may come from teaching and research institutions in France or abroad, or from public or private research centers.
L'archive ouverte pluridisciplinaire HAL, est destinée au dépôt et à la diffusion de documents scientifiques de niveau recherche, publiés ou non, émanant des établissements d'enseignement et de recherche français ou étrangers, des laboratoires publics ou privés. 


\title{
Revisiting carbenium chemistry
}

\section{on Amorphous Silica Alumina:}

\section{unraveling their milder acidity as compared to zeolites}

\author{
Fabien Leydier ${ }^{1,2}$, Céline Chizallet ${ }^{1, *}$, Dominique Costa $^{2}$, Pascal Raybaud ${ }^{1}$ \\ ${ }^{1}$ IFP Energies nouvelles, Rond-Point de l'échangeur de Solaize, BP3, 69360 Solaize, France \\ ${ }^{2}$ Institut de Recherches de Chimie de Paris, Chimie Paristech CNRS, UMR 8247, 11 rue \\ Pierre et Marie Curie, 75005 Paris, France \\ * Corresponding author, Tel: +33 4.37.70.22.42 ; Fax: +33 4.37.70.20.66 \\ E-mail: celine.chizallet@ifpen.fr
}

fabien.leydier@idris.fr ； dominique-costa@chimie-paristech.fr ； celine.chizallet@ifpen.fr; pascal.raybaud@ifpen.fr 


\begin{abstract}
Amorphous silica-aluminas (ASA) are prominent solids for their acidic properties. They are of first interest in catalysis, in particular for cracking reactions. Their relative acidity as compared to zeolites is a long-lasting general issue, driven by the limited knowledge of the surface structure of ASA, due to their amorphous nature. In the present contribution, thanks to a first principles approach based on our original ASA surface model, and on the study of a model mordenite zeolite (MOR), we propose a rational explanation for this feature. We compare by density functional theory calculations the ability of ASA and MOR to generate carbenium species from isobutene, versus $\pi$-complexes and alkoxide species. On ASA, carbenium species can be formed on Pseudo-Bridging Silanols (PBS) only, and are much less stable than in MOR. Then, we investigate the cracking pathway of a model alkene, 2,4,4trimethyl-2-pentene (DIB), by quantifying the stability of relevant intermediates of carbocationic and alkoxide natures. The carbocationic pathway is favored in MOR, whereas on ASA this pathway is strongly activated. By contrast, the PBS on ASA initiate preferentially a tertiary alkoxide route, or possibly a combined carbocationic/alkoxy route. Finally, the $i$-butene desorption step is also limiting in MOR due to the confinement effect induced by the zeolite pores. As a result, the higher cracking reactivity of zeolites as compared to ASA is mainly attributed to the favored nature of the carbenium route, stabilized by higher electrostatic confinement effect.
\end{abstract}

\title{
Keywords
}

Heterogeneous catalysis, amorphous silica alumina, zeolite, cracking, carbocation, carbenium, alkoxide, DFT, isobutene, 2,4,4-trimethyl-2-pentene, confinement effect. 


\section{Introduction}

The concept of Brønsted acidity[1] has been studied for a long time in the context of aqueous solutions, but its application to solid surfaces still remains an issue. One significant example is the case of amorphous silica-alumina (ASA) materials, which are widely used in fine chemistry,[2-4] biomass conversion[5, 6] and in industrial refining processes.[7] Indeed, the intimate chemical mixing of silica with alumina in ASA materials is empirically known to promote an enhanced surface Brønsted acidity with respect to the reference alumina or silica solids themselves. At the same time, this acidity is milder than the one observed in microporous alumino-silicates such as zeolites.[8, 9] This milder acidity is targeted in industrial processes such as hydrocracking (HCK) in order to reach optimal cracking selectivity into middle distillate products.[10, 11] However, the origin of this milder acidity as compared to zeolites is still questioned, due to the difficulty to reach a resolution at the molecular scale of ASA surfaces - amorphous systems - and of the intermediates involved during reactions even by using cutting edge operando techniques. Understanding the acidity of ASA is moreover of prominent interest because they are also suspected to be present as debris in (non-leached) de-aluminated zeolites.[12, 13] Interestingly, ASA and ultrastable-Y zeolite have been found to exhibit similar hydrocracking patterns in the conversion of the pristane model molecule.[14]

Bifunctional catalysts such as used in HCK processes require an optimal control of the balance between the hydrogenation function and the acidic function.[7, 15-18] For that purpose, various types of catalytic supports exhibiting a tuneable acidity scale (mesoporous alumina, ASA or zeolites) are industrially used in combination with a hydrogenating active phase (either a transition metal sulfide or a reduced metal).[7, 17] Indeed, it is crucial to optimize the acidic function of the HCK support: the milder acidity of ASA as compared to 
zeolites is profitably used to convert petroleum feeds containing also refractory compounds (such as nitrogen organic ones) in a selective way.

Many experimental studies[19-29] have analyzed the nature of possible Brønsted acid sites (BAS) on ASA: zeolite-like Si- $(\mathrm{OH})-\mathrm{Al}$ groups,[19-22] silanols in the vicinity of aluminum atoms[23-25] and water molecules adsorbed on aluminum atoms[26, 27]. However, at a molecular scale, it remains crucial to understand the precise chemical behavior of the ASA Brønsted acid sites, how they catalyze cracking reactions and the reasons of their milder acidity of ASA as compared to zeolites. In particular, it is proposed that ASA surface would exhibit very few BAS with an acidic character very similar to BAS found in zeolite.[21, 30] However, this interpretation remains questionable because it is also well known that the strength depends on both the local BAS atomic structure and the confinement effect depending on the pores' size surrounding the BAS.[31, 32]

Using density functional theory (DFT) calculations, some of us recently established a relevant ASA surface model which renders the diversity of surface Brønsted acid sites and highlights the existence of pseudo-bridging silanols (PBS).[33] In particular, PBS-Al sites consist of silanols whose oxygen atom is close to an aluminum atom, and able to be bonded to it upon deprotonation (Figure 1). The surface model also shows the presence of silicic PBS site (PBS-Si, see Figure 1), other silanols, for which Si-O-Al bridges coexist (called hereafter Silanol-Al), and water molecules adsorbed on aluminum atoms ( $\mathrm{III}_{1}$ in figure 1).

\section{-- HERE FIGURE 1 --}

So far, DFT approaches investigated the surface Brønsted acidity by calculating the adsorption of probe molecules,[34] in particular 2,6-lutidine[35] and CO.[36] The lutidine protonation was shown to be favored when the conjugated base of the BAS is stabilized. In 
particular, the PBS-Si site depicted in Figure 1 and the PBS-Al site called $\mathrm{Si}\left(\mathrm{V}_{1}\right)-\mathrm{OH} \cdots \mathrm{Al}_{\mathrm{IV}}$ $\left(\mathrm{III}_{3}\right)$ are both able to transfer their proton to lutidine, thanks to the formation of a new Si-O and Al-O bond after deprotonation, respectively. Other PBS-Al sites were not able to do so due to angular constraints,[33] while Silanol-Al sites are not deprotonated because the conjugated silanolate cannot be stabilized. The water molecule adsorbed on the $\mathrm{III}_{1}$ aluminum atom can generate lutidinium, as an Al-OH group is formed. Cascade proton transfer can also occur from this adsorbed water molecule to the neighboring silanol group.[35] The vibrational stretching of $\mathrm{CO}$ was found sensitive to the electrostatic field at the surface of aluminosilicic materials.[36] In the course of a reaction involving a hydrocarbon, both aspects (proton transfer and local electrostatic field) are expected to play a role. In particular the relation between the structure of the sites, the protonation ability towards lutidine, the electrostatic field, and the reactivity in cracking reactions, remains to be understood.

For zeolite catalysts, it is quite widely admitted that olefin cracking proceeds via carbenium ions (formed on protonic sites of the material),[37] well known in organic chemistry.[38-40] After formation, and possible isomerization, these species are considered to undergo $\beta$-scission (Figure 2).[16, 18, 41-44]

-- HERE FIGURE 2 --

However, isolating carbocations within or on the surface of aluminosilicates remains a challenging task due to their supposed short lifetime.[45, 46] Highly resonant species mainly could be observed in zeolites, $[45,47,48]$ even if indirect evidence was provided by ${ }^{13} \mathrm{C}$ NMR for the transient formation of short-live carbenium ions in the case of long-chain or branched 
olefins.[49-51] Alternative proposals to carbenium $\beta$-scission can thus be recorded. Sie[52] proposes a pathway invoking hydride shifts and highly non classical cyclopropane-like intermediates, by analogy with what is known about isomerization reactions.[53] Up to date, no confirmation of this kind of pathway could ever be given.[16] Moreover, alkoxides (Figure 3) were characterized in zeolites thanks to ${ }^{13} \mathrm{C}$ NMR [51, 54, 55] and FTIR[56, 57] and were calculated as stable species by earlier quantum chemical calculations,[58-64] which led some authors to refute the existence of carbenium ions as cracking intermediates in zeolites, even for tertiary carbenium. The intermediate or transition state nature of carbenium in zeolites as calculated by quantum chemistry however appears to be strongly dependant on the alkene considered,[65] but also on the level of theory (DFT, with or without dispersion corrections, versus post-Hartree-Fock methods for example),[66, 67] on the kind of model small clusters versus periodic cells - and on the local geometry of the active site.[65, 68-75] Indeed, taking into account the electrostatics induced by the porosity leads to a stabilization of charged species whereas the stability of the neutral species remains almost unchanged. Temperature effects are also shown to affect the alkoxide existence probability over that of carbenium, according to DFT calculations.[76-78]

\section{-- HERE FIGURE 3 --}

In the case of ASA, the formation of carbenium ions thanks to surface proton transfers was also invoked for a long time[15, 79, 80] even if alkoxides are mainly observed by FTIR.[56] Generally, the catalytic activity of ASA in acid-catalyzed reactions is lower than zeolites.[18, 81] This could be either due to intrinsically weaker sites,[82] or to a lower amount of the same kind of sites (bridging $\mathrm{Si}-(\mathrm{OH})-\mathrm{Al}$ groups) than in zeolites.[21, 30] There 
is however a lack of detailed data both on the stability of carbenium species on these solids, and on their implication in the conversion of hydrocarbons, in particular cracking of alkenes.

In the present work, we propose to determine the energetics of carbocation / alkoxide formation followed by C-C $\beta$-scission for BAS located on ASA surface and in a reference zeolite (mordenite, MOR), thanks to periodic DFT $+\mathrm{D}$ calculations. As indicated above, we suspect that confinement effects may play a key role, so it is mandatory to include dispersion corrections. We chose a model molecule, 2,4,4-trimethyl-2-pentene (DIB, 8 carbon atoms), as its branched character is expected to render the formation of carbocations and the $\beta$-scission favorable, with stable intermediates (Figure 2). Indeed, the $\beta$-scission of carbocations produced from linear alkenes generates a primary carbenium ion, which is energetically highly unfavorable.[16] Thus, the n-alkylcarbenium ions undergo skeletal isomerization first, leading to branched isomers. In the present study, we do not address any preliminary isomerization reaction, but focus on the $\mathrm{C}-\mathrm{C}$ bond cleavage from a multi-branched species. This is the archetype of the so-called "type A" $\beta$-scission,[83] which both starts and ends up with tertiary carbenium ions.

So as to investigate such mechanism, the physicochemical interaction of isobutene (formed upon DIB cracking) with the acid sites of the surface has to be understood first. Stable intermediates and energy profiles will be investigated, but at this stage activation energies are not evaluated: however we will show that the quantitative analysis of the thermodynamic profiles is very much insightful for discriminating the two acid solids. The relative stabilities of the $\pi$-complex, the tert-butyl cation and isobutoxy species (Figure 3) were studied on various sites of the ASA surface model, in comparison with mordenite. The existence of a possible link between the lutidine (results from ref. [35]) versus alkene protonation abilities will be looked at, also in comparison with $\mathrm{CO}$ adsorption features (results from ref. [36]). Then, the implication of the stability of the various intermediates in the shape 
of the reaction profile for the cracking of DIB will be detailed on the most important site of ASA, PBS-Al (Figure 1), in comparison with the acid site of mordenite. PBS-Al site was indeed shown to protonate lutidine,[35] and induces a strong electrostatic field.[36] Temperature effects are taken into account thanks to a statistical thermodynamic approach to understand the nature of the preferred pathways. We will finally show how the confinement effects, $[31,32,69,84]$ existing in zeolites but absent from ASA, are determining the nature of the preferred pathway on each solid and the lower acidity of ASA as compared to the reference MOR zeolite.

\section{Methodology}

The ASA supercell was the same as in references $[33,35]$ for $\theta_{\mathrm{OH}}=5.4 \mathrm{~nm}^{-2}$ (Figure 1), with dimensions of $11.13 \times 16.77 \times 43 \AA^{3}$. The $\mathrm{OH}$ content chosen represents typical reaction conditions used in catalysis and characterization experiments (see ref. [33] for a full thermodynamic diagram). Model mordenite (with a main channel circumscribed by twelvemembered rings - $12 \mathrm{MR}$ ) was considered to depict the behavior of a typical proton exchanged zeolite. The primitive cell, doubled in the $c$ direction was considered (dimensions: 13.78x13.78x $15.15 \AA^{3}$ ). One $\mathrm{T}_{4}$ site was substituted by an $\mathrm{Al}$ atom, with a proton in the $\mathrm{O}_{10}$ position (see for example reference [85] for structural details).

Periodic DFT calculations were carried out with the PBE exchange-correlation functional[86] as implemented in VASP 5.2.[87, 88] The projected augmented wave (PAW) method[89] was used to describe the core-electrons interactions and the plane wave basis set was limited to a kinetic cut-off energy of $500 \mathrm{eV}$. Van der Waals corrections as proposed within the Grimme formalism[90] was applied. These corrections are mandatory to evaluate reasonably accurate energies of hydrocarbons adsorbed on aluminosilicates, even if systematic deviation from experimental values is still observed.[66, 91, 92] 2x1x1 and $2 \times 2 \times 2$ 
K-points meshes were used for the ASA and MOR supercells respectively. A dipolar correction was applied to account for the arbitrary interaction between asymmetric - thus polar - slabs in the case of ASA. We achieved geometry optimizations with a convergence criterion on forces on relaxed atoms $\left(<2.10^{-2} \mathrm{eV} . \AA^{-1}\right)$. We also explored the energy potential landscape by performing velocity scaled molecular dynamics (time step: 3 fs, artificial mass for hydrogen: 5 u.a., $500 \mathrm{~K}$, energy cutoff: $400 \mathrm{eV}$ ), followed by quenching performed with an energy cutoff of $500 \mathrm{eV}$, to solve the configurations of the DIB molecule adsorbed on BAS.

The stability of each adsorbed species was first quantified by an energetic approach, calculating the reaction energy $\Delta_{\mathrm{r}} \mathrm{U}$, and decomposing it into Kohn-Sham (DFT-PBE, $\Delta_{\mathrm{r}} \mathrm{U}_{\mathrm{DFT}}$ term) and dispersion (Grimme, $\Delta_{\mathrm{r}} \mathrm{U}_{\mathrm{disp}}$ term) contributions, according to equation 1.

$$
\Delta_{\mathrm{r}} \mathrm{U}=\mathrm{U}_{\text {adsorbed-species }}-\mathrm{U}_{\text {alkene }}-\mathrm{U}_{\text {site }}=\Delta_{\mathrm{r}} \mathrm{U}_{\mathrm{DFT}}+\Delta_{\mathrm{r}} \mathrm{U}_{\text {disp }}
$$

Equation 1

Thermal corrections were then introduced as explained below, to obtain the reaction Gibbs energy $\Delta_{\mathrm{r}} \mathrm{G}$. Then, we considered DIB cracking (equation 2).

$$
\text { Surface }_{(\mathrm{s})}+\mathrm{DIB}_{(\mathrm{g})}=\text { Surface }_{(\mathrm{s})}-\text { Molecule- }_{(\mathrm{ads})}+\mathrm{n} \times \text { Molecule- }_{(\mathrm{g})} \quad \text { Equation } 2
$$

Surface $_{(\mathrm{s})}$ depicts the surface (external surface of ASA or internal surface of MOR) on which the $\operatorname{DIB}_{(\mathrm{g})}$ adsorbs to generate the Surface $(\mathrm{s})-$ Molecule- $1_{(\text {ads })}$ system, together with the possible release of $n$ Molecule- $2_{(\mathrm{g})}$ (when DIB is transformed into isobutene, $n=1$ then $n=2$ ). Thermal and entropic corrections were then included for evaluating the reaction Gibbs free energy (equation 3 and 4), considering a temperature of $500 \mathrm{~K}$, which is typical of hydrocracking reaction conditions,[16] and standard pressure.

$$
\Delta_{\mathrm{r}} \mathrm{G}_{(\mathrm{T})}=\mathrm{G}_{\text {Surface }_{(\mathrm{s})}-{\text { Molecule- } 1_{\text {(ads) }}}}+\mathrm{n} \times \mathrm{G}_{\text {Molecule-2 }_{(\mathrm{g})}}-\mathrm{G}_{\text {Surface }_{(\mathrm{s})}}-\mathrm{G}_{\mathrm{DIB}_{(\mathrm{g})}} \quad \text { Equation } 3
$$

with : $\quad \mathrm{G}_{(\mathrm{T})}=\mathrm{U}_{\text {elec }}+\mathrm{F}_{\text {trans }(\mathrm{T})}+\mathrm{F}_{\mathrm{rot}(\mathrm{T})}+\mathrm{F}_{\mathrm{vib}(\mathrm{T})}+\mathrm{PV}_{\mathrm{m}}+\mathrm{ZPE} \quad$ Equation 4 where $F_{\text {trans(T) }}, F_{\text {rot(T) }}$ and $F_{\operatorname{vib}(T)}$ are translational, rotational and vibrational contributions to free energy, $V_{m}$ the molar volume, $U_{\text {elec }}$ the electronic $(D F T+D)$ contribution to energy, $\mathrm{ZPE}$ 
the zero-point vibrational energy.

To evaluate $\mathrm{F}_{\text {trans(T) }}, \mathrm{F}_{\mathrm{rot}(\mathrm{T})}$ and $\mathrm{F}_{\mathrm{vib}(\mathrm{T})}$, we calculated the partition function of each gaseous and adsorbed species, considering each gaseous species as ideal gas (see Supporting Information S1.1.) and adsorbed species as partially immobile gaseous species. For each adsorbed molecules (see Supporting Information S1.2. for details), we assumed that some translational contributions to enthalpy and entropy are lost upon adsorption: one over three degree of freedom is lost for carbenium and $\pi$-complexes (2D gas model), whereas alkoxy species are supposed to be immobile from a translational point of view. Indeed, the latter are linked to the surface by an additional C-O bond. In the same spirit, we also considered that one rotational degree of freedom over three is lost upon adsorption for $\pi$-complexes and carbenium species, two degrees of freedom being lost for alkoxides. Indeed, partial rotational freedom has been experimentally evidenced on related systems.[93] In a first approach, we kept unchanged the vibrational contribution to enthalpy $\mathrm{H}$ and entropy $\mathrm{S}$ as compared to gas phase species. Although some of the approximations performed here differ from previously published calculations dealing with isobutene reactivity in zeolites, [77, 78] it can be noted that difference in Gibbs free energy corrections between the tertiary alkoxides and carbenium species are of the same order of magnitude: $\left(\Delta_{\mathrm{r}} \mathrm{G}-\Delta_{\mathrm{r}} \mathrm{U}\right)_{\text {carbenium }}-\left(\Delta_{\mathrm{r}} \mathrm{G}-\Delta_{\mathrm{r}} \mathrm{U}\right)_{\text {alkoxide }}$ equals to $70 \mathrm{~kJ} \mathrm{~mol}^{-1}$ at $500 \mathrm{~K}$ in our case, whereas it was evaluated around $70 \mathrm{~kJ} \cdot \mathrm{mol}^{-1}$ in mordenite by

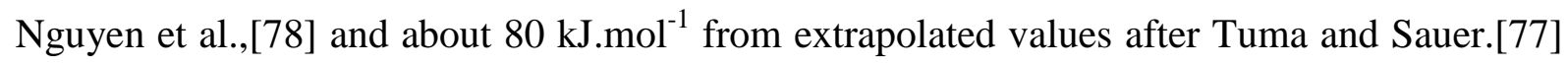
This validates the $\Delta_{\mathrm{r}} \mathrm{G}$ values which will be presented in the following, even if refinements could be brought to check the assumptions made for their calculation.

As mentioned in Introduction, activation reaction energies were not evaluated at this stage but we will show that the chemical behaviors of the two acid materials can already be differentiated according to the trends revealed by the energy profiles. Note also that the present study lacks from self-interactions corrections due to the level of theory used (DFT), 
which is expected to affect the overall energetics.[67] Main trends regarding the ASA versus MOR comparison might however not be strongly affected.

Electrostatic field was calculated in the surrounding of the $\mathrm{C}=\mathrm{C}$ bond of the isobutene molecule when adsorbed as a $\pi$-complex mode. For that purpose, we calculated the local electrostatic potential on the system, after removing the isobutene molecule, but keeping all other geometric features constrained. The gradient of the electrostatic potential (ionic plus Hartree plus exchange-correlation) was estimated between two points A and B distant by $1 \AA$, aligned perpendicular to the $\pi$-bonding of the molecule, the middle of the $[\mathrm{AB}]$ segment being also the middle of the $[\mathrm{C}=\mathrm{C}]$ one. Note that the electrostatic field determined in the present work differs from previous values reported in ref. [36] due to the inclusion of the exchangecorrelation potential (available in the present version of VASP) and to the different geometry of the acid site after $\mathrm{CO}$ versus isobutene adsorption.

\section{Results and discussion}

\subsection{Isobutene interaction with surface acid sites}

We firstly investigate the stability of key intermediates obtained upon interaction of isobutene with the acid sites of ASA and MOR. The stability and structures of the $\pi$-complex, carbenium and alkoxides are compared in Table 1. 
Table 1. Formation energies $\Delta_{\mathrm{r}} \mathrm{U}$ of the $\pi$-complex (physisorption), the carbenium ion and the alkoxide from isobutene on various sites of the ASA surface and in MOR ; decomposition (see eq. 1) of $\Delta_{\mathrm{r}} \mathrm{U}$ into the DFT energy $\left(\Delta_{\mathrm{r}} \mathrm{U}_{\mathrm{DFT}}\right)$ and dispersion correction terms $\left(\Delta_{\mathrm{r}} \mathrm{U}_{\text {disp }}\right)$; distance between the $\pi$-complex (terminal $\mathrm{C}$ atom of the $\mathrm{C}=\mathrm{C}$ bond) and the acid site $(\mathrm{H} \cdots \pi)$, and $\mathrm{O}_{\text {site }}-\mathrm{C}_{\text {alkoxide }}$ bond length ;

\begin{tabular}{|c|c|c|c|c|c|c|c|c|c|c|c|}
\hline \multirow{2}{*}{ Acid site $^{1}$} & \multirow{2}{*}{$\begin{array}{c}\text { Protonation } \\
\text { ability of } \\
\text { lutidine }^{2}\end{array}$} & \multirow{2}{*}{$\begin{array}{l}\text { Location and } \\
\text { terminology } \\
\text { (see figure 1) }\end{array}$} & \multicolumn{3}{|c|}{$\Delta_{\mathbf{r}} \mathbf{U}(\mathbf{k J} / \mathbf{m o l})$} & \multicolumn{3}{|c|}{$\begin{array}{c}\text { Decomposition of } \Delta_{\mathrm{r}} \mathrm{U}: \\
\Delta_{\mathrm{r}} \mathrm{U}_{\text {DFT }}\left(\Delta_{\mathrm{r}} \mathrm{U}_{\text {disp }}\right)(\mathrm{kJ} / \mathrm{mol})\end{array}$} & \multicolumn{2}{|c|}{$\begin{array}{c}\mathrm{H} \cdots \pi \text { distance, } \\
\mathrm{O}_{\text {site }}-\mathrm{C}_{\text {alkoxide }} \text { bond length }(\AA)\end{array}$} & \multirow{2}{*}{$\begin{array}{c}\text { Electrostatic } \\
\text { field } \\
\left(\mathrm{V} \cdot \mathrm{nm}^{-1}\right)^{\mathbf{3}}\end{array}$} \\
\hline & & & $\pi$-complex & Carbenium & Alkoxide & $\pi$-complex & Carbenium & Alkoxide & $\pi$-complex & Alkoxide & \\
\hline PBS-Si & protonation & $\mathrm{Si}\left(\mathrm{U}_{1}\right)-\mathrm{OH} \cdots \mathrm{Si}\left(\mathrm{V}_{2}\right)$ & -13 & +61 & -16 & $+19(-32)$ & $+112(-51)$ & $+40(-56)$ & 2.179 & 1.502 & 21.48 \\
\hline PBS-Al & protonation & $\mathrm{Si}\left(\mathrm{V}_{1}\right)-\mathrm{OH} \cdots \mathrm{Al}_{\mathrm{IV}}\left(\mathrm{III}_{3}\right)$ & -18 & +30 & -59 & $+37(-55)$ & $+100(-70)$ & $+5(-64)$ & 2.042 & 1.484 & 27.64 \\
\hline \multirow{2}{*}{ PBS-Al } & \multirow{2}{*}{ No protonation } & $\mathrm{Si}\left(\mathrm{Z}_{2}\right)-\mathrm{OH} \cdots \mathrm{Al}_{\mathrm{V}}\left(\mathrm{III}_{2}\right)$ & -50 & - & -75 & $-12(-38)$ & - & $-19(-56)$ & 2.053 & 1.482 & 30.18 \\
\hline & & $\mathrm{Si}\left(\mathrm{Y}_{2}\right)-\mathrm{OH} \cdots \mathrm{Al}_{\mathrm{V}}\left(\mathrm{III}_{4}\right)$ & -26 & - & -33 & $+8(-34)$ & - & $+21(-54)$ & 2.364 & 1.447 & 16.44 \\
\hline Silanol-Al & No protonation & $\mathrm{Si}\left(\mathrm{W}_{2}\right)-\mathrm{OH}$ & -9 & - & -54 & $+7(-16)$ & - & $-12(-42)$ & 2.052 & 1.480 & 24.59 \\
\hline $\mathrm{Al}-\mathrm{OH}$ & protonation & $\mathrm{Al}\left(\mathrm{III}_{1}\right)-\mathrm{HOH}_{(2)}$ & -11 & - & -6 & $+16(-27)$ & - & $+49(-55)$ & 2.785 & 1.521 & 5.32 \\
\hline Mordenite & protonation & & -95 & -88 & -95 & $-44(-51)$ & $-1(-87)$ & $-22(-73)$ & 1.821 & 1.624 & 34.10 \\
\hline
\end{tabular}

${ }^{1}$ For the nature of acid sites, the reader is referred to Figure 1 (and introduction) $:$ PBS $=$ pseudo-bridging silanols, $\mathrm{PBS}-\mathrm{Si}=$ silicic $\mathrm{PBS}, \mathrm{PBS}-\mathrm{Al}=\mathrm{aluminic} \mathrm{PBS}, \mathrm{Silanol}-\mathrm{Al}=$ silanol with Si-O-Al bridges.

${ }^{2}$ From ref. [35]

${ }^{3}$ At the surrounding of the isobutene molecule adsorbed according to a $\pi$-complex mode, see text. 


\subsubsection{Structure and stability of the $\pi$-complex}

The physisorption of isobutene takes place through the interaction of the $\mathrm{C}=\mathrm{C}$ double bond with the acidic proton of the surface. After geometry optimisation, the molecule slightly orients the terminal $\mathrm{C}$ atom of the $\mathrm{C}=\mathrm{C}$ bond near the surface proton (see structures in Figure 4).

\section{-- HERE FIGURE 4 --}

The distance between this carbon atom and the proton (table 1) is as high as 2.0-2.8 whereas it is only $1.8 \AA$ in mordenite. On ASA, the adsorption is most favorable $\left(-50 \mathrm{~kJ}^{\circ} \mathrm{mol}^{-}\right.$ $\left.{ }^{1}\right)$ on $\mathrm{Si}\left(\mathrm{Z}_{2}\right)-\mathrm{OH} \cdots \mathrm{Al}_{\mathrm{V}}\left(\mathrm{III}_{2}\right)$, which belongs to the PBS-Al family, but does not protonate lutidine.[35] The adsorption is however much more favorable in mordenite $\left(-95 \mathrm{~kJ}^{\mathrm{mol}}{ }^{-1}\right)$. This effect is assigned to:

- van der Waals interactions between isobutene and the surrounding framework of MOR. For the $\pi$-complexes in weak interaction as mainly found on ASA, van der Waals forces represent the major part of the attractive part of the interaction but they are weaker by about 13 to $35 \mathrm{~kJ} \cdot \mathrm{mol}^{-1}$ than in MOR with one exception: the PBS-Al site $\left(\mathrm{V}_{1}\right)$ for which van der Waals interactions are as high as in MOR.

- Electrostatic interactions, which are weaker on the planar surface of ASA than in MOR (see electrostatic field values in Table 1). Even for this neutral complex, it is interesting to notice that the electrostatic contributions exert a strongly stabilizing effect in MOR $\left(-44 \mathrm{~kJ}^{\mathrm{mol}}{ }^{-1}\right)$ whereas they are destabilizing on ASA $(+7$ to +37 $\left.\mathrm{kJ} . \mathrm{mol}^{-1}\right)$ with the exception of the PBS-Al site $\left(\mathrm{Z}_{2}\right)$.

Note that the two $\pi$-complexes with higher $\left|\Delta_{\mathrm{r}} \mathrm{U}\right|$ and $\left|\Delta_{\mathrm{r}} \mathrm{U}_{\mathrm{DFT}}\right|$ values (MOR and PBS-Al site $\left(\mathrm{Z}_{2}\right)$ ) exhibit high frequency shifts of CO [36] (see Supporting Information S2) and the 
highest electrostatic field value (see Table 1), which suggests that the electrostatic part of the interaction becomes critical in their case. Moreover, the value of the electrostatic field was found to be directly correlated to the distance between the molecule and the framework (Figure 5). By formally displacing the position of the molecule within the MOR main channel, perpendicularly to it, we calculate a depletion of the electrostatic field (line in Figure 5), in a very similar fashion as the values obtained on the various sites of ASA. This demonstrates that the enhanced electrostatic field in the zeolite in the surrounding of the molecule, at the origin of more stable adsorption complexes, is a direct consequence of the proximity of the molecule with the framework.

\section{-- HERE FIGURE 5 -}

As expected, no correlation could be found between the adsorption energy of isobutene into $\pi$-complex and the lutidine protonation ability of the acid site involved (see Supporting Information S2). As previously discussed in Ref. [36], in which no direct correlation between $\mathrm{CO}$ adsorption and lutidine protonation was reported, the stability of the $\pi$-complexes cannot be deduced from the analysis of BAS strength probed by lutidine or CO molecule because it results from multi-contribution effects.

Note that regarding mordenite, our energy results compare well with previous ab initio reports in the literature,[78, 94] in particular DFT+D.[59, 66, 67, 95-97]

\subsubsection{Carbenium formation}

The $\mathrm{C}_{4} \mathrm{H}_{9}{ }^{+}$species is well-known to be planar. For the systems investigated here the $\mathrm{C}_{\text {molecule }}{ }^{\cdots} \mathrm{O}_{\text {surf }}$ distance is higher than $1.7 \AA$. In mordenite, we obtained a carbenium as local energy minimum $\left(-88 \mathrm{~kJ} \cdot \mathrm{mol}^{-1}\right)$, depicted in Figure 6-a. Note that the carbocation is 
positioned, after geometry optimization, above the aluminum atom, each of the methyl groups being oriented towards framework oxygen atoms, similarly to previous results reported for $\mathrm{H}-$ ZSM-5.[74] The energetics are comparable to most recent reports on various zeolite frameworks using similar levels of theory[78, 94] (although $\Delta_{\mathrm{r}} \mathrm{U}$ being more negative than most advanced hybrid MP2/DFT results[66, 67]).

On ASA however, it appeared to be very difficult to obtain carbenium as local energy minimum, as the geometry optimizations very often led to the restitution of the proton to the surface, to regenerate isobutene and the acid site, or to the formation of a $\mathrm{C}-\mathrm{O}_{\text {surf }}$ bond to provide the alkoxide (see next section). Only two local energy minima were obtained on the PBS-Al $\mathrm{Si}\left(\mathrm{V}_{1}\right)-\mathrm{OH} \cdots \mathrm{Al}_{\mathrm{IV}}\left(\mathrm{III}_{3}\right)$ and on the PBS-Si (Figure 6-b-to d), although they correspond to endothermic formation reactions. The existence of this carbenium ion as a transition state on this site or on other ASA sites cannot be excluded at present time, but it is beyond the scope of the present work.

The protonation of isobutene to form a carbenium species is quite comparable with the formation of lutidinium from lutidine: it involves a concomitant stabilization of the silanolate species in the case of ASA. For a given site (bridging $\mathrm{OH}$ group of mordenite or PBS-Al of ASA), the formation of lutidinium is favored over that of the tert-butyl carbenium ion (Table 2). This is an illustration of the different basic characters of the two molecules, linked to different stabilization of the protonated species on the surface. When comparing the $\operatorname{Si}\left(\mathrm{V}_{1}\right)-$ $\mathrm{OH}^{\cdots} \mathrm{Al}_{\mathrm{IV}}\left(\mathrm{III}_{3}\right)$ site of ASA (PBS-Al which protonates lutidine) with the bridging $\mathrm{OH}$ group in mordenite, it appears that both ionic species are more strongly stabilized in the zeolite than on the ASA surface. As the dispersion interaction corrections are rather comparable for both solids, this trend must be assigned to electrostatic effects, also measured by the electrostatic field on the different solids. Table 2 reports electrostatic field values calculated for $\pi$ - 
complexes. Note that the protocol we applied to calculate this field -see section 2- cannot be transposed to systems in which atom transfers have occurred, and that the value of the electrostatic field is expected to be significantly different after proton transfer (negatively charged framework). The electrostatic field evolves from $27.6 \mathrm{~V} \cdot \mathrm{nm}^{-1}$ on the PBS-Al site up to $34.1 \mathrm{~V} \cdot \mathrm{nm}^{-1}$ on the MOR site, which induces a stabilizing effect on charged species such as tert-butyl carbenium ions. As mentioned in section 3.1.1., the electrostatic interaction is conditioned by the proximity of the molecule with the framework, allowed in MOR by the three hydrogen bonds illustrated in Figure 6a) and b) for the most stable tert-butyl carbenium ion. A similar trend was inferred for aromatics isomerization reactions on the basis of a charge analysis, which reveals that charged species are stabilized by the surrounding negatively charged oxygen atoms of the zeolitic framework.[69] So the higher acidity of zeolites with regard to ASA can thus be attributed mainly to electrostatic confinement effect rather than van der Waals confinement. This different stabilization of ions pairs is expected to have significant impact on catalysis, $[98,99]$ as it will be shown further.

Table 2. Formation energy of tert-butyl carbenium ion and of lutidinium on the bridging $\mathrm{OH}$ group of mordenite and on the $\mathrm{Si}\left(\mathrm{V}_{1}\right)-\mathrm{OH} \cdots \mathrm{Al}_{\mathrm{IV}}\left(\mathrm{III}_{3}\right)$ PBS-Al site of the ASA surface. All energies are expressed in $\mathrm{kJ} / \mathrm{mol}$.

\begin{tabular}{|c|c|c|c|c|c|}
\hline \multirow{2}{*}{ Site } & \multicolumn{2}{|c|}{$\Delta_{\mathbf{r}} \mathbf{U}$} & \multicolumn{2}{|c|}{$\begin{array}{c}\text { Decomposition of } \Delta_{\mathrm{r}} \mathrm{U}: \\
\Delta_{\mathrm{r}} \mathrm{U}_{\text {DFT }}\left(\Delta_{\mathrm{r}} \mathrm{U}_{\text {disp }}\right)\end{array}$} & \multirow{2}{*}{$\begin{array}{l}\text { Electrostatic field } * \\
\left(\mathbf{V} \cdot \mathbf{n m}^{-1}\right)\end{array}$} \\
\hline & $\begin{array}{l}\text { tert-butyl } \\
\text { carbenium }\end{array}$ & Lutidinium* & $\begin{array}{l}\text { tert-butyl } \\
\text { carbenium }\end{array}$ & Lutidinium* & \\
\hline PBS-Al & +30 & -101 & $+100(-70)$ & $-41(-60)$ & 27.6 \\
\hline Mordenite & -88 & -241 & $-1(-87)$ & $-172(-69)$ & 34.1 \\
\hline
\end{tabular}

\footnotetext{
* These calculations were performed with the same functional (PBE) as for the formation of tert-butyl carbenium ion, and include dispersion corrections (DFT-D2).

* as calculated for $\pi$-complexes, see Table 1 .
} 


\subsubsection{Tert-butoxide formation}

Contrary to the carbenium ion, the alkoxide species is not planar anymore and exhibits an umbrella like conformation with the formation of a covalent $\mathrm{C}-\mathrm{O}_{\text {surf }}$ bond (Table 1). The newly formed $\mathrm{C}-\mathrm{O}_{\text {surf }}$ bond length is almost constant from one site to another $(1.48 \pm 0.04 \AA)$. We consider here that the alkoxide intermediates is anchored either on the $\mathrm{O}_{\text {surf }}$ atom of the BAS itself or on the neighboring $\mathrm{O}_{\text {surf }}$ atom as reported in the literature devoted to zeolitic materials.[58, 59, 65, 68, 70, 77, 95, 97, 100-103] The various possible kinds of intermediates and their energetics are exemplified in Figure 7 without making any assumptions on the mechanisms leading to them. These mechanisms will be briefly discussed in the next section.

In the case of the ASA site $\mathrm{Si}\left(\mathrm{V}_{1}\right)-\mathrm{OH} \cdots \mathrm{Al}_{\mathrm{IV}}\left(\mathrm{III}_{3}\right)$ (PBS-Al which protonates lutidine) the energy difference between the two plausible products (Figure 7-c and d) is higher than for mordenite (32 kJ.mol ${ }^{-1}$ versus $7 \mathrm{~kJ} \mathrm{~mol}^{-1}$ respectively), in line with the different local structures. Once the proton of PBS-Al is transferred to isobutene, the silanolate has to be stabilized, by analogy to the proton transfer to lutidine,[35] and this stabilization occurs as follows:

- the tert-butyl cation is the stabilizing group by forming the new $\mathrm{C}-\mathrm{O}_{\text {surf }}$ bond capping the PBS (Figure 7-c)

- or the PBS-Al closes its bridge (in the same way as after proton transfer to lutidine), by forming a new $\mathrm{O}-\mathrm{Al}$ bond, while the alkoxide forms a new $\mathrm{C}-\mathrm{O}$ bond on the neighboring site (Figure 7-d).

-- HERE FIGURE 7 --

The intermediate formed on the BAS appears to be more stable, likely in relation with 
the less sterically hindered nature of the terminal Si-O-R group (Figure 7-c) as compared to the multicoordinated nature of the $\mathrm{O}$ atom of the alkoxide formed on the neighboring $\mathrm{O}$ site (Figure 7-d). This is illustrated by the higher position of carbon atoms along the perpendicular to the surface for the configuration reported in Figure 7-c as compared to the one shown in Figure 7-d (central carbon atom : difference in height of $0.47 \AA$, other carbon atoms : mean difference in height of $0.44 \AA$ ). Note that the stabilization of the silanolate obtained after proton transfer by the protonated species itself (the possible carbenium ion) instead of the implication of surface atoms (as for lutidine) is an illustration of the amphoteric character of the alkene molecule.

The alkoxide intermediates formed on the BAS itself were then systematically investigated for the whole set of ASA sites under study. The formation energy of the tertbutoxide species lies between -6 and $-75 \mathrm{~kJ} \mathrm{~mol}^{-1}$ (Table 1), whereas it is more negative in mordenite $\left(-95 \mathrm{~kJ}_{\mathrm{mol}}{ }^{-1}\right)$, which is due to both dispersion and electronic contributions. Note that this energy interval is compatible with previous reports, $[59,66,67,70,95-97]$ including $\mathrm{DFT}+\mathrm{D},[78,94]$ in various zeolitic frameworks and site positions.

No correlation could be revealed between $\Delta_{\mathrm{r}} \mathrm{U}$ value and the adsorption energy of lutidine or the shift of its $v_{8 \mathrm{a}}$ mode, even if a proton transfer occurs in each case. This is due to the strong differences in the adsorbed structures, with a strong charge separation in the case of the lutidinium-deprotonated surface interaction. Moreover, due to the amphoteric nature of isobutene, the formation energy of the alkoxide according to a 4-center mechanism is not dependent on the ability of the silanolate to stabilize on other surface atoms, which was critical in the case of lutidine.

Surprisingly, a good correlation is obtained between the formation energy of the tertbutoxy species and the CO frequency shift (Figure 8). In our previous work, we showed that the $\mathrm{CO}$ frequency shift is directly related to the local electrostatic field.[36] Such a correlation 
is quite unexpected because contrary to the case of $\mathrm{CO}$, the surface proton was transferred to the isobutene molecule. This indicates that not only the proton, but also a large piece of the surface, play a role in the local electrostatic field, as already shown in reference [36]. Table 1 also shows that dispersion forces also play a significant role in the interaction, but the fluctuation from one site to another (from -42 to $-64 \mathrm{~kJ} \cdot \mathrm{mol}^{-1}$ ) is lower than for the $\pi$-complex due to the rather constrained $\mathrm{C}-\mathrm{O}_{\text {surf }}$ distance $(1.48 \pm 0.04 \AA)$. However, as for the $\pi$-complex, variation of the electronic contribution is rather large and impacts strongly the overall adsorption energy.

\section{-- HERE FIGURE 8 --}

\subsubsection{Protonation energy profiles at $0 \mathrm{~K}$}

The adsorption energies of isobutene according to the three modes previously exposed are gathered in Figure 9-a and b. Note that this representation does not imply any consequence on the mechanisms involved in the formation of the carbocation and alkoxide species (see further).

Without dispersion corrections (Figure 9-a), the $\pi$-complex is the most stable species within mordenite, whereas the alkoxide is the most stable on some sites of ASA. Including dispersion corrections (Figure 9-b), all profiles are lower in energy. In mordenite, the energy profile becomes also nearly isoenergetic for the protonation reaction and much more favorable than all profiles calculated on the ASA sites. On ASA, the formation of the carbenium ion seems to be preferentially formed on the single PBS-Al able to protonate lutidine. In any case, Figure 9-b unambiguously shows that carbenium ions and alkoxides formation is much easier in zeolites than on ASA. It could also be that carbenium ions are energetically stronger competitors to alkoxide species in zeolites.

Comparing the various sites of the ASA surface (taking into account dispersion forces), it appears that the two sites stabilizing carbenium ion are one PBS-Al $\left(\mathrm{V}_{1}\right)$ and the PBS-Si 
$\left(\mathrm{U}_{1}\right)$, which are also able to protonate lutidine. Thus the proton transfer ability as formerly revealed by the lutidine probe molecule is qualitatively linked with the carbenium formation. By contrast, the more stable alkoxide species are not directly linked to it, as already discussed: some sites exhibiting high frequency shifts when $\mathrm{CO}$ is used as a probe molecule, lead to the formation of stable alkoxides but neither to carbenium ions nor lutidinium ions. This is the case of one PBS-Al which is not able to protonate lutidine, $\mathrm{Si}\left(\mathrm{Z}_{2}\right)-\mathrm{OH} \cdots \mathrm{Al} l_{\mathrm{V}}\left(\mathrm{III}_{2}\right)$.

\section{-- HERE FIGURE 9 --}

If the lifetime of the tert-butyl carbenium species is already questioned in the case of zeolites,[51, 67] it can be anticipated that the lifetime of such species on ASA will be even shorter. Whereas carbenium chemistry is often supposed to be at the core of hydrocarbon conversion for aluminosilicates, our present results suggest that it would hardly be the case on ASA.

Regarding the possible mechanisms connecting the tertiary alkoxide to the two other intermediates ( $\pi$-complex and carbocation), three pathways are usually invoked for the formation of tertiary alkoxides:

- one "indirect" mechanism as investigated in Ref. [67]: the carbocation is a precursor of the tertiary alkoxide either anchored on the $\mathrm{O}$ site of the BAS itself or on the neighbouring $\mathrm{O}$ site (see also previous section)

- two "direct" mechanisms (without formation of carbocation):

o the so-called "4-center mechanism": the acid site protonates isobutene and stabilizes subsequently the alkoxide on the conjugated base (deprotonated $\mathrm{O}$ atom).

o the so-called "6-center mechanism": the alkoxide species is formed on a 
neighbouring $\mathrm{O}$ atom.

Regarding zeolites, the 6-center mechanism is mostly presented in the literature.[58, 59, 65, 70, 77, 95, 97, 100-103] Bhan et al.[101] compared the two mechanisms in the case of propene and hexene adsorbed on 10T-clusters of ZSM-5 and concluded that the stability of both the transition states and the products are in favor of a 6-center mechanism, although the reaction and activation energies differ from less than $20 \mathrm{~kJ} \cdot \mathrm{mol}^{-1}$. In addition, they report that the activation energy is about $80 \mathrm{~kJ} \cdot \mathrm{mol}^{-1}$ and involves a carbocation like transition state. This value is close to the one reported by Tuma et al. in Ref. [67], involved in the formation of the tert-butyl carbenium species in H-ferrierite. In MOR, Rozanska et al. [70] reported also moderate activation energies (similar to the one leading to the carbocation intermediates, $\sim 30$ $\mathrm{kJ} . \mathrm{mol}^{-1}$ ) leading to butoxy species via a carbocation like transition state. So even if these results are much dependant on the location of the acid site, the nature of the alumina-silicate materials and the level of theory itself, the formation of alkoxy species is possible from a kinetic point of view and might occur according to the two mechanisms listed above. We will thus consider alkoxy and carbocation pathways in the forthcoming sections.

\subsubsection{Protonation energy profiles: thermal effects}

Figure 9-c depicts the $\Delta_{\mathrm{r}} \mathrm{G}$ values for all species derived from isobutene adsorption on ASA and MOR, according to the methods exposed in section 2. For mordenite, the alkoxides become much less favorable (their formation becomes an endergonic reaction) than the carbenium, due to their lower number of degree of freedom (translational and rotational). On ASA, despite the application of similar thermal corrections, the carbenium remains a highly improbable species as compared to the alkoxide, although the formation of both compounds is endergonic. This trend will have a direct impact on the cracking reaction pathway investigated in the forthcoming section. 


\subsection{DIB cracking pathway}

To further investigate this aspect, we calculate the DFT energy and Gibbs energy profiles of the cracking reaction of a relevant olefin molecule: DIB (2,4,4-trimethyl-2pentene). Our main aim is to compare cracking pathways between MOR and ASA. As carbenium ions are very often invoked in the cracking pathways of alkenes on zeolites, one may choose to investigate first on ASA a site which is able to stabilize both carbenium and alkoxide intermediates. For this reason, we focus on the PBS-Al site $\left(\mathrm{Si}\left(\mathrm{V}_{1}\right)-\mathrm{OH} \cdots \mathrm{Al}_{\mathrm{IV}}\left(\mathrm{III}_{3}\right)\right)$, which allows the investigation of carbenium versus alkoxides routes, and according to the previous section, with intermediates being more stable on it than on the PBS-Si sites (see Figure 9). We compare it with the bridging $\mathrm{Si}-(\mathrm{OH})-\mathrm{Al}$ group in mordenite. Note that in the future, other ASA sites will deserve attention, even if they do not stabilize carbenium ions as intermediates, in particular those leading to most stable alkoxides (PBS-Al $\mathrm{Si}\left(\mathrm{Z}_{2}\right)$ $\mathrm{OH} \cdots \mathrm{Al}_{\mathrm{V}}\left(\mathrm{III}_{2}\right)$.

\subsubsection{Reaction pathways under study}

The various intermediates and reaction pathways considered are depicted in Figure 10, on the PBS-Al site $\mathrm{Si}\left(\mathrm{V}_{1}\right)-\mathrm{OH} \cdots \mathrm{Al}_{\mathrm{IV}}\left(\mathrm{III}_{3}\right)$ of ASA. Strictly analogous pathways are investigated on MOR. They involve either secondary or tertiary carbocations/alkoxides according to six formal steps (likely not elementary steps):

- physisorption of DIB on the BAS of the ASA surface or mordenite

- proton transfer from PBS-Al (ASA) or $\mathrm{Si}-(\mathrm{OH})-\mathrm{Al}(\mathrm{MOR})$ to $\mathrm{DIB}$, leading either to a carbenium or to an alkoxide. We consider here tertiary carbenium $\left(\mathrm{C}_{\mathrm{III}}\right)$ only and either secondary $\left(\mathrm{A}_{\mathrm{II}}, \mathrm{B}_{\mathrm{II}}\right)$ or tertiary alkoxides $\left(\mathrm{A}_{\mathrm{III}}, \mathrm{B}_{\mathrm{III}}\right)$. The alkoxide can be stabilized either on the deprotonated oxygen atom (A), or on a neighboring one (B) 
according to the previous analysis made for isobutene.

- the cracking step occurs via $\beta$-scission leading to a physisorbed isobutene molecule and either a tert-butyl cation (5) or isobutoxy species $(1,2,3,4)$. In the following, for sake of clarity, we report pathways involving only either carbenium intermediate or alkoxides. Note that according to the discussion on 3.1.4, we cannot exclude that carbenium intermediate may be precursor of alkoxide species, however for sake or clarity the energy profiles presented in Figure 11 will not illustrate explicitly the combination of the two routes. However, we will discuss this point in 3.2.2.

- the first isobutene molecule formed is then desorbed (loss of the physisorption energy) leading either to adsorbed tert-butyl cation (3') or isobutoxy species (1', 2')

- the carbenium or alkoxide remaining at the surface then transfers one proton to regenerate the surface BAS (A or B) and produces a second physisorbed isobutene molecule.

- finally, the secondly formed isobutene molecule desorbs.

-- HERE FIGURE 10 --

\subsubsection{Gibbs free energy profiles}

In what follows, we directly report the $\Delta_{\mathrm{r}} \mathrm{G}$ profiles of the adsorbed intermediates (including thermal and entropy contributions) as explained in the Methods section. Note that in the conditions considered $(500 \mathrm{~K}$, all partial pressures of gaseous compounds equal to 1 bar), the overall $\Delta_{\mathrm{r}} \mathrm{G}$ value for the cracking reaction of DIB into isobutene is almost zero (-3 $\mathrm{kJ} . \mathrm{mol}^{-1}$ ). Energy profiles at $0 \mathrm{~K}$, as well as $\Delta_{\mathrm{r}} \mathrm{U}$ and $\Delta_{\mathrm{r}} \mathrm{G}$ values of all intermediates for ASA and MOR are reported in Supporting Information S3. Considering firstly the alkoxide routes only, it appears on ASA that the $\mathrm{A}_{\text {III }}$ pathway is favored over the $\mathrm{A}_{\text {II }}$ one, as well as the 
$\mathrm{B}_{\text {III }}$ route over the $\mathrm{B}_{\text {II }}$ one. Thus on a given site, tertiary alkoxides are more stabilized. In the following, for the sake of clarity, we plot in Figure 11 mainly the $\mathrm{A}_{\mathrm{III}}$ and $\mathrm{B}_{\mathrm{II}}$ routes, as being representative for the reactivity of tertiary and secondary alkoxide routes. Regarding mordenite, the $\mathrm{A}_{\text {III }}$ and $\mathrm{A}_{\mathrm{II}}$ routes will be shown. Considering the whole set of energy data reported in Supporting Information S3 does not affect the conclusions drawn in the following. From a general point of view, it appears that all pathways are favored $\left(\Delta_{\mathrm{r}} \mathrm{U}\right.$ and $\left.\Delta_{\mathrm{r}} \mathrm{G}\right)$ in mordenite as compared to ASA. This is related to the electrostatic confinement effect already mentioned in section 3.1.2. Moreover in MOR, the carbenium route is favored over all other routes, at each stage of the reaction. This is due to higher entropy of adsorbed carbenium species as compared to alkoxides, as shown by the comparison of $\Delta_{\mathrm{r}} \mathrm{G}$ and $\Delta_{\mathrm{r}} \mathrm{U}$ profiles (Supporting Information S3). This finding is in agreement with the classical description of carbenium chemistry considered to take place in zeolites.[16, 41-43, 80, 104] The drawing is not the same for ASA, and to understand this, we detail each reaction step in the following.

\section{a) DIB adsorption and protonation}

In a similar way as for isobutene, the adsorption of the DIB molecule as a $\pi$-complex is much stronger in mordenite $\left(\Delta_{\mathrm{r}} \mathrm{G}=-88 \mathrm{~kJ} \cdot \mathrm{mol}^{-1}\right)$ than on ASA $\left(\Delta_{\mathrm{r}} \mathrm{G}=+12 \mathrm{~kJ} \cdot \mathrm{mol}^{-1}\right)$. Moreover, on ASA, protonated DIB is much more stabilized in a tertiary alkoxide form $\left(\Delta_{\mathrm{r}} \mathrm{G}\right.$ $\left.=36 \mathrm{~kJ} \cdot \mathrm{mol}^{-1}\right)$ rather than in a secondary alkoxide one $\left(\Delta_{\mathrm{r}} \mathrm{G}=86 \mathrm{~kJ} \cdot \mathrm{mol}^{-1}\right)$ or than in a carbenium one $\left(\Delta_{\mathrm{r}} \mathrm{G}=63 \mathrm{~kJ} \cdot \mathrm{mol}^{-1}\right)$. In MOR, the trend is the opposite, with the carbenium ion being the most favorable species at this stage $\left(\Delta_{\mathrm{r}} \mathrm{G}=-87 \mathrm{~kJ} \cdot \mathrm{mol}^{-1}\right.$, almost iso-G with the $\pi$ complex) with regard to alkoxides.

Considering kinetic data available on zeolites (see section 3.1.4), the activation energy for the formation of the alkoxy intermediate would be similar with the one leading to the 
carbenium. Assuming that this trend is still valid for ASA, and that barriers will all be higher in ASA than in MOR (if a Brønsted-Evans-Polanyi relationship exists), kinetics may not change our conclusions for the comparison of MOR and ASA, neither for the probability of alkoxides versus carbenium formation.

\section{b) Cracking step}

Considering first $\Delta_{\mathrm{r}} \mathrm{U}$ values, the cracking reaction is most of the time endothermic on ASA and in MOR $\left(>70 \mathrm{~kJ} \cdot \mathrm{mol}^{-1}\right)$. Note that this is also the case for the gas phase cracking reaction $\left(\mathrm{DIB}=2\right.$ isobutene, computed $\left.\Delta_{\mathrm{r}} \mathrm{U}:+87 \mathrm{~kJ} \cdot \mathrm{mol}^{-1}\right)$. This trend is explained from the energy loss of one $\mathrm{C}-\mathrm{C}$ bond due to $\beta$-scission, which is not compensated by the energy gain induced by the conversion of a $\mathrm{C}-\mathrm{C}$ bond into a $\mathrm{C}=\mathrm{C}$ one. Calculated reaction energies for $\beta$ scission steps of various alkenes reported in the literature for zeolites differ quite strongly from one study to another: from $\sim 10 \mathrm{~kJ} \mathrm{~mol}^{-1}$ to more than $100 \mathrm{~kJ} \cdot \mathrm{mol}^{-1}$ (in the same interval as our results) $[64,75,105-110]$ with a very strong impact of the size of the model and the type of zeolite, thus of steric and confinement effects. Coming back to $\Delta_{\mathrm{r}} \mathrm{G}$ profiles, it appears that including thermal and entropic effects counterbalances the internal energy variations (as observed for gas phase, for which $\Delta_{\mathrm{r}} \mathrm{G} \approx 0$ at $500 \mathrm{~K}$ ).

Analysing the $\Delta_{\mathrm{r}} \mathrm{G}$ profiles on ASA shows that the most favorable one involves the tertiary alkoxy: $A_{I I I} \rightarrow 2\left(+12 \mathrm{~kJ} \cdot \mathrm{mol}^{-1}\right)$. The scenario involving $\mathrm{B}_{\mathrm{II}} \rightarrow 3\left(-53 \mathrm{~kJ}^{\mathrm{mol}} \mathrm{m}^{-1}\right)$ should be

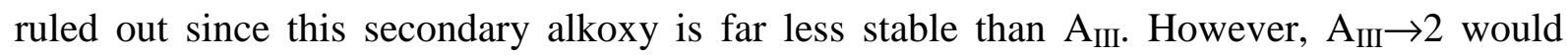
occur on the same site for the formation of the first and second alkoxide species (before and after cracking) which might be mechanistically difficult to envisage (to be confirmed by more detailed mechanistic studies). So, we report in figure 11 also the $\mathrm{A}_{\mathrm{III}} \rightarrow 1$ reaction $\left(+50 \mathrm{~kJ}^{\mathrm{m}} \mathrm{mol}^{-}\right.$ ${ }^{1}$ ) in which two neighboring sites are involved during cracking (as it is for $\mathrm{B}_{\mathrm{II}} \rightarrow 3$ ). In MOR, the most favorable pathway would be $\mathrm{A}_{\mathrm{II}} \rightarrow 2\left(+7 \mathrm{~kJ} \mathrm{~mol}^{-1}\right)$ or $\mathrm{A}_{\mathrm{III}} \rightarrow 1\left(+3 \mathrm{~kJ} \cdot \mathrm{mol}^{-1}\right)$. For the 
same reason, we also plot $\mathrm{B}_{\mathrm{III}} \rightarrow 3\left(-13 \mathrm{~kJ} \cdot \mathrm{mol}^{-1}\right)$. The pathway involving the carbocationic intermediate $\mathrm{C}_{\mathrm{III}} \rightarrow 5$, is slightly more endergonic (+28 $\left.\mathrm{kJ} \cdot \mathrm{mol}^{-1}\right)$.

At this stage, one could also suggest the existence of an alternative cracking pathway combining carbocation and alkoxy routes during the cracking step. For MOR, this would not bring any gain in Gibbs energy, as carbenium species are by far more stable than alkoxides: a purely carbenium pathway remains the most probable. By contrast, on ASA we can propose that the optimal cracking step could occur along the route $\mathrm{C}_{\mathrm{III}} \rightarrow 1$ or $\mathrm{C}_{\mathrm{III}} \rightarrow 3,1$ and 3 being significantly more stable than the carbocation 5. Although we previously underlined that the probability of existence of $\mathrm{C}_{\mathrm{III}}$ is certainly lower than $\mathrm{A}_{\mathrm{III}}, \mathrm{C}_{\mathrm{III}}$ may offer a favorable alternative if too high barriers have to be overcome for the cracking of alkoxide $\mathrm{A}_{\text {III. }}$ In that case, the energy demanding step would be the formation of $\mathrm{C}_{\mathrm{III}}$, whereas it is the cracking step for the $A_{\text {III }}$ pathway. In such a scenario, $A_{\text {III }}$ would be a dormant species and the relative concentration of $\mathrm{C}_{\mathrm{III}}$ versus $\mathrm{A}_{\mathrm{III}}$ species on the ASA surface would be the determining parameter for cracking.

\section{-- HERE FIGURE 11 --}

In the literature, energy barriers for $\beta$-scission steps in zeolites were evaluated mainly from alkoxides, with values as high as $150-200 \mathrm{~kJ} \mathrm{~mol}^{-1}$.[108-112] Earlier studies performed on small clusters (neglecting confinement effect) even concluded to barriers above 200 $\mathrm{kJ}^{\mathrm{mol}}{ }^{-1} .[62-64,105-107]$ Starting from a carbenium species,[75, 112] barriers between 65 and $115 \mathrm{~kJ}_{\mathrm{mol}}{ }^{-1}$ were reported in H-ZSM5. Kinetic modelling (compared to octane hydrocracking experiments on Pt/H-USY) leads to energy barriers for $\beta$-scission close to 125 $\mathrm{kJ} \mathrm{mol}{ }^{-1}$, however with strong dependence on the hypothesis retained for the kinetic model used for fitting the experimental data.[104] On ASA, the identification of transition states and activation energies is still required to determine which of the cracking step and carbocation 
formation is the kinetic limiting step on ASA. Even in absence of such kinetic values, considering the higher energy levels of the carbocationic intermediates $\left(\mathrm{C}_{\mathrm{III}}\right.$ and 5) on ASA (both $\Delta_{\mathrm{r}} \mathrm{U}$ and $\Delta_{\mathrm{r}} \mathrm{G}$ profiles), this route seems to be thermodynamically difficult to envisage. Thus we suggest in the future to also explore kinetic parameters of the mixed route leading from carbocationic intermediate to cracked alkoxide intermediates particularly on ASA, as this route seems to be thermodynamically relevant.

\section{c)Deprotonation/Desorption steps}

On ASA and MOR, the deprotonation steps are all exergonic (opposite trends as the protonation step discussed before). In MOR, the isobutene desorption step is endergonic $\left(\Delta_{\mathrm{r}} \mathrm{G}\right.$ $=+68 \mathrm{~kJ} \cdot \mathrm{mol}^{-1}$ ), which can be a limitation to the reaction rate due to the confinement effect induced by the zeolite pores, whereas it is generally admitted that the $\beta$-scission step is rate controlling.[16-18] It is beyond the scope of the present study to identify the rate limiting step, however it is clear from this $\Delta_{\mathrm{r}} \mathrm{G}$ profile, that product desorption limitation in MOR may also be at the origin of hydrocarbon overcracking. As a complementary analysis, let us consider the situation in which a protonated DIB intermediate is formed in the zeolitic micropore, from the cracking of longer chain hydrocarbons. In that case, after deprotonation, the desorption of DIB is also strongly limiting $\left(\Delta_{\mathrm{r}} \mathrm{G}=+87 \mathrm{~kJ} \cdot \mathrm{mol}^{-1}\right)$ which implies that DIB would preferentially remain in the pore and be cracked according to the pathway described above. The situation is different on ASA in which the desorption steps of DIB and isobutene molecules are slightly exergonic: it appears thermodynamically more favorable to reversely deprotonate and desorb it, than to further crack it into lighter hydrocarbons. This is first theoretical explanation of the origin of the milder acidic character of ASA wished for specific applications in which overcracking must be avoided. In particular, cracking being activated, the corresponding energy barrier is expected to reinforce this trend, which is contrasting with 
the scenario in MOR. To a certain extent this interpretation is also coherent with previous kinetic modelling of phenantrene hydrocracking which has highlighted that an optimal pore size is required for controlling the selectivity towards targeted products of intermediate molecular weight in hydrocracking.[10, 32] In particular, the relative positions of the $\Delta_{\mathrm{r}} \mathrm{G}$ profiles as plotted in Figure $\mathbf{1 1}$ may directly be correlated on the size of micropores and mesopores of the considered aluminosilicates, hopefully leading to the discovery of an optimal pocket size.[113, 114] This concept may open new routes for future investigations.

\section{Conclusions}

Thanks to DFT+D2 calculations led on original Amorphous Silica Alumina surface models, we propose a rational interpretation of the lower acidity of ASA surface with respect to zeolite, as probed by alkene cracking reactivity.

Regarding the interaction of isobutene with aluminosilicates, three key intermediates ( $\pi$-complex, carbocation and alkoxide) are less stable on the ASA surface than in MOR. This is mainly due to the combined effect of the electrostatic and van der Waals contributions, being stronger in zeolites by confinement effect. Moreover, the tert-butyl carbenium ion is significantly higher in energy on the ASA surface than in MOR. Since the calculated van der Waals contributions are close in both cases, the origin of the carbocation stabilization in MOR is attributed to an electrostatic confinement effect as quantified by the large electrostatic field in the zeolite[36]. As a consequence, the formation of the tert-butyl carbenium ion is nearly isoenergetic in MOR, whereas it is endergonic on the PBS-Al site of ASA. By contrast, alkoxy species are more stabilized on the PBS-Al site than carbocationic ones: this is a first indication discriminating the chemical reactivity of BAS located in MOR versus BAS on ASA surface. 
By investigating the cracking pathway of DIB, we highlighted that the carbocationic pathways are less favored on ASA with respect to the alkoxy route. A reverse trend was found in MOR, in which the formation of carbocationic intermediates is stabilized again by electrostatic confinement effects. In the case of ASA, the reaction profile goes through slightly positive $\Delta_{\mathrm{r}} \mathrm{G}$ values. The preferred nature of the tertiary alkoxy route was also revealed, whereas the carbenium route appears very unlikely. From the stability of the intermediates, the protonation and $\beta$-scission steps are likely the limiting ones for the "pure" carbocationic or alkoxy routes. For the cracking step, on ASA we also highlighted an alternative pathway combining minority carbocationic species and cracked alkoxy intermediates. Further kinetic analyses are mandatory to confirm this proposal.

Moreover, we furnished a quantified picture based on the Gibbs free enthalpy of each intermediate, which reveal a thermodynamic reason for the formation of overcracked products in zeolites with small micropores while these are less stabilized on ASA. This thermodynamic analysis provides the first theoretical explanation of the origin of the empirically observed milder acidic character of ASA targeted for specific applications in which selective cracking is sought.

\section{Acknowledgments}

We thank Hervé Toulhoat, Christophe Bouchy and Emanuelle Guillon (IFP Energies nouvelles) for fruitful discussions. All calculations were performed at IFPEN HPC center and at IDRIS/CINES HPC centers within projects funded by GENCI. 


\section{References}

[1] J.N. Brønsted, Recl. Trav. Chim. Pays-Bas 42 (1923) 718-727.

[2] C. Thieuleux, A. Maraval, L. Veyre, C. Copéret, D. Soulivong, J.M. Basset, G.J. Sunley, Angew. Chem. Int. Ed. 46 (2007) 2288-2290.

[3] A.W. Moses, C. Raab, A.E. Nelson, H.D. Leifeste, N.A. Ramsahye, S. Chattopadhyay, J. Eckert, B.F. Chmelka, S.L. Scott J. Am. Chem. Soc. 129 (2007) 8912-8920.

[4] K. Motokura, M. Tada, Y. Iwasawa, J. Am. Chem. Soc. 129 (2007) 9540-9541.

[5] J.Q. Bond, D.M. Alonso, D. Wang, R.W. West, J.A. Dumesic, Science 327 (2010) 11101114.

[6] G.W. Huber, R.D. Cortright, J.A. Dumesic, Angew. Chem. Int. Ed. 43 (2004) 1549-1551.

[7] F. Bertoncini, A. Bonduelle-Skrzypcak, J. Francis, E. Guillon, Hydrocracking, in: H.

Toulhoat, P. Raybaud (Eds.) Catalysis by transition metal sulphides: from molecular theory to industrial applications, Technip Eds., 2013, pp. 609-677.

[8] M. Caillot, A. Chaumonnot, M. Digne, J.A.V. Bokhoven, ChemCatChem 5 (2013) 36443656.

[9] A. Ishihara, H. Negura, T. Hashimoto, H. Nasu, Appl. Catal. A 388 (2010) 68-76.

[10] E. Benazzi, L. Leite, N. Marchal-George, H. Toulhoat, P. Raybaud, J. Catal. 217 (2003) 376-387.

[11] L. Leite, E. Benazzi, N. Marchal-George, Catal. Today 65 (2001) 241-247.

[12] D. Barthomeuf, Zeolites 10 (1990) 131-133.

[13] A. Omegna, R. Prins, J.A. van Bokhoven, J. Phys. Chem. B 109 (2005) 9280-9283.

[14] G. Burnens, C. Bouchy, E. Guillon, J.A. Martens, J. Catal. 282 (2011) 145-154.

[15] H.L. Coornradt, W.E. Garwood, Ind. Eng. Chem. Res. Proc. Des. Dev. 3 (1964) 38-45.

[16] J. Weitkamp, ChemCatChem 4 (2012) 292-306. 
[17] C. Bouchy, G. Hastoy, E. Guillon, J.A. Martens, Oil Gas Sci. Technol. - Rev. IFP 64 (2009) 91-112.

[18] C. Marcilly, Acido-basic Catalysis, Technip Eds., Paris, 2005.

[19] A. Corma, V. Fornes, M.T. Navarro, J. Perez-Pariente, J. Catal. 148 (1994) 569-574.

[20] K. Gora-Marek, M. Derewinski, P. Sarv, J. Datka, Catal. Today 101 (2005) 131-138.

[21] B. Xu, C. Sievers, J.A. Lercher, J.A.R. van Veen, P. Giltay, R. Prins, J.A. van Bokhoven, J. Phys. Chem. C 111 (2007) 12075-12079.

[22] E.J.M. Hensen, D.G. Poduval, P.C.M.M. Magusin, A.E. Coumans, J.A.R. van Veen, J. Catal. 269 (2010) 201-218.

[23] B.P. Nielsen, J.H. Onuferko, B.C. Gates, Ind. Eng. Chem. Fundam. 25 (1986) 337-343. [24] M. Trombetta, G. Busca, S. Rossini, V. Piccoli, U. Cornaro, A. Guercio, R. Catani, R.J. Willey, J. Catal. 179 (1998) 581-596.

[25] G. Crépeau, V. Montouillout, A. Vimont, L. Mariey, T. Cseri, F. Maugé, J. Phys. Chem. B 110 (2006) 15172-15185.

[26] E. Garrone, B. Onida, B. Bonelli, C. Busco, P. Ugliengo, J. Phys. Chem. B 110 (2006) 19087-19092.

[27] M.F. Williams, B. Fonfé, C. Sievers, A. Abraham, J.A. van Bokhoven, A. Jentys, J.A.R. van Veen, J.A. Lercher, J. Catal. 251 (2007) 485-496.

[28] Y. Jiang, J. Huang, W. Dai, M. Hunger, Solid State Nucl. Magn. Reson. 39 (2011) 116141.

[29] T. Yamamoto, T. Kudo, T. Yamamoto, J. Kawai, Mic. Mes. Mat. 182 (2013) 239-243.

[30] D.G. Poduval, J.A.R. van Veen, M.S. Rigutto, E.J.M. Hensen, Chem. Commun. 46 (2010) 3466-3468.

[31] E.G. Derouane, J.M. André, A.A. Lucas, J. Catal. 110 (1988) 58-73.

[32] H. Toulhoat, P. Raybaud, E. Benazzi, J. Catal. 221 (2004) 500-509. 
[33] C. Chizallet, P. Raybaud, Angew. Chem. Int. Ed. 48 (2009) 2891-2893.

[34] C. Chizallet, P. Raybaud, ChemPhysChem 11 (2010) 105-108.

[35] F. Leydier, C. Chizallet, A. Chaumonnot, M. Digne, E. Soyer, A.A. Quoineaud, D.

Costa, P. Raybaud, J. Catal. 284 (2011) 215-229.

[36] F. Leydier, C. Chizallet, D. Costa, P. Raybaud, Chem. Commun. 48 (2012) 4076-4078.

[37] W.O. Haag, R.M. Lago, P.B. Weisz, Nature 309 (1984) 589-591.

[38] F.C. Whitmore, J. Am. Chem. Soc. 54 (1932) 3274-3283.

[39] G.A. Olah, Angew. Chem. Int. Ed. 34 (1995) 1393-1405.

[40] R.R. Naredla, D.A. Klumpp, Chem. Rev. 113 (2013) 6905-6948.

[41] I.E. Maxwell, Catal. Today 1 (1987) 385-413.

[42] I. Kiricsi, H. Förster, G. Tasi, J.B. Nagy, Chem. Rev. 99 (1999) 2085-2114.

[43] J.F. Denayer, G.V. Baron, G. Vanbutsele, P.A. Jacobs, J.A. Martens, J. Catal. 190 (2000) 469-473.

[44] Y.V. Kissin, J. Catal. 163 (1996) 50-62.

[45] E.A. Lombardo, J.M. Dereppe, G. Marcelin, W. Keith Hall, J. Catal. 114 (1988) 167-175.

[46] A. Corma, Chem. Rev. 95 (1995) 559-614.

[47] T. Xu, J.F. Haw, J. Am. Chem. Soc. 116 (1994) 10188-10195.

[48] I.L.C. Buurmans, E.A. Pidko, J.M. de Groot, E. Stavitski, R.A. van Santen, B.M.

Weckhuysen, Phys. Chem. Chem. Phys. 12 (2010) 7032-7040.

[49] N.D. Lazo, B.R. Richardson, P.D. Schettler, J.L. White, E.J. Munson, J.F. Haw, J. Phys. Chem. 95 (1991) 9420-9425.

[50] A. Stepanov, K. Zamaraev, J. Thomas, Catal. Lett. 13 (1992) 407-422.

[51] I.I. Ivanova, Y.G. Kolyagin, Chem. Soc. Rev. 39 (2010) 5018-5050.

[52] S.T. Sie, Ind. Eng. Chem. Res. 31 (1992) 1881-1889.

[53] J. Weitkamp, Ind. Eng. Chem. Proc. Res. Dev. 21 (1982) 550-558. 
[54] J.F. Haw, J.B. Nicholas, T. Xu, L.W. Beck, D.B. Ferguson, Acc. Chem. Res. 29 (1996) 259-267.

[55] W. Wang, M. Hunger, Acc. Chem. Res. 41 (2008) 895-904.

[56] J.N. Kondo, H. Ishikawa, E. Yoda, F. Wakabayashi, K. Domen, J. Phys. Chem. B 103 (1999) 8538-8543.

[57] H. Ishikawa, E. Yoda, J.N. Kondo, F. Wakabayashi, K. Domen, J. Phys. Chem. B 103 (1999) 5681-5686.

[58] V.B. Kazansky, I.N. Senchenya, J. Catal. 119 (1989) 108-120.

[59] P. Viruela-Martin, C.M. Zicovich-Wilson, A. Corma, J. Phys. Chem. 97 (1993) 1371313719.

[60] I.N. Senchenya, V.B. Kazansky, Catal. Lett. 8 (1991) 317-326.

[61] V.B. Kazansky, M.V. Frash, R.A. van Santen, Appl. Catal. A 146 (1996) 225-247.

[62] A.M. Rigby, G.J. Kramer, R.A. van Santen, J. Catal. 170 (1997) 1-10.

[63] M.V. Frash, V.B. Kazansky, A.M. Rigby, R.A. van Santen, J. Phys. Chem. B 102 (1998) 2232-2238.

[64] P.J. Hay, A. Redondo, Y. Guo, Catal. Today 50 (1999) 517-523.

[65] M. Boronat, P.M. Viruela, A. Corma, J. Am. Chem. Soc. 126 (2004) 3300-3309.

[66] C. Tuma, J. Sauer, Phys. Chem. Chem. Phys. 8 (2006) 3955-3965.

[67] C. Tuma, T. Kerber, J. Sauer, Angew. Chem. Int. Ed. 49 (2010) 4678-4680.

[68] M. Boronat, C.M. Zicovich-Wilson, A. Corma, P. Viruela, Phys. Chem. Chem. Phys. 1 (1999) 537-543.

[69] X. Rozanska, R. van Santen, F. Hutschka, J. Hafner, J. Am. Chem. Soc. 123 (2001) $7655-7667$.

[70] X. Rozanska, R.A. van Santen, T. Demuth, F. Hutschka, J. Hafner, J. Phys. Chem. B 107 (2003) 1309-1315. 
[71] L. Benco, J. Hafner, F. Hutschka, H. Toulhoat, J. Phys. Chem. B 107 (2003) 9756-9762.

[72] M. Boronat, A. Corma, Appl. Catal. A 336 (2008) 2-10.

[73] H. Fang, A. Zheng, S. Li, J. Xu, L. Chen, F. Deng, J. Phys. Chem. C 114 (2010) 1025410264.

[74] H. Fang, A. Zheng, J. Xu, S. Li, Y. Chu, L. Chen, F. Deng, J. Phys. Chem. C 115 (2011) 7429-7439.

[75] Y.-H. Guo, M. Pu, B.-H. Chen, F. Cao, Appl. Catal. A 455 (2013) 65-70.

[76] L. Benco, T. Demuth, J. Hafner, F. Hutschka, H. Toulhoat, J. Catal. 205 (2002) 147-156.

[77] C. Tuma, J. Sauer, Angew. Chem. Int. Ed. 44 (2005) 4769-4771.

[78] C.M. Nguyen, B.A. De Moor, M.-F. Reyniers, G.B. Marin, J. Phys. Chem. C 116 (2012) 18236-18249.

[79] B.S. Greensfelder, H.H. Voge, G.M. Good, Ind. Eng. Chem. 41 (1949) 2573-2584.

[80] C.L. Thomas, Ind. Eng. Chem. 41 (1949) 2564-2573.

[81] G. Busca, Phys. Chem. Chem. Phys. 1 (1999) 723-736.

[82] E.J.M. Hensen, D.G. Poduval, V. Degirmenci, D.A.J.M. Ligthart, W. Chen, F. Maugé, M.S. Rigutto, J.A.R. van Veen, J. Phys. Chem. C 116 (2012) 21416-21429.

[83] J. Weitkamp, P.A. Jacobs, J.A. Martens, Appl. Catal. 8 (1983) 123-141.

[84] D. Barthomeuf, Mater. Chem. Phys. 17 (1987) 49-71.

[85] T. Demuth, J. Hafner, L. Benco, H. Toulhoat, J. Phys. Chem. B 104 (2000) 4593-4607.

[86] J. Perdew, K. Burke, M. Ernzerhof, Phys. Rev. Lett. 77 (1996) 3865-3868.

[87] G. Kresse, J. Hafner, Phys. Rev. B 49 (1994) 14251-14269.

[88] G. Kresse, J. Furthmüller, Phys. Rev. B 54 (1996) 11169, 1-8.

[89] G. Kresse, D. Joubert, Phys. Rev. B 59 (1999) 1758-1775.

[90] S. Grimme, J. Comput. Chem. 27 (2006) 1787-1799.

[91] F. Göltl, A. Grüneis, T. Bucko, J. Hafner, J. Chem. Phys. 137 (2012) 114111, 1-7. 
[92] C.C. Chiu, G.N. Vayssilov, A. Genest, A. Borgna, N. Rosch, J. Comput. Chem. (2014).

[93] Z. Zhang, R. Rousseau, J. Gong, B.D. Kay, Z. Dohnálek, J. Am. Chem. Soc. 131 (2009) 17926-17932.

[94] Y. Chu, B. Han, A. Zheng, X. Yi, F. Deng, J. Phys. Chem. C 117 (2013) 2194-2202.

[95] V. Nieminen, M. Sierka, D.Y. Murzin, J. Sauer, J. Catal. 231 (2005) 393-404.

[96] B.A. De Moor, M.-F.o. Reyniers, M. Sierka, J. Sauer, G.B. Marin, J. Phys. Chem. C 112 (2008) 11796-11812.

[97] P. E. Sinclair, A. de Vries, P. Sherwood, C. Richard A. Catlow, R. A. van Santen, J. Chem. Soc., Faraday Trans. 94 (1998) 3401-3408.

[98] R.T. Carr, M. Neurock, E. Iglesia, J. Catal. 278 (2011) 78-93.

[99] J. Macht, R.T. Carr, E. Iglesia, J. Am. Chem. Soc. 131 (2009) 6554-6565.

[100] V.B. Kazansky, Acc. Chem. Res. 24 (1991) 379-383.

[101] A. Bhan, Y.V. Joshi, W.N. Delgass, K.T. Thomson, J. Phys. Chem. B 107 (2003) 10476-10487.

[102] B.A. De Moor, M.-F. Reyniers, G.B. Marin, Phys. Chem. Chem. Phys. 11 (2009) 29392958.

[103] X. Rozanska, T. Demuth, F. Hutschka, J. Hafner, R.A. van Santen, J. Phys. Chem. B 106 (2002) 3248-3254.

[104] J. Thybaut, C.S.L. Narasimhan, G. Marin, J.M. Denayer, G. Baron, P. Jacobs, J. Martens, Catal. Lett. 94 (2004) 81-88.

[105] S. Svelle, S. Kolboe, O. Swang, J. Phys. Chem. B 108 (2004) 2953-2962.

[106] Q. Li, A.L.L. East, Can. J. Chem. 83 (2005) 1146-1157.

[107] Q. Li, A.L.L. East, Can. J. Chem. 84 (2006) 1159-1166.

[108] Y. Chu, B. Han, A. Zheng, F. Deng, J. Phys. Chem. C 116 (2012) 12687-12695.

[109] S. Namuangruk, P. Pantu, J. Limtrakul, ChemPhysChem 6 (2005) 1333-1339. 
[110] Y.-X. Sun, J. Yang, L.-F. Zhao, J.-X. Dai, H. Sun, J. Phys. Chem. C 114 (2010) 59755984.

[111] Y.-H. Guo, M. Pu, J.-Y. Wu, J.-Y. Zhang, B.-H. Chen, Appl. Surf. Sci. 254 (2007) 604609.

[112] M.N. Mazar, S.H. Al Hashimi, M. Cococcioni, A. Bhan, J. Phys. Chem. C (2013) $23609-23620$

[113] R. Gounder, E. Iglesia, J. Am. Chem. Soc. 131 (2009) 1958-1971.

[114] H. Toulhoat, M. Lontsi Fomena, T. de Bruin, J. Am. Chem. Soc. 133 (2011) 2481-2491. 


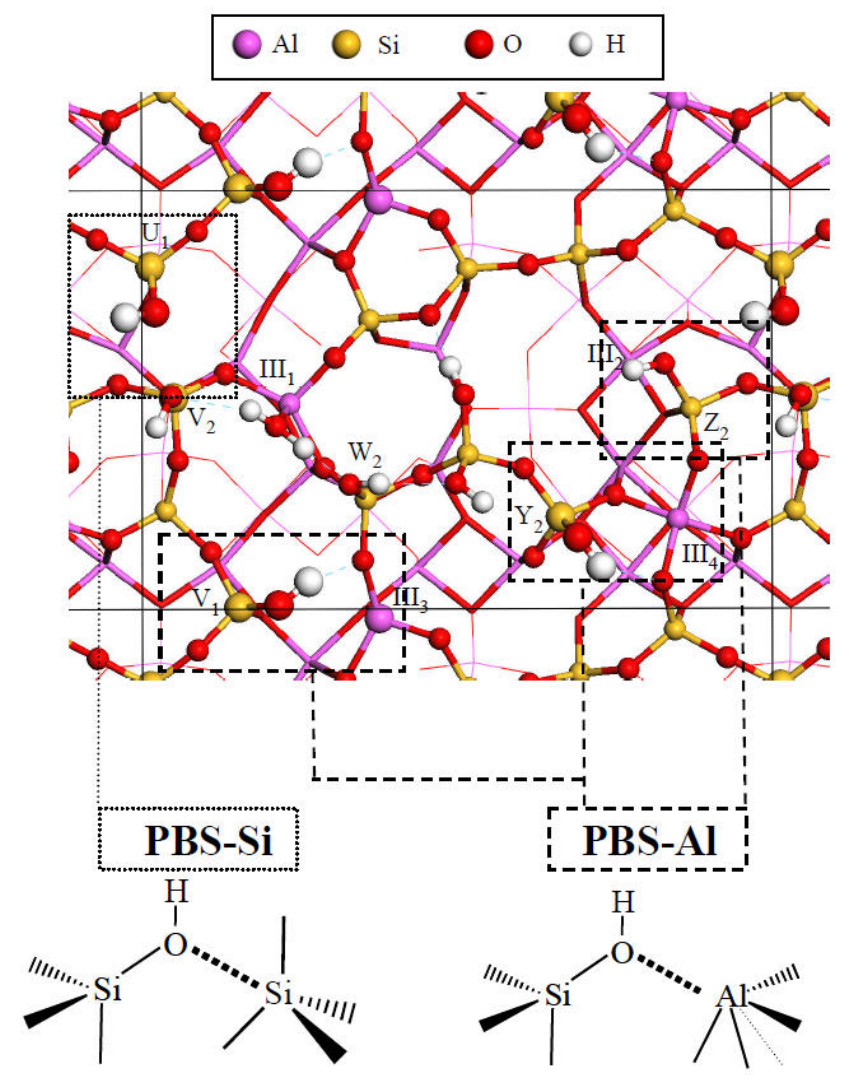

Figure 1. ASA surface model for $\theta_{\mathrm{OH}}=5.4 \mathrm{~nm}^{-2}$, as established in ref. [37, 38]. Some Pseudo-Bridging Silanols (PBS) are shown, in particular silicic PBS (PBS-Si, $\mathrm{Si}\left(\mathrm{U}_{1}\right)-\mathrm{OH} \cdots \mathrm{Si}\left(\mathrm{V}_{2}\right)$ ) and aluminic PBS (PBS-Al, $\left.\mathrm{Si}\left(\mathrm{V}_{1}\right)-\mathrm{OH} \cdots \mathrm{Al}_{\mathrm{IV}}\left(\mathrm{III}_{3}\right)\right)$ are able to protonate lutidine, others are not. 


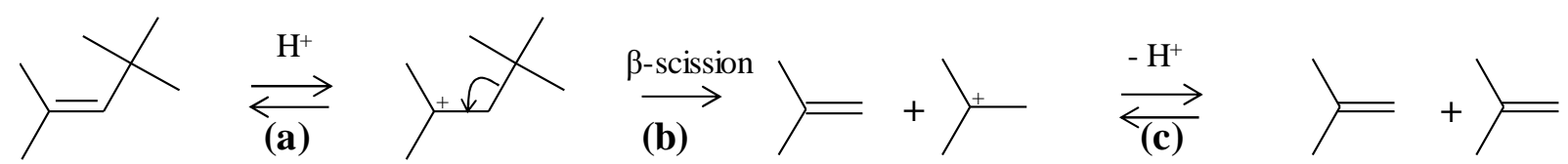

Figure 2. Expected reaction steps leading to the cracking of 2,4,4-trimethyl-2-pentene (DIB): (a) protonation step, (b) $\beta$-scission step, (c) product formation and regeneration of the protonic site. 
(a)

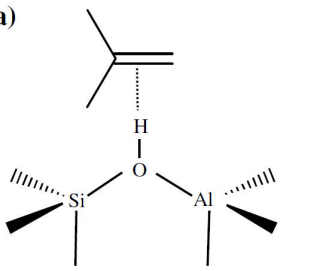

(b)

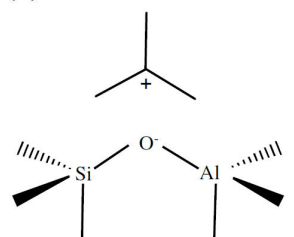

(c)

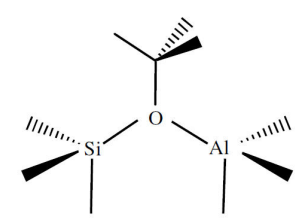

Figure 3. Species likely formed upon interaction of isobutene with the protonic site of a zeolite (omitting further reactions, like dimerization $[63,64])$ : (a) $\pi$-complex, (b) carbenium ion - tert-butyl - , (c) alkoxide - isobutoxide - also called $\sigma$-complex. The tertiary species is exemplified here. 
(a)

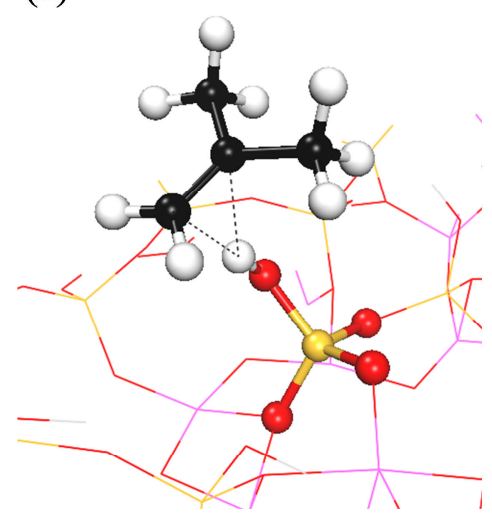

(b)

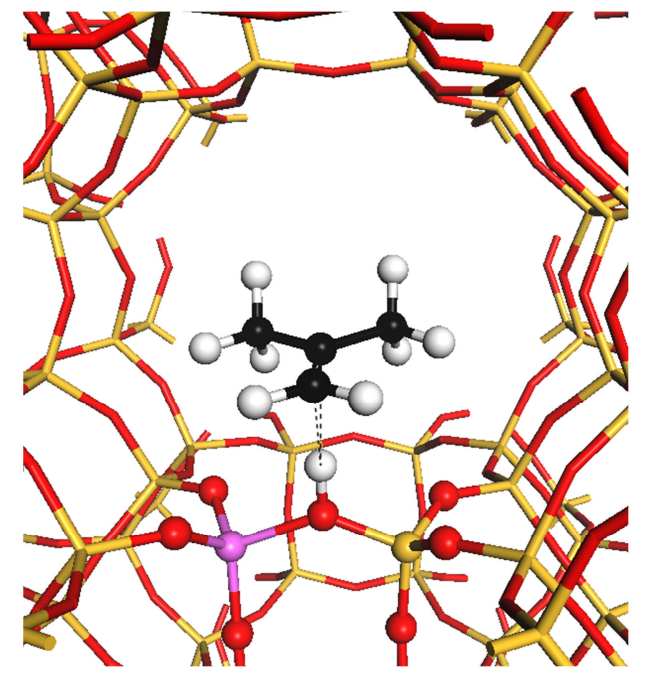

Figure 4. $\pi$-complex adsorption mode of isobutene on ASA ( $\mathrm{Si}\left(\mathrm{Z}_{2}\right)-\mathrm{OH}$ site) (a) and MOR (b). Same color code as figure 1, carbon atoms are in black. 


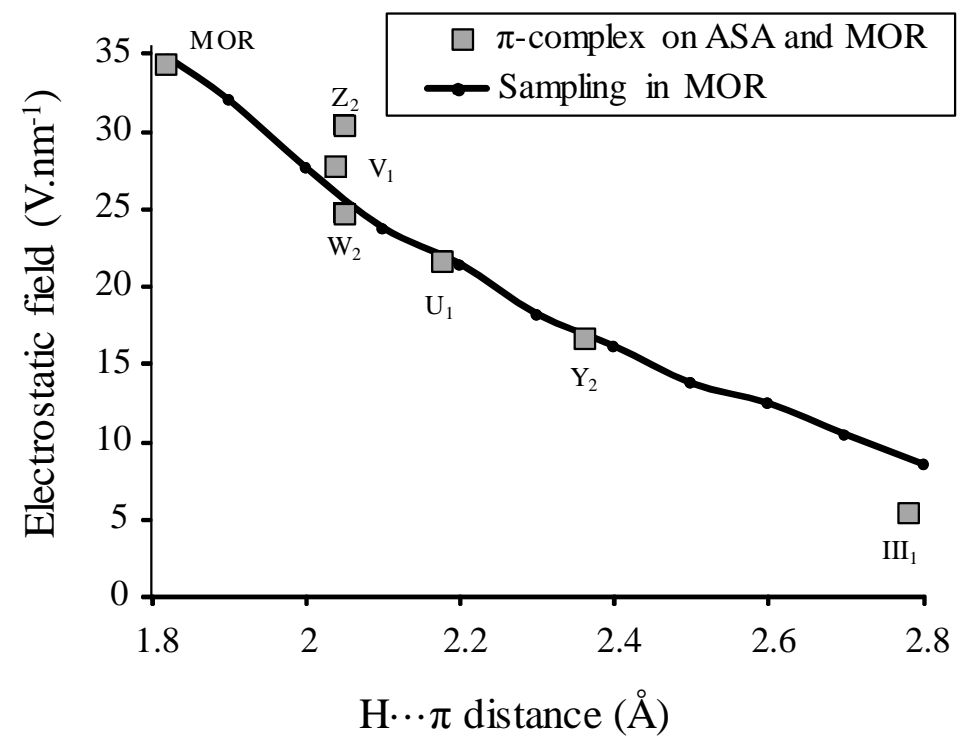

Figure 5. Electrostatic field evaluated in the surrounding of the isobutene molecule adsorbed in a $\pi$-complex mode, as a function of the distance between the acid site $(\mathrm{H})$ and the nearer $\mathrm{C}$ atom of the $\mathrm{C}=\mathrm{C}$ bond (terminal atom) (grey squares). The electrostatic field was also evaluated in the cavity of MOR, by formally increasing the $\mathrm{H} \cdots \mathrm{C}$ distance, perpendicularly to the channel direction, and is depicted by the solid line (increment: $0.1 \AA$ ). 


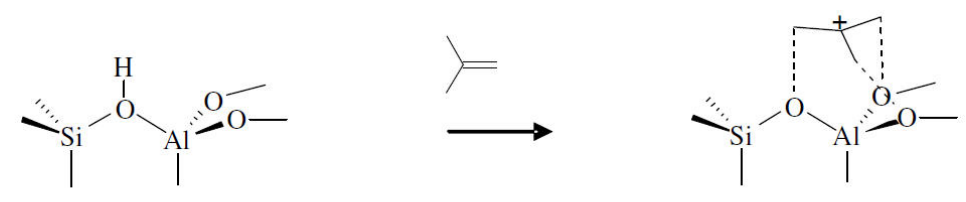

(a)
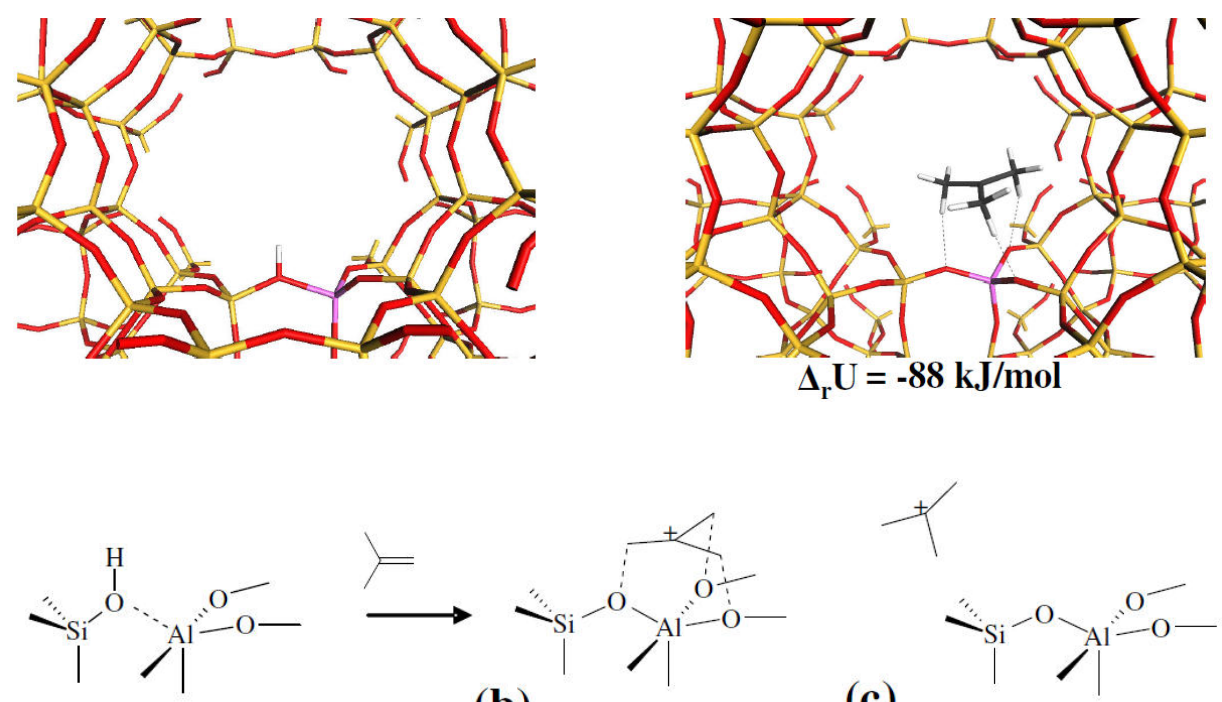

(b)

(c)
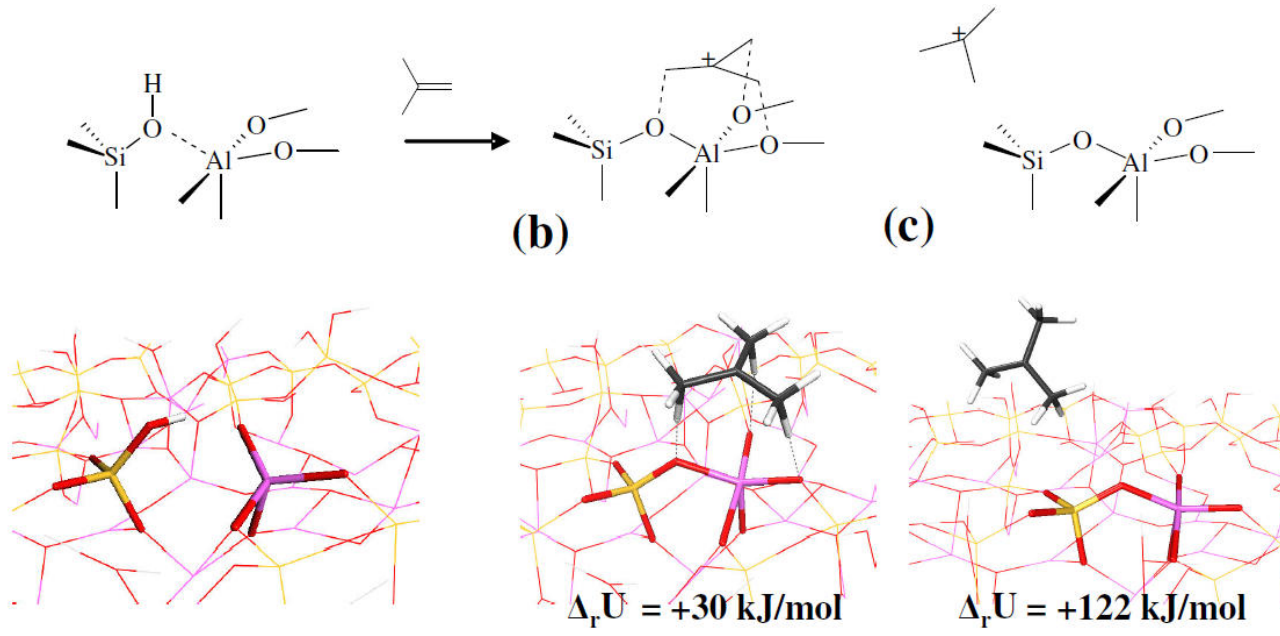<smiles>C[Si](C)(O)O[Si](C)(C)O</smiles><smiles>C=CC[CH2-]</smiles>

(d)<smiles>C[SiH](C)O[Si](C)(C)O</smiles>
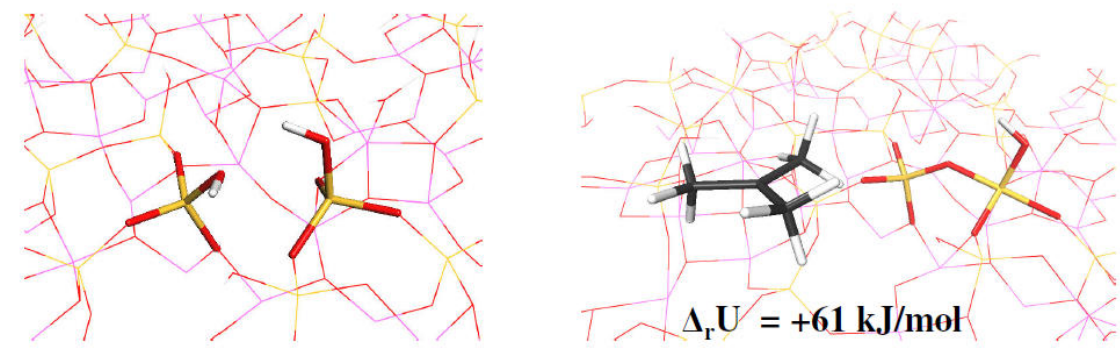

Figure 6. Geometries and energetics of the formation of tertiobutoxonium from isobutene on mordenite (a), PBS-Al site of ASA (this site is able to protonate lutidine) (b and c), and PBS-Si site of ASA (d). 
<smiles>C=C(C)C[C@H](C)N(C)OC(C)(C)[Al](C)(C)OC</smiles>

(a)
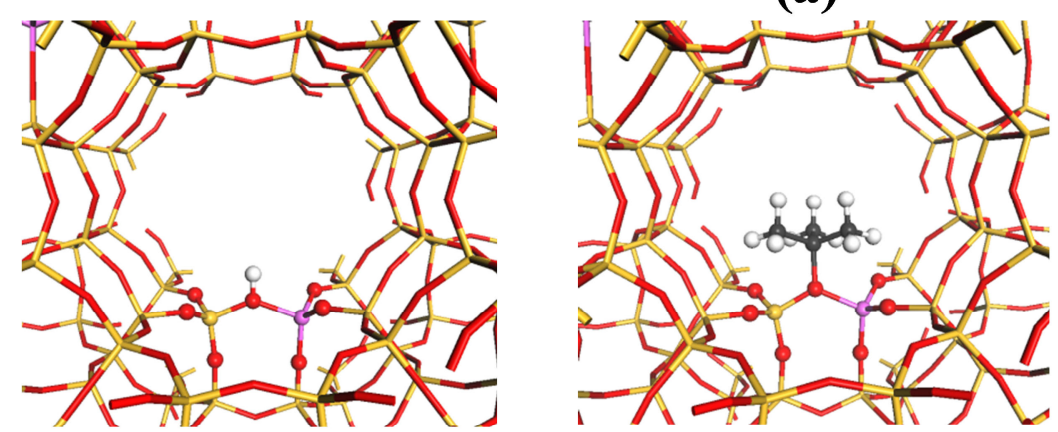

$\Delta_{\mathbf{r}} \mathrm{U}=\mathbf{- 9 5} \mathrm{kJ} / \mathrm{mol}$

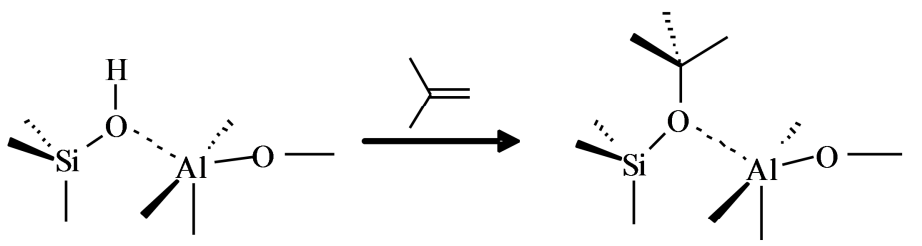

(c)

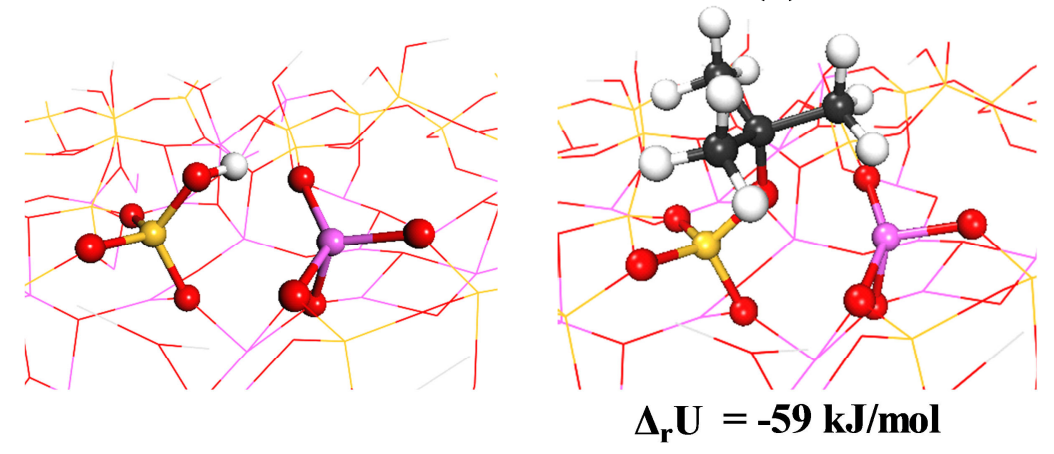<smiles>C[Si](C)O[Al](C)(C)OC(C)(C)C</smiles>

(b)

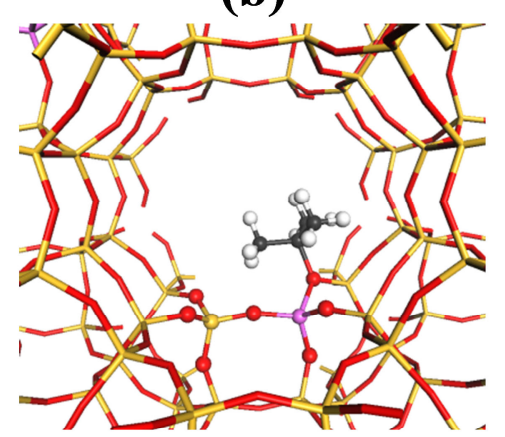

$\Delta_{\mathrm{r}} \mathrm{U}=\mathbf{- 8 8} \mathrm{kJ} / \mathrm{mol}$

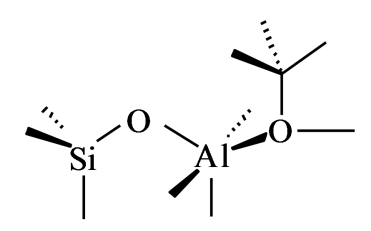

(d)

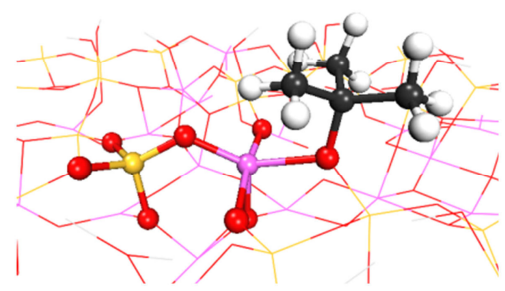

$\Delta_{\mathbf{r}} \mathrm{U}=-27 \mathrm{~kJ} / \mathrm{mol}$

Figure 7. Geometries and adsorption energies of isobutene to form an alkoxide on mordenite (a and $b$ ) and ASA (PBS-Al which protonates lutidine, $\mathrm{c}$ and d), according to a 4-center (a and c) or 6-center (b and d) mechanism. Same color code as figure 1, carbon atoms are in black. 


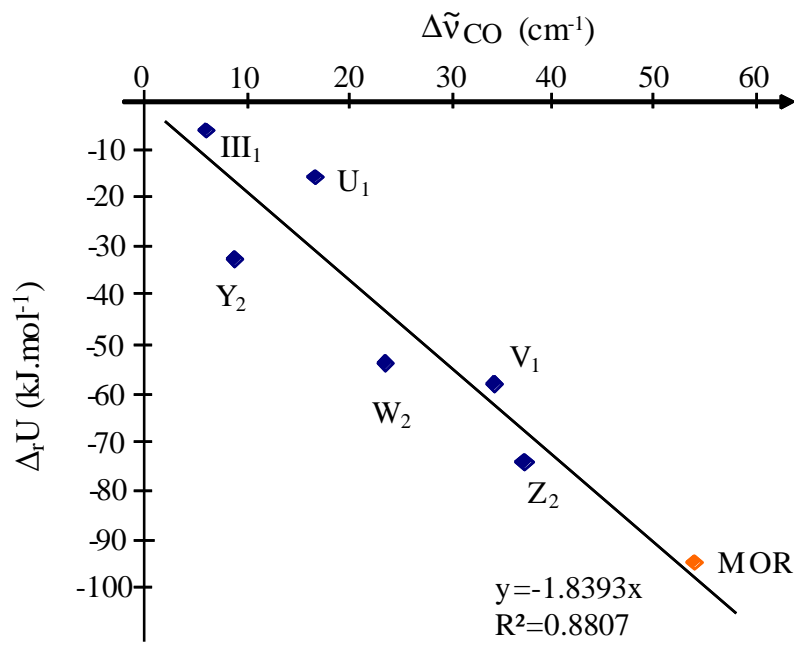

Figure 8. Formation energy of tert-butoxy on ASA (blue) and mordenite (orange) from isobutene, as a function of the frequency shift of $\mathrm{CO}$ when adsorbed on the same surface site. Values of CO frequency shifts are extracted from [36]. 
(a)

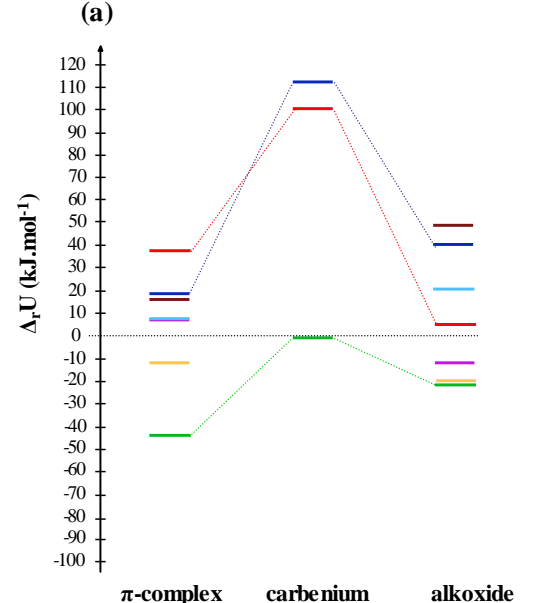

(b)

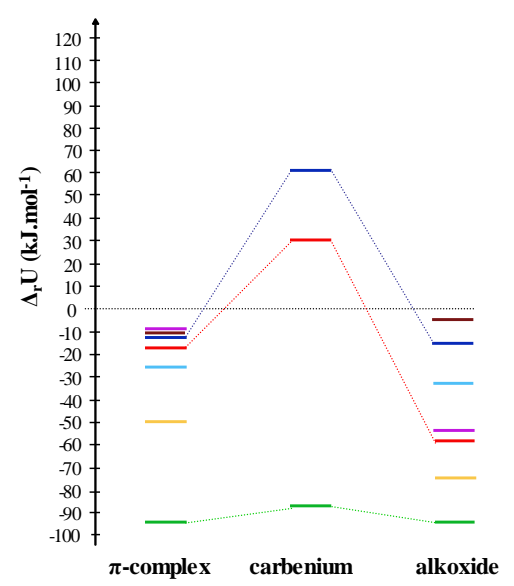

(c)

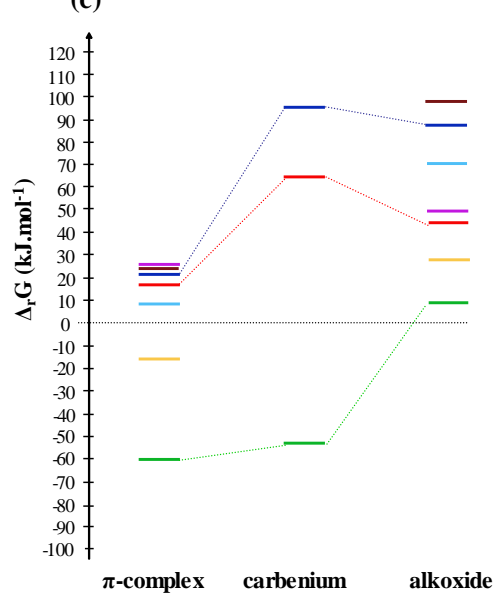

ASA

Mordenite

— PBS-Si Si( $\left.\mathrm{U}_{1}\right)-\mathrm{OH} \cdots \mathrm{Si}\left(\mathrm{V}_{2}\right.$

_ Silanol Al Si( $\left(\mathrm{W}_{2}\right)-\mathrm{OH}$

- Al-OH from $\mathrm{Al}\left(\mathrm{III}_{1}\right)-\mathrm{HOH}_{(2)}$

— PBS-Al Si( $\left.\mathrm{V}_{1}\right)-\mathrm{OH} \cdots \mathrm{Al}_{\mathrm{IV}}\left(\mathrm{III}_{3}\right)$ (protonates lutidine)

- PBS-Al Si( $\left.\mathrm{Z}_{2}\right)-\mathrm{OH} \cdots \mathrm{Al}_{\mathrm{V}}\left(\mathrm{III}_{2}\right)$ (does not protonate lutidine)

— PBS-Al Si( $\left(\mathrm{Y}_{2}\right)-\mathrm{OH} \cdots \mathrm{Al}_{\mathrm{V}}\left(\mathrm{III}_{4}\right)$ (does not protonate lutidine)

Figure 9. Energy diagram representing the formation energy of adsorbed isobutene according to the three modes investigated, without dispersion corrections (a) or taking into account dispersion corrections (b). Gibbs energy diagram (c) at $500 \mathrm{~K}$. 


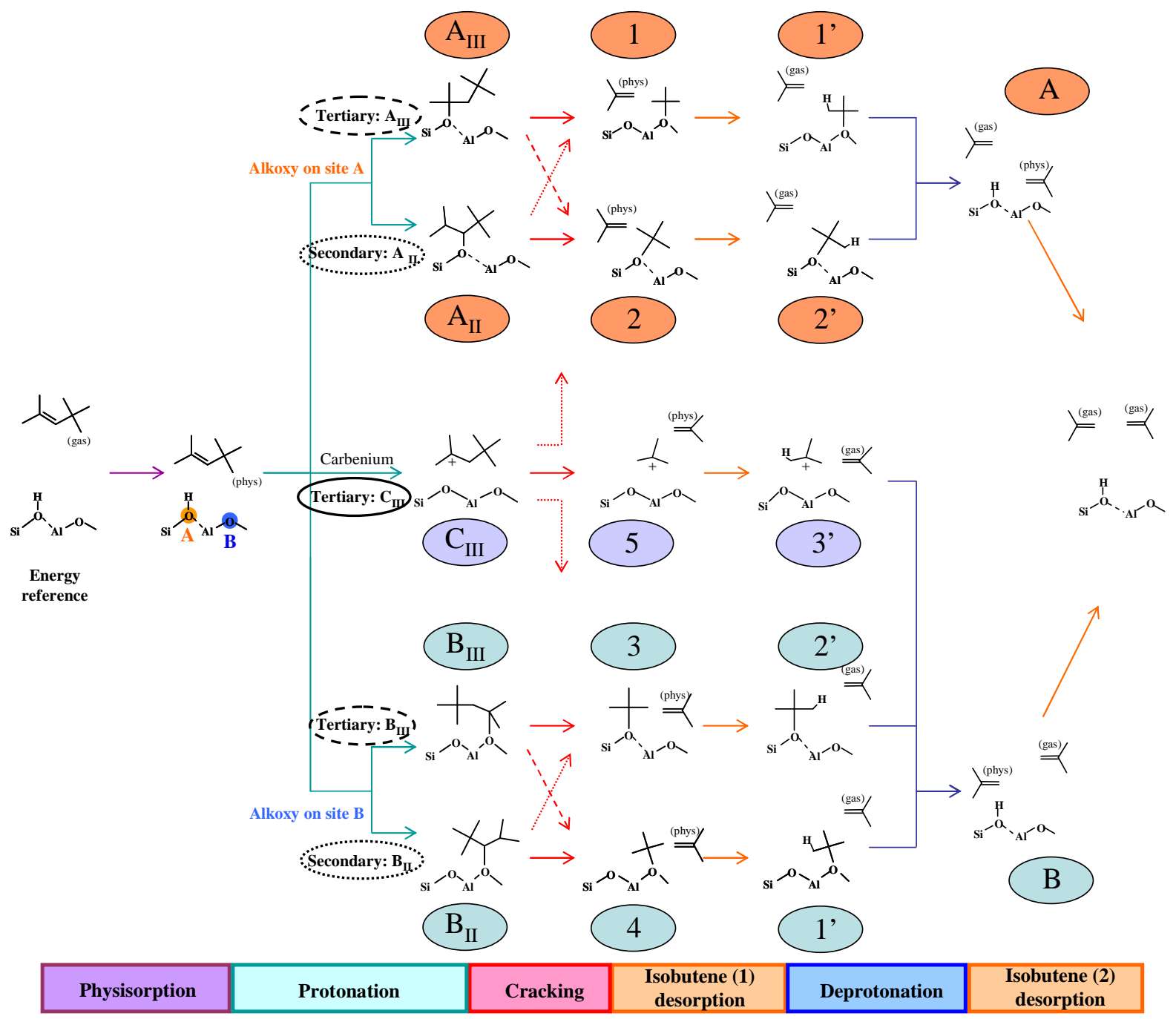

Figure 10. Reaction pathway investigated for DIB cracking reaction, from tertiary and secondary alkoxides or from a tertiary carbenium ion $\left(\mathrm{C}_{\mathrm{III}}\right)$. Reactions are illustrated in the case of the strongest PBS-Al site of ASA. 


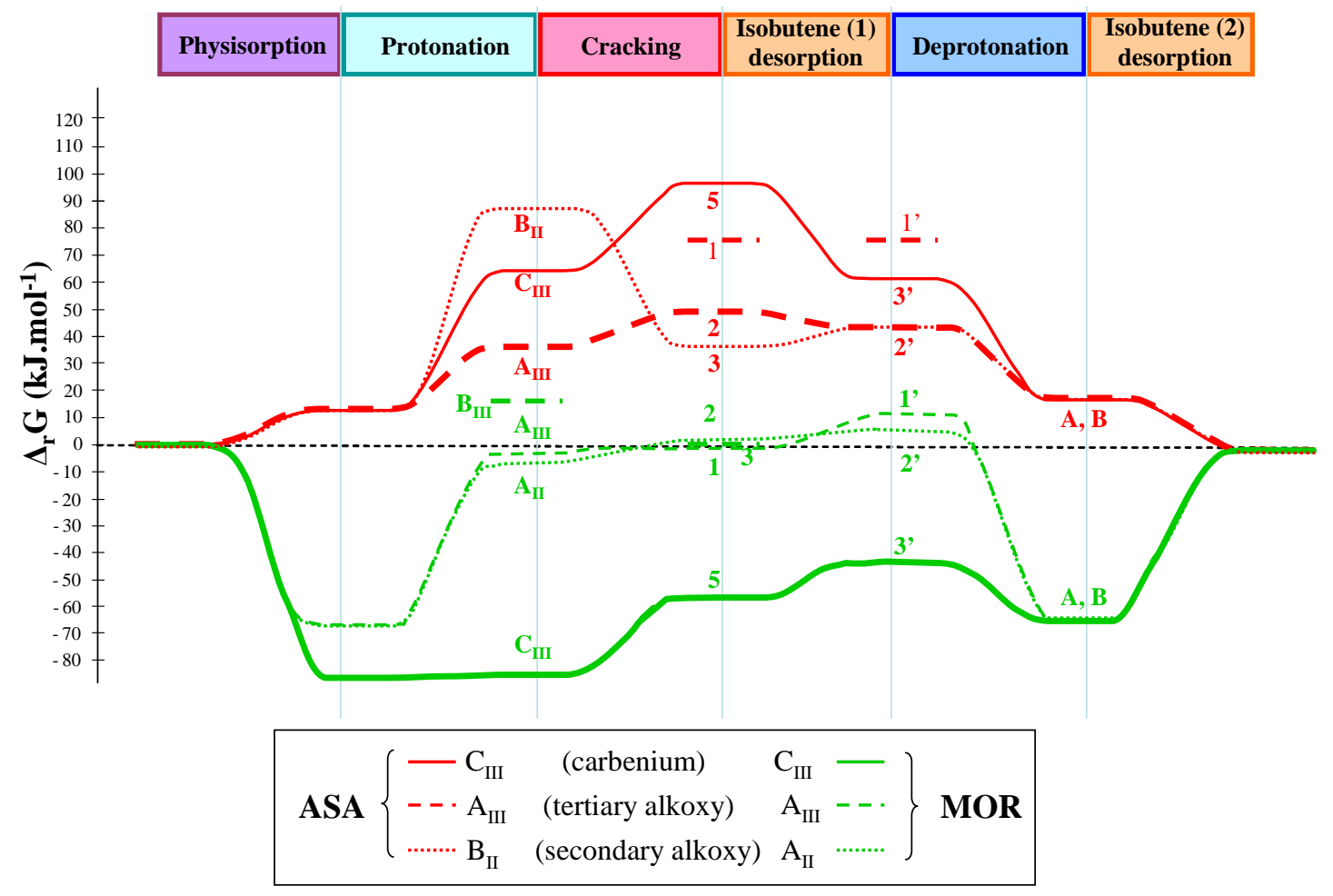

Figure 11. Gibbs free energy profiles for the selected cracking pathways of diisobutene on the ASA surface (PBS-Al site) and in mordenite, at $500 \mathrm{~K}$ and all partial pressures equal to 1 bar. Labels refer to intermediates depicted in Figure 10. Lines relate to the selected pathways, as explained in the main text. 


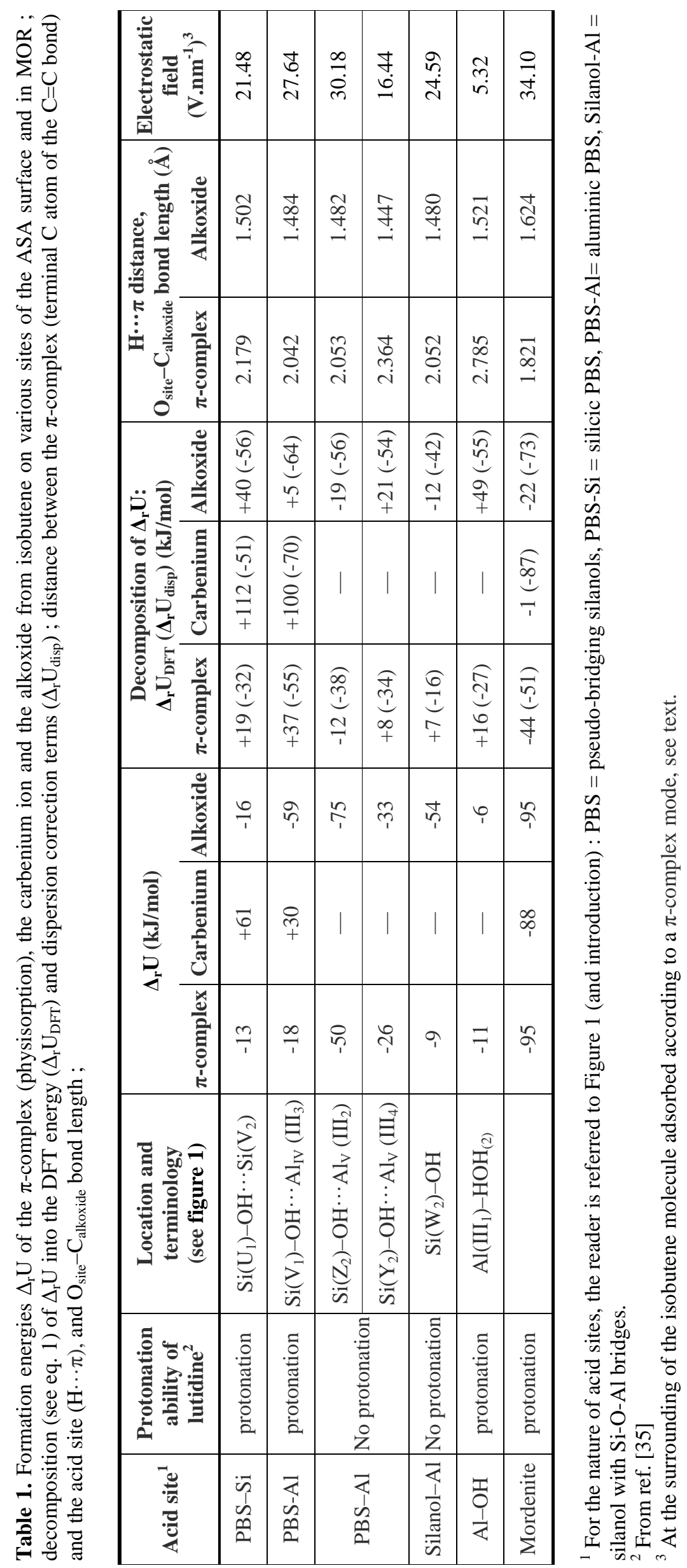


Table 2. Formation energy of tert-butyl carbenium ion and of lutidinium on the bridging $\mathrm{OH}$ group of mordenite and on the $\mathrm{Si}\left(\mathrm{V}_{1}\right)-\mathrm{OH} \cdots \mathrm{Al}_{\mathrm{IV}}\left(\mathrm{III}_{3}\right)$ PBS-Al site of the ASA surface. All energies are expressed in $\mathrm{kJ} / \mathrm{mol}$.

\begin{tabular}{|c|c|c|c|c|c|}
\hline \multirow{2}{*}{ Site } & \multicolumn{2}{|c|}{$\Delta_{\mathbf{r}} \mathbf{U}$} & \multicolumn{2}{|c|}{$\begin{array}{c}\text { Decomposition of } \Delta_{\mathrm{r}} \mathrm{U}: \\
\Delta_{\mathrm{r}} \mathbf{U}_{\text {DFT }}\left(\Delta_{\mathrm{r}} \mathbf{U}_{\text {disp }}\right)\end{array}$} & \multirow{2}{*}{$\begin{array}{l}\text { Electrostatic field* } \\
\quad\left(\mathbf{V} \cdot \mathbf{n m}^{-1}\right)\end{array}$} \\
\hline & $\begin{array}{l}\text { tert-butyl } \\
\text { carbenium }\end{array}$ & Lutidinium* & $\begin{array}{l}\text { tert-butyl } \\
\text { carbenium }\end{array}$ & Lutidinium* & \\
\hline PBS-Al & +30 & -101 & $+100(-70)$ & $-41(-60)$ & 27.6 \\
\hline Mordenite & -88 & -241 & $-1(-87)$ & $-172(-69)$ & 34.1 \\
\hline
\end{tabular}

* These calculations were performed with the same functional (PBE) as for the formation of tert-butyl carbenium ion, and include dispersion corrections (DFT-D2).

$*$ as calculated for $\pi$-complexes, see Table 1 . 


\title{
SUPPORTING INFORMATION
}

\author{
Revisiting carbenium chemistry
}

on Amorphous Silica Alumina:

unraveling their milder acidity as compared to zeolites

\author{
Fabien Leydier ${ }^{1,2}$, Céline Chizallet ${ }^{1}$, Dominique Costa ${ }^{2}$, Pascal Raybaud ${ }^{1}$ \\ ${ }^{1}$ IFP Energies nouvelles, Rond-Point de l'échangeur de Solaize, BP3, 69360 Solaize, France \\ ${ }^{2}$ ENSCP, Chimie-Paristech, 11 rue Pierre et Marie Curie, 75005 Paris, France
}




\section{S1. Gibbs free energy calculations}

\section{S1.1. Calculation of the various corrections ( $H, S$, translational, rotational and vibrational) for ideal gases}

To take into account temperature $(\mathrm{T})$ and pressure $(\mathrm{P})$ effects from the energies $\mathrm{U}$ calculated with VASP, the Gibbs free energy $G$ has to be estimated thanks to equations S1., S2. and S3, with $\mathrm{H}$ the enthalpy, $\mathrm{S}$ the entropy, $\mathrm{V}_{\mathrm{m}}$ the molar volume, $\mathrm{U}_{\text {elec }}$ the electronic $(\mathrm{DFT}+\mathrm{D})$ contribution to energy, $\mathrm{U}_{\text {trans }}, \mathrm{U}_{\text {rot, }}, \mathrm{U}_{\mathrm{vib}}$ the translational, rotational and vibrational contributions to enthalpy respectively, ZPE the zero-point vibrational energy, $S_{\text {trans, }} S_{\text {rot, }} S_{\text {vib(T) }}$ the translational, rotational and vibrational contributions to entropy respectively.

$$
\begin{array}{cr}
\mathrm{G}_{(\mathrm{T})}=\mathrm{H}_{(\mathrm{T})}-\mathrm{T} \times \mathrm{S}_{(\mathrm{T})} & \text { Equation } \mathrm{S} \text { 1 } \\
\mathrm{H}_{(\mathrm{T})}=\mathrm{U}_{(\mathrm{T})}+\mathrm{PV}_{\mathrm{m}}=\mathrm{U}_{\text {elec }}+\mathrm{U}_{\operatorname{trans}(\mathrm{T})}+\mathrm{U}_{\mathrm{rot}(\mathrm{T})}+\mathrm{U}_{\mathrm{vib}(\mathrm{T})}+\mathrm{ZPE}+\mathrm{PV}_{\mathrm{m}} & \text { Equation S2 } \\
\mathrm{S}_{(\mathrm{T})}=\mathrm{S}_{\operatorname{trans}(\mathrm{T})}+\mathrm{S}_{\mathrm{rot}(\mathrm{T})}+\mathrm{S}_{\mathrm{vib}(\mathrm{T})} & \text { Equation S3 }
\end{array}
$$

This can also be written as :

$$
\mathrm{G}_{(\mathrm{T})}=\mathrm{U}_{\text {elec }}+\mathrm{F}_{\text {trans(T) }}+\mathrm{F}_{\text {rot( }(\mathrm{T})}+\mathrm{F}_{\mathrm{vib}(\mathrm{T})}+\mathrm{PV}_{\mathrm{m}}+\mathrm{ZPE}
$$

Equation S4

where $\mathrm{F}_{\text {trans(T) }}, \mathrm{F}_{\mathrm{rot}(\mathrm{T})}$ and $\mathrm{F}_{\mathrm{vib}(\mathrm{T})}$ are translational, rotational and vibrational contributions to free energy.

Each term was evaluated thanks to statistical thermodynamics, within the ideal gas model for gaseous species, according to equations S4-S8. In particular, the $\mathrm{S}_{\text {trans }}$ term is given by the Sackur-Tetrode equation (equation S7). ${ }^{1-3}$

$$
\begin{aligned}
& \mathrm{U}_{\operatorname{trans}(\mathrm{T})}=\mathrm{U}_{\operatorname{rot}(\mathrm{T})}=\frac{3}{2} \mathcal{N} \mathrm{kT}
\end{aligned}
$$

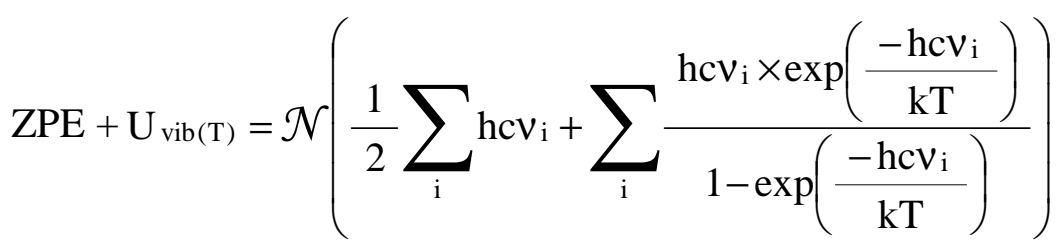

$$
\begin{aligned}
& \mathrm{S}_{\text {trans }(\mathrm{T})}=\mathcal{N} \mathrm{k}\left(\frac{5}{2} \ln \mathrm{T}-\ln \mathrm{P}+\frac{3}{2} \ln \mathcal{M}-1,165\right) \\
& \mathrm{S}_{\mathrm{rot}(\mathrm{T})}=\mathcal{N} \mathrm{k} \ln \left(\frac{\sqrt{\pi}}{\sigma}\left(\frac{8 \pi^{2} \mathrm{kT}}{\mathrm{h}^{2}}\right)^{3 / 2} \sqrt{\mathrm{A}_{\mathrm{e}} \times \mathrm{B}_{\mathrm{e}} \times \mathrm{C}_{\mathrm{e}}}\right) \\
& \mathrm{S}_{\mathrm{vib}(\mathrm{T})}=\mathcal{N} \mathrm{k} \sum_{\mathrm{i}} \frac{\frac{\mathrm{hc} v_{\mathrm{i}}}{\mathrm{kT}} \times \exp \left(\frac{-h c v_{\mathrm{i}}}{\mathrm{kT}}\right)}{1-\exp \left(\frac{-\mathrm{hcv_{ \textrm {i } }}}{\mathrm{kT}}\right)}-\mathcal{N} \mathrm{k} \sum_{\mathrm{i}} \ln \left(1-\exp \left(\frac{-\mathrm{hc} v_{\mathrm{i}}}{\mathrm{kT}}\right)\right)
\end{aligned}
$$

Equation S5

Equation S6

Equation S7

Equation S8

Equation S9

with $\mathcal{N}$ : Avogadro constant; k: Boltzmann constant; h : Planck constant; c : speed of light; $v_{\mathrm{i}}$ : vibration eigenvalues; $\mathcal{M}$ : molar mass; $\sigma$ : symmetry number; $\mathrm{A}_{\mathrm{e}}, \mathrm{B}_{\mathrm{e}}, \mathrm{C}_{\mathrm{e}}$ : moments of inertia according to the three eigenaxis of the molecule. 


\section{S1.2. Calculation of Gibbs free energy of reaction for DIB cracking into isobutene}

The reaction profiles, taking into account temperature and pressure effects, are estimated for the following reaction :

$$
\text { Surface }_{(\mathrm{s})}+\mathrm{DIB}_{(\mathrm{g})}=\text { Surface }_{(\mathrm{s})}-\text { Molecule- }_{(\mathrm{ads})}+\mathrm{n} \text { Molecule- } 2_{(\mathrm{g})} \quad \text { Equation S10 }
$$

Surface $_{(\mathrm{s})}$ depicts the surface (external surface of ASA or internal surface of MOR) on which the $\mathrm{DIB}_{(\mathrm{g})}$ adsorbs to generate the Surface $(\mathrm{s})-$ Molecule- $_{(\mathrm{ads})}$ system, with n Molecule- $2_{(\mathrm{g})}$ in case of DIB $(n=1)$ or isobutene $(n=1$ or 2$)$ possible release.

$\mathrm{DIB}_{(\mathrm{g})}$ and Molecule- $2_{(\mathrm{g})}$ are considered as ideal gases, their Gibbs free energies are calculated according to section S1.1. The pressure of each compound is taken equal to 1 bar. The entropy and PV terms relative to Surface $_{(\mathrm{s})}$ are supposed to be compensated along the reaction depicted by Equation S10.

Regarding the adsorbed molecule Molecule- $1_{(\mathrm{ads})}$, the translational contributions (Equations S5 and S7) present for the gas phase are supposed to be lost for alkoxide species, whereas two degrees of freedom over 3 are expected to be kept in the adsorbed phase for carbenium and $\pi$ complexes. ZPE variations (Equation S6) are neglected between gas phase and adsorbed state.

The reaction Gibbs free energy $\Delta_{\mathrm{r}} \mathrm{G}$ corresponding to equation $\mathrm{S} 10$ is given in equations $\mathrm{S} 11$ and $\mathrm{S} 12, \mathrm{G}_{\mathrm{i}}$ representing the molar Gibbs free energy for each compound $\mathrm{i}, \Delta_{\mathrm{r}} \mathrm{U}_{\text {elec }}$ being the electronic contribution to the reaction energy, $\Delta \mathrm{F}$ the corresponding thermal correction in free energy, $\Delta_{\mathrm{ZPE}}$ the variation in ZPE.

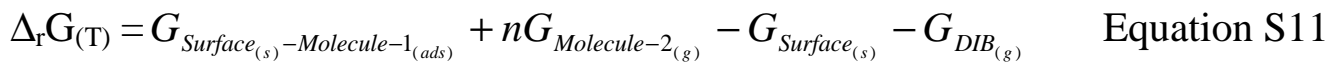

$$
\begin{aligned}
& \Delta_{\mathrm{r}} \mathrm{G}_{(\mathrm{T})} \approx \Delta_{\mathrm{r}} \mathrm{U}_{\text {elec }}+\Delta \mathrm{F}+((\mathrm{n}-1) \mathrm{RT})+\Delta_{\mathrm{ZPE}} \quad \text { Equation } \mathrm{S} 12
\end{aligned}
$$




\section{S2. Isobutene adsorption according to the $\pi$-complex mode: variation of adsorption energies with spectroscopic features}

(a)

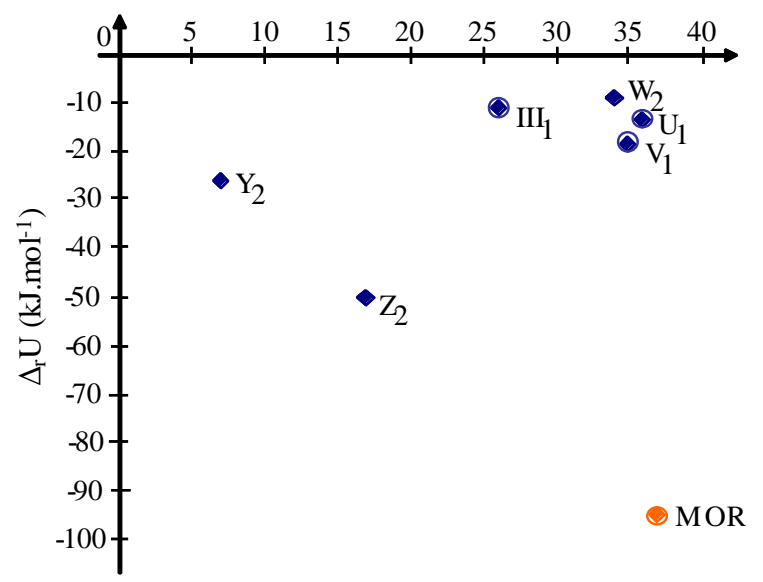

(b)

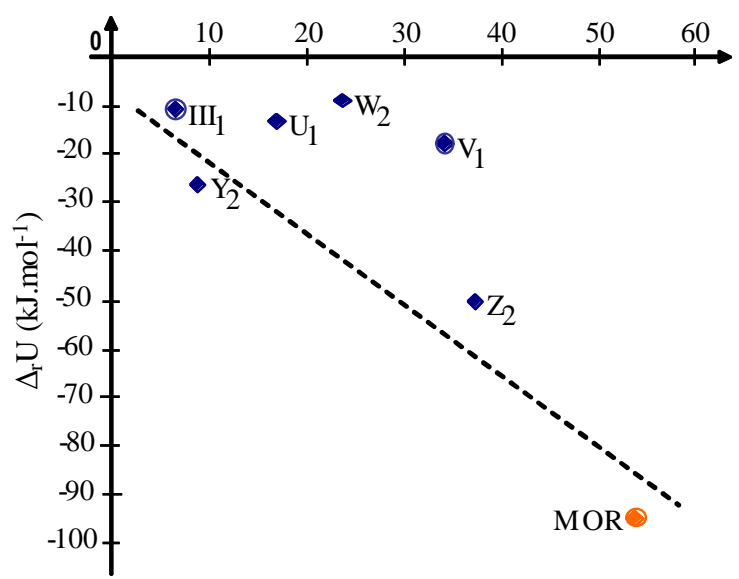

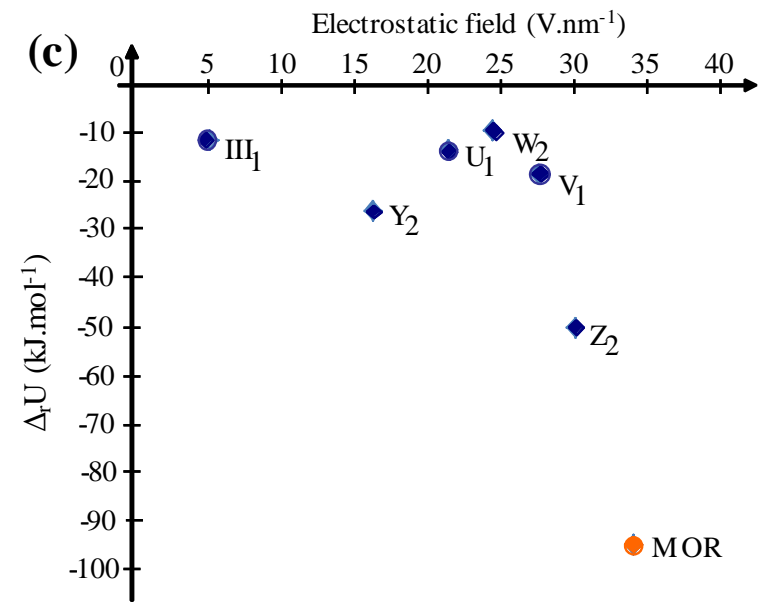

Lutidine protonation

- No lutidine protonation

Figure S2.1. Formation energy of the $\pi$-complex on ASA (blue) and mordenite (orange) from isobutene, as a function of one vibration frequency of lutidine (a), the frequency shift of $\mathrm{CO}$ (b) when adsorbed on the same surface site, (c) the electrostatic field in the pi-complex adsorption configuration. Values of lutidine frequencies and $\mathrm{CO}$ frequency shifts are extracted from ref. 4 and 5 respectively. 


\section{S3. Numerical data for DIB cracking profiles, and energy plot}

Table S3.1. $\Delta_{\mathrm{r}} \mathrm{U}$ and $\Delta_{\mathrm{r}} \mathrm{G}(\mathrm{T}=500 \mathrm{~K}, 1$ bar of each gas species) values for the various intermediates calculated on ASA (PBS-Al) and in MOR, for DIB cracking into isobutene (see figure 9 in the manuscript for the terminology). The reference state is the isolated surface site and DIB molecule in gas phase.

\begin{tabular}{|c|c|c|c|c|}
\hline \hline \multirow{2}{*}{ Species } & \multicolumn{2}{|c|}{$\left.\boldsymbol{\Delta}_{\mathbf{r}} \mathbf{U}(\mathbf{k J . m o l})^{\mathbf{1}}\right)$} & \multicolumn{2}{|c|}{$\boldsymbol{\Delta}_{\mathbf{r}} \mathbf{G}\left(\mathbf{k J . m o l} \mathbf{~}^{\mathbf{1}}\right)$} \\
\cline { 2 - 5 } & MOR & ASA & MOR & ASA \\
\hline DIB $\left._{\text {(phys }}\right)$ & -131 & -31 & -88 & 12 \\
$\mathrm{~A}_{\mathrm{III}}$ & -122 & -82 & -4 & 36 \\
$\mathrm{~A}_{\mathrm{II}}$ & -124 & -78 & -6 & 40 \\
$\mathrm{C}_{\mathrm{III}}$ & -130 & 20 & -87 & 63 \\
$\mathrm{~B}_{\mathrm{III}}$ & -104 & -55 & 14 & 63 \\
$\mathrm{~B}_{\mathrm{II}}$ & -77 & -32 & 41 & 86 \\
1 & -54 & 23 & -1 & 76 \\
2 & -52 & -5 & 1 & 48 \\
3 & -52 & -20 & 1 & 33 \\
4 & -54 & 24 & -1 & 77 \\
5 & -44 & 111 & -59 & 96 \\
$1^{\prime}$ & -2 & 60 & 10 & 75 \\
$2^{\prime}$ & -9 & 27 & 3 & 43 \\
$3^{\prime}$ & 11 & 117 & -45 & 61 \\
$\mathrm{~A}$ & -8 & 68 & -65 & 16 \\
$\mathrm{~B}$ & -8 & 68 & -65 & 16 \\
Final state & 87 & 87 & -3 & -3 \\
\hline \hline
\end{tabular}




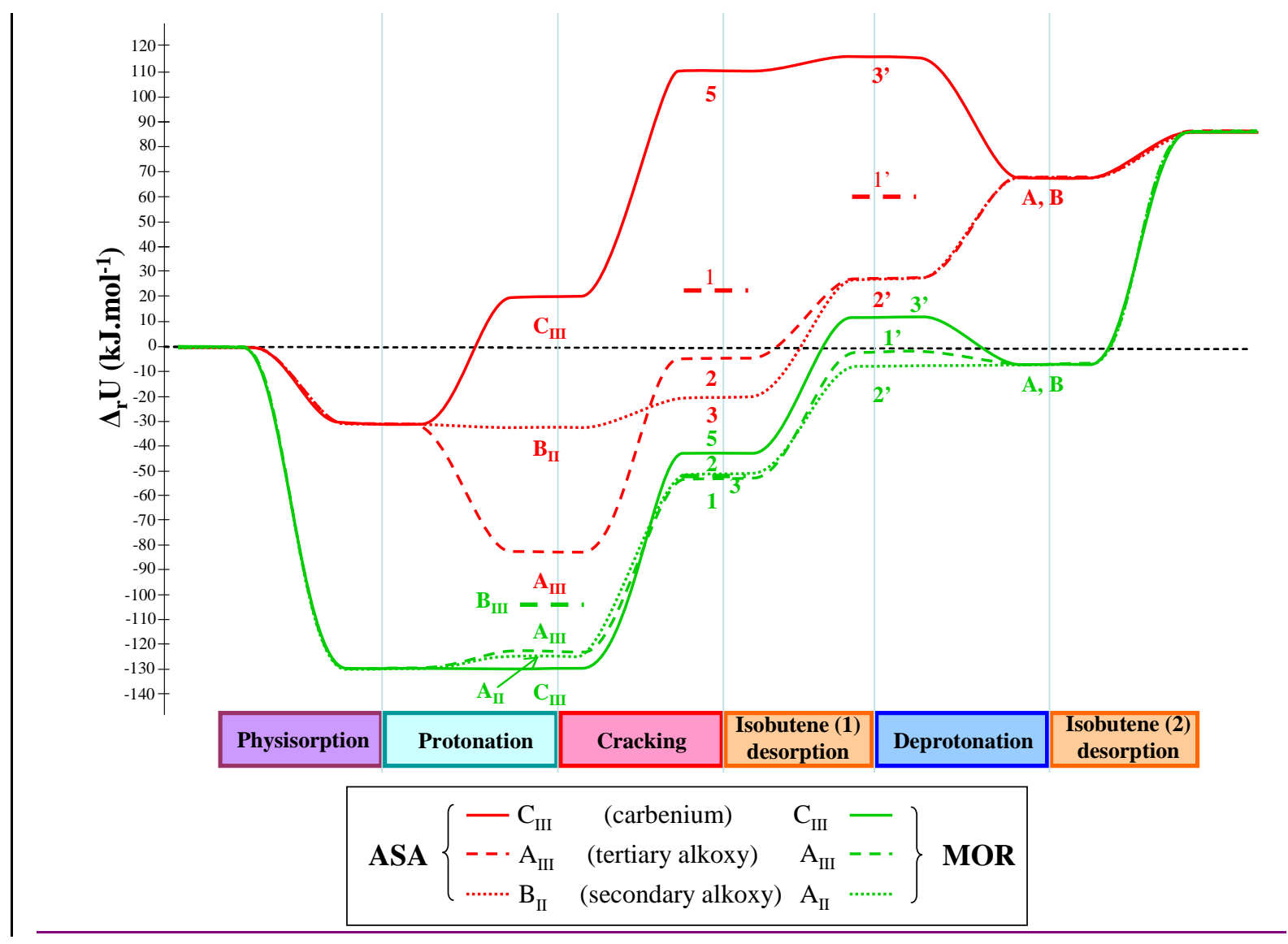

Figure S3.1. Energy profiles for the selected cracking pathways of diisobutene on the ASA surface (PBS-Al site) and in mordenite. Labels refer to intermediates depicted in Figure 9. Lines relate to the selected pathways, as explained in the main text. 


\section{S4. .cif files for most important structures involved in DIB reaction on ASA and in MOR}

Table S4.1 indicates the number of the file reported below, corresponding to the structures on MOR and ASA.

Table S4.1. Number of the files for the systems which coordinate are reported below.

\begin{tabular}{|c|c|c|}
\hline \hline \multirow{2}{*}{ Species } & \multicolumn{2}{|c|}{ File number } \\
\cline { 2 - 3 } & MOR & ASA \\
\hline DIB $\left._{\text {(phys }}\right)$ & 1 & 11 \\
$\mathrm{~A}_{\text {III }}$ & 2 & 12 \\
$\mathrm{C}_{\text {III }}$ & 3 & 13 \\
1 & 4 & 14 \\
2 & 5 & 15 \\
5 & 6 & 16 \\
$1^{\prime}$ & 7 & 17 \\
$2^{\prime}$ & 8 & 18 \\
$3^{\prime}$ & 9 & 19 \\
$\mathrm{~A}$ & 10 & 20 \\
\hline \hline
\end{tabular}

The common first lines for files $\mathrm{n}^{\circ} 1$ to 10 are the following in the .cif files:

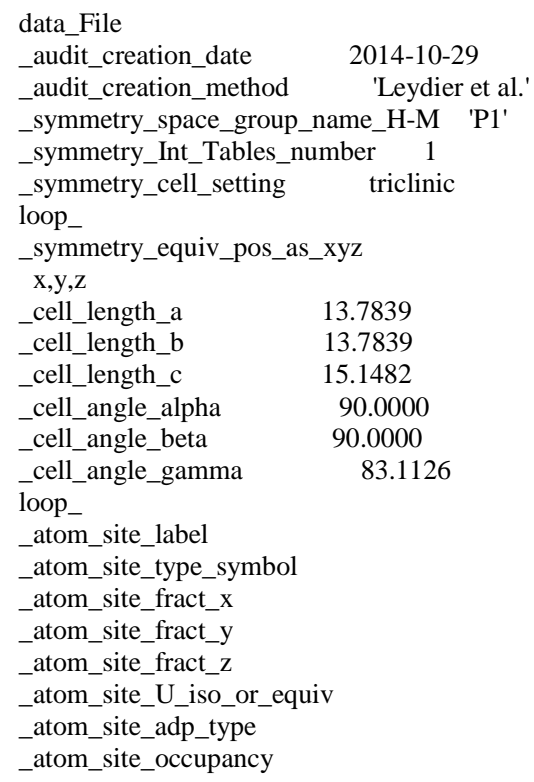

The common first lines for files $n^{\circ} 11$ to 20 are the following in the cif files:

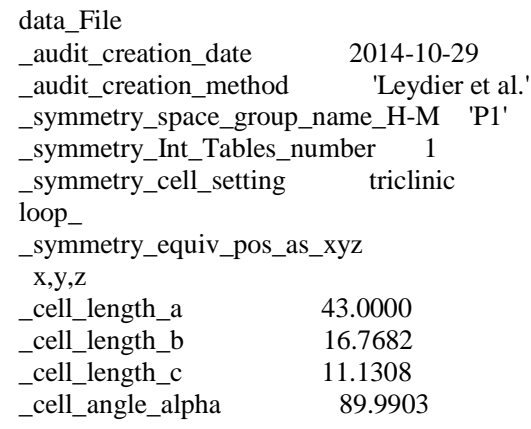


loop_

_atom_site_label

_atom_site_type_symbol

_atom_site_fract_x

_atom_site_fract_y

_atom_site_fract_z

_atom_site_U_iso_or_equiv

_atom_site_adp_type

_atom_site_occupancy

\section{Then come the coordinates :}

\section{File $\mathbf{n}^{\circ} \mathbf{1}$}

$\begin{array}{llllllll}\text { Si2 } & \text { Si } & 0.37635 & 0.76224 & 0.02399 & 0.00000 & \text { Uiso } & 1.00\end{array}$ $\begin{array}{llllllll}\mathrm{Si} 3 & \mathrm{Si} & 0.60998 & 0.00605 & 0.02213 & 0.00000 & \text { Uiso } & 1.00\end{array}$ $\begin{array}{llllllll}\mathrm{Si} 4 & \mathrm{Si} & 0.61276 & 0.23289 & 0.27342 & 0.00000 & \text { Uiso } & 1.00\end{array}$ $\begin{array}{llllllll}\mathrm{Si} 4 & \mathrm{Si} & 0.61276 & 0.23289 & 0.27342 & 0.00000 & \text { Uiso } & 1.00 \\ \mathrm{Si} 5 & \mathrm{Si} & 0.37603 & 0.98743 & 0.27484 & 0.00000 & \text { Uiso } & 1.00 \\ \mathrm{Si6} & \mathrm{Si} & 0.76183 & 0.37630 & 0.23162 & 0.00000 & \text { Uiso } & 1.00\end{array}$ $\begin{array}{llllllll}\mathrm{Si6} & \mathrm{Si} & 0.76183 & 0.37630 & 0.23162 & 0.00000 & \text { Uiso } & 1.00 \\ \mathrm{Si7} & \mathrm{Si} & 0.00743 & 0.60133 & 0.22567 & 0.00000 & \text { Uiso } & 1.00\end{array}$ $\begin{array}{lllllllll}\text { S17 } & \text { Si } & 0.00743 & 0.60133 & 0.22567 & 0.00000 & \text { Uiso } & 1.00\end{array}$ $\begin{array}{llllllll}\mathrm{Si} 8 & \mathrm{Si} & 0.23320 & 0.61310 & 0.47975 & 0.00000 & \text { Uiso } & 1.00 \\ \mathrm{Si} 9 & \mathrm{Si} & 0.98763 & 0.38190 & 0.47806 & 0.00000 & \text { Uis } & 1.00\end{array}$ $\begin{array}{llllllll}\mathrm{Si} 9 & \mathrm{Si} & 0.98763 & 0.38190 & 0.47806 & 0.00000 & \text { Uiso } & 1.00\end{array}$ $\begin{array}{llllllll}\text { Si10 } & \text { Si } & 0.61599 & 0.23035 & 0.48027 & 0.00000 & \text { Uiso } & 1.00\end{array}$ $\begin{array}{llllllll}\text { Si11 } & \text { Si } & 0.37397 & 0.99002 & 0.48146 & 0.00000 & \text { Uiso } & 1.00\end{array}$ $\begin{array}{lllllllll}\mathrm{Si} 12 & \mathrm{Si} & 0.37363 & 0.76351 & 0.23056 & 0.00000 & \text { Uiso } & 1.00\end{array}$ $\begin{array}{llllllll}\text { Si13 } & \text { Si } & 0.60226 & 0.00744 & 0.22857 & 0.00000 & \text { Uiso } & 1.00\end{array}$ $\begin{array}{llllllll}\text { Si14 } & \text { Si } & 0.23068 & 0.61073 & 0.27227 & 0.00000 & \text { Uiso } & 1.00\end{array}$ $\begin{array}{lllllllll}\text { Si15 } & \text { Si } & 0.98867 & 0.37519 & 0.27338 & 0.00000 & \text { Uiso } & 1.00\end{array}$ $\begin{array}{lllllllll}\text { Sil6 } & \text { Si } & 0.76363 & 0.37945 & 0.02694 & 0.00000 & \text { Uiso } & 1.00\end{array}$ $\begin{array}{lllllllll}\text { Si17 } & \text { Si } & 0.00587 & 0.60456 & 0.01884 & 0.00000 & \text { Uiso } & 1.00\end{array}$ $\begin{array}{llllllll}\text { Sil8 } & \text { Si } & 0.46337 & 0.28870 & 0.12564 & 0.00000 & \text { Uiso } & 1.00\end{array}$

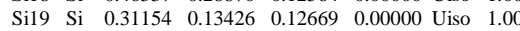
$\begin{array}{llllllll}\mathrm{Si} 20 & \mathrm{Si} & 0.53622 & 0.70496 & 0.37567 & 0.00000 & \text { Uiso } & 1.00\end{array}$ $\begin{array}{llllllll}\mathrm{Si} 21 & \mathrm{Si} & 0.68767 & 0.86587 & 0.37482 & 0.00000 & \text { Uiso } & 1.00\end{array}$ $\begin{array}{llllllll}\mathrm{Si} 22 & \mathrm{Si} & 0.28800 & 0.46222 & 0.12705 & 0.00000 & \text { Uiso } & 1.00\end{array}$ $\begin{array}{llllllll}\mathrm{Si} 23 & \mathrm{Si} & 0.13541 & 0.30797 & 0.12751 & 0.00000 & \text { Uiso } & 1.00\end{array}$ $\begin{array}{lllllllll}\mathrm{Si} 24 & \mathrm{Si} & 0.69847 & 0.53397 & 0.37986 & 0.00000 & \text { Uiso } & 1.00\end{array}$

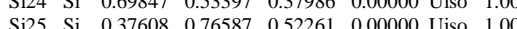
$\begin{array}{llllllllllll}\mathrm{Si} 26 & \mathrm{Si} & 0.60173 & 0.00601 & 0.52554 & 0.00000 & \text { Uiso } & 1.00\end{array}$ $\begin{array}{llllllllll}\text { Si26 Si } & 0.60173 & 0.00601 & 0.52554 & 0.00000 & \text { Uiso } & 1.00\end{array}$ $\begin{array}{llllllllllllllll}\text { Si27 Si } & 0.62203 & 0.22622 & 0.77484 & 0.00000 & \text { Uiso } & 1.00\end{array}$ $\begin{array}{llllllllll}\text { Si28 Si } & 0.37891 & 0.99077 & 0.77306 & 0.00000 & \text { Uiso } & 1.00\end{array}$ $\begin{array}{llllllll}\mathrm{Si} 29 & \mathrm{Si} & 0.76380 & 0.37305 & 0.72890 & 0.00000 & \text { Uiso } & 1.00 \\ \mathrm{Si} 30 & \mathrm{Si} & 0.00658 & 0.60475 & 0.73461 & 0.0000 & \text { Uiso } & 1.00\end{array}$ $\begin{array}{llllllll}\mathrm{Si30} & \mathrm{Si} & 0.00658 & 0.60475 & 0.73461 & 0.00000 & \text { Uiso } & 1.00\end{array}$ $\begin{array}{llllllll}\mathrm{Si} 31 & \mathrm{Si} & 0.22787 & 0.62046 & 0.97997 & 0.00000 & \text { Uiso } & 1.00\end{array}$ $\begin{array}{llllllll}\mathrm{Si32} & \mathrm{Si} & 0.98997 & 0.37863 & 0.97926 & 0.00000 & \text { Uiso } & 1.00\end{array}$ $\begin{array}{llllllll}\mathrm{Si33} & \mathrm{Si} & 0.62282 & 0.22881 & 0.98133 & 0.00000 & \text { Uiso } & 1.00\end{array}$ $\begin{array}{lllllllll}\mathrm{Si3} 4 & \mathrm{Si} & 0.38365 & 0.98899 & 0.97769 & 0.00000 & \text { Uiso } & 1.00\end{array}$ $\begin{array}{lllllllll}\mathrm{Si35} & \mathrm{Si} & 0.37449 & 0.76504 & 0.72878 & 0.00000 & \text { Uiso } & 1.00\end{array}$ $\begin{array}{lllllllll}\text { Si36 Si } & 0.60737 & 0.00346 & 0.73141 & 0.00000 & \text { Uiso } & 1.00\end{array}$ $\begin{array}{lllllllll}\text { Si37 } & \text { Si } & 0.22999 & 0.61899 & 0.77447 & 0.00000 & \text { Uiso } & 1.00\end{array}$ $\begin{array}{lllllllll}\text { Si38 } & \text { Si } & 0.98887 & 0.37996 & 0.77686 & 0.00000 & \text { Uiso } & 1.00\end{array}$ $\begin{array}{llllllll}\mathrm{Si} 39 & \mathrm{Si} & 0.76054 & 0.37520 & 0.52324 & 0.00000 & \text { Uiso } & 1.00\end{array}$ $\begin{array}{llllllll}\mathrm{Si} 40 & \mathrm{Si} & 0.01029 & 0.60608 & 0.52675 & 0.00000 & \text { Uiso } & 1.00\end{array}$ $\begin{array}{llllllll}\mathrm{Si} 41 & \mathrm{Si} & 0.46504 & 0.28842 & 0.62868 & 0.00000 & \text { Uiso } & 1.00\end{array}$ $\begin{array}{llllllll}\mathrm{Si} 42 & \mathrm{Si} & 0.30836 & 0.13835 & 0.62655 & 0.00000 & \text { Uiso } & 1.00\end{array}$ $\begin{array}{llllllll}\mathrm{Si} 43 & \mathrm{Si} & 0.53108 & 0.70222 & 0.87713 & 0.00000 & \text { Uiso } & 1.00\end{array}$ $\begin{array}{lllllllll}\mathrm{Si} 44 & \mathrm{Si} & 0.68734 & 0.85795 & 0.87837 & 0.00000 & \text { Uiso } & 1.00\end{array}$ $\begin{array}{llllllll}S \mathrm{Si} 45 & \mathrm{Si} & 0.29148 & 0.46445 & 0.62603 & 0.00000 & \text { Uiso } & 1.00\end{array}$ $\begin{array}{lllllll}\text { Si46 Si } & 0.13457 & 0.31433 & 0.62610 & 0.00000 & \text { Uiso } & 1.00\end{array}$ $\begin{array}{lllllllll}\mathrm{Si} 47 & \mathrm{Si} & 0.13457 & 0.31433 & 0.62610 & 0.00000 & \text { Uso } & 1.00\end{array}$ $\begin{array}{llllllll}\mathrm{Si47} & \mathrm{Si} & 0.70264 & 0.53078 & 0.87549 & 0.00000 & \text { Uiso } & 1.00 \\ \mathrm{Si} 8 & \mathrm{Si} & 0.85897 & 0.68625 & 0.87467 & 0.00000 & \text { Uiso } & 1.00\end{array}$

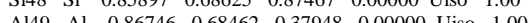
$\begin{array}{llllllll}\text { Al49 } & \text { Al } & 0.86746 & 0.68462 & 0.37948 & 0.00000 & \text { Uiso } & 1.00\end{array}$ $\begin{array}{lllllllll}\mathrm{O} 50 & \mathrm{O} & 0.52569 & 0.29401 & 0.21653 & 0.00000 & \text { Uiso } & 1.00 \\ \mathrm{O} 51 & \mathrm{O} & 0.31053 & 0.06899 & 0.21610 & 0.00000 & \text { Uiso } & 1.00\end{array}$ $\begin{array}{llllllll}051 & 0 & 0.31053 & 0.06899 & 0.21610 & 0.00000 & \text { Uiso } & 1.00\end{array}$ $\begin{array}{llllllll}\mathrm{O} 52 & \mathrm{O} & 0.34754 & 0.88294 & 0.51000 & 0.00000 & \text { Uiso } & 1.00 \\ \mathrm{O} 53 & \mathrm{O} & 0.47331 & 0.72886 & 0.46536 & 0.00000 & \text { Uiso } & 1.00\end{array}$ $\begin{array}{llllllll}\mathrm{O} 54 & \mathrm{O} & 0.67113 & 0.92884 & 0.46462 & 0.00000 & \text { Uiso } & 1.00\end{array}$ $\begin{array}{llllllll}0555 & \mathrm{O} & 0.62541 & 0.11870 & 0.24321 & 0.00000 & \text { Uiso } & 1.00\end{array}$ $\begin{array}{llllllll}\mathrm{O} 56 & \mathrm{O} & 0.28182 & 0.53375 & 0.04164 & 0.00000 & \text { Uiso } & 1.00\end{array}$ $\begin{array}{llllllll}057 & \mathrm{O} & 0.06881 & 0.31067 & 0.03889 & 0.00000 & \text { Uiso } & 1.00\end{array}$ $\begin{array}{llllllll}0588 & \mathrm{O} & 0.87886 & 0.35436 & 0.24673 & 0.00000 & \text { Uiso } & 1.00\end{array}$ $\begin{array}{lllllllll}059 & \mathrm{O} & 0.71188 & 0.46544 & 0.29181 & 0.00000 & \text { Uiso } & 1.00\end{array}$ $\begin{array}{llllllll}\mathrm{O} 60 & \mathrm{O} & 0.93517 & 0.67508 & 0.28361 & 0.00000 & \text { Uiso } & 1.00\end{array}$ $\begin{array}{llllllll}\text { O61 } & \text { O } & 0.11219 & 0.64077 & 0.00197 & 0.00000 & \text { Uiso } & 1.00\end{array}$ $\begin{array}{llllllll}\mathrm{O} 62 & \mathrm{O} & 0.46711 & 0.71990 & 0.28922 & 0.00000 & \text { Uiso } & 1.00 \\ 063 & \mathrm{O} & 0.67864 & 0.93539 & 0.28843 & 0.00000 & \text { Usiso } & 1.00\end{array}$ $\begin{array}{lllllllll}063 & 0 & 0.67864 & 0.93539 & 0.28843 & 0.00000 & \text { Uiso } & 1.00 \\ 064 & 0 & 0.64304 & 0.11409 & 0.00844 & 0.00000 & \text { Uiso } & 1.00\end{array}$ $\begin{array}{llllllll}064 & 0 & 0.64304 & 0.1140 & 0.0084 & 0.0000 & \text { Uiso } & 1.00 \\ 065 & \mathrm{O} & 0.53422 & 0.28450 & 0.03981 & 0.00000 & \text { Uiso } & 1.00\end{array}$ \begin{tabular}{lllllllll}
065 & 0 & 0.53422 & 0.28450 & 0.03981 & 0.00000 & Uiso & 1.00 \\
\hline 666 & 0 & 0.31495 & 0.06460 & 0.03996 & 0.00000 & Uiso & 1.00
\end{tabular} \begin{tabular}{lllllllll}
$\mathrm{O} 66$ & $\mathrm{O}$ & 0.31495 & 0.06460 & 0.03996 & 0.00000 & Uiso & 1.00 \\
\hline $\mathrm{O} 67$ & $\mathrm{O}$ & 0.34865 & 0.88030 & 0.24749 & 0.00000 & Uiso & 1.00
\end{tabular} $\begin{array}{llllllll}\text { O67 } & \mathrm{O} & 0.34865 & 0.88030 & 0.24749 & 0.00000 & \text { Uiso } & 1.00\end{array}$ $\begin{array}{llllllllll}0688 & 0 & 0.7264 & 0.46299 & 0.46632 & 0.00000 & \text { Uiso } & 1.00\end{array}$ $\begin{array}{llllllllll}069 & 0 & 0.93866 & 0.68475 & 0.47312 & 0.00000 & \text { Uiso } & 1.00\end{array}$ $\begin{array}{llllllll}070 & \mathrm{O} & 0.12034 & 0.62542 & 0.23423 & 0.00000 & \text { Uiso } & 1.00 \\ 071 & \mathrm{O} & 0.29568 & 0.52351 & 0.21807 & 0.00000 & \text { Uiso } & 1.00\end{array}$ $\begin{array}{llllllll}\mathrm{O} 71 & \mathrm{O} & 0.29568 & 0.52351 & 0.21807 & 0.00000 & \text { Uiso } & 1.00\end{array}$ $\begin{array}{llllllll}\mathrm{O} 72 & \mathrm{O} & 0.06727 & 0.30445 & 0.21483 & 0.00000 & \text { Uiso } & 1.00 \\ \mathrm{O} 73 & \mathrm{O} & 0.87722 & 0.36380 & 0.50522 & 0.00000 & \text { Uiso } & 1.00\end{array}$ $\begin{array}{llllllll}073 & \mathrm{O} & 0.87722 & 0.36380 & 0.50522 & 0.00000 & \text { Uiso } & 1.00\end{array}$ $\begin{array}{llllllll}074 & \mathrm{O} & 0.49209 & -0.00679 & 0.25929 & 0.00000 & \text { Uiso } & 1.00\end{array}$ $\begin{array}{llllllll}075 & \mathrm{O} & 0.49796 & 0.00466 & 0.98923 & 0.00000 & \text { Uiso } & 1.00\end{array}$

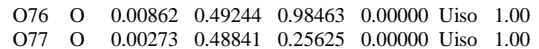


$\begin{array}{llllllll}\text { O78 } & \mathrm{O} & 0.38537 & 0.38511 & 0.11773 & 0.00000 & \text { Uiso } & 1.00\end{array}$ $\begin{array}{lllllllll}079 & \mathrm{O} & 0.21314 & 0.21035 & 0.12433 & 0.00000 & \text { Uiso } & 1.00\end{array}$ $\begin{array}{llllllllll}080 & \mathrm{O} & 0.58840 & 0.59369 & 0.37740 & 0.00000 & \text { Uiso } & 1.00\end{array}$ $\begin{array}{llllllll}081 & \mathrm{O} & 0.80482 & 0.81387 & 0.37784 & 0.00000 & \text { Uiso } & 1.00\end{array}$ $\begin{array}{lllllllll}082 & 0 & 0.40918 & 0.19003 & 0.12780 & 0.00000 & \text { Uiso } & 1.00\end{array}$ $\begin{array}{lllllllll}083 & \mathrm{O} & 0.61873 & 0.78052 & 0.36889 & 0.00000 & \text { Uiso } & 1.00\end{array}$ $\begin{array}{lllllllllll}084 & \mathrm{O} & 0.19096 & 0.40572 & 0.13054 & 0.00000 & \text { Uiso } & 1.00\end{array}$ $\begin{array}{lllllllll}084 & \mathrm{O} & 0.19096 & 0.40572 & 0.13054 & 0.00000 & \text { Uiso } & 1.00 \\ 085 & \mathrm{O} & 0.77863 & 0.60817 & 0.38461 & 0.00000 & \text { Uiso } & 1.00\end{array}$ $\begin{array}{lllllllll}085 & 0 & 0.77863 & 0.60817 & 0.38461 & 0.00000 & \text { Uiso } & 1.00 \\ 0.00000 & \text { Uiso } & 1.00\end{array}$ $\begin{array}{llllllll}086 & \mathrm{O} & 0.3494 & 0.00931 & 0.37778 & 0.00000 & \text { Uiso } & 1.00\end{array}$ $\begin{array}{llllllllll}087 & 0 & 0.58349 & 0.24166 & 0.37728 & 0.00000 & \text { Uiso } & 1.00\end{array}$ $\begin{array}{lllllllll}088 & \mathrm{O} & 0.61936 & 0.97614 & 0.12603 & 0.00000 & \text { Unso } & 1.00\end{array}$ $\begin{array}{llllllll}089 & \mathrm{O} & 0.40202 & 0.74263 & 0.12761 & 0.00000 & \text { Uiso } & 1.00\end{array}$ $\begin{array}{lllllllll}090 & \mathrm{O} & 0.00853 & 0.34747 & 0.37658 & 0.00000 & \text { Uiso } & 1.00\end{array}$ $\begin{array}{llllllll}\mathrm{O} 91 & \mathrm{O} & 0.23271 & 0.58145 & 0.37637 & 0.00000 & \text { Uiso } & 1.00 \\ 092 & \mathrm{O} & 0.97640 & 0.61222 & 0.12194 & 0.00000 & \text { Uiso } & 1.00\end{array}$ $\begin{array}{lllllllll}092 & \mathrm{O} & 0.97640 & 0.61222 & 0.12194 & 0.00000 & \text { Uiso } & 1.00\end{array}$ $\begin{array}{lllllllll}093 & \mathrm{O} & 0.73804 & 0.40589 & 0.12927 & 0.00000 & \text { Uiso } & 1.00\end{array}$ $\begin{array}{llllllll}094 & \mathrm{O} & 0.71596 & 0.27544 & 0.25577 & 0.00000 & \text { Uiso } & 1.00\end{array}$ $\begin{array}{llllllll}095 & \mathrm{O} & 0.27335 & 0.72223 & -0.00066 & 0.00000 & \text { Uiso } & 1.00\end{array}$ $\begin{array}{llllllll}096 & \mathrm{O} & 0.27786 & 0.71253 & 0.25812 & 0.00000 & \text { Uiso } & 1.00\end{array}$ $\begin{array}{llllllll}097 & \mathrm{O} & 0.72251 & 0.27752 & -0.00012 & 0.00000 & \text { Uiso } & 1.00\end{array}$ $\begin{array}{llllllll}098 & \mathrm{O} & 0.53404 & 0.28296 & 0.71597 & 0.00000 & \text { Uiso } & 1.00\end{array}$ $\begin{array}{lllllllll}099 & \mathrm{O} & 0.31035 & 0.07022 & 0.71429 & 0.00000 & \text { Uiso } & 1.00\end{array}$ $\begin{array}{lllllllll}0100 & \mathrm{O} & 0.36638 & 0.87844 & 0.00373 & 0.00000 & \text { Uiso } & 1.00\end{array}$ $\begin{array}{llllllll}0101 & \mathrm{O} & 0.46336 & 0.70443 & 0.96504 & 0.00000 & \text { Uiso } & 1.00\end{array}$ $\begin{array}{lllllllll}0102 & 0 & 0.68223 & 0.92761 & 0.96555 & 0.00000 & \text { Uiso } & 1.00\end{array}$ $\begin{array}{lllllllll}0103 & \mathrm{O} & 0.63916 & 0.11100 & 0.74987 & 0.00000 & \text { Uiso } & 1.00\end{array}$ $\begin{array}{llllllllll}0104 & 0 & 0.29834 & 0.52739 & 0.743576 & 0.00000 & \text { Uiso } & 1.00\end{array}$

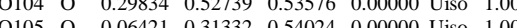

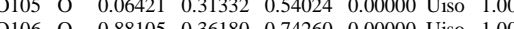
$\begin{array}{lllllllll}0.0 .0000 & 0 & 0.88105 & 0.36180 & 0.74260 & 0.0000 & \text { Uiso } & 1.00\end{array}$ $\begin{array}{llllllllll}0107 & \mathrm{O} & 0.70877 & 0.45931 & 0.79016 & 0.00000 & \text { Uiso } & 1.00\end{array}$ $\begin{array}{llllllllll}0108 & 0 & 0.92754 & 0.68127 & 0.7865 & 0.00000 & \text { Uiso } & 1.00\end{array}$ $\begin{array}{lllllllll}0109 & 0 & 0.12343 & 0.63039 & 0.51940 & 0.00000 & \text { Uiso } & 1.00 \\ 0110 & 0 & 0.46500 & 0.7146 & 0.78817 & 0.00000 & \text { Uiso } & 1.00\end{array}$ $\begin{array}{lllllllll}0110 & \mathrm{O} & 0.46500 & 0.71461 & 0.78817 & 0.00000 & \text { Uiso } & 1.00\end{array}$ $\begin{array}{lllllllll}0111 & \mathrm{O} & 0.67683 & 0.92353 & 0.78872 & 0.00000 & \text { Uiso } & 1.00\end{array}$ $\begin{array}{lllllllll}0112 & \mathrm{O} & 0.62938 & 0.11525 & 0.50756 & 0.00000 & \text { Uiso } & 1.00\end{array}$ $\begin{array}{llllllll}0113 & \mathrm{O} & 0.53084 & 0.29070 & 0.53969 & 0.00000 & \text { Uiso } & 1.00\end{array}$ $\begin{array}{llllllll}0114 & \mathrm{O} & 0.30600 & 0.07199 & 0.53786 & 0.00000 & \text { Uiso } & 1.00\end{array}$ $\begin{array}{lllllllll}0115 & \mathrm{O} & 0.35719 & 0.88214 & 0.74458 & 0.00000 & \text { Uiso } & 1.00\end{array}$ $\begin{array}{llllllll}0116 & \mathrm{O} & 0.71123 & 0.46807 & 0.96648 & 0.00000 & \text { Uiso } & 1.00\end{array}$ $\begin{array}{lllllllll}0117 & \mathrm{O} & 0.92494 & 0.67573 & 0.96374 & 0.00000 & \text { Uiso } & 1.00\end{array}$ $\begin{array}{llllllll}0118 & \mathrm{O} & 0.11470 & 0.63663 & 0.75071 & 0.00000 & \text { Uiso } & 1.00\end{array}$ $\begin{array}{lllllllll}0119 & \mathrm{O} & 0.28703 & 0.53349 & 0.71324 & 0.00000 & \text { Uiso } & 1.00\end{array}$ $\begin{array}{llllllll}0120 & \mathrm{O} & 0.07027 & 0.31690 & 0.71603 & 0.00000 & \text { Uiso } & 1.00\end{array}$ $\begin{array}{lllllllll}0121 & \mathrm{O} & 0.88089 & 0.36534 & 0.01323 & 0.00000 & \text { Uiso } & 1.00\end{array}$ $\begin{array}{lllllllll}0122 & \mathrm{O} & 0.49362 & 0.00183 & 0.75785 & 0.00000 & \text { Uiso } & 1.00\end{array}$ $\begin{array}{llllllllll}0123 & \mathrm{O} & 0.48878 & -0.00167 & 0.50011 & 0.00000 & \text { Uiso } & 1.00\end{array}$ $\begin{array}{lllllllll}0124 & \mathrm{O} & 0.00388 & 0.49581 & 0.48855 & 0.00000 & \text { Uiso } & 1.00\end{array}$ $\begin{array}{lllllllll}0125 & 0 & 0.00205 & 0.49532 & 0.77329 & 0.00000 & \text { Uiso } & 1.00\end{array}$ $\begin{array}{lllllllll}0125 & 0 & 0.00205 & 0.4532 & 0.77329 & 0.00000 & \text { Uiso } & 1.00\end{array}$ $\begin{array}{llllllllllll}0126 & 0 & 0.38865 & 0.38556 & 0.63332 & 0.00000 & \text { Uiso } & 1.00\end{array}$ $\begin{array}{lllllllll}0127 & \mathrm{O} & 0.21080 & 0.21555 & 0.62739 & 0.00000 & \text { Uiso } & 1.00\end{array}$ $\begin{array}{lllllllll}0128 & 0 & 0.59768 & 0.59742 & 0.87347 & 0.00000 & \text { Uiso } & 1.00\end{array}$ $\begin{array}{lllllllll}0129 & 0 & 0.79227 & 0.79148 & 0.87664 & 0.00000 & \text { Uiso } & 1.00 \\ 0130 & 0 & 0.40733 & 0.19211 & 0.62532 & 0.00000 & \text { Uiso } & 1.00\end{array}$ $\begin{array}{llllllllll}0130 & \mathrm{O} & 0.40733 & 0.19211 & 0.62532 & 0.0000 & \text { Uiso } & 1.00 \\ 0131 & \mathrm{O} & 0.59808 & 0.79114 & 0.88271 & 0.0000 & \text { Uiso } & 1.00\end{array}$

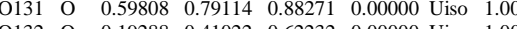
$\begin{array}{llllllll}0132 & \mathrm{O} & 0.19288 & 0.41022 & 0.62232 & 0.00000 & \text { Uiso } & 1.00\end{array}$ $\begin{array}{llllllll}0133 & \mathrm{O} & 0.79113 & 0.59792 & 0.87073 & 0.00000 & \text { Uiso } & 1.00\end{array}$ $\begin{array}{llllllll}0134 & \mathrm{O} & 0.35114 & 0.01102 & 0.87601 & 0.00000 & \text { Uiso } & 1.00\end{array}$ $\begin{array}{llllllll}0135 & \mathrm{O} & 0.59148 & 0.24021 & 0.87807 & 0.00000 & \text { Uiso } & 1.00\end{array}$ $\begin{array}{llllllll}0136 & \mathrm{O} & 0.62336 & 0.97558 & 0.62759 & 0.00000 & \text { Uiso } & 1.00\end{array}$ $\begin{array}{llllllll}0137 & \mathrm{O} & 0.40079 & 0.74128 & 0.62570 & 0.00000 & \text { Uiso } & 1.00\end{array}$ $\begin{array}{lllllllll}0138 & \mathrm{O} & 0.00380 & 0.34156 & 0.87776 & 0.00000 & \text { Uiso } & 1.00\end{array}$ $\begin{array}{lllllllll}0139 & \mathrm{O} & 0.24586 & 0.58732 & 0.87747 & 0.00000 & \text { Uiso } & 1.00\end{array}$ $\begin{array}{lllllllll}0140 & \mathrm{O} & 0.97996 & 0.60817 & 0.63082 & 0.00000 & \text { Uiso } & 1.00\end{array}$ $\begin{array}{llllllllll}0141 & \mathrm{O} & 0.73784 & 0.39955 & 0.62670 & 0.00000 & \text { Uiso } & 1.00\end{array}$ $\begin{array}{llllllll}\mathrm{O} 142 & \mathrm{O} & 0.72440 & 0.26995 & 0.75530 & 0.00000 & \text { Uiso } & 1.00\end{array}$ $\begin{array}{lllllllll}0143 & \mathrm{O} & 0.28278 & 0.71417 & 0.48928 & 0.00000 & \text { Uiso } & 1.00\end{array}$ $\begin{array}{lllllllll}0144 & \mathrm{O} & 0.27423 & 0.72149 & 0.75615 & 0.00000 & \text { Uiso } & 1.00\end{array}$ $\begin{array}{llllllllll}0145 & \mathrm{O} & 0.72025 & 0.27180 & 0.49689 & 0.00000 & \text { Uiso } & 1.00\end{array}$ \begin{tabular}{llllllllll}
\hline 145 & $\mathrm{O}$ & 0.72025 & 0.27180 & 0.4988 & 0.00000 & Uiso & 1.00
\end{tabular} $\begin{array}{llllllllll}\mathrm{H} 146 & \mathrm{H} & 0.85327 & 0.86547 & 0.38240 & 0.00000 & \text { Uiso } & 1.00 \\ \mathrm{H} 147 & \mathrm{H} & 0.85747 & 0.96686 & 0.19324 & 0.00000 & \text { Uis } & 1.00\end{array}$ $\begin{array}{lllllllll}\mathrm{H} 147 & \mathrm{H} & 0.85747 & 0.9686 & 0.19324 & 0.00000 & \text { Uiso } & 1.00\end{array}$ $\begin{array}{lllllllll}H 148 & \mathrm{H} & 0.96343 & 0.10052 & 0.23380 & 0.00000 & \text { Uiso } & 1.00\end{array}$ $\begin{array}{lllllllll}\mathrm{H} 149 & \mathrm{H} & 0.86295 & 0.02357 & 0.34200 & 0.00000 & \text { Uiso } & 1.00 \\ \mathrm{H} 150 & \mathrm{H} & 0.81455 & 0.05802 & 0.47907 & 0.0000 & \text { Uiso } & 1.00\end{array}$ $\begin{array}{llllllll}\mathrm{H} 150 & \mathrm{H} & 0.81455 & 0.05802 & 0.47507 & 0.00000 & \text { Uiso } & 1.00 \\ \mathrm{H} 151 & \mathrm{H} & 0.91449 & 0.05603 & 0.55378 & 0.0000 & \text { Uiso } & 1.00\end{array}$ $\begin{array}{llllllll}\mathrm{H} 151 & \mathrm{H} & 0.91449 & 0.05603 & 0.55378 & 0.00000 & \text { Uiso } & 1.00\end{array}$ $\begin{array}{lllllllll}\mathrm{H} 152 & \mathrm{H} & 0.89795 & 0.84203 & 0.21932 & 0.00000 & \text { Unso } & 1.00\end{array}$ $\begin{array}{lllllllll}\mathrm{H} 153 & \mathrm{H} & 0.96267 & 0.90483 & 0.14038 & 0.00000 & \text { Uiso } & 1.00\end{array}$ $\begin{array}{llllllll}\mathrm{H} 154 & \mathrm{H} & 0.06599 & 0.03088 & 0.18319 & 0.00000 & \text { Uiso } & 1.00\end{array}$

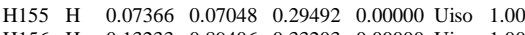
$\begin{array}{llllllll}\mathrm{H} 156 & \mathrm{H} & 0.13233 & 0.89406 & 0.33203 & 0.00000 & \text { Uiso } & 1.00\end{array}$ $\begin{array}{llllllll}\mathrm{H} 157 & \mathrm{H} & 0.11307 & 0.85544 & 0.22245 & 0.00000 & \text { Uiso } & 1.00\end{array}$ $\begin{array}{lllllllll}\text { H158 } & \mathrm{H} & 0.05636 & 0.79987 & 0.31077 & 0.00000 & \text { Uiso } & 1.00\end{array}$ $\begin{array}{llllllll}\mathrm{H} 159 & \mathrm{H} & 0.99938 & 0.85608 & 0.54620 & 0.00000 & \text { Uiso } & 1.00\end{array}$ $\begin{array}{llllllll}\mathrm{H} 160 & \mathrm{H} & 0.07824 & 0.94300 & 0.51662 & 0.00000 & \text { Uiso } & 1.00\end{array}$ $\begin{array}{lllllllll}\mathrm{H} 161 & \mathrm{H} & 0.06750 & 0.84097 & 0.44675 & 0.00000 & \text { Uiso } & 1.00\end{array}$ $\begin{array}{llllllll}\mathrm{H} 162 & \mathrm{H} & 0.84170 & 0.95893 & 0.55519 & 0.00000 & \text { Uiso } & 1.00\end{array}$ $\begin{array}{llllllll}\mathrm{C} 163 & \mathrm{C} & 0.02507 & 0.04342 & 0.24542 & 0.00000 & \text { Uiso } & 1.00\end{array}$ $\begin{array}{llllllll}\mathrm{C} 164 & \mathrm{C} & 0.87359 & 0.01030 & 0.51115 & 0.00000 & \text { Uiso } & 1.00\end{array}$ $\begin{array}{lllllllll}\mathrm{C} 165 & \mathrm{C} & 0.98897 & 0.94681 & 0.27587 & 0.00000 & \text { Uiso } & 1.00\end{array}$ $\begin{array}{lllllllllll}\text { C166 } & \text { C } & 0.92222 & 0.91298 & 0.20311 & 0.00000 & \text { Uiso } & 1.00\end{array}$ $\begin{array}{lllllllll}\mathrm{C} 166 & \mathrm{C} & 0.92222 & 0.91298 & 0.20311 & 0.0000 & \text { Uiso } & 1.00\end{array}$ $\begin{array}{lllllllll}\mathrm{C} 167 & \mathrm{C} & 0.07772 & 0.8654 & 0.28702 & 0.0000 & \text { Uiso } & 1.00 \\ \mathrm{C} 168 & \mathrm{C} & 0.92805 & 0.97239 & 0.35799 & 0.0000 & \text { Uiso } & 1.00\end{array}$ $\begin{array}{lllllllll}\mathrm{C} 168 & \mathrm{C} & 0.92805 & 0.97239 & 0.35799 & 0.00000 & \text { Uiso } & 1.00\end{array}$ $\begin{array}{lllllllll}\mathrm{C} 169 & \mathrm{C} & 0.94323 & 0.95679 & 0.44580 & 0.00000 & \text { Uiso } & 1.00 \\ \mathrm{C} 170 & \mathrm{C} & 0.02693 & 0.89488 & 0.48974 & 0.00000 & \text { Uiso } & 1.00\end{array}$

\section{File $n^{\circ} 2$}

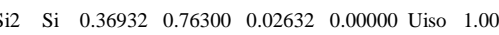

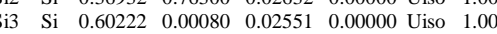
$\begin{array}{lllllllll}\mathrm{Si} 4 & \mathrm{Si} & 0.61474 & 0.23027 & 0.27519 & 0.00000 & \text { Uiso } & 1.00\end{array}$ $\begin{array}{llllllll}\mathrm{Si} 5 & \mathrm{Si} & 0.37466 & 0.98716 & 0.27724 & 0.00000 & \text { Uiso } & 1.00\end{array}$ 
$\begin{array}{llllllll}\text { Si6 } & \text { Si } & 0.75690 & 0.37866 & 0.23196 & 0.00000 & \text { Uiso } & 1.00\end{array}$ $\begin{array}{lllllllll}\mathrm{Si} 7 & \mathrm{Si} & 0.00858 & 0.60622 & 0.23057 & 0.00000 & \text { Uiso } & 1.00\end{array}$ $\begin{array}{lllllllll}\text { Si8 } & \text { Si } & 0.22783 & 0.61377 & 0.48225 & 0.00000 & \text { Uiso } & 1.00\end{array}$ $\begin{array}{llllllllll}\text { Si9 } & \text { Si } & 0.98908 & 0.37392 & 0.48201 & 0.00000 & \text { Uiso } & 1.00\end{array}$ $\begin{array}{llllllll}\mathrm{Si} 10 & \mathrm{Si} & 0.61465 & 0.22547 & 0.48223 & 0.00000 & \text { Uiso } & 1.00\end{array}$ $\begin{array}{llllllll}\text { Sil1 } & \text { Si } & 0.36684 & 0.99097 & 0.48339 & 0.00000 & \text { Uiso } & 1.00\end{array}$ $\begin{array}{llllllll}\text { Si12 } & \text { Si } & 0.37814 & 0.76019 & 0.23182 & 0.00000 & \text { Uiso } & 1.00\end{array}$ $\begin{array}{lllllllllll} & \mathrm{Si} 13 & \mathrm{Si} & 0.59952 & 0.00798 & 0.23195 & 0.00000 & \text { Uiso } & 1.00\end{array}$

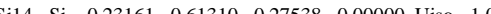
$\begin{array}{lllllllll}\text { Si14 } & \text { Si } & 0.23161 & 0.61310 & 0.27538 & 0.00000 & \text { Uiso } & 1.00\end{array}$ $\begin{array}{lllllllllll}\text { Si15 Si } & 0.98495 & 0.38163 & 0.27690 & 0.00000 & \text { Uiso } & 1.00\end{array}$ $\begin{array}{llllllll}\text { Sil6 Si } & 0.76389 & 0.36923 & 0.02685 & 0.00000 & \text { Uiso } & 1.00\end{array}$ $\begin{array}{lllllllll}\text { Sil7 } & \text { Si } & 0.00139 & 0.60138 & 0.02255 & 0.00000 & \text { Uiso } & 1.00\end{array}$ $\begin{array}{llllllll}\text { Si18 } & \text { Si } & 0.46230 & 0.28680 & 0.12849 & 0.00000 & \text { Uiso } & 1.00\end{array}$ $\begin{array}{llllllll}\text { Si19 } & \text { Si } & 0.30744 & 0.13481 & 0.13016 & 0.00000 & \text { Uiso } & 1.00\end{array}$ $\begin{array}{lllllllll}\text { Si20 } & \text { Si } & 0.53784 & 0.70334 & 0.37696 & 0.00000 & \text { Uiso } & 1.00\end{array}$ $\begin{array}{lllllllll}\text { Si21 } & \text { Si } & 0.68826 & 0.86440 & 0.37876 & 0.00000 & \text { Uiso } & 1.00\end{array}$

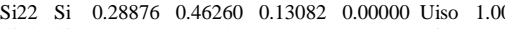
$\begin{array}{llllllll}\mathrm{Si} 23 & \mathrm{Si} & 0.13353 & 0.31086 & 0.13060 & 0.00000 & \text { Uiso } & 1.00\end{array}$ $\begin{array}{lllllllll}\mathrm{Si} 24 & \mathrm{Si} & 0.69863 & 0.53489 & 0.37711 & 0.00000 & \text { Uiso } & 1.00\end{array}$ $\begin{array}{lllllllll}\mathrm{Si22} & \mathrm{Si} & 0.37368 & 0.76625 & 0.52344 & 0.00000 & \text { Uiso } & 1.00\end{array}$ $\begin{array}{llllllll}\text { Si26 } & \text { Si } & 0.59425 & 0.00428 & 0.52863 & 0.00000 & \text { Uiso } & 1.00\end{array}$ $\begin{array}{llllllll}\text { Si27 } & \text { Si } & 0.61753 & 0.22628 & 0.77556 & 0.00000 & \text { Uiso } & 1.00\end{array}$ $\begin{array}{llllllll}\mathrm{Si28} & \mathrm{Si} & 0.37945 & 0.98516 & 0.77781 & 0.00000 & \text { Uiso } & 1.00\end{array}$ $\begin{array}{llllllll}\text { Si29 } & \text { Si } & 0.75701 & 0.37791 & 0.72935 & 0.00000 & \text { Uiso } & 1.00\end{array}$ $\begin{array}{llllllll}\mathrm{Si30} & \mathrm{Si} & 0.00627 & 0.60346 & 0.73944 & 0.00000 & \text { Uiso } & 1.00\end{array}$ $\begin{array}{lllllllll}\text { Si31 } & \text { Si } & 0.22597 & 0.61677 & 0.98183 & 0.00000 & \text { Uiso } & 1.00\end{array}$ $\begin{array}{lllllllll}\text { Si32 } & \text { Si } & 0.98701 & 0.37368 & 0.98182 & 0.00000 & \text { Uiso } & 1.00\end{array}$ $\begin{array}{lllllllll}\mathrm{Si} 33 & \mathrm{Si} & 0.61807 & 0.22313 & 0.98239 & 0.00000 & \text { Uiso } & 1.00\end{array}$ $\begin{array}{llllllllll}\mathrm{Si} 34 & \mathrm{Si} & 0.37463 & 0.98793 & 0.98185 & 0.00000 & \text { Uiso } & 1.00\end{array}$ $\begin{array}{llllllll} & \text { Si35 Si } & 0.37922 & 0.75738 & 0.72909 & 0.00000 & \text { Uiso } & 1.00\end{array}$ $\begin{array}{lllllllll}\text { Sis } & \text { Si } & 0.37922 & 0.75738 & 0.72919 & 0.00000 & \text { Uiso } & 1.00\end{array}$ $\begin{array}{lllllll}\text { Si36 Si } & 0.60422 & 0.00461 & 0.73497 & 0.00000 & \text { Uiso } & 1.00\end{array}$ $\begin{array}{lllllllll}\text { S137 } & \text { Si } & 0.23029 & 0.61605 & 0.77631 & 0.00000 & \text { Uiso } & 1.00\end{array}$ $\begin{array}{lllllllll}\text { Si38 } & \text { Si } & 0.98366 & 0.38029 & 0.77940 & 0.00000 & \text { Uiso } & 1.00\end{array}$ $\begin{array}{lllllllll}\text { Si39 } & \text { Si } & 0.76232 & 0.37208 & 0.52338 & 0.00000 & \text { Uiso } & 1.00\end{array}$ $\begin{array}{lllllllll}\text { Si40 } & \text { Si } & 0.00599 & 0.59964 & 0.53078 & 0.00000 & \text { Uiso } & 1.00\end{array}$ $\begin{array}{lllllllll}\mathrm{Si} 41 & \mathrm{Si} & 0.46062 & 0.28486 & 0.62982 & 0.00000 & \text { Uiso } & 1.00\end{array}$ $\begin{array}{lllllllll}\mathrm{Si} 42 & \mathrm{Si} & 0.30453 & 0.13348 & 0.63070 & 0.00000 & \text { Uiso } & 1.00\end{array}$ $\begin{array}{lllllllll}\mathrm{Si} 43 & \mathrm{Si} & 0.52936 & 0.69908 & 0.87826 & 0.00000 & \text { Uiso } & 1.00\end{array}$ $\begin{array}{llllllll}\mathrm{Si} 44 & \mathrm{Si} & 0.68443 & 0.85649 & 0.87908 & 0.00000 & \text { Uiso } & 1.00\end{array}$ $\begin{array}{lllllllll}\mathrm{Si} 45 & \mathrm{Si} & 0.28694 & 0.46094 & 0.62789 & 0.00000 & \text { Uiso } & 1.00\end{array}$ $\begin{array}{llllllll}\text { Si46 } & \text { Si } & 0.13158 & 0.30892 & 0.62948 & 0.00000 & \text { Uiso } & 1.00\end{array}$ $\begin{array}{llllllll}\mathrm{Si} 47 & \mathrm{Si} & 0.70119 & 0.52936 & 0.87780 & 0.00000 & \text { Uiso } & 1.00\end{array}$ $\begin{array}{lllllllll}\mathrm{Si} 48 & \mathrm{Si} & 0.85701 & 0.68608 & 0.87803 & 0.00000 & \text { Uiso } & 1.00\end{array}$ $\begin{array}{lllllllll}\mathrm{A} 149 & \mathrm{Al} & 0.86386 & 0.69007 & 0.37981 & 0.00000 & \text { Uiso } & 1.00\end{array}$ $\begin{array}{llllllll}050 & \mathrm{O} & 0.52796 & 0.29084 & 0.21756 & 0.00000 & \text { Uiso } & 1.00\end{array}$ $\begin{array}{lllllllll}051 & \mathrm{O} & 0.30637 & 0.06446 & 0.21635 & 0.00000 & \text { Uiso } & 1.00\end{array}$ $\begin{array}{llllllll}052 & \mathrm{O} & 0.34366 & 0.88379 & 0.51555 & 0.00000 & \text { Uiso } & 1.00\end{array}$ $\begin{array}{llllllllll}053 & 0 & 0.46828 & 0.73423 & 0.46144 & 0.00000 & \text { Uiso } & 1.00\end{array}$ $\begin{array}{llllllllll}054 & \mathrm{O} & 0.46828 & 0.73423 & 0.46144 & 0.00000 & \text { Uiso } & 1.00\end{array}$ $\begin{array}{llllllllll}055 & 0 & 0.65142 & 0.11701 & 0.24185 & 0.00000 & \text { Uiso } & 1.00\end{array}$ $\begin{array}{llllllll}055 & \mathrm{O} & 0.63142 & 0.11701 & 0.24185 & 0.00000 & \text { Uiso } & 1.00\end{array}$ $\begin{array}{llllllllllll}056 & \mathrm{O} & 0.28279 & 0.53195 & 0.04387 & 0.00000 & \text { Uiso } & 1.00\end{array}$ $\begin{array}{lllllllll}057 & 0 & 0.07286 & 0.31134 & 0.03865 & 0.00000 & \text { Uiso } & -1.00\end{array}$ $\begin{array}{lllllllllll}058 & 0 & 0.87369 & 0.36673 & 0.25000 & 0.0000 & \text { Uiso } & 1.00\end{array}$ $\begin{array}{lllllllll}059 & \mathrm{O} & 0.70006 & 0.46835 & 0.28749 & 0.00000 & \text { Uiso } & 1.00\end{array}$ $\begin{array}{llllllll}\mathrm{O} 60 & \mathrm{O} & 0.93939 & 0.68366 & 0.28791 & 0.00000 & \text { Uiso } & 1.00\end{array}$ $\begin{array}{llllllll}\mathrm{O} 61 & \mathrm{O} & 0.10967 & 0.63096 & 0.00169 & 0.00000 & \text { Uiso } & 1.00\end{array}$ $\begin{array}{lllllllll}\text { O62 } & \mathrm{O} & 0.47377 & 0.71392 & 0.28674 & 0.00000 & \text { Uiso } & 1.00\end{array}$ $\begin{array}{lllllllll}063 & \mathrm{O} & 0.67321 & 0.93494 & 0.29293 & 0.00000 & \text { Uiso } & 1.00\end{array}$ $\begin{array}{llllllll}\mathrm{O} 64 & \mathrm{O} & 0.63539 & 0.10861 & 0.01062 & 0.00000 & \text { Uiso } & 1.00\end{array}$ $\begin{array}{llllllll}065 & \mathrm{O} & 0.53054 & 0.28118 & 0.04079 & 0.00000 & \text { Uiso } & 1.00\end{array}$ $\begin{array}{llllllll}066 & \mathrm{O} & 0.30868 & 0.07025 & 0.04018 & 0.00000 & \text { Uiso } & 1.00\end{array}$ $\begin{array}{llllllllll}067 & \mathrm{O} & 0.35875 & 0.87748 & 0.24748 & 0.00000 & \text { Uiso } & 1.00\end{array}$ $\begin{array}{llllllll}\mathrm{O} 68 & \mathrm{O} & 0.71635 & 0.46132 & 0.46159 & 0.00000 & \text { Uiso } & 1.00\end{array}$ $\begin{array}{lllllllll}\mathrm{O} 69 & \mathrm{O} & 0.92604 & 0.67024 & 0.47829 & 0.00000 & \text { Uiso } & 1.00\end{array}$ $\begin{array}{llllllll}\mathrm{O} 70 & \mathrm{O} & 0.12190 & 0.63072 & 0.23565 & 0.00000 & \text { Uiso } & 1.00\end{array}$ $\begin{array}{llllllll}\mathrm{O} 71 & \mathrm{O} & 0.29632 & 0.52606 & 0.22059 & 0.00000 & \text { Uiso } & 1.00 \\ 072 & \mathrm{O} & 0.05988 & 0.31150 & 0.21438 & 0.00000 & \text { Usiso } & 1.00\end{array}$ $\begin{array}{lllllllll}072 & \mathrm{O} & 0.05988 & 0.31150 & 0.21438 & 0.00000 & \text { Uiso } & 1.00 \\ 073 & \mathrm{O} & 0.88027 & 0.35247 & 0.51204 & 0.00000 & \text { Uiso } & 1.00\end{array}$

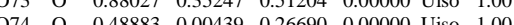
$\begin{array}{lllllllll}075 & 0 & 0.49002 & 0.00088 & 0.99335 & 0.00000 & \text { Uiso } & 1.00\end{array}$ $\begin{array}{lllllllll}075 & 0 & 0.49072 & 0.00438 & 0.26690 & 0.00000 & \text { Uiso } & 1.00\end{array}$ $\begin{array}{lllllllll}076 & \mathrm{O} & -0.00517 & 0.48983 & 0.99036 & 0.00000 & \text { Uiso } & 1.00 \\ 077 & \mathrm{O} & 0.00393 & 0.49471 & 0.26603 & 0.00000 & \text { Uiso } & 1.00\end{array}$ $\begin{array}{lllllllll}077 & 0 & 0.00393 & 0.4947 & 0.26363 & 0.00000 & \text { Uiso } & 1.00\end{array}$ $\begin{array}{lllllllllll}078 & 0 & 0.38576 & 0.38467 & 0.12287 & 0.00000 & \text { Uiso } & 1.00\end{array}$ $\begin{array}{lllllllll}079 & 0 & 0.20950 & 0.21173 & 0.13313 & 0.00000 & \text { Uiso } & 1.00\end{array}$ $\begin{array}{llllllll}080 & \mathrm{O} & 0.58766 & 0.59172 & 0.38776 & 0.00000 & \text { Uiso } & 1.00\end{array}$ $\begin{array}{lllllllll}081 & \mathrm{O} & 0.80853 & 0.82315 & 0.38306 & 0.00000 & \text { Uiso } & 1.00\end{array}$ $\begin{array}{lllllllll}082 & \mathrm{O} & 0.40571 & 0.18964 & 0.13309 & 0.00000 & \text { Uiso } & 1.00\end{array}$ $\begin{array}{llllllll}083 & \mathrm{O} & 0.62354 & 0.77527 & 0.36950 & 0.00000 & \text { Uiso } & 1.00\end{array}$ $\begin{array}{lllllllll}084 & \mathrm{O} & 0.19101 & 0.40715 & 0.13530 & 0.00000 & \text { Uiso } & 1.00\end{array}$ $\begin{array}{llllllll}085 & \mathrm{O} & 0.77630 & 0.61158 & 0.37125 & 0.00000 & \text { Uiso } & 1.00\end{array}$ $\begin{array}{llllllll}086 & \mathrm{O} & 0.34175 & 0.00559 & 0.37927 & 0.00000 & \text { Uiso } & 1.00\end{array}$ $\begin{array}{llllllll}087 & \mathrm{O} & 0.58310 & 0.23464 & 0.37872 & 0.00000 & \text { Uiso } & 1.00\end{array}$ $\begin{array}{lllllllll}088 & \mathrm{O} & 0.61157 & 0.97225 & 0.12995 & 0.00000 & \text { Uiso } & 1.00\end{array}$ $\begin{array}{lllllllll}\mathrm{O} 89 & \mathrm{O} & 0.39979 & 0.73687 & 0.12805 & 0.00000 & \text { Uiso } & 1.00\end{array}$ $\begin{array}{llllllll}090 & \mathrm{O} & 0.00536 & 0.34639 & 0.37830 & 0.00000 & \text { Uiso } & 1.00\end{array}$ $\begin{array}{llllllllll}091 & \mathrm{O} & 0.23099 & 0.58176 & 0.37882 & 0.00000 & \text { Uiso } & 1.00\end{array}$ $\begin{array}{lllllllll}\mathrm{O} 92 & \mathrm{O} & 0.97648 & 0.61234 & 0.12667 & 0.00000 & \text { Uiso } & 1.00\end{array}$ $\begin{array}{lllllllll}\mathrm{O} 93 & \mathrm{O} & 0.73432 & 0.39953 & 0.12778 & 0.00000 & \text { Uiso } & 1.00\end{array}$ $\begin{array}{llllllllll}094 & 0 & 0.71714 & 0.27587 & 0.26095 & 0.00000 & \text { Uiso } & 1.00\end{array}$ $\begin{array}{llllllll}095 & 0 & 0.71714 & 0.27587 & 0.26095 & 0.00000 & \text { Uiso } & 1.00\end{array}$ $\begin{array}{llllllllll}096 & 0 & 0.28111 & 0.71413 & 0.26471 & 0.00000 & \text { Uiso } & 1.00\end{array}$ $\begin{array}{llllllllll}096 & 0 & 0.28111 & 0.71413 & 0.2647 & 0.00000 & \text { Uiso } & 1.00 \\ 097 & 0 & 0.71974 & 0.26876 & 0.99994 & 0.00000 & \text { Uis } & 1.00\end{array}$ $\begin{array}{llllllllll}097 & 0 & 0.71974 & 0.26876 & 0.9994 & 0.00000 & \text { Uiso } & 1.00\end{array}$ $\begin{array}{llllllllll}098 & 0 & 0.52811 & 0.28257 & 0.71803 & 0.00000 & \text { Uiso } & 1.00\end{array}$ $\begin{array}{lllllllll}099 & 0 & 0.31063 & 0.05894 & 0.71417 & 0.00000 & \text { Uiso } & 1.00\end{array}$ $\begin{array}{lllllllll}0100 & \mathrm{O} & 0.35251 & 0.88076 & 0.01433 & 0.00000 & \text { Uiso } & 1.00\end{array}$ $\begin{array}{lllllllll}0101 & \mathrm{O} & 0.45618 & 0.71388 & 0.96243 & 0.00000 & \text { Uiso } & 1.00\end{array}$ $\begin{array}{lllllllll}0102 & \mathrm{O} & 0.67409 & 0.92160 & 0.96929 & 0.00000 & \text { Uiso } & 1.00\end{array}$ $\begin{array}{llllllll}0103 & \mathrm{O} & 0.63894 & 0.11238 & 0.74585 & 0.00000 & \text { Uiso } & 1.00\end{array}$ $\begin{array}{lllllllll}\mathrm{O} 104 & \mathrm{O} & 0.28977 & 0.52692 & 0.53920 & 0.00000 & \text { Uiso } & 1.00 \\ \mathrm{O} 105 & \mathrm{O} & 0.06878 & 0.30239 & 0.53914 & 0.00000 & \text { Uiso } & 1.00\end{array}$ $\begin{array}{llllllll}0106 & \mathrm{O} & 0.87347 & 0.36820 & 0.74777 & 0.00000 & \text { Uiso } & 1.00\end{array}$ 
$\begin{array}{llllllll}0107 & \mathrm{O} & 0.70006 & 0.46711 & 0.78667 & 0.00000 & \text { Uiso } & 1.00\end{array}$ $\begin{array}{lllllllll}0108 & \mathrm{O} & 0.92745 & 0.68077 & 0.79143 & 0.00000 & \text { Uiso } & 1.00\end{array}$ $\begin{array}{lllllllll}0109 & \mathrm{O} & 0.11590 & 0.63241 & 0.51723 & 0.00000 & \text { Uiso } & 1.00\end{array}$ $\begin{array}{llllllll}0110 & \mathrm{O} & 0.46811 & 0.69967 & 0.78637 & 0.00000 & \text { Uiso } & 1.00\end{array}$ $\begin{array}{lllllllll}0111 & \mathrm{O} & 0.67668 & 0.92658 & 0.79201 & 0.00000 & \text { Uiso } & 1.00\end{array}$ $\begin{array}{lllllllll}0112 & 0 & 0.63094 & 0.11033 & 0.51051 & 0.00000 & \text { Uiso } & 1.00\end{array}$ $\begin{array}{llllllllll}0113 & 0 & 0.52781 & 0.28369 & 0.54129 & 0.00000 & \text { Uiso } & 1.00\end{array}$ $\begin{array}{llllllllll}0113 & 0 & 0.52781 & 0.2836 & 0.54129 & 0.00000 & \text { Uiso } & 1.00\end{array}$

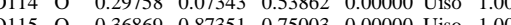
$\begin{array}{lllllllll}0115 & 0 & 0.36869 & 0.87351 & 0.75003 & 0.00000 & \text { Uiso } & 1.00\end{array}$ $\begin{array}{lllllllll}0116 & 0 & 0.71877 & 0.45728 & 0.96235 & 0.00000 & \text { Uiso } & 1.00 \\ 0117 & 0 & 0.92156 & 0.67508 & 0.96823 & 0.0000 & \text { Uis } & 1.00\end{array}$ $\begin{array}{lllllllll}0117 & \mathrm{O} & 0.72156 & 0.67508 & 0.96823 & 0.00000 & \text { Uiso } & 1.00 \\ 0118 & \mathrm{O} & 0.1454 & 0.6358 & 0.75460 & 0.00000 & \text { Us } & 1.00\end{array}$ $\begin{array}{llllllllll}0118 & \mathrm{O} & 0.11454 & 0.63586 & 0.75460 & 0.00000 & \text { Uiso } & 1.00\end{array}$ $\begin{array}{lllllllll}0119 & 0 & 0.28469 & 0.52799 & 0.71626 & 0.00000 & \text { Uiso } & 1.00\end{array}$ $\begin{array}{llllllll}0120 & \mathrm{O} & 0.06002 & 0.31617 & 0.71488 & 0.00000 & \text { Uiso } & 1.00\end{array}$ $\begin{array}{lllllllll}0121 & 0 & 0.88185 & 0.34927 & 0.01834 & 0.00000 & \text { Uiso } & 1.00\end{array}$ $\begin{array}{lllllllll}0122 & \mathrm{O} & 0.49277 & 0.00597 & 0.77009 & 0.00000 & \text { Uiso } & 1.00\end{array}$ $\begin{array}{llllllll}0123 & \mathrm{O} & 0.48058 & 0.00533 & 0.50103 & 0.00000 & \text { Uiso } & 1.00\end{array}$

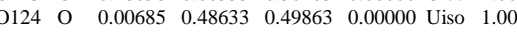

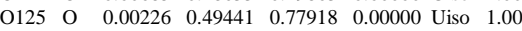
$\begin{array}{llllllll}0126 & \mathrm{O} & 0.38495 & 0.38367 & 0.63047 & 0.00000 & \text { Uiso } & 1.00\end{array}$ $\begin{array}{llllllll}0127 & \mathrm{O} & 0.20734 & 0.21048 & 0.63993 & 0.00000 & \text { Uiso } & 1.00\end{array}$ $\begin{array}{llllllll}0128 & 0 & 0.59591 & 0.59466 & 0.88889 & 0.00000 & \text { Uiso } & 1.00\end{array}$ $\begin{array}{llllllll}0129 & \mathrm{O} & 0.79051 & 0.79166 & 0.87926 & 0.00000 & \text { Uiso } & 1.00\end{array}$ $\begin{array}{llllllll}0130 & \mathrm{O} & 0.40287 & 0.18843 & 0.62938 & 0.00000 & \text { Uiso } & 1.00\end{array}$ $\begin{array}{llllllll}0131 & \mathrm{O} & 0.59665 & 0.78790 & 0.87584 & 0.00000 & \text { Uiso } & 1.00\end{array}$ $\begin{array}{llllllll}0132 & \mathrm{O} & 0.18949 & 0.40505 & 0.62619 & 0.00000 & \text { Uiso } & 1.00\end{array}$ $\begin{array}{lllllllll}0133 & 0 & 0.78816 & 0.59848 & 0.87279 & 0.00000 & \text { Uiso } & 1.00\end{array}$ $\begin{array}{llllllllllll}0134 & 0 & 0.34356 & 0.00457 & 0.87908 & 0.00000 & \text { Uiso } & 1.00\end{array}$ $\begin{array}{lllllllllll}0134 & 0 & 0.34356 & 0.0848 & 0.87279 & 0.00000 & \text { Uiso } & 1.00\end{array}$ $\begin{array}{lllllllll}0136 & 0 & 0.58675 & 0.23419 & 0.87912 & 0.00000 & \text { Uno } & 1.00 \\ 0136 & 0 & 0.61111 & 0.97332 & 0.63135 & 0.0000 & \text { Uiso } & 1.00\end{array}$ $\begin{array}{lllllllllll}0136 & \mathrm{O} & 0.61111 & 0.97332 & 0.63135 & 0.00000 & \text { Uiso } & 1.00 \\ 0137 & \mathrm{O} & 0.40360 & 0.73814 & 0.62502 & 0.00000 & \text { Uso } & 1.00\end{array}$ $\begin{array}{llllllllll}0137 & \mathrm{O} & 0.40360 & 0.73814 & 0.62502 & 0.00000 & \text { Uiso } & 1.00 \\ 0138 & \mathrm{O} & 0.00052 & 0.33939 & 0.87953 & 0.00000 & \text { Us } & 1.00\end{array}$ $\begin{array}{lllllllll}0138 & \mathrm{O} & 0.00052 & 0.33939 & 0.87953 & 0.00000 & \text { Uiso } & 1.00\end{array}$ $\begin{array}{llllllllll}0139 & \mathrm{O} & 0.24736 & 0.58541 & 0.87943 & 0.00000 & \text { Uiso } & 1.00\end{array}$ $\begin{array}{llllllllll}0140 & \mathrm{O} & 0.97999 & 0.60544 & 0.63576 & 0.00000 & \text { Uiso } & 1.00\end{array}$ $\begin{array}{lllllllll}0141 & \mathrm{O} & 0.73503 & 0.40018 & 0.62560 & 0.00000 & \text { Uiso } & 1.00\end{array}$ $\begin{array}{llllllll}0142 & \mathrm{O} & 0.71732 & 0.27562 & 0.75822 & 0.00000 & \text { Uiso } & 1.00\end{array}$ $\begin{array}{lllllllll}0143 & \mathrm{O} & 0.27934 & 0.71375 & 0.49345 & 0.00000 & \text { Uiso } & 1.00\end{array}$ $\begin{array}{llllllll}0144 & \mathrm{O} & 0.27674 & 0.71695 & 0.75517 & 0.00000 & \text { Uiso } & 1.00\end{array}$ $\begin{array}{llllllll}0145 & \mathrm{O} & 0.71693 & 0.27115 & 0.49769 & 0.00000 & \text { Uiso } & 1.00\end{array}$ $\begin{array}{lllllllll}\mathrm{H} 146 & \mathrm{H} & 0.92204 & 0.12467 & 0.29672 & 0.00000 & \text { Uiso } & 1.00\end{array}$ $\begin{array}{llllllll}\mathrm{H} 147 & \mathrm{H} & 0.89374 & 0.05117 & 0.43246 & 0.00000 & \text { Uiso } & 1.00\end{array}$ $\begin{array}{lllllllll}\mathrm{H} 148 & \mathrm{H} & 0.82077 & 0.98035 & 0.49642 & 0.00000 & \text { Uiso } & 1.00\end{array}$ $\begin{array}{lllllllll}\mathrm{H} 149 & \mathrm{H} & 0.77662 & 0.03387 & 0.39347 & 0.00000 & \text { Uiso } & 1.00\end{array}$ $\begin{array}{lllllllll}\mathrm{H} 150 & \mathrm{H} & 0.91969 & 0.85129 & 0.25233 & 0.00000 & \text { Uiso } & 1.00\end{array}$ $\begin{array}{llllllll}\mathrm{H} 151 & \mathrm{H} & 0.90326 & 0.04642 & 0.14301 & 0.00000 & \text { Uiso } & 1.00\end{array}$ $\begin{array}{lllllllll}\mathrm{H} 152 & \mathrm{H} & 0.97117 & 0.93125 & 0.11752 & 0.00000 & \text { Uiso } & 1.00\end{array}$ $\begin{array}{llllllll}\mathrm{H} 153 & \mathrm{H} & 0.03195 & 0.03820 & 0.12522 & 0.00000 & \text { Uiso } & 1.00\end{array}$ $\begin{array}{llllllllll}\mathrm{H} 154 & \mathrm{H} & 0.04615 & 0.12225 & 0.26413 & 0.00000 & \text { Uiso } & 1.00\end{array}$ $\begin{array}{lllllllll}\mathrm{H} 154 & \mathrm{H} & 0.04615 & 0.12225 & 0.26413 & 0.00000 & \text { Uiso } & 1.00 \\ \mathrm{H} 155 & \mathrm{H} & 0.01877 & 0.07274 & 0.36737 & 0.00000 & \text { Uis } & 1.00\end{array}$ $\begin{array}{llllllllll}1155 & \mathrm{H} & 0.01877 & 0.07274 & 0.36737 & 0.00000 & \text { Uiso } & 1.00\end{array}$ $\begin{array}{lllllllll}4156 & \mathrm{H} & 0.14776 & 0.95851 & 0.22674 & 0.00000 & \text { Uiso } & 1.00\end{array}$ $\begin{array}{llllllllll}\mathrm{H} 157 & \mathrm{H} & 0.09334 & 0.84774 & 0.23285 & 0.00000 & \text { Uiso } & 1.00 \\ \mathrm{H} 158 & \mathrm{H} & 0.11747 & 0.91226 & 0.33061 & 0.00000 & \text { Uiso } & 1.00\end{array}$ $\begin{array}{llllllllll}4158 & \mathrm{H} & 0.11747 & 0.91226 & 0.33061 & 0.00000 & \text { Uiso } & 1.00 \\ \mathrm{H} 159 & \mathrm{H} & 0.8386 & 0.96167 & 0.25567 & 0.00000 & \text { Us } & 1.00\end{array}$ $\begin{array}{lllllllll}\mathrm{H} 159 & \mathrm{H} & 0.83869 & 0.96167 & 0.25567 & 0.00000 & \text { Uiso } & 1.00\end{array}$ $\begin{array}{lllllllllll} & \text { H160 H } & 0.01774 & 0.80138 & 0.39904 & 0.00000 & \text { Uiso } & 1.00\end{array}$ $\begin{array}{lllllllll}\mathrm{H} 161 & \mathrm{H} & 0.95549 & 0.84180 & 0.49891 & 0.00000 & \text { Uiso } & 1.00\end{array}$ $\begin{array}{llllllll}\mathrm{H} 162 & \mathrm{H} & 0.02596 & 0.92072 & 0.44035 & 0.00000 & \text { Uiso } & 1.00\end{array}$ $\begin{array}{llllllll}\text { C163 } & \text { C } & 0.84053 & 0.99812 & 0.42880 & 0.00000 & \text { Uiso } & 1.00\end{array}$ $\begin{array}{llllllll}\mathrm{C} 164 & \mathrm{C} & 0.99230 & 0.98142 & 0.25262 & 0.00000 & \text { Uiso } & 1.00\end{array}$ $\begin{array}{lllllllll}\mathrm{C} 165 & \mathrm{C} & 0.97326 & 0.00037 & 0.15349 & 0.00000 & \text { Uiso } & 1.00\end{array}$ $\begin{array}{llllllll}\text { C166 } & \mathrm{C} & 0.99415 & 0.08062 & 0.29831 & 0.00000 & \text { Uiso } & 1.00\end{array}$ $\begin{array}{lllllllll}\mathrm{C} 167 & \mathrm{C} & 0.09308 & 0.92098 & 0.26177 & 0.00000 & \text { Uiso } & 1.00\end{array}$ $\begin{array}{lllllllll}\mathrm{C} 168 & \mathrm{C} & 0.90671 & 0.92387 & 0.28310 & 0.00000 & \text { Uiso } & 1.00\end{array}$ $\begin{array}{lllllllll}\text { C169 } & \mathrm{C} & 0.89058 & 0.90986 & 0.38105 & 0.00000 & \text { Uiso } & 1.00\end{array}$ $\begin{array}{llllllll}\mathrm{C} 170 & \mathrm{C} & 0.97710 & 0.86369 & 0.43294 & 0.00000 & \text { Uiso } & 1.00\end{array}$

\section{File $\mathbf{n}^{\circ} 3$}

$\begin{array}{llllllll}\mathrm{Si} 2 & \mathrm{Si} & 0.37548 & 0.76534 & 0.02423 & 0.00000 & \text { Uiso } & 1.00\end{array}$ $\begin{array}{llllllll}\text { Si3 } & \text { Si } & 0.60800 & 0.00145 & 0.02323 & 0.00000 & \text { Uiso } & 1.00\end{array}$

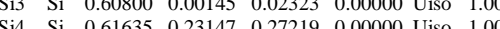
$\begin{array}{llllllll}\mathrm{Si} 5 & \mathrm{Si} & 0.61635 & 0.23147 & 0.27219 & 0.00000 & \text { Uiso } & 1.00\end{array}$ $\begin{array}{llllllll}\text { Sif } & \text { Si } & 0.37983 & 0.98552 & 0.27478 & 0.00000 & \text { Uiso } & 1.00\end{array}$

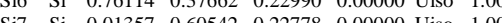

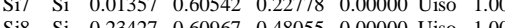
$\begin{array}{llllllll}\text { Si8 } & \text { Si } & 0.23427 & 0.60967 & 0.48055 & 0.00000 & \text { Uiso } & 1.00\end{array}$ $\begin{array}{llllllll}\text { Si9 } & \mathrm{Si} & 0.99035 & 0.37861 & 0.47958 & 0.00000 & \text { Uiso } & 1.00\end{array}$ $\begin{array}{llllllll}\text { Si10 } & \text { Si } & 0.61630 & 0.22984 & 0.47939 & 0.00000 & \text { Uiso } & 1.00\end{array}$ $\begin{array}{llllllll}\text { Si11 } & \text { Si } & 0.37751 & 0.98724 & 0.47951 & 0.00000 & \text { Uiso } & 1.00\end{array}$ $\begin{array}{llllllll}\mathrm{Si} 12 & \mathrm{Si} & 0.38184 & 0.75958 & 0.23047 & 0.00000 & \text { Uiso } & 1.00\end{array}$ $\begin{array}{llllllll}\text { Si13 } & \text { Si } & 0.60552 & 0.00615 & 0.22981 & 0.00000 & \text { Uiso } & 1.00\end{array}$ $\begin{array}{llllllll}\text { Sil4 } & \text { Si } & 0.23821 & 0.60808 & 0.27239 & 0.00000 & \text { Uiso } & 1.00\end{array}$ $\begin{array}{lllllllll}\text { Si15 } & \text { Si } & 0.98843 & 0.38117 & 0.27492 & 0.00000 & \text { Uiso } & 1.00\end{array}$

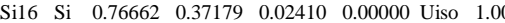
$\begin{array}{llllllll}\text { Sil7 } & \text { Si } & 0.00665 & 0.60349 & 0.02052 & 0.00000 & \text { Uiso } & 1.00\end{array}$ $\begin{array}{llllllll}\text { Si18 } & \text { Si } & 0.46717 & 0.28731 & 0.12494 & 0.00000 & \text { Uiso } & 1.00\end{array}$ $\begin{array}{llllllll}\text { Si19 } & \text { Si } & 0.31260 & 0.13507 & 0.12757 & 0.00000 & \text { Uiso } & 1.00\end{array}$ $\begin{array}{llllllll}\mathrm{Si} 20 & \mathrm{Si} & 0.54023 & 0.70672 & 0.37655 & 0.00000 & \text { Uiso } & 1.00\end{array}$ $\begin{array}{llllllll}\mathrm{Si} 21 & \mathrm{Si} & 0.69456 & 0.86498 & 0.37653 & 0.00000 & \text { Uiso } & 1.00\end{array}$ $\begin{array}{lllllllllll}\mathrm{Si} 22 & \mathrm{Si} & 0.29297 & 0.46144 & 0.12610 & 0.00000 & \text { Uiso } & 1.00\end{array}$ $\begin{array}{llllllll}\mathrm{Si} 23 & \mathrm{Si} & 0.13729 & 0.30999 & 0.12893 & 0.00000 & \text { Uiso } & 1.00\end{array}$ $\begin{array}{llllllll} & \text { Si24 Si } & 0.70840 & 0.53248 & 0.37611 & 0.00000 & \text { Uiso } & 1.00\end{array}$ $\begin{array}{lllllllll} & \text { S.25 Si } & 0.70840 & 0.53248 & 0.37611 & 0.00000 & \text { Uiso } & 1.00\end{array}$ $\begin{array}{llllllllll}\text { Si2s Si } & 0.37591 & 0.76305 & 0.52174 & 0.00000 & \text { Uiso } & 1.00\end{array}$ $\begin{array}{llllllllll}S_{12} 6 & S_{1} & 0.60451 & 0.00435 & 0.52411 & 0.00000 & \text { Uiso } & 1.00\end{array}$ $\begin{array}{lllllllll}\mathrm{Si} 27 & \mathrm{Si} & 0.62648 & 0.22546 & 0.77344 & 0.00000 & \text { Uiso } & 1.00\end{array}$ $\begin{array}{lllllllll}\mathrm{Si} 28 & \mathrm{Si} & 0.38533 & 0.98943 & 0.77424 & 0.00000 & \text { Uiso } & 1.00\end{array}$ $\begin{array}{lllllllll}\mathrm{Si} 29 & \mathrm{Si} & 0.76504 & 0.37634 & 0.72813 & 0.00000 & \text { Uiso } & 1.00\end{array}$ $\begin{array}{llllllll}\text { Si30 } & \text { Si } & 0.00987 & 0.60490 & 0.73555 & 0.00000 & \text { Uiso } & 1.00\end{array}$ $\begin{array}{lllllllll}\mathrm{Si} 31 & \mathrm{Si} & 0.22973 & 0.62026 & 0.97992 & 0.00000 & \text { Uiso } & 1.00\end{array}$ $\begin{array}{llllllll}\mathrm{Si} 32 & \mathrm{Si} & 0.99238 & 0.37619 & 0.97918 & 0.00000 & \text { Uiso } & 1.00\end{array}$ $\begin{array}{llllllll}\mathrm{Si33} & \mathrm{Si} & 0.62468 & 0.22248 & 0.97971 & 0.00000 & \text { Uiso } & 1.00\end{array}$ $\begin{array}{llllllll}\mathrm{Si} 34 & \mathrm{Si} & 0.38039 & 0.99062 & 0.97866 & 0.00000 & \text { Uiso } & 1.00\end{array}$ 
$\begin{array}{llllllll}\text { Si35 } & \text { Si } & 0.37960 & 0.76219 & 0.72795 & 0.00000 & \text { Uiso } & 1.00\end{array}$

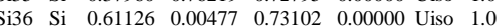
$\begin{array}{llllllll}\text { Si37 } & \text { Si } & 0.23224 & 0.62201 & 0.77446 & 0.00000 & \text { Uiso } & 1.00\end{array}$ $\begin{array}{lllllllll}\mathrm{Si} 38 & \mathrm{Si} & 0.99202 & 0.37990 & 0.77630 & 0.00000 & \text { Uiso } & 1.00\end{array}$ $\begin{array}{llllllll}\text { Si39 } & \text { Si } & 0.76512 & 0.37141 & 0.52206 & 0.00000 & \text { Uiso } & 1.00\end{array}$ $\begin{array}{llllllll}\mathrm{Si} 40 & \mathrm{Si} & 0.00978 & 0.60385 & 0.52763 & 0.00000 & \text { Uiso } & 1.00\end{array}$ $\begin{array}{llllllllll} & \text { Si41 Si } & 0.46887 & 0.28937 & 0.62806 & 0.00000 & \text { Uiso } & 1.00\end{array}$

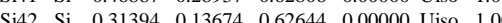
$\begin{array}{lllllll}\mathrm{Si4} 3 & \mathrm{Si} & 0.353265 & 0.70076 & 0.87563 & 0.00000 \\ \text { Ulso } & 1.00\end{array}$ $\begin{array}{lllllllll}\text { Sif4 Si } & 0.53265 & 0.70076 & 0.87563 & 0.00000 & \text { Uiso } & 1.00\end{array}$

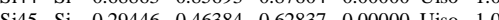
$\begin{array}{lllllllll}\mathrm{Si} 45 & \mathrm{Si} & 0.29446 & 0.46384 & 0.62837 & 0.00000 & \text { Uiso } & 1.00\end{array}$ $\begin{array}{lllllllll}\mathrm{Si46} & \mathrm{Si} & 0.13886 & 0.31181 & 0.62697 & 0.00000 & \text { Uiso } & 1.00\end{array}$ $\begin{array}{lllllllll}\text { Si47 } & \text { Si } & 0.70368 & 0.52960 & 0.87564 & 0.00000 & \text { Uiso } & 1.00\end{array}$ $\begin{array}{llllllll}\mathrm{Si} 48 & \mathrm{Si} & 0.86011 & 0.68555 & 0.87668 & 0.00000 & \text { Uiso } & 1.00\end{array}$ $\begin{array}{llllllllll}\mathrm{Al} 49 & \mathrm{Al} & 0.86916 & 0.68984 & 0.37611 & 0.00000 & \text { Uiso } & 1.00\end{array}$ $\begin{array}{llllllll}\mathrm{O} 50 & \mathrm{O} & 0.53082 & 0.29485 & 0.21471 & 0.00000 & \text { Uiso } & 1.00\end{array}$ $\begin{array}{lllllllll}051 & \mathrm{O} & 0.31294 & 0.06285 & 0.21264 & 0.00000 & \text { Uiso } & 1.00\end{array}$ $\begin{array}{lllllllll}052 & \mathrm{O} & 0.34881 & 0.88036 & 0.50767 & 0.00000 & \text { Uiso } & 1.00\end{array}$ $\begin{array}{llllllll}053 & \mathrm{O} & 0.46805 & 0.72015 & 0.46180 & 0.00000 & \text { Uiso } & 1.00\end{array}$ $\begin{array}{llllllll}054 & \mathrm{O} & 0.68146 & 0.93352 & 0.46539 & 0.00000 & \text { Uiso } & 1.00\end{array}$ $\begin{array}{lllllllll}055 & \mathrm{O} & 0.62842 & 0.11852 & 0.23923 & 0.00000 & \text { Uiso } & 1.00\end{array}$ $\begin{array}{lllllllll}056 & \mathrm{O} & 0.28531 & 0.53433 & 0.04156 & 0.00000 & \text { Uiso } & 1.00\end{array}$ $\begin{array}{lllllllll}057 & \mathrm{O} & 0.07377 & 0.31036 & 0.03839 & 0.00000 & \text { Uiso } & 1.00\end{array}$ $\begin{array}{llllllll}058 & \mathrm{O} & 0.87822 & 0.36268 & 0.24779 & 0.00000 & \text { Uiso } & 1.00\end{array}$ $\begin{array}{llllllllll}059 & \mathrm{O} & 0.70474 & 0.46504 & 0.28717 & 0.00000 & \text { Uiso } & 1.00\end{array}$ $\begin{array}{lllllllll}\mathrm{O} 60 & \mathrm{O} & 0.94965 & 0.68555 & 0.28630 & 0.00000 & \text { Uiso } & 1.00\end{array}$ $\begin{array}{lllllllll}061 & \mathrm{O} & 0.11369 & 0.63714 & 0.00097 & 0.00000 & \text { Uiso } & 1.00\end{array}$ $\begin{array}{llllllllll}062 & 0 & 0.47615 & 0.71230 & 0.28586 & 0.00000 & \text { Uiso } & 1.00\end{array}$ $\begin{array}{lllllllllll}062 & 0 & 0.47615 & 0.71230 & 0.28586 & 0.00000 & \text { Uiso } & 1.00\end{array}$ $\begin{array}{llllllllll}063 & \mathrm{O} & 0.68233 & 0.93662 & 0.28962 & 0.00000 & \text { Uiso } & 1.00\end{array}$ \begin{tabular}{llllllllll}
064 & $\mathrm{O}$ & 0.64375 & 0.10807 & 0.00745 & 0.0000 & Uiso & 1.00 \\
\hline
\end{tabular} $\begin{array}{llllllllll}065 & 0 & 0.53670 & 0.27929 & 0.03818 & 0.00000 & \text { Uiso } & 1.00\end{array}$ $\begin{array}{lllllllll}066 & 0 & 0.31304 & 0.07205 & 0.03669 & 0.0000 & \text { Uiso } & 1.00\end{array}$ $\begin{array}{llllllllll}067 & \mathrm{O} & 0.35647 & 0.87605 & 0.24986 & 0.0000 & \text { Uiso } & 1.00\end{array}$ $\begin{array}{llllllllll}068 & \mathrm{O} & 0.71449 & 0.46018 & 0.46214 & 0.00000 & \text { Uiso } & 1.00\end{array}$ $\begin{array}{llllllll}069 & \mathrm{O} & 0.94020 & 0.68407 & 0.47323 & 0.00000 & \text { Uiso } & 1.00\end{array}$ $\begin{array}{llllllll}070 & \mathrm{O} & 0.12863 & 0.62532 & 0.23229 & 0.00000 & \text { Uiso } & 1.00\end{array}$ $\begin{array}{lllllllll}071 & \mathrm{O} & 0.30291 & 0.52092 & 0.21814 & 0.00000 & \text { Uiso } & 1.00\end{array}$ $\begin{array}{lllllllll}072 & \mathrm{O} & 0.06563 & 0.31112 & 0.21414 & 0.00000 & \text { Uiso } & 1.00\end{array}$ $\begin{array}{lllllllll}073 & \mathrm{O} & 0.88298 & 0.35315 & 0.50921 & 0.00000 & \text { Uiso } & 1.00\end{array}$ $\begin{array}{llllllll}074 & \mathrm{O} & 0.49473 & 0.99598 & 0.26208 & 0.00000 & \text { Uiso } & 1.00\end{array}$ $\begin{array}{llllllll}\mathrm{O} 75 & \mathrm{O} & 0.49532 & 0.00268 & 0.99141 & 0.00000 & \text { Uiso } & 1.00\end{array}$ $\begin{array}{lllllllll}076 & \mathrm{O} & 0.00524 & 0.49135 & 0.98751 & 0.00000 & \text { Uiso } & 1.00\end{array}$ $\begin{array}{llllllll}\mathrm{O} 77 & \mathrm{O} & 0.00551 & 0.49429 & 0.26185 & 0.00000 & \text { Uiso } & 1.00\end{array}$ $\begin{array}{llllllll}078 & \mathrm{O} & 0.38992 & 0.38426 & 0.11453 & 0.00000 & \text { Uiso } & 1.00\end{array}$ $\begin{array}{lllllllll}079 & \mathrm{O} & 0.21394 & 0.21124 & 0.13113 & 0.00000 & \text { Uiso } & 1.00\end{array}$ $\begin{array}{llllllll}080 & \mathrm{O} & 0.60327 & 0.60066 & 0.38253 & 0.00000 & \text { Uiso } & 1.00\end{array}$ $\begin{array}{lllllllll}\mathrm{O} 81 & \mathrm{O} & 0.80144 & 0.80571 & 0.37506 & 0.00000 & \text { Uiso } & 1.00\end{array}$ $\begin{array}{llllllllll}082 & 0 & 0.41043 & 0.19031 & 0.13127 & 0.00000 & \text { Uiso } & 1.00\end{array}$ $\begin{array}{llllllllll}082 & 0 & 0.41043 & 0.19031 & 0.13127 & 0.00000 & \text { Uiso } & 1.00 \\ 083 & 0 & 0.60709 & 0.79540 & 0.37551 & 0.00000 & \text { Uiso } & 1.00\end{array}$ $\begin{array}{llllllllll}083 & \mathrm{O} & 0.60709 & 0.79540 & 0.37551 & 0.00000 & \text { Uiso } & 1.00 \\ 084 & \mathrm{O} & 0.19507 & 0.40630 & 0.13071 & 0.00000 & \text { Uis } & 1.00\end{array}$ $\begin{array}{lllllllll}084 & \mathrm{O} & 0.19507 & 0.40630 & 0.13071 & 0.00000 & \text { Uiso } & 1.00\end{array}$ $\begin{array}{lllllllll}085 & \mathrm{O} & 0.79843 & 0.59453 & 0.37278 & 0.00000 & \text { Uiso } & 1.00\end{array}$ $\begin{array}{llllllllll}086 & \mathrm{O} & 0.34826 & 0.00831 & 0.37671 & 0.00000 & \text { Uiso } & 1.00\end{array}$ $\begin{array}{lllllllll}087 & \mathrm{O} & 0.58537 & 0.23847 & 0.37581 & 0.00000 & \text { Uiso } & 1.00\end{array}$ $\begin{array}{lllllllll}088 & \mathrm{O} & 0.61833 & 0.97243 & 0.12712 & 0.00000 & \text { Uiso } & 1.00\end{array}$ $\begin{array}{lllllllll}089 & \mathrm{O} & 0.40513 & 0.74084 & 0.12637 & 0.00000 & \text { Uiso } & 1.00\end{array}$ $\begin{array}{llllllll}090 & \mathrm{O} & 0.00934 & 0.34819 & 0.37683 & 0.00000 & \text { Uiso } & 1.00\end{array}$ $\begin{array}{lllllllll}\mathrm{O} 91 & \mathrm{O} & 0.23702 & 0.57947 & 0.37663 & 0.00000 & \text { Uiso } & 1.00\end{array}$ $\begin{array}{lllllllll}092 & \mathrm{O} & 0.98045 & 0.61333 & 0.12429 & 0.00000 & \text { Uiso } & 1.00\end{array}$ $\begin{array}{llllllll}093 & \mathrm{O} & 0.73952 & 0.40001 & 0.12592 & 0.00000 & \text { Uiso } & 1.00\end{array}$ $\begin{array}{llllllll}094 & \mathrm{O} & 0.72048 & 0.27357 & 0.25653 & 0.00000 & \text { Uiso } & 1.00\end{array}$ $\begin{array}{llllllll}095 & \mathrm{O} & 0.27356 & 0.72275 & -0.00003 & 0.00000 & \text { Uiso } & 1.00\end{array}$ $\begin{array}{llllllllll}096 & \mathrm{O} & 0.28531 & 0.71024 & 0.26019 & 0.00000 & \text { Uiso } & 1.00\end{array}$ $\begin{array}{lllllllll}097 & \mathrm{O} & 0.72568 & 0.26957 & 0.99736 & 0.00000 & \text { Uiso } & 1.00\end{array}$ $\begin{array}{lllllllll}\mathrm{O} 98 & \mathrm{O} & 0.53944 & 0.28318 & 0.71426 & 0.00000 & \text { Uiso } & 1.00\end{array}$ $\begin{array}{lllllllll}099 & \mathrm{O} & 0.31712 & 0.06686 & 0.71318 & 0.00000 & \text { Uiso } & 1.00\end{array}$ $\begin{array}{llllllllll}0100 & \mathrm{O} & 0.35899 & 0.88277 & 0.01017 & 0.00000 & \text { Uiso } & 1.00\end{array}$ $\begin{array}{llllllll}\mathrm{O} 101 & \mathrm{O} & 0.46303 & 0.71420 & 0.96221 & 0.00000 & \text { Uiso } & 1.00\end{array}$ $\begin{array}{llllllllll}0102 & 0 & 0.67816 & 0.92148 & 0.96628 & 0.00000 & \text { Uiso } & 1.00\end{array}$ $\begin{array}{llllllllll}0103 & 0 & 0.67819 & 0.92148 & 0.96228 & 0.0000 & \text { Uiso } & 1.00\end{array}$ $\begin{array}{llllllllll}0104 & 0 & 0.2948 & 0.1182 & 0.74325 & 0.00000 & \text { Uiso } & 1.00\end{array}$

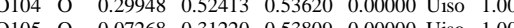
$\begin{array}{llllllll}0105 & \mathrm{O} & 0.07268 & 0.31220 & 0.53809 & 0.00000 & \text { Uiso } & 1.00\end{array}$ $\begin{array}{lllllllll}0106 & \mathrm{O} & 0.88205 & 0.36769 & 0.74402 & 0.00000 & \text { Uiso } & 1.00 \\ 0107 & \mathrm{O} & 0.70814 & 0.4656 & 0.78539 & 0.00000 & \text { Uiso } & 1.00\end{array}$ $\begin{array}{lllllllllll}0107 & \mathrm{O} & 0.70814 & 0.46556 & 0.78539 & 0.00000 & \text { Uiso } & 1.00 \\ 0108 & \mathrm{O} & 0.92974 & 0.6727 & 0.7893 & 0.0000 & \text { Uiso } & 1.00\end{array}$ $\begin{array}{llllllllll}0108 & \mathrm{O} & 0.92974 & 0.67827 & 0.78963 & 0.00000 & \text { Uiso } & 1.00\end{array}$ $\begin{array}{llllllll}0109 & \mathrm{O} & 0.12358 & 0.62566 & 0.51785 & 0.00000 & \text { Uiso } & 1.00\end{array}$ $\begin{array}{lllllllll}0110 & \mathrm{O} & 0.46759 & 0.70448 & 0.78586 & 0.00000 & \text { Uiso } & 1.00\end{array}$ $\begin{array}{llllllll}0111 & \mathrm{O} & 0.68241 & 0.92623 & 0.78868 & 0.00000 & \text { Uiso } & 1.00\end{array}$ $\begin{array}{lllllllll}0112 & \mathrm{O} & 0.62637 & 0.11659 & 0.51125 & 0.00000 & \text { Uiso } & 1.00\end{array}$ $\begin{array}{lllllllll}0113 & \mathrm{O} & 0.53168 & 0.29384 & 0.53741 & 0.00000 & \text { Uiso } & 1.00\end{array}$

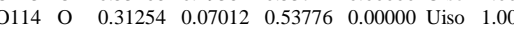
$\begin{array}{lllllllll}0115 & \mathrm{O} & 0.37103 & 0.87911 & 0.74449 & 0.00000 & \text { Uiso } & 1.00\end{array}$ $\begin{array}{lllllllll}0116 & \mathrm{O} & 0.71574 & 0.45927 & 0.96176 & 0.00000 & \text { Uiso } & 1.00\end{array}$ $\begin{array}{llllllll}0117 & \mathrm{O} & 0.92559 & 0.67562 & 0.96625 & 0.00000 & \text { Uiso } & 1.00\end{array}$ $\begin{array}{llllllll}0118 & \mathrm{O} & 0.11688 & 0.64021 & 0.75076 & 0.00000 & \text { Uiso } & 1.00\end{array}$ $\begin{array}{lllllllll}0119 & \mathrm{O} & 0.28935 & 0.53677 & 0.71291 & 0.00000 & \text { Uiso } & 1.00\end{array}$ $\begin{array}{lllllllll}0120 & \mathrm{O} & 0.06978 & 0.31356 & 0.71407 & 0.00000 & \text { Uiso } & 1.00\end{array}$ $\begin{array}{llllllll}0121 & \mathrm{O} & 0.88435 & 0.35676 & 0.01202 & 0.00000 & \text { Uiso } & 1.00\end{array}$ $\begin{array}{lllllllll}0122 & 0 & 0.49934 & 0.00714 & 0.76574 & 0.00000 & \text { Uiso } & 1.00\end{array}$ $\begin{array}{lllllllllll}0123 & 0 & 0.49333 & 0.99220 & 0.49381 & 0.00000 & \text { Uiso } & 1.00\end{array}$ $\begin{array}{llllllllll}0124 & 0 & 0.00049 & 0.49343 & 0.49288 & 0.00000 & \text { Uiso } & 1.00\end{array}$ $\begin{array}{lllllllllll}0124 & 0 & 0.01075 & 0.4343 & 0.77236 & 0.00000 & \text { Uiso } & 1.00\end{array}$ $\begin{array}{lllllllllll}0125 & 0 & 0.01075 & 0.49395 & 0.77236 & 0.00000 & \text { Uiso } & 1.00\end{array}$ $\begin{array}{llllllllll}0126 & 0 & 0.39241 & 0.38734 & 0.63628 & 0.00000 & \text { Uiso } & 1.00\end{array}$ $\begin{array}{llllllllll}0127 & 0 & 0.21515 & 0.21277 & 0.62882 & 0.00000 & \text { Uiso } & 1.00\end{array}$ $\begin{array}{lllllllll}0128 & 0 & 0.59814 & 0.5953 & 0.88133 & 0.00000 & \text { Uiso } & 1.00\end{array}$ $\begin{array}{llllllllllll}0129 & 0 & 0.79438 & 0.79136 & 0.87684 & 0.00000 & \text { Uiso } & 1.00\end{array}$ $\begin{array}{lllllllll}0130 & \mathrm{O} & 0.41134 & 0.19275 & 0.62512 & 0.00000 & \text { Uiso } & 1.00\end{array}$ $\begin{array}{llllllll}0131 & \mathrm{O} & 0.60068 & 0.78870 & 0.87223 & 0.00000 & \text { Uiso } & 1.00\end{array}$ $\begin{array}{lllllllll}0132 & \mathrm{O} & 0.19693 & 0.40794 & 0.62773 & 0.00000 & \text { Uiso } & 1.00\end{array}$ $\begin{array}{lllllllll}\mathrm{O} 133 & \mathrm{O} & 0.79132 & 0.59799 & 0.87397 & 0.00000 & \text { Uiso } & 1.00 \\ \mathrm{O} 134 & \mathrm{O} & 0.35032 & 0.00739 & 0.87583 & 0.00000 & \text { Uiso } & 1.00\end{array}$ $\begin{array}{llllllll}0135 & \mathrm{O} & 0.59316 & 0.23352 & 0.87640 & 0.00000 & \text { Uiso } & 1.00\end{array}$ 
$\begin{array}{llllllll}0136 & \mathrm{O} & 0.61873 & 0.97352 & 0.62754 & 0.00000 & \text { Uiso } & 1.00\end{array}$ $\begin{array}{lllllllll}0137 & \mathrm{O} & 0.40305 & 0.73917 & 0.62442 & 0.00000 & \text { Uiso } & 1.00\end{array}$ $\begin{array}{lllllllll}0138 & \mathrm{O} & 0.00823 & 0.34148 & 0.87718 & 0.00000 & \text { Uiso } & 1.00\end{array}$ $\begin{array}{llllllll}0139 & \mathrm{O} & 0.24868 & 0.58903 & 0.87709 & 0.00000 & \text { Uiso } & 1.00\end{array}$ $\begin{array}{lllllllll}0140 & 0 & 0.98137 & 0.61027 & 0.63212 & 0.00000 & \text { Uiso } & 1.00\end{array}$ $\begin{array}{lllllllll}0141 & \mathrm{O} & 0.73997 & 0.39762 & 0.62494 & 0.00000 & \text { Uiso } & 1.00\end{array}$ $\begin{array}{llllllllll}0142 & 0 & 0.72746 & 0.27373 & 0.75895 & 0.00000 & \text { Uiso } & 1.00\end{array}$ $\begin{array}{llllllllll}0143 & 0 & 0.27906 & 0.71323 & 0.49333 & 0.00000 & \text { Uis } & 1.00\end{array}$ $\begin{array}{llllllllll}0144 & 0 & 0.277629 & 0.72483 & 0.75616 & 0.00000 & \text { UST } & 1.00\end{array}$ $\begin{array}{lllllllll}0144 & 0 & 0.27629 & 0.72483 & 0.75616 & 0.00000 & \text { Uiso } & 1.00\end{array}$ \begin{tabular}{llllllllll}
0145 & $\mathrm{O}$ & 0.72131 & 0.27010 & 0.49535 & 0.00000 & Uiso & 1.00 \\
\hline 146 & $\mathrm{H}$ & 0.15807 & 0.81047 & 0.41595 & 0.00000 & Uiso & 1.00
\end{tabular} $\begin{array}{lllllllll}\mathrm{H} 146 & \mathrm{H} & 0.15807 & 0.81047 & 0.41595 & 0.00000 & \text { Uiso } & 1.00 \\ \mathrm{H} 147 & \mathrm{H} & 0.05455 & 0.89052 & 0.52419 & 0.00000 & \text { Uis } & 1.00\end{array}$ $\begin{array}{llllllll}\text { H147 } & \mathrm{H} & 0.05455 & 0.89052 & 0.52419 & 0.00000 & \text { Uiso } & 1.00 \\ \mathrm{H} 148 & \mathrm{H} & 0.92986 & 0.94505 & 0.49739 & 0.00000 & \text { Uis } & 1.00\end{array}$ $\begin{array}{lllllllll}\mathrm{H} 148 & \mathrm{H} & 0.92986 & 0.94505 & 0.49739 & 0.00000 & \text { Uiso } & 1.00\end{array}$ $\begin{array}{llllllll}\text { H1 } 149 & \mathrm{H} & 0.97029 & 0.81623 & 0.48320 & 0.00000 & \text { Uiso } & 1.00\end{array}$ $\begin{array}{llllllllll}\mathrm{H} 150 & \mathrm{H} & 0.91380 & 0.01349 & 0.35839 & 0.00000 & \text { Uiso } & 1.00\end{array}$ $\begin{array}{llllllll}\mathrm{H} 151 & \mathrm{H} & 0.90104 & 0.89793 & 0.31890 & 0.00000 & \text { Uiso } & 1.00\end{array}$ $\begin{array}{llllllll}\mathrm{H} 152 & \mathrm{H} & 0.15598 & 0.87688 & 0.31265 & 0.00000 & \text { Uiso } & 1.00\end{array}$ $\begin{array}{lllllllll}\mathrm{H} 153 & \mathrm{H} & 0.08834 & 0.77813 & 0.32201 & 0.00000 & \text { Uiso } & 1.00\end{array}$ $\begin{array}{llllllll}\mathrm{H} 154 & \mathrm{H} & 0.86363 & 0.09990 & 0.23010 & 0.00000 & \text { Uiso } & 1.00\end{array}$ $\begin{array}{lllllllll}\mathrm{H} 155 & \mathrm{H} & 0.83639 & 0.98803 & 0.18207 & 0.00000 & \text { Uiso } & 1.00\end{array}$ $\begin{array}{lllllllll}\mathrm{H} 156 & \mathrm{H} & 0.91024 & 0.06452 & 0.12373 & 0.00000 & \text { Uiso } & 1.00\end{array}$ $\begin{array}{llllllll}\mathrm{H} 157 & \mathrm{H} & 0.09486 & 0.85947 & 0.18862 & 0.00000 & \text { Uiso } & 1.00\end{array}$ $\begin{array}{lllllllll}\mathrm{H} 158 & \mathrm{H} & 0.02657 & 0.92139 & 0.10259 & 0.00000 & \text { Uiso } & 1.00\end{array}$

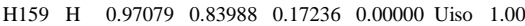
$\begin{array}{llllllll}\mathrm{H} 160 & \mathrm{H} & 0.03694 & 0.11822 & 0.27746 & 0.00000 & \text { Uiso } & 1.00\end{array}$ $\begin{array}{llllllll}\mathrm{H} 161 & \mathrm{H} & 0.08132 & 0.07928 & 0.17170 & 0.00000 & \text { Uiso } & 1.00\end{array}$ $\begin{array}{llllllllll}\mathrm{H} 162 & \mathrm{H} & 0.13266 & 0.01996 & 0.26727 & 0.00000 & \text { Uiso } & 1.00\end{array}$ \begin{tabular}{lllllllll}
\hline 162 & $\mathrm{H}$ & 0.13266 & 0.01996 & 0.26727 & 0.0000 & Uiso & 1.00
\end{tabular} $\begin{array}{lllllllll}\mathrm{C} 163 & \mathrm{C} & 0.95655 & 0.95185 & 0.32406 & 0.00000 & \text { Uiso } & 1.00\end{array}$ $\begin{array}{llllllllll}C 164 & C & 0.02303 & 0.89898 & 0.38696 & 0.00000 & \text { Uiso } & 1.00\end{array}$ $\begin{array}{lllllllll}\mathrm{C} 165 & \mathrm{C} & 0.99243 & 0.89274 & 0.47826 & 0.00000 & \text { Uiso } & 1.00\end{array}$ \begin{tabular}{lllllllll}
$C 166$ & $C$ & 0.11319 & 0.83955 & 0.36022 & 0.00000 & Uiso & 1.00 \\
\hline & 167 & $C$ & 0.98641 & 0.98258 & 0.23071 & 0.00000 & Uso & 1.00
\end{tabular} $\begin{array}{lllllllll}\mathrm{C} 167 & \mathrm{C} & 0.98641 & 0.98258 & 0.23071 & 0.00000 & \text { Uiso } & 1.00 \\ \mathrm{C} 168 & \mathrm{C} & 0.89329 & 0.03703 & 0.18942 & 0.00000 & \text { Uiso } & 1.00\end{array}$ $\begin{array}{llllllll}\mathrm{C} 168 & \mathrm{C} & 0.89329 & 0.03703 & 0.18942 & 0.00000 & \text { Uiso } & 1.00\end{array}$ $\begin{array}{llllllll}\mathrm{C} 169 & \mathrm{C} & 0.02201 & 0.89524 & 0.17090 & 0.00000 & \text { Uiso } & 1.00 \\ \mathrm{C} 170 & \mathrm{C} & 0.06383 & 0.05360 & 0.23787 & 0.00000 & \text { Uiso } & 1.00\end{array}$

\section{File $n^{\circ} 4$}

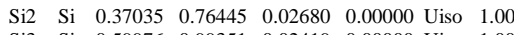
$\begin{array}{lllllllll}\text { Si3 } & \text { Si } & 0.59976 & 0.99351 & 0.02419 & 0.00000 & \text { Uiso } & 1.00\end{array}$ $\begin{array}{lllllllll}\mathrm{Si} 4 & \mathrm{Si} & 0.61038 & 0.22169 & 0.27670 & 0.00000 & \text { Uiso } & 1.00\end{array}$ $\begin{array}{llllllll}\text { Si5 } & \text { Si } & 0.37576 & 0.97702 & 0.27525 & 0.00000 & \text { Uiso } & 1.00\end{array}$ $\begin{array}{llllllll}\text { Si6 } & \text { Si } & 0.75209 & 0.36992 & 0.23605 & 0.00000 & \text { Uiso } & 1.00\end{array}$ $\begin{array}{llllllll}\text { Si7 } & \text { Si } & 0.01298 & 0.60193 & 0.23256 & 0.00000 & \text { Uiso } & 1.00\end{array}$ $\begin{array}{lllllllll}\text { Si8 } & \text { Si } & 0.23129 & 0.60338 & 0.48670 & 0.00000 & \text { Uiso } & 1.00\end{array}$ $\begin{array}{lllllllll}\text { Si9 } & \text { Si } & 0.98443 & 0.36701 & 0.48821 & 0.00000 & \text { Uiso } & 1.00\end{array}$ $\begin{array}{lllllllll}\mathrm{Si} 10 & \mathrm{Si} & 0.60486 & 0.22951 & 0.48329 & 0.00000 & \text { Uiso } & 1.00\end{array}$

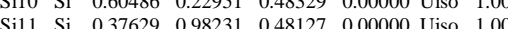
$\begin{array}{llllllllll}\mathrm{Si1} & \mathrm{Si} & 0.37940 & 0.75102 & 0.23224 & 0.00000 & \text { Uiso } & 1.00\end{array}$ $\begin{array}{llllllll}\mathrm{S} 112 & \mathrm{Si} & 0.37940 & 0.75102 & 0.23224 & 0.00000 & \text { Uiso } & 1.00\end{array}$ $\begin{array}{llllllllll}\mathrm{Si1} & \mathrm{Si} & 0.60248 & 0.00313 & 0.23007 & 0.00000 & \text { Uiso } & 1.00\end{array}$ $\begin{array}{lllllllll}\text { Sil4 } & \text { Si } & 0.24164 & 0.59481 & 0.27804 & 0.00000 & \text { Uiso } & 1.00\end{array}$ $\begin{array}{llllllll}\mathrm{Si15} & \mathrm{Si} & 0.97699 & 0.38602 & 0.28358 & 0.00000 & \text { Uiso } & 1.00\end{array}$ $\begin{array}{lllllllll}\text { Sil6 } & \text { Si } & 0.75958 & 0.36067 & 0.02965 & 0.00000 & \text { Uiso } & 1.00\end{array}$ $\begin{array}{llllllll}\text { Si17 } & \text { Si } & 0.00352 & 0.59635 & 0.02508 & 0.00000 & \text { Uiso } & 1.00\end{array}$ $\begin{array}{llllllll}\mathrm{Si} 18 & \mathrm{Si} & 0.45948 & 0.27537 & 0.12759 & 0.00000 & \text { Uiso } & 1.00\end{array}$ $\begin{array}{llllllll}\text { Si19 } & \text { Si } & 0.29655 & 0.12870 & 0.13429 & 0.00000 & \text { Uiso } & 1.00\end{array}$ $\begin{array}{lllllllll}\mathrm{Si} 20 & \mathrm{Si} & 0.53083 & 0.70170 & 0.38174 & 0.00000 & \text { Uiso } & 1.00\end{array}$ $\begin{array}{llllllll}\mathrm{Si} 21 & \mathrm{Si} & 0.67772 & 0.85909 & 0.38352 & 0.00000 & \text { Uiso } & 1.00\end{array}$ $\begin{array}{llllllll}\mathrm{Si22} & \mathrm{Si} & 0.29295 & 0.45219 & 0.12843 & 0.00000 & \text { Uiso } & 1.00\end{array}$ $\begin{array}{lllllllll}\mathrm{Si} 23 & \mathrm{Si} & 0.12856 & 0.30655 & 0.13317 & 0.00000 & \text { Uiso } & 1.00\end{array}$ $\begin{array}{lllllllll}\mathrm{Si} 24 & \mathrm{Si} & 0.70857 & 0.53055 & 0.37279 & 0.00000 & \text { Uiso } & 1.00\end{array}$ $\begin{array}{llllllll}\mathrm{Si} 25 & \mathrm{Si} & 0.36935 & 0.75641 & 0.52472 & 0.00000 & \text { Uiso } & 1.00\end{array}$ $\begin{array}{llllllll}\mathrm{Si} 26 & \mathrm{Si} & 0.60156 & 0.00495 & 0.53025 & 0.00000 & \text { Uiso } & 1.00\end{array}$ $\begin{array}{llllllll}\mathrm{Si} 27 & \mathrm{Si} & 0.62276 & 0.22115 & 0.77496 & 0.00000 & \text { Uiso } & 1.00\end{array}$ $\begin{array}{llllllll}\mathrm{Si} 28 & \mathrm{Si} & 0.38479 & 0.97855 & 0.78066 & 0.00000 & \text { Uiso } & 1.00\end{array}$ $\begin{array}{llllllll}\mathrm{Si} 29 & \mathrm{Si} & 0.75956 & 0.37493 & 0.72950 & 0.00000 & \text { Uiso } & 1.00\end{array}$ $\begin{array}{llllllll}\mathrm{Si} 30 & \mathrm{Si} & 0.00212 & 0.60262 & 0.74082 & 0.00000 & \text { Uiso } & 1.00\end{array}$ $\begin{array}{llllllll}\mathrm{Si} 31 & \mathrm{Si} & 0.22727 & 0.61534 & 0.98562 & 0.00000 & \text { Uiso } & 1.00\end{array}$ $\begin{array}{llllllllll}\mathrm{Si} 32 & \mathrm{Si} & 0.98513 & 0.36920 & 0.98259 & 0.00000 & \text { Uiso } & 1.00\end{array}$ $\begin{array}{llllllll}S 32 & \text { Si } & 0.98513 & 0.36920 & 0.98259 & 0.00000 & \text { Sis } & 1.00\end{array}$ $\begin{array}{llllllll}\mathrm{Si33} & \mathrm{Si} & 0.61951 & 0.21141 & 0.98037 & 0.00000 & \text { Uiso } & 1.00\end{array}$ $\begin{array}{llllllllll}\mathrm{S} 134 & \mathrm{Si} & 0.36997 & 0.9961 & 0.98590 & 0.00000 & \text { Uiso } & 1.00\end{array}$ $\begin{array}{llllllll}\mathrm{Si35} & \mathrm{Si} & 0.37485 & 0.75152 & 0.73198 & 0.00000 & \text { Uiso } & 1.00\end{array}$

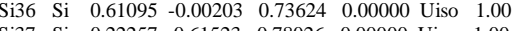
$\begin{array}{llllllll}\mathrm{Si} 37 & \mathrm{Si} & 0.22257 & 0.61523 & 0.78026 & 0.00000 & \text { Uiso } & 1.00\end{array}$ $\begin{array}{lllllllll}\mathrm{Si} 38 & \mathrm{Si} & 0.98691 & 0.37554 & 0.77801 & 0.00000 & \text { Uiso } & 1.00\end{array}$ $\begin{array}{lllllllll}\mathrm{Si} 39 & \mathrm{Si} & 0.76045 & 0.37251 & 0.52405 & 0.00000 & \text { Uiso } & 1.00\end{array}$ $\begin{array}{lllllllll}\mathrm{Si} 40 & \mathrm{Si} & 0.00437 & 0.59381 & 0.53277 & 0.00000 & \text { Uiso } & 1.00\end{array}$ $\begin{array}{lllllllll}\mathrm{Si} 41 & \mathrm{Si} & 0.45811 & 0.28163 & 0.63226 & 0.00000 & \text { Uiso } & 1.00\end{array}$ $\begin{array}{llllllll}\mathrm{Si} 42 & \mathrm{Si} & 0.30657 & 0.12345 & 0.63038 & 0.00000 & \text { Uiso } & 1.00\end{array}$ $\begin{array}{llllllll}\mathrm{Si} 43 & \mathrm{Si} & 0.52503 & 0.69004 & 0.87513 & 0.00000 & \text { Uiso } & 1.00\end{array}$ $\begin{array}{llllllll}\mathrm{Si} 44 & \mathrm{Si} & 0.67953 & 0.84467 & 0.87625 & 0.00000 & \text { Uiso } & 1.00\end{array}$ $\begin{array}{llllllll}\mathrm{Si} 45 & \mathrm{Si} & 0.28469 & 0.45361 & 0.63352 & 0.00000 & \text { Uiso } & 1.00\end{array}$ $\begin{array}{llllllll}\mathrm{Si} 46 & \mathrm{Si} & 0.13230 & 0.29666 & 0.62972 & 0.00000 & \text { Uiso } & 1.00\end{array}$ $\begin{array}{llllllllll}\mathrm{Si} 47 & \mathrm{Si} & 0.69502 & 0.51980 & 0.88331 & 0.00000 & \text { Uiso } & 1.00\end{array}$ $\begin{array}{llllllll}\mathrm{Si} 48 & \mathrm{Si} & 0.85132 & 0.67593 & 0.88533 & 0.00000 & \text { Uiso } & 1.00\end{array}$ $\begin{array}{lllllllll}\mathrm{Al} 49 & \mathrm{Al} & 0.86031 & 0.68424 & 0.37936 & 0.00000 & \text { Uiso } & 1.00\end{array}$ $\begin{array}{lllllllll}\mathrm{O} 50 & \mathrm{O} & 0.52559 & 0.28137 & 0.21585 & 0.00000 & \text { Uiso } & 1.00\end{array}$ $\begin{array}{lllllllll}051 & \mathrm{O} & 0.30176 & 0.04989 & 0.21464 & 0.00000 & \text { Uiso } & 1.00\end{array}$ $\begin{array}{llllllll}051 & 0 & 0.30176 & 0.04989 & 0.21464 & 0.00000 & \text { Uiso } & 1.00 \\ 052 & 0 & 0.35154 & 0.87245 & 0.50291 & 0.00000 & \text { Uiso } & 1.00\end{array}$ $\begin{array}{lllllllllll}052 & 0 & 0.35154 & 0.87245 & 0.50291 & 0.00000 & \text { Uiso } & 1.00 \\ 053 & 0 & 0.45468 & 0.69971 & 0.46368 & 0.00000 & \text { Uiso } & 1.00\end{array}$ $\begin{array}{lllllllll}053 & \mathrm{O} & 0.45468 & 0.6977 & 0.46368 & 0.00000 & \text { Uiso } & 1.00 \\ 054 & \mathrm{O} & 0.67408 & 0.93621 & 0.46593 & 0.00000 & \text { Uis } & 1.00\end{array}$ $\begin{array}{llllllll}054 & 0 & 0.67408 & 0.93621 & 0.46593 & 0.00000 & \text { Uiso } & 1.00 \\ 055 & 0 & 0.62661 & 0.10747 & 0.24834 & 0.00000 & \text { Uis } & 1.00\end{array}$ $\begin{array}{llllllllllll}055 & 0 & 0.62661 & 0.10747 & 0.24834 & 0.00000 & \text { Uiso } & 1.00\end{array}$ $\begin{array}{llllllll}056 & 0 & 0.28368 & 0.53134 & 0.04858 & 0.00000 & \text { Uiso } & 1.00 \\ 057 & 0 & 0.06605 & 0.30321 & 0.04225 & 0.0000 & \text { Uiso } & 1.00\end{array}$ $\begin{array}{llllllll}057 & \mathrm{O} & 0.06605 & 0.30321 & 0.04225 & 0.00000 & \text { Uiso } & 1.00\end{array}$ $\begin{array}{lllllllll}058 & \mathrm{O} & 0.86797 & 0.36192 & 0.25965 & 0.00000 & \text { Uiso } & 1.00\end{array}$ $\begin{array}{llllllll}\text { O59 } & \mathrm{O} & 0.68923 & 0.45638 & 0.29166 & 0.00000 & \text { Uiso } & 1.00\end{array}$ $\begin{array}{llllllll}\mathrm{O} 60 & \mathrm{O} & 0.95301 & 0.70056 & 0.28550 & 0.00000 & \text { Uiso } & 1.00\end{array}$ $\begin{array}{llllllll}\text { O61 } & \mathrm{O} & 0.11072 & 0.62908 & 0.00628 & 0.00000 & \text { Uiso } & 1.00\end{array}$ $\begin{array}{llllllll}\mathrm{O} 62 & \mathrm{O} & 0.47239 & 0.70020 & 0.28792 & 0.00000 & \text { Uiso } & 1.00 \\ \mathrm{O} 63 & \mathrm{O} & 0.67226 & 0.92115 & 0.29079 & 0.00000 & \text { Uiso } & 1.00\end{array}$ 
$\begin{array}{llllllll}\text { O64 } & \mathrm{O} & 0.64090 & 0.09377 & -0.00685 & 0.00000 & \text { Uiso } & 1.00\end{array}$ $\begin{array}{llllllll}\mathrm{O} 65 & \mathrm{O} & 0.52762 & 0.25585 & 0.04076 & 0.00000 & \text { Uiso } & 1.00\end{array}$ $\begin{array}{lllllllll}066 & \mathrm{O} & 0.29666 & 0.07158 & 0.03973 & 0.00000 & \text { Uiso } & 1.00\end{array}$ $\begin{array}{llllllll}067 & \mathrm{O} & 0.35283 & 0.86545 & 0.25857 & 0.00000 & \text { Uiso } & 1.00\end{array}$ $\begin{array}{llllllllll}068 & \mathrm{O} & 0.72493 & 0.46693 & 0.46390 & 0.00000 & \text { Uiso } & 1.00\end{array}$ $\begin{array}{lllllllll}069 & \mathrm{O} & 0.93419 & 0.66454 & 0.47071 & 0.00000 & \text { Uiso } & 1.00\end{array}$ $\begin{array}{llllllllll}070 & 0 & 0.12887 & 0.60488 & 0.24376 & 0.00000 & \text { Uiso } & 1.00\end{array}$ $\begin{array}{lllllllll}071 & \mathrm{O} & 0.12887 & 0.60488 & 0.24376 & 0.00000 & \text { Uiso } & 1.00\end{array}$

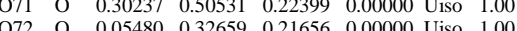
$\begin{array}{lllllllll}072 & \mathrm{O} & 0.05480 & 0.32659 & 0.21656 & 0.00000 & \text { Uiso } & 1.00 \\ 073 & \mathrm{O} & 0.87668 & 0.33782 & 0.51174 & 0.00000 & \text { Uiso } & 1.00\end{array}$ $\begin{array}{llllllllll}073 & 0 & 0.87688 & 0.33782 & 0.51174 & 0.0000 & \text { Uiso } & 1.00\end{array}$ $\begin{array}{llllllllll}074 & 0 & 0.48806 & 0.98850 & 0.25042 & 0.0000 & \text { Uiso } & 1.00\end{array}$ $\begin{array}{llllllllll}075 & \mathrm{O} & 0.48208 & 0.00506 & 0.01005 & 0.0000 & \text { Uiso } & 1.00\end{array}$ $\begin{array}{llllllll}076 & \mathrm{O} & -0.00184 & 0.48424 & 0.99552 & 0.00000 & \text { Uiso } & 1.00\end{array}$ $\begin{array}{llllllll}\mathrm{O} 77 & \mathrm{O} & 0.98303 & 0.50382 & 0.27716 & 0.00000 & \text { Uiso } & 1.00 \\ \mathrm{O} 78 & \mathrm{O} & 0.39147 & 0.37826 & 0.11301 & 0.00000 & \text { Uso } & 1.00\end{array}$ $\begin{array}{lllllllll}078 & \mathrm{O} & 0.39147 & 0.37826 & 0.11301 & 0.00000 & \text { Uiso } & 1.00\end{array}$ $\begin{array}{llllllll}\mathrm{O} 79 & \mathrm{O} & 0.19601 & 0.20242 & 0.14466 & 0.00000 & \text { Uiso } & 1.00\end{array}$ $\begin{array}{llllllll}\mathrm{O} 80 & \mathrm{O} & 0.60937 & 0.60565 & 0.38689 & 0.00000 & \text { Uiso } & 1.00\end{array}$ $\begin{array}{llllllll}\mathrm{O} 81 & \mathrm{O} & 0.77635 & 0.78627 & 0.38762 & 0.00000 & \text { Uiso } & 1.00\end{array}$ $\begin{array}{llllllll}082 & \mathrm{O} & 0.39164 & 0.18772 & 0.13985 & 0.00000 & \text { Uiso } & 1.00\end{array}$ $\begin{array}{llllllll}083 & \mathrm{O} & 0.57971 & 0.80296 & 0.38862 & 0.00000 & \text { Uiso } & 1.00\end{array}$ $\begin{array}{llllllll}084 & \mathrm{O} & 0.19592 & 0.39570 & 0.12794 & 0.00000 & \text { Uiso } & 1.00\end{array}$ $\begin{array}{llllllll}085 & \mathrm{O} & 0.80179 & 0.58510 & 0.35002 & 0.00000 & \text { Uiso } & 1.00\end{array}$ $\begin{array}{llllllll}086 & \mathrm{O} & 0.35441 & 0.00630 & 0.37777 & 0.00000 & \text { Uiso } & 1.00\end{array}$ $\begin{array}{llllllll}087 & \mathrm{O} & 0.57557 & 0.23188 & 0.37920 & 0.00000 & \text { Uiso } & 1.00\end{array}$ $\begin{array}{llllllllll}088 & \mathrm{O} & 0.62540 & 0.97011 & 0.12717 & 0.00000 & \text { Uiso } & 1.00\end{array}$ $\begin{array}{lllllllll}\mathrm{O} 89 & \mathrm{O} & 0.40535 & 0.73947 & 0.12778 & 0.00000 & \text { Uiso } & 1.00\end{array}$ $\begin{array}{llllllllll}090 & \mathrm{O} & 0.00597 & 0.35112 & 0.38319 & 0.00000 & \text { Uiso } & 1.00\end{array}$ $\begin{array}{llllllllllll}091 & \mathrm{O} & 0.07690 & 0.57306 & 0.38297 & 0.00000 & \text { Uiso } & 1.00\end{array}$ $\begin{array}{llllllllll}091 & 0 & 0.24690 & 0.57306 & 0.38297 & 0.00000 & \text { Uiso } & 1.00\end{array}$ $\begin{array}{llllllllll}092 & \mathrm{O} & 0.97981 & 0.60851 & 0.13025 & 0.00000 & \text { Uiso } & 1.00 \\ 093 & \mathrm{O} & 0.73641 & 0.39279 & 0.13127 & 0.00000 & \text { Uis } & 1.00\end{array}$ \begin{tabular}{lllllllll}
093 & $\mathrm{O}$ & 0.73641 & 0.39279 & 0.13127 & 0.00000 & Uiso & 1.00 \\
\hline 994 & $\mathrm{O}$ & 0.71363 & 0.26575 & 0.26104 & 0.00000 & Uiso & 1.00
\end{tabular} $\begin{array}{llllllllll}095 & 0 & 0.71363 & 0.26575 & 0.26104 & 0.0000 & \text { Uiso } & 1.00\end{array}$ $\begin{array}{lllllllllll}095 & 0 & 0.26887 & 0.71876 & 0.00628 & 0.00000 & \text { Uiso } & 1.00\end{array}$ \begin{tabular}{lllllllll}
096 & $\mathrm{O}$ & 0.2827 & 0.69771 & 0.25503 & 0.00000 & Uiso & 1.00 \\
\hline 97 & $\mathrm{O}$ & 0.71871 & 0.25676 & 0.00935 & 0.00000 & Uso & 1.00
\end{tabular} $\begin{array}{lllllllll}097 & \mathrm{O} & 0.71871 & 0.25676 & 0.00935 & 0.00000 & \text { Uiso } & 1.00 \\ 098 & \mathrm{O} & 0.5386 & 0.27241 & 0.71457 & 0.00000 & \text { Uso } & 1.00\end{array}$ $\begin{array}{lllllllll}098 & \mathrm{O} & 0.53386 & 0.27241 & 0.71457 & 0.00000 & \text { Uiso } & 1.00\end{array}$ $\begin{array}{llllllll}099 & \text { O } & 0.31599 & 0.04878 & 0.71403 & 0.00000 & \text { Uiso } & 1.00\end{array}$ $\begin{array}{lllllllll}0100 & \mathrm{O} & 0.34774 & 0.88185 & 0.01455 & 0.00000 & \text { Uiso } & 1.00\end{array}$ $\begin{array}{llllllll}0101 & \mathrm{O} & 0.45702 & 0.71813 & 0.96126 & 0.00000 & \text { Uiso } & 1.00\end{array}$ $\begin{array}{llllllll}0102 & \mathrm{O} & 0.65235 & 0.90303 & 0.96731 & 0.00000 & \text { Uiso } & 1.00\end{array}$

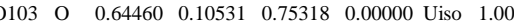
$\begin{array}{llllllll}\mathrm{O} 104 & \mathrm{O} & 0.29505 & 0.51929 & 0.54536 & 0.00000 & \text { Uiso } & 1.00\end{array}$ $\begin{array}{lllllllll}0105 & \mathrm{O} & 0.06574 & 0.29333 & 0.54125 & 0.00000 & \text { Uiso } & 1.00\end{array}$ $\begin{array}{llllllll}0106 & \mathrm{O} & 0.87618 & 0.36448 & 0.74758 & 0.00000 & \text { Uiso } & 1.00\end{array}$ $\begin{array}{lllllllll}0107 & \mathrm{O} & 0.70237 & 0.46167 & 0.78968 & 0.00000 & \text { Uiso } & 1.00\end{array}$ $\begin{array}{lllllllll}0108 & 0 & 0.91559 & 0.66739 & 0.79525 & 0.00000 & \text { Uiso } & 1.00\end{array}$ $\begin{array}{llllllll}0109 & 0 & 0.11778 & 0.61056 & 0.51401 & 0.00000 & \text { Uiso } & 1.00\end{array}$ $\begin{array}{llllllllll}0110 & \mathrm{O} & 0.45809 & 0.68307 & 0.78748 & 0.00000 & \text { Uiso } & 1.00\end{array}$ $\begin{array}{llllllllllllllll}0111 & 0 & 0.68283 & 0.91879 & 0.79291 & 0.00000 & \text { Uiso } & 1.00\end{array}$ $\begin{array}{lllllllllll}0111 & 0 & 0.68283 & 0.91879 & 0.79291 & 0.00000 & \text { Uiso } & 1.00\end{array}$ $\begin{array}{llllllllll}0113 & \mathrm{O} & 0.62302 & 0.127519 & 0.28912 & 0.53872 & 0.00000 & \text { Uiso } & 1.00\end{array}$ $\begin{array}{lllllllll}0114 & 0 & 0.51519 & 0.28912 & 0.53872 & 0.00000 & \text { Uiso } & 1.00\end{array}$ $\begin{array}{llllllllll}0114 & 0 & 0.30506 & 0.06089 & 0.53924 & 0.00000 & \text { Uiso } & 1.00 \\ 0115 & 0 & 0.37110 & 0.86495 & 0.76224 & 0.00000 & \text { Uiso } & 1.00\end{array}$ $\begin{array}{lllllllll}0115 & 0 & 0.37110 & 0.86495 & 0.76224 & 0.00000 & \text { Uiso } & 1.00 \\ 0116 & 0 & 0.70422 & 0.44379 & 0.96546 & 0.00000 & \text { Uiso } & 1.00\end{array}$ $\begin{array}{lllllllllll}0116 & \mathrm{O} & 0.70422 & 0.44379 & 0.96546 & 0.00000 & \text { Uiso } & 1.00 \\ 0117 & \mathrm{O} & 0.92201 & 0.6867 & 0.97216 & 0.0000 & \text { Ulso } & 1.00\end{array}$ $\begin{array}{lllllllllll}0117 & 0 & 0.92201 & 0.66867 & 0.97216 & 0.0000 & \text { Uiso } & 1.00\end{array}$ $\begin{array}{lllllllll}0118 & \mathrm{O} & 0.10554 & 0.64057 & 0.76698 & 0.00000 & \text { Uiso } & 1.00\end{array}$ $\begin{array}{llllllll}0119 & \mathrm{O} & 0.26746 & 0.52335 & 0.71991 & 0.00000 & \text { Uiso } & 1.00\end{array}$ $\begin{array}{llllllll}0120 & \mathrm{O} & 0.06353 & 0.30630 & 0.71733 & 0.00000 & \text { Uiso } & 1.00\end{array}$ $\begin{array}{lllllllll}0121 & \mathrm{O} & 0.87674 & 0.34815 & 0.01289 & 0.00000 & \text { Uiso } & 1.00\end{array}$ $\begin{array}{llllllll}0122 & \mathrm{O} & 0.49865 & -0.00385 & 0.76925 & 0.00000 & \text { Uiso } & 1.00\end{array}$ $\begin{array}{llllllllll}0123 & \mathrm{O} & 0.48887 & -0.00606 & 0.50495 & 0.00000 & \text { Uiso } & 1.00\end{array}$ $\begin{array}{llllllllll}0124 & \mathrm{O} & 0.99230 & 0.47883 & 0.51671 & 0.00000 & \text { Uiso } & 1.00\end{array}$ $\begin{array}{lllllllll}0125 & \mathrm{O} & 0.00736 & 0.48856 & 0.76998 & 0.00000 & \text { Uiso } & 1.00\end{array}$ $\begin{array}{llllllll}0126 & \mathrm{O} & 0.38466 & 0.38091 & 0.64686 & 0.00000 & \text { Uiso } & 1.00\end{array}$ $\begin{array}{lllllllll}0127 & \mathrm{O} & 0.20524 & 0.19568 & 0.63786 & 0.00000 & \text { Uiso } & 1.00\end{array}$ $\begin{array}{lllllllll}0128 & \mathrm{O} & 0.58916 & 0.58494 & 0.89104 & 0.00000 & \text { Uiso } & 1.00\end{array}$ $\begin{array}{lllllllll}0129 & \mathrm{O} & 0.78577 & 0.78142 & 0.88677 & 0.00000 & \text { Uiso } & 1.00\end{array}$ $\begin{array}{lllllllll}\mathrm{O} 130 & \mathrm{O} & 0.39983 & 0.18567 & 0.63042 & 0.00000 & \text { Uiso } & 1.00\end{array}$ $\begin{array}{llllllllll}0131 & 0 & 0.59619 & 0.77344 & 0.85712 & 0.00000 & \text { Uiso } & 1.00\end{array}$ $\begin{array}{lllllllllll}0132 & 0 & 0.19275 & 0.39090 & 0.62283 & 0.00000 & \text { Uiso } & 1.00\end{array}$ $\begin{array}{llllllllllllllllll}0132 & 0 & 0.19275 & 0.3974 & 0.62283 & 0.00000 & \text { Uiso } & 1.00\end{array}$ $\begin{array}{llllllllllll}0133 & 0 & 0.78326 & 0.58767 & 0.88956 & 0.00000 & 0.000 & \text { Ulso } & 1.00\end{array}$ $\begin{array}{lllllllll}0134 & \mathrm{O} & 0.35019 & 0.00685 & 0.88101 & 0.00000 & \text { Uiso } & 1.00 \\ 0135 & \mathrm{O} & 0.59200 & 0.23771 & 0.87813 & 0.0000 & \text { Uiso } & 1.00\end{array}$ $\begin{array}{lllllllllllll}0135 & 0 & 0.59200 & 0.23771 & 0.87813 & 0.00000 & \text { Uiso } & 1.00\end{array}$ $\begin{array}{lllllllll}0136 & 0 & 0.62134 & 0.97054 & 0.63217 & 0.00000 & \text { Uiso } & 1.00 \\ 0137 & 0 & 0.40057 & 0.74031 & 0.62770 & 0.00000 & \text { Uiso } & 1.00\end{array}$ $\begin{array}{lllllllllll}0137 & 0 & 0.40057 & 0.74031 & 0.6277 & 0.00000 & \text { Uiso } & 1.00 \\ 0138 & \mathrm{O} & 0.0034 & 0.3394 & 0.87982 & 0.00000 & \text { Uis } & 1.00\end{array}$ $\begin{array}{lllllllll}0138 & \mathrm{O} & 0.00314 & 0.33948 & 0.87982 & 0.00000 & \text { Uiso } & 1.00\end{array}$ $\begin{array}{lllllllll}0139 & \mathrm{O} & 0.24709 & 0.58539 & 0.88285 & 0.00000 & \text { Uiso } & 1.00\end{array}$ $\begin{array}{llllllll}0140 & \mathrm{O} & 0.98019 & 0.61729 & 0.63668 & 0.00000 & \text { Uiso } & 1.00\end{array}$ $\begin{array}{llllllll}0141 & \mathrm{O} & 0.73691 & 0.40215 & 0.62675 & 0.00000 & \text { Uiso } & 1.00\end{array}$ $\begin{array}{lllllllll}0142 & \mathrm{O} & 0.72180 & 0.27066 & 0.75458 & 0.00000 & \text { Uiso } & 1.00\end{array}$ $\begin{array}{llllllll}0143 & \mathrm{O} & 0.26812 & 0.70984 & 0.50458 & 0.00000 & \text { Uiso } & 1.00\end{array}$ $\begin{array}{llllllll}0144 & \mathrm{O} & 0.26958 & 0.71403 & 0.75295 & 0.00000 & \text { Uiso } & 1.00\end{array}$ $\begin{array}{lllllllll}0145 & \mathrm{O} & 0.70447 & 0.27938 & 0.49703 & 0.00000 & \text { Uiso } & 1.00\end{array}$ $\begin{array}{lllllllll}\mathrm{H} 146 & \mathrm{H} & 0.11314 & 0.75667 & 0.17930 & 0.00000 & \text { Uiso } & 1.00\end{array}$ $\begin{array}{llllllllll}\mathrm{H} 147 & \mathrm{H} & 0.08741 & 0.88456 & 0.35119 & 0.00000 & \text { Uiso } & 1.00\end{array}$ $\begin{array}{lllllllll}\mathrm{H} 148 & \mathrm{H} & 0.02548 & 0.80215 & 0.41484 & 0.00000 & \text { Uiso } & 1.00\end{array}$ $\begin{array}{lllllllll}\mathrm{H} 149 & \mathrm{H} & 0.12868 & 0.75793 & 0.34841 & 0.00000 & \text { Uiso } & 1.00\end{array}$ $\begin{array}{llllllll}\mathrm{H} 150 & \mathrm{H} & 0.88020 & 0.89542 & 0.34388 & 0.00000 & \text { Uiso } & 1.00\end{array}$ $\begin{array}{llllllll}\mathrm{H} 151 & \mathrm{H} & 0.84945 & 0.86598 & 0.23339 & 0.00000 & \text { Uiso } & 1.00\end{array}$ $\begin{array}{llllllllll}\mathrm{H} 152 & \mathrm{H} & 0.93422 & 0.95080 & 0.25319 & 0.00000 & \text { Uiso } & 1.00\end{array}$ $\begin{array}{llllllll}\mathrm{H} 152 & \mathrm{H} & 0.93422 & 0.95080 & 0.25319 & 0.0000 & \text { Uiso } & 1.00\end{array}$ $\begin{array}{llllllll}153 & \mathrm{H} & 0.06629 & 0.88158 & 0.17823 & 0.00000 & \text { Uiso } & 1.00\end{array}$ $\begin{array}{lllllllll}\mathrm{H} 154 & \mathrm{H} & -0.00400 & 0.7592 & 0.13079 & 0.00000 & \text { Uiso } & 1.00 \\ \mathrm{H} 155 & \mathrm{H} & 0.88390 & 0.90663 & 0.08416 & 0.00000 & \text { Uis } & 1.00\end{array}$

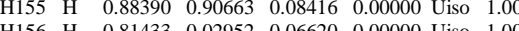
$\begin{array}{llllllll}1156 & \mathrm{H} & 0.81433 & 0.02952 & 0.06620 & 0.00000 & \text { Uiso } & 1.00\end{array}$ $\begin{array}{llllllll}\mathrm{H} 157 & \mathrm{H} & 0.96673 & 0.87118 & 0.86682 & 0.00000 & \text { Uiso } & 1.00\end{array}$ $\begin{array}{llllllll}H 158 & \mathrm{H} & 0.05939 & 0.92812 & 0.91947 & 0.00000 & \text { Uiso } & 1.00\end{array}$ $\begin{array}{llllllll}\mathrm{H} 159 & \mathrm{H} & 0.00175 & 0.83723 & -0.02305 & 0.00000 & \text { Uiso } & 1.00\end{array}$ $\begin{array}{llllllll}\mathrm{H} 160 & \mathrm{H} & 0.85980 & 0.04305 & 0.84251 & 0.00000 & \text { Uiso } & 1.00\end{array}$ $\begin{array}{llllllll}\mathrm{H} 161 & \mathrm{H} & 0.95823 & 0.09740 & 0.88833 & 0.00000 & \text { Uiso } & 1.00\end{array}$ $\begin{array}{llllllll}\mathrm{H} 162 & \mathrm{H} & 0.83920 & 0.12181 & 0.93618 & 0.00000 & \text { Uiso } & 1.00\end{array}$ $\begin{array}{llllllll}\text { C163 } & \mathrm{C} & 0.90864 & 0.88293 & 0.27696 & 0.00000 & \text { Uiso } & 1.00\end{array}$ $\begin{array}{llllllll}\text { C164 } & \mathrm{C} & -0.00457 & 0.80409 & 0.27430 & 0.00000 & \text { Uiso } & 1.00\end{array}$ 
$\begin{array}{llllllll}\text { C165 } & \mathrm{C} & 0.06329 & 0.81130 & 0.35214 & 0.00000 & \text { Uiso } & 1.00\end{array}$ $\begin{array}{lllllllll}\mathrm{C} 166 & \mathrm{C} & 0.04600 & 0.80723 & 0.18510 & 0.00000 & \text { Uiso } & 1.00\end{array}$ $\begin{array}{llllllllll}\mathrm{C} 167 & \mathrm{C} & 0.91384 & 0.97810 & -0.03518 & 0.00000 & \text { Uiso } & 1.00\end{array}$ $\begin{array}{lllllllll}\mathrm{C} 168 & \mathrm{C} & 0.86871 & 0.97124 & 0.04258 & 0.00000 & \text { Uiso } & 1.00\end{array}$ $\begin{array}{llllllllll}\mathrm{C} 169 & \mathrm{C} & 0.98929 & 0.89902 & 0.93097 & 0.00000 & \text { Uiso } & 1.00\end{array}$ $\begin{array}{llllllll}\text { C170 } & \text { C } & 0.89114 & 0.06523 & 0.90537 & 0.00000 & \text { Uiso } & 1.00\end{array}$

\section{File $n^{\circ} 5$}

$\begin{array}{llllllll}\text { Si2 } & \text { Si } & 0.37058 & 0.75561 & 0.02941 & 0.00000 & \text { Uiso } & 1.00\end{array}$ $\begin{array}{lllllllll}\mathrm{Si} 3 & \mathrm{Si} & 0.60129 & -0.00578 & 0.02805 & 0.00000 & \text { Uiso } & 1.00\end{array}$ $\begin{array}{lllllllll}\mathrm{Si} 4 & \mathrm{Si} & 0.61952 & 0.22494 & 0.27603 & 0.00000 & \text { Uiso } & 1.00\end{array}$ $\begin{array}{llllllll}\mathrm{Si5} & \mathrm{Si} & 0.37673 & 0.98556 & 0.27968 & 0.00000 & \text { Uiso } & 1.00 \\ \mathrm{Si6} & \mathrm{Si} & 0.75839 & 0.37696 & 0.23121 & 0.00000 \text { Uiso } & 1.00\end{array}$ $\begin{array}{llllllll}\text { Si6 } & \text { Si } & 0.75839 & 0.37696 & 0.23121 & 0.00000 & \text { Uiso } & 1.00\end{array}$ $\begin{array}{llllllll}\mathrm{Si} 7 & \mathrm{Si} & 0.01273 & 0.60127 & 0.23073 & 0.00000 & \text { Uiso } & 1.00\end{array}$ $\begin{array}{llllllll}\mathrm{Si} 8 & \mathrm{Si} & 0.22972 & 0.60578 & 0.48032 & 0.00000 & \text { Uiso } & 1.00\end{array}$ $\begin{array}{lllllllll}\text { Si9 } & \text { Si } & 0.98993 & 0.36849 & 0.48302 & 0.00000 & \text { Uiso } & 1.00\end{array}$ $\begin{array}{llllllll}\mathrm{Si} 10 & \mathrm{Si} & 0.61371 & 0.22282 & 0.48328 & 0.00000 & \text { Uiso } & 1.00\end{array}$ $\begin{array}{llllllll}\text { Sil1 } & \text { Si } & 0.36946 & 0.98462 & 0.48601 & 0.00000 & \text { Uiso } & 1.00\end{array}$ $\begin{array}{llllllll}\text { Si12 } & \text { Si } & 0.38483 & 0.75727 & 0.23416 & 0.00000 & \text { Uiso } & 1.00\end{array}$ $\begin{array}{llllllll}\text { Si13 } & \text { Si } & 0.60181 & 0.00453 & 0.23468 & 0.00000 & \text { Uiso } & 1.00\end{array}$ $\begin{array}{lllllllll}\text { Sil4 } & \text { Si } & 0.23481 & 0.61045 & 0.27370 & 0.00000 & \text { Uiso } & 1.00\end{array}$ $\begin{array}{lllllllll}\text { Si15 } & \text { Si } & 0.98749 & 0.37785 & 0.27728 & 0.00000 & \text { Uiso } & 1.00\end{array}$ $\begin{array}{lllllllll}\text { Sil6 } & \text { Si } & 0.76154 & 0.36266 & 0.02591 & 0.00000 & \text { Uiso } & 1.00\end{array}$ $\begin{array}{lllllllll}\text { Si17 } & \text { Si } & 0.00319 & 0.59498 & 0.02275 & 0.00000 & \text { Uiso } & 1.00\end{array}$ $\begin{array}{lllllllll}\text { Si18 } & \text { Si } & 0.46182 & 0.27766 & 0.12905 & 0.00000 & \text { Uiso } & 1.00\end{array}$ $\begin{array}{lllllllll} & \text { Si19 } & \text { Si } & 0.30598 & 0.12752 & 0.13165 & 0.00000 & \text { Uiso } & 1.00\end{array}$

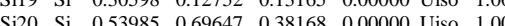
$\begin{array}{lllllllll} & \text { Si20 Si } & 0.53985 & 0.69647 & 0.38168 & 0.00000 & \text { Uiso } & 1.00\end{array}$ $\begin{array}{llllllll}\text { Si21 } & \text { Si } & 0.68882 & 0.85903 & 0.38311 & 0.00000 & \text { Uiso } & 1.00\end{array}$

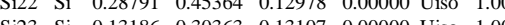
$\begin{array}{llllllll}\text { Si23 } & \text { Si } & 0.13186 & 0.30363 & 0.13107 & 0.00000 & \text { Uiso } & 1.00\end{array}$ $\begin{array}{llllllll}\text { Si24 } & \text { Si } & 0.69990 & 0.52845 & 0.37755 & 0.00000 & \text { Uiso } & 1.00\end{array}$ $\begin{array}{lllllllll}\text { Si25 } & \text { Si } & 0.37198 & 0.76058 & 0.52433 & 0.00000 & \text { Uiso } & 1.00\end{array}$ $\begin{array}{llllllll}\mathrm{Si} 26 & \mathrm{Si} & 0.59710 & -0.00081 & 0.53188 & 0.00000 & \text { Uiso } & 1.00\end{array}$ $\begin{array}{llllllll}\text { Si27 } & \text { Si } & 0.62314 & 0.22324 & 0.77526 & 0.00000 & \text { Uiso } & 1.00\end{array}$ $\begin{array}{lllllllll}\text { Si28 } & \text { Si } & 0.38515 & 0.98319 & 0.78079 & 0.00000 & \text { Uiso } & 1.00\end{array}$ $\begin{array}{llllllll}\mathrm{Si} 29 & \mathrm{Si} & 0.75881 & 0.37703 & 0.72902 & 0.00000 & \text { Uiso } & 1.00\end{array}$ $\begin{array}{llllllll}\mathrm{Si} 30 & \mathrm{Si} & 0.00848 & 0.60217 & 0.74113 & 0.00000 & \text { Uiso } & 1.00\end{array}$ $\begin{array}{lllllllll} & \mathrm{Si3} & \mathrm{Si} & 0.22921 & 0.60581 & 0.98191 & 0.00000 & \text { Uiso } & 1.00\end{array}$ $\begin{array}{llllllll}\mathrm{Si} 32 & \mathrm{Si} & 0.98564 & 0.36781 & 0.98141 & 0.00000 & \text { Uiso } & 1.00\end{array}$ $\begin{array}{lllllllll}\mathrm{Si} 33 & \mathrm{Si} & 0.61479 & 0.21838 & 0.98193 & 0.00000 & \text { Uiso } & 1.00\end{array}$ $\begin{array}{llllllll}\text { Si34 } & \text { Si } & 0.37314 & 0.97929 & 0.98510 & 0.00000 & \text { Uiso } & 1.00\end{array}$ $\begin{array}{llllllll}\mathrm{Si35} & \mathrm{Si} & 0.38027 & 0.75614 & 0.73014 & 0.00000 & \text { Uiso } & 1.00\end{array}$ $\begin{array}{lllllllll}\mathrm{Si36} & \mathrm{Si} & 0.60973 & 0.00173 & 0.73855 & 0.00000 & \text { Uiso } & 1.00\end{array}$ $\begin{array}{lllllllll}\text { Si37 } & \text { Si } & 0.23069 & 0.61844 & 0.77662 & 0.00000 & \text { Uiso } & 1.00\end{array}$ $\begin{array}{lllllllll}\text { Si38 } & \text { Si } & 0.98625 & 0.37894 & 0.77965 & 0.00000 & \text { Uiso } & 1.00\end{array}$ $\begin{array}{llllllllll}\text { Si39 } & \text { Si } & 0.76536 & 0.36467 & 0.52388 & 0.00000 & \text { Uiso } & 1.00\end{array}$ $\begin{array}{lllllllll} & \mathrm{Si39} & \mathrm{Si} & 0.76536 & 0.36467 & 0.52388 & 0.00000 & \text { Uiso } & 1.00\end{array}$ $\begin{array}{llllllllll} & \mathrm{Si} 41 & \mathrm{Si} & 0.46269 & 0.28398 & 0.63069 & 0.00000 & \text { Uiso } & 1.00\end{array}$ $\begin{array}{lllllllll} & \text { Si41 } & \text { Si } & 0.46269 & 0.28398 & 0.63069 & 0.00000 & \text { Uiso } & 1.00\end{array}$

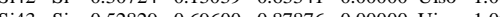
$\begin{array}{llllllll} & \text { Si43 Si } & 0.52829 & 0.69600 & 0.87876 & 0.00000 & \text { Unso } & 1.00\end{array}$

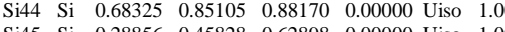
$\begin{array}{lllllllll}\mathrm{Si} 45 & \mathrm{Si} & 0.28856 & 0.45828 & 0.62898 & 0.00000 & \text { Uiso } & 1.00\end{array}$ $\begin{array}{llllllll}\mathrm{Si} 46 & \mathrm{Si} & 0.13345 & 0.30414 & 0.63059 & 0.00000 & \text { Uiso } & 1.00\end{array}$ $\begin{array}{lllllllll}\mathrm{Si} 47 & \mathrm{Si} & 0.70066 & 0.52346 & 0.87788 & 0.00000 & \text { Uiso } & 1.00\end{array}$ $\begin{array}{llllllll}\mathrm{Si} 48 & \mathrm{Si} & 0.85693 & 0.67764 & 0.88032 & 0.00000 & \text { Uiso } & 1.00\end{array}$ $\begin{array}{lllllllll}\mathrm{Al} 49 & \mathrm{Al} & 0.86537 & 0.68285 & 0.38072 & 0.00000 & \text { Uiso } & 1.00\end{array}$ $\begin{array}{lllllllll}\mathrm{O} 50 & \mathrm{O} & 0.53196 & 0.27916 & 0.21534 & 0.00000 & \text { Uiso } & 1.00\end{array}$ $\begin{array}{lllllllll}\mathrm{O} 51 & \mathrm{O} & 0.30415 & 0.05924 & 0.21916 & 0.00000 & \text { Uiso } & 1.00\end{array}$ $\begin{array}{lllllllll}052 & \mathrm{O} & 0.34120 & 0.87765 & 0.51329 & 0.00000 & \text { Uiso } & 1.00\end{array}$ $\begin{array}{llllllllll}053 & \mathrm{O} & 0.46674 & 0.72579 & 0.46363 & 0.00000 & \text { Uiso } & 1.00\end{array}$ $\begin{array}{llllllll}054 & \mathrm{O} & 0.66766 & 0.92048 & 0.47348 & 0.00000 & \text { Uiso } & 1.00\end{array}$ $\begin{array}{llllllllll}055 & \mathrm{O} & 0.64115 & 0.10978 & 0.24885 & 0.00000 & \text { Uiso } & 1.00\end{array}$ $\begin{array}{llllllll}056 & \mathrm{O} & 0.28453 & 0.51476 & 0.03789 & 0.00000 & \text { Uiso } & 1.00\end{array}$ $\begin{array}{llllllll}\mathrm{O} 57 & \mathrm{O} & 0.06973 & 0.30480 & 0.03982 & 0.00000 & \text { Uiso } & 1.00\end{array}$ $\begin{array}{lllllllll}058 & \mathrm{O} & 0.87471 & 0.36881 & 0.25088 & 0.00000 & \text { Uiso } & 1.00\end{array}$ $\begin{array}{lllllllll}059 & \mathrm{O} & 0.69886 & 0.46616 & 0.28567 & 0.00000 & \text { Uiso } & 1.00\end{array}$ $\begin{array}{llllllll}\mathrm{O} 60 & \mathrm{O} & 0.93922 & 0.67428 & 0.28822 & 0.00000 & \text { Uiso } & 1.00 \\ 061 & \mathrm{O} & 0.11265 & 0.61875 & -0.00100 & 0.00000 & \text { Uiso } & 1.00\end{array}$

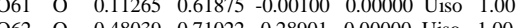
$\begin{array}{llllllll}062 & \mathrm{O} & 0.48039 & 0.71022 & 0.28901 & 0.00000 & \text { Uiso } & 1.00\end{array}$ $\begin{array}{llllllll}063 & \mathrm{O} & 0.66814 & 0.92498 & 0.29544 & 0.00000 & \text { Uiso } & 1.00\end{array}$ $\begin{array}{lllllllll}064 & \mathrm{O} & 0.62844 & 0.10286 & 0.00649 & 0.00000 & \text { Uiso } & 1.00\end{array}$ $\begin{array}{lllllllll}065 & \mathrm{O} & 0.52528 & 0.27563 & 0.03851 & 0.00000 & \text { Uiso } & 1.00\end{array}$ $\begin{array}{lllllllll}066 & \mathrm{O} & 0.30662 & 0.06121 & 0.04284 & 0.00000 & \text { Uiso } & 1.00\end{array}$ $\begin{array}{llllllll}067 & \mathrm{O} & 0.36500 & 0.87397 & 0.25265 & 0.00000 & \text { Uiso } & 1.00\end{array}$ $\begin{array}{llllllll}068 & \mathrm{O} & 0.72104 & 0.45150 & 0.45893 & 0.00000 & \text { Uiso } & 1.00\end{array}$ $\begin{array}{llllllll}069 & \mathrm{O} & 0.92727 & 0.66803 & 0.47965 & 0.00000 & \text { Uiso } & 1.00\end{array}$ $\begin{array}{llllllll}070 & \mathrm{O} & 0.12362 & 0.63220 & 0.23785 & 0.00000 & \text { Uiso } & 1.00\end{array}$ $\begin{array}{llllllll}071 & \mathrm{O} & 0.29501 & 0.52420 & 0.21493 & 0.00000 & \text { Uiso } & 1.00\end{array}$ $\begin{array}{llllllll}\mathrm{O} 72 & \mathrm{O} & 0.05893 & 0.30339 & 0.21541 & 0.00000 & \text { Uiso } & 1.00\end{array}$ $\begin{array}{llllllll}073 & \mathrm{O} & 0.88308 & 0.34125 & 0.51327 & 0.00000 & \text { Uiso } & 1.00\end{array}$ $\begin{array}{llllllll}074 & \mathrm{O} & 0.48893 & 0.00804 & 0.26545 & 0.00000 & \text { Uiso } & 1.00\end{array}$ $\begin{array}{lllllllll}075 & \mathrm{O} & 0.48862 & 0.98513 & 0.00223 & 0.00000 & \text { Uiso } & 1.00\end{array}$ $\begin{array}{llllllll}076 & \mathrm{O} & 0.99165 & 0.48366 & 0.99298 & 0.00000 & \text { Uiso } & 1.00\end{array}$ $\begin{array}{lllllllll}077 & \mathrm{O} & 0.01350 & 0.48855 & 0.26495 & 0.00000 & \text { Uiso } & 1.00\end{array}$ $\begin{array}{llllllll}078 & \mathrm{O} & 0.38455 & 0.37498 & 0.12902 & 0.00000 & \text { Uiso } & 1.00\end{array}$ $\begin{array}{lllllllll}079 & \mathrm{O} & 0.20907 & 0.20558 & 0.13235 & 0.00000 & \text { Uiso } & 1.00\end{array}$ $\begin{array}{lllllllll}080 & 0 & 0.58909 & 0.58458 & 0.39172 & 0.00000 & \text { Uiso } & 1.00\end{array}$ $\begin{array}{llllllllll}080 & 0 & 0.58909 & 0.58458 & 0.39172 & 0.0000 & \text { Uiso } & 1.00\end{array}$ $\begin{array}{llllllllll}081 & \mathrm{O} & 0.80994 & 0.81753 & 0.38093 & 0.00000 & \text { Uiso } & 1.00\end{array}$ $\begin{array}{llllllllll}082 & \mathrm{O} & 0.40563 & 0.18037 & 0.13332 & 0.00000 & \text { Uiso } & 1.00\end{array}$ $\begin{array}{lllllllll}083 & \mathrm{O} & 0.62611 & 0.76830 & 0.38163 & 0.00000 & \text { Uiso } & 1.00\end{array}$ $\begin{array}{llllllllll}084 & 0 & 0.18911 & 0.40022 & 0.13665 & 0.00000 & \text { Uiso } & 1.00\end{array}$ $\begin{array}{lllllllll}085 & 0 & 0.77705 & 0.60570 & 0.37328 & 0.00000 & \text { Uiso } & 1.00\end{array}$ $\begin{array}{llllllll}086 & 0 & 0.34644 & 0.00500 & 0.38217 & 0.00000 & \text { Uiso } & 1.00\end{array}$ $\begin{array}{llllllll}087 & \mathrm{O} & 0.58590 & 0.23346 & 0.37902 & 0.00000 & \text { Uiso } & 1.00\end{array}$ $\begin{array}{llllllll}088 & \mathrm{O} & 0.61722 & 0.97046 & 0.13272 & 0.00000 & \text { Uiso } & 1.00\end{array}$ $\begin{array}{lllllllll}\text { O89 } & \text { O } & 0.40841 & 0.73668 & 0.13029 & 0.00000 & \text { Uiso } & 1.00\end{array}$ $\begin{array}{llllllll}090 & \mathrm{O} & 0.00639 & 0.34341 & 0.37901 & 0.00000 & \text { Uiso } & 1.00\end{array}$ $\begin{array}{llllllll}\mathrm{O} 91 & \mathrm{O} & 0.23666 & 0.57595 & 0.37646 & 0.00000 & \text { Uiso } & 1.00 \\ \mathrm{O} 92 & \mathrm{O} & 0.98094 & 0.60796 & 0.12706 & 0.00000 & \text { Uiso } & 1.00\end{array}$ 
$\begin{array}{llllllll}093 & \mathrm{O} & 0.73705 & 0.39717 & 0.12681 & 0.00000 & \text { Uiso } & 1.00\end{array}$ $\begin{array}{lllllllll}094 & \mathrm{O} & 0.71997 & 0.27355 & 0.26033 & 0.00000 & \text { Uiso } & 1.00\end{array}$ $\begin{array}{lllllllll}095 & \mathrm{O} & 0.27057 & 0.70636 & 0.01330 & 0.00000 & \text { Uiso } & 1.00\end{array}$ $\begin{array}{llllllll}096 & \mathrm{O} & 0.28754 & 0.70980 & 0.26440 & 0.00000 & \text { Uiso } & 1.00\end{array}$ $\begin{array}{llllllll}097 & \mathrm{O} & 0.71666 & 0.26082 & 0.00572 & 0.00000 & \text { Uiso } & 1.00\end{array}$ $\begin{array}{lllllllll}098 & \mathrm{O} & 0.53469 & 0.27774 & 0.71578 & 0.00000 & \text { Uiso } & 1.00\end{array}$ $\begin{array}{lllllllllllllllll}099 & 0 & 0.31581 & 0.05788 & 0.71831 & 0.00000 & \text { Uiso } & 1.00\end{array}$ $\begin{array}{lllllllllll}0100 & 0 & 0.31581 & 0.34576 & 0.87208 & 0.01216 & 0.00000 & \text { Uiso } & 1.00\end{array}$

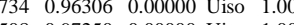
$\begin{array}{lllllllllll}0102 & 0 & 0.67421 & 0.91508 & 0.97250 & 0.00000 & \text { Uiso } & 1.00\end{array}$ $\begin{array}{lllllllll}0103 & \mathrm{O} & 0.64673 & 0.10856 & 0.74874 & 0.00000 & \text { Uiso } & 1.00 \\ 0104 & \mathrm{O} & 0.29118 & 0.51793 & 0.53637 & 0.00000 & \text { Uis } & 1.00\end{array}$ $\begin{array}{llllllllll}0104 & \mathrm{O} & 0.29118 & 0.51793 & 0.53637 & 0.00000 & \text { Uiso } & 1.00 \\ 0105 & \mathrm{O} & 0.07332 & 0.2959 & 0.5384 & 0.0000 & \text { Uiso } & 1.00\end{array}$ $\begin{array}{lllllllll}0105 & 0 & 0.07332 & 0.23959 & 0.53849 & 0.00000 & \text { Uiso } & 1.00\end{array}$ $\begin{array}{llllllll}0106 & \mathrm{O} & 0.87442 & 0.37252 & 0.74964 & 0.00000 & \text { Uiso } & 1.00\end{array}$ $\begin{array}{llllllllll}0107 & \mathrm{O} & 0.69746 & 0.46508 & 0.78472 & 0.00000 & \text { Uiso } & 1.00\end{array}$ $\begin{array}{lllllllll}0108 & \mathrm{O} & 0.92514 & 0.67515 & 0.79234 & 0.00000 & \text { Uiso } & 1.00\end{array}$ $\begin{array}{llllllll}0109 & \mathrm{O} & 0.11720 & 0.62261 & 0.51340 & 0.00000 & \text { Uiso } & 1.00\end{array}$ $\begin{array}{lllllllll}\mathrm{O} 110 & \mathrm{O} & 0.46725 & 0.69445 & 0.78691 & 0.00000 & \text { Uiso } & 1.00\end{array}$ $\begin{array}{llllllll}0111 & \mathrm{O} & 0.68092 & 0.92248 & 0.79557 & 0.00000 & \text { Uiso } & 1.00\end{array}$

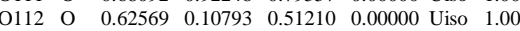
$\begin{array}{lllllllll}0113 & \mathrm{O} & 0.52602 & 0.28374 & 0.53972 & 0.00000 & \text { Uiso } & 1.00\end{array}$ $\begin{array}{llllllll}0114 & \mathrm{O} & 0.30285 & 0.06739 & 0.54280 & 0.00000 & \text { Uiso } & 1.00\end{array}$ $\begin{array}{llllllll}0115 & \mathrm{O} & 0.37620 & 0.87200 & 0.75026 & 0.00000 & \text { Uiso } & 1.00\end{array}$ $\begin{array}{llllllll}0116 & \mathrm{O} & 0.71209 & 0.44742 & 0.96004 & 0.00000 & \text { Uiso } & 1.00\end{array}$ $\begin{array}{llllllll}0117 & \mathrm{O} & 0.92369 & 0.66916 & 0.96884 & 0.00000 & \text { Uiso } & 1.00\end{array}$ $\begin{array}{llllllll}0118 & \mathrm{O} & 0.11404 & 0.63968 & 0.75947 & 0.00000 & \text { Uiso } & 1.00\end{array}$ $\begin{array}{llllllllll}0119 & 0 & 0.28232 & 0.53277 & 0.71243 & 0.00000 & \text { Uiso } & 1.00\end{array}$ $\begin{array}{llllllllll}01120 & 0 & 0.28232 & 0.53277 & 0.71243 & 0.00000 & \text { Uiso } & 1.00\end{array}$ $\begin{array}{llllllllllll}0121 & 0 & 0.05881 & 0.31213 & 0.71372 & 0.00000 & 0.07892 & 0.34187 & 0.01243 & 0.00000 & \text { Uiso } & 1.00\end{array}$ $\begin{array}{llllllllll}0121 & 0 & 0.87892 & 0.3413 & 0.71372 & 0.00000 & \text { Uiso } & 1.00 \\ 0122 & 0 & 0.49811 & 0.00594 & 0.77439 & 0.00000 & \text { Unso } & 1.00\end{array}$ $\begin{array}{lllllllllll}0122 & 0 & 0.49811 & 0.00594 & 0.77439 & 0.00000 & \text { Uiso } & 1.00\end{array}$ $\begin{array}{llllllllll}0123 & \mathrm{O} & 0.48465 & 0.00898 & 0.50506 & 0.00000 & \text { Uiso } & 1.00\end{array}$ $\begin{array}{lllllllll}0124 & \mathrm{O} & 0.00087 & 0.48212 & 0.50185 & 0.00000 & \text { Uiso } & 1.00 \\ 0125 & \mathrm{O} & 0.01022 & 0.49155 & 0.77930 & 0.0000 & \text { Uis } & 1.00\end{array}$ $\begin{array}{llllllllll}0125 & \mathrm{O} & 0.01020 & 0.49155 & 0.77930 & 0.00000 & \text { Uiso } & 1.00\end{array}$ $\begin{array}{lllllllll}0126 & \mathrm{O} & 0.38864 & 0.38394 & 0.63672 & 0.00000 & \text { Uiso } & 1.00\end{array}$ $\begin{array}{lllllllll}0127 & \mathrm{O} & 0.20762 & 0.20443 & 0.64135 & 0.00000 & \text { Uiso } & 1.00\end{array}$ $\begin{array}{llllllll}0128 & \mathrm{O} & 0.59869 & 0.59402 & 0.88928 & 0.00000 & \text { Uiso } & 1.00\end{array}$ $\begin{array}{llllllll}0129 & \mathrm{O} & 0.78572 & 0.78031 & 0.88219 & 0.00000 & \text { Uiso } & 1.00\end{array}$ $\begin{array}{lllllllll}0130 & \mathrm{O} & 0.40312 & 0.18883 & 0.63031 & 0.00000 & \text { Uiso } & 1.00\end{array}$ $\begin{array}{llllllll}0131 & \mathrm{O} & 0.59087 & 0.78849 & 0.87594 & 0.00000 & \text { Uiso } & 1.00\end{array}$ $\begin{array}{lllllllll}0132 & \mathrm{O} & 0.19339 & 0.39905 & 0.63037 & 0.00000 & \text { Uiso } & 1.00\end{array}$ $\begin{array}{llllllll}\mathrm{O} 133 & \mathrm{O} & 0.79287 & 0.58609 & 0.87691 & 0.00000 & \text { Uiso } & 1.00\end{array}$ $\begin{array}{lllllllll}0134 & \mathrm{O} & 0.34781 & 0.00010 & 0.88172 & 0.00000 & \text { Uiso } & 1.00\end{array}$ $\begin{array}{llllllll}0135 & \mathrm{O} & 0.58916 & 0.23392 & 0.87791 & 0.00000 & \text { Uiso } & 1.00\end{array}$ $\begin{array}{lllllllll}0136 & \mathrm{O} & 0.61560 & 0.97026 & 0.63488 & 0.00000 & \text { Uiso } & 1.00\end{array}$ $\begin{array}{lllllllll}0137 & \mathrm{O} & 0.40253 & 0.73523 & 0.62614 & 0.00000 & \text { Uiso } & 1.00\end{array}$ $\begin{array}{lllllllll}0138 & \mathrm{O} & 0.00383 & 0.33593 & 0.87891 & 0.00000 & \text { Uiso } & 1.00\end{array}$ $\begin{array}{llllllllll}0139 & \mathrm{O} & 0.25297 & 0.58425 & 0.87813 & 0.00000 & \text { Uiso } & 1.00\end{array}$ $\begin{array}{llllllllll}0140 & 0 & 0.98450 & 0.60448 & 0.63701 & 0.00000 & \text { Uiso } & 1.00\end{array}$ $\begin{array}{llllllllll}0141 & 0 & 0.73826 & 0.39787 & 0.62495 & 0.00000 & \text { Uiso } & 1.00\end{array}$

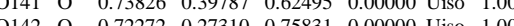
$\begin{array}{lllllllll}0142 & \mathrm{O} & 0.72272 & 0.27310 & 0.75831 & 0.00000 & \text { Uiso } & 1.00\end{array}$ $\begin{array}{lllllllll}0143 & 0 & 0.27833 & 0.70681 & 0.45540 & 0.00000 & \text { Uiso } & 1.00\end{array}$ $\begin{array}{llllllllll}0144 & \mathrm{O} & 0.27583 & 0.72049 & 0.75720 & 0.00000 & \text { Uiso } & 1.00 \\ 0145 & \mathrm{O} & 0.71745 & 0.26431 & 0.50200 & 0.00000 & \text { Uiso } & 1.00\end{array}$ $\begin{array}{lllllllll}0145 & \mathrm{O} & 0.71745 & 0.26431 & 0.50200 & 0.00000 & \text { Uiso } & 1.00\end{array}$ $\begin{array}{lllllllll}\mathrm{H} 146 & \mathrm{H} & 0.92151 & 0.83244 & 0.2368 & 0.00000 & \text { Uiso } & 1.00\end{array}$ $\begin{array}{lllllllll}\mathrm{H} 147 & \mathrm{H} & 0.02998 & 0.91416 & 0.40483 & 0.00000 & \text { Uiso } & 1.00\end{array}$ $\begin{array}{llllllll}\mathrm{H} 148 & \mathrm{H} & 0.96888 & 0.84360 & 0.48106 & 0.00000 & \text { Uiso } & 1.00\end{array}$ $\begin{array}{llllllll}\mathrm{H} 149 & \mathrm{H} & 0.01631 & 0.79103 & 0.37934 & 0.00000 & \text { Uiso } & 1.00\end{array}$

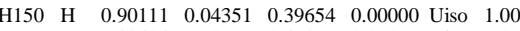
$\begin{array}{llllllll}\mathrm{H} 151 & \mathrm{H} & 0.83404 & 0.98691 & 0.47812 & 0.00000 & \text { Uiso } & 1.00\end{array}$ $\begin{array}{llllllll}\mathrm{H} 152 & \mathrm{H} & 0.77808 & 0.02763 & 0.37532 & 0.00000 & \text { Uiso } & 1.00\end{array}$ $\begin{array}{llllllll}\mathrm{H} 153 & \mathrm{H} & 0.95453 & 0.95226 & 0.25324 & 0.00000 & \text { Uiso } & 1.00\end{array}$ $\begin{array}{llllllll}\mathrm{H} 154 & \mathrm{H} & 0.82990 & 0.93654 & 0.23421 & 0.00000 & \text { Uiso } & 1.00\end{array}$ $\begin{array}{llllllll}\mathrm{H} 155 & \mathrm{H} & 0.10577 & 0.81701 & 0.07724 & 0.00000 & \text { Uiso } & 1.00\end{array}$ $\begin{array}{lllllllll}\mathrm{H} 156 & \mathrm{H} & 0.97738 & 0.86779 & 0.10407 & 0.00000 & \text { Uiso } & 1.00\end{array}$ $\begin{array}{llllllll}\mathrm{H} 157 & \mathrm{H} & 0.08143 & 0.83441 & 0.85033 & 0.00000 & \text { Uiso } & 1.00\end{array}$ $\begin{array}{lllllllll}\mathrm{H} 158 & \mathrm{H} & 0.11821 & 0.94942 & 0.87697 & 0.00000 & \text { Uiso } & 1.00\end{array}$ $\begin{array}{lllllllll}\mathrm{H} 159 & \mathrm{H} & 0.17245 & 0.84248 & 0.93316 & 0.00000 & \text { Uiso } & 1.00\end{array}$ $\begin{array}{lllllllll}\mathrm{H} 160 & \mathrm{H} & 0.90416 & 0.91765 & 0.88308 & 0.00000 & \text { Uiso } & 1.00\end{array}$ $\begin{array}{lllllllll}H 161 & \mathrm{H} & 0.90416 & 0.9165 & 0.88308 & 0.00000 & \text { Uiso } & 1.00 \\ \mathrm{H} 16115 & 0.02574 & 0.92792 & 0.00000 & \text { Uiso } & 1.00\end{array}$ $\begin{array}{llllllll}\mathrm{H} 161 & \mathrm{H} & 0.94115 & 0.02574 & 0.92792 & 0.00000 & \text { Uiso } & 1.00 \\ \mathrm{H} 162 & \mathrm{H} & 0.87282 & 0.95071 & 0.99431 & 0.00000 & \text { Uis } & 1.00\end{array}$ Uiso 1.00 $\begin{array}{lllllllll}\text { C163 } & \text { C } & 0.84538 & -0.00615 & 0.40689 & 0.00000 & \text { Uiso } & 1.00\end{array}$ C164 C 0.88602 $\begin{array}{lllllllll}\mathrm{C} 165 & \mathrm{C} & 0.98056 & 0.85752 & 0.41084 & 0.00000 & \text { Uiso } & 1.00 \\ \mathrm{C} 166 & \mathrm{C} & 0.89763 & 0.90445 & 0.2601 & 0.00000 & \text { Uis } & 1.00\end{array}$ $\begin{array}{lllllllll}C 166 & \mathrm{C} & 0.89763 & 0.90445 & 0.26601 & 0.00000 & \text { Uiso } & 1.00\end{array}$ $\begin{array}{llllllllll}\mathrm{C} 167 & \mathrm{C} & 0.02474 & 0.89342 & -0.02592 & 0.00000 & \text { Uiso } & 1.00\end{array}$ $\begin{array}{llllllll}\mathrm{C} 168 & \mathrm{C} & 0.03689 & 0.85785 & 0.05621 & 0.00000 & \text { Uiso } & 1.00\end{array}$ $\begin{array}{llllllll}\mathrm{C} 169 & \mathrm{C} & 0.10406 & 0.87887 & 0.90557 & 0.00000 & \text { Uiso } & 1.00 \\ \mathrm{C} 170 & \mathrm{C} & 0.93033 & 0.94969 & 0.94400 & 0.00000 & \text { Uiso } & 1.00\end{array}$

\section{File $n^{\circ} 6$}

$\begin{array}{llllllll}\mathrm{Si} 2 & \mathrm{Si} & 0.37940 & 0.75931 & 0.02356 & 0.00000 & \text { Uiso } & 1.00\end{array}$ $\begin{array}{lllllllll}\mathrm{Si} 3 & \mathrm{Si} & 0.60962 & -0.00533 & 0.02306 & 0.00000 & \text { Uiso } & 1.00\end{array}$ $\begin{array}{llllllll}\mathrm{Si} 4 & \mathrm{Si} & 0.61990 & 0.22601 & 0.27345 & 0.00000 & \text { Uiso } & 1.00\end{array}$ $\begin{array}{llllllll}\mathrm{Si} 5 & \mathrm{Si} & 0.38319 & 0.98265 & 0.27462 & 0.00000 & \text { Uiso } & 1.00\end{array}$ $\begin{array}{llllllll}\text { Si6 } & \text { Si } & 0.76165 & 0.37476 & 0.22880 & 0.00000 & \text { Uiso } & 1.00\end{array}$ $\begin{array}{llllllll}\mathrm{Si} 7 & \mathrm{Si} & 0.01465 & 0.60036 & 0.22672 & 0.00000 & \text { Uiso } & 1.00\end{array}$ $\begin{array}{llllllll}\mathrm{Si} 8 & \mathrm{Si} & 0.23611 & 0.60587 & 0.47819 & 0.00000 & \text { Uiso } & 1.00\end{array}$ $\begin{array}{llllllll}\text { Si9 } & \text { Si } & 0.99208 & 0.37273 & 0.47770 & 0.00000 & \text { Uiso } & 1.00\end{array}$

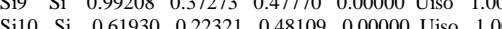
$\begin{array}{llllllll}\text { Si1 } & \text { Si } & 0.61930 & 0.22321 & 0.48109 & 0.00000 & \text { Uiso } & 1.00\end{array}$ $\begin{array}{llllllll}\text { Si11 } & \text { Si } & 0.37913 & 0.98318 & 0.47986 & 0.00000 & \text { Uiso } & 1.00\end{array}$ $\begin{array}{llllllllll}\text { Sil2 Si } & 0.38374 & 0.75602 & 0.22997 & 0.00000 & \text { Uiso } & 1.00\end{array}$

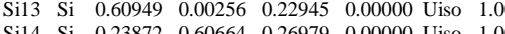
$\begin{array}{llllllll}\text { Si14 } & \text { Si } & 0.23872 & 0.60664 & 0.26979 & 0.00000 & \text { Uiso } & 1.00\end{array}$ $\begin{array}{lllllllll}\text { Si15 } & \text { Si } & 0.98991 & 0.37689 & 0.27370 & 0.00000 & \text { Uiso } & 1.00\end{array}$ $\begin{array}{llllllll}\text { Si16 Si } & 0.76750 & 0.36571 & 0.02321 & 0.00000 & \text { Uiso } & 1.00\end{array}$ $\begin{array}{llllllll}\text { Si17 } & \text { Si } & 0.01027 & 0.59840 & 0.01953 & 0.00000 & \text { Uiso } & 1.00\end{array}$ $\begin{array}{llllllll}\text { Si18 } & \text { Si } & 0.46889 & 0.28027 & 0.12659 & 0.00000 & \text { Uiso } & 1.00\end{array}$ $\begin{array}{llllllll}\text { Si19 } & \text { Si } & 0.31241 & 0.12953 & 0.12794 & 0.00000 & \text { Uiso } & 1.00\end{array}$ $\begin{array}{llllllll}\mathrm{Si} 20 & \mathrm{Si} & 0.54043 & 0.70205 & 0.37696 & 0.00000 & \text { Uiso } & 1.00\end{array}$ 
$\begin{array}{llllllll}\text { Si21 } & \text { Si } & 0.69470 & 0.85992 & 0.37728 & 0.00000 & \text { Uiso } & 1.00\end{array}$ $\begin{array}{llllllll}\mathrm{Si} 22 & \mathrm{Si} & 0.29529 & 0.45553 & 0.12550 & 0.00000 & \text { Uiso } & 1.00\end{array}$ $\begin{array}{llllllll}\mathrm{Si} 23 & \mathrm{Si} & 0.13819 & 0.30529 & 0.12756 & 0.00000 & \text { Uiso } & 1.00\end{array}$ $\begin{array}{llllllll}\mathrm{Si} 24 & \mathrm{Si} & 0.70836 & 0.52769 & 0.37587 & 0.00000 & \text { Uiso } & 1.00\end{array}$ $\begin{array}{llllllll}\mathrm{Si} 25 & \mathrm{Si} & 0.37636 & 0.75968 & 0.52165 & 0.00000 & \text { Uiso } & 1.00\end{array}$ $\begin{array}{lllllllll}\text { Si26 Si } & 0.60610 & -0.00163 & 0.52638 & 0.00000 & \text { Uiso } & 1.00\end{array}$ $\begin{array}{llllllllll} & \mathrm{Si} 27 & \mathrm{Si} & 0.62660 & 0.22312 & 0.57328 & 0.00000 & \text { Uis } & 1.00\end{array}$ $\begin{array}{llllllll}\mathrm{Si} 28 & \mathrm{Si} & 0.38743 & 0.98507 & 0.77544 & 0.00000 & \text { Uiso } & 1.00\end{array}$

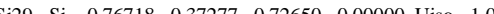
$\begin{array}{llllllll}\text { Si30 Si } & 0.01063 & 0.59841 & 0.73389 & 0.0000 & 0.0000 & \text { Uiso } & 1.00\end{array}$

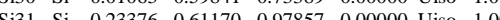
$\begin{array}{llllllll} & \text { Si31 Si } & 0.23376 & 0.61170 & 0.97857 & 0.00000 & \text { Uiso } & 1.00\end{array}$ $\begin{array}{llllllllll}\mathrm{Si32} & \text { Si } & 0.99314 & 0.37211 & 0.97760 & 0.00000 & \text { Uiso } & 1.00\end{array}$ $\begin{array}{llllllll}\mathrm{Si33} & \mathrm{Si} & 0.62635 & 0.21625 & 0.97957 & 0.00000 & \text { Uiso } & 1.00\end{array}$ $\begin{array}{lllllllll}\mathrm{Si34} & \mathrm{Si} & 0.38084 & 0.98481 & 0.97970 & 0.00000 & \text { Uiso } & 1.00 \\ \mathrm{Si35} & \mathrm{Si} & 0.37928 & 0.75833 & 0.72831 & 0.00000 & \text { Uis } & 1.00\end{array}$ $\begin{array}{llllllll}\mathrm{S} 135 & \mathrm{Si} & 0.37928 & 0.75833 & 0.72831 & 0.00000 & \text { Uiso } & 1.00\end{array}$ $\begin{array}{lllllllll}\mathrm{Si} 36 & \mathrm{Si} & 0.61362 & 0.00085 & 0.73225 & 0.00000 & \text { Uiso } & 1.00\end{array}$ $\begin{array}{llllllll}\mathrm{Si} 37 & \mathrm{Si} & 0.23227 & 0.61698 & 0.77290 & 0.00000 & \text { Uiso } & 1.00\end{array}$ $\begin{array}{lllllllll}\mathrm{Si} 38 & \mathrm{Si} & 0.99363 & 0.37366 & 0.77476 & 0.00000 & \text { Uiso } & 1.00\end{array}$ $\begin{array}{llllllll}\mathrm{Si39} & \mathrm{Si} & 0.76638 & 0.36603 & 0.52109 & 0.00000 & \text { Uiso } & 1.00\end{array}$ $\begin{array}{llllllll}\text { Si40 } & \text { Si } & 0.01229 & 0.59751 & 0.52570 & 0.00000 & \text { Uiso } & 1.00\end{array}$ $\begin{array}{lllllllll}\text { Si41 } & \text { Si } & 0.47003 & 0.28565 & 0.62769 & 0.00000 & \text { Uiso } & 1.00\end{array}$

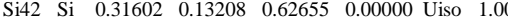
$\begin{array}{lllllllll}\mathrm{Si} 43 & \mathrm{Si} & 0.53525 & 0.69578 & 0.87348 & 0.00000 & \text { Uiso } & 1.00\end{array}$ $\begin{array}{llllllll}\mathrm{Si} 44 & \mathrm{Si} & 0.69180 & 0.85127 & 0.87497 & 0.00000 & \text { Uiso } & 1.00\end{array}$ $\begin{array}{llllllll}\text { Si45 } & \text { Si } & 0.29497 & 0.45953 & 0.62546 & 0.00000 & \text { Uiso } & 1.00\end{array}$ $\begin{array}{lllllllll}\text { Si46 Si } & 0.14029 & 0.30629 & 0.62540 & 0.00000 & \text { Uiso } & 1.00\end{array}$ $\begin{array}{lllllllll}\mathrm{Si} 47 & \mathrm{Si} & 0.70555 & 0.52481 & 0.87565 & 0.00000 & \text { Uiso } & 1.00\end{array}$ $\begin{array}{llllllll}\mathrm{Si} 48 & \mathrm{Si} & 0.86286 & 0.68035 & 0.87710 & 0.00000 & \text { Uiso } & 1.00\end{array}$ \begin{tabular}{lllllllllll}
\hline 4149 & Al & 0.86972 & 0.68429 & 0.37582 & 0.00000 & Uiso & 1.00
\end{tabular} \begin{tabular}{lllllllll}
\hline O149 & Al & 0.86972 & 0.68429 & 0.37582 & 0.00000 & Uiso & 1.00
\end{tabular} $\begin{array}{lllllllll}051 & 0 & 0.53188 & 0.28676 & 0.21685 & 0.0000 & \text { Uiso } & 1.00\end{array}$ $\begin{array}{lllllllll}051 & 0 & 0.31276 & 0.05849 & 0.21382 & 0.00000 & \text { Uiso } & 1.00\end{array}$ $\begin{array}{llllllllll}052 & 0 & 0.34865 & 0.87669 & 0.50687 & 0.00000 & \text { Uiso } & 1.00\end{array}$ $\begin{array}{llllllll}053 & \mathrm{O} & 0.46922 & 0.71654 & 0.46281 & 0.00000 & \text { Uiso } & 1.00\end{array}$ $\begin{array}{lllllllll}054 & \mathrm{O} & 0.68200 & 0.92748 & 0.46667 & 0.00000 & \text { Uiso } & 1.00 \\ 055 & \mathrm{O} & 0.6359 & 0.11370 & 0.23870 & 0.00000 & \text { Uso } & 1.00\end{array}$ $\begin{array}{llllllll}055 & \mathrm{O} & 0.63598 & 0.11370 & 0.23870 & 0.00000 & \text { Uiso } & 1.00\end{array}$ $\begin{array}{lllllllll}056 & \mathrm{O} & 0.28963 & 0.52382 & 0.03778 & 0.00000 & \text { Uiso } & 1.00\end{array}$ $\begin{array}{lllllllll}057 & \mathrm{O} & 0.07562 & 0.30727 & 0.03641 & 0.00000 & \text { Uiso } & 1.00\end{array}$ $\begin{array}{lllllllll}058 & \mathrm{O} & 0.87869 & 0.36252 & 0.24641 & 0.00000 & \text { Uiso } & 1.00\end{array}$ $\begin{array}{lllllllll}059 & \mathrm{O} & 0.70487 & 0.46449 & 0.28438 & 0.00000 & \text { Uiso } & 1.00\end{array}$ $\begin{array}{llllllll}060 & \mathrm{O} & 0.94834 & 0.67916 & 0.28485 & 0.00000 & \text { Uiso } & 1.00\end{array}$ $\begin{array}{llllllll}\mathrm{O} 61 & \mathrm{O} & 0.11864 & 0.62986 & 0.00285 & 0.00000 & \text { Uiso } & 1.00\end{array}$ $\begin{array}{lllllllll}062 & \mathrm{O} & 0.47559 & 0.70549 & 0.28685 & 0.00000 & \text { Uiso } & 1.00\end{array}$ $\begin{array}{llllllll}063 & \mathrm{O} & 0.68370 & 0.93204 & 0.29086 & 0.00000 & \text { Uiso } & 1.00\end{array}$ $\begin{array}{lllllllll}\mathrm{O} 64 & \mathrm{O} & 0.64503 & 0.10067 & 0.00334 & 0.00000 & \text { Uiso } & 1.00\end{array}$ $\begin{array}{llllllll}065 & \mathrm{O} & 0.53929 & 0.27160 & 0.04021 & 0.00000 & \text { Uiso } & 1.00\end{array}$ $\begin{array}{llllllll}066 & \mathrm{O} & 0.31285 & 0.06537 & 0.03780 & 0.00000 & \text { Uiso } & 1.00\end{array}$ $\begin{array}{llllllll}\text { O667 } & \mathrm{O} & 0.36260 & 0.87246 & 0.24973 & 0.00000 & \text { Uiso } & 1.00\end{array}$ $\begin{array}{llllllllll}068 & 0 & 0.71340 & 0.45172 & 0.45918 & 0.00000 & \text { Uiso } & 1.00\end{array}$ $\begin{array}{llllllllll}068 & 0 & 0.71340 & 0.45172 & 0.45918 & 0.00000 & \text { Uiso } & 1.00\end{array}$ $\begin{array}{llllllll}069 & \mathrm{O} & 0.94257 & 0.6787 & 0.47210 & 0.00000 & \text { Uiso } & 1.00\end{array}$ $\begin{array}{llllllllll}070 & \mathrm{O} & 0.12860 & 0.62363 & 0.23126 & 0.00000 & \text { Uiso } & 1.00\end{array}$ $\begin{array}{lllllllll}071 & 0 & 0.30347 & 0.52011 & 0.21481 & 0.00000 & \text { Uiso } & 1.00\end{array}$ $\begin{array}{lllllllll}072 & 0 & 0.06524 & 0.30625 & 0.21182 & 0.00000 & \text { Uiso } & 1.00\end{array}$ $\begin{array}{llllllllll}073 & 0 & 0.88412 & 0.34966 & 0.50784 & 0.00000 & \text { Uiso } & 1.00\end{array}$ $\begin{array}{lllllllll}074 & \mathrm{O} & 0.49718 & 0.99651 & 0.26002 & 0.00000 & \text { Uiso } & 1.00\end{array}$ $\begin{array}{lllllllll}075 & \mathrm{O} & 0.49576 & -0.00445 & 0.99479 & 0.00000 & \text { Uiso } & 1.00\end{array}$ $\begin{array}{llllllll}076 & \mathrm{O} & 0.00638 & 0.48737 & 0.98372 & 0.00000 & \text { Uiso } & 1.00\end{array}$ $\begin{array}{llllllll}077 & \mathrm{O} & 0.01036 & 0.48932 & 0.26299 & 0.00000 & \text { Uiso } & 1.00\end{array}$ $\begin{array}{llllllll}078 & \mathrm{O} & 0.39251 & 0.37810 & 0.11754 & 0.00000 & \text { Uiso } & 1.00\end{array}$ $\begin{array}{llllllll}079 & \mathrm{O} & 0.21391 & 0.20592 & 0.12998 & 0.00000 & \text { Uiso } & 1.00\end{array}$ $\begin{array}{llllllll}080 & \mathrm{O} & 0.60372 & 0.59629 & 0.38397 & 0.00000 & \text { Uiso } & 1.00\end{array}$ $\begin{array}{llllllll}\mathrm{O} 81 & \mathrm{O} & 0.80150 & 0.80007 & 0.37636 & 0.00000 & \text { Uiso } & 1.00\end{array}$ $\begin{array}{lllllllll}082 & \mathrm{O} & 0.41062 & 0.18428 & 0.13110 & 0.00000 & \text { Uiso } & 1.00\end{array}$ $\begin{array}{llllllll}083 & \mathrm{O} & 0.60673 & 0.79116 & 0.37440 & 0.00000 & \text { Uiso } & 1.00\end{array}$ $\begin{array}{lllllllll}084 & \mathrm{O} & 0.19719 & 0.40081 & 0.13136 & 0.00000 & \text { Uiso } & 1.00\end{array}$ $\begin{array}{llllllll}\mathrm{O} 85 & \mathrm{O} & 0.79892 & 0.58913 & 0.37588 & 0.00000 & \text { Uiso } & 1.00\end{array}$ $\begin{array}{llllllll}086 & \mathrm{O} & 0.35294 & 0.00503 & 0.37683 & 0.00000 & \text { Uiso } & 1.00\end{array}$ $\begin{array}{llllllll}087 & \mathrm{O} & 0.58934 & 0.23031 & 0.37721 & 0.00000 & \text { Uiso } & 1.00\end{array}$ $\begin{array}{llllllll}088 & \mathrm{O} & 0.62352 & 0.96780 & 0.12711 & 0.00000 & \text { Uiso } & 1.00\end{array}$ $\begin{array}{lllllllll}\mathrm{O} 88 & \mathrm{O} & 0.40863 & 0.73676 & 0.12614 & 0.00000 & \text { Uiso } & 1.00\end{array}$ $\begin{array}{llllllll}\mathrm{O} 90 & \mathrm{O} & 0.01039 & 0.34149 & 0.37517 & 0.00000 & \text { Uiso } & 1.00\end{array}$ $\begin{array}{lllllllll}091 & 0 & 0.01039 & 0.34149 & 0.37517 & 0.00000 & \text { Uiso } & 1.00\end{array}$ \begin{tabular}{lllllllll}
091 & $\mathrm{O}$ & 0.23927 & 0.57721 & 0.37402 & 0.00000 & Uiso & 1.00 \\
\hline 92 & $\mathrm{O}$ & 0.98187 & 0.60434 & 0.12307 & 0.00000 & Uiso & 1.00
\end{tabular} $\begin{array}{lllllllllll}09 & 0 & 0.98187 & 0.60434 & 0.12307 & 0.00000 & \text { Uiso } & 1.00\end{array}$ $\begin{array}{llllllllll}093 & 0 & 0.73948 & 0.39539 & 0.12450 & 0.00000 & \text { Uiso } & 1.00\end{array}$ $\begin{array}{lllllllll}094 & \mathrm{O} & 0.72172 & 0.27231 & 0.25809 & 0.00000 & \text { Uiso } & 1.00\end{array}$ $\begin{array}{llllllll}095 & \mathrm{O} & 0.28001 & 0.71274 & -0.00134 & 0.00000 & \text { Uiso } & 1.00\end{array}$ $\begin{array}{llllllll}096 & \mathrm{O} & 0.28476 & 0.70946 & 0.25756 & 0.00000 & \text { Uiso } & 1.00\end{array}$ $\begin{array}{lllllllll}097 & \mathrm{O} & 0.72798 & 0.26216 & 0.99811 & 0.00000 & \text { Uiso } & 1.00\end{array}$ $\begin{array}{llllllll}098 & \mathrm{O} & 0.53909 & 0.28118 & 0.71488 & 0.00000 & \text { Uiso } & 1.00\end{array}$ $\begin{array}{llllllll}099 & \mathrm{O} & 0.32015 & 0.06239 & 0.71348 & 0.00000 & \text { Uiso } & 1.00\end{array}$ $\begin{array}{llllllll}0100 & \mathrm{O} & 0.35808 & 0.87649 & 0.00858 & 0.00000 & \text { Uiso } & 1.00\end{array}$ $\begin{array}{llllllll}0101 & \mathrm{O} & 0.46953 & 0.71203 & 0.96240 & 0.00000 & \text { Uiso } & 1.00\end{array}$ $\begin{array}{lllllllll}0102 & \mathrm{O} & 0.67741 & 0.91212 & 0.96696 & 0.00000 & \text { Uiso } & 1.00\end{array}$ $\begin{array}{llllllll}0103 & \mathrm{O} & 0.64654 & 0.10951 & 0.74246 & 0.00000 & \text { Uiso } & 1.00\end{array}$ $\begin{array}{llllllll}0104 & \mathrm{O} & 0.30113 & 0.51972 & 0.53333 & 0.00000 & \text { Uiso } & 1.00\end{array}$ $\begin{array}{lllllllll}0105 & \mathrm{O} & 0.07336 & 0.30583 & 0.53701 & 0.00000 & \text { Uiso } & 1.00\end{array}$ $\begin{array}{lllllllll}0106 & \mathrm{O} & 0.88454 & 0.36070 & 0.74062 & 0.00000 & \text { Uiso } & 1.00\end{array}$ $\begin{array}{lllllllll}0107 & \mathrm{O} & 0.71418 & 0.46411 & 0.78351 & 0.00000 & \text { Uiso } & 1.00\end{array}$ $\begin{array}{llllllll}0108 & 0 & 0.92826 & 0.67009 & 0.78729 & 0.00000 & \text { Uiso } & 1.00\end{array}$ $\begin{array}{lllllllll}0109 & 0 & 0.12548 & 0.62078 & 0.51582 & 0.00000 & \text { Uiso } & 1.00\end{array}$

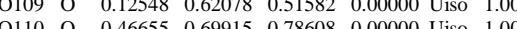
$\begin{array}{lllllllllll}0111 & 0 & 0.68693 & 0.92508 & 0.79072 & 0.00000 & \text { Uiso } & 1.00\end{array}$ $\begin{array}{llllllllll}01112 & 0 & 0.68693 & 0.9250 & 0.7808 & 0.0000 & \text { Uiso } & 1.00 \\ 0112 & 0 & 0.62837 & 0.11048 & 0.51404 & 0.00000 & \text { Uiso } & 1.00\end{array}$ $\begin{array}{lllllllllll}0112 & 0 & 0.62837 & 0.11048 & 0.51404 & 0.00000 & \text { Uiso } & 1.00\end{array}$ $\begin{array}{lllllllll}0113 & 0 & 0.53441 & 0.28857 & 0.53780 & 0.00000 & \text { Uiso } & 1.00\end{array}$ $\begin{array}{lllllllll}0114 & \mathrm{O} & 0.31381 & 0.06593 & 0.53776 & 0.00000 & \text { Uiso } & 1.00\end{array}$ $\begin{array}{llllllllll}0115 & \mathrm{O} & 0.37130 & 0.87487 & 0.74643 & 0.000000 & \text { Uiso } & 1.00 \\ 0116 & \mathrm{O} & 0.7185 & 0.4503 & 0.9593 & 0.0000 & \text { Uno } & 1.00\end{array}$ $\begin{array}{lllllllll}0116 & \mathrm{O} & 0.71485 & 0.45093 & 0.95934 & 0.00000 & \text { Uiso } & 1.00\end{array}$ $\begin{array}{lllllllll}0117 & \mathrm{O} & 0.93218 & 0.67280 & 0.96414 & 0.00000 & \text { Uiso } & 1.00\end{array}$ $\begin{array}{lllllllll}0118 & \mathrm{O} & 0.11653 & 0.63580 & 0.75038 & 0.00000 & \text { Uiso } & 1.00\end{array}$ $\begin{array}{llllllll}\mathrm{O} 119 & \mathrm{O} & 0.28860 & 0.53283 & 0.70978 & 0.00000 & \text { Uiso } & 1.00 \\ \mathrm{O} 120 & \mathrm{O} & 0.07248 & 0.30775 & 0.71328 & 0.00000 & \text { Uiso } & 1.00\end{array}$ $\begin{array}{llllllll}0121 & \mathrm{O} & 0.88532 & 0.35299 & 0.01153 & 0.00000 & \text { Uiso } & 1.00\end{array}$ 
$\begin{array}{llllllll}0122 & \mathrm{O} & 0.50201 & 0.00080 & 0.76721 & 0.00000 & \text { Uiso } & 1.00\end{array}$ $\begin{array}{lllllllll}0123 & \mathrm{O} & 0.49467 & 0.98665 & 0.49716 & 0.00000 & \text { Uiso } & 1.00\end{array}$ $\begin{array}{lllllllll}\mathrm{O} 124 & \mathrm{O} & 0.00372 & 0.48751 & 0.49002 & 0.00000 & \text { Uiso } & 1.00\end{array}$ $\begin{array}{llllllll}0125 & \mathrm{O} & 0.01273 & 0.48751 & 0.77069 & 0.00000 & \text { Uiso } & 1.00\end{array}$ $\begin{array}{llllllll}0126 & \mathrm{O} & 0.39315 & 0.38345 & 0.63400 & 0.00000 & \text { Uiso } & 1.00\end{array}$ $\begin{array}{lllllllll}0127 & 0 & 0.21696 & 0.20769 & 0.62843 & 0.00000 & \text { Uiso } & 1.00\end{array}$ $\begin{array}{lllllllllll}0128 & 0 & 0.59928 & 0.58949 & 0.87923 & 0.00000 & \text { Uiso } & 1.00\end{array}$

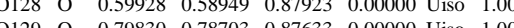

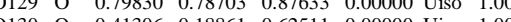
$\begin{array}{lllllllll}0130 & \mathrm{O} & 0.41306 & 0.1886 & 0.62511 & 0.00000 & \text { Uiso } & 1.00\end{array}$ $\begin{array}{lllllllll}0131 & \mathrm{O} & 0.60528 & 0.78182 & 0.86519 & 0.0000 & \text { Uiso } & 1.00 \\ 0132 & \mathrm{O} & 0.19778 & 0.40296 & 0.62429 & 0.0000 & \text { Uiso } & 1.00\end{array}$ $\begin{array}{lllllllll}0132 & \mathrm{O} & 0.19778 & 0.40296 & 0.62429 & 0.00000 & \text { Uiso } & 1.00 \\ 0133 & \mathrm{O} & 0.79234 & 0.5920 & 0.88051 & 0.0000 & \text { Uiso } & 1.00\end{array}$ $\begin{array}{lllllllll}0133 & \mathrm{O} & 0.79234 & 0.5920 & 0.88051 & 0.00000 & \text { Uiso } & 1.00\end{array}$ $\begin{array}{lllllllll}0134 & 0 & 0.35237 & 0.00422 & 0.87678 & 0.00000 & \text { Uiso } & 1.00\end{array}$ $\begin{array}{lllllllll}0135 & \mathrm{O} & 0.59419 & 0.23082 & 0.87657 & 0.00000 & \text { Uiso } & 1.00\end{array}$ $\begin{array}{lllllllll}0136 & 0 & 0.62192 & 0.96710 & 0.62948 & 0.00000 & \text { Uiso } & 1.00\end{array}$ $\begin{array}{lllllllll}0137 & \mathrm{O} & 0.40260 & 0.73649 & 0.62458 & 0.00000 & \text { Uiso } & 1.00\end{array}$ $\begin{array}{lllllllll}0138 & \mathrm{O} & 0.00778 & 0.33566 & 0.87588 & 0.00000 & \text { Uiso } & 1.00\end{array}$ $\begin{array}{llllllll}0139 & \mathrm{O} & 0.24944 & 0.58230 & 0.87497 & 0.00000 & \text { Uiso } & 1.00\end{array}$ $\begin{array}{llllllll}0140 & \mathrm{O} & 0.98360 & 0.60333 & 0.63013 & 0.00000 & \text { Uiso } & 1.00\end{array}$ $\begin{array}{llllllll}0141 & \mathrm{O} & 0.74113 & 0.39457 & 0.62353 & 0.00000 & \text { Uiso } & 1.00\end{array}$ $\begin{array}{llllllll}\mathrm{O} 142 & \mathrm{O} & 0.72733 & 0.27163 & 0.75835 & 0.00000 & \text { Uiso } & 1.00\end{array}$ $\begin{array}{llllllll}0143 & \mathrm{O} & 0.27975 & 0.70985 & 0.49232 & 0.00000 & \text { Uiso } & 1.00\end{array}$ $\begin{array}{lllllllll}0144 & \mathrm{O} & 0.27584 & 0.72035 & 0.75559 & 0.00000 & \text { Uiso } & 1.00\end{array}$ $\begin{array}{llllllll}0145 & \mathrm{O} & 0.72454 & 0.26282 & 0.49733 & 0.00000 & \text { Uiso } & 1.00\end{array}$ $\begin{array}{lllllllll}\mathrm{H} 146 & \mathrm{H} & 0.16331 & 0.81087 & 0.40183 & 0.00000 & \text { Uiso } & 1.00\end{array}$ $\begin{array}{llllllllll}\mathrm{H} 147 & \mathrm{H} & 0.07241 & 0.86142 & 0.53257 & 0.00000 & \text { Uiso } & 1.00\end{array}$ $\begin{array}{llllllllllll}\mathrm{H} 148 & \mathrm{H} & 0.94935 & 0.93000 & 0.52577 & 0.00000 & \text { Uiso } & 1.00\end{array}$ $\begin{array}{llllllll}\mathrm{H} 148 & \mathrm{H} & 0.9435 & 0.93001 & 0.52577 & 0.00000 & \text { Uiso } & 1.00 \\ \mathrm{H} 149 & \mathrm{H} & 0.97447 & 0.80410 & 0.49077 & 0.00000 & \text { Uiso } & 1.00\end{array}$

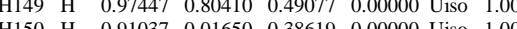
$\begin{array}{lllllllllll}150 & H & 0.91037 & 0.01650 & 0.38619 & 0.00000 & \text { Uiso } & 1.00\end{array}$ $\begin{array}{llllllll}\mathrm{H} 151 & \mathrm{H} & 0.88965 & 0.90235 & 0.34288 & 0.00000 & \text { Uiso } & 1.00\end{array}$ $\begin{array}{llllllllll}1152 & \mathrm{H} & 0.97074 & 0.97132 & 0.28322 & 0.00000 & \text { Uiso } & 1.00\end{array}$ \begin{tabular}{lllllllll}
$\mathrm{H} 153$ & $\mathrm{H}$ & 0.13110 & 0.88043 & 0.30116 & 0.00000 & Uiso & 1.00 \\
\hline 154 & $\mathrm{H}$ & 0.07264 & 0.77790 & 0.3258 & 0.00000 & Us & 1.00
\end{tabular} $\begin{array}{lllllllll}\mathrm{H} 154 & \mathrm{H} & 0.07264 & 0.77790 & 0.32589 & 0.00000 & \text { Uiso } & 1.00\end{array}$ $\begin{array}{llllllll}\mathrm{H} 155 & \mathrm{H} & 0.07893 & 0.80598 & 0.16417 & 0.00000 & \text { Uiso } & 1.00\end{array}$ $\begin{array}{llllllll}\mathrm{H} 156 & \mathrm{H} & 0.94380 & 0.83233 & 0.17723 & 0.00000 & \text { Uiso } & 1.00\end{array}$ $\begin{array}{llllllll}\mathrm{H} 157 & \mathrm{H} & 0.08979 & 0.87794 & 0.94664 & 0.00000 & \text { Uiso } & 1.00\end{array}$ $\begin{array}{llllllll}\mathrm{H} 158 & \mathrm{H} & 0.10445 & 0.98679 & 0.00429 & 0.00000 & \text { Uiso } & 1.00\end{array}$ $\begin{array}{llllllll}H 159 & \mathrm{H} & 0.16191 & 0.87196 & 0.04439 & 0.00000 & \text { Uiso } & 1.00\end{array}$ $\begin{array}{llllllll}\mathrm{H} 160 & \mathrm{H} & 0.89756 & 0.91699 & 0.96369 & 0.00000 & \text { Uiso } & 1.00\end{array}$ $\begin{array}{lllllllll}\mathrm{H} 161 & \mathrm{H} & 0.91372 & 0.02544 & 0.02138 & 0.00000 & \text { Uiso } & 1.00\end{array}$ $\begin{array}{llllllll}\mathrm{H} 162 & \mathrm{H} & 0.84864 & 0.93511 & 0.07204 & 0.00000 & \text { Uiso } & 1.00\end{array}$ $\begin{array}{lllllllll}\mathrm{C} 163 & \mathrm{C} & 0.94712 & 0.95357 & 0.34978 & 0.00000 & \text { Uiso } & 1.00\end{array}$ $\begin{array}{llllllll}\mathrm{C} 164 & \mathrm{C} & 0.02136 & 0.89532 & 0.40171 & 0.00000 & \text { Uiso } & 1.00\end{array}$ $\begin{array}{lllllllll}\mathrm{C} 165 & \mathrm{C} & 0.00487 & 0.87789 & 0.49411 & 0.00000 & \text { Uiso } & 1.00\end{array}$ $\begin{array}{lllllllll}\mathrm{C} 166 & \mathrm{C} & 0.10455 & 0.84034 & 0.35713 & 0.00000 & \text { Uiso } & 1.00\end{array}$ $\begin{array}{lllllllll}\mathrm{C} 167 & \mathrm{C} & 0.00568 & 0.89707 & 0.06621 & 0.00000 & \text { Uiso } & 1.00\end{array}$ $\begin{array}{lllllllll}\mathrm{C} 168 & \mathrm{C} & 0.00960 & 0.84360 & 0.14040 & 0.00000 & \text { Uiso } & 1.00\end{array}$ $\begin{array}{lllllllllll}C 169 & C & 0.09569 & 0.90892 & 0.01332 & 0.00000 & \text { Uiso } & 1.00\end{array}$ $\begin{array}{llllllll}\mathrm{C} 169 & \mathrm{C} & 0.09569 & 0.90892 & 0.01332 & 0.00000 & \text { Uiso } & 1.00 \\ \mathrm{C} 170 & \mathrm{C} & 0.91103 & 0.94630 & 0.02970 & 0.00000 & \text { Uiso } & 1.00\end{array}$

\section{File $\mathbf{n}^{\circ} 7$}

$\begin{array}{llllllll}\mathrm{Si} 2 & \mathrm{Si} & 0.37526 & 0.76740 & 0.02615 & 0.00000 & \text { Uiso } & 1.00\end{array}$ $\begin{array}{llllllll}\mathrm{Si} 3 & \mathrm{Si} & 0.60590 & -0.00255 & 0.02375 & 0.00000 & \text { Uiso } & 1.00\end{array}$ $\begin{array}{llllllll}\mathrm{Si} 4 & \mathrm{Si} & 0.61248 & 0.22733 & 0.27424 & 0.00000 & \text { Uiso } & 1.00\end{array}$ $\begin{array}{lllllllll}\mathrm{Si5} & \mathrm{Si} & 0.37588 & 0.98111 & 0.27519 & 0.00000 & \text { Uiso } & 1.00\end{array}$ $\begin{array}{llllllll}\text { Si6 } & \text { Si } & 0.75567 & 0.37319 & 0.23123 & 0.00000 & \text { Uiso } & 1.00\end{array}$ $\begin{array}{llllllll}\mathrm{Si} 7 & \mathrm{Si} & 0.01434 & 0.60325 & 0.22821 & 0.00000 & \text { Uiso } & 1.00\end{array}$ $\begin{array}{llllllll}\mathrm{Si} 8 & \mathrm{Si} & 0.23527 & 0.60785 & 0.48351 & 0.00000 & \text { Uiso } & 1.00\end{array}$ $\begin{array}{lllllllll}\text { Si9 } & \text { Si } & 0.98987 & 0.37276 & 0.48268 & 0.00000 & \text { Uiso } & 1.00\end{array}$ $\begin{array}{llllllll}\text { Si10 } & \text { Si } & 0.61420 & 0.22912 & 0.48150 & 0.00000 & \text { Uiso } & 1.00\end{array}$ $\begin{array}{llllllll}\text { Sil1 } & \mathrm{Si} & 0.37792 & 0.98598 & 0.48019 & 0.00000 & \text { Uiso } & 1.00\end{array}$ $\begin{array}{lllllllll}\text { Si12 } & \text { Si } & 0.37875 & 0.75473 & 0.23153 & 0.00000 & \text { Uiso } & 1.00\end{array}$ $\begin{array}{llllllll}\text { Si13 } & \text { Si } & 0.60204 & 0.00245 & 0.22956 & 0.00000 & \text { Uiso } & 1.00\end{array}$ $\begin{array}{lllllllll}\text { Sil4 } & \text { Si } & 0.24241 & 0.59864 & 0.27528 & 0.00000 & \text { Uiso } & 1.00\end{array}$ $\begin{array}{lllllllll}\text { Si15 } & \text { Si } & 0.98027 & 0.38742 & 0.27886 & 0.00000 & \text { Uiso } & 1.00\end{array}$ $\begin{array}{llllllll}\text { Si16 } & \mathrm{Si} & 0.76528 & 0.36723 & 0.02554 & 0.00000 & \text { Uiso } & 1.00\end{array}$

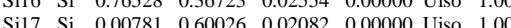

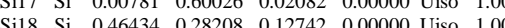
$\begin{array}{llllllll}S 118 & \mathrm{Si} & 0.46434 & 0.28208 & 0.12742 & 0.00000 & \text { Uiso } & 1.00\end{array}$ $\begin{array}{llllllll}\text { S119 } & \text { Si } & 0.30355 & 0.13370 & 0.13244 & 0.00000 & \text { Uiso } & 1.00\end{array}$ $\begin{array}{lllllllll} & \text { Si20 } & \text { Si } & 0.53455 & 0.70435 & 0.37851 & 0.00000 & \text { Uiso } & 1.00\end{array}$ $\begin{array}{llllllll}\text { Si21 } & \text { Si } & 0.68129 & 0.86278 & 0.38023 & 0.00000 & \text { Uiso } & 1.00\end{array}$ $\begin{array}{llllllll}\mathrm{Si} 22 & \mathrm{Si} & 0.29647 & 0.45831 & 0.12515 & 0.00000 & \text { Uiso } & 1.00\end{array}$ $\begin{array}{lllllllll}\mathrm{Si23} & \mathrm{Si} & 0.13398 & 0.31166 & 0.12913 & 0.00000 & \text { Uiso } & 1.00\end{array}$ $\begin{array}{lllllllll}\text { Si24 } & \text { Si } & 0.71174 & 0.53230 & 0.37072 & 0.00000 & \text { Uiso } & 1.00\end{array}$ $\begin{array}{llllllll}\mathrm{Si25} & \mathrm{Si} & 0.37570 & 0.75873 & 0.52333 & 0.00000 & \text { Uiso } & 1.00\end{array}$ $\begin{array}{lllllllll}\mathrm{Si} 26 & \mathrm{Si} & 0.60351 & 0.00631 & 0.52771 & 0.00000 & \text { Uiso } & 1.00\end{array}$ $\begin{array}{llllllll}\text { Si27 } & \text { Si } & 0.62387 & 0.22498 & 0.77508 & 0.00000 & \text { Uiso } & 1.00\end{array}$ $\begin{array}{llllllll}\mathrm{Si} 28 & \mathrm{Si} & 0.38532 & 0.98507 & 0.77871 & 0.00000 & \text { Uiso } & 1.00\end{array}$ $\begin{array}{llllllll}\mathrm{Si} 29 & \mathrm{Si} & 0.76208 & 0.37739 & 0.72707 & 0.00000 & \text { Uiso } & 1.00\end{array}$ $\begin{array}{llllllll}\mathrm{Si} 30 & \mathrm{Si} & 0.00642 & 0.60434 & 0.73517 & 0.00000 & \text { Uiso } & 1.00\end{array}$ $\begin{array}{lllllllll}\mathrm{Si} 31 & \mathrm{Si} & 0.23061 & 0.62104 & 0.98254 & 0.00000 & \text { Uiso } & 1.00\end{array}$ $\begin{array}{llllllll}\mathrm{Si32} & \mathrm{Si} & 0.99080 & 0.37376 & 0.97870 & 0.00000 & \text { Uiso } & 1.00\end{array}$ $\begin{array}{llllllll}\mathrm{Si33} & \mathrm{Si} & 0.62556 & 0.21585 & 0.98112 & 0.00000 & \text { Uiso } & 1.00\end{array}$ $\begin{array}{lllllllll}\mathrm{Si3} & \mathrm{Si} & 0.37634 & 0.99337 & 0.98327 & 0.00000 & \text { Uiso } & 1.00\end{array}$ $\begin{array}{llllllll}\mathrm{Si} 35 & \mathrm{Si} & 0.37810 & 0.75769 & 0.73022 & 0.00000 & \text { Uiso } & 1.00\end{array}$ $\begin{array}{lllllllll}\text { Si36 } & \text { Si } & 0.61064 & 0.00322 & 0.73433 & 0.00000 & \text { Uiso } & 1.00\end{array}$ $\begin{array}{lllllllll}\mathrm{Si} 37 & \mathrm{Si} & 0.22871 & 0.61869 & 0.77665 & 0.00000 & \text { Uiso } & 1.00\end{array}$ $\begin{array}{lllllllll} & \text { Si38 } & \text { Si } & 0.98917 & 0.37807 & 0.77466 & 0.00000 & \text { Uiso } & 1.00\end{array}$

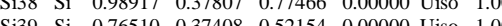
$\begin{array}{lllllllll}\text { Si39 } & \text { Si } & 0.76510 & 0.37408 & 0.52154 & 0.00000 & \text { Uiso } & 1.00\end{array}$ $\begin{array}{llllllll} & \text { Si40 Si } & 0.00903 & 0.59965 & 0.52750 & 0.00000 & \text { Uiso } & 1.00\end{array}$ $\begin{array}{lllllllll} & \text { Si41 Si } & 0.46471 & 0.28443 & 0.63009 & 0.00000 & 0.000 & 1.00\end{array}$ $\begin{array}{llllllll}\mathrm{Si} 42 & \mathrm{Si} & 0.31160 & 0.12997 & 0.62855 & 0.00000 & \text { Uiso } & 1.00\end{array}$ $\begin{array}{lllllllll}\mathrm{Si} 43 & \mathrm{Si} & 0.53085 & 0.69695 & 0.87534 & 0.00000 & \text { Uiso } & 1.00\end{array}$ $\begin{array}{lllllllll}\mathrm{Si} 44 & \mathrm{Si} & 0.68673 & 0.85176 & 0.87497 & 0.00000 & \text { Uiso } & 1.00\end{array}$ $\begin{array}{llllllll}\mathrm{Si} 45 & \mathrm{Si} & 0.29064 & 0.45935 & 0.63116 & 0.00000 & \text { Uiso } & 1.00\end{array}$ $\begin{array}{llllllll}\mathrm{Si} 46 & \mathrm{Si} & 0.13703 & 0.30542 & 0.62718 & 0.00000 & \text { Uiso } & 1.00\end{array}$ $\begin{array}{lllllllll}\mathrm{Si} 47 & \mathrm{Si} & 0.70116 & 0.52627 & 0.87837 & 0.00000 & \text { Uiso } & 1.00\end{array}$ $\begin{array}{lllllllll}\text { Si48 } & \mathrm{Si} & 0.85806 & 0.68209 & 0.87980 & 0.00000 & \text { Uiso } & 1.00\end{array}$

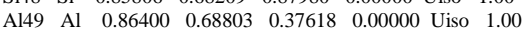


$\begin{array}{llllllll}\text { O50 } & \mathrm{O} & 0.52625 & 0.29142 & 0.21790 & 0.00000 & \text { Uiso } & 1.00\end{array}$ $\begin{array}{lllllllll}051 & \mathrm{O} & 0.30523 & 0.05595 & 0.21341 & 0.00000 & \text { Uiso } & 1.00\end{array}$ $\begin{array}{lllllllll}\mathrm{O} 52 & \mathrm{O} & 0.35804 & 0.87549 & 0.50475 & 0.00000 & \text { Uiso } & 1.00\end{array}$ $\begin{array}{llllllll}053 & \mathrm{O} & 0.46444 & 0.70435 & 0.46485 & 0.00000 & \text { Uiso } & 1.00\end{array}$ $\begin{array}{llllllll}054 & \mathrm{O} & 0.67625 & 0.93273 & 0.46770 & 0.00000 & \text { Uiso } & 1.00\end{array}$ $\begin{array}{lllllllll}055 & \mathrm{O} & 0.62409 & 0.11482 & 0.24046 & 0.00000 & \text { Uiso } & 1.00\end{array}$ $\begin{array}{llllllllll}056 & \mathrm{O} & 0.28817 & 0.53749 & 0.04539 & 0.00000 & \text { Uiso } & 1.00\end{array}$ $\begin{array}{llllllll}056 & 0 & 0.28817 & 0.53749 & 0.04539 & 0.00000 & \text { Uiso } & 1.00 \\ 057 & \mathrm{O} & 0.07238 & 0.30792 & 0.03775 & 0.00000 & \text { Uiso } & 1.00\end{array}$ $\begin{array}{lllllllll}058 & 0 & 0.87199 & 0.36285 & 0.037706 & 0.00000 & \text { Uiso } & 1.00 \\ 0.00\end{array}$ $\begin{array}{llllllll}058 & 0 & 0.87199 & 0.36285 & 0.25306 & 0.00000 & \text { Uiso } & 1.00 \\ 059 & 0 & 0.69473 & 0.45980 & 0.28788 & 0.00000 & \text { Uiso } & 1.00\end{array}$ $\begin{array}{lllllllll}059 & 0 & 0.69473 & 0.45980 & 0.28788 & 0.00000 & \text { Uiso } & 1.00\end{array}$ $\begin{array}{llllllllll}060 & 0 & 0.95383 & 0.70276 & 0.27935 & 0.00000 & \text { Uiso } & 1.00\end{array}$ $\begin{array}{lllllllll}061 & \mathrm{O} & 0.11466 & 0.63566 & 0.00588 & 0.00000 & \text { Uiso } & 1.00\end{array}$ $\begin{array}{lllllllll}062 & \mathrm{O} & 0.46967 & 0.70219 & 0.28854 & 0.00000 & \text { Uiso } & 1.00\end{array}$ $\begin{array}{llllllll}\mathrm{O} 63 & \mathrm{O} & 0.67570 & 0.93235 & 0.29196 & 0.00000 & \text { Uiso } & 1.00 \\ 064 & \mathrm{O} & 0.64826 & 0.09962 & 0.00117 & 0.00000 & \text { Uiso } & 1.00\end{array}$ $\begin{array}{llllllll}064 & \mathrm{O} & 0.64826 & 0.09962 & 0.00117 & 0.00000 & \text { Uiso } & 1.00\end{array}$ $\begin{array}{lllllllll}\mathrm{O} 65 & \mathrm{O} & 0.53630 & 0.26417 & 0.04288 & 0.00000 & \text { Uiso } & 1.00\end{array}$ $\begin{array}{llllllll}\mathrm{O} 66 & \mathrm{O} & 0.30602 & 0.07531 & 0.03859 & 0.00000 & \text { Uiso } & 1.00\end{array}$ $\begin{array}{llllllll}\mathrm{O} 67 & \mathrm{O} & 0.35365 & 0.86990 & 0.25534 & 0.00000 & \text { Uiso } & 1.00\end{array}$ $\begin{array}{llllllll}068 & \mathrm{O} & 0.72477 & 0.46710 & 0.46121 & 0.00000 & \text { Uiso } & 1.00\end{array}$ $\begin{array}{llllllll}069 & \mathrm{O} & 0.94018 & 0.67286 & 0.46633 & 0.00000 & \text { Uiso } & 1.00\end{array}$ $\begin{array}{llllllll}070 & \mathrm{O} & 0.13015 & 0.60657 & 0.23981 & 0.00000 & \text { Uiso } & 1.00\end{array}$ $\begin{array}{lllllllll}071 & \mathrm{O} & 0.30591 & 0.51119 & 0.22085 & 0.00000 & \text { Uiso } & 1.00\end{array}$ $\begin{array}{llllllll}072 & \mathrm{O} & 0.05945 & 0.32955 & 0.21211 & 0.00000 & \text { Uiso } & 1.00\end{array}$ $\begin{array}{llllllll}073 & \mathrm{O} & 0.88252 & 0.34629 & 0.51098 & 0.00000 & \text { Uiso } & 1.00\end{array}$ $\begin{array}{llllllllll}074 & \mathrm{O} & 0.48928 & -0.00747 & 0.25496 & 0.00000 & \text { Uiso } & 1.00\end{array}$ $\begin{array}{lllllllll}075 & \mathrm{O} & 0.49036 & 0.00742 & 0.99920 & 0.00000 & \text { Uiso } & 1.00\end{array}$ $\begin{array}{lllllllll}076 & \mathrm{O} & 0.00572 & 0.48886 & 0.98761 & 0.00000 & \text { Uiso } & 1.00\end{array}$ $\begin{array}{lllllllllll}077 & \mathrm{O} & 0.00572 & 0.48886 & 0.98761 & 0.00000 & \text { Uiso } & 1.00\end{array}$ $\begin{array}{lllllllll}078 & 0 & -0.01527 & 0.50563 & 0.27410 & 0.00000 & \text { Uiso } & 1.00 \\ 078 & 0.39391 & 0.38288 & 0.10993 & 0.00000 & \text { Uiso } & 1.00\end{array}$ $\begin{array}{lllllllll}078 & 0 & 0.39391 & 0.38288 & 0.10993 & 0.00000 & \text { Uiso } & 1.00 \\ 079 & 0 & 0.20359 & 0.20874 & 0.14014 & 0.00000 & \text { Uis } & 1.00\end{array}$ $\begin{array}{lllllllll}079 & \mathrm{O} & 0.20359 & 0.20874 & 0.14014 & 0.00000 & \text { Uiso } & 1.00\end{array}$ $\begin{array}{lllllllll}080 & 0 & 0.61229 & 0.60764 & 0.38199 & 0.00000 & \text { Uiso } & 1.00\end{array}$ $\begin{array}{llllllll}081 & \mathrm{O} & 0.78049 & 0.79062 & 0.38136 & 0.00000 & \text { Uiso } & 1.00\end{array}$ $\begin{array}{lllllllll}082 & \mathrm{O} & 0.39955 & 0.19155 & 0.13857 & 0.00000 & \text { Uiso } & 1.00\end{array}$ $\begin{array}{llllllll}083 & \mathrm{O} & 0.58425 & 0.80521 & 0.37912 & 0.00000 & \text { Uiso } & 1.00\end{array}$ $\begin{array}{llllllll}084 & \mathrm{O} & 0.19873 & 0.40285 & 0.12469 & 0.00000 & \text { Uiso } & 1.00\end{array}$ $\begin{array}{lllllllll}085 & \mathrm{O} & 0.80580 & 0.58714 & 0.35200 & 0.00000 & \text { Uiso } & 1.00\end{array}$ $\begin{array}{llllllll}086 & \mathrm{O} & 0.34977 & 0.00802 & 0.37736 & 0.00000 & \text { Uiso } & 1.00\end{array}$ $\begin{array}{lllllllll}087 & \mathrm{O} & 0.58217 & 0.23319 & 0.37799 & 0.00000 & \text { Uiso } & 1.00\end{array}$ $\begin{array}{llllllll}088 & \mathrm{O} & 0.62191 & 0.96952 & 0.12732 & 0.00000 & \text { Uiso } & 1.00\end{array}$ $\begin{array}{lllllllll}089 & \mathrm{O} & 0.40605 & 0.74017 & 0.12759 & 0.00000 & \text { Uiso } & 1.00\end{array}$ $\begin{array}{llllllll}\mathrm{O} 90 & \mathrm{O} & 0.00820 & 0.35035 & 0.37808 & 0.00000 & \text { Uiso } & 1.00\end{array}$ $\begin{array}{lllllllll}091 & \mathrm{O} & 0.24660 & 0.57533 & 0.37997 & 0.00000 & \text { Uiso } & 1.00\end{array}$ $\begin{array}{lllllllll}092 & \mathrm{O} & 0.98147 & 0.60771 & 0.12577 & 0.00000 & \text { Uiso } & 1.00\end{array}$ $\begin{array}{lllllllll}093 & \mathrm{O} & 0.73826 & 0.39754 & 0.12693 & 0.00000 & \text { Uiso } & 1.00\end{array}$ $\begin{array}{llllllll}094 & \mathrm{O} & 0.71633 & 0.26943 & 0.25649 & 0.00000 & \text { Uiso } & 1.00\end{array}$ $\begin{array}{llllllll}095 & \mathrm{O} & 0.27353 & 0.72426 & 0.00106 & 0.00000 & \text { Uiso } & 1.00\end{array}$ $\begin{array}{llllllll}096 & \mathrm{O} & 0.28070 & 0.70334 & 0.25389 & 0.00000 & \text { Uiso } & 1.00\end{array}$ $\begin{array}{lllllllllll}097 & 0 & 0.72553 & 0.26367 & 0.00211 & 0.00000 & \text { Uiso } & 1.00\end{array}$ $\begin{array}{llllllllll}098 & 0 & 0.73441 & 0.27928 & 0.71679 & 0.00000 & \text { Uiso } & 1.00\end{array}$ $\begin{array}{llllllllll}098 & 0 & 0.53441 & 0.27928 & 0.71679 & 0.00000 & \text { Uiso } & 1.00\end{array}$ $\begin{array}{lllllllll}099 & 0 & 0.31727 & 0.05938 & 0.71491 & 0.00000 & \text { Uiso } & 1.00\end{array}$ $\begin{array}{lllllllllll}0100 & \mathrm{O} & 0.35617 & 0.88527 & 0.01585 & 0.00000 & \text { Uiso } & 1.00\end{array}$ $\begin{array}{llllllllll}0101 & \mathrm{O} & 0.46344 & 0.72012 & 0.96265 & 0.00000 & \text { Uiso } & 1.00 \\ 0102 & \mathrm{O} & 0.66767 & 0.91152 & 0.96697 & 0.0000 & \text { Uiso } & 1.00\end{array}$ $\begin{array}{llllllllll}0102 & \mathrm{O} & 0.66767 & 0.91152 & 0.96697 & 0.00000 & \text { Uiso } & 1.00 \\ 0103 & \mathrm{O} & 0.6549 & 0.11061 & 0.74758 & 0.0000 & \text { Uiso } & 1.00\end{array}$ $\begin{array}{lllllllll}0103 & \mathrm{O} & 0.64549 & 0.11061 & 0.74758 & 0.0000 & \text { Uiso } & 1.00\end{array}$ $\begin{array}{lllllllll}0104 & \mathrm{O} & 0.29929 & 0.52402 & 0.54207 & 0.00000 & \text { Uiso } & 1.00\end{array}$

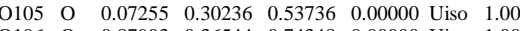
$\begin{array}{llllllll}0106 & \mathrm{O} & 0.87903 & 0.36544 & 0.74348 & 0.00000 & \text { Uiso } & 1.00\end{array}$ $\begin{array}{llllllll}0107 & \mathrm{O} & 0.70716 & 0.46702 & 0.78544 & 0.00000 & \text { Uiso } & 1.00\end{array}$ $\begin{array}{llllllll}0108 & \mathrm{O} & 0.92454 & 0.67368 & 0.79109 & 0.00000 & \text { Uiso } & 1.00\end{array}$ $\begin{array}{llllllll}0109 & \mathrm{O} & 0.12269 & 0.61911 & 0.51453 & 0.00000 & \text { Uiso } & 1.00\end{array}$ $\begin{array}{lllllllll}0110 & \mathrm{O} & 0.46320 & 0.69448 & 0.78777 & 0.00000 & \text { Uiso } & 1.00\end{array}$ $\begin{array}{llllllllll}0111 & \mathrm{O} & 0.68407 & 0.92499 & 0.79055 & 0.00000 & \text { Uiso } & 1.00\end{array}$ $\begin{array}{llllllll}0112 & \mathrm{O} & 0.63152 & 0.11691 & 0.51632 & 0.00000 & \text { Uiso } & 1.00\end{array}$ $\begin{array}{lllllllll}0113 & \mathrm{O} & 0.52753 & 0.29015 & 0.53953 & 0.00000 & \text { Uiso } & 1.00\end{array}$ $\begin{array}{llllllll}0114 & \mathrm{O} & 0.30793 & 0.06378 & 0.53982 & 0.00000 & \text { Uiso } & 1.00\end{array}$ $\begin{array}{lllllllll}0115 & \mathrm{O} & 0.37210 & 0.87307 & 0.75348 & 0.00000 & \text { Uiso } & 1.00\end{array}$ $\begin{array}{llllllll}0116 & \mathrm{O} & 0.71240 & 0.45175 & 0.96146 & 0.00000 & \text { Uiso } & 1.00\end{array}$ $\begin{array}{llllllllllll}0117 & \mathrm{O} & 0.92677 & 0.67357 & 0.96807 & 0.00000 & \text { Uiso } & 1.00\end{array}$ $\begin{array}{lllllllllll}0118 & 0 & 0.11283 & 0.63866 & 0.75564 & 0.00000 & \text { Uiso } & 1.00\end{array}$ $\begin{array}{lllllllll}0118 & 0 & 0.11283 & 0.63862 & 0.75564 & 0.00000 & \text { Uiso } & 1.00\end{array}$

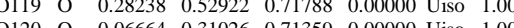
$\begin{array}{llllllllll}0121 & \mathrm{O} & 0.06664 & 0.31026 & 0.71359 & 0.00000 & \text { Uiso } & 1.00 \\ 0.121 & \mathrm{O} & 0.88301 & 0.35538 & 0.01281 & 0.0000 & \text { Uiso } & 1.00\end{array}$ $\begin{array}{lllllllllllll}0121 & 0 & 0.88301 & 0.3538 & 0.01281 & 0.00000 & \text { Uiso } & 1.00\end{array}$ $\begin{array}{lllllllll}0122 & 0 & 0.49945 & 0.00297 & 0.77061 & 0.00000 & \text { Uiso } & 1.00 \\ 0123 & 0 & 0.49122 & 0.00124 & 0.49712 & 0.00000 & \text { Uis } & 1.00\end{array}$ $\begin{array}{lllllllll}0123 & 0 & 0.49122 & 0.00124 & 0.49712 & 0.00000 \\ 0124 & 0 & -0.00018 & 0.48592 & 0.50362 & 0.0000 & 1.00\end{array}$ $\begin{array}{lllllllll}0124 & \mathrm{O} & -0.00018 & 0.48592 & 0.50362 & 0.00000 & \text { Uiso } & 1.00\end{array}$ $\begin{array}{llllllll}0125 & \mathrm{O} & 0.00759 & 0.49181 & 0.76779 & 0.00000 & \text { Uiso } & 1.00\end{array}$

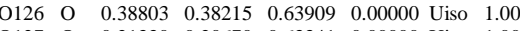
$\begin{array}{llllllll}0127 & \mathrm{O} & 0.21330 & 0.20670 & 0.63341 & 0.00000 & \text { Uiso } & 1.00\end{array}$

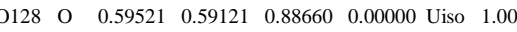

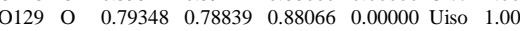

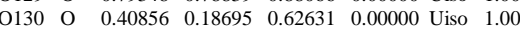
$\begin{array}{lllllllll}0131 & \mathrm{O} & 0.60152 & 0.78150 & 0.86210 & 0.00000 & \text { Uiso } & 1.00\end{array}$ $\begin{array}{lllllllll}0132 & \mathrm{O} & 0.19399 & 0.40244 & 0.62514 & 0.00000 & \text { Uiso } & 1.00\end{array}$ $\begin{array}{lllllllll}0133 & \mathrm{O} & 0.78874 & 0.59499 & 0.88187 & 0.00000 & \text { Uiso } & 1.00\end{array}$ $\begin{array}{lllllllll}0134 & \mathrm{O} & 0.34934 & 0.00745 & 0.87955 & 0.00000 & \text { Uiso } & 1.00\end{array}$ $\begin{array}{llllllllll}0135 & \mathrm{O} & 0.59337 & 0.23524 & 0.87874 & 0.00000 & \text { Uiso } & 1.00\end{array}$ $\begin{array}{llllllll}0136 & \mathrm{O} & 0.61660 & 0.97335 & 0.63064 & 0.00000 & \text { Uiso } & 1.00\end{array}$ $\begin{array}{llllllll}0137 & 0 & 0.40319 & 0.73929 & 0.62660 & 0.00000 & \text { Uiso } & 1.00\end{array}$ $\begin{array}{lllllllllll}0138 & 0 & 0.00533 & 0.34129 & 0.87625 & 0.00000 & \text { Uiso } & 1.00\end{array}$ $\begin{array}{llllllllll}0138 & 0 & 0.00533 & 0.34129 & 0.87625 & 0.00000 & \text { Uiso } & 1.00 \\ 0139 & 0 & 0.24713 & 0.58880 & 0.88001 & 0.00000 & \text { Uis } & 1.00\end{array}$ $\begin{array}{lllllllllll}0140 & 0 & 0.24713 & 0.58880 & 0.8801 & 0.0000 & \text { Uiso } & 1.00 \\ 0.03030 & 0.61632 & 0.63151 & 0.00000 & \text { Uiso } & 1.00\end{array}$ $\begin{array}{llllllllll}0140 & 0 & 0.98030 & 0.61632 & 0.63151 & 0.0000 & \text { Uiso } & 1.00\end{array}$ $\begin{array}{llllllll}0141 & \mathrm{O} & 0.73821 & 0.40158 & 0.62402 & 0.00000 & \text { Uiso } & 1.00\end{array}$ $\begin{array}{llllllllll}0142 & 0 & 0.72276 & 0.27529 & 0.75648 & 0.00000 & \text { Uiso } & 1.00\end{array}$ $\begin{array}{lllllllll}0143 & \mathrm{O} & 0.27542 & 0.71332 & 0.49777 & 0.00000 & \text { Uiso } & 1.00\end{array}$ $\begin{array}{lllllllllll}0144 & \mathrm{O} & 0.27369 & 0.72001 & 0.75444 & 0.00000 & \text { Unso } & 1.00 \\ 0145 & \mathrm{O} & 0.71528 & 0.27729 & 0.49317 & 0.00000 & \text { USo } & 1.00\end{array}$ $\begin{array}{lllllllll}0145 & \mathrm{O} & 0.71528 & 0.27729 & 0.49317 & 0.00000 & \text { Uiso } & 1.00\end{array}$ $\begin{array}{llllllll}\mathrm{H} 146 & \mathrm{H} & 0.11043 & 0.75366 & 0.16821 & 0.00000 & \text { Uiso } & 1.00\end{array}$ $\begin{array}{llllllll}\mathrm{H} 147 & \mathrm{H} & 0.08808 & 0.88929 & 0.33840 & 0.00000 & \text { Uiso } & 1.00\end{array}$ $\begin{array}{llllllll}\mathrm{H} 148 & \mathrm{H} & 0.02458 & 0.81106 & 0.40531 & 0.00000 & \text { Uiso } & 1.00\end{array}$ $\begin{array}{llllllll}\mathrm{H} 149 & \mathrm{H} & 0.12781 & 0.76240 & 0.34098 & 0.00000 & \text { Uiso } & 1.00\end{array}$ $\begin{array}{llllllll}\mathrm{H} 150 & \mathrm{H} & 0.87923 & 0.89978 & 0.33136 & 0.00000 & \text { Uiso } & 1.00\end{array}$ 
$\begin{array}{llllllll}\mathrm{H} 151 & \mathrm{H} & 0.84994 & 0.86659 & 0.22144 & 0.00000 & \text { Uiso } & 1.00\end{array}$ $\begin{array}{llllllll}\mathrm{H} 152 & \mathrm{H} & 0.93472 & 0.95205 & 0.24001 & 0.00000 & \text { Uiso } & 1.00\end{array}$ $\begin{array}{llllllll}\mathrm{H} 153 & \mathrm{H} & 0.07054 & 0.87978 & 0.16946 & 0.00000 & \text { Uiso } & 1.00\end{array}$ $\begin{array}{llllllll}\mathrm{H} 154 & \mathrm{H} & -0.00597 & 0.79956 & 0.12125 & 0.00000 & \text { Uiso } & 1.00\end{array}$ $\begin{array}{llllllll}\mathrm{C} 155 & \mathrm{C} & 0.90852 & 0.88499 & 0.26509 & 0.00000 & \text { Uiso } & 1.00\end{array}$ $\begin{array}{llllllll}\mathrm{C} 156 & \mathrm{C} & -0.00460 & 0.80620 & 0.26486 & 0.00000 & \text { Uiso } & 1.00\end{array}$ $\begin{array}{llllllllll}C 157 & C & 0.06297 & 0.81659 & 0.34235 & 0.00000 & \text { Uiso } & 1.00\end{array}$ $\begin{array}{llllllll}\mathrm{C} 157 & \mathrm{C} & 0.06297 & 0.81659 & 0.34235 & 0.00000 & \text { Uiso } & 1.00 \\ \mathrm{C} 158 & \mathrm{C} & 0.04565 & 0.80720 & 0.17527 & 0.00000 & \text { Uiso } & 1.00\end{array}$

\section{File $\mathbf{n}^{\circ} 8$}

$\begin{array}{llllllll}\text { Si2 } & \text { Si } & 0.37320 & 0.76592 & 0.02462 & 0.00000 & \text { Uiso } & 1.00\end{array}$ $\begin{array}{llllllll}\mathrm{Si3} & \mathrm{Si} & 0.60534 & 0.00367 & 0.02389 & 0.00000 & \text { Uiso } & 1.00 \\ \mathrm{Si} & \mathrm{Si} & 0.61757 & 0.23342 & 0.27309 & 0.00000 & \text { Uiso } & 1.00\end{array}$ $\begin{array}{lllllllll}\mathrm{Si} 4 & \mathrm{Si} & 0.61757 & 0.23342 & 0.27309 & 0.00000 & \text { Uiso } & 1.00\end{array}$ $\begin{array}{lllllllll}\text { Si5 } & \text { Si } & 0.37656 & 0.99083 & 0.27479 & 0.00000 & \text { Uiso } & 1.00\end{array}$ $\begin{array}{llllllll}\text { Si6 } & \text { Si } & 0.75941 & 0.38199 & 0.23051 & 0.00000 & \text { Uiso } & 1.00\end{array}$ $\begin{array}{lllllllll}\text { Si7 } & \text { Si } & 0.01407 & 0.60637 & 0.22805 & 0.00000 & \text { Uiso } & 1.00\end{array}$ $\begin{array}{llllllll}\mathrm{Si} 8 & \mathrm{Si} & 0.23179 & 0.61721 & 0.48117 & 0.00000 & \text { Uiso } & 1.00\end{array}$ $\begin{array}{lllllllll}\mathrm{Si} 9 & \mathrm{Si} & 0.99291 & 0.37735 & 0.48064 & 0.00000 & \text { Uiso } & 1.00\end{array}$ $\begin{array}{llllllll}\mathrm{Si} 10 & \mathrm{Si} & 0.61895 & 0.22962 & 0.48005 & 0.00000 & \text { Uiso } & 1.00\end{array}$ $\begin{array}{llllllll}\text { Sil1 } & \text { Si } & 0.37419 & 0.99408 & 0.48130 & 0.00000 & \text { Uiso } & 1.00\end{array}$ $\begin{array}{llllllll}\text { Si12 } & \text { Si } & 0.38276 & 0.76458 & 0.23008 & 0.00000 & \text { Uiso } & 1.00\end{array}$ $\begin{array}{lllllllll}\text { Si13 } & \text { Si } & 0.60211 & 0.01088 & 0.22969 & 0.00000 & \text { Uiso } & 1.00\end{array}$ $\begin{array}{lllllllll}\text { Sil4 } & \text { Si } & 0.23653 & 0.61504 & 0.27427 & 0.00000 & \text { Uiso } & 1.00\end{array}$ $\begin{array}{lllllllll}\text { Si15 } & \text { Si } & 0.98839 & 0.38297 & 0.27546 & 0.00000 & \text { Uiso } & 1.00\end{array}$ $\begin{array}{lllllllll}\text { Si16 } & \text { Si } & 0.76562 & 0.37322 & 0.02566 & 0.00000 & \text { Uiso } & 1.00\end{array}$

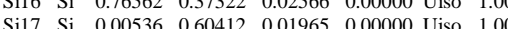
$\begin{array}{lllllllll}\text { Si18 } & \mathrm{Si} & 0.46402 & 0.28836 & 0.12704 & 0.00000 & \text { Uiso } & 1.00\end{array}$ $\begin{array}{lllllllll}\text { Sil } & \text { Si } & 0.46402 & 0.28836 & 0.12704 & 0.00000 & \text { Uiso } & 1.00\end{array}$

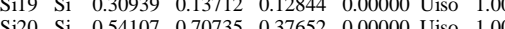

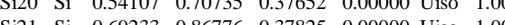
$\begin{array}{llllllll}\mathrm{Si21} & \mathrm{Si} & 0.69233 & 0.86776 & 0.37825 & 0.00000 & \text { Uiso } & 1.00\end{array}$ $\begin{array}{llllllll}\mathrm{Si22} & \mathrm{Si} & 0.28940 & 0.46366 & 0.12935 & 0.00000 & \text { Uiso } & 1.00\end{array}$ $\begin{array}{lllllllll}\mathrm{Si23} & \mathrm{Si} & 0.13444 & 0.31251 & 0.12893 & 0.00000 & \text { Uiso } & 1.00\end{array}$ $\begin{array}{lllllllll}\text { Si24 } & \text { Si } & 0.69987 & 0.53739 & 0.37569 & 0.00000 & \text { Uiso } & 1.00\end{array}$ $\begin{array}{llllllll}\mathrm{Si} 25 & \mathrm{Si} & 0.37621 & 0.77051 & 0.52230 & 0.00000 & \text { Uiso } & 1.00\end{array}$ $\begin{array}{llllllll}\mathrm{Si26} & \mathrm{Si} & 0.60196 & 0.00741 & 0.52679 & 0.00000 & \text { Uiso } & 1.00\end{array}$ $\begin{array}{llllllll}\mathrm{Si} 27 & \mathrm{Si} & 0.62335 & 0.23012 & 0.77449 & 0.00000 & \text { Uiso } & 1.00\end{array}$ $\begin{array}{llllllll}\text { Si28 } & \text { Si } & 0.38429 & 0.98827 & 0.77645 & 0.00000 & \text { Uiso } & 1.00\end{array}$ $\begin{array}{lllllllll}\mathrm{Si2} 2 & \mathrm{Si} & 0.76124 & 0.38101 & 0.72773 & 0.00000 & \text { Uiso } & 1.00\end{array}$ $\begin{array}{llllllll}\text { Si30 } & \text { Si } & 0.00995 & 0.60751 & 0.73798 & 0.00000 & \text { Uiso } & 1.00\end{array}$ $\begin{array}{llllllll}\mathrm{Si} 31 & \mathrm{Si} & 0.22953 & 0.61906 & 0.98015 & 0.00000 & \text { Uiso } & 1.00\end{array}$ $\begin{array}{llllllll}\mathrm{Si32} & \mathrm{Si} & 0.98931 & 0.37719 & 0.97910 & 0.00000 & \text { Uiso } & 1.00\end{array}$ $\begin{array}{lllllllll}\mathrm{Si} 33 & \mathrm{Si} & 0.62067 & 0.22664 & 0.98147 & 0.00000 & \text { Uiso } & 1.00\end{array}$ $\begin{array}{llllllll}\mathrm{Si34} & \mathrm{Si} & 0.37735 & 0.99107 & 0.98023 & 0.00000 & \text { Uiso } & 1.00\end{array}$ $\begin{array}{lllllllll}\mathrm{Si} 35 & \mathrm{Si} & 0.38211 & 0.76066 & 0.72775 & 0.00000 & \text { Uiso } & 1.00\end{array}$ $\begin{array}{llllllll}\text { Si36 Si } & 0.60926 & 0.00806 & 0.73391 & 0.00000 & \text { Uiso } & 1.00\end{array}$ $\begin{array}{lllllllll}\mathrm{Si} 37 & \mathrm{Si} & 0.23345 & 0.61988 & 0.77486 & 0.00000 & \text { Uiso } & 1.00\end{array}$

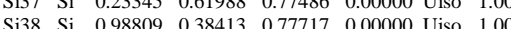

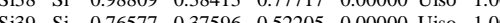

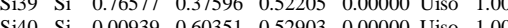
$\begin{array}{lllllllll} & \text { Si4 } & \text { Si } & 0.00939 & 0.60351 & 0.52903 & 0.00000 & \text { Uiso } & 1.00\end{array}$ $\begin{array}{llllllll} & \text { Si41 Si } & 0.46624 & 0.28949 & 0.62879 & 0.00000 & \text { Uiso } & 1.00\end{array}$ $\begin{array}{lllllllll}\mathrm{Si} 42 & \text { Si } & 0.31006 & 0.13720 & 0.62944 & 0.00000 & \text { Uiso } & 1.00\end{array}$ $\begin{array}{lllllllll}\mathrm{Si} 43 & \mathrm{Si} & 0.53175 & 0.70221 & 0.87604 & 0.00000 & \text { Uiso } & 1.00\end{array}$ $\begin{array}{llllllllll}\text { Si44 } & \mathrm{Si} & 0.68671 & 0.85905 & 0.87714 & 0.00000 & \text { Uiso } & 1.00\end{array}$ $\begin{array}{llllllll}\mathrm{Si} 45 & \mathrm{Si} & 0.29163 & 0.46386 & 0.62660 & 0.00000 & \text { Uiso } & 1.00\end{array}$ $\begin{array}{lllllllll}\text { Si46 } & \text { Si } & 0.13598 & 0.31084 & 0.62770 & 0.00000 & \text { Uiso } & 1.00\end{array}$ $\begin{array}{llllllll}\mathrm{Si} 47 & \mathrm{Si} & 0.70346 & 0.53163 & 0.87598 & 0.00000 & \text { Uiso } & 1.00\end{array}$ $\begin{array}{llllllll}\mathrm{Si} 48 & \mathrm{Si} & 0.85933 & 0.68763 & 0.87710 & 0.00000 & \text { Uiso } & 1.00\end{array}$ $\begin{array}{lllllllll}\mathrm{Al} 49 & \mathrm{Al} & 0.86732 & 0.68969 & 0.37704 & 0.00000 & \text { Uiso } & 1.00\end{array}$ $\begin{array}{llllllll}\mathrm{O} 50 & \mathrm{O} & 0.53000 & 0.29324 & 0.21580 & 0.00000 & \text { Uiso } & 1.00\end{array}$ $\begin{array}{lllllllll}051 & \mathrm{O} & 0.30677 & 0.06822 & 0.21538 & 0.00000 & \text { Uiso } & 1.00\end{array}$ $\begin{array}{llllllll}052 & \mathrm{O} & 0.34776 & 0.88811 & 0.51386 & 0.00000 & \text { Uiso } & 1.00\end{array}$ $\begin{array}{llllllllll}053 & \mathrm{O} & 0.47001 & 0.73665 & 0.46002 & 0.00000 & \text { Uiso } & 1.00\end{array}$ $\begin{array}{llllllll}\mathrm{O} 54 & \mathrm{O} & 0.67187 & 0.92673 & 0.46995 & 0.00000 & \text { Uiso } & 1.00\end{array}$ $\begin{array}{llllllll}055 & \mathrm{O} & 0.63415 & 0.11951 & 0.24150 & 0.00000 & \text { Uiso } & 1.00\end{array}$ $\begin{array}{llllllll}056 & \mathrm{O} & 0.28352 & 0.53255 & 0.04208 & 0.00000 & \text { Uiso } & 1.00\end{array}$ $\begin{array}{llllllll}057 & \mathrm{O} & 0.07367 & 0.31407 & 0.03696 & 0.00000 & \text { Uiso } & 1.00\end{array}$ $\begin{array}{llllllll}\mathrm{O} 58 & \mathrm{O} & 0.87584 & 0.37128 & 0.25034 & 0.00000 & \text { Uiso } & 1.00\end{array}$ $\begin{array}{llllllll}058 & 0 & 0.87584 & 0.37128 & 0.25034 & 0.00000 & \text { Uiso } & 1.00 \\ 059 & 0 & 0.70062 & 0.46994 & 0.28683 & 0.00000 & \text { Uiso } & 1.00\end{array}$ $\begin{array}{lllllllll}059 & 0 & 0.70062 & 0.4694 & 0.28683 & 0.00000 & \text { Uiso } & 1.00\end{array}$ $\begin{array}{lllllllll}060 & \mathrm{O} & 0.94143 & 0.68119 & 0.28466 & 0.00000 & \text { Uiso } & 1.00\end{array}$ $\begin{array}{lllllllll}061 & 0 & 0.11276 & 0.63489 & 0.99748 & 0.00000 & \text { Uiso } & 1.00\end{array}$ $\begin{array}{lllllllll}062 & \mathrm{O} & 0.4798 & 0.72224 & 0.28490 & 0.00000 & \text { Uiso } & 1.00\end{array}$ $\begin{array}{lllllllll}063 & \mathrm{O} & 0.67279 & 0.93648 & 0.29199 & 0.00000 & \text { Uiso } & 1.00\end{array}$ $\begin{array}{llllllll}064 & \mathrm{O} & 0.63747 & 0.11153 & 0.00741 & 0.00000 & \text { Uiso } & 1.00\end{array}$ $\begin{array}{llllllll}\mathrm{O} 65 & \mathrm{O} & 0.53196 & 0.28359 & 0.03916 & 0.00000 & \text { Uiso } & 1.00\end{array}$ $\begin{array}{llllllll}066 & \mathrm{O} & 0.31046 & 0.07201 & 0.03889 & 0.00000 & \text { Uiso } & 1.00\end{array}$ $\begin{array}{llllllll}067 & \mathrm{O} & 0.35843 & 0.88140 & 0.24613 & 0.00000 & \text { Uiso } & 1.00\end{array}$ $\begin{array}{llllllll}\mathrm{O} 68 & \mathrm{O} & 0.71816 & 0.46524 & 0.46102 & 0.00000 & \text { Uiso } & 1.00\end{array}$ $\begin{array}{llllllll}\mathrm{O} 69 & \mathrm{O} & 0.92941 & 0.67388 & 0.47590 & 0.00000 & \text { Uiso } & 1.00\end{array}$ $\begin{array}{llllllll}070 & \mathrm{O} & 0.12611 & 0.63408 & 0.23581 & 0.00000 & \text { Uiso } & 1.00\end{array}$ $\begin{array}{llllllll}071 & \mathrm{O} & 0.29890 & 0.52700 & 0.21891 & 0.00000 & \text { Uiso } & 1.00\end{array}$ $\begin{array}{lllllllll}072 & \mathrm{O} & 0.06088 & 0.31051 & 0.21267 & 0.00000 & \text { Uiso } & 1.00\end{array}$ $\begin{array}{llllllll}073 & \mathrm{O} & 0.88364 & 0.35729 & 0.51062 & 0.00000 & \text { Uiso } & 1.00\end{array}$ $\begin{array}{llllllll}074 & \mathrm{O} & 0.49036 & 0.00718 & 0.26079 & 0.00000 & \text { Uiso } & 1.00\end{array}$ $\begin{array}{lllllllll}075 & \mathrm{O} & 0.49254 & 0.00139 & 0.99426 & 0.00000 & \text { Uiso } & 1.00\end{array}$ $\begin{array}{llllllll}076 & \mathrm{O} & 0.99886 & 0.49299 & 0.98651 & 0.00000 & \text { Uiso } & 1.00\end{array}$ $\begin{array}{lllllllll}077 & \mathrm{O} & 0.01139 & 0.49449 & 0.26276 & 0.00000 & \text { Uiso } & 1.00\end{array}$ $\begin{array}{llllllllll}078 & \mathrm{O} & 0.38582 & 0.38496 & 0.12124 & 0.00000 & \text { Uiso } & 1.00\end{array}$ $\begin{array}{llllllllll}078 & 0 & 0.3858 & 0.38496 & 0.12124 & 0.00000 & \text { Uiso } & 1.00 \\ 079 & 0 & 0.21230 & 0.21498 & 0.12976 & 0.00000 & \text { Uiso } & 1.00\end{array}$ $\begin{array}{lllllllll}079 & 0 & 0.21230 & 0.21498 & 0.12976 & 0.00000 & \text { Uiso } & 1.00 \\ 080 & 0 & 0.58916 & 0.59497 & 0.38550 & 0.00000 & \text { Uiso } & 1.00\end{array}$ $\begin{array}{llllllllll}080 & \mathrm{O} & 0.58916 & 0.59497 & 0.38550 & 0.00000 & \text { Uiso } & 1.00\end{array}$ $\begin{array}{lllllllll}081 & \mathrm{O} & 0.81315 & 0.82484 & 0.37608 & 0.00000 & \text { Uiso } & 1.00\end{array}$ $\begin{array}{llllllllllll}082 & 0 & 0.40872 & 0.19041 & 0.13160 & 0.00000 & \text { Uiso } & 1.00\end{array}$ $\begin{array}{lllllllll}083 & 0 & 0.62831 & 0.77803 & 0.37462 & 0.00000 & \text { Uiso } & 1.00\end{array}$ $\begin{array}{llllllll}084 & \mathrm{O} & 0.19074 & 0.40973 & 0.13496 & 0.00000 & \text { Uiso } & 1.00\end{array}$ $\begin{array}{lllllllll}085 & \mathrm{O} & 0.77797 & 0.61365 & 0.36934 & 0.00000 & \text { Uiso } & 1.00\end{array}$ $\begin{array}{lllllllll}086 & \mathrm{O} & 0.34777 & 0.00963 & 0.37760 & 0.00000 & \text { Uiso } & 1.00\end{array}$ $\begin{array}{llllllll}087 & \mathrm{O} & 0.58678 & 0.23932 & 0.37677 & 0.00000 & \text { Uiso } & 1.00\end{array}$ $\begin{array}{llllllll}088 & \mathrm{O} & 0.61816 & 0.97532 & 0.12809 & 0.00000 & \text { Uiso } & 1.00\end{array}$ $\begin{array}{llllllll}\mathrm{O} 89 & \mathrm{O} & 0.40567 & 0.74228 & 0.12635 & 0.00000 & \text { Uiso } & 1.00 \\ \mathrm{O} 90 & \mathrm{O} & 0.00908 & 0.34903 & 0.37714 & 0.00000 & \text { Uiso } & 1.00\end{array}$ 
$\begin{array}{llllllll}091 & \mathrm{O} & 0.23673 & 0.58454 & 0.37789 & 0.00000 & \text { Uiso } & 1.00\end{array}$ $\begin{array}{lllllllll}092 & \mathrm{O} & 0.98282 & 0.61313 & 0.12418 & 0.00000 & \text { Uiso } & 1.00\end{array}$ $\begin{array}{lllllllll}093 & \mathrm{O} & 0.73824 & 0.40531 & 0.12658 & 0.00000 & \text { Uiso } & 1.00\end{array}$ $\begin{array}{lllllllll}094 & \mathrm{O} & 0.71992 & 0.27838 & 0.25699 & 0.00000 & \text { Uiso } & 1.00\end{array}$ $\begin{array}{llllllll}095 & \mathrm{O} & 0.27159 & 0.72172 & 0.00294 & 0.00000 & \text { Uiso } & 1.00\end{array}$ $\begin{array}{lllllllll}096 & \mathrm{O} & 0.28793 & 0.71476 & 0.26274 & 0.00000 & \text { Uiso } & 1.00\end{array}$ $\begin{array}{llllllllll}097 & 0 & 0.72211 & 0.27154 & 0.00190 & 0.00000 & \text { Uiso } & 1.00\end{array}$ $\begin{array}{lllllllllll}097 & 0 & 0.72211 & 0.27154 & 0.00190 & 0.00000 & \text { Uiso } & 1.00 \\ 098 & 0 & 0.53526 & 0.28719 & 0.71595 & 0.00000 & \text { Uiso } & 1.00\end{array}$

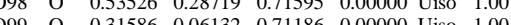
$\begin{array}{lllllllll}0.00 & 0.00000\end{array}$ $\begin{array}{llllllll}0100 & 0 & 0.35463 & 0.88316 & 0.00999 & 0.00000 & \text { Uiso } & 1.00\end{array}$ $\begin{array}{lllllllll}0101 & \mathrm{O} & 0.45946 & 0.71597 & 0.96071 & 0.00000 & \text { Uiso } & 1.00\end{array}$ $\begin{array}{lllllllll}0102 & \mathrm{O} & 0.67633 & 0.92424 & 0.96718 & 0.00000 & \text { Uiso } & 1.00 \\ 0103 & \mathrm{O} & 0.6437 & 0.11579 & 0.7468 & 0.0000 & \text { Uis } & 1.00\end{array}$ $\begin{array}{lllllllll}0103 & 0 & 0.64347 & 0.11579 & 0.74608 & 0.00000 & \text { Uiso } & 1.00\end{array}$ $\begin{array}{lllllllll}0104 & \mathrm{O} & 0.29374 & 0.53064 & 0.53832 & 0.00000 & \text { Uiso } & 1.00\end{array}$ $\begin{array}{lllllllll}0105 & 0 & 0.07230 & 0.30555 & 0.53774 & 0.00000 & \text { Uiso } & 1.00\end{array}$ $\begin{array}{lllllllll}0106 & \mathrm{O} & 0.87775 & 0.37376 & 0.74483 & 0.00000 & \text { Uiso } & 1.00\end{array}$ $\begin{array}{llllllll}0107 & \mathrm{O} & 0.70279 & 0.46942 & 0.78489 & 0.00000 & \text { Uiso } & 1.00\end{array}$ $\begin{array}{llllllll}0108 & \mathrm{O} & 0.92965 & 0.68260 & 0.79047 & 0.00000 & \text { Uiso } & 1.00\end{array}$

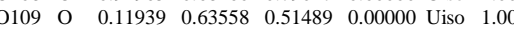
$\begin{array}{lllllllll}0110 & \mathrm{O} & 0.47022 & 0.70115 & 0.78452 & 0.00000 & \text { Uiso } & 1.00\end{array}$ $\begin{array}{lllllllll}0111 & \mathrm{O} & 0.68154 & 0.92931 & 0.79025 & 0.00000 & \text { Uiso } & 1.00\end{array}$ $\begin{array}{llllllll}0112 & \mathrm{O} & 0.63555 & 0.11446 & 0.50804 & 0.00000 & \text { Uiso } & 1.00\end{array}$ $\begin{array}{lllllllll}0113 & \mathrm{O} & 0.53186 & 0.28713 & 0.53929 & 0.00000 & \text { Uiso } & 1.00\end{array}$ $\begin{array}{lllllllll}0114 & \mathrm{O} & 0.30750 & 0.07821 & 0.53647 & 0.00000 & \text { Uiso } & 1.00\end{array}$ $\begin{array}{lllllllll}0115 & \mathrm{O} & 0.37287 & 0.87632 & 0.75040 & 0.00000 & \text { Uiso } & 1.00\end{array}$ $\begin{array}{llllllll}0116 & \mathrm{O} & 0.71770 & 0.45935 & 0.96061 & 0.00000 & \text { Uiso } & 1.00\end{array}$ $\begin{array}{llllllllll}0117 & 0 & 0.92394 & 0.67805 & 0.96731 & 0.00000 & \text { Uiso } & 1.00\end{array}$ $\begin{array}{llllllllllll}0117 & 0 & 0.92394 & 0.6785 & 0.96731 & 0.00000 & \text { Uso } & 1.00\end{array}$ $\begin{array}{lllllllllll}0118 & 0 & 0.11749 & 0.6405 & 0.96731 & 0.00000 & 0.75427 & 0.00000 & 1.00\end{array}$ $\begin{array}{llllllll}0119 & 0 & 0.28661 & 0.53122 & 0.71485 & 0.00000 & \text { Uiso } & 1.00\end{array}$ $\begin{array}{lllllllllll}0120 & 0 & 0.06460 & 0.31918 & 0.71323 & 0.00000 & \text { Uiso } & 1.00\end{array}$ $\begin{array}{lllllllllll}0121 & \mathrm{O} & 0.88331 & 0.35455 & 0.01471 & 0.00000 & \text { Uiso } & 1.00 \\ 0122 & \mathrm{O} & 0.49772 & 0.00845 & 0.76843 & 0.00000 & \text { Uis } & 1.00\end{array}$ $\begin{array}{lllllllll}0122 & 0 & 0.49772 & 0.00845 & 0.76843 & 0.00000 & \text { Uiso } & 1.00\end{array}$ $\begin{array}{llllllllll}0123 & \mathrm{O} & 0.48954 & 0.00359 & 0.49671 & 0.00000 & \text { Uiso } & 1.00 \\ 0124 & \mathrm{O} & 0.0104 & 0.4893 & 0.49723 & 0.0000 & \text { Uis } & 1.00\end{array}$ $\begin{array}{llllllll}0124 & \mathrm{O} & 0.01044 & 0.48993 & 0.49723 & 0.00000 & \text { Uiso } & 1.00\end{array}$ $\begin{array}{lllllllll}0125 & \mathrm{O} & 0.00818 & 0.49769 & 0.77672 & 0.00000 & \text { Uiso } & 1.00\end{array}$ $\begin{array}{llllllll}0126 & \mathrm{O} & 0.39134 & 0.38897 & 0.62975 & 0.00000 & \text { Uiso } & 1.00\end{array}$ $\begin{array}{llllllll}0127 & \mathrm{O} & 0.21083 & 0.21158 & 0.63749 & 0.00000 & \text { Uiso } & 1.00\end{array}$ $\begin{array}{lllllllll}0128 & \mathrm{O} & 0.59924 & 0.59850 & 0.88618 & 0.00000 & \text { Uiso } & 1.00\end{array}$ $\begin{array}{llllllll}0129 & \mathrm{O} & 0.79163 & 0.79237 & 0.87808 & 0.00000 & \text { Uiso } & 1.00\end{array}$ $\begin{array}{lllllllll}0130 & \mathrm{O} & 0.40711 & 0.19407 & 0.63061 & 0.00000 & \text { Uiso } & 1.00\end{array}$ $\begin{array}{llllllll}0131 & \mathrm{O} & 0.59742 & 0.79233 & 0.87269 & 0.00000 & \text { Uiso } & 1.00\end{array}$ $\begin{array}{llllllllll}0132 & \mathrm{O} & 0.19579 & 0.40565 & 0.62378 & 0.00000 & \text { Uiso } & 1.00\end{array}$ $\begin{array}{lllllllll}0133 & \mathrm{O} & 0.79224 & 0.59862 & 0.87246 & 0.00000 & \text { Uiso } & 1.00\end{array}$ $\begin{array}{lllllllll}0134 & \mathrm{O} & 0.34825 & 0.00992 & 0.87733 & 0.00000 & \text { Uiso } & 1.00\end{array}$ $\begin{array}{lllllllll}0135 & \mathrm{O} & 0.59161 & 0.23994 & 0.87777 & 0.00000 & \text { Uiso } & 1.00\end{array}$ $\begin{array}{lllllllll}0136 & \mathrm{O} & 0.61669 & 0.97808 & 0.62999 & 0.00000 & \text { Uiso } & 1.00\end{array}$ $\begin{array}{lllllllll}0137 & \mathrm{O} & 0.40678 & 0.74268 & 0.62368 & 0.00000 & \text { Uiso } & 1.00\end{array}$ $\begin{array}{lllllllll}0138 & 0 & 0.00363 & 0.34233 & 0.87706 & 0.00000 & \text { Uiso } & 1.00\end{array}$ $\begin{array}{llllllllllll}0138 & 0 & 0.00363 & 0.34233 & 0.87706 & 0.0000 & \text { Uiso } & 1.00 \\ 0139 & 0 & 0.25241 & 0.58907 & 0.87770 & 0.0000 & \text { Uis } & 1.00\end{array}$

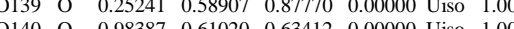
$\begin{array}{llllllllll}0140 & 0 & 0.98387 & 0.61020 & 0.63412 & 0.00000 & \text { Uiso } & 1.00\end{array}$ $\begin{array}{lllllllllll}0141 & \mathrm{O} & 0.73784 & 0.40297 & 0.62424 & 0.00000 & \text { Uiso } & 1.00\end{array}$ $\begin{array}{llllllllll}0142 & 0 & 0.72417 & 0.27770 & 0.75720 & 0.00000 & \text { Uiso } & 1.00\end{array}$ $\begin{array}{llllllllll}0143 & \mathrm{O} & 0.28164 & 0.71811 & 0.49294 & 0.00000 & \text { Uiso } & 1.00 \\ 0144 & \mathrm{O} & 0.27923 & 0.72084 & 0.75289 & 0.0000 & \text { Uiso } & 1.00\end{array}$ $\begin{array}{llllllllll}0144 & \mathrm{O} & 0.27623 & 0.72084 & 0.72289 & 0.00000 & \text { Uiso } & 1.00 \\ 0145 & 0 & 0.72128 & 0.2788 & 0.4551 & 0.0000 & \text { Uiso } & 1.00\end{array}$ $\begin{array}{llllllll}0145 & \mathrm{O} & 0.72128 & 0.27488 & 0.49551 & 0.00000 & \text { Uiso } & 1.00\end{array}$

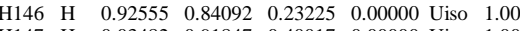
$\begin{array}{llllllll}\mathrm{H} 147 & \mathrm{H} & 0.03482 & 0.91847 & 0.40017 & 0.00000 & \text { Uiso } & 1.00\end{array}$ $\begin{array}{llllllll}\mathrm{H} 148 & \mathrm{H} & 0.97321 & 0.84818 & 0.47617 & 0.00000 & \text { Uiso } & 1.00\end{array}$ $\begin{array}{lllllllll}\mathrm{H} 149 & \mathrm{H} & 0.01951 & 0.79589 & 0.37398 & 0.00000 & \text { Uiso } & 1.00\end{array}$

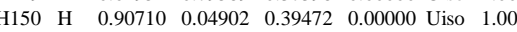
$\begin{array}{lllllllll}\mathrm{H} 151 & \mathrm{H} & 0.83927 & 0.99154 & 0.47510 & 0.00000 & \text { Uiso } & 1.00\end{array}$ $\begin{array}{llllllllll}\mathrm{H} 152 & \mathrm{H} & 0.78368 & 0.03494 & 0.37301 & 0.00000 & \text { Uiso } & 1.00\end{array}$ $\begin{array}{llllllll}\mathrm{H} 153 & \mathrm{H} & 0.95821 & 0.96079 & 0.24992 & 0.00000 & \text { Uiso } & 1.00\end{array}$ $\begin{array}{lllllllll}\mathrm{H} 154 & \mathrm{H} & 0.83372 & 0.94492 & 0.23053 & 0.00000 & \text { Uiso } & 1.00\end{array}$ $\begin{array}{llllllll}\mathrm{C} 155 & \mathrm{C} & 0.85075 & 0.99983 & 0.40403 & 0.00000 & \text { Uiso } & 1.00\end{array}$ $\begin{array}{lllllllll}\mathrm{C} 156 & \mathrm{C} & 0.89027 & 0.90353 & 0.36083 & 0.00000 & \text { Uiso } & 1.00\end{array}$ $\begin{array}{lllllllll}\mathrm{C} 158 & \mathrm{C} & 0.90160 & 0.91237 & 0.26174 & 0.00000 & \text { Uiso } & 1.00\end{array}$

\section{File $\mathbf{n}^{\circ} 9$}

$\begin{array}{llllllll}\text { Si2 } & \text { Si } & 0.37386 & 0.76556 & 0.02404 & 0.00000 & \text { Uiso } & 1.00\end{array}$ $\begin{array}{llllllll}\mathrm{Si} 3 & \mathrm{Si} & 0.60721 & 0.00176 & 0.02280 & 0.00000 & \text { Uiso } & 1.00\end{array}$

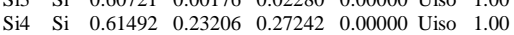
$\begin{array}{lllllllll}\text { Si5 } & \text { Si } & 0.37956 & 0.98518 & 0.27457 & 0.00000 & \text { Uiso } & 1.00\end{array}$ $\begin{array}{lllllllll}\mathrm{Si} 6 & \mathrm{Si} & 0.75919 & 0.37838 & 0.22987 & 0.00000 & \text { Uiso } & 1.00\end{array}$ $\begin{array}{lllllllll}\mathrm{Si} 7 & \mathrm{Si} & 0.01304 & 0.60514 & 0.22668 & 0.00000 & \text { Uiso } & 1.00\end{array}$ $\begin{array}{llllllll}\text { Si8 } & \text { Si } & 0.23281 & 0.61169 & 0.47956 & 0.00000 & \text { Uiso } & 1.00\end{array}$ $\begin{array}{lllllllll}\mathrm{Si} 9 & \mathrm{Si} & 0.98957 & 0.37920 & 0.47938 & 0.00000 & \text { Uiso } & 1.00\end{array}$ $\begin{array}{llllllll}\mathrm{Si10} & \mathrm{Si} & 0.61651 & 0.22928 & 0.48026 & 0.00000 & \text { Uiso } & 1.00\end{array}$ $\begin{array}{lllllllll}\text { Sil1 } & \text { Si } & 0.37746 & 0.98885 & 0.47964 & 0.00000 & \text { Uiso } & 1.00\end{array}$ $\begin{array}{llllllll}\text { Si12 } & \text { Si } & 0.38174 & 0.75893 & 0.22996 & 0.00000 & \text { Uiso } & 1.00\end{array}$ $\begin{array}{llllllll}\mathrm{Si} 13 & \mathrm{Si} & 0.60503 & 0.00706 & 0.22906 & 0.00000 & \text { Uiso } & 1.00\end{array}$ $\begin{array}{llllllll}\text { Si14 } & \text { Si } & 0.23722 & 0.60808 & 0.27158 & 0.00000 & \text { Uiso } & 1.00\end{array}$ $\begin{array}{lllllllll}\text { Si15 } & \text { Si } & 0.98700 & 0.38160 & 0.27458 & 0.00000 & \text { Uiso } & 1.00\end{array}$ $\begin{array}{llllllll}\mathrm{Si} 16 & \mathrm{Si} & 0.76548 & 0.37277 & 0.02438 & 0.00000 & \text { Uiso } & 1.00\end{array}$ $\begin{array}{lllllllll}\text { Si17 } & \text { Si } & 0.00542 & 0.60354 & 0.01962 & 0.00000 & \text { Uiso } & 1.00\end{array}$ $\begin{array}{llllllllll}\text { Si18 } & \text { Si } & 0.46515 & 0.28670 & 0.12602 & 0.00000 & \text { Uiso } & 1.00\end{array}$

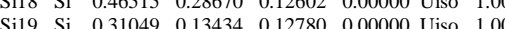
$\begin{array}{llllllllll} & \text { Sir } & \text { Si } & 0.53959 & 0.70770 & 0.37567 & 0.00000 & \text { Uiso } & 1.00\end{array}$ $\begin{array}{lllllllll} & \text { Si20 Si } & 0.53959 & 0.70770 & 0.37567 & 0.00000 & \text { Uiso } & 1.00\end{array}$ $\begin{array}{llllllll}S i 21 & \text { Si } & 0.69386 & 0.8689 & 0.37582 & 0.00000 & \text { Uiso } & 1.00\end{array}$ $\begin{array}{llllllll}\mathrm{Si}_{2} 2 & \text { Si } & 0.29128 & 0.46075 & 0.12610 & 0.00000 & \text { Uiso } & 1.00\end{array}$ $\begin{array}{lllllllll}\mathrm{Si} 23 & \mathrm{Si} & 0.13548 & 0.30924 & 0.12870 & 0.00000 & \text { Uiso } & 1.00\end{array}$ $\begin{array}{llllllllll}\mathrm{Si} 24 & \mathrm{Si} & 0.70759 & 0.53350 & 0.37603 & 0.00000 & \text { Uiso } & 1.00\end{array}$ $\begin{array}{lllllllll}\mathrm{Si} 25 & \mathrm{Si} & 0.37430 & 0.76477 & 0.52035 & 0.00000 & \text { Uiso } & 1.00\end{array}$ $\begin{array}{llllllll}\mathrm{Si} 26 & \mathrm{Si} & 0.60486 & 0.00420 & 0.52495 & 0.00000 & \text { Uiso } & 1.00\end{array}$ $\begin{array}{llllllll}\mathrm{Si27} & \mathrm{Si} & 0.62535 & 0.22642 & 0.77346 & 0.00000 & \text { Uiso } & 1.00\end{array}$ $\begin{array}{llllllll}\mathrm{Si} 28 & \mathrm{Si} & 0.38467 & 0.98887 & 0.77412 & 0.00000 & \text { Uiso } & 1.00\end{array}$ $\begin{array}{lllllllll}\text { Si29 } & \text { Si } & 0.76317 & 0.37732 & 0.72792 & 0.00000 & \text { Uiso } & 1.00\end{array}$ $\begin{array}{llllllll}\mathrm{Si} 30 & \mathrm{Si} & 0.00858 & 0.60607 & 0.73486 & 0.00000 & \text { Uiso } & 1.00\end{array}$ 
$\begin{array}{llllllll}\text { Si31 } & \text { Si } & 0.22816 & 0.62026 & 0.98003 & 0.00000 & \text { Uiso } & 1.00\end{array}$ $\begin{array}{llllllll}\mathrm{Si} 32 & \mathrm{Si} & 0.99105 & 0.37638 & 0.97901 & 0.00000 & \text { Uiso } & 1.00\end{array}$ $\begin{array}{llllllll}\mathrm{Si33} & \mathrm{Si} & 0.62330 & 0.22306 & 0.98006 & 0.00000 & \text { Uiso } & 1.00\end{array}$ $\begin{array}{lllllllll}\mathrm{Si3} 4 & \mathrm{Si} & 0.37938 & 0.99066 & 0.97876 & 0.00000 & \text { Uiso } & 1.00\end{array}$ $\begin{array}{lllllllll}\mathrm{Si35} & \mathrm{Si} & 0.37902 & 0.76190 & 0.72671 & 0.00000 & \text { Uiso } & 1.00\end{array}$ $\begin{array}{llllllll}\text { Si36 } & \mathrm{Si} & 0.61066 & 0.00492 & 0.73138 & 0.00000 & \text { Uiso } & 1.00\end{array}$ $\begin{array}{llllllll}\mathrm{Si} 37 & \mathrm{Si} & 0.23117 & 0.62213 & 0.77436 & 0.00000 & \text { Uiso } & 1.00\end{array}$ $\begin{array}{lllllllllll} & \mathrm{Si} 38 & \mathrm{Si} & 0.99014 & 0.38141 & 0.77611 & 0.00000 & \text { Uiso } & 1.00\end{array}$ $\begin{array}{lllllllll} & \text { Si39 } & \text { Si } & 0.76447 & 0.37255 & 0.52223 & 0.00000 & \text { Uiso } & 1.00\end{array}$

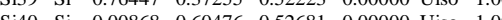

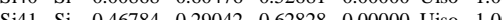
$\begin{array}{lllllllll}\text { Si41 } & \text { Si } & 0.46784 & 0.29042 & 0.62828 & 0.00000 & \text { Uiso } & 1.00\end{array}$ $\begin{array}{llllllll}\mathrm{Si} 42 & \mathrm{Si} & 0.31267 & 0.13723 & 0.62720 & 0.00000 & \text { Uiso } & 1.00\end{array}$ $\begin{array}{lllllllll}\text { Si43 Si } & 0.53110 & 0.70098 & 0.87474 & 0.00000 & \text { Uiso } & 1.00\end{array}$ $\begin{array}{llllllll}\mathrm{Si} 44 & \mathrm{Si} & 0.68753 & 0.85687 & 0.87607 & 0.00000 & \text { Uiso } & 1.00 \\ \mathrm{Si45} & \mathrm{Si} & 0.29353 & 0.46496 & 0.62761 & 0.00000 & \text { Us } & 1.00\end{array}$ $\begin{array}{lllllllll}\text { Si45 } & \text { Si } & 0.29353 & 0.46496 & 0.62761 & 0.00000 & \text { Uiso } & 1.00\end{array}$ $\begin{array}{llllllll}\text { Si46 } & \text { Si } & 0.13779 & 0.31216 & 0.62686 & 0.00000 & \text { Uiso } & 1.00\end{array}$

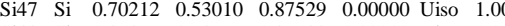
$\begin{array}{llllllll}\text { Si48 } & \text { Si } & 0.85877 & 0.68579 & 0.87580 & 0.00000 & \text { Uiso } & 1.00\end{array}$ $\begin{array}{lllllllll}\mathrm{Al} 49 & \mathrm{Al} & 0.86826 & 0.69062 & 0.37443 & 0.00000 & \text { Uiso } & 1.00\end{array}$ $\begin{array}{llllllll}\mathrm{O} 50 & \mathrm{O} & 0.52798 & 0.29516 & 0.21618 & 0.00000 & \text { Uiso } & 1.00\end{array}$ $\begin{array}{lllllllll}051 & \mathrm{O} & 0.31091 & 0.06093 & 0.21215 & 0.00000 & \text { Uiso } & 1.00\end{array}$ $\begin{array}{lllllllll}052 & \mathrm{O} & 0.34789 & 0.88231 & 0.50798 & 0.00000 & \text { Uiso } & 1.00\end{array}$ $\begin{array}{llllllll}053 & \mathrm{O} & 0.46661 & 0.72272 & 0.46029 & 0.00000 & \text { Uiso } & 1.00\end{array}$ $\begin{array}{llllllll}054 & \mathrm{O} & 0.68075 & 0.93300 & 0.46555 & 0.00000 & \text { Uiso } & 1.00\end{array}$ $\begin{array}{lllllllll}055 & \mathrm{O} & 0.62746 & 0.11965 & 0.23785 & 0.00000 & \text { Uiso } & 1.00\end{array}$ $\begin{array}{lllllllll}056 & \mathrm{O} & 0.28277 & 0.53372 & 0.04155 & 0.00000 & \text { Uiso } & 1.00\end{array}$ $\begin{array}{lllllllll}057 & \mathrm{O} & 0.07291 & 0.31039 & 0.03758 & 0.00000 & \text { Uiso } & 1.00\end{array}$ $\begin{array}{lllllllll}058 & 0 & 0.87612 & 0.36481 & 0.24830 & 0.00000 & \text { Uiso } & 1.00\end{array}$ $\begin{array}{llllllllll}058 & 0 & 0.87612 & 0.36481 & 0.24830 & 0.0000 & \text { Uiso } & 1.00 \\ 0.756 & 0 & 0.70233 & 0.46676 & 0.28693 & 0.00000 & \text { Uiso } & 1.00\end{array}$ $\begin{array}{llllllllll}059 & 0 & 0.70233 & 0.46676 & 0.28693 & 0.00000 & \text { Uiso } & 1.00\end{array}$ $\begin{array}{lllllllll}060 & \mathrm{O} & 0.94966 & 0.68572 & 0.28535 & 0.00000 & \text { Uiso } & 1.00\end{array}$

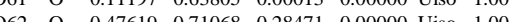
$\begin{array}{llllllllll}062 & 0 & 0.47619 & 0.71068 & 0.28471 & 0.00000 & \text { Uiso } & 1.00\end{array}$ $\begin{array}{lllllllll}063 & \mathrm{O} & 0.68197 & 0.93871 & 0.28981 & 0.00000 & \text { Uiso } & 1.00\end{array}$ \begin{tabular}{lllllllll}
064 & $\mathrm{O}$ & 0.64294 & 0.10815 & 0.00599 & 0.00000 & Uiso & 1.00 \\
\hline 065 & $\mathrm{O}$ & 0.5351 & 0.27793 & 0.03948 & 0.00000 & Uso & 1.00
\end{tabular} $\begin{array}{llllllll}\mathrm{O} 65 & \mathrm{O} & 0.53511 & 0.27793 & 0.03948 & 0.00000 & \text { Uiso } & 1.00\end{array}$ $\begin{array}{llllllll}066 & \mathrm{O} & 0.31197 & 0.07259 & 0.03615 & 0.00000 & \text { Uiso } & 1.00\end{array}$ $\begin{array}{llllllll}067 & \mathrm{O} & 0.35722 & 0.87510 & 0.25088 & 0.00000 & \text { Uiso } & 1.00\end{array}$ $\begin{array}{lllllllll}068 & \mathrm{O} & 0.71446 & 0.46101 & 0.46179 & 0.00000 & \text { Uiso } & 1.00\end{array}$ $\begin{array}{llllllll}069 & \mathrm{O} & 0.93938 & 0.68548 & 0.47206 & 0.00000 & \text { Uiso } & 1.00\end{array}$ $\begin{array}{llllllll}070 & \mathrm{O} & 0.12799 & 0.62552 & 0.23062 & 0.00000 & \text { Uiso } & 1.00\end{array}$ $\begin{array}{llllllll}071 & \mathrm{O} & 0.30183 & 0.52037 & 0.21800 & 0.00000 & \text { Uiso } & 1.00\end{array}$ $\begin{array}{lllllllll}072 & \mathrm{O} & 0.06291 & 0.31071 & 0.21336 & 0.00000 & \text { Uiso } & 1.00\end{array}$ $\begin{array}{llllllll}073 & \mathrm{O} & 0.88239 & 0.35423 & 0.51000 & 0.00000 & \text { Uiso } & 1.00\end{array}$ $\begin{array}{lllllllll}074 & \mathrm{O} & 0.49404 & -0.00325 & 0.26069 & 0.00000 & \text { Uiso } & 1.00\end{array}$ $\begin{array}{lllllllll}075 & \mathrm{O} & 0.49424 & 0.00277 & 0.99176 & 0.00000 & \text { Uiso } & 1.00\end{array}$ $\begin{array}{lllllllll}076 & \mathrm{O} & 0.00461 & 0.49136 & 0.98682 & 0.00000 & \text { Uiso } & 1.00\end{array}$ $\begin{array}{llllllll}\mathrm{O} 77 & \mathrm{O} & 0.00550 & 0.49434 & 0.26152 & 0.00000 & \text { Uiso } & 1.00\end{array}$ $\begin{array}{llllllllll}078 & \mathrm{O} & 0.38830 & 0.38382 & 0.11419 & 0.00000 & \text { Uiso } & 1.00\end{array}$ $\begin{array}{llllllllll}078 & 0 & 0.38830 & 0.38382 & 0.11419 & 0.00000 & \text { Uiso } & 1.00\end{array}$ $\begin{array}{llllllll}080 & 0 & 0.21150 & 0.21007 & 0.13153 & 0.00000 & \text { Uiso } & 1.00\end{array}$ $\begin{array}{lllllllll}080 & \mathrm{O} & 0.60287 & 0.60203 & 0.38396 & 0.00000 & \text { Uiso } & 1.00\end{array}$ \begin{tabular}{lllllllllll}
\hline 881 & 0 & 0.80076 & 0.80656 & 0.37420 & 0.00000 & Uiso & 1.00
\end{tabular} $\begin{array}{lllllllll}082 & 0 & 0.40795 & 0.19011 & 0.13285 & 0.00000 & \text { Uiso } & 1.00\end{array}$ $\begin{array}{lllllllll}083 & \mathrm{O} & 0.60620 & 0.79657 & 0.37337 & 0.00000 & \text { Uiso } & 1.00\end{array}$ $\begin{array}{llllllllll}084 & \mathrm{O} & 0.19367 & 0.40530 & 0.13139 & 0.00000 & \text { Uiso } & 1.00\end{array}$ $\begin{array}{llllllll}085 & \mathrm{O} & 0.79802 & 0.59507 & 0.37183 & 0.00000 & \text { Uiso } & 1.00\end{array}$ $\begin{array}{llllllll}086 & \mathrm{O} & 0.34920 & 0.00930 & 0.37659 & 0.00000 & \text { Uiso } & 1.00\end{array}$ $\begin{array}{llllllll}087 & \mathrm{O} & 0.58564 & 0.23697 & 0.37652 & 0.00000 & \text { Uiso } & 1.00\end{array}$ $\begin{array}{lllllllll}088 & \mathrm{O} & 0.61851 & 0.97283 & 0.12657 & 0.00000 & \text { Uiso } & 1.00\end{array}$ $\begin{array}{llllllll}\mathrm{O} 89 & \mathrm{O} & 0.40498 & 0.74088 & 0.12580 & 0.00000 & \text { Uiso } & 1.00\end{array}$ $\begin{array}{llllllll}090 & \mathrm{O} & 0.00794 & 0.34876 & 0.37659 & 0.00000 & \text { Uiso } & 1.00\end{array}$ $\begin{array}{lllllllll}091 & \mathrm{O} & 0.23518 & 0.58010 & 0.37597 & 0.00000 & \text { Uiso } & 1.00\end{array}$ $\begin{array}{llllllllll}092 & \mathrm{O} & 0.97919 & 0.61278 & 0.12346 & 0.00000 & \text { Uiso } & 1.00\end{array}$ $\begin{array}{llllllll}093 & \mathrm{O} & 0.73806 & 0.40201 & 0.12588 & 0.00000 & \text { Uiso } & 1.00\end{array}$ $\begin{array}{lllllllll}094 & \mathrm{O} & 0.71822 & 0.27550 & 0.25623 & 0.00000 & \text { Uiso } & 1.00\end{array}$ $\begin{array}{llllllll}095 & \mathrm{O} & 0.27195 & 0.72256 & 0.00093 & 0.00000 & \text { Uiso } & 1.00\end{array}$ $\begin{array}{llllllll}096 & \mathrm{O} & 0.28502 & 0.70979 & 0.25936 & 0.00000 & \text { Uiso } & 1.00\end{array}$ $\begin{array}{llllllll}\mathrm{O} 97 & \mathrm{O} & 0.72386 & 0.27073 & -0.00169 & 0.00000 & \text { Uiso } & 1.00\end{array}$ $\begin{array}{llllllll}098 & \mathrm{O} & 0.53829 & 0.28464 & 0.71454 & 0.00000 & \text { Uiso } & 1.00\end{array}$ $\begin{array}{lllllllll}099 & \mathrm{O} & 0.31619 & 0.06624 & 0.71315 & 0.00000 & \text { Uiso } & 1.00\end{array}$ $\begin{array}{llllllllll}0100 & 0 & 0.31619 & 0.06624 & 0.71315 & 0.00000 & \text { Uiso } & 1.00\end{array}$

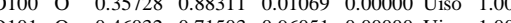
\begin{tabular}{llllllllll}
\hline & 0.0102 & 0 & 0.46032 & 0.71503 & 0.96051 & 0.00000 & Uiso & 1.00
\end{tabular} $\begin{array}{lllllllllll}0102 & 0 & 0.67688 & 0.92111 & 0.96628 & 0.00000 & \text { Uiso } & 1.00\end{array}$ $\begin{array}{lllllllll}0103 & \mathrm{O} & 0.64527 & 0.11259 & 0.74361 & 0.00000 & \text { Uiso } & 1.00 \\ 0104 & \mathrm{O} & 0.29798 & 0.52715 & 0.53636 & 0.00000 & \text { Uis } & 1.00\end{array}$ $\begin{array}{llllllllll}0104 & \mathrm{O} & 0.29798 & 0.5271 & 0.5363 & 0.0000 & \text { Uiso } & 1.00\end{array}$ $\begin{array}{lllllllll}0105 & \mathrm{O} & 0.07201 & 0.31277 & 0.53768 & 0.00000 & \text { Uiso } & 1.00\end{array}$ $\begin{array}{llllllll}0106 & \mathrm{O} & 0.88013 & 0.36951 & 0.74378 & 0.00000 & \text { Uiso } & 1.00\end{array}$ $\begin{array}{llllllll}0107 & \mathrm{O} & 0.70528 & 0.46571 & 0.78534 & 0.00000 & \text { Uiso } & 1.00\end{array}$ $\begin{array}{lllllllll}\mathrm{O} 108 & \mathrm{O} & 0.92847 & 0.67964 & 0.78876 & 0.00000 & \text { Uiso } & 1.00\end{array}$ $\begin{array}{lllllllll}0109 & \mathrm{O} & 0.12208 & 0.62767 & 0.51696 & 0.00000 & \text { Uiso } & 1.00\end{array}$

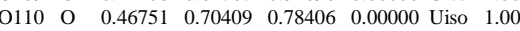
$\begin{array}{llllllll}0111 & \mathrm{O} & 0.68190 & 0.92687 & 0.78922 & 0.00000 & \text { Uiso } & 1.00\end{array}$ $\begin{array}{llllllll}0112 & \mathrm{O} & 0.62789 & 0.11618 & 0.51256 & 0.00000 & \text { Uiso } & 1.00\end{array}$ $\begin{array}{lllllllll}0113 & \mathrm{O} & 0.53104 & 0.29273 & 0.53766 & 0.00000 & \text { Uiso } & 1.00\end{array}$ $\begin{array}{lllllllll}0114 & \mathrm{O} & 0.31232 & 0.07197 & 0.53752 & 0.00000 & \text { Uiso } & 1.00\end{array}$ $\begin{array}{llllllll}0115 & \mathrm{O} & 0.37064 & 0.87872 & 0.74367 & 0.00000 & \text { Uiso } & 1.00\end{array}$ $\begin{array}{lllllllll}0116 & 0 & 0.71546 & 0.46010 & 0.96149 & 0.00000 & \text { Uiso } & 1.00\end{array}$ $\begin{array}{llllllll}0117 & \mathrm{O} & 0.92398 & 0.67511 & 0.96542 & 0.00000 & \text { Uiso } & 1.00\end{array}$ $\begin{array}{llllllll}0118 & 0 & 0.11587 & 0.64085 & 0.75014 & 0.00000 & \text { Uiso } & 1.00\end{array}$ $\begin{array}{llllllllll}0119 & 0 & 0.28790 & 0.53639 & 0.71317 & 0.00000 & \text { Uiso } & 1.00\end{array}$ $\begin{array}{llllllllllll}01120 & 0 & 0.06814 & 0.31559 & 0.71363 & 0.00000 & \text { Uiso } & 1.00\end{array}$ $\begin{array}{lllllllllll}0121 & 0 & 0.88326 & 0.35720 & 0.01265 & 0.00000 & \text { Uiso } & 1.00\end{array}$ $\begin{array}{llllllllll}0121 & 0 & 0.88326 & 0.35720 & 0.01265 & 0.00000 & \text { Uiso } & 1.00 \\ 0122 & 0 & 0.49855 & 0.00685 & 0.76538 & 0.00000 & \text { Uiso } & 1.00\end{array}$ $\begin{array}{lllllllll}0122 & 0 & 0.49855 & 0.00685 & 0.01265 & 0.00000 & \text { Uiso } & 1.00 \\ 0123 & 0 & 0.49322 & 0.00649 & 0.49496 & 0.00000 & \text { Uiso } & 1.00\end{array}$

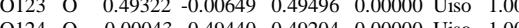
$\begin{array}{llllllllll}0124 & 0 & -0.00043 & 0.49440 & 0.49204 & 0.00000 & \text { Uiso } & 1.00\end{array}$

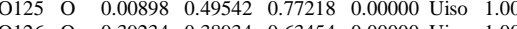
$\begin{array}{lllllllll}0126 & \mathrm{O} & 0.39234 & 0.38934 & 0.63454 & 0.00000 & \text { Uiso } & 1.00\end{array}$ $\begin{array}{llllllll}0127 & \mathrm{O} & 0.21306 & 0.21237 & 0.62985 & 0.00000 & \text { Uiso } & 1.00\end{array}$ $\begin{array}{lllllllll}0128 & \mathrm{O} & 0.59661 & 0.59568 & 0.88195 & 0.00000 & \text { Uiso } & 1.00\end{array}$ $\begin{array}{lllllllll}\mathrm{O} 129 & \mathrm{O} & 0.79324 & 0.79153 & 0.87675 & 0.00000 & \text { Uiso } & 1.00 \\ \mathrm{O} 130 & \mathrm{O} & 0.40920 & 0.19453 & 0.62695 & 0.00000 & \text { Uiso } & 1.00\end{array}$ $\begin{array}{llllllll}\mathrm{O} 131 & \mathrm{O} & 0.59931 & 0.78890 & 0.87161 & 0.00000 & \text { Uiso } & 1.00\end{array}$ 
$\begin{array}{llllllll}0132 & \mathrm{O} & 0.19693 & 0.40765 & 0.62635 & 0.00000 & \text { Uiso } & 1.00\end{array}$ $\begin{array}{lllllllll}0133 & \mathrm{O} & 0.78976 & 0.59846 & 0.87198 & 0.00000 & \text { Uiso } & 1.00\end{array}$ $\begin{array}{lllllllll}0134 & \mathrm{O} & 0.34978 & 0.00655 & 0.87572 & 0.00000 & \text { Uiso } & 1.00\end{array}$ $\begin{array}{llllllll}0135 & \mathrm{O} & 0.59231 & 0.23539 & 0.87659 & 0.00000 & \text { Uiso } & 1.00\end{array}$ $\begin{array}{llllllll}0136 & \mathrm{O} & 0.61946 & 0.97294 & 0.62815 & 0.00000 & \text { Uiso } & 1.00\end{array}$ $\begin{array}{lllllllll}0137 & \mathrm{O} & 0.40087 & 0.73899 & 0.62286 & 0.00000 & \text { Uiso } & 1.00\end{array}$ $\begin{array}{lllllllll}0138 & 0 & 0.00605 & 0.34224 & 0.87675 & 0.00000 & \text { Uiso } & 1.00\end{array}$

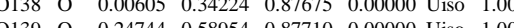

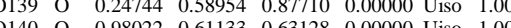
$\begin{array}{lllllllll}0140 & \mathrm{O} & 0.98022 & 0.61133 & 0.63128 & 0.0000 & \text { Uiso } & 1.00 \\ 0141 & \mathrm{O} & 0.73831 & 0.39939 & 0.62479 & 0.0000 & \text { Uiso } & 1.00\end{array}$ $\begin{array}{lllllllllll}0141 & 0 & 0.73831 & 0.39939 & 0.62479 & 0.00000 & \text { Uiso } & 1.00\end{array}$ \begin{tabular}{llllllllll}
0142 & $\mathrm{O}$ & 0.72654 & 0.27404 & 0.75805 & 0.00000 & Uiso & 1.00 \\
\hline 1443 & $\mathrm{O}$ & 0.27710 & 0.71595 & 0.49042 & 0.0000 & Uiso & 1.00
\end{tabular} $\begin{array}{lllllllll}0143 & \mathrm{O} & 0.27710 & 0.71595 & 0.49042 & 0.00000 & \text { Uiso } & 1.00\end{array}$ $\begin{array}{lllllllll}0144 & \mathrm{O} & 0.27588 & 0.72455 & 0.75574 & 0.00000 & \text { Uiso } & 1.00\end{array}$

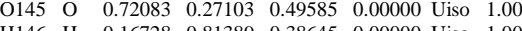
$\begin{array}{lllllllll}\mathrm{H} 146 & \mathrm{H} & 0.16728 & 0.81389 & 0.38645 & 0.00000 & \text { Uiso } & 1.00\end{array}$ $\begin{array}{lllllllll}\mathrm{H} 147 & \mathrm{H} & 0.07476 & 0.86798 & 0.51557 & 0.00000 & \text { Uiso } & 1.00\end{array}$ $\begin{array}{lllllllll}\mathrm{H} 148 & \mathrm{H} & 0.95072 & 0.93441 & 0.50654 & 0.00000 & \text { Uiso } & 1.00\end{array}$ $\begin{array}{lllllllll}\mathrm{H} 149 & \mathrm{H} & -0.02169 & 0.80787 & 0.47533 & 0.00000 & \text { Uiso } & 1.00\end{array}$ $\begin{array}{llllllll}\mathrm{H} 150 & \mathrm{H} & 0.92142 & 0.02467 & 0.36499 & 0.00000 & \text { Uiso } & 1.00\end{array}$ $\begin{array}{llllllll}\mathrm{H} 151 & \mathrm{H} & 0.89012 & 0.91182 & 0.32761 & 0.00000 & \text { Uiso } & 1.00\end{array}$ $\begin{array}{lllllllll}\mathrm{H} 152 & \mathrm{H} & 0.97622 & 0.97167 & 0.26364 & 0.00000 & \text { Uiso } & 1.00\end{array}$ $\begin{array}{lllllllll}\mathrm{H} 153 & \mathrm{H} & 0.13896 & 0.88575 & 0.28685 & 0.00000 & \text { Uiso } & 1.00\end{array}$ $\begin{array}{lllllllll}\mathrm{H} 154 & \mathrm{H} & 0.07837 & 0.78318 & 0.30776 & 0.00000 & \text { Uiso } & 1.00\end{array}$ $\begin{array}{llllllll}\mathrm{C} 155 & \mathrm{C} & 0.95210 & 0.95748 & 0.33081 & 0.00000 & \text { Uiso } & 1.00\end{array}$ $\begin{array}{llllllll}\mathrm{C} 156 & \mathrm{C} & 0.02546 & 0.89892 & 0.38415 & 0.00000 & \text { Uiso } & 1.00\end{array}$ $\begin{array}{llllllll}\mathrm{C} 157 & \mathrm{C} & 0.00758 & 0.88264 & 0.47626 & 0.00000 & \text { Uiso } & 1.00\end{array}$ $\begin{array}{lllllllll}\text { C158 } & \mathrm{C} & 0.10987 & 0.84471 & 0.34101 & 0.00000 & \text { Uiso } & 1.00\end{array}$

\section{File $\mathbf{n}^{\circ} 10$}

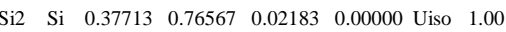
$\begin{array}{lllllllll}\mathrm{Si} 3 & \mathrm{Si} & 0.60853 & 0.00662 & 0.02057 & 0.00000 & \text { Uiso } & 1.00\end{array}$ $\begin{array}{llllllll}\mathrm{Si} 4 & \mathrm{Si} & 0.61971 & 0.23182 & 0.27137 & 0.00000 & \text { Uiso } & 1.00\end{array}$ $\begin{array}{lllllllll}\text { Si5 } & \text { Si } & 0.37651 & 0.99281 & 0.27158 & 0.00000 & \text { Uiso } & 1.00\end{array}$ $\begin{array}{llllllll}\text { Si6 } & \text { Si } & 0.76326 & 0.37763 & 0.22794 & 0.00000 & \text { Uiso } & 1.00\end{array}$ $\begin{array}{llllllll}\mathrm{Si} 7 & \mathrm{Si} & 0.01192 & 0.60690 & 0.22447 & 0.00000 & \text { Uiso } & 1.00\end{array}$ $\begin{array}{llllllll}\text { Si8 } & \text { Si } & 0.23047 & 0.61638 & 0.47839 & 0.00000 & \text { Uiso } & 1.00\end{array}$ $\begin{array}{lllllllll}\text { Si9 } & \text { Si } & 0.98902 & 0.38177 & 0.47683 & 0.00000 & \text { Uiso } & 1.00\end{array}$ $\begin{array}{llllllll}\mathrm{Si} 10 & \mathrm{Si} & 0.61757 & 0.23182 & 0.47876 & 0.00000 & \text { Uiso } & 1.00\end{array}$ $\begin{array}{llllllll}\mathrm{Si11} & \mathrm{Si} & 0.37761 & 0.99039 & 0.47771 & 0.00000 & \text { Uiso } & 1.00\end{array}$ $\begin{array}{llllllll}\text { Si12 } & \text { Si } & 0.37784 & 0.76793 & 0.22795 & 0.00000 & \text { Uiso } & 1.00\end{array}$ $\begin{array}{lllllllll}\text { Si13 } & \text { Si } & 0.60338 & 0.00905 & 0.22653 & 0.00000 & \text { Uiso } & 1.00\end{array}$ $\begin{array}{llllllll}\text { Sil4 } & \text { Si } & 0.23388 & 0.61712 & 0.27062 & 0.00000 & \text { Uiso } & 1.00\end{array}$ $\begin{array}{llllllll}\text { Si15 } & \text { Si } & 0.99092 & 0.38219 & 0.27233 & 0.00000 & \text { Uiso } & 1.00\end{array}$ $\begin{array}{llllllll}\text { Si16 } & \text { Si } & 0.76482 & 0.37687 & 0.02242 & 0.00000 & \text { Uiso } & 1.00\end{array}$ $\begin{array}{lllllllll}\text { Sil7 } & \text { Si } & 0.00782 & 0.60673 & 0.01721 & 0.00000 & \text { Uiso } & 1.00\end{array}$ $\begin{array}{lllllllllll}\text { Si18 } & \text { Si } & 0.46776 & 0.29077 & 0.12376 & 0.00000 & \text { Uis } & 1.00\end{array}$

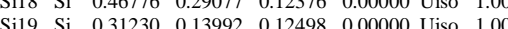
$\begin{array}{lllllllll} & \text { Si20 Si } & 0.53631 & 0.70569 & 0.37562 & 0.00000 & \text { Uiso } & 1.00\end{array}$ $\begin{array}{lllllllll}S 120 & \mathrm{Si} & 0.53631 & 0.70569 & 0.37562 & 0.00000 & \text { Uiso } & 1.00\end{array}$ $\begin{array}{llllll}\text { Si21 } & \text { Si } & 0.68785 & 0.86700 & 0.37553 & 0.00000 \\ \text { Uiso } & 1.00\end{array}$ $\begin{array}{lllllllll}\mathrm{Si22} & \mathrm{Si} & 0.29322 & 0.46651 & 0.12621 & 0.00000 & \text { Uiso } & 1.00\end{array}$ $\begin{array}{llllllll}\mathrm{Si} 23 & \mathrm{Si} & 0.13763 & 0.31546 & 0.12546 & 0.00000 & \text { Uiso } & 1.00\end{array}$ $\begin{array}{lllllllll}\mathrm{Si24} & \mathrm{Si} & 0.69978 & 0.53447 & 0.37361 & 0.00000 & \text { Uiso } & 1.00\end{array}$ $\begin{array}{lllllllll}\mathrm{Si} 25 & \mathrm{Si} & 0.37433 & 0.76624 & 0.52124 & 0.00000 & \text { Uiso } & 1.00\end{array}$ $\begin{array}{llllllll}\mathrm{Si} 26 & \mathrm{Si} & 0.60440 & 0.00738 & 0.52318 & 0.00000 & \text { Uiso } & 1.00\end{array}$ $\begin{array}{lllllllll}\text { Si27 } & \text { Si } & 0.62290 & 0.23017 & 0.77072 & 0.00000 & \text { Uiso } & 1.00\end{array}$ $\begin{array}{llllllll}\mathrm{Si} 28 & \mathrm{Si} & 0.38159 & 0.99234 & 0.77291 & 0.00000 & \text { Uiso } & 1.00\end{array}$ $\begin{array}{llllllll}\text { Si29 } & \text { Si } & 0.76528 & 0.37859 & 0.72580 & 0.00000 & \text { Uiso } & 1.00\end{array}$ $\begin{array}{llllllll}\text { Si30 } & \text { Si } & 0.00727 & 0.60584 & 0.73332 & 0.00000 & \text { Uiso } & 1.00\end{array}$ $\begin{array}{llllllll}\text { Si31 } & \text { Si } & 0.23155 & 0.62048 & 0.97772 & 0.00000 & \text { Uiso } & 1.00\end{array}$ $\begin{array}{lllllllll}\mathrm{Si32} & \mathrm{Si} & 0.99045 & 0.38169 & 0.97567 & 0.00000 & \text { Uiso } & 1.00\end{array}$ $\begin{array}{llllllll}\mathrm{Si33} & \mathrm{Si} & 0.62317 & 0.22898 & 0.97743 & 0.00000 & \text { Uiso } & 1.00\end{array}$ $\begin{array}{llllllll}\text { Si34 } & \text { Si } & 0.38131 & 0.99212 & 0.97738 & 0.00000 & \text { Uiso } & 1.00\end{array}$ $\begin{array}{llllllll}\mathrm{Si} 35 & \mathrm{Si} & 0.37591 & 0.76583 & 0.72752 & 0.00000 & \text { Uiso } & 1.00\end{array}$ $\begin{array}{lllllllll}\text { Si36 Si } & 0.60880 & 0.00734 & 0.72910 & 0.00000 & \text { Uiso } & 1.00\end{array}$ $\begin{array}{llllllll}\mathrm{Si} 36 & \mathrm{Si} & 0.23029 & 0.62152 & 0.77232 & 0.00000 & \text { Uiso } & 1.00\end{array}$ $\begin{array}{llllllllll}\text { Si38 } & \text { Si } & 0.99082 & 0.38043 & 0.77379 & 0.00000 & \text { Uiso } & 1.00\end{array}$ $\begin{array}{llllllllll}\mathrm{Si} 39 & \mathrm{Si} & 0.76241 & 0.37706 & 0.52065 & 0.00000 & \text { Uiso } & 1.00\end{array}$ $\begin{array}{lllllllll} & \mathrm{Si} 40 & \mathrm{Si} & 0.00779 & 0.60670 & 0.52579 & 0.00000 & \text { Uiso } & 1.00\end{array}$ $\begin{array}{llllllllll} & \text { Si41 } & \text { Si } & 0.46634 & 0.29209 & 0.62583 & 0.00000 & \text { Uiso } & 1.00\end{array}$ $\begin{array}{lllllll} & \text { Si41 } & \text { Si } & 0.46634 & 0.29209 & 0.62583 & 0.00000 \\ \text { Uiso } & 1.00\end{array}$ $\begin{array}{llllllll}S i 42 & \text { Si } & 0.31221 & 0.13935 & 0.62493 & 0.00000 & \text { Uiso } & 1.00\end{array}$

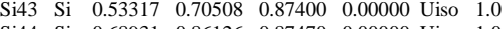
$\begin{array}{lllllllll}\mathrm{Si} 44 & \mathrm{Si} & 0.68931 & 0.86126 & 0.87470 & 0.00000 & \text { Uiso } & 1.00\end{array}$ $\begin{array}{lllllllll}\mathrm{Si} 45 & \mathrm{Si} & 0.29123 & 0.46680 & 0.62369 & 0.00000 & \text { Uiso } & 1.00\end{array}$ $\begin{array}{lllllllll}\mathrm{Si} 46 & \mathrm{Si} & 0.13680 & 0.31404 & 0.62402 & 0.00000 & \text { Uiso } & 1.00\end{array}$ $\begin{array}{llllllllll}\mathrm{Si} 47 & \mathrm{Si} & 0.70466 & 0.53374 & 0.87500 & 0.00000 & \text { Uiso } & 1.00\end{array}$ $\begin{array}{llllllll}\mathrm{Si} 48 & \mathrm{Si} & 0.86100 & 0.68970 & 0.87558 & 0.00000 & \text { Uiso } & 1.00\end{array}$ $\begin{array}{llllllll}\text { Al49 } & \text { Al } & 0.86864 & 0.68551 & 0.37485 & 0.00000 & \text { Uiso } & 1.00\end{array}$ $\begin{array}{lllllllll}050 & \mathrm{O} & 0.53375 & 0.29245 & 0.21285 & 0.00000 & \text { Uiso } & 1.00\end{array}$ $\begin{array}{lllllllll}051 & \mathrm{O} & 0.30962 & 0.07479 & 0.21437 & 0.00000 & \text { Uiso } & 1.00\end{array}$ $\begin{array}{llllllllll}052 & \mathrm{O} & 0.35138 & 0.88353 & 0.50684 & 0.00000 & \text { Uiso } & 1.00\end{array}$ $\begin{array}{lllllllll}053 & \mathrm{O} & 0.46777 & 0.72190 & 0.46255 & 0.00000 & \text { Uiso } & 1.00\end{array}$ $\begin{array}{llllllll}054 & \mathrm{O} & 0.67989 & 0.93390 & 0.46370 & 0.00000 & \text { Uiso } & 1.00\end{array}$ $\begin{array}{llllllll}055 & \mathrm{O} & 0.63440 & 0.11752 & 0.24146 & 0.00000 & \text { Uiso } & 1.00\end{array}$ $\begin{array}{llllllllll}056 & \mathrm{O} & 0.28930 & 0.53510 & 0.03870 & 0.00000 & \text { Uiso } & 1.00\end{array}$ $\begin{array}{llllllll}\mathrm{O} 57 & \mathrm{O} & 0.07175 & 0.31840 & 0.03645 & 0.00000 & \text { Uiso } & 1.00\end{array}$ $\begin{array}{lllllllll}058 & \mathrm{O} & 0.88049 & 0.36553 & 0.24351 & 0.00000 & \text { Uiso } & 1.00\end{array}$ $\begin{array}{lllllllll}059 & 0 & 0.70702 & 0.46574 & 0.28564 & 0.00000 & \text { Uiso } & 1.00\end{array}$ $\begin{array}{llllllllll}060 & 0 & 0.9406 & 0.46574 & 0.28564 & 0.00000 & \text { Uiso } & 1.00\end{array}$ $\begin{array}{llllllllll}060 & 0 & 0.94061 & 0.68306 & 0.28127 & 0.00000 & \text { Uiso } & 1.00\end{array}$ $\begin{array}{llllllll}061 & \mathrm{O} & 0.11636 & 0.63829 & 0.00213 & 0.00000 & \text { Uiso } & 1.00\end{array}$ $\begin{array}{llllllll}062 & 0 & 0.47294 & 0.72681 & 0.28602 & 0.00000 & \text { Uiso } & 1.00\end{array}$ $\begin{array}{lllllllll}063 & 0 & 0.67465 & 0.93258 & 0.28686 & 0.00000 & \text { Uiso } & 1.00\end{array}$ $\begin{array}{lllllllll}064 & \mathrm{O} & 0.64098 & 0.11480 & 0.00658 & 0.00000 & \text { Uiso } & 1.00\end{array}$ $\begin{array}{lllllllll}065 & \mathrm{O} & 0.53604 & 0.28768 & 0.03605 & 0.00000 & \text { Uiso } & 1.00\end{array}$ $\begin{array}{lllllllll}066 & \mathrm{O} & 0.31398 & 0.07039 & 0.03819 & 0.00000 & \text { Uiso } & 1.00\end{array}$ $\begin{array}{lllllllll}067 & \mathrm{O} & 0.35324 & 0.88534 & 0.24094 & 0.00000 & \text { Uiso } & 1.00\end{array}$ $\begin{array}{llllllll}\text { O68 } & \text { O } & 0.70795 & 0.46419 & 0.46076 & 0.00000 & \text { Uiso } & 1.00\end{array}$ $\begin{array}{llllllll}069 & \text { O } & 0.93461 & 0.68361 & 0.47174 & 0.00000 & \text { Uiso } & 1.00\end{array}$

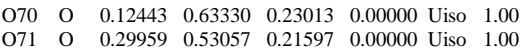


$\begin{array}{llllllll}\mathrm{O} 72 & \mathrm{O} & 0.06851 & 0.31236 & 0.21212 & 0.00000 & \text { Uiso } & 1.00\end{array}$ $\begin{array}{lllllllll}073 & \mathrm{O} & 0.87951 & 0.36152 & 0.50474 & 0.00000 & \text { Uiso } & 1.00\end{array}$ $\begin{array}{llllllllll}074 & \mathrm{O} & 0.49165 & 0.00344 & 0.25707 & 0.00000 & \text { Uiso } & 1.00\end{array}$ $\begin{array}{llllllll}075 & \mathrm{O} & 0.49616 & 0.00517 & 0.98926 & 0.00000 & \text { Uiso } & 1.00\end{array}$ $\begin{array}{lllllllll}076 & \mathrm{O} & 0.00422 & 0.49687 & 0.97915 & 0.00000 & \text { Uiso } & 1.00\end{array}$ $\begin{array}{llllllllll}077 & \mathrm{O} & 0.00863 & 0.49546 & 0.26051 & 0.00000 & \text { Uiso } & 1.00\end{array}$ $\begin{array}{lllllllll}078 & 0 & 0.39020 & 0.38815 & 0.12049 & 0.00000 & \text { Uiso } & 1.00\end{array}$ $\begin{array}{lllllllll}078 & 0 & 0.39020 & 0.38815 & 0.12049 & 0.00000 & \text { Uiso } & 1.00\end{array}$ $\begin{array}{lllllllll}078 & 0 & 0.21527 & 0.21779 & 0.12271 & 0.00000 & \text { Uiso } & 1.00 \\ 080 & 0 & 0.59014 & 0.59516 & 0.37449 & 0.00000 & \text { Uis } & 1.00\end{array}$ $\begin{array}{lllllllll}080 & \mathrm{O} & 0.59014 & 0.59516 & 0.37449 & 0.00000 & \text { Uiso } & 1.00\end{array}$ $\begin{array}{llllllllll}081 & 0 & 0.80388 & 0.81303 & 0.3744 & 0.00000 & \text { Uiso } & 1.00\end{array}$ $\begin{array}{lllllllll}082 & \mathrm{O} & 0.41161 & 0.19327 & 0.12572 & 0.00000 & \text { Uiso } & 1.00\end{array}$ $\begin{array}{llllllllll}083 & 0 & 0.61717 & 0.78316 & 0.37977 & 0.0000 & \text { Uiso } & 1.00\end{array}$ $\begin{array}{llllllll}084 & \mathrm{O} & 0.19457 & 0.41232 & 0.12946 & 0.00000 & \text { Uiso } & 1.00\end{array}$ $\begin{array}{llllllll}\mathrm{O} 85 & \mathrm{O} & 0.78141 & 0.60717 & 0.37348 & 0.00000 & \text { Uiso } & 1.00\end{array}$ $\begin{array}{lllllllll}086 & \mathrm{O} & 0.34924 & 0.01052 & 0.37491 & 0.00000 & \text { Uiso } & 1.00\end{array}$ $\begin{array}{llllllll}\mathrm{O} 87 & \mathrm{O} & 0.58797 & 0.24030 & 0.37481 & 0.00000 & \text { Uiso } & 1.00\end{array}$ $\begin{array}{llllllll}088 & \mathrm{O} & 0.61962 & 0.97643 & 0.12433 & 0.00000 & \text { Uiso } & 1.00\end{array}$ $\begin{array}{llllllll}\mathrm{O} 89 & \mathrm{O} & 0.40316 & 0.74297 & 0.12510 & 0.00000 & \text { Uiso } & 1.00\end{array}$ $\begin{array}{llllllll}090 & \mathrm{O} & 0.00893 & 0.34934 & 0.37464 & 0.00000 & \text { Uiso } & 1.00\end{array}$ $\begin{array}{llllllll}091 & \mathrm{O} & 0.23236 & 0.58663 & 0.37438 & 0.00000 & \text { Uiso } & 1.00\end{array}$ $\begin{array}{lllllllll}092 & \mathrm{O} & 0.97944 & 0.61137 & 0.12077 & 0.00000 & \text { Uiso } & 1.00\end{array}$ $\begin{array}{lllllllll}093 & \mathrm{O} & 0.73800 & 0.40154 & 0.12484 & 0.00000 & \text { Uiso } & 1.00\end{array}$ $\begin{array}{llllllll}094 & \mathrm{O} & 0.72341 & 0.27456 & 0.25569 & 0.00000 & \text { Uiso } & 1.00\end{array}$ $\begin{array}{lllllllll}095 & \mathrm{O} & 0.27607 & 0.72310 & 0.99512 & 0.00000 & \text { Uiso } & 1.00\end{array}$ $\begin{array}{lllllllll}096 & \mathrm{O} & 0.28243 & 0.71866 & 0.26002 & 0.00000 & \text { Uiso } & 1.00\end{array}$ $\begin{array}{lllllllll}097 & \mathrm{O} & 0.72515 & 0.27465 & 0.99364 & 0.00000 & \text { Uiso } & 1.00\end{array}$ $\begin{array}{llllllll}098 & 0 & 0.53547 & 0.28928 & 0.71287 & 0.00000 & \text { Uiso } & 1.00\end{array}$ $\begin{array}{llllllllll}099 & 0 & 0.53547 & 0.28928 & 0.71287 & 0.00000 & \text { Uiso } & 1.00\end{array}$ $\begin{array}{llllllllll}0100 & 0 & 0.31389 & 0.07084 & 0.71242 & 0.0000 & \text { Uiso } & 1.00\end{array}$

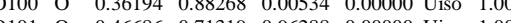
$\begin{array}{llllllllll}0101 & \mathrm{O} & 0.46686 & 0.71319 & 0.96288 & 0.00000 & \text { Uiso } & 1.00\end{array}$ $\begin{array}{llllllllll}0102 & 0 & 0.68035 & 0.92801 & 0.96377 & 0.00000 & \text { Uiso } & 1.00 \\ 0103 & 0 & 0.64066 & 0.1618 & 0.74077 & 0.00000 & \text { Uiso } & 1.00\end{array}$ $\begin{array}{lllllllll}0103 & \mathrm{O} & 0.64066 & 0.11618 & 0.74077 & 0.00000 & \text { Uiso } & 1.00\end{array}$ $\begin{array}{llllllllll}0104 & \mathrm{O} & 0.29589 & 0.52981 & 0.53321 & 0.00000 & \text { Uiso } & 1.00\end{array}$ $\begin{array}{lllllllll}0105 & \mathrm{O} & 0.06731 & 0.31297 & 0.53741 & 0.00000 & \text { Uiso } & 1.00\end{array}$ $\begin{array}{lllllllll}0106 & \mathrm{O} & 0.88275 & 0.36431 & 0.73874 & 0.00000 & \text { Uiso } & 1.00\end{array}$ $\begin{array}{llllllll}0107 & \mathrm{O} & 0.71367 & 0.46765 & 0.78604 & 0.00000 & \text { Uiso } & 1.00\end{array}$ $\begin{array}{llllllll}0108 & 0 & 0.92773 & 0.68047 & 0.78682 & 0.00000 & \text { Uiso } & 1.00\end{array}$ $\begin{array}{lllllllll}0109 & \mathrm{O} & 0.12033 & 0.63197 & 0.51691 & 0.00000 & \text { Uiso } & 1.00\end{array}$ $\begin{array}{llllllll}0110 & \mathrm{O} & 0.46553 & 0.71208 & 0.78597 & 0.00000 & \text { Uiso } & 1.00\end{array}$ $\begin{array}{llllllllll}0111 & \mathrm{O} & 0.68131 & 0.93054 & 0.78714 & 0.00000 & \text { Uiso } & 1.00\end{array}$ $\begin{array}{llllllll}0112 & \mathrm{O} & 0.63039 & 0.11779 & 0.50921 & 0.00000 & \text { Uiso } & 1.00\end{array}$ $\begin{array}{llllllll}0113 & \mathrm{O} & 0.53106 & 0.29351 & 0.53594 & 0.00000 & \text { Uiso } & 1.00\end{array}$

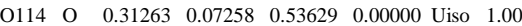
$\begin{array}{llllllll}0115 & \mathrm{O} & 0.36265 & 0.88287 & 0.74435 & 0.00000 & \text { Uiso } & 1.00\end{array}$ $\begin{array}{lllllllll}0116 & \mathrm{O} & 0.71132 & 0.46527 & 0.96246 & 0.00000 & \text { Uiso } & 1.00\end{array}$ $\begin{array}{llllllll}0117 & 0 & 0.92930 & 0.68255 & 0.96353 & 0.00000 & \text { Uiso } & 1.00\end{array}$ $\begin{array}{llllllll}0118 & 0 & 0.11488 & 0.63953 & 0.74885 & 0.00000 & \text { Uiso } & 1.00\end{array}$ $\begin{array}{lllllllll}0119 & 0 & 0.28721 & 0.53663 & 0.71034 & 0.00000 & \text { Uiso } & 1.00\end{array}$ $\begin{array}{lllllllllll}0119 & \mathrm{O} & 0.28721 & 0.53663 & 0.71034 & 0.0000 & \text { Uiso } & 1.00 \\ 0120 & 0 & 0.07163 & 0.31570 & 0.71346 & 0.00000 & \text { Uiso } & 1.00\end{array}$

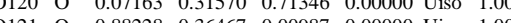
$\begin{array}{llllllllll}0121 & 0 & 0.88228 & 0.36467 & 0.0087 & 0.00000 & \text { Uiso } & 1.00\end{array}$ $\begin{array}{lllllllllll}0122 & \mathrm{O} & 0.49650 & 0.00584 & 0.76111 & 0.00000 & \text { Uiso } & 1.00 \\ 0123 & \mathrm{O} & 0.49383 & 0.99690 & 0.49112 & 0.00000 & \text { Uiso } & 1.00\end{array}$ $\begin{array}{llllllllll}0123 & \mathrm{O} & 0.49383 & 0.99690 & 0.49112 & 0.00000 & \text { Uiso } & 1.00 \\ 0124 & \mathrm{O} & 0.00320 & 0.49569 & 0.48919 & 0.00000 & \text { Uis } & 1.00\end{array}$ $\begin{array}{lllllllll}0124 & \mathrm{O} & 0.00320 & 0.4969 & 0.48919 & 0.00000 & \text { Uiso } & 1.00 \\ 0125 & \mathrm{O} & 0.0596 & 0.4530 & 0.77033 & 0.0000 & \text { Uiso } & 1.00\end{array}$ $\begin{array}{lllllllll}0125 & 0 & 0.00596 & 0.45530 & 0.77033 & 0.00000 & \text { Uiso } & 1.00\end{array}$ $\begin{array}{lllllllll}0126 & \mathrm{O} & 0.38912 & 0.38967 & 0.62963 & 0.00000 & \text { Uiso } & 1.00\end{array}$ $\begin{array}{llllllll}0127 & \mathrm{O} & 0.21386 & 0.21569 & 0.62478 & 0.00000 & \text { Uiso } & 1.00\end{array}$ $\begin{array}{lllllllll}0128 & \mathrm{O} & 0.59931 & 0.59985 & 0.87430 & 0.00000 & \text { Uiso } & 1.00\end{array}$ $\begin{array}{llllllll}0129 & \mathrm{O} & 0.79459 & 0.79523 & 0.87475 & 0.00000 & \text { Uiso } & 1.00\end{array}$ $\begin{array}{llllllll}0130 & \mathrm{O} & 0.41017 & 0.19464 & 0.62471 & 0.00000 & \text { Uiso } & 1.00\end{array}$ $\begin{array}{lllllllll}0131 & \mathrm{O} & 0.60060 & 0.79374 & 0.87296 & 0.00000 & \text { Uiso } & 1.00\end{array}$ $\begin{array}{llllllll}0132 & \mathrm{O} & 0.19369 & 0.41091 & 0.62170 & 0.00000 & \text { Uiso } & 1.00\end{array}$ $\begin{array}{llllllllll}0133 & \mathrm{O} & 0.79251 & 0.60181 & 0.87731 & 0.00000 & \text { Uiso } & 1.00\end{array}$

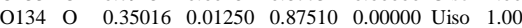
$\begin{array}{lllllllll}0135 & \mathrm{O} & 0.59131 & 0.23868 & 0.87414 & 0.00000 & \text { Uiso } & 1.00\end{array}$ $\begin{array}{llllllll}0136 & \mathrm{O} & 0.61953 & 0.97528 & 0.62571 & 0.00000 & \text { Uiso } & 1.00\end{array}$ $\begin{array}{lllllllll}0137 & 0 & 0.40080 & 0.74284 & 0.62416 & 0.00000 & \text { Uiso } & 1.00\end{array}$ $\begin{array}{llllllll}0138 & \mathrm{O} & 0.00514 & 0.34248 & 0.87492 & 0.00000 & \text { Uiso } & 1.00\end{array}$ $\begin{array}{llllllllll}0139 & 0 & 0.24678 & 0.58786 & 0.87483 & 0.00000 & \text { Uiso } & 1.00\end{array}$ $\begin{array}{llllllllll}0140 & 0 & 0.97859 & 0.61041 & 0.63002 & 0.00000 & \text { Uiso } & 1.00\end{array}$ $\begin{array}{llllllllllll}0141 & 0 & 0.978596 & 0.61041 & 0.63002 & 0.00000 & \text { Uiso } & 1.00\end{array}$ $\begin{array}{llllllllllll}0142 & \mathrm{O} & 0.7385 & 0.72431 & 0.27684 & 0.75438 & 0.00000 & \text { Uiso } & 1.00\end{array}$ \begin{tabular}{lllllllll}
\hline 142 & $\mathrm{O}$ & 0.72431 & 0.27684 & 0.75438 & 0.00000 & Uiso & 1.00
\end{tabular} $\begin{array}{lllllllll}0143 & \mathrm{O} & 0.27734 & 0.71856 & 0.49134 & 0.00000 & \text { Uiso } & 1.00\end{array}$ $\begin{array}{lllllllll}0144 & \mathrm{O} & 0.27450 & 0.72438 & 0.75508 & 0.00000 & \text { Uiso } & 1.00 \\ 0145 & \mathrm{O} & 0.72118 & 0.27388 & 0.49607 & 0.00000 & \text { Uso } & 1.00\end{array}$ $\begin{array}{lllllllll}0145 & \mathrm{O} & 0.72118 & 0.27388 & 0.49607 & 0.00000 & \text { Uiso } & 1.00\end{array}$ $\begin{array}{lllllllllll}\mathrm{H} 146 & \mathrm{H} & 0.85214 & 0.86197 & 0.35368 & 0.00000 & \text { Uiso } & 1.00\end{array}$ $\begin{array}{llllllll}\mathrm{H} 147 & \mathrm{H} & 0.08811 & 0.94954 & 0.45722 & 0.00000 & \text { Uiso } & 1.00\end{array}$ $\begin{array}{llllllll}\mathrm{H} 148 & \mathrm{H} & 0.00512 & 0.86931 & 0.49322 & 0.00000 & \text { Uiso } & 1.00\end{array}$ $\begin{array}{llllllll}\mathrm{H} 149 & \mathrm{H} & 0.07179 & 0.84353 & 0.39325 & 0.00000 & \text { Uiso } & 1.00\end{array}$ $\begin{array}{llllllll}\mathrm{H} 150 & \mathrm{H} & 0.94951 & 0.09833 & 0.45353 & 0.00000 & \text { Uiso } & 1.00\end{array}$ $\begin{array}{lllllllll}H 151 & \mathrm{H} & 0.86761 & 0.02037 & 0.49519 & 0.00000 & \text { Uiso } & 1.00\end{array}$ $\begin{array}{lllllllll}\mathrm{H} 152 & \mathrm{H} & 0.84015 & 0.08498 & 0.39396 & 0.00000 & \text { Uiso } & 1.00\end{array}$ $\begin{array}{lllllllll}\mathrm{H} 153 & \mathrm{H} & 0.97304 & 0.88104 & 0.26454 & 0.00000 & \text { Uiso } & 1.00\end{array}$ $\begin{array}{llllllll}\mathrm{H} 154 & \mathrm{H} & 0.87411 & 0.98667 & 0.26445 & 0.00000 & \text { Uiso } & 1.00\end{array}$ $\begin{array}{lllllllll}\mathrm{C} 155 & \mathrm{C} & 0.89900 & 0.04598 & 0.43376 & 0.00000 & \text { Uiso } & 1.00\end{array}$ $\begin{array}{lllllllll}\mathrm{C} 156 & \mathrm{C} & 0.95384 & 0.96212 & 0.38451 & 0.00000 & \text { Uiso } & 1.00\end{array}$ $\begin{array}{llllllll}\mathrm{C} 157 & \mathrm{C} & 0.03423 & 0.90227 & 0.43406 & 0.00000 & \text { Uiso } & 1.00\end{array}$

\section{File $n^{\circ} 11$}

$\begin{array}{llllllll}\mathrm{H} 2 & \mathrm{H} & 0.46876 & 0.44985 & 0.76074 & 0.00000 & \text { Uiso } & 1.00\end{array}$ $\begin{array}{llllllll}\mathrm{H} 3 & \mathrm{H} & 0.45149 & 0.29296 & 0.88021 & 0.00000 & \text { Uiso } & 1.00\end{array}$ $\begin{array}{llllllll}\mathrm{H} 4 & \mathrm{H} & 0.46784 & 0.62771 & 0.70636 & 0.00000 & \text { Uiso } & 1.00\end{array}$ $\begin{array}{llllllll}\mathrm{H} 5 & \mathrm{H} & 0.43215 & 0.51104 & 0.41816 & 0.00000 & \text { Uiso } & 1.00\end{array}$ $\begin{array}{lllllllll}\mathrm{H} 6 & \mathrm{H} & 0.43802 & 0.21671 & 0.43750 & 0.00000 & \text { Uiso } & 1.00\end{array}$ $\begin{array}{llllllll}\mathrm{H} 7 & \mathrm{H} & 0.45680 & 0.94704 & 0.40636 & 0.00000 & \text { Uiso } & 1.00\end{array}$ $\begin{array}{llllllll}\mathrm{H} 8 & \mathrm{H} & 0.44601 & 0.77295 & 0.01946 & 0.00000 & \text { Uiso } & 1.00\end{array}$ $\begin{array}{lllllllll}\mathrm{H} 9 & \mathrm{H} & 0.43378 & 0.04830 & 0.30083 & 0.00000 & \text { Uiso } & 1.00\end{array}$

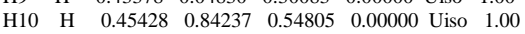


$\begin{array}{llllllll}\mathrm{H} 11 & \mathrm{H} & 0.45323 & 0.75218 & 0.61816 & 0.00000 & \text { Uiso } & 1.00\end{array}$ $\begin{array}{llllllllll}\mathrm{H} 12 & \mathrm{H} & 0.46681 & 0.62248 & 0.94418 & 0.00000 & \text { Uiso } & 1.00\end{array}$ $\begin{array}{lllllllll}\mathrm{H} 13 & \mathrm{H} & 0.45510 & 0.63074 & 0.09838 & 0.00000 & \text { Uiso } & 1.00\end{array}$ $\begin{array}{lllllllll}\mathrm{H} 14 & \mathrm{H} & 0.48169 & 0.55575 & 0.05496 & 0.00000 & \text { Uiso } & 1.00\end{array}$ $\begin{array}{llllllllll}\mathrm{H} 15 & \mathrm{H} & 0.52607 & 0.64852 & 0.89677 & 0.00000 & \text { Uiso } & 1.00\end{array}$ $\begin{array}{llllllll}\mathrm{H} 16 & \mathrm{H} & 0.53692 & 0.58671 & 0.02031 & 0.00000 & \text { Uiso } & 1.00\end{array}$ $\begin{array}{lllllllll}\mathrm{H} 17 & \mathrm{H} & 0.55063 & 0.68718 & 0.01213 & 0.00000 & \text { Uiso } & 1.00\end{array}$ $\begin{array}{llllllll}\mathrm{H} 17 & \mathrm{H} & 0.55063 & 0.68718 & 0.01213 & 0.00000 & \text { Uiso } & 1.00\end{array}$ $\begin{array}{llllllll}\mathrm{H} 18 & \mathrm{H} & 0.52763 & 0.7118 & 0.22641 & 0.00000 \\ \mathrm{H} 19 & \mathrm{H} & 0.51713 & 0.60883 & 0.21988 & 0.00000 & \text { Uiso } & 1.00\end{array}$

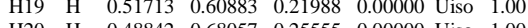
$\begin{array}{llllllll}\mathrm{H} 20 & \mathrm{H} & 0.48842 & 0.68057 & 0.25555 & 0.00000 & \text { Uiso } & 1.00\end{array}$

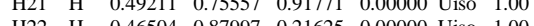
$\begin{array}{lllllllll}\mathrm{H} 22 & \mathrm{H} & 0.46504 & 0.87997 & 0.21625 & 0.00000 & \text { Uiso } & 1.00 \\ \mathrm{H} 23 & \mathrm{H} & 0.50553 & 0.89754 & 0.21501 & 0.0000 & \text { Uis } & 1.00\end{array}$ $\begin{array}{llllllllll}\mathrm{H} 23 & \mathrm{H} & 0.50553 & 0.89754 & 0.21501 & 0.00000 & \text { Uiso } & 1.00\end{array}$

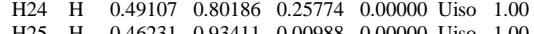
$\begin{array}{llllllll}\mathrm{H} 25 & \mathrm{H} & 0.46231 & 0.93411 & 0.00988 & 0.00000 & \text { Uiso } & 1.00 \\ \mathrm{H} 26 & \mathrm{H} & 0.48135 & 0.88299 & 0.88882 & 0.00000 & \text { Uiso } & 1.00\end{array}$ $\begin{array}{llllllll}\mathrm{H} 26 & \mathrm{H} & 0.48135 & 0.88299 & 0.88882 & 0.00000 & \text { Uiso } & 1.00 \\ \mathrm{H} 27 & \mathrm{H} & 0.50286 & 0.94276 & 0.99200 & 0.00000 & \text { Uiso } & 1.00\end{array}$ $\begin{array}{llllllll}\mathrm{Al} 28 & \mathrm{Al} & 0.35127 & 0.05697 & 0.89405 & 0.00000 & \text { Uiso } & 1.00\end{array}$

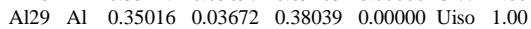
$\begin{array}{llllllll}\mathrm{Al} 30 & \mathrm{Al} & 0.34427 & 0.56633 & 0.89986 & 0.00000 & \text { Uiso } & 1.00\end{array}$ $\begin{array}{llllllll}\mathrm{Al} 31 & \mathrm{Al} & 0.34388 & 0.53285 & 0.38242 & 0.00000 & \text { Uiso } & 1.00\end{array}$ $\begin{array}{llllllll}\mathrm{Al} 32 & \mathrm{Al} & 0.34430 & 0.87459 & 0.89718 & 0.00000 & \text { Uiso } & 1.00\end{array}$ $\begin{array}{llllllll}\text { Al33 } & \text { Al } & 0.34999 & 0.85096 & 0.41039 & 0.00000 & \text { Uiso } & 1.00\end{array}$ $\begin{array}{llllllll}\mathrm{Al} 34 & \mathrm{Al} & 0.35322 & 0.40171 & 0.88391 & 0.00000 & \text { Uiso } & 1.00\end{array}$ $\begin{array}{llllllll}\mathrm{Al} 35 & \mathrm{Al} & 0.32880 & 0.38674 & 0.51910 & 0.00000 & \text { Uiso } & 1.00\end{array}$ $\begin{array}{lllllllll}\mathrm{Al} 36 & \mathrm{Al} & 0.30475 & 0.80226 & 0.26181 & 0.00000 & \text { Uiso } & 1.00\end{array}$ $\begin{array}{llllllllll}A 137 & \text { Al } & 0.30074 & 0.80731 & 0.76480 & 0.00000 & \text { Uiso } & 1.00\end{array}$ $\begin{array}{llllllll}\text { Al38 } & \text { Al } & 0.30453 & 0.30669 & 0.26025 & 0.00000 & \text { Uiso } & 1.00\end{array}$ \begin{tabular}{llllllll}
\hline Al38 Al & 0.30453 & 0.30669 & 0.26025 & 0.00000 & Uiso & 1.00
\end{tabular}

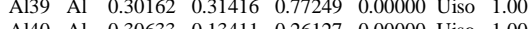
$\begin{array}{llllllll}\text { Al40 Al } & 0.30633 & 0.1341 & 0.77249 & 0.00000 & \text { Uiso } & 1.00\end{array}$ $\begin{array}{llllllllll}\mathrm{Al} 41 & \mathrm{Al} & 0.30316 & 0.13612 & 0.75846 & 0.00000 & \text { Uns } & 1.00\end{array}$ $\begin{array}{lllllllll}\text { Al42 } & \text { Al } & 0.29754 & 0.63493 & 0.26782 & 0.00000 & \text { Uiso } & 1.00\end{array}$ $\begin{array}{lllllllll}\text { Al43 Al } & 0.2992 & 0.64130 & 0.76050 & 0.00000 & \text { Uiso } & 1.00\end{array}$ $\begin{array}{llllllll}\mathrm{Al} 44 & \mathrm{Al} & 0.21191 & 0.97110 & 0.25381 & 0.00000 & \text { Uiso } & 1.00\end{array}$ $\begin{array}{llllllll}\mathrm{A} 145 & \mathrm{Al} & 0.21176 & 0.97245 & 0.75450 & 0.00000 & \text { Uiso } & 1.00\end{array}$ $\begin{array}{llllllll}\mathrm{Al} 46 & \mathrm{Al} & 0.20935 & 0.47056 & 0.25063 & 0.00000 & \text { Uiso } & 1.00\end{array}$ $\begin{array}{llllllll}\mathrm{Al} 47 & \mathrm{Al} & 0.21015 & 0.47068 & 0.75172 & 0.00000 & \text { Uiso } & 1.00\end{array}$ $\begin{array}{llllllll}\mathrm{Al} 48 & \mathrm{Al} & 0.32931 & 0.97024 & 0.12991 & 0.00000 & \text { Uiso } & 1.00\end{array}$ $\begin{array}{llllllll}\mathrm{A} 149 & \mathrm{Al} & 0.32690 & 0.96657 & 0.62732 & 0.00000 & \text { Uiso } & 1.00\end{array}$

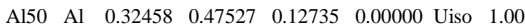
$\begin{array}{llllllll}\mathrm{A} 151 & \mathrm{Al} & 0.33979 & 0.53546 & 0.65873 & 0.00000 & \text { Uiso } & 1.00\end{array}$ $\begin{array}{llllllll}\mathrm{Al} 52 & \mathrm{Al} & 0.28081 & 0.22013 & 0.02363 & 0.00000 & \text { Uiso } & 1.00\end{array}$ $\begin{array}{llllllll}\mathrm{Al} 53 & \mathrm{Al} & 0.28186 & 0.22140 & 0.51692 & 0.00000 & \text { Uiso } & 1.00\end{array}$ $\begin{array}{llllllll}\mathrm{Al} 54 & \mathrm{Al} & 0.28101 & 0.71859 & 0.00729 & 0.00000 & \text { Uiso } & 1.00\end{array}$ $\begin{array}{llllllll}\mathrm{Al} 55 & \mathrm{Al} & 0.28101 & 0.72099 & 0.50702 & 0.00000 & \text { Uiso } & 1.00\end{array}$ $\begin{array}{llllllll}\mathrm{A} 156 & \mathrm{Al} & 0.34591 & 0.23237 & 0.91679 & 0.00000 & \text { Uiso } & 1.00\end{array}$ $\begin{array}{llllllll}\mathrm{A} 157 & \mathrm{Al} & 0.35637 & 0.24065 & 0.65621 & 0.00000 & \text { Uiso } & 1.00\end{array}$ $\begin{array}{lllllllll}4158 & \mathrm{Al} & 0.35266 & 0.73115 & 0.12544 & 0.00000 & \text { Uiso } & 1.00\end{array}$ $\begin{array}{llllllll}\text { Al58 Al } & 0.35266 & 0.73115 & 0.12544 & 0.00000 & \text { Uiso } & 1.00 \\ \text { Al59 } & \text { Al } & 0.35167 & 0.73154 & 0.66594 & 0.00000 & \text { Uiso } & 1.00\end{array}$ $\begin{array}{llllllll}\text { Al59 Al } & 0.35167 & 0.73154 & 0.66594 & 0.00000 & \text { Uiso } & 1.00\end{array}$

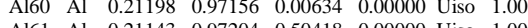
$\begin{array}{llllllll}\text { Al61 Al } & 0.2143 & 0.97204 & 0.50418 & 0.00000 & \text { Uiso } & 1.00\end{array}$

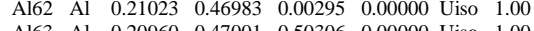
$\begin{array}{llllllll}\text { Al63 Al } & 0.20960 & 0.47001 & 0.50306 & 0.00000 & \text { Uiso } & 1.00\end{array}$ $\begin{array}{llllllll}\text { Al64 Al } & 0.28284 & 0.96110 & 0.37883 & 0.00000 & \text { Uiso } & 1.00\end{array}$ $\begin{array}{llllllll}\mathrm{Al65} & \mathrm{Al} & 0.28189 & 0.97621 & 0.87927 & 0.00000 & \text { Uiso } & 1.00\end{array}$ $\begin{array}{llllllll}\text { Al66 Al } & 0.27839 & 0.46744 & 0.37677 & 0.00000 & \text { Uiso } & 1.00\end{array}$ $\begin{array}{llllllll}\mathrm{Al} 67 & \mathrm{Al} & 0.28082 & 0.47475 & 0.87728 & 0.00000 & \text { Uiso } & 1.00\end{array}$ $\begin{array}{llllllll}\mathrm{Al} 68 & \mathrm{Al} & 0.23655 & 0.21902 & 0.25537 & 0.00000 & \text { Uiso } & 1.00\end{array}$ $\begin{array}{llllllll}\mathrm{Al} 69 & \mathrm{Al} & 0.23539 & 0.22002 & 0.75396 & 0.00000 & \text { Uiso } & 1.00\end{array}$

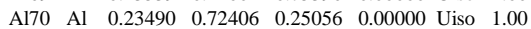
$\begin{array}{llllllll}\text { Al71 } & \text { Al } & 0.23417 & 0.72320 & 0.74999 & 0.00000 & \text { Uiso } & 1.00\end{array}$ $\begin{array}{llllllll}\mathrm{Al} 72 & \mathrm{Al} & 0.26186 & 0.05810 & 0.13329 & 0.00000 & \text { Uiso } & 1.00\end{array}$ $\begin{array}{llllllll}\text { Al73 } & \text { Al } & 0.26127 & 0.05827 & 0.62615 & 0.00000 & \text { Uiso } & 1.00\end{array}$ $\begin{array}{llllllll}\text { Al74 } & \text { Al } & 0.25841 & 0.55813 & 0.12481 & 0.00000 & \text { Uiso } & 1.00\end{array}$ $\begin{array}{llllllll}\text { Al75 } & \text { Al } & 0.25901 & 0.55861 & 0.62456 & 0.00000 & \text { Uiso } & 1.00\end{array}$ $\begin{array}{llllllll}\text { Al76 } & \text { Al } & 0.26183 & 0.88315 & 0.12666 & 0.00000 & \text { Uiso } & 1.00\end{array}$ $\begin{array}{llllllll}\text { Al77 } & \text { Al } & 0.25962 & 0.88322 & 0.62709 & 0.00000 & \text { Uiso } & 1.00\end{array}$ $\begin{array}{lllllllll}\text { Al78 } & \text { Al } & 0.25952 & 0.38232 & 0.13199 & 0.00000 & \text { Uiso } & 1.00 \\ \text { Al79 } & \text { Al } & 0.25925 & 0.38332 & 0.63006 & 0.00000 & \text { Uiso } & 1.00\end{array}$ $\begin{array}{lllllllll}\text { Al7 Al } & 0.25925 & 0.38332 & 0.63006 & 0.00000 & \text { Uiso } & 1.00\end{array}$

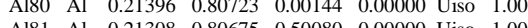
$\begin{array}{lllllll}\text { Al81 Al } & 0.21308 & 0.80675 & 0.50080 & 0.00000 & \text { Uiso } & 1.00\end{array}$ Al82 Al 0.212990 .305930 .500800 .00000 Uiso 1.00 $\begin{array}{llllllll}\text { Al83 Al } & 0.21299 & 0.30600 & 0.50084 & 0.00000 & \text { Uiso } & 1.00\end{array}$ $\begin{array}{lllllll}\text { Al84 Al } & 0.21369 & 0.13512 & 0.00596 & 0.00000 & \text { Uiso } & 1.00\end{array}$ $\begin{array}{lllllllll}\text { Al85 } & \text { Al } & 0.21368 & 0.13572 & 0.50169 & 0.00000 & \text { Uiso } & 1.00\end{array}$ $\begin{array}{llllllll}\mathrm{Al} 86 & \mathrm{Al} & 0.21270 & 0.63517 & 0.99915 & 0.00000 & \text { Uiso } & 1.00\end{array}$ $\begin{array}{llllllll}\mathrm{Al} 87 & \mathrm{Al} & 0.21272 & 0.63523 & 0.49872 & 0.00000 & \text { Uiso } & 1.00\end{array}$ $\begin{array}{llllllll}\text { Al88 } & \mathrm{Al} & 0.40261 & 0.15166 & 0.78370 & 0.00000 & \text { Uiso } & 1.00\end{array}$ $\begin{array}{llllllll}\text { Al89 } & \text { Al } & 0.35104 & 0.22617 & 0.39951 & 0.00000 & \text { Uiso } & 1.00\end{array}$ $\begin{array}{llllllll}\mathrm{Al} 90 & \mathrm{Al} & 0.39731 & 0.62849 & 0.01946 & 0.00000 & \text { Uiso } & 1.00\end{array}$ $\begin{array}{lllllllll}\mathrm{Al} 91 & \mathrm{Al} & 0.40237 & 0.76534 & 0.50808 & 0.00000 & \text { Uiso } & 1.00\end{array}$ $\begin{array}{llllllll}092 & \mathrm{O} & 0.30862 & 0.87737 & 0.38125 & 0.00000 & \text { Uiso } & 1.00\end{array}$ $\begin{array}{lllllllll}093 & \mathrm{O} & 0.30392 & 0.88794 & 0.88223 & 0.00000 & \text { Uiso } & 1.00\end{array}$ $\begin{array}{lllllllll}094 & \mathrm{O} & 0.30654 & 0.38908 & 0.38504 & 0.00000 & \text { Uiso } & 1.00\end{array}$ $\begin{array}{llllllll}095 & \mathrm{O} & 0.30650 & 0.39350 & 0.88053 & 0.00000 & \text { Uiso } & 1.00\end{array}$ $\begin{array}{llllllll}\mathrm{O} 96 & \mathrm{O} & 0.30842 & 0.04362 & 0.36946 & 0.00000 & \text { Uiso } & 1.00\end{array}$ $\begin{array}{llllllll}\mathrm{O} 97 & \mathrm{O} & 0.30942 & 0.05812 & 0.87192 & 0.00000 & \text { Uiso } & 1.00\end{array}$ $\begin{array}{llllllll}098 & \mathrm{O} & 0.30385 & 0.55061 & 0.37163 & 0.00000 & \text { Uiso } & 1.00\end{array}$ $\begin{array}{llllllll}\text { O99 } & \text { O } & 0.30298 & 0.56353 & 0.87893 & 0.00000 & \text { Uiso } & 1.00\end{array}$ \begin{tabular}{llllllllll}
0100 & $\mathrm{O}$ & 0.30737 & 0.22367 & 0.38766 & 0.00000 & Uiso & 1.00 \\
\hline 0101 & $\mathrm{O}$ & 0.30455 & 0.21997 & 0.88853 & 0.00000 & Uiso & 1.00
\end{tabular}

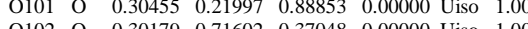
$\begin{array}{llllllllllll}0102 & \mathrm{O} & 0.30179 & 0.71602 & 0.37048 & 0.00000 & \text { Uiso } & 1.00\end{array}$ \begin{tabular}{llllllllll}
0103 & $\mathrm{O}$ & 0.30064 & 0.72221 & 0.87094 & 0.00000 & Uiso & 1.00 \\
\hline & 104 & $\mathrm{O}$ & 0.35154 & 0.13442 & 0.31388 & 0.00000 & Uso & 1.00
\end{tabular} $\begin{array}{llllllllll}0104 & 0 & 0.35154 & 0.13442 & 0.31388 & 0.00000 & \text { Uiso } & 1.00 \\ 0105 & 0 & 0.35608 & 0.15404 & 0.78218 & 0.00000 & \text { Us } & 1.00\end{array}$ $\begin{array}{llllllllll}0105 & 0 & 0.35608 & 0.15404 & 0.78218 & 0.00000 & \text { Uiso } & 1.00\end{array}$ $\begin{array}{llllllll}0106 & \mathrm{O} & 0.35953 & 0.62700 & 0.36776 & 0.00000 & \text { Uiso } & 1.00\end{array}$ $\begin{array}{llllllll}0107 & \mathrm{O} & 0.34596 & 0.63203 & 0.73639 & 0.00000 & \text { Uiso } & 1.00\end{array}$ $\begin{array}{lllllllll}\mathrm{O} 108 & \mathrm{O} & 0.34948 & 0.79796 & 0.26277 & 0.00000 & \text { Uiso } & 1.00\end{array}$ $\begin{array}{lllllllll}0109 & \mathrm{O} & 0.34562 & 0.80661 & 0.77475 & 0.00000 & \text { Uiso } & 1.00\end{array}$ $\begin{array}{llllllll}0110 & \mathrm{O} & 0.35603 & 0.31052 & 0.02384 & 0.00000 & \text { Uiso } & 1.00\end{array}$ $\begin{array}{llllllll}\mathrm{O} 111 & \mathrm{O} & 0.41707 & 0.39839 & 0.64539 & 0.00000 & \text { Uiso } & 1.00\end{array}$ 
$\begin{array}{llllllll}0112 & \mathrm{O} & 0.35372 & 0.96574 & 0.75580 & 0.00000 & \text { Uiso } & 1.00\end{array}$ $\begin{array}{lllllllll}0113 & \mathrm{O} & 0.35538 & 0.48785 & 0.23987 & 0.00000 & \text { Uiso } & 1.00\end{array}$ $\begin{array}{llllllll}0114 & \mathrm{O} & 0.35415 & 0.48758 & 0.78379 & 0.00000 & \text { Uiso } & 1.00\end{array}$ $\begin{array}{lllllllll}0115 & \mathrm{O} & 0.30491 & 0.88681 & 0.14315 & 0.00000 & \text { Uiso } & 1.00\end{array}$ $\begin{array}{llllllll}0116 & 0 & 0.30157 & 0.88685 & 0.64343 & 0.00000 & \text { Uiso } & 1.00\end{array}$ $\begin{array}{llllllllll}0117 & 0 & 0.30234 & 0.38934 & 0.15024 & 0.00000 & \text { Uiso } & 1.00\end{array}$

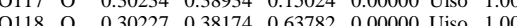
$\begin{array}{llllllll}0118 & 0 & 0.30227 & 0.38174 & 0.63782 & 0.0000 & \text { Uiso } & 1.00\end{array}$

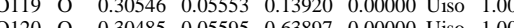
$\begin{array}{lllllllll}0120 & \mathrm{O} & 0.30485 & 0.05595 & 0.63897 & 0.00000 & \text { Uiso } & 1.00 \\ 0121 & \mathrm{O} & 0.30108 & 0.55970 & 0.14383 & 0.00000 & \text { Uiso } & 1.00\end{array}$ $\begin{array}{llllllllllll}0121 & 0 & 0.30108 & 0.55950 & 0.14383 & 0.00000 & \text { Uiso } & 1.00\end{array}$ $\begin{array}{llllllllll}0122 & \mathrm{O} & 0.30062 & 0.55970 & 0.63915 & 0.00000 & 0.3150 & 1.00 \\ 0123 & 0 & 0.30655 & 0.22066 & 0.15021 & 0.0000 & \text { Uiso } & 1.00\end{array}$ $\begin{array}{lllllllllll}0123 & 0 & 0.30655 & 0.22066 & 0.15021 & 0.00000 & \text { Uiso } & 1.00 \\ 0124 & 0 & 0.31017 & 0.21890 & 0.64661 & 0.0000 & \text { Uis } & 1.00\end{array}$ $\begin{array}{lllllllll}0124 & 0 & 0.31017 & 0.21890 & 0.64661 & 0.00000 & \text { Uiso } & 1.00\end{array}$ $\begin{array}{llllllll}0125 & \mathrm{O} & 0.30982 & 0.71777 & 0.13652 & 0.00000 & \text { Uiso } & 1.00 \\ 0126 & \mathrm{O} & 0.30662 & 0.72451 & 0.63823 & 0.00000 & \text { Uiso } & 1.00\end{array}$ $\begin{array}{lllllllll}0126 & 0 & 0.30662 & 0.72451 & 0.63823 & 0.00000 & \text { Uiso } & 1.00\end{array}$ $\begin{array}{lllllllll}0127 & \mathrm{O} & 0.35921 & 0.14313 & 0.00323 & 0.00000 & \text { Uiso } & 1.00\end{array}$ $\begin{array}{lllllllll}0128 & \mathrm{O} & 0.35671 & 0.64583 & 0.00027 & 0.00000 & \text { Uiso } & 1.00\end{array}$ $\begin{array}{llllllll}0129 & \mathrm{O} & 0.36447 & 0.81150 & 0.01687 & 0.00000 & \text { Uiso } & 1.00\end{array}$ $\begin{array}{lllllllll}0130 & \mathrm{O} & 0.35991 & 0.77779 & 0.52186 & 0.00000 & \text { Uiso } & 1.00\end{array}$ $\begin{array}{lllllllll}0131 & \mathrm{O} & 0.34501 & 0.30492 & 0.78759 & 0.00000 & \text { Uiso } & 1.00\end{array}$ $\begin{array}{llllllll}0132 & \mathrm{O} & 0.35332 & 0.30376 & 0.52021 & 0.00000 & \text { Uiso } & 1.00\end{array}$ $\begin{array}{lllllllll}0133 & \mathrm{O} & 0.35086 & 0.96791 & 0.99185 & 0.00000 & \text { Uiso } & 1.00\end{array}$ $\begin{array}{llllllll}0134 & \mathrm{O} & 0.34971 & 0.95664 & 0.49366 & 0.00000 & \text { Uiso } & 1.00\end{array}$ $\begin{array}{llllllll}0135 & \mathrm{O} & 0.34838 & 0.47876 & 0.00201 & 0.00000 & \text { Uiso } & 1.00\end{array}$ $\begin{array}{llllllll}0136 & \mathrm{O} & 0.34939 & 0.48202 & 0.52098 & 0.00000 & \text { Uiso } & 1.00\end{array}$ $\begin{array}{llllllll}0137 & \mathrm{O} & 0.20714 & 0.89410 & 0.88748 & 0.00000 & \text { Uiso } & 1.00\end{array}$ $\begin{array}{lllllllllll}0138 & 0 & 0.20694 & 0.89349 & 0.38613 & 0.00000 & \text { Uiso } & 1.00\end{array}$ $\begin{array}{llllllllll}0138 & 0 & 0.20694 & 0.89349 & 0.38613 & 0.0000 & \text { Uiso } & 1.00\end{array}$ $\begin{array}{llllllllll}0140 & 0 & 0.20585 & 0.39218 & 0.88554 & 0.00000 & 0.38447 & 0.00000 & \text { Uiso } & 1.00\end{array}$

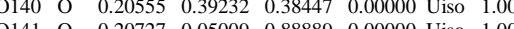
$\begin{array}{llllllll}0141 & \mathrm{O} & 0.20727 & 0.05009 & 0.88889 & 0.00000 & \text { Uiso } & 1.00\end{array}$ $\begin{array}{llllllllll}0142 & \mathrm{O} & 0.20705 & 0.04986 & 0.38642 & 0.00000 & \text { Uiso } & 1.00\end{array}$ $\begin{array}{lllllllll}0143 & \mathrm{O} & 0.20574 & 0.54782 & 0.88445 & 0.00000 & \text { Uiso } & 1.00\end{array}$ $\begin{array}{llllllllll}0144 & \mathrm{O} & 0.20531 & 0.54773 & 0.38375 & 0.00000 & \text { Uiso } & 1.00 \\ 0145 & 0 & 0.21095 & 0.22046 & 0.8865 & 0.0000 & \text { Uiso } & 1.00\end{array}$ $\begin{array}{llllllllll}0145 & \mathrm{O} & 0.21095 & 0.22046 & 0.88651 & 0.00000 & \text { Uiso } & 1.00\end{array}$ $\begin{array}{lllllllll}0146 & \mathrm{O} & 0.21090 & 0.22066 & 0.38494 & 0.00000 & \text { Uiso } & 1.00\end{array}$ $\begin{array}{llllllll}0147 & \mathrm{O} & 0.21040 & 0.72146 & 0.88361 & 0.00000 & \text { Uiso } & 1.00\end{array}$ $\begin{array}{lllllllll}0148 & \mathrm{O} & 0.21029 & 0.72184 & 0.38227 & 0.00000 & \text { Uiso } & 1.00\end{array}$ $\begin{array}{llllllll}0149 & \mathrm{O} & 0.26215 & 0.13521 & 0.25359 & 0.00000 & \text { Uiso } & 1.00\end{array}$ $\begin{array}{llllllll}0150 & \mathrm{O} & 0.26141 & 0.13781 & 0.74601 & 0.00000 & \text { Uiso } & 1.00\end{array}$ $\begin{array}{lllllllll}0151 & \mathrm{O} & 0.25707 & 0.63595 & 0.24359 & 0.00000 & \text { Uiso } & 1.00\end{array}$ $\begin{array}{llllllll}\mathrm{O} 152 & \mathrm{O} & 0.25710 & 0.63729 & 0.74183 & 0.00000 & \text { Uiso } & 1.00\end{array}$ $\begin{array}{llllllll}0153 & \mathrm{O} & 0.26170 & 0.80403 & 0.24292 & 0.00000 & \text { Uiso } & 1.00\end{array}$ $\begin{array}{lllllllll}0154 & \mathrm{O} & 0.25846 & 0.80743 & 0.74601 & 0.00000 & \text { Uiso } & 1.00\end{array}$ $\begin{array}{llllllll}\mathrm{O} 155 & \mathrm{O} & 0.26074 & 0.30527 & 0.25416 & 0.00000 & \text { Uiso } & 1.00\end{array}$ $\begin{array}{llllllll}0156 & \mathrm{O} & 0.25984 & 0.30508 & 0.75079 & 0.00000 & \text { Uiso } & 1.00\end{array}$ $\begin{array}{llllllll}0157 & \mathrm{O} & 0.25642 & 0.96842 & 0.25014 & 0.00000 & \text { Uiso } & 1.00\end{array}$ $\begin{array}{llllllll}0158 & \mathrm{O} & 0.25571 & 0.97390 & 0.75027 & 0.00000 & \text { Uiso } & 1.00\end{array}$ $\begin{array}{llllllllllll}0159 & 0 & 0.25338 & 0.47022 & 0.24795 & 0.00000 & \text { Uiso } & 1.00\end{array}$ $\begin{array}{llllllllll}0159 & \mathrm{O} & 0.25338 & 0.47022 & 0.24795 & 0.00000 & \text { Uiso } & 1.00 \\ 0160 & 0 & 0.25437 & 0.47040 & 0.74746 & 0.00000 & \text { Uiso } & 1.00\end{array}$ $\begin{array}{llllllllllll}0160 & 0 & 0.25437 & 0.47040 & 0.74746 & 0.00000 & \text { Uiso } & 1.00\end{array}$ $\begin{array}{lllllllll}0161 & \mathrm{O} & 0.21592 & 0.8300 & 0.12150 & 0.00000 & \text { Uiso } & 1.00\end{array}$ $\begin{array}{lllllllll}0162 & \mathrm{O} & 0.21497 & 0.89461 & 0.62121 & 0.00000 & \text { Uiso } & 1.00 \\ 0163 & \mathrm{O} & 0.21420 & 0.39217 & 0.12082 & 0.00000 & \text { Uis } & 1.00\end{array}$ $\begin{array}{llllllllll}0163 & 0 & 0.21420 & 0.39217 & 0.12082 & 0.00000 & \text { Uiso } & 1.00\end{array}$ $\begin{array}{lllllllll}0164 & \mathrm{O} & 0.21407 & 0.3200 & 0.62043 & 0.00000 & \text { Uiso } & 1.00 \\ 0165 & \mathrm{O} & 0.21590 & 0.0453 & 0.1245 & 0.0000 & \text { Uiso } & 1.00\end{array}$ $\begin{array}{lllllllll}0165 & \mathrm{O} & 0.21590 & 0.04853 & 0.12485 & 0.0000 & \text { Uiso } & 1.00\end{array}$ $\begin{array}{lllllllll}0166 & \mathrm{O} & 0.21583 & 0.04998 & 0.62089 & 0.00000 & \text { Uiso } & 1.00\end{array}$ $\begin{array}{lllllllll}\mathrm{O} 167 & \mathrm{O} & 0.21366 & 0.54798 & 0.11865 & 0.00000 & \text { Uiso } & 1.00\end{array}$ $\begin{array}{llllllll}0168 & \mathrm{O} & 0.21380 & 0.54805 & 0.61841 & 0.00000 & \text { Uiso } & 1.00\end{array}$ $\begin{array}{llllllll}0169 & \mathrm{O} & 0.20848 & 0.21991 & 0.12777 & 0.00000 & \text { Uiso } & 1.00\end{array}$ $\begin{array}{llllllll}0170 & \mathrm{O} & 0.20818 & 0.22101 & 0.62578 & 0.00000 & \text { Uiso } & 1.00\end{array}$ $\begin{array}{llllllll}0171 & \mathrm{O} & 0.20815 & 0.72182 & 0.12286 & 0.00000 & \text { Uiso } & 1.00\end{array}$ $\begin{array}{llllllll}0172 & \mathrm{O} & 0.20752 & 0.72170 & 0.62177 & 0.00000 & \text { Uiso } & 1.00\end{array}$ $\begin{array}{lllllllll}0173 & \mathrm{O} & 0.25600 & 0.13646 & 0.01719 & 0.00000 & \text { Uiso } & 1.00\end{array}$ $\begin{array}{lllllllll}0174 & \mathrm{O} & 0.25648 & 0.13831 & 0.51274 & 0.00000 & \text { Uiso } & 1.00\end{array}$ $\begin{array}{lllllllll}0175 & \mathrm{O} & 0.25471 & 0.63737 & 0.01157 & 0.00000 & \text { Uiso } & 1.00\end{array}$ $\begin{array}{lllllllll}0176 & \mathrm{O} & 0.25487 & 0.63733 & 0.50809 & 0.00000 & \text { Uiso } & 1.00\end{array}$ $\begin{array}{lllllllll}0177 & \mathrm{O} & 0.25632 & 0.80362 & 0.01316 & 0.00000 & \text { Uiso } & 1.00\end{array}$ $\begin{array}{lllllllll}0178 & 0 & 0.25525 & 0.80440 & 0.51081 & 0.00000 & \text { Uiso } & 1.00\end{array}$ $\begin{array}{llllllllllll}0179 & \mathrm{O} & 0.25541 & 0.30358 & 0.01769 & 0.00000 & \text { Uiso } & 1.00\end{array}$ $\begin{array}{lllllllllll}0180 & 0 & 0.25553 & 0.30338 & 0.51503 & 0.00000 & \text { Uiso } & 1.00\end{array}$ $\begin{array}{lllllllll}0180 & 0 & 0.25553 & 0.30338 & 0.51503 & 0.00000 & \text { Uiso } & 1.00\end{array}$

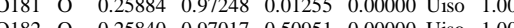
$\begin{array}{llllllllll}0182 & \mathrm{O} & 0.25840 & 0.97017 & 0.50951 & 0.00000 & \text { Uiso } & 1.00 \\ 0183 & \mathrm{O} & 0.25710 & 0.47055 & 0.01117 & 0.00000 & \text { Uiso } & 1.00\end{array}$ $\begin{array}{lllllllll}0183 & \mathrm{O} & 0.25710 & 0.47055 & 0.01117 & 0.00000 & \text { Uiso } & 1.00\end{array}$ $\begin{array}{lllllllll}0184 & \mathrm{O} & 0.25616 & 0.46930 & 0.51001 & 0.00000 & \text { Uiso } & 1.00 \\ 0185 & \mathrm{O} & 0.35220 & 0.29827 & 0.27093 & 0.00000 & \text { Uiso } & 1.00\end{array}$ \begin{tabular}{llllllllll}
0185 & 0 & 0.35220 & 0.29827 & 0.27093 & 0.00000 & Uiso & 1.00 \\
\hline 186 & 0 & 0.39615 & 0.71319 & 0.65902 & 0.0000 & Uis & 1.00
\end{tabular} $\begin{array}{llllllll}0186 & \mathrm{O} & 0.39615 & 0.71319 & 0.65902 & 0.00000 & \text { Uiso } & 1.00\end{array}$ $\begin{array}{lllllllll}0187 & \mathrm{O} & 0.41533 & 0.18288 & 0.92845 & 0.00000 & \text { Uiso } & 1.00\end{array}$ $\begin{array}{lllllllll}0188 & \mathrm{O} & 0.39887 & 0.88720 & 0.86768 & 0.00000 & \text { Uiso } & 1.00\end{array}$ $\begin{array}{lllllllll}0189 & \mathrm{O} & 0.39758 & 0.38902 & 0.17666 & 0.00000 & \text { Uiso } & 1.00\end{array}$ $\begin{array}{llllllll}0190 & \mathrm{O} & 0.39440 & 0.37950 & 0.87131 & 0.00000 & \text { Uiso } & 1.00\end{array}$ $\begin{array}{llllllll}0191 & \mathrm{O} & 0.39821 & 0.25448 & 0.71337 & 0.00000 & \text { Uiso } & 1.00\end{array}$ $\begin{array}{lllllllll}0192 & \mathrm{O} & 0.41036 & 0.55941 & 0.65147 & 0.00000 & \text { Uiso } & 1.00\end{array}$ $\begin{array}{llllllll}0193 & \mathrm{O} & 0.46490 & 0.49488 & 0.70722 & 0.00000 & \text { Uiso } & 1.00\end{array}$ $\begin{array}{llllllll}0194 & \mathrm{O} & 0.40838 & 0.54454 & 0.29831 & 0.00000 & \text { Uiso } & 1.00\end{array}$ $\begin{array}{llllllll}0195 & \mathrm{O} & 0.40542 & 0.53194 & 0.07049 & 0.00000 & \text { Uiso } & 1.00\end{array}$

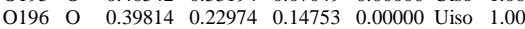
$\begin{array}{llllllll}0197 & \mathrm{O} & 0.39556 & 0.04527 & 0.84347 & 0.00000 & \text { Uiso } & 1.00\end{array}$ $\begin{array}{llllllll}0198 & \mathrm{O} & 0.40602 & 0.07249 & 0.10519 & 0.00000 & \text { Uiso } & 1.00\end{array}$ $\begin{array}{lllllllll}\mathrm{O} 199 & \mathrm{O} & 0.41427 & 0.69615 & 0.39808 & 0.00000 & \text { Uiso } & 1.00\end{array}$ $\begin{array}{lllllllllllllll}0200 & 0 & 0.45041 & 0.32191 & 0.80448 & 0.00000 & \text { Uiso } & 1.00\end{array}$ \begin{tabular}{llllllllll}
\hline 0200 & $\mathrm{O}$ & 0.45041 & 0.32191 & 0.80448 & 0.0000 & Uiso & 1.00 \\
\hline 2201 & 0 & 0.45313 & 0.67218 & 0.70669 & 0.00000 & Uiso & 1.00
\end{tabular} \begin{tabular}{llllllllll}
0201 & $\mathrm{O}$ & 0.45313 & 0.67218 & 0.70669 & 0.0000 & Uiso & 1.00 \\
\hline & 0.000 & 0.46941 & 0.0000 & Uis & 1.00
\end{tabular} \begin{tabular}{lllllllll}
0202 & $\mathrm{O}$ & 0.40983 & 0.63102 & 0.86941 & 0.00000 & Uiso & 1.00 \\
\hline & 0203 & $\mathrm{O}$ & 0.44577 & 0.48979 & 0.48168 & 0.000000 & Uiso & 1.00
\end{tabular} $\begin{array}{lllllllll}0203 & \mathrm{O} & 0.44577 & 0.4897 & 0.48168 & 0.00000 & \text { Uiso } & 1.00\end{array}$ $\begin{array}{llllllllll}0204 & \mathrm{O} & 0.37041 & 0.16780 & 0.53181 & 0.00000 & \text { Uiso } & 1.00\end{array}$ $\begin{array}{lllllllll}0205 & \mathrm{O} & 0.42078 & 0.11417 & 0.65222 & 0.00000 & \text { Uiso } & 1.00\end{array}$ $\begin{array}{llllllllllll}0206 & 0 & 0.40930 & 0.94029 & 0.64891 & 0.00000 & \text { Uiso } & 1.00\end{array}$ $\begin{array}{lllllllll}\mathrm{O} 207 & \mathrm{O} & 0.39804 & 0.87015 & 0.43272 & 0.00000 & \text { Uiso } & 1.00\end{array}$ $\begin{array}{llllllll}\mathrm{O} 208 & \mathrm{O} & 0.42688 & 0.16799 & 0.41824 & 0.00000 & \text { Uiso } & 1.00\end{array}$ $\begin{array}{lllllllll}\mathrm{O} 209 & \mathrm{O} & 0.39735 & 0.03752 & 0.46012 & 0.00000 & \text { Uiso } & 1.00\end{array}$ $\begin{array}{lllllllll}\mathrm{O} 210 & \mathrm{O} & 0.45077 & 0.94591 & 0.49098 & 0.00000 & \text { Uiso } & 1.00\end{array}$ $\begin{array}{llllllll}\mathrm{O} 211 & \mathrm{O} & 0.40940 & 0.91022 & 0.10291 & 0.00000 & \text { Uiso } & 1.00\end{array}$

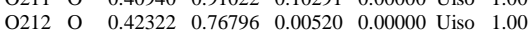


$\begin{array}{llllllll}\mathrm{O} 213 & \mathrm{O} & 0.42959 & 0.99346 & 0.27573 & 0.00000 & \text { Uiso } & 1.00\end{array}$ $\begin{array}{lllllllll}\mathrm{O} 214 & \mathrm{O} & 0.39039 & 0.67837 & 0.17301 & 0.00000 & \text { Uiso } & 1.00\end{array}$ $\begin{array}{llllllll}0215 & \mathrm{O} & 0.36448 & 0.98267 & 0.23685 & 0.00000 & \text { Uiso } & 1.00\end{array}$ $\begin{array}{llllllll}\mathrm{O} 216 & \mathrm{O} & 0.44356 & 0.79092 & 0.56005 & 0.00000 & \text { Uiso } & 1.00\end{array}$ $\begin{array}{llllllll}\mathrm{Si} 217 & \mathrm{Si} & 0.39217 & 0.96056 & 0.77267 & 0.00000 & \text { Uiso } & 1.00\end{array}$ $\begin{array}{lllllllll}\mathrm{Si} 218 & \mathrm{Si} & 0.41382 & 0.34052 & 0.76471 & 0.00000 & \text { Uiso } & 1.00\end{array}$

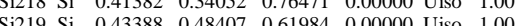
$\begin{array}{lllllllll} & \mathrm{Si} 220 \mathrm{Si} & 0.39302 & 0.48642 & 0.18758 & 0.00000 & \text { Uiso } & 1.00\end{array}$ $\begin{array}{lllllllll} & \text { Si221 Si } 0.39615 & 0.15801 & 0.04837 & 0.00000 & \text { Uiso } & 1.00\end{array}$ $\begin{array}{llllllll} & \text { Si221 Si } & 0.39615 & 0.15801 & 0.04837 & 0.0000 & \text { Ulso } & 1.00\end{array}$ $\begin{array}{lllllllll}51222 & \text { Si } & 0.39421 & 0.63974 & 0.31212 & 0.00000 & \text { Uiso } & 1.00\end{array}$ $\begin{array}{llllllllll}S i 223 & \text { Si } & 0.37542 & 0.30908 & 0.15306 & 0.0000 & \text { Uiso } & 1.00\end{array}$ $\begin{array}{lllllllll}\mathrm{Si} 224 & \mathrm{Si} & 0.41604 & 0.64200 & 0.72877 & 0.0000 & \text { Uiso } & 1.00\end{array}$ $\begin{array}{lllllll}\mathrm{Si} 225 \mathrm{Si} & 0.41210 & 0.94838 & 0.50192 & 0.00000 & \text { Uiso } & 1.00\end{array}$

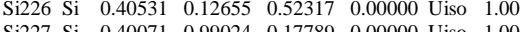
$\begin{array}{llllllll}\text { Si227 Si } & 0.40071 & 0.99024 & 0.17789 & 0.00000 & \text { Uiso } & 1.00\end{array}$ $\begin{array}{llllllll}\mathrm{Si} 228 & \mathrm{Si} & 0.40039 & 0.84501 & 0.00175 & 0.00000 & \text { Uiso } & 1.00\end{array}$ $\begin{array}{llllllll}\mathrm{C} 229 & \mathrm{C} & 0.50175 & 0.67541 & 0.06542 & 0.00000 & \text { Uiso } & 1.00\end{array}$ $\begin{array}{lllllllll}\mathrm{C} 230 & \mathrm{C} & 0.47474 & 0.61785 & 0.03777 & 0.00000 & \text { Uiso } & 1.00\end{array}$ $\begin{array}{lllllllll}\mathrm{C} 231 & \mathrm{C} & 0.53065 & 0.64793 & 0.99407 & 0.00000 & \text { Uiso } & 1.00\end{array}$ $\begin{array}{lllllllll}\mathrm{C} 232 & \mathrm{C} & 0.50913 & 0.66976 & 0.19994 & 0.00000 & \text { Uiso } & 1.00\end{array}$ $\begin{array}{llllllll}\mathrm{C} 233 & \mathrm{C} & 0.49342 & 0.75711 & 0.01632 & 0.00000 & \text { Uiso } & 1.00\end{array}$ $\begin{array}{lllllllll}\mathrm{C} 234 & \mathrm{C} & 0.48854 & 0.82990 & 0.06543 & 0.00000 & \text { Uiso } & 1.00\end{array}$ $\begin{array}{lllllllll}\mathrm{C} 235 & \mathrm{C} & 0.48766 & 0.85192 & 0.19650 & 0.00000 & \text { Uiso } & 1.00\end{array}$ $\begin{array}{lllllllll}\mathrm{C} 236 & \mathrm{C} & 0.48328 & 0.90063 & 0.98363 & 0.00000 & \text { Uiso } & 1.00\end{array}$

\section{File $\mathbf{n}^{\circ} 12$ :}

$\begin{array}{llllllll}\mathrm{H} 2 & \mathrm{H} & 0.46805 & 0.44900 & 0.75979 & 0.00000 & \text { Uiso } & 1.00\end{array}$

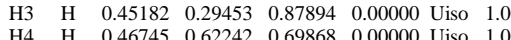

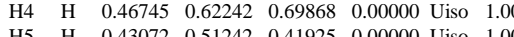

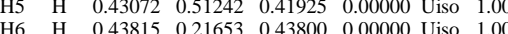

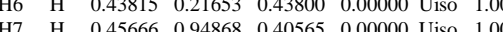
$\begin{array}{llllllll}\mathrm{H} 7 & \mathrm{H} & 0.45666 & 0.94868 & 0.40565 & 0.00000 & \text { Uiso } & 1.00\end{array}$ $\begin{array}{llllllll}48 & \mathrm{H} & 0.43380 & 0.04936 & 0.30189 & 0.00000 & \text { Uiso } & 1.00\end{array}$ $\begin{array}{llllllllll}\mathrm{H} & \mathrm{H} & 0.45459 & 0.84210 & 0.54554 & 0.00000 & \text { Uiso } & 1.00\end{array}$ $\begin{array}{llllllll}\mathrm{H} 10 & \mathrm{H} & 0.45383 & 0.75055 & 0.61136 & 0.00000 & \text { Uiso } & 1.00\end{array}$ $\begin{array}{llllllll}\mathrm{H} 11 & \mathrm{H} & 0.44932 & 0.68806 & 0.16097 & 0.00000 & \text { Uiso } & 1.00 \\ \mathrm{H} 12 & \mathrm{H} & 0.48870 & 0.54395 & 0.08260 & 0.00000 & \text { Uiso } & 1.00\end{array}$ $\begin{array}{llllllll}\mathrm{H} 12 & \mathrm{H} & 0.48870 & 0.54395 & 0.08260 & 0.00000 & \text { Uiso } & 1.00\end{array}$ $\begin{array}{llllllll}\mathrm{H} 13 & \mathrm{H} & 0.47490 & 0.56568 & 0.23002 & 0.00000 & \text { Uiso } & 1.00\end{array}$ $\begin{array}{llllllll}\mathrm{H} 14 & \mathrm{H} & 0.51546 & 0.55176 & 0.20498 & 0.00000 & \text { Uiso } & 1.00\end{array}$ $\begin{array}{llllllll}\mathrm{H} 15 & \mathrm{H} & 0.52027 & 0.65271 & 0.96636 & 0.00000 & \text { Uiso } & 1.00\end{array}$ $\begin{array}{llllllll}\mathrm{H} 16 & \mathrm{H} & 0.54615 & 0.64739 & 0.09122 & 0.00000 & \text { Uiso } & 1.00\end{array}$ $\begin{array}{llllllll}\mathrm{H} 17 & \mathrm{H} & 0.53142 & 0.74124 & 0.04436 & 0.00000 & \text { Uiso } & 1.00\end{array}$ $\begin{array}{llllllll}\mathrm{H} 18 & \mathrm{H} & 0.52471 & 0.67432 & 0.30678 & 0.00000 & \text { Uiso } & 1.00\end{array}$ $\begin{array}{lllllllll}\mathrm{H} 19 & \mathrm{H} & 0.48506 & 0.70338 & 0.32364 & 0.00000 & \text { Uiso } & 1.00\end{array}$ $\begin{array}{lllllllll}\mathrm{H} 20 & \mathrm{H} & 0.51237 & 0.76877 & 0.25392 & 0.00000 & \text { Uiso } & 1.00\end{array}$ $\begin{array}{lllllllll}\mathrm{H} 21 & \mathrm{H} & 0.46036 & 0.65153 & 0.01644 & 0.00000 & \text { Uiso } & 1.00\end{array}$ $\begin{array}{llllllll}\mathrm{H} 22 & \mathrm{H} & 0.45938 & 0.83907 & 0.20724 & 0.00000 & \text { Uiso } & 1.00\end{array}$ $\begin{array}{lllllllll}\mathrm{H} 23 & \mathrm{H} & 0.46433 & 0.90343 & 0.08076 & 0.00000 & \text { Uiso } & 1.00\end{array}$ $\begin{array}{lllllllll}\mathrm{H} 24 & \mathrm{H} & 0.49585 & 0.84390 & 0.12999 & 0.00000 & \text { Uiso } & 1.00\end{array}$ $\begin{array}{llllllll}\mathrm{H} 25 & \mathrm{H} & 0.50169 & 0.79384 & 0.91969 & 0.00000 & \text { Uiso } & 1.00\end{array}$ $\begin{array}{llllllllll}\mathrm{H} 26 & \mathrm{H} & 0.46802 & 0.84695 & 0.87290 & 0.00000 & \text { Uiso } & 1.00\end{array}$ $\begin{array}{llllllll}\mathrm{H} 26 & \mathrm{H} & 0.46802 & 0.84695 & 0.87290 & 0.00000 & \text { Uiso } & 1.00 \\ \mathrm{H} 27 & \mathrm{H} & 0.47023 & 0.74165 & 0.85026 & 0.00000 & \text { Uiso } & 1.00\end{array}$

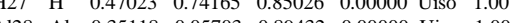
$\begin{array}{llllllll}\mathrm{Al} 28 & \mathrm{Al} & 0.35118 & 0.05703 & 0.89432 & 0.00000 & \text { Uiso } & 1.00\end{array}$ $\begin{array}{llllllllll}A 129 & \text { Al } & 0.35989 & 0.03737 & 0.38127 & 0.00000 & \text { Uiso } & 1.00\end{array}$ $\begin{array}{lllllllll}\text { Al30 Al } & 0.34382 & 0.56544 & 0.90029 & 0.00000 & \text { Uiso } & 1.00\end{array}$

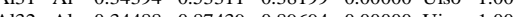
$\begin{array}{llllllll}\text { Al32 } & \text { Al } & 0.34488 & 0.87439 & 0.89694 & 0.00000 & \text { Uiso } & 1.00\end{array}$ $\begin{array}{llllllll} & \text { Al33 Al } & 0.34999 & 0.85134 & 0.40989 & 0.00000 & \text { Uiso } & 1.00\end{array}$ $\begin{array}{lllllllll}\mathrm{Al} 34 & \mathrm{Al} & 0.35313 & 0.40125 & 0.88438 & 0.00000 & \text { Uiso } & 1.00\end{array}$ $\begin{array}{llllllll}\text { Al35 Al } & 0.32885 & 0.38705 & 0.51942 & 0.00000 & \text { Uiso } & 1.00\end{array}$ $\begin{array}{llllllll}\text { Al36 } & \text { Al } & 0.30483 & 0.80232 & 0.26139 & 0.00000 & \text { Uiso } & 1.00\end{array}$ $\begin{array}{llllllll}\mathrm{Al} 37 & \mathrm{Al} & 0.30084 & 0.80741 & 0.76492 & 0.00000 & \text { Uiso } & 1.00\end{array}$ $\begin{array}{llllllll}\mathrm{Al} 38 & \mathrm{Al} & 0.30446 & 0.30687 & 0.26077 & 0.00000 & \text { Uiso } & 1.00\end{array}$ $\begin{array}{lllllllll}\mathrm{Al} 39 & \mathrm{Al} & 0.30159 & 0.31414 & 0.77253 & 0.00000 & \text { Uiso } & 1.00\end{array}$ $\begin{array}{lllllllll}\mathrm{Al} 40 & \mathrm{Al} & 0.30629 & 0.13426 & 0.26135 & 0.00000 & \text { Uiso } & 1.00\end{array}$ $\begin{array}{lllllllll}\mathrm{Al} 41 & \mathrm{Al} & 0.30316 & 0.13607 & 0.75859 & 0.00000 & \text { Uiso } & 1.00\end{array}$ $\begin{array}{llllllll}\mathrm{Al} 42 & \mathrm{Al} & 0.29764 & 0.63510 & 0.26726 & 0.00000 & \text { Uiso } & 1.00\end{array}$ $\begin{array}{lllllllll}\mathrm{Al} 43 & \mathrm{Al} & 0.29903 & 0.64138 & 0.76010 & 0.00000 & \text { Uiso } & 1.00\end{array}$ $\begin{array}{lllllllll}\mathrm{Al} 44 & \mathrm{Al} & 0.21191 & 0.97107 & 0.25381 & 0.00000 & \text { Uiso } & 1.00\end{array}$ $\begin{array}{lllllllll}\text { Al45 } & \mathrm{Al} & 0.21176 & 0.97236 & 0.75450 & 0.00000 & \text { Uiso } & 1.00\end{array}$ $\begin{array}{llllllll}\mathrm{A} 146 & \mathrm{Al} & 0.20935 & 0.47053 & 0.25063 & 0.00000 & \text { Uiso } & 1.00\end{array}$ $\begin{array}{lllllllll}\text { Al47 } & \text { Al } & 0.21015 & 0.47060 & 0.75172 & 0.00000 & \text { Uiso } & 1.00\end{array}$ $\begin{array}{llllllllll}A 48 & \text { Al } & 0.32927 & 0.97034 & 0.12995 & 0.00000 & \text { Uiso } & 1.00\end{array}$ $\begin{array}{lllllll} & 0.9659 & 0.62752 & 0.00000 & \text { Uiso } & 1.00\end{array}$

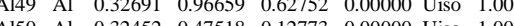
\begin{tabular}{lllllllllll}
\hline & $\mathrm{Al}$ & 0.32452 & 0.47518 & 0.12773 & 0.00000 & Uiso & 1.00
\end{tabular} $\begin{array}{lllllllll}\text { Al51 Al } & 0.33998 & 0.53568 & 0.65872 & 0.00000 & \text { Uiso } & 1.00\end{array}$ $\begin{array}{lllllllll} & \mathrm{Al5} 2 & \mathrm{Al} & 0.28078 & 0.22011 & 0.02383 & 0.00000 & \text { Uiso } & 1.00\end{array}$ $\begin{array}{lllllllllll}A 153 & \text { Al } & 0.28184 & 0.22134 & 0.5163 & 0.00000 & \text { Uiso } & 1.00\end{array}$ $\begin{array}{llllllll}\mathrm{Al} 54 & \text { Al } & 0.28100 & 0.71861 & 0.00697 & 0.00000 & \text { Uiso } & 1.00\end{array}$ $\begin{array}{llllllllll}A 155 & \text { Al } & 0.28103 & 0.72100 & 0.50676 & 0.00000 & \text { Ulso } & 1.00\end{array}$ $\begin{array}{lllllllll}\mathrm{Al} 56 & \mathrm{Al} & 0.34579 & 0.23238 & 0.91688 & 0.00000 & \text { Uiso } & 1.00\end{array}$ $\begin{array}{llllllll}\text { Al57 } & \text { Al } & 0.35622 & 0.24057 & 0.65625 & 0.00000 & \text { Uiso } & 1.00\end{array}$ $\begin{array}{llllllll}\mathrm{Al} 58 & \mathrm{Al} & 0.35294 & 0.73191 & 0.12421 & 0.00000 & \text { Uiso } & 1.00\end{array}$ $\begin{array}{llllllll}\mathrm{Al} 59 & \mathrm{Al} & 0.35172 & 0.73161 & 0.66542 & 0.00000 & \text { Uiso } & 1.00\end{array}$ $\begin{array}{llllllll}\mathrm{Al} 60 & \mathrm{Al} & 0.21198 & 0.97156 & 0.00634 & 0.00000 & \text { Uiso } & 1.00\end{array}$ $\begin{array}{llllllll}\text { Al61 } & \text { Al } & 0.21143 & 0.97199 & 0.50418 & 0.00000 & \text { Uiso } & 1.00\end{array}$ $\begin{array}{llllllll}\mathrm{Al} 62 & \mathrm{Al} & 0.21023 & 0.46983 & 0.00295 & 0.00000 & \text { Uiso } & 1.00\end{array}$ $\begin{array}{llllllll}\text { Al63 } & \mathrm{Al} & 0.20960 & 0.46996 & 0.50306 & 0.00000 & \text { Uiso } & 1.00\end{array}$ $\begin{array}{lllllllll}\mathrm{Al} 64 & \mathrm{Al} & 0.28282 & 0.96130 & 0.37880 & 0.00000 & \text { Uiso } & 1.00\end{array}$ $\begin{array}{lllllllll}\mathrm{Al} 65 & \mathrm{Al} & 0.28200 & 0.97575 & 0.87935 & 0.00000 & \text { Uiso } & 1.00\end{array}$ $\begin{array}{lllllllll}\text { Al66 } & \mathrm{Al} & 0.27841 & 0.46774 & 0.37687 & 0.00000 & \text { Uiso } & 1.00\end{array}$ $\begin{array}{llllllllll}\text { Al67 Al } & 0.28072 & 0.47447 & 0.87717 & 0.00000 & \text { Uiso } & 1.00\end{array}$ $\begin{array}{lllllllll}\text { Al67 } & \text { Al } & 0.28072 & 0.47447 & 0.87717 & 0.00000 & \text { Uiso } & 1.00\end{array}$

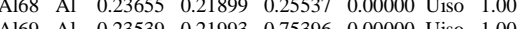
$\begin{array}{lllllllll} & 169 & \text { Al } & 0.23539 & 0.21893 & 0.75396 & 0.00000 & \text { Uiso } & 1.00\end{array}$

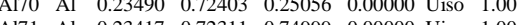

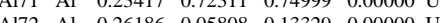


$\begin{array}{llllllll}\text { Al73 } & \text { Al } & 0.26127 & 0.05820 & 0.62615 & 0.00000 & \text { Uiso } & 1.00\end{array}$ $\begin{array}{lllllllll}\text { Al74 } & \mathrm{Al} & 0.25841 & 0.55811 & 0.12481 & 0.00000 & \text { Uiso } & 1.00\end{array}$ $\begin{array}{lllllllll}\text { Al75 } & \text { Al } & 0.25901 & 0.55854 & 0.62456 & 0.00000 & \text { Uiso } & 1.00\end{array}$ $\begin{array}{lllllllll}\text { Al76 } & \mathrm{Al} & 0.26183 & 0.88314 & 0.12666 & 0.00000 & \text { Uiso } & 1.00\end{array}$ $\begin{array}{lllllllll}\mathrm{Al} 77 & \mathrm{Al} & 0.25962 & 0.88314 & 0.62709 & 0.00000 & \text { Uiso } & 1.00\end{array}$ $\begin{array}{lllllllll}\text { Al78 } & \text { Al } & 0.25952 & 0.38231 & 0.13199 & 0.00000 & \text { Uiso } & 1.00\end{array}$ $\begin{array}{lllllllll}\text { Al79 Al } & 0.25952 & 0.38231 & 0.13199 & 0.00000 & \text { Uiso } & 1.00\end{array}$ $\begin{array}{llllllll}\text { Al79 Al } & 0.25925 & 0.38325 & 0.63006 & 0.00000 & \text { Uiso } & 1.00\end{array}$ $\begin{array}{llllllll}A 181 & \mathrm{Al} & 0.21396 & 0.80723 & 0.00144 & 0.00000 & \text { Uiso } & 1.00\end{array}$ $\begin{array}{llllllllll}\text { Al81 Al } & 0.21308 & 0.80669 & 0.50080 & 0.00000 & \text { Uiso } & 1.00\end{array}$

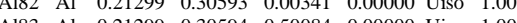
\begin{tabular}{llllllll}
\hline Al83 Al & 0.21299 & 0.30594 & 0.50084 & 0.00000 & Uiso & 1.00
\end{tabular} $\begin{array}{llllllll}\text { Al84 Al } & 0.21369 & 0.13512 & 0.00596 & 0.00000 & \text { Uiso } & 1.00\end{array}$ $\begin{array}{llllllll}\text { Al85 Al } & 0.21368 & 0.13566 & 0.50169 & 0.00000 & \text { Uiso } & 1.00\end{array}$ $\begin{array}{llllllll}\text { Al86 } & \text { Al } & 0.21270 & 0.63505 & 0.99915 & 0.00000 & \text { Uiso } & 1.00\end{array}$ $\begin{array}{lllllllll}\text { Al87 } & \text { Al } & 0.21272 & 0.63517 & 0.49872 & 0.00000 & \text { Uiso } & 1.00\end{array}$ $\begin{array}{llllllll}\mathrm{Al} 88 & \mathrm{Al} & 0.40253 & 0.15197 & 0.78416 & 0.00000 & \text { Uiso } & 1.00\end{array}$ $\begin{array}{llllllll}\text { Al89 } & \text { Al } & 0.35102 & 0.22655 & 0.39955 & 0.00000 & \text { Uiso } & 1.00\end{array}$ $\begin{array}{llllllll}\mathrm{Al} 90 & \mathrm{Al} & 0.39734 & 0.62760 & 0.01827 & 0.00000 & \text { Uiso } & 1.00\end{array}$ $\begin{array}{llllllll}\mathrm{Al} 91 & \mathrm{Al} & 0.40233 & 0.76575 & 0.50699 & 0.00000 & \text { Uiso } & 1.00\end{array}$ $\begin{array}{lllllllll}092 & \mathrm{O} & 0.30863 & 0.87757 & 0.38072 & 0.00000 & \text { Uiso } & 1.00\end{array}$ $\begin{array}{lllllllll}093 & \mathrm{O} & 0.30430 & 0.88773 & 0.88239 & 0.00000 & \text { Uiso } & 1.00\end{array}$ $\begin{array}{llllllllll}094 & \mathrm{O} & 0.30655 & 0.38942 & 0.38531 & 0.00000 & \text { Uiso } & 1.00\end{array}$ $\begin{array}{llllllll}095 & \mathrm{O} & 0.30640 & 0.39331 & 0.88095 & 0.00000 & \text { Uiso } & 1.00\end{array}$

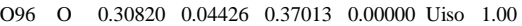
$\begin{array}{lllllllll}097 & \mathrm{O} & 0.30935 & 0.05807 & 0.87211 & 0.00000 & \text { Uiso } & 1.00\end{array}$ $\begin{array}{lllllllll}\mathrm{O} 98 & \mathrm{O} & 0.30391 & 0.55084 & 0.37136 & 0.00000 & \text { Uiso } & 1.00\end{array}$ $\begin{array}{llllllllll}099 & 0 & 0.30272 & 0.56347 & 0.87878 & 0.00000 & \text { Uiso } & 1.00\end{array}$ $\begin{array}{lllllllllll}0100 & 0 & 0.30272 & 0.56347 & 0.87878 & 0.0000 & \text { Uso } & 1.00\end{array}$

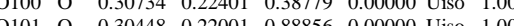
$\begin{array}{llllllllllllll}0101 & 0 & 0.30448 & 0.22001 & 0.38779 & 0.00000 & \text { Uis } & 1.00 \\ 0102 & 0 & 0.30187 & 0.71614 & 0.37014 & 0.00000 & \text { Uiso } & 1.00\end{array}$ $\begin{array}{lllllllll}0102 & \mathrm{O} & 0.30187 & 0.71614 & 0.37014 & 0.00000 & \text { Uiso } & 1.00\end{array}$ $\begin{array}{llllllllll}0103 & \mathrm{O} & 0.30076 & 0.72214 & 0.8707 & 0.00000 & \text { Uiso } & 1.00 \\ 0104 & \mathrm{O} & 0.35143 & 0.13491 & 0.31410 & 0.00000 & \text { Uso } & 1.00\end{array}$ $\begin{array}{lllllllllll}0104 & \mathrm{O} & 0.35143 & 0.13491 & 0.31410 & 0.00000 & \text { Uiso } & 1.00 \\ 0105 & \mathrm{O} & 0.35589 & 0.15406 & 0.78238 & 0.00000 & \text { US } & 1.00\end{array}$ $\begin{array}{lllllllll}0105 & 0 & 0.35589 & 0.15406 & 0.78238 & 0.00000 & \text { Unso } & 1.00\end{array}$ $\begin{array}{lllllllll}0106 & \mathrm{O} & 0.35951 & 0.62731 & 0.36522 & 0.00000 & \text { Uiso } & 1.00\end{array}$ $\begin{array}{lllllllll}0107 & \mathrm{O} & 0.34609 & 0.63209 & 0.73608 & 0.00000 & \text { Uiso } & 1.00\end{array}$ $\begin{array}{llllllll}0108 & \mathrm{O} & 0.34960 & 0.79839 & 0.26189 & 0.00000 & \text { Uiso } & 1.00\end{array}$ $\begin{array}{llllllll}0109 & \mathrm{O} & 0.34577 & 0.80666 & 0.77414 & 0.00000 & \text { Uiso } & 1.00\end{array}$ $\begin{array}{lllllllll}0110 & \mathrm{O} & 0.35584 & 0.31084 & 0.02376 & 0.00000 & \text { Uiso } & 1.00\end{array}$ $\begin{array}{llllllll}0111 & \mathrm{O} & 0.41614 & 0.39782 & 0.64461 & 0.00000 & \text { Uiso } & 1.00\end{array}$ $\begin{array}{lllllllll}0112 & \mathrm{O} & 0.35385 & 0.96631 & 0.75560 & 0.00000 & \text { Uiso } & 1.00\end{array}$ $\begin{array}{llllllll}0113 & \mathrm{O} & 0.35548 & 0.48806 & 0.23940 & 0.00000 & \text { Uiso } & 1.00\end{array}$ $\begin{array}{lllllllll}0114 & \mathrm{O} & 0.35382 & 0.48723 & 0.78431 & 0.00000 & \text { Uiso } & 1.00\end{array}$ $\begin{array}{lllllllll}0115 & \mathrm{O} & 0.30494 & 0.88671 & 0.14277 & 0.00000 & \text { Uiso } & 1.00\end{array}$ $\begin{array}{llllllll}\mathrm{O} 116 & \mathrm{O} & 0.30157 & 0.88684 & 0.64353 & 0.00000 & \text { Uiso } & 1.00\end{array}$ $\begin{array}{lllllllll}0117 & \mathrm{O} & 0.30231 & 0.38938 & 0.15052 & 0.00000 & \text { Uiso } & 1.00\end{array}$ $\begin{array}{lllllllll}0118 & \mathrm{O} & 0.30227 & 0.38186 & 0.63800 & 0.00000 & \text { Uiso } & 1.00\end{array}$ $\begin{array}{llllllll}0119 & \mathrm{O} & 0.30542 & 0.05556 & 0.13945 & 0.00000 & \text { Uiso } & 1.00\end{array}$ $\begin{array}{lllllllll}0120 & 0 & 0.30485 & 0.05596 & 0.63908 & 0.00000 & \text { Uiso } & 1.00\end{array}$ $\begin{array}{llllllllllllllll}0121 & 0 & 0.30411 & 0.55981 & 0.14353 & 0.00000 & \text { Uiso } & 1.00\end{array}$ $\begin{array}{llllllllll}0121 & 0 & 0.3011 & 0.55981 & 0.14353 & 0.00000 & \text { Uiso } & 1.00\end{array}$ $\begin{array}{lllllllll}0122 & 0 & 0.30071 & 0.55968 & 0.6395 & 0.00000 & \text { Uiso } & 1.00\end{array}$ $\begin{array}{lllllllllll}0123 & \mathrm{O} & 0.30656 & 0.22082 & 0.15038 & 0.00000 & \text { Uiso } & 1.00\end{array}$ $\begin{array}{llllllllll}0124 & 0 & 0.31013 & 0.21897 & 0.64666 & 0.00000 & \text { Uiso } & 1.00 \\ 0125 & 0 & 0.31004 & 0.71788 & 0.13568 & 0.00000 & \text { Uiso } & 1.00\end{array}$ $\begin{array}{llllllllll}0125 & 0 & 0.31004 & 0.71788 & 0.13568 & 0.00000 & \text { Uiso } & 1.00\end{array}$ $\begin{array}{llllllllll}0126 & 0 & 0.30664 & 0.72450 & 0.63800 & 0.00000 & \text { Uiso } & 1.00\end{array}$ $\begin{array}{lllllllll}0127 & \mathrm{O} & 0.35910 & 0.14353 & 0.00354 & 0.00000 & \text { Uiso } & 1.00\end{array}$ $\begin{array}{lllllllll}0128 & \mathrm{O} & 0.35686 & 0.64480 & -0.00155 & 0.00000 & \text { Uiso } & 1.00\end{array}$ $\begin{array}{lllllllll}0129 & \mathrm{O} & 0.36594 & 0.81016 & 0.01492 & 0.00000 & \text { Uiso } & 1.00\end{array}$ $\begin{array}{lllllllll}0130 & \mathrm{O} & 0.35986 & 0.77773 & 0.52099 & 0.00000 & \text { Uiso } & 1.00\end{array}$ $\begin{array}{llllllll}0131 & \mathrm{O} & 0.34496 & 0.30499 & 0.78773 & 0.00000 & \text { Uiso } & 1.00\end{array}$ $\begin{array}{llllllll}0132 & \mathrm{O} & 0.35332 & 0.30402 & 0.52042 & 0.00000 & \text { Uiso } & 1.00\end{array}$ $\begin{array}{lllllllll}0133 & \mathrm{O} & 0.35075 & 0.96789 & 0.99187 & 0.00000 & \text { Uiso } & 1.00\end{array}$ $\begin{array}{llllllllll}0134 & \mathrm{O} & 0.34957 & 0.95680 & 0.49369 & 0.00000 & \text { Uiso } & 1.00\end{array}$ $\begin{array}{lllllllll}0135 & \mathrm{O} & 0.34838 & 0.47839 & 0.00253 & 0.00000 & \text { Uiso } & 1.00\end{array}$ $\begin{array}{llllllll}0136 & \mathrm{O} & 0.34954 & 0.48239 & 0.52070 & 0.00000 & \text { Uiso } & 1.00\end{array}$ $\begin{array}{llllllll}0137 & \mathrm{O} & 0.20714 & 0.89400 & 0.88748 & 0.00000 & \text { Uiso } & 1.00\end{array}$ $\begin{array}{lllllllll}0138 & \mathrm{O} & 0.20694 & 0.89345 & 0.38613 & 0.00000 & \text { Uiso } & 1.00\end{array}$ $\begin{array}{llllllll}0139 & \mathrm{O} & 0.20580 & 0.39208 & 0.88554 & 0.00000 & \text { Uiso } & 1.00\end{array}$ $\begin{array}{lllllllllll}0140 & 0 & 0.20555 & 0.39227 & 0.38447 & 0.00000 & \text { Uiso } & 1.00\end{array}$ $\begin{array}{lllllllllllll}0141 & 0 & 0.20727 & 0.04999 & 0.88889 & 0.00000 & \text { Uiso } & 1.00\end{array}$ $\begin{array}{llllllllll}0141 & 0 & 0.20727 & 0.0492 & 0.88889 & 0.00000 & \text { Uiso } & 1.00\end{array}$ $\begin{array}{lllllllll}0143 & 0 & 0.2070574 & 0.04981 & 0.38642 & 0.00000 & \text { Uiso } & 1.00\end{array}$ $\begin{array}{llllllll}0143 & \mathrm{O} & 0.20574 & 0.54772 & 0.88445 & 0.00000 & \text { Uiso } & 1.00 \\ 0144 & \mathrm{O} & 0.20531 & 0.54768 & 0.38375 & 0.00000 & \text { Uiso } & 1.00\end{array}$ $\begin{array}{llllllllll}0144 & 0 & 0.20531 & 0.54768 & 0.38375 & 0.00000 & \text { Uiso } & 1.00 \\ 0145 & 0 & 0.21095 & 0.22036 & 0.88651 & 0.0000 & \text { Uiso } & 1.00\end{array}$ $\begin{array}{llllllllll}0145 & \mathrm{O} & 0.21095 & 0.22036 & 0.88651 & 0.00000 & \text { Uiso } & 1.00 \\ 0146 & \mathrm{O} & 0.21090 & 0.22062 & 0.3849 & 0.0000 & \text { Uiso } & 1.00\end{array}$ $\begin{array}{lllllllllll}0146 & 0 & 0.21090 & 0.22062 & 0.38494 & 0.00000 & \text { Uiso } & 1.00\end{array}$ $\begin{array}{lllllllll}0147 & \mathrm{O} & 0.21040 & 0.72136 & 0.88361 & 0.00000 & \text { Uiso } & 1.00\end{array}$ $\begin{array}{lllllllll}0148 & \mathrm{O} & 0.21029 & 0.72180 & 0.38227 & 0.00000 & \text { Uiso } & 1.00\end{array}$ $\begin{array}{lllllllll}0149 & \mathrm{O} & 0.26215 & 0.13518 & 0.25359 & 0.00000 & \text { Uiso } & 1.00\end{array}$ $\begin{array}{llllllll}0150 & \mathrm{O} & 0.26141 & 0.13773 & 0.74601 & 0.00000 & \text { Uiso } & 1.00\end{array}$ $\begin{array}{llllllll}0151 & \mathrm{O} & 0.25707 & 0.63592 & 0.24359 & 0.00000 & \text { Uiso } & 1.00\end{array}$ $\begin{array}{llllllll}0152 & \mathrm{O} & 0.25710 & 0.63721 & 0.74183 & 0.00000 & \text { Uiso } & 1.00\end{array}$ $\begin{array}{llllllll}\mathrm{O} 153 & \mathrm{O} & 0.26170 & 0.80400 & 0.24292 & 0.00000 & \text { Uiso } & 1.00\end{array}$ $\begin{array}{lllllllll}0154 & \mathrm{O} & 0.25846 & 0.80735 & 0.74601 & 0.00000 & \text { Uiso } & 1.00\end{array}$ $\begin{array}{llllllll}0155 & \mathrm{O} & 0.26074 & 0.30524 & 0.25416 & 0.00000 & \text { Uiso } & 1.00\end{array}$ $\begin{array}{lllllllll}0156 & \mathrm{O} & 0.25984 & 0.30500 & 0.75079 & 0.00000 & \text { Uiso } & 1.00\end{array}$ $\begin{array}{llllllll}0157 & \mathrm{O} & 0.25642 & 0.96839 & 0.25014 & 0.00000 & \text { Uiso } & 1.00\end{array}$ $\begin{array}{llllllll}0158 & \mathrm{O} & 0.25570 & 0.97382 & 0.75027 & 0.00000 & \text { Uiso } & 1.00\end{array}$ $\begin{array}{llllllllll}0159 & \mathrm{O} & 0.25338 & 0.47019 & 0.24795 & 0.00000 & \text { Uiso } & 1.00\end{array}$ $\begin{array}{lllllllll}0160 & \mathrm{O} & 0.25437 & 0.47031 & 0.74746 & 0.00000 & \text { Uiso } & 1.00\end{array}$ $\begin{array}{llllllllll}0161 & 0 & 0.21592 & 0.89298 & 0.12150 & 0.00000 & \text { Uiso } & 1.00\end{array}$ $\begin{array}{llllllllll}0161 & 0 & 0.21592 & 0.89298 & 0.12150 & 0.00000 & \text { Uiso } & 1.00\end{array}$ $\begin{array}{lllllllll}0162 & \mathrm{O} & 0.21497 & 0.89454 & 0.62121 & 0.00000 & \text { Uiso } & 1.00 \\ 0163 & 0 & 0.21420 & 0.39216 & 0.12082 & 0.00000 & \text { Uiso } & 1.00\end{array}$ $\begin{array}{llllllll}0163 & \mathrm{O} & 0.21420 & 0.39216 & 0.12082 & 0.00000 & \text { Uiso } & 1.00 \\ 0164 & \mathrm{O} & 0.21407 & 0.39193 & 0.62043 & 0.000000 & \text { Uiso } & 1.00\end{array}$ $\begin{array}{lllllllll}0164 & \mathrm{O} & 0.21407 & 0.39193 & 0.62043 & 0.00000 & \text { Uiso } & 1.00 \\ 0165 & \mathrm{O} & 0.21590 & 0.0452 & 0.12485 & 0.00000 & \text { Uis } & 1.00\end{array}$ $\begin{array}{llllllllll}0165 & \mathrm{O} & 0.21590 & 0.04852 & 0.12485 & 0.00000 & \text { Uiso } & 1.00\end{array}$ $\begin{array}{lllllllll}0166 & \mathrm{O} & 0.21583 & 0.04991 & 0.62089 & 0.00000 & \text { Uiso } & 1.00\end{array}$ $\begin{array}{lllllllll}0167 & 0 & 0.21366 & 0.54797 & 0.11865 & 0.00000 & \text { Uiso } & 1.00 \\ 0168 & 0 & 0.21380 & 0.54798 & 0.61841 & 0.00000 & \text { Uis } & 1.00\end{array}$ $\begin{array}{lllllllll}0168 & \mathrm{O} & 0.21380 & 0.54798 & 0.61841 & 0.00000 & \text { Uiso } & 1.00\end{array}$ $\begin{array}{lllllllll}0169 & \mathrm{O} & 0.20848 & 0.21989 & 0.12777 & 0.00000 & \text { Uiso } & 1.00\end{array}$ $\begin{array}{llllllll}0170 & \mathrm{O} & 0.20818 & 0.22094 & 0.62578 & 0.00000 & \text { Uiso } & 1.00\end{array}$ $\begin{array}{llllllll}0171 & \mathrm{O} & 0.20815 & 0.72181 & 0.12286 & 0.00000 & \text { Uiso } & 1.00\end{array}$ $\begin{array}{llllllll}0172 & \mathrm{O} & 0.20752 & 0.72163 & 0.62177 & 0.00000 & \text { Uiso } & 1.00\end{array}$ $\begin{array}{llllllll}0173 & \mathrm{O} & 0.25600 & 0.13645 & 0.01719 & 0.00000 & \text { Uiso } & 1.00\end{array}$ 
$\begin{array}{llllllll}\mathrm{O} 174 & \mathrm{O} & 0.25648 & 0.13825 & 0.51274 & 0.00000 & \text { Uiso } & 1.00\end{array}$ $\begin{array}{lllllllll}0175 & \mathrm{O} & 0.25471 & 0.63737 & 0.01157 & 0.00000 & \text { Uiso } & 1.00\end{array}$ $\begin{array}{llllllll}0176 & \mathrm{O} & 0.25487 & 0.63727 & 0.50809 & 0.00000 & \text { Uiso } & 1.00\end{array}$ $\begin{array}{llllllllll}0177 & \mathrm{O} & 0.25632 & 0.80362 & 0.01316 & 0.00000 & \text { Uiso } & 1.00\end{array}$ $\begin{array}{llllllll}0178 & 0 & 0.25525 & 0.80434 & 0.51081 & 0.00000 & \text { Uiso } & 1.00\end{array}$ $\begin{array}{llllllll}0179 & \mathrm{O} & 0.25541 & 0.30358 & 0.01769 & 0.00000 & \text { Uiso } & 1.00\end{array}$ $\begin{array}{llllllll}0180 & 0 & 0.25553 & 0.30333 & 0.51503 & 0.00000 & \text { Uiso } & 1.00\end{array}$ $\begin{array}{lllllllll}0181 & 0 & 0.25553 & 0.30333 & 0.51503 & 0.00000 & \text { Uiso } & 1.00\end{array}$

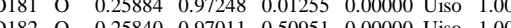
$\begin{array}{lllllllll}0182 & 0 & 0.25840 & 0.97011 & 0.5051 & 0.00000 & \text { Uiso } & 1.00\end{array}$ $\begin{array}{llllllllll}0183 & 0 & 0.25710 & 0.47055 & 0.01117 & 0.00000 & \text { Uiso } & 1.00\end{array}$ $\begin{array}{llllllll}0184 & \mathrm{O} & 0.25616 & 0.46925 & 0.51001 & 0.00000 & \text { Uiso } & 1.00 \\ 0185 & \mathrm{O} & 0.35212 & 0.29894 & 0.27115 & 0.00000 & \text { Uso } & 1.00\end{array}$ $\begin{array}{llllllllll}0185 & 0 & 0.35212 & 0.29894 & 0.27115 & 0.00000 & \text { Uiso } & 1.00 \\ 0186 & 0 & 0.39627 & 0.71350 & 0.65805 & 0.00000 & \text { Uis } & 1.00\end{array}$ $\begin{array}{llllllllll}0186 & 0 & 0.39627 & 0.71350 & 0.65805 & 0.00000 & \text { Uiso } & 1.00\end{array}$ $\begin{array}{llllllll}\mathrm{O} 187 & \mathrm{O} & 0.41511 & 0.18402 & 0.92863 & 0.00000 & \text { Uiso } & 1.00 \\ 0188 & \mathrm{O} & 0.39904 & 0.88817 & 0.86715 & 0.00000 & \text { Uis } & 1.00\end{array}$ $\begin{array}{llllllll}0188 & 0 & 0.39904 & 0.88817 & 0.86715 & 0.00000 & \text { Uiso } & 1.00\end{array}$ $\begin{array}{lllllllll}\mathrm{O} 189 & \mathrm{O} & 0.39760 & 0.38938 & 0.17623 & 0.00000 & \text { Uiso } & 1.00\end{array}$ $\begin{array}{llllllll}\mathrm{O} 190 & \mathrm{O} & 0.39436 & 0.37955 & 0.87173 & 0.00000 & \text { Uiso } & 1.00\end{array}$ $\begin{array}{lllllllll}0191 & \mathrm{O} & 0.39801 & 0.25434 & 0.71357 & 0.00000 & \text { Uiso } & 1.00\end{array}$ $\begin{array}{llllllll}0192 & \mathrm{O} & 0.40870 & 0.55811 & 0.65763 & 0.00000 & \text { Uiso } & 1.00\end{array}$ $\begin{array}{llllllll}0193 & \mathrm{O} & 0.46383 & 0.49450 & 0.70755 & 0.00000 & \text { Uiso } & 1.00\end{array}$ $\begin{array}{llllllll}0194 & \mathrm{O} & 0.40848 & 0.54523 & 0.29641 & 0.00000 & \text { Uiso } & 1.00\end{array}$ $\begin{array}{llllllll}0195 & \mathrm{O} & 0.40577 & 0.53152 & 0.06883 & 0.00000 & \text { Uiso } & 1.00\end{array}$ $\begin{array}{lllllllll}0196 & \mathrm{O} & 0.39808 & 0.23091 & 0.14785 & 0.00000 & \text { Uiso } & 1.00\end{array}$ $\begin{array}{llllllll}\mathrm{O} 197 & \mathrm{O} & 0.39557 & 0.04603 & 0.84409 & 0.00000 & \text { Uiso } & 1.00\end{array}$ $\begin{array}{llllllll}0198 & \mathrm{O} & 0.40592 & 0.07490 & 0.10790 & 0.00000 & \text { Uiso } & 1.00\end{array}$ $\begin{array}{llllllll}0199 & \mathrm{O} & 0.41419 & 0.69679 & 0.39610 & 0.00000 & \text { Uiso } & 1.00\end{array}$ $\begin{array}{llllllllll}0200 & 0 & 0.45028 & 0.32264 & 0.80263 & 0.00000 & \text { Uiso } & 1.00\end{array}$ $\begin{array}{lllllllllll}0200 & 0 & 0.45028 & 0.32264 & 0.80263 & 0.00000 & \text { Uiso } & 1.00\end{array}$

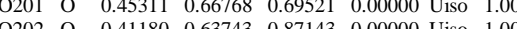
$\begin{array}{llllllllll}0202 & 0 & 0.41180 & 0.63743 & 0.87143 & 0.00000 & \text { Uiso } & 1.00\end{array}$ $\begin{array}{lllllllll}0204 & 0 & 0.44437 & 0.49255 & 0.48361 & 0.00000 & \text { Uiso } & 1.00\end{array}$ $\begin{array}{llllllllll}0204 & \mathrm{O} & 0.37027 & 0.16792 & 0.53208 & 0.00000 & \text { Uiso } & 1.00 \\ 0205 & \mathrm{O} & 0.42074 & 0.11456 & 0.65262 & 0.00000 & \text { Uis } & 1.00\end{array}$ $\begin{array}{lllllllll}0205 & \mathrm{O} & 0.42074 & 0.1456 & 0.65262 & 0.00000 & \text { Uiso } & 1.00\end{array}$ $\begin{array}{lllllllll}0206 & 0 & 0.40946 & 0.94100 & 0.64881 & 0.00000 & \text { Uiso } & 1.00\end{array}$ $\begin{array}{llllllll}0207 & 0 & 0.39799 & 0.87062 & 0.43279 & 0.00000 & \text { Uiso } & 1.00\end{array}$ $\begin{array}{lllllllll}\mathrm{O} 208 & \mathrm{O} & 0.42675 & 0.16824 & 0.41856 & 0.00000 & \text { Uiso } & 1.00\end{array}$ $\begin{array}{lllllllll}0209 & \mathrm{O} & 0.39717 & 0.03790 & 0.46093 & 0.00000 & \text { Uiso } & 1.00\end{array}$

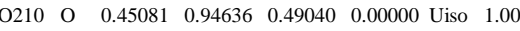
$\begin{array}{llllllll}0211 & \mathrm{O} & 0.40786 & 0.91443 & 0.10255 & 0.00000 & \text { Uiso } & 1.00\end{array}$

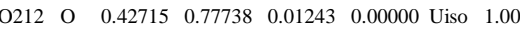
$\begin{array}{lllllllll}0213 & \mathrm{O} & 0.42929 & 0.99462 & 0.27714 & 0.00000 & \text { Uiso } & 1.00\end{array}$ $\begin{array}{llllllll}\mathrm{O} 214 & \mathrm{O} & 0.39051 & 0.67875 & 0.17060 & 0.00000 & \text { Uiso } & 1.00\end{array}$ $\begin{array}{llllllll}0215 & \mathrm{O} & 0.36399 & 0.98405 & 0.23827 & 0.00000 & \text { Uiso } & 1.00\end{array}$

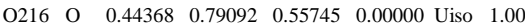
$\begin{array}{llllllll}\mathrm{Si} 217 & \mathrm{Si} & 0.39233 & 0.96161 & 0.77246 & 0.00000 & \text { Uiso } & 1.00\end{array}$ $\begin{array}{llllllll}\mathrm{Si} 218 \mathrm{Si} & 0.41351 & 0.34037 & 0.76463 & 0.00000 & \text { Uiso } & 1.00\end{array}$

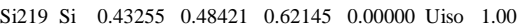
$\begin{array}{llllllll}\mathrm{Si} 220 & \mathrm{Si} & 0.39308 & 0.48663 & 0.18630 & 0.00000 & \text { Uiso } & 1.00\end{array}$ $\begin{array}{lllllllll} & \mathrm{Si} 221 & \mathrm{Si} & 0.39600 & 0.15932 & 0.04873 & 0.00000 & \text { Uiso } & 1.00\end{array}$ $\begin{array}{llllllllll} & \text { Si222 Si } & 0.39421 & 0.64021 & 0.31010 & 0.00000 & \text { Uiso } & 1.00\end{array}$

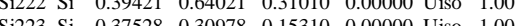
$\begin{array}{lllllll} & \text { Si223 Si } 0.37528 & 0.30978 & 0.15310 & 0.00000 & \text { Uiso } & 1.00\end{array}$ $\begin{array}{lllllll}\text { Si224 Si } & 0.41611 & 0.64252 & 0.72839 & 0.00000 & \text { Uiso } & 1.00 \\ \text { Si225 Si } & 0.41216 & 0.94890 & 0.50182 & 0.00000 & \text { Uiso } & 1.00\end{array}$ $\begin{array}{llllll} & \text { Si225 Si } & 0.41216 & 0.94890 & 0.50182 & 0.00000 \\ \text { Siso } & 1.00\end{array}$ $\begin{array}{lllllllll}\mathrm{Si226} & \mathrm{Si} & 0.40522 & 0.12684 & 0.52364 & 0.00000 & \text { Uiso } & 1.00\end{array}$ $\begin{array}{lllllllll}S i 227 & \text { Si } & 0.40020 & 0.99281 & 0.17995 & 0.00000 & \text { Uiso } & 1.00\end{array}$ $\begin{array}{llllllll}\mathrm{Si} 228 & \mathrm{Si} & 0.40155 & 0.84735 & 0.00242 & 0.00000 & \text { Uiso } & 1.00\end{array}$ $\begin{array}{llllllllll}\mathrm{C} 229 & \mathrm{C} & 0.49802 & 0.66632 & 0.14198 & 0.00000 & \text { Uiso } & 1.00\end{array}$ $\begin{array}{llllllll}\mathrm{C} 230 & \mathrm{C} & 0.49398 & 0.57640 & 0.16611 & 0.00000 & \text { Uiso } & 1.00\end{array}$ $\begin{array}{lllllllll}\mathrm{C} 231 & \mathrm{C} & 0.52544 & 0.67787 & 0.05551 & 0.00000 & \text { Uiso } & 1.00\end{array}$ $\begin{array}{lllllllll}\mathrm{C} 232 & \mathrm{C} & 0.50533 & 0.70616 & 0.26337 & 0.00000 & \text { Uiso } & 1.00\end{array}$ $\begin{array}{llllllll}\mathrm{C} 233 & \mathrm{C} & 0.46655 & 0.69415 & 0.08829 & 0.00000 & \text { Uiso } & 1.00\end{array}$ $\begin{array}{llllllll}\mathrm{C} 234 & \mathrm{C} & 0.46136 & 0.77776 & 0.03428 & 0.00000 & \text { Uiso } & 1.00\end{array}$ $\begin{array}{llllllll}\mathrm{C} 235 & \mathrm{C} & 0.47050 & 0.84520 & 0.11894 & 0.00000 & \text { Uiso } & 1.00\end{array}$ $\begin{array}{llllllllll}\text { C236 } & \mathrm{C} & 0.47631 & 0.79034 & 0.91151 & 0.00000 & \text { Uiso } & 1.00\end{array}$

\section{File $\mathbf{n}^{\circ} 13$}

$\begin{array}{llllllll}\text { Al2 } & \mathrm{Al} & 0.35118 & 0.05661 & 0.89454 & 0.00000 & \text { Uiso } & 1.00\end{array}$ $\begin{array}{llllllll}\mathrm{Al} 3 & \mathrm{Al} & 0.35054 & 0.03692 & 0.37826 & 0.00000 & \text { Uiso } & 1.00\end{array}$

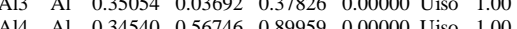
$\begin{array}{llllllllll}\mathrm{Al} 5 & \mathrm{Al} & 0.34540 & 0.56746 & 0.89959 & 0.00000 & \text { Uiso } & 1.00\end{array}$

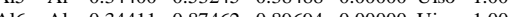
$\begin{array}{llllllll}\text { Al6 } & \text { Al } & 0.34411 & 0.87462 & 0.89694 & 0.00000 & \text { Uiso } & 1.00\end{array}$ $\begin{array}{llllllll}\mathrm{Al7} & \mathrm{Al} & 0.34964 & 0.85220 & 0.41146 & 0.00000 & \text { Uiso } & 1.00\end{array}$ $\begin{array}{lllllllll}\text { Al8 } & \text { Al } & 0.35361 & 0.40152 & 0.88455 & 0.00000 & \text { Uiso } & 1.00\end{array}$ $\begin{array}{llllllll}\text { Al9 Al } & 0.32882 & 0.38549 & 0.51913 & 0.00000 & \text { Uiso } & 1.00\end{array}$ $\begin{array}{llllllll}\mathrm{Al} 10 & \mathrm{Al} & 0.30447 & 0.80309 & 0.26366 & 0.00000 & \text { Uiso } & 1.00\end{array}$ $\begin{array}{lllllllll}\mathrm{Al11} & \mathrm{Al} & 0.30077 & 0.80705 & 0.76467 & 0.00000 & \text { Uiso } & 1.00\end{array}$ $\begin{array}{lllllllll}\mathrm{Al} 12 & \mathrm{Al} & 0.30463 & 0.30702 & 0.25967 & 0.00000 & \text { Uiso } & 1.00\end{array}$

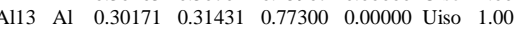
$\begin{array}{llllllll}\mathrm{Al} 14 & \mathrm{Al} & 0.30645 & 0.13419 & 0.26050 & 0.00000 & \text { Uiso } & 1.00\end{array}$ $\begin{array}{llllllll}\text { Al15 } & \text { Al } & 0.30313 & 0.13586 & 0.75840 & 0.00000 & \text { Uiso } & 1.00\end{array}$ $\begin{array}{llllllll}\text { Al16 } & \text { Al } & 0.29740 & 0.63518 & 0.26728 & 0.00000 & \text { Uiso } & 1.00\end{array}$ $\begin{array}{llllllll}\text { Al17 } & \text { Al } & 0.29913 & 0.64080 & 0.76102 & 0.00000 & \text { Uiso } & 1.00\end{array}$ $\begin{array}{lllllllll}\text { Al18 } & \text { Al } & 0.21191 & 0.97110 & 0.25381 & 0.00000 & \text { Uiso } & 1.00\end{array}$ $\begin{array}{lllllllll}\text { Al19 } & \text { Al } & 0.21176 & 0.97245 & 0.75450 & 0.00000 & \text { Uiso } & 1.00\end{array}$ $\begin{array}{llllllll}\mathrm{Al} 20 & \mathrm{Al} & 0.20935 & 0.47056 & 0.25063 & 0.00000 & \text { Uiso } & 1.00\end{array}$ $\begin{array}{lllllllll}\mathrm{Al} 21 & \mathrm{Al} & 0.21015 & 0.47068 & 0.75172 & 0.00000 & \text { Uiso } & 1.00\end{array}$ $\begin{array}{llllllllll}\mathrm{Al} 22 & \mathrm{Al} & 0.32934 & 0.96977 & 0.12968 & 0.00000 & \text { Uiso } & 1.00\end{array}$

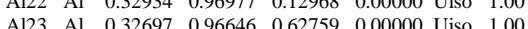
\begin{tabular}{llllllllll}
\hline & Al & 0.32453 & 0.947611 & 0.12630 & 0.00000 & Uiso & 1.00
\end{tabular}

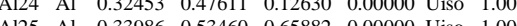
$\begin{array}{lllllllll}\text { Al25 Al } & 0.33986 & 0.53460 & 0.65882 & 0.00000 & \text { Uiso } & 1.00\end{array}$ \begin{tabular}{llllllll}
\hline Al26 & Al & 0.28083 & 0.22011 & 0.02343 & 0.00000 & Uiso & 1.00
\end{tabular} $\begin{array}{llllllll}\text { Al27 } & \text { Al } & 0.28199 & 0.22129 & 0.51628 & 0.00000 & \text { Uiso } & 1.00\end{array}$

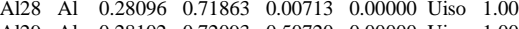
$\begin{array}{llllllll}\mathrm{Al} 29 & \mathrm{Al} & 0.28102 & 0.72093 & 0.50720 & 0.00000 & \text { Uiso } & 1.00\end{array}$ $\begin{array}{lllllllll}\mathrm{Al} 30 & \mathrm{Al} & 0.34600 & 0.23208 & 0.91654 & 0.00000 & \text { Uiso } & 1.00\end{array}$ $\begin{array}{llllllll}\text { Al31 } & \text { Al } & 0.35615 & 0.23923 & 0.65562 & 0.00000 & \text { Uiso } & 1.00\end{array}$ $\begin{array}{llllllll}\mathrm{Al} 32 & \mathrm{Al} & 0.35161 & 0.73334 & 0.12742 & 0.00000 & \text { Uiso } & 1.00\end{array}$ $\begin{array}{llllllll}\mathrm{Al} 33 & \mathrm{Al} & 0.35156 & 0.73100 & 0.66497 & 0.00000 & \text { Uiso } & 1.00\end{array}$

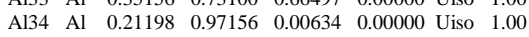


$\begin{array}{llllllll}\text { Al35 } & \text { Al } & 0.21143 & 0.97204 & 0.50418 & 0.00000 & \text { Uiso } & 1.00\end{array}$ $\begin{array}{lllllllll}\text { Al36 } & \text { Al } & 0.21023 & 0.46983 & 0.00295 & 0.00000 & \text { Uiso } & 1.00\end{array}$ $\begin{array}{llllllll}\mathrm{Al} 37 & \mathrm{Al} & 0.20960 & 0.47001 & 0.50306 & 0.00000 & \text { Uiso } & 1.00\end{array}$ $\begin{array}{llllllllll}\mathrm{A} 338 & \mathrm{Al} & 0.28282 & 0.96215 & 0.37888 & 0.00000 & \text { Uiso } & 1.00\end{array}$ $\begin{array}{lllllllll}\text { Al39 } & \text { Al } & 0.28188 & 0.97617 & 0.87928 & 0.00000 & \text { Uiso } & 1.00\end{array}$ $\begin{array}{lllllllll}\mathrm{Al} 40 & \mathrm{Al} & 0.27849 & 0.46755 & 0.37671 & 0.00000 & \text { Uiso } & 1.00\end{array}$ $\begin{array}{lllllllllll}\mathrm{Al} 41 & \mathrm{Al} & 0.28100 & 0.47512 & 0.87725 & 0.00000 & \text { Uiso } & 1.00\end{array}$ $\begin{array}{llllllll}\text { Al42 } & \text { Al } & 0.23655 & 0.21902 & 0.25537 & 0.00000 & \text { Uiso } & 1.00\end{array}$

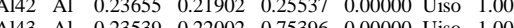
$\begin{array}{llllllll}\text { Al43 Al } & 0.23539 & 0.22002 & 0.75396 & 0.00000 & \text { Uiso } & 1.00\end{array}$ $\begin{array}{lllllllllll} & \text { Al45 } & \text { Al } & 0.23490 & 0.72406 & 0.25056 & 0.00000 & \text { Uiso } & 1.00\end{array}$ $\begin{array}{lllllllll}\text { Al45 Al } & 0.23417 & 0.72320 & 0.74999 & 0.00000 & \text { Uiso } & 1.00\end{array}$ \begin{tabular}{llllllll} 
Al46 Al & 0.26186 & 0.05810 & 0.13329 & 0.00000 & Uiso & 1.00 \\
\hline Al47 & Al & 0.26127 & 0.05827 & 0.62615 & 0.00000 & Uis & 1.00
\end{tabular} $\begin{array}{lllllllll}\text { Al47 } & \text { Al } & 0.26127 & 0.05827 & 0.62615 & 0.00000 & \text { Uiso } & 1.00\end{array}$ $\begin{array}{llllllll}\text { Al48 } & \text { Al } & 0.25841 & 0.55813 & 0.12481 & 0.00000 & \text { Uiso } & 1.00\end{array}$ $\begin{array}{lllllllll}\text { Al49 } & \text { Al } & 0.25901 & 0.55861 & 0.62456 & 0.00000 & \text { Uiso } & 1.00\end{array}$ $\begin{array}{lllllllll}\mathrm{Al} 50 & \mathrm{Al} & 0.26183 & 0.88315 & 0.12666 & 0.00000 & \text { Uiso } & 1.00\end{array}$ $\begin{array}{llllllll}\mathrm{A} 151 & \mathrm{Al} & 0.25962 & 0.88322 & 0.62709 & 0.00000 & \text { Uiso } & 1.00\end{array}$ $\begin{array}{llllllll}\mathrm{Al52} & \mathrm{Al} & 0.25952 & 0.38232 & 0.13199 & 0.00000 & \text { Uiso } & 1.00\end{array}$ $\begin{array}{llllllll}\mathrm{Al} 53 & \mathrm{Al} & 0.25925 & 0.38332 & 0.63006 & 0.00000 & \text { Uiso } & 1.00\end{array}$ $\begin{array}{llllllll}\mathrm{Al} 54 & \mathrm{Al} & 0.21396 & 0.80723 & 0.00144 & 0.00000 & \text { Uiso } & 1.00\end{array}$ $\begin{array}{llllllll}\mathrm{Al} 55 & \mathrm{Al} & 0.21308 & 0.80675 & 0.50080 & 0.00000 & \text { Uiso } & 1.00\end{array}$ $\begin{array}{llllllll}\text { Al56 } & \mathrm{Al} & 0.21299 & 0.30593 & 0.00341 & 0.00000 & \text { Uiso } & 1.00\end{array}$ $\begin{array}{llllllll}\mathrm{Al} 57 & \mathrm{Al} & 0.21299 & 0.30600 & 0.50084 & 0.00000 & \text { Uiso } & 1.00\end{array}$ $\begin{array}{llllllll}\text { Al58 } & \mathrm{Al} & 0.21369 & 0.13512 & 0.00596 & 0.00000 & \text { Uiso } & 1.00\end{array}$ $\begin{array}{lllllllll}\mathrm{Al} 59 & \mathrm{Al} & 0.21368 & 0.13572 & 0.50169 & 0.00000 & \text { Uiso } & 1.00\end{array}$ $\begin{array}{lllllllll}\mathrm{Al} 60 & \mathrm{Al} & 0.21270 & 0.63517 & 0.99915 & 0.00000 & \text { Uiso } & 1.00\end{array}$ $\begin{array}{lllllllll}A 161 & \text { Al } & 0.21272 & 0.63523 & 0.49872 & 0.00000 & \text { Uiso } & 1.00\end{array}$ \begin{tabular}{lllllllll}
\hline 161 & Al & 0.21272 & 0.63523 & 0.49872 & 0.00000 & Uiso & 1.00
\end{tabular}

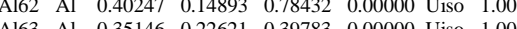
$\begin{array}{lllllllll}163 & \text { Al } & 0.35146 & 0.22621 & 0.39783 & 0.00000 & \text { Uiso } & 1.00\end{array}$ $\begin{array}{llllllll}\text { A164 Al } & 0.39867 & 0.64549 & 0.01221 & 0.00000 & \text { Uiso } & 1.00\end{array}$ $\begin{array}{lllllllll}165 & \text { Al } & 0.40216 & 0.76638 & 0.50612 & 0.00000 & \text { Uiso } & 1.00\end{array}$ $\begin{array}{lllllllll}066 & \mathrm{O} & 0.30820 & 0.87817 & 0.38335 & 0.00000 & \text { Uiso } & 1.00\end{array}$ $\begin{array}{llllllllll}067 & \mathrm{O} & 0.30372 & 0.88782 & 0.88192 & 0.00000 & \text { Uiso } & 1.00\end{array}$ $\begin{array}{lllllllll}068 & \mathrm{O} & 0.30662 & 0.38890 & 0.38465 & 0.00000 & \text { Uiso } & 1.00\end{array}$ $\begin{array}{llllllll}069 & \mathrm{O} & 0.30686 & 0.39398 & 0.88103 & 0.00000 & \text { Uiso } & 1.00\end{array}$ $\begin{array}{lllllllll}070 & \mathrm{O} & 0.30868 & 0.04425 & 0.36912 & 0.00000 & \text { Uiso } & 1.00\end{array}$ $\begin{array}{lllllllll}071 & \mathrm{O} & 0.30930 & 0.05825 & 0.87203 & 0.00000 & \text { Uiso } & 1.00\end{array}$ $\begin{array}{lllllllll}072 & \mathrm{O} & 0.30403 & 0.55067 & 0.37087 & 0.00000 & \text { Uiso } & 1.00\end{array}$ $\begin{array}{lllllllll}073 & \mathrm{O} & 0.30374 & 0.56312 & 0.87970 & 0.00000 & \text { Uiso } & 1.00\end{array}$ $\begin{array}{lllllllll}074 & \mathrm{O} & 0.30767 & 0.22401 & 0.38678 & 0.00000 & \text { Uiso } & 1.00\end{array}$ $\begin{array}{llllllll}075 & \mathrm{O} & 0.30461 & 0.22016 & 0.88832 & 0.00000 & \text { Uiso } & 1.00\end{array}$ $\begin{array}{lllllllll}076 & \mathrm{O} & 0.30187 & 0.71578 & 0.37075 & 0.00000 & \text { Uiso } & 1.00\end{array}$ $\begin{array}{lllllllll}077 & \mathrm{O} & 0.30046 & 0.72188 & 0.87072 & 0.00000 & \text { Uiso } & 1.00\end{array}$ $\begin{array}{lllllllll}078 & \mathrm{O} & 0.35187 & 0.13470 & 0.31167 & 0.00000 & \text { Uiso } & 1.00\end{array}$ $\begin{array}{lllllllll}079 & \mathrm{O} & 0.35605 & 0.15271 & 0.78245 & 0.00000 & \text { Uiso } & 1.00\end{array}$ $\begin{array}{llllllll}\mathrm{O} 80 & \mathrm{O} & 0.36089 & 0.62507 & 0.38877 & 0.00000 & \text { Uiso } & 1.00\end{array}$ $\begin{array}{llllllll}\mathrm{O} 81 & \mathrm{O} & 0.34631 & 0.63135 & 0.73471 & 0.00000 & \text { Uiso } & 1.00\end{array}$ $\begin{array}{lllllllllll}082 & \mathrm{O} & 0.34870 & 0.79974 & 0.26501 & 0.00000 & \text { Uiso } & 1.00\end{array}$ $\begin{array}{lllllllll}082 & 0 & 0.3487 & 0.75974 & 0.26501 & 0.00000 & \text { Uiso } & 1.00\end{array}$ $\begin{array}{lllllllll}084 & 0 & 0.34545 & 0.80562 & 0.77462 & 0.00000 & \text { Uiso } & 1.00\end{array}$ $\begin{array}{llllllll}084 & \mathrm{O} & 0.35606 & 0.31136 & 0.02176 & 0.00000 & \text { Uiso } & 1.00\end{array}$ $\begin{array}{llllllll}085 & \mathrm{O} & 0.41561 & 0.39468 & 0.64054 & 0.00000 & \text { Uiso } & 1.00 \\ 086 & \mathrm{O} & 0.35358 & 0.96393 & 0.75707 & 0.00000 & \text { Us } & 1.00\end{array}$ $\begin{array}{lllllllll}086 & 0 & 0.35358 & 0.96393 & 0.75707 & 0.00000 & \text { Uiso } & 1.00\end{array}$ \begin{tabular}{llllllll}
087 & $\mathrm{O}$ & 0.35519 & 0.49313 & 0.23777 & 0.00000 & Uiso & 1.00 \\
\hline 888 & $\mathrm{O}$ & 0.35462 & 0.48736 & 0.78434 & 0.00000 & Uiso & 1.00
\end{tabular} $\begin{array}{lllllllll}088 & \mathrm{O} & 0.35462 & 0.48736 & 0.78434 & 0.00000 & \text { Uiso } & 1.00\end{array}$ $\begin{array}{llllllll}089 & \mathrm{O} & 0.30464 & 0.88703 & 0.14405 & 0.00000 & \text { Uiso } & 1.00\end{array}$ $\begin{array}{llllllll}\mathrm{O} 90 & \mathrm{O} & 0.30160 & 0.88669 & 0.64312 & 0.00000 & \text { Uiso } & 1.00\end{array}$ $\begin{array}{llllllll}\mathrm{O} 91 & \mathrm{O} & 0.30248 & 0.38982 & 0.14986 & 0.00000 & \text { Uiso } & 1.00\end{array}$ $\begin{array}{lllllllll}\mathrm{O} 92 & \mathrm{O} & 0.30227 & 0.38118 & 0.63791 & 0.00000 & \text { Uiso } & 1.00\end{array}$ $\begin{array}{llllllll}093 & \mathrm{O} & 0.30548 & 0.05549 & 0.13906 & 0.00000 & \text { Uiso } & 1.00\end{array}$ $\begin{array}{llllllll}094 & \mathrm{O} & 0.30484 & 0.05574 & 0.63912 & 0.00000 & \text { Uiso } & 1.00\end{array}$ $\begin{array}{llllllll}\mathrm{O} 95 & \mathrm{O} & 0.30093 & 0.56005 & 0.14281 & 0.00000 & \text { Uiso } & 1.00\end{array}$ $\begin{array}{llllllllll}096 & \mathrm{O} & 0.30062 & 0.55890 & 0.63995 & 0.00000 & \text { Uiso } & 1.00\end{array}$ $\begin{array}{llllllll}\mathrm{O} 97 & \mathrm{O} & 0.30669 & 0.22108 & 0.14987 & 0.00000 & \text { Uiso } & 1.00\end{array}$ $\begin{array}{lllllllll}098 & \mathrm{O} & 0.31023 & 0.21842 & 0.64623 & 0.00000 & \text { Uiso } & 1.00\end{array}$ $\begin{array}{lllllllll}099 & \mathrm{O} & 0.30937 & 0.71722 & 0.13753 & 0.00000 & \text { Uiso } & 1.00\end{array}$ $\begin{array}{llllllll}\mathrm{O} 100 & \mathrm{O} & 0.30656 & 0.72386 & 0.63819 & 0.00000 & \text { Uiso } & 1.00 \\ \mathrm{O} 101 & \mathrm{O} & 0.35936 & 0.14351 & 0.00350 & 0.00000 & \text { Uiso } & 1.00\end{array}$ $\begin{array}{lllllllll}0102 & 0 & 0.35762 & 0.65026 & 0.99194 & 0.00000 & \text { Uiso } & 1.00\end{array}$ $\begin{array}{lllllllllll}0102 & 0 & 0.35762 & 0.65026 & 0.9194 & 0.00000 & \text { Uiso } & 1.00 \\ 0103 & 0 & 0.36177 & 0.81390 & 0.01978 & 0.0000 & \text { Uiso } & 1.00\end{array}$ $\begin{array}{llllllllll}0103 & \mathrm{O} & 0.36177 & 0.81390 & 0.01978 & 0.00000 & \text { Uiso } & 1.00\end{array}$ $\begin{array}{lllllllll}0104 & 0 & 0.35979 & 0.77919 & 0.52257 & 0.00000 & \text { Uiso } & 1.00\end{array}$ $\begin{array}{lllllllll}0105 & \mathrm{O} & 0.34516 & 0.30444 & 0.78689 & 0.00000 & \text { Uiso } & 1.00 \\ 0106 & \mathrm{O} & 0.35352 & 0.30285 & 0.51967 & 0.0000 & \text { Uiso } & 1.00\end{array}$ $\begin{array}{lllllllll}0106 & \mathrm{O} & 0.35352 & 0.30285 & 0.51967 & 0.00000 & \text { Uiso } & -1.00\end{array}$ $\begin{array}{lllllllll}0107 & \mathrm{O} & 0.35068 & 0.96862 & 0.99178 & 0.00000 & \text { Uiso } & 1.00 \\ 0108 & \mathrm{O} & 0.35001 & 0.9582 & 0.49383 & 0.0000 & \text { Uiso } & 1.00\end{array}$ $\begin{array}{llllllllll}0108 & \mathrm{O} & 0.35001 & 0.95782 & 0.49383 & 0.00000 & \text { Uiso } & 1.00\end{array}$ $\begin{array}{lllllllll}0109 & 0 & 0.34979 & 0.47962 & 0.00402 & 0.00000 & \text { Uiso } & 1.00\end{array}$ $\begin{array}{lllllllll}0110 & \mathrm{O} & 0.34930 & 0.47987 & 0.52203 & 0.00000 & \text { Uiso } & 1.00\end{array}$ $\begin{array}{llllllll}0111 & \mathrm{O} & 0.20714 & 0.89410 & 0.88748 & 0.00000 & \text { Uiso } & 1.00\end{array}$ $\begin{array}{lllllllll}0112 & \mathrm{O} & 0.20694 & 0.89349 & 0.38613 & 0.00000 & \text { Uiso } & 1.00\end{array}$ $\begin{array}{llllllll}0113 & \mathrm{O} & 0.20580 & 0.39218 & 0.88554 & 0.00000 & \text { Uiso } & 1.00\end{array}$ $\begin{array}{llllllll}0114 & \mathrm{O} & 0.20555 & 0.39232 & 0.38447 & 0.00000 & \text { Uiso } & 1.00\end{array}$

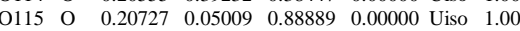
$\begin{array}{lllllllll}0116 & \mathrm{O} & 0.20705 & 0.04986 & 0.38642 & 0.00000 & \text { Uiso } & 1.00\end{array}$ $\begin{array}{lllllllll}0117 & \mathrm{O} & 0.20574 & 0.54782 & 0.88445 & 0.00000 & \text { Uiso } & 1.00\end{array}$ $\begin{array}{lllllllll}0118 & \mathrm{O} & 0.20531 & 0.54773 & 0.38375 & 0.00000 & \text { Uiso } & 1.00\end{array}$ $\begin{array}{llllllll}0119 & \mathrm{O} & 0.21095 & 0.22046 & 0.88651 & 0.00000 & \text { Uiso } & 1.00\end{array}$ $\begin{array}{lllllllll}0120 & 0 & 0.21090 & 0.22066 & 0.38494 & 0.00000 & \text { Uiso } & 1.00\end{array}$

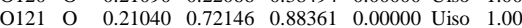
$\begin{array}{lllllllll}0122 & \mathrm{O} & 0.21029 & 0.72184 & 0.38227 & 0.00000 & \text { Uiso } & 1.00\end{array}$ $\begin{array}{llllllllll}0123 & 0 & 0.26215 & 0.13521 & 0.25359 & 0.00000 & \text { Uiso } & 1.00\end{array}$ $\begin{array}{llllllllll}0124 & 0 & 0.26141 & 0.13781 & 0.74601 & 0.00000 & \text { Uiso } & 1.00\end{array}$ $\begin{array}{lllllllllll}0125 & 0 & 0.25707 & 0.63595 & 0.24359 & 0.00000 & \text { Uiso } & 1.00\end{array}$ $\begin{array}{llllllll}0125 & 0 & 0.25707 & 0.6359 & 0.24359 & 0.00000 & \text { Uiso } & 1.00\end{array}$ $\begin{array}{lllllllllll}0126 & \mathrm{O} & 0.25710 & 0.63729 & 0.74183 & 0.00000 & \text { Uiso } & 1.00\end{array}$ $\begin{array}{lllllllll}0127 & \mathrm{O} & 0.26170 & 0.80403 & 0.24292 & 0.00000 & \text { Uiso } & 1.00\end{array}$ $\begin{array}{llllllllll}0128 & 0 & 0.25846 & 0.80743 & 0.74601 & 0.00000 & \text { Uiso } & 1.00\end{array}$ $\begin{array}{lllllllll}0129 & 0 & 0.26074 & 0.30527 & 0.25416 & 0.00000 & \text { Uiso } & 1.00 \\ 0130 & 0 & 0.2584 & 0.30508 & 0.75079 & 0.0000 & \text { Uns } & 1.00\end{array}$ $\begin{array}{lllllllll}0130 & \mathrm{O} & 0.25984 & 0.30508 & 0.75079 & 0.00000 & \text { Uiso } & 1.00\end{array}$ $\begin{array}{llllllll}0131 & \mathrm{O} & 0.25642 & 0.96842 & 0.25014 & 0.00000 & \text { Uiso } & 1.00\end{array}$ $\begin{array}{lllllllll}0132 & \mathrm{O} & 0.25571 & 0.97390 & 0.75027 & 0.00000 & \text { Uiso } & 1.00\end{array}$ $\begin{array}{lllllllll}\mathrm{O} 133 & \mathrm{O} & 0.25338 & 0.47022 & 0.24795 & 0.00000 & \text { Uiso } & 1.00 \\ \mathrm{O} 134 & \mathrm{O} & 0.25437 & 0.47040 & 0.74746 & 0.00000 & \text { Uiso } & 1.00\end{array}$ $\begin{array}{llllllll}\mathrm{O} 135 & \mathrm{O} & 0.21592 & 0.89300 & 0.12150 & 0.00000 & \text { Uiso } & 1.00\end{array}$ 
$\begin{array}{llllllll}0136 & \mathrm{O} & 0.21497 & 0.89461 & 0.62121 & 0.00000 & \text { Uiso } & 1.00\end{array}$ $\begin{array}{llllllll}0137 & \mathrm{O} & 0.21420 & 0.39217 & 0.12082 & 0.00000 & \text { Uiso } & 1.00\end{array}$ $\begin{array}{llllllll}0138 & \mathrm{O} & 0.21407 & 0.39200 & 0.62043 & 0.00000 & \text { Uiso } & 1.00\end{array}$ $\begin{array}{llllllll}0139 & \mathrm{O} & 0.21590 & 0.04853 & 0.12485 & 0.00000 & \text { Uiso } & 1.00\end{array}$ $\begin{array}{lllllllll}0140 & \mathrm{O} & 0.21583 & 0.04998 & 0.62089 & 0.00000 & \text { Uiso } & 1.00\end{array}$ $\begin{array}{llllllllll}0141 & 0 & 0.21366 & 0.54798 & 0.11865 & 0.00000 & \text { Uiso } & 1.00\end{array}$ $\begin{array}{llllllllll}0142 & 0 & 0.21380 & 0.54805 & 0.6184 & 0.00000 & \text { Ulsc }\end{array}$ $\begin{array}{lllllllllll}0142 & 0 & 0.21380 & 0.5480 & 0.61841 & 0.00000 & \text { Uiso } & 1.00\end{array}$ $\begin{array}{llllllllll}0144 & 0 & 0.2088 & 0.21991 & 0.12777 & 0.00000 & \text { Uiso } & 1.00\end{array}$ $\begin{array}{lllllllll}0144 & \mathrm{O} & 0.20818 & 0.22101 & 0.62578 & 0.00000 & \text { Uiso } & 1.00\end{array}$ $\begin{array}{llllllllll}0145 & \mathrm{O} & 0.20815 & 0.72182 & 0.12286 & 0.0000 & \text { Uiso } & 1.00 \\ 0146 & \mathrm{O} & 0.20752 & 0.72170 & 0.62177 & 0.00000 & \text { Uiso } & 1.00\end{array}$ $\begin{array}{lllllllll}0146 & \mathrm{O} & 0.20752 & 0.72170 & 0.62177 & 0.00000 & \text { Uiso } & 1.00 \\ 0147 & \mathrm{O} & 0.25600 & 0.13646 & 0.01719 & 0.0000 & \text { Uiso } & 1.00\end{array}$ $\begin{array}{lllllllllll}0147 & \mathrm{O} & 0.25600 & 0.13646 & 0.01719 & 0.00000 & \text { Uiso } & 1.00\end{array}$ $\begin{array}{lllllllll}0148 & \mathrm{O} & 0.25648 & 0.13831 & 0.51274 & 0.00000 & \text { Uiso } & 1.00\end{array}$ $\begin{array}{llllllll}0149 & \mathrm{O} & 0.25471 & 0.63737 & 0.01157 & 0.00000 & \text { Uiso } & 1.00 \\ 0150 & \mathrm{O} & 0.25487 & 0.63733 & 0.50809 & 0.00000 & \text { Uis } & 1.00\end{array}$ $\begin{array}{lllllllllll}0150 & 0 & 0.25487 & 0.63733 & 0.50809 & 0.00000 & \text { Uiso } & 1.00\end{array}$ $\begin{array}{lllllllll}0151 & \mathrm{O} & 0.25632 & 0.80362 & 0.01316 & 0.00000 & \text { Uiso } & 1.00\end{array}$ $\begin{array}{llllllll}0152 & \mathrm{O} & 0.25525 & 0.80440 & 0.51081 & 0.00000 & \text { Uiso } & 1.00\end{array}$ $\begin{array}{lllllllll}0153 & \mathrm{O} & 0.25541 & 0.30358 & 0.01769 & 0.00000 & \text { Uiso } & 1.00\end{array}$ $\begin{array}{llllllll}0154 & \mathrm{O} & 0.25553 & 0.30338 & 0.51503 & 0.00000 & \text { Uiso } & 1.00\end{array}$

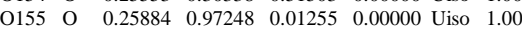
$\begin{array}{lllllllll}0156 & \mathrm{O} & 0.25840 & 0.97017 & 0.50951 & 0.00000 & \text { Uiso } & 1.00\end{array}$ $\begin{array}{llllllll}0157 & \mathrm{O} & 0.25710 & 0.47055 & 0.01117 & 0.00000 & \text { Uiso } & 1.00\end{array}$ $\begin{array}{llllllll}0158 & \mathrm{O} & 0.25616 & 0.46930 & 0.51001 & 0.00000 & \text { Uiso } & 1.00\end{array}$ $\begin{array}{lllllllll}0159 & \mathrm{O} & 0.35318 & 0.29961 & 0.26949 & 0.00000 & \text { Uiso } & 1.00\end{array}$ $\begin{array}{lllllllll}0160 & \mathrm{O} & 0.39627 & 0.71160 & 0.65503 & 0.00000 & \text { Uiso } & 1.00\end{array}$ $\begin{array}{llllllll}0161 & \mathrm{O} & 0.41567 & 0.18064 & 0.92833 & 0.00000 & \text { Uiso } & 1.00\end{array}$ $\begin{array}{lllllllllllll}0162 & 0 & 0.39840 & 0.88200 & 0.86854 & 0.00000 & \text { Uiso } & 1.00\end{array}$ $\begin{array}{lllllllll}0162 & 0 & 0.39840 & 0.88200 & 0.8685 & 0.00000 & \text { Uiso } & 1.00 \\ 0163 & 0 & 0.39699 & 0.39498 & 0.16672 & 0.00000 & \text { Uiso } & 1.00\end{array}$ $\begin{array}{llllllllll}0164 & 0 & 0.3969 & 0.3998 & 0.16672 & 0.0000 & 0.37 \text { Uso } & 1.00\end{array}$ $\begin{array}{lllllllll}0164 & 0 & 0.39491 & 0.37891 & 0.87008 & 0.00000 & \text { Unso } & 1.00\end{array}$ $\begin{array}{llllllll}0165 & \mathrm{O} & 0.39811 & 0.25215 & 0.71380 & 0.00000 & \text { Uiso } & 1.00 \\ 0166 & \mathrm{O} & 0.41002 & 0.5561 & 0.64165 & 0.00000 & \text { Uiso } & 1.00\end{array}$ $\begin{array}{lllllllll}0166 & \mathrm{O} & 0.41002 & 0.55651 & 0.64165 & 0.00000 & \text { Uiso } & 1.00\end{array}$ $\begin{array}{lllllllll}0167 & \mathrm{O} & 0.46436 & 0.48822 & 0.69383 & 0.00000 & \text { Uiso } & 1.00\end{array}$ $\begin{array}{lllllllll}0168 & 0 & 0.40821 & 0.54618 & 0.29450 & 0.00000 & \text { Unso } & 1.00\end{array}$ $\begin{array}{llllllllll}0169 & \mathrm{O} & 0.40643 & 0.54442 & 0.06724 & 0.00000 & \text { Uiso } & 1.00\end{array}$ $\begin{array}{lllllllll}0170 & \mathrm{O} & 0.39876 & 0.23165 & 0.14426 & 0.00000 & \text { Uiso } & 1.00\end{array}$ $\begin{array}{lllllllll}0171 & \mathrm{O} & 0.39554 & 0.04281 & 0.84465 & 0.00000 & \text { Uiso } & 1.00\end{array}$ $\begin{array}{llllllll}0172 & \mathrm{O} & 0.40561 & 0.07058 & 0.10616 & 0.00000 & \text { Uiso } & 1.00\end{array}$ $\begin{array}{lllllllll}0173 & \mathrm{O} & 0.41511 & 0.69761 & 0.39662 & 0.00000 & \text { Uiso } & 1.00\end{array}$

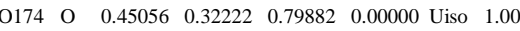
$\begin{array}{lllllllll}0175 & \mathrm{O} & 0.45363 & 0.66701 & 0.68972 & 0.00000 & \text { Uiso } & 1.00\end{array}$ $\begin{array}{llllllll}0176 & \mathrm{O} & 0.41246 & 0.62653 & 0.86044 & 0.00000 & \text { Uiso } & 1.00\end{array}$ $\begin{array}{lllllllll}0177 & \mathrm{O} & 0.44332 & 0.48279 & 0.47014 & 0.00000 & \text { Uiso } & 1.00\end{array}$ $\begin{array}{lllllllll}0178 & \mathrm{O} & 0.37005 & 0.16636 & 0.53177 & 0.00000 & \text { Uiso } & 1.00\end{array}$ $\begin{array}{lllllllll}0179 & \mathrm{O} & 0.42063 & 0.11264 & 0.65213 & 0.00000 & \text { Uiso } & 1.00\end{array}$ $\begin{array}{llllllll}0180 & \mathrm{O} & 0.40888 & 0.93985 & 0.64988 & 0.00000 & \text { Uiso } & 1.00\end{array}$ $\begin{array}{llllllll}0181 & \mathrm{O} & 0.39775 & 0.87075 & 0.43236 & 0.00000 & \text { Uiso } & 1.00\end{array}$ $\begin{array}{llllllllll}0182 & \mathrm{O} & 0.42659 & 0.17172 & 0.42106 & 0.00000 & \text { Uiso } & 1.00\end{array}$ $\begin{array}{lllllllllllllll}0182 & 0 & 0.42659 & 0.17172 & 0.42106 & 0.0000 & \text { Uiso } & 1.00\end{array}$ $\begin{array}{lllllllll}0184 & 0 & 0.3789 & 0.03810 & 0.45713 & 0.00000 & \text { Uiso } & 1.00\end{array}$ $\begin{array}{llllllllll}0185 & 0 & 0.4027 & 0.94626 & 0.49009 & 0.0000 & 0.000 & \text { Uiso } & 1.00\end{array}$ $\begin{array}{lllllllll}0185 & \mathrm{O} & 0.40926 & 0.90416 & 0.09851 & 0.00000 & \text { Uiso } & 1.00\end{array}$ $\begin{array}{lllllllll}0186 & 0 & 0.41775 & 0.75132 & 0.00970 & 0.00000 & \text { Uiso } & 1.00 \\ 0187 & 0 & 0.42992 & 0.98810 & 0.27056 & 0.00000 & \text { Uis } & 1.00\end{array}$ $\begin{array}{lllllllll}0187 & \mathrm{O} & 0.42992 & 0.98810 & 0.27056 & 0.00000 & \text { Uiso } & 1.00 \\ 0188 & \mathrm{O} & 0.38605 & 0.67739 & 0.18190 & 0.00000 & \text { Us } & 1.00\end{array}$ $\begin{array}{llllllllll}0188 & \mathrm{O} & 0.38605 & 0.67739 & 0.18190 & 0.00000 & \text { Uiso } & 1.00 \\ 0189 & \mathrm{O} & 0.36460 & 0.98070 & 0.23557 & 0.00000 & \text { Uis } & 1.00\end{array}$ $\begin{array}{llllllllllll}0189 & 0 & 0.36460 & 0.98070 & 0.23557 & 0.00000 & \text { Uiso } & 1.00\end{array}$ $\begin{array}{lllllllll}\mathrm{O} 190 & \mathrm{O} & 0.44319 & 0.79328 & 0.55943 & 0.00000 & \text { Uiso } & 1.00\end{array}$ $\begin{array}{lllllll}\text { Si191 Si } & 0.39204 & 0.95722 & 0.77573 & 0.00000 & \text { Uiso } & 1.00\end{array}$ $\begin{array}{llllllll}\mathrm{Si} 192 & \mathrm{Si} & 0.41340 & 0.33859 & 0.76236 & 0.00000 & \text { Uiso } & 1.00\end{array}$ $\begin{array}{llllllll}\text { Si193 Si } & 0.43237 & 0.47934 & 0.61008 & 0.00000 & \text { Uiso } & 1.00\end{array}$ $\begin{array}{llllllll}\text { Si194 Si } & 0.39217 & 0.49334 & 0.17784 & 0.00000 & \text { Uiso } & 1.00\end{array}$ $\begin{array}{lllllll}\text { Si195 Si } & 0.39637 & 0.15689 & 0.04905 & 0.00000 & \text { Uiso } & 1.00\end{array}$ $\begin{array}{lllllll}\text { Si196 Si } & 0.39331 & 0.64040 & 0.31744 & 0.00000 & \text { Uiso } & 1.00\end{array}$ $\begin{array}{llllllll}\text { Si197 } & \mathrm{Si} & 0.37601 & 0.31144 & 0.15008 & 0.00000 & \text { Uiso } & 1.00\end{array}$ $\begin{array}{llllllll}\text { Si198 Si } & 0.41641 & 0.63907 & 0.71852 & 0.00000 & \text { Uiso } & 1.00\end{array}$ $\begin{array}{llllllll}\text { Si199 Si } & 0.41154 & 0.94867 & 0.50310 & 0.00000 & \text { Uiso } & 1.00\end{array}$ $\begin{array}{llllllll}\mathrm{Si} 200 \mathrm{Si} & 0.40525 & 0.12655 & 0.52321 & 0.00000 & \text { Uiso } & 1.00\end{array}$ $\begin{array}{llllllll}\mathrm{Si} 201 & \mathrm{Si} & 0.40077 & 0.98490 & 0.17301 & 0.00000 & \text { Uiso } & 1.00\end{array}$ $\begin{array}{llllllll}\mathrm{Si} 202 & \mathrm{Si} & 0.39941 & 0.83477 & 0.00202 & 0.00000 & \text { Uiso } & 1.00\end{array}$ $\begin{array}{llllllll}\mathrm{H} 203 & \mathrm{H} & 0.46820 & 0.44066 & 0.74254 & 0.00000 & \text { Uiso } & 1.00\end{array}$ $\begin{array}{llllllll}\mathrm{H} 204 & \mathrm{H} & 0.45220 & 0.29182 & 0.87317 & 0.00000 & \text { Uiso } & 1.00\end{array}$ $\begin{array}{lllllllllll}1204 & \mathrm{H} & 0.45220 & 0.2182 & 0.87317 & 0.00000 & \text { Uiso } & 1.00\end{array}$

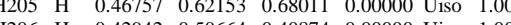
\begin{tabular}{llllllll}
$\mathrm{H} 206$ & $\mathrm{H}$ & 0.42942 & 0.50664 & 0.40874 & 0.00000 & Uiso & 1.00 \\
\hline
\end{tabular} $\begin{array}{lllllllll}\mathrm{H} 207 & \mathrm{H} & 0.43680 & 0.22100 & 0.4444 & 0.00000 & \text { Uiso } & 1.00\end{array}$ $\begin{array}{lllllllll}\mathrm{H} 208 & \mathrm{H} & 0.45520 & 0.9471 & 0.40419 & 0.00000 & \text { Uiso } & 1.00\end{array}$ $\begin{array}{lllllllll}\mathrm{H} 209 & \mathrm{H} & 0.4337 & 0.04324 & 0.29449 & 0.00000 & \text { Uiso } & 1.00 \\ \mathrm{H} 210 & \mathrm{H} & 0.45387 & 0.8479 & 0.54635 & 0.0000 & \text { Uis } & 1.00\end{array}$

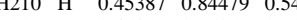
$\begin{array}{llllllll}\mathrm{H} 211 & \mathrm{H} & 0.45359 & 0.75256 & 0.61161 & 0.00000 & \text { Uiso } & 1.00\end{array}$

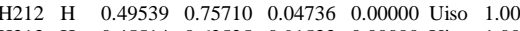
$\begin{array}{llllllll}\mathrm{H} 213 & \mathrm{H} & 0.45514 & 0.62525 & 0.91533 & 0.00000 & \text { Uiso } & 1.00\end{array}$ $\begin{array}{llllllll}\mathrm{H} 214 & \mathrm{H} & 0.48492 & 0.55409 & 0.91981 & 0.00000 & \text { Uiso } & 1.00\end{array}$ $\begin{array}{llllllll}\mathrm{H} 215 & \mathrm{H} & 0.47034 & 0.59817 & 0.24588 & 0.00000 & \text { Uiso } & 1.00\end{array}$ $\begin{array}{llllllll}\mathrm{H} 216 & \mathrm{H} & 0.48499 & 0.72810 & 0.20155 & 0.00000 & \text { Uiso } & 1.00\end{array}$ $\begin{array}{lllllllll}\mathrm{H} 217 & \mathrm{H} & 0.45631 & 0.73810 & 0.08344 & 0.00000 & \text { Uiso } & 1.00\end{array}$ $\begin{array}{lllllllll}\mathrm{H} 218 & \mathrm{H} & 0.45359 & 0.54372 & 0.12450 & 0.00000 & \text { Uiso } & 1.00\end{array}$ $\begin{array}{llllllll}\mathrm{H} 219 & \mathrm{H} & 0.49326 & 0.65884 & 0.88523 & 0.00000 & \text { Uiso } & 1.00\end{array}$ $\begin{array}{lllllllll}\mathrm{H} 220 & \mathrm{H} & 0.52434 & 0.50655 & 0.99286 & 0.00000 & \text { Uiso } & 1.00\end{array}$ $\begin{array}{llllllll}\mathrm{H} 221 & \mathrm{H} & 0.47725 & 0.42048 & 0.05020 & 0.00000 & \text { Uiso } & 1.00\end{array}$ $\begin{array}{lllllllll}\mathrm{H} 222 & \mathrm{H} & 0.46635 & 0.41175 & 0.20397 & 0.00000 & \text { Uiso } & 1.00\end{array}$ $\begin{array}{llllllll}\mathrm{H} 223 & \mathrm{H} & 0.50324 & 0.37531 & 0.15525 & 0.00000 & \text { Uiso } & 1.00\end{array}$ $\begin{array}{lllllllllll}\mathrm{H} 224 & \mathrm{H} & 0.54727 & 0.47165 & 0.11388 & 0.00000 & \text { Uiso } & 1.00\end{array}$ $\begin{array}{lllllllll}\mathrm{H} 224 & \mathrm{H} & 0.54727 & 0.4765 & 0.11388 & 0.0000 & \text { Uiso } & 1.00 \\ \mathrm{H} 225 & \mathrm{H} & 0.53980 & 0.57562 & 0.09926 & 0.00000 & \text { Uiso } & 1.00\end{array}$ $\begin{array}{llllllllll}1225 & \mathrm{H} & 0.53980 & 0.57562 & 0.11388 & 0.00000 & 0.00000 & \text { Uiso } & 1.00\end{array}$ \begin{tabular}{lllllllll}
\hline 226 & $\mathrm{H}$ & 0.52926 & 0.45964 & 0.31636 & 0.00000 & Uiso & 1.00
\end{tabular} $\begin{array}{lllllllll}\mathrm{H} 227 & \mathrm{H} & 0.49239 & 0.49791 & 0.36365 & 0.00000 & \text { Uiso } & 1.00\end{array}$ $\begin{array}{llllllllll}14228 & \mathrm{H} & 0.52301 & 0.56469 & 0.32159 & 0.00000 & \text { Uiso } & 1.00\end{array}$ $\begin{array}{llllllll}C 229 & \mathrm{C} & 0.48046 & 0.61664 & 0.34195 & 0.00000 & \text { Uiso } & 1.00\end{array}$ $\begin{array}{lllllllll}C 230 & \mathrm{C} & 0.48094 & 0.63548 & 0.06904 & 0.00000 & \text { Uiso } & 1.00\end{array}$ $\begin{array}{llllllll}\mathrm{C} 231 & \mathrm{C} & 0.48116 & 0.71859 & 0.10532 & 0.00000 & \text { Uiso } & 1.00\end{array}$ $\begin{array}{lllllllll}\mathrm{C} 232 & \mathrm{C} & 0.47530 & 0.57278 & 0.15713 & 0.00000 & \text { Uiso } & 1.00\end{array}$ $\begin{array}{llllllll}\mathrm{C} 233 & \mathrm{C} & 0.50104 & 0.50414 & 0.16990 & 0.00000 & \text { Uiso } & 1.00\end{array}$ $\begin{array}{llllllll}\mathrm{C} 234 & \mathrm{C} & 0.48599 & 0.42318 & 0.14309 & 0.00000 & \text { Uiso } & 1.00\end{array}$ $\begin{array}{lllllllll}\mathrm{C} 235 & \mathrm{C} & 0.52953 & 0.51582 & 0.08844 & 0.00000 & \text { Uiso } & 1.00\end{array}$

$\begin{array}{llllllll}\mathrm{C} 236 & \mathrm{C} & 0.51193 & 0.50699 & 0.30137 & 0.00000 & \text { Uiso } & 1.00\end{array}$ 


\section{File n ${ }^{\circ} 14$}

$\begin{array}{llllllll}\text { Al2 } & \text { Al } & 0.35095 & 0.05625 & 0.89194 & 0.00000 & \text { Uiso } & 1.00\end{array}$ $\begin{array}{llllllll}\mathrm{Al} 3 & \mathrm{Al} & 0.35065 & 0.03573 & 0.37578 & 0.00000 & \text { Uiso } & 1.00\end{array}$ $\begin{array}{llllllll}\mathrm{Al} 4 & \mathrm{Al} & 0.34899 & 0.57666 & 0.89097 & 0.00000 & \text { Uiso } & 1.00\end{array}$ $\begin{array}{llllllllll}\text { Al5 } & \text { Al } & 0.34315 & 0.53139 & 0.38215 & 0.00000 & \text { Uiso } & 1.00\end{array}$ $\begin{array}{llllllllll}\text { Al6 } & \text { Al } & 0.34457 & 0.87518 & 0.89523 & 0.00000 & \text { Uiso } & 1.00\end{array}$ $\begin{array}{llllllllllll}A 17 & \mathrm{Al} & 0.34933 & 0.85223 & 0.40954 & 0.00000 & \text { Uiso } & 1.00\end{array}$ $\begin{array}{lllllllllll}\mathrm{A} 18 & \mathrm{Al} & 0.35527 & 0.359912 & 0.88560 & 0.00000 & \text { Uiso } & 1.00\end{array}$

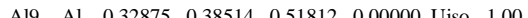
$\begin{array}{lllllllllll}A 110 & \text { Al } & 0.30426 & 0.80287 & 0.26330 & 0.00000 & \text { Uiso } & 1.00\end{array}$

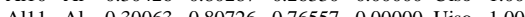
$\begin{array}{llllllll}\mathrm{A} 11 & \mathrm{Al} & 0.30063 & 0.80726 & 0.76557 & 0.00000 & \text { Uiso } & 1.00 \\ \mathrm{All} 2 & \mathrm{Al} & 0.30462 & 0.30637 & 0.25882 & 0.00000 & \text { Uiso } & 1.00\end{array}$ $\begin{array}{llllllll}\mathrm{Al13} & \mathrm{Al} & 0.30182 & 0.31595 & 0.77543 & 0.00000 & \text { Uiso } & 1.00\end{array}$ $\begin{array}{llllllll}\mathrm{All} & \mathrm{Al} & 0.30643 & 0.13377 & 0.26004 & 0.00000 & \text { Uiso } & 1.00\end{array}$ $\begin{array}{llllllll}\text { Al15 Al } & 0.30314 & 0.13597 & 0.75641 & 0.00000 & \text { Uiso } & 1.00\end{array}$ $\begin{array}{lllllll}\text { Al16 Al } & 0.29776 & 0.63497 & 0.26944 & 0.00000 & \text { Uiso } & 1.00\end{array}$ $\begin{array}{llllllll}\mathrm{Al} 17 & \mathrm{Al} & 0.29900 & 0.64023 & 0.76314 & 0.00000 & \text { Uiso } & 1.00\end{array}$ $\begin{array}{llllllll}\text { Al18 } & \mathrm{Al} & 0.21191 & 0.97110 & 0.25381 & 0.00000 & \text { Uiso } & 1.00\end{array}$ $\begin{array}{llllllll}\mathrm{Al} 19 & \mathrm{Al} & 0.21176 & 0.97245 & 0.75450 & 0.00000 & \text { Uiso } & 1.00\end{array}$ $\begin{array}{llllllll}\mathrm{Al} 20 & \mathrm{Al} & 0.20935 & 0.47056 & 0.25063 & 0.00000 & \text { Uiso } & 1.00\end{array}$ $\begin{array}{llllllll}\mathrm{Al} 21 & \mathrm{Al} & 0.21015 & 0.47068 & 0.75172 & 0.00000 & \text { Uiso } & 1.00\end{array}$ $\begin{array}{llllllll}\mathrm{Al} 22 & \mathrm{Al} & 0.32927 & 0.96962 & 0.12789 & 0.00000 & \text { Uiso } & 1.00\end{array}$ $\begin{array}{llllllll}\mathrm{Al} 23 & \mathrm{Al} & 0.32671 & 0.96628 & 0.62493 & 0.00000 & \text { Uiso } & 1.00\end{array}$ $\begin{array}{llllllllll}\mathrm{A} 124 & \mathrm{Al} & 0.32385 & 0.47562 & 0.12615 & 0.00000 & \text { Uiso } & 1.00\end{array}$

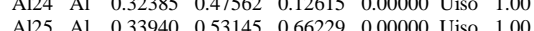
\begin{tabular}{llllllllll}
\hline & $\mathrm{A} 25$ & $\mathrm{Al}$ & 0.33940 & 0.53145 & 0.66229 & 0.00000 & Uiso & 1.00
\end{tabular} $\begin{array}{lllllll}\text { Al26 Al } & 0.28080 & 0.2200 & 0.02295 & 0.00000 & \text { Uiso } & 1.00\end{array}$ $\begin{array}{llllllll} & \text { Al27 Al } & 0.28199 & 0.22108 & 0.51564 & 0.00000 & \text { Uiso } & 1.00\end{array}$ $\begin{array}{lllllllll}\text { Al28 Al } & 0.28097 & 0.71872 & 0.00838 & 0.0000 & \text { Uiso } & 1.00\end{array}$ $\begin{array}{llllllll}\mathrm{Al} 29 & \mathrm{Al} & 0.28083 & 0.72114 & 0.50740 & 0.00000 & \text { Uiso } & 1.00\end{array}$ $\begin{array}{llllllll}\text { Al30 Al } & 0.34598 & 0.23073 & 0.91316 & 0.00000 & \text { Uiso } & 1.00\end{array}$ $\begin{array}{llllllll}\mathrm{Al} 31 & \mathrm{Al} & 0.35608 & 0.23881 & 0.65352 & 0.00000 & \text { Uiso } & 1.00\end{array}$ $\begin{array}{llllllll}\text { Al32 Al } & 0.35146 & 0.73459 & 0.12630 & 0.00000 & \text { Uiso } & 1.00\end{array}$ $\begin{array}{llllllll}\mathrm{Al} 33 & \mathrm{Al} & 0.35055 & 0.73165 & 0.66252 & 0.00000 & \text { Uiso } & 1.00\end{array}$ $\begin{array}{llllllll}\mathrm{Al} 34 & \mathrm{Al} & 0.21198 & 0.97156 & 0.00634 & 0.00000 & \text { Uiso } & 1.00\end{array}$ $\begin{array}{llllllll}\mathrm{Al} 35 & \mathrm{Al} & 0.21143 & 0.97204 & 0.50418 & 0.00000 & \text { Uiso } & 1.00\end{array}$ $\begin{array}{llllllll}\mathrm{Al} 36 & \mathrm{Al} & 0.21023 & 0.46983 & 0.00295 & 0.00000 & \text { Uiso } & 1.00\end{array}$ $\begin{array}{llllllll}\mathrm{Al} 37 & \mathrm{Al} & 0.20960 & 0.47001 & 0.50306 & 0.00000 & \text { Uiso } & 1.00\end{array}$ $\begin{array}{llllllll}\text { Al38 } & \text { Al } & 0.28274 & 0.96214 & 0.37849 & 0.00000 & \text { Uiso } & 1.00\end{array}$ $\begin{array}{lllllllll}\mathrm{Al} 39 & \mathrm{Al} & 0.28189 & 0.97627 & 0.87919 & 0.00000 & \text { Uiso } & 1.00\end{array}$

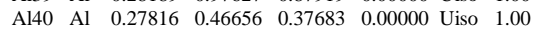
$\begin{array}{lllllllll}\mathrm{Al} 41 & \mathrm{Al} & 0.28161 & 0.47735 & 0.87798 & 0.00000 & \text { Uiso } & 1.00\end{array}$ $\begin{array}{llllllll}\mathrm{Al} 42 & \mathrm{Al} & 0.23655 & 0.21902 & 0.25537 & 0.00000 & \text { Uiso } & 1.00\end{array}$ $\begin{array}{llllllll}\mathrm{Al} 43 & \mathrm{Al} & 0.23539 & 0.22002 & 0.75396 & 0.00000 & \text { Uiso } & 1.00\end{array}$ $\begin{array}{lllllllll}\mathrm{Al} 44 & \mathrm{Al} & 0.23490 & 0.72406 & 0.25056 & 0.00000 & \text { Uiso } & 1.00\end{array}$ $\begin{array}{lllllllllll}\text { Al45 Al } & 0.23417 & 0.72320 & 0.74999 & 0.00000 & \text { Uiso } & 1.00\end{array}$ $\begin{array}{lllllllll}\mathrm{Al} 4 & \mathrm{Al} & 0.23417 & 0.72320 & 0.7498 & 0.00000 & \text { Uiso } & 1.00\end{array}$

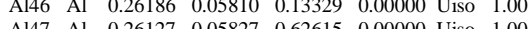
$\begin{array}{lllllllll}\text { Al47 Al } & 0.26127 & 0.05827 & 0.62615 & 0.00000 & \text { Uiso } & 1.00\end{array}$ $\begin{array}{lllllll}\text { Al48 Al } & 0.25841 & 0.55813 & 0.12481 & 0.00000 & \text { Uiso } & 1.00\end{array}$ $\begin{array}{lllllllll}\text { Al49 Al } & 0.25901 & 0.55861 & 0.62456 & 0.00000 & \text { Uiso } & 1.00\end{array}$ $\begin{array}{llllllll}\text { Al50 Al } & 0.26183 & 0.88315 & 0.12666 & 0.00000 & \text { Uiso } & 1.00\end{array}$ $\begin{array}{llllllll}\text { Al51 } & \text { Al } & 0.25962 & 0.88322 & 0.62709 & 0.00000 & \text { Uiso } & 1.00\end{array}$ $\begin{array}{llllllll}\text { Al52 Al } & 0.25952 & 0.38232 & 0.13199 & 0.00000 & \text { Uiso } & 1.00\end{array}$ $\begin{array}{llllllll}\text { Al53 } & \mathrm{Al} & 0.25925 & 0.38332 & 0.63006 & 0.00000 & \text { Uiso } & 1.00\end{array}$ $\begin{array}{llllllll}\mathrm{Al} 54 & \mathrm{Al} & 0.21396 & 0.80723 & 0.00144 & 0.00000 & \text { Uiso } & 1.00\end{array}$

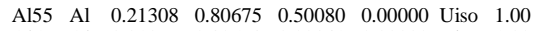

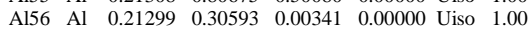

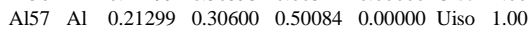
$\begin{array}{llllllll}\mathrm{Al} 58 & \mathrm{Al} & 0.21369 & 0.13512 & 0.00596 & 0.00000 & \text { Uiso } & 1.00\end{array}$ $\begin{array}{llllllll}\mathrm{Al} 59 & \mathrm{Al} & 0.21368 & 0.13572 & 0.50169 & 0.00000 & \text { Uiso } & 1.00\end{array}$

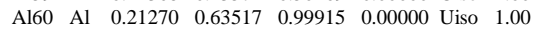
$\begin{array}{llllllll}\mathrm{Al} 61 & \mathrm{Al} & 0.21272 & 0.63523 & 0.49872 & 0.00000 & \text { Uiso } & 1.00\end{array}$ $\begin{array}{llllllll}\mathrm{Al} 62 & \mathrm{Al} & 0.40271 & 0.14853 & 0.78208 & 0.00000 & \text { Uiso } & 1.00\end{array}$ $\begin{array}{lllllllll}\text { Al63 } & \mathrm{Al} & 0.35141 & 0.22520 & 0.39640 & 0.00000 & \text { Uiso } & 1.00\end{array}$ $\begin{array}{llllllll}\mathrm{Al} 63 & \mathrm{Al} & 0.35141 & 0.22520 & 0.39640 & 0.00000 & \text { Uiso } & 1.00 \\ \mathrm{Al} 64 & \mathrm{Al} & 0.39903 & 0.65408 & 0.00629 & 0.00000 & \text { Uiso } & 1.00\end{array}$ $\begin{array}{llllllllll}\text { Al65 Al } & 0.40093 & 0.76264 & 0.50291 & 0.00000 & \text { Uiso } & 1.00\end{array}$ $\begin{array}{llllllll}\text { Al65 } & \text { Al } & 0.4073 & 0.76264 & 0.50291 & 0.00000 & \text { Uiso } & 1.00 \\ 0667 & 0 & 0.30797 & 0.87818 & 0.38238 & 0.00000 & \text { Uiso } & 1.00\end{array}$ $\begin{array}{llllllll}066 & \mathrm{O} & 0.30797 & 0.87818 & 0.38238 & 0.00000 & \text { Uiso } & 1.00 \\ 067 & \mathrm{O} & 0.30398 & 0.88828 & 0.88142 & 0.00000 & \text { Uiso } & 1.00\end{array}$

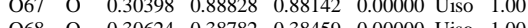

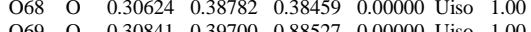
$\begin{array}{lllllllll}069 & \mathrm{O} & 0.30841 & 0.39700 & 0.88527 & 0.00000 & \text { Uiso } & 1.00 \\ 070 & 0 & 0.30873 & 0.04393 & 0.36833 & 0.00000 & \text { Uso } & 1.00\end{array}$ $\begin{array}{lllllllll}070 & \mathrm{O} & 0.30873 & 0.04393 & 0.36833 & 0.00000 & \text { Uiso } & 1.00\end{array}$ $\begin{array}{lllllllllllllllll}071 & 0 & 0.30910 & 0.05884 & 0.87085 & 0.00000 & \text { Uiso } & 1.00\end{array}$ $\begin{array}{lllllllll}\mathrm{O} 72 & \mathrm{O} & 0.30301 & 0.55005 & 0.37334 & 0.00000 & \text { Uiso } & 1.00\end{array}$ $\begin{array}{llllllll}\mathrm{O} 73 & \mathrm{O} & 0.30603 & 0.56290 & 0.88443 & 0.00000 & \text { Uiso } & 1.00\end{array}$ $\begin{array}{llllllll}\mathrm{O} 74 & \mathrm{O} & 0.30762 & 0.22322 & 0.38595 & 0.00000 & \text { Uiso } & 1.00\end{array}$ $\begin{array}{llllllll}\mathrm{O} 75 & \mathrm{O} & 0.30446 & 0.22153 & 0.88719 & 0.00000 & \text { Uiso } & 1.00\end{array}$ $\begin{array}{llllllll}\mathrm{O} 76 & \mathrm{O} & 0.30129 & 0.71663 & 0.37126 & 0.00000 & \text { Uiso } & 1.00\end{array}$ $\begin{array}{llllllll}\mathrm{O} 77 & \mathrm{O} & 0.30038 & 0.72174 & 0.87142 & 0.00000 & \text { Uiso } & 1.00\end{array}$ $\begin{array}{llllllll}078 & \text { o } & 0.35189 & 0.13379 & 0.31009 & 0.00000 & \text { Uiso } & 1.00\end{array}$

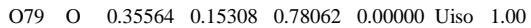
$\begin{array}{llllllll}\mathrm{O} 80 & \mathrm{O} & 0.35639 & 0.62761 & 0.36037 & 0.00000 & \text { Uiso } & 1.00\end{array}$ $\begin{array}{lllllllll}081 & \mathrm{O} & 0.34541 & 0.63081 & 0.73466 & 0.00000 & \text { Uiso } & 1.00\end{array}$ $\begin{array}{lllllllll}082 & \mathrm{O} & 0.34853 & 0.79976 & 0.26387 & 0.00000 & \text { Uiso } & 1.00\end{array}$ $\begin{array}{llllllll}\mathrm{O} 83 & \mathrm{O} & 0.34510 & 0.80536 & 0.77353 & 0.00000 & \text { Uiso } & 1.00\end{array}$ $\begin{array}{llllllll}084 & \mathrm{O} & 0.35560 & 0.31133 & 0.01737 & 0.00000 & \text { Uiso } & 1.00\end{array}$ $\begin{array}{llllllll}085 & \mathrm{O} & 0.41396 & 0.39390 & 0.63695 & 0.00000 & \text { Uiso } & 1.00\end{array}$ $\begin{array}{llllllll}\mathrm{O} 86 & \mathrm{O} & 0.35335 & 0.96551 & 0.75324 & 0.00000 & \text { Uiso } & 1.00 \\ \mathrm{O} 87 & \mathrm{O} & 0.35256 & 0.4826 & 0.24275 & 0.00000 & \text { Usis } & 1.00\end{array}$ $\begin{array}{llllllll}087 & \mathrm{O} & 0.35246 & 0.48260 & 0.24275 & 0.00000 & \text { Uiso } & 1.00\end{array}$ $\begin{array}{llllllll}088 & \mathrm{O} & 0.35517 & 0.48753 & 0.79128 & 0.00000 & \text { Uiso } & 1.00\end{array}$ $\begin{array}{llllllll}089 & \mathrm{O} & 0.30457 & 0.88708 & 0.14340 & 0.00000 & \text { Uiso } & 1.00\end{array}$ $\begin{array}{llllllll}090 & \mathrm{O} & 0.30154 & 0.88634 & 0.64264 & 0.00000 & \text { Uiso } & 1.00\end{array}$ $\begin{array}{llllllll}091 & \mathrm{O} & 0.30229 & 0.38865 & 0.14651 & 0.00000 & \text { Uiso } & 1.00\end{array}$ $\begin{array}{lllllllll}0922 & \mathrm{O} & 0.30254 & 0.38203 & 0.63937 & 0.00000 & \text { Uiso } & 1.00\end{array}$ $\begin{array}{llllllll}093 & \mathrm{O} & 0.30542 & 0.05537 & 0.13840 & 0.00000 & \text { Uiso } & 1.00\end{array}$ $\begin{array}{llllllll}\mathrm{O} 94 & \mathrm{O} & 0.30471 & 0.05571 & 0.63766 & 0.00000 & \text { Uiso } & 1.00\end{array}$ $\begin{array}{llllllll}\mathrm{O} 95 & \mathrm{O} & 0.30088 & 0.55983 & 0.14270 & 0.00000 & \text { Uiso } & 1.00\end{array}$ $\begin{array}{llllllll}\mathrm{O} 96 & \mathrm{O} & 0.30037 & 0.55714 & 0.64413 & 0.00000 & \text { Uiso } & 1.00\end{array}$ $\begin{array}{llllllll}\mathrm{O} 97 & \mathrm{O} & 0.30662 & 0.22062 & 0.14898 & 0.00000 & \text { Uiso } & 1.00 \\ \mathrm{O} 98 & \mathrm{O} & 0.31033 & 0.21840 & 0.64464 & 0.00000 & \text { Uiso } & 1.00\end{array}$ 
$\begin{array}{llllllll}099 & \mathrm{O} & 0.30915 & 0.71772 & 0.13809 & 0.00000 & \text { Uiso } & 1.00\end{array}$ $\begin{array}{lllllllll}0100 & \mathrm{O} & 0.30624 & 0.72299 & 0.63858 & 0.00000 & \text { Uiso } & 1.00\end{array}$ $\begin{array}{llllllll}\mathrm{O} 101 & \mathrm{O} & 0.35912 & 0.14276 & 0.00139 & 0.00000 & \text { Uiso } & 1.00\end{array}$ $\begin{array}{llllllll}0102 & 0 & 0.35716 & 0.65145 & 0.00121 & 0.00000 & \text { Uiso } & 1.00\end{array}$ $\begin{array}{lllllllll}0103 & \mathrm{O} & 0.36185 & 0.81489 & 0.01887 & 0.00000 & \text { Uiso } & 1.00\end{array}$ $\begin{array}{llllllllll}0104 & 0 & 0.35865 & 0.77816 & 0.51940 & 0.00000 & \text { Uiso } & 1.00\end{array}$ $\begin{array}{llllllllllll}0105 & 0 & 0.34534 & 0.30522 & 0.78541 & 0.00000 & \text { Uiso } & 1.00\end{array}$ $\begin{array}{llllllllll}0105 & 0 & 0.34534 & 0.30522 & 0.78541 & 0.00000 & \text { Uiso } & 1.00\end{array}$ $\begin{array}{llllllllllll}0106 & 0 & 0.3534 & 0.30522 & 0.78541 & 0.00000 & \text { Uiso } & 1.00 \\ 0107 & 0 & 0.35053 & 0.96923 & 0.98991 & 0.00000 & \text { Ulso } & 1.00\end{array}$ $\begin{array}{llllllllll}0107 & \mathrm{O} & 0.35053 & 0.96923 & 0.9891 & 0.00000 & \text { Uiso } & 1.00\end{array}$ $\begin{array}{lllllllll}0108 & 0 & 0.34985 & 0.95714 & 0.49153 & 0.00000 & \text { Uiso } & 1.00 \\ 0109 & 0 & 0.35737 & 0.48433 & 0.02535 & 0.00000 & \text { Uiso } & 1.00\end{array}$ $\begin{array}{lllllllll}0109 & \mathrm{O} & 0.35737 & 0.48433 & 0.02535 & 0.00000 & \text { Uiso } & 1.00 \\ 0110 & \mathrm{O} & 0.3487 & 0.4899 & 0.52227 & 0.0000 & \text { Uiso } & 1.00\end{array}$ $\begin{array}{llllllllll}0111 & \mathrm{O} & 0.34887 & 0.48099 & 0.52227 & 0.00000 & \text { Uiso } & 1.00\end{array}$ $\begin{array}{lllllllll}0111 & 0 & 0.20714 & 0.89410 & 0.88748 & 0.00000 & \text { Uiso } & 1.00\end{array}$ $\begin{array}{lllllllll}0112 & \mathrm{O} & 0.20694 & 0.89349 & 0.38613 & 0.00000 & \text { Uiso } & 1.00\end{array}$ $\begin{array}{llllllllllllll}0113 & 0 & 0.20580 & 0.39218 & 0.88554 & 0.00000 & \text { Uiso } & 1.00\end{array}$ $\begin{array}{lllllllll}0114 & \mathrm{O} & 0.20555 & 0.39232 & 0.38447 & 0.00000 & \text { Uiso } & 1.00\end{array}$ $\begin{array}{llllllll}0115 & \mathrm{O} & 0.20727 & 0.05009 & 0.88889 & 0.00000 & \text { Uiso } & 1.00\end{array}$ $\begin{array}{lllllllll}0116 & \mathrm{O} & 0.20705 & 0.04986 & 0.38642 & 0.00000 & \text { Uiso } & 1.00\end{array}$ $\begin{array}{llllllll}0117 & \mathrm{O} & 0.20574 & 0.54782 & 0.88445 & 0.00000 & \text { Uiso } & 1.00\end{array}$ $\begin{array}{lllllllll}0118 & \mathrm{O} & 0.20531 & 0.54773 & 0.38375 & 0.00000 & \text { Uiso } & 1.00\end{array}$ $\begin{array}{lllllllll}0119 & \mathrm{O} & 0.21095 & 0.22046 & 0.88651 & 0.00000 & \text { Uiso } & 1.00\end{array}$ $\begin{array}{lllllllll}0120 & \mathrm{O} & 0.21090 & 0.22066 & 0.38494 & 0.00000 & \text { Uiso } & 1.00\end{array}$ $\begin{array}{llllllll}0121 & \mathrm{O} & 0.21040 & 0.72146 & 0.88361 & 0.00000 & \text { Uiso } & 1.00\end{array}$ $\begin{array}{llllllll}0122 & \mathrm{O} & 0.21029 & 0.72184 & 0.38227 & 0.00000 & \text { Uiso } & 1.00\end{array}$ $\begin{array}{llllllll}0123 & \mathrm{O} & 0.26215 & 0.13521 & 0.25359 & 0.00000 & \text { Uiso } & 1.00\end{array}$ $\begin{array}{llllllll}0124 & \mathrm{O} & 0.26141 & 0.13781 & 0.74601 & 0.00000 & \text { Uiso } & 1.00\end{array}$ $\begin{array}{lllllllll}0125 & 0 & 0.25707 & 0.63595 & 0.24359 & 0.00000 & \text { Uiso } & 1.00\end{array}$ $\begin{array}{lllllllllllll}0126 & 0 & 0.25710 & 0.63729 & 0.74183 & 0.00000 & \text { Uiso } & 1.00\end{array}$ $\begin{array}{llllllllll}0126 & 0 & 0.25710 & 0.63729 & 0.74183 & 0.00000 & \text { Uiso } & 1.00\end{array}$ $\begin{array}{llllllllll}0127 & 0 & 0.26170 & 0.80403 & 0.24292 & 0.00000 & \text { Uiso } & 1.00\end{array}$ $\begin{array}{llllllllll}0128 & \mathrm{O} & 0.25846 & 0.80743 & 0.74601 & 0.00000 & \text { Uiso } & 1.00\end{array}$ $\begin{array}{llllllllll}0129 & 0 & 0.26074 & 0.30527 & 0.25416 & 0.00000 & \text { Uiso } & 1.00 \\ 0130 & 0 & 0.25984 & 0.30508 & 0.75079 & 0.00000 & \text { Uis } & 1.00\end{array}$ $\begin{array}{llllllllll}0130 & 0 & 0.25984 & 0.30508 & 0.75079 & 0.00000 & \text { Uiso } & 1.00\end{array}$ $\begin{array}{llllllllll}0131 & \mathrm{O} & 0.25642 & 0.96842 & 0.25014 & 0.00000 & \text { Uiso } & 1.00\end{array}$ $\begin{array}{lllllllll}0132 & 0 & 0.25571 & 0.97390 & 0.75027 & 0.00000 & \text { Uiso } & 1.00\end{array}$ $\begin{array}{llllllll}0133 & \mathrm{O} & 0.25338 & 0.47022 & 0.24795 & 0.00000 & \text { Uiso } & 1.00\end{array}$ $\begin{array}{llllllll}0134 & \mathrm{O} & 0.25437 & 0.47040 & 0.74746 & 0.00000 & \text { Uiso } & 1.00\end{array}$ $\begin{array}{llllllll}0135 & \mathrm{O} & 0.21592 & 0.89300 & 0.12150 & 0.00000 & \text { Uiso } & 1.00\end{array}$ $\begin{array}{llllllll}0136 & \mathrm{O} & 0.21497 & 0.89461 & 0.62121 & 0.00000 & \text { Uiso } & 1.00\end{array}$ $\begin{array}{llllllll}0137 & \mathrm{O} & 0.21420 & 0.39217 & 0.12082 & 0.00000 & \text { Uiso } & 1.00\end{array}$ $\begin{array}{lllllllll}0138 & \mathrm{O} & 0.21407 & 0.39200 & 0.62043 & 0.00000 & \text { Uiso } & 1.00\end{array}$ $\begin{array}{llllllll}0139 & \mathrm{O} & 0.21590 & 0.04853 & 0.12485 & 0.00000 & \text { Uiso } & 1.00\end{array}$ $\begin{array}{lllllllll}0140 & \mathrm{O} & 0.21583 & 0.04998 & 0.62089 & 0.00000 & \text { Uiso } & 1.00\end{array}$ $\begin{array}{llllllll}0141 & \mathrm{O} & 0.21366 & 0.54798 & 0.11865 & 0.00000 & \text { Uiso } & 1.00\end{array}$ $\begin{array}{llllllll}\mathrm{O} 142 & \mathrm{O} & 0.21380 & 0.54805 & 0.61841 & 0.00000 & \text { Uiso } & 1.00\end{array}$ $\begin{array}{llllllll}0143 & \mathrm{O} & 0.20848 & 0.21991 & 0.12777 & 0.00000 & \text { Uiso } & 1.00\end{array}$ $\begin{array}{llllllll}0144 & \mathrm{O} & 0.20818 & 0.22101 & 0.62578 & 0.00000 & \text { Uiso } & 1.00\end{array}$ $\begin{array}{lllllllll}0145 & \mathrm{O} & 0.20815 & 0.72182 & 0.12286 & 0.00000 & \text { Uiso } & 1.00\end{array}$ $\begin{array}{lllllllll}0146 & 0 & 0.20752 & 0.72170 & 0.62177 & 0.00000 & \text { Uiso } & 1.00\end{array}$ $\begin{array}{lllllllllll}0146 & 0 & 0.20752 & 0.72170 & 0.62177 & 0.00000 & \text { Uiso } & 1.00\end{array}$

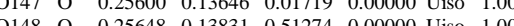
$\begin{array}{llllllllll}0148 & \mathrm{O} & 0.25648 & 0.13831 & 0.51274 & 0.00000 & \text { Uiso } & 1.00\end{array}$ $\begin{array}{llllllllll}0149 & 0 & 0.25471 & 0.63737 & 0.01157 & 0.00000 & \text { Uiso } & 1.00\end{array}$ $\begin{array}{lllllllll}0150 & 0 & 0.25487 & 0.63733 & 0.5080 & 0.00000 & \text { Uiso } & 1.00 \\ 0151 & 0 & 0.25632 & 0.80362 & 0.01316 & 0.00000 & \text { Uiso } & 1.00\end{array}$ $\begin{array}{lllllllll}0151 & \mathrm{O} & 0.25632 & 0.80362 & 0.01316 & 0.00000 & \text { Uiso } & 1.00 \\ 0152 & \mathrm{O} & 0.25525 & 0.80440 & 0.51081 & 0.00000 & \text { Uis } & 1.00\end{array}$ $\begin{array}{lllllllllll}0152 & 0 & 0.25525 & 0.80440 & 0.51081 & 0.00000 & \text { Unso } & 1.00\end{array}$ $\begin{array}{lllllllll}0153 & \mathrm{O} & 0.25541 & 0.30358 & 0.01769 & 0.00000 & \text { Uiso } & 1.00\end{array}$

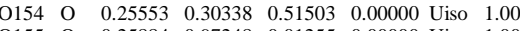
$\begin{array}{lllllllll}0155 & \mathrm{O} & 0.25884 & 0.97248 & 0.01255 & 0.00000 & \text { Uiso } & 1.00\end{array}$ $\begin{array}{llllllll}0156 & \mathrm{O} & 0.25840 & 0.97017 & 0.50951 & 0.00000 & \text { Uiso } & 1.00\end{array}$ $\begin{array}{llllllll}0157 & \mathrm{O} & 0.25710 & 0.47055 & 0.01117 & 0.00000 & \text { Uiso } & 1.00\end{array}$ $\begin{array}{llllllll}0158 & \mathrm{O} & 0.25616 & 0.46930 & 0.51001 & 0.00000 & \text { Uiso } & 1.00\end{array}$ $\begin{array}{llllllll}0159 & \mathrm{O} & 0.35254 & 0.29799 & 0.26721 & 0.00000 & \text { Uiso } & 1.00\end{array}$ $\begin{array}{lllllllll}0160 & \mathrm{O} & 0.39427 & 0.70991 & 0.65187 & 0.00000 & \text { Uiso } & 1.00\end{array}$ $\begin{array}{llllllll}0161 & \mathrm{O} & 0.41542 & 0.18027 & 0.92659 & 0.00000 & \text { Uiso } & 1.00\end{array}$ $\begin{array}{llllllll}0162 & \mathrm{O} & 0.39660 & 0.88333 & 0.86483 & 0.00000 & \text { Uiso } & 1.00\end{array}$ $\begin{array}{lllllllll}\mathrm{O} 163 & \mathrm{O} & 0.39560 & 0.39273 & 0.15853 & 0.00000 & \text { Uiso } & 1.00\end{array}$ $\begin{array}{llllllllll}0164 & 0 & 0.39633 & 0.37785 & 0.86947 & 0.00000 & \text { Uiso } & 1.00\end{array}$ $\begin{array}{lllllllll}0165 & \mathrm{O} & 0.39794 & 0.25142 & 0.71249 & 0.00000 & \text { Uiso } & 1.00\end{array}$ $\begin{array}{lllllllll}0166 & \mathrm{O} & 0.40671 & 0.55469 & 0.63246 & 0.00000 & \text { Uiso } & 1.00\end{array}$ $\begin{array}{lllllllll}0166 & \mathrm{O} & 0.40671 & 0.55469 & 0.63246 & 0.00000 & \text { Uiso } & 1.00 \\ 0167 & \mathrm{O} & 0.46254 & 0.49226 & 0.66659 & 0.00000 & \text { Uiso } & 1.00\end{array}$ $\begin{array}{lllllllll}0167 & \mathrm{O} & 0.46254 & 0.49226 & 0.66659 & 0.00000 & \text { Uiso } & 1.00\end{array}$ $\begin{array}{llllllllll}0168 & 0 & 0.40258 & 0.5454 & 0.27621 & 0.00000 & \text { Uiso } & 1.00\end{array}$ $\begin{array}{lllllllll}0169 & \mathrm{O} & 0.41240 & 0.54482 & 0.06055 & 0.00000 & \text { Uiso } & 1.00 \\ 0170 & \mathrm{O} & 0.39790 & 0.23001 & 0.14236 & 0.00000 & \text { Uiso } & 1.00\end{array}$

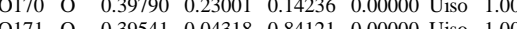
$\begin{array}{lllllllll}0171 & \mathrm{O} & 0.39541 & 0.04318 & 0.84121 & 0.00000 & \text { Uiso } & 1.00 \\ 0172 & \mathrm{O} & 0.0566 & 0.0621 & 0.10315 & 0.0000 & \text { Uiso } & 1.00\end{array}$ $\begin{array}{llllllllll}0172 & 0 & 0.40566 & 0.06921 & 0.10315 & 0.00000 & \text { Uiso } & 1.00\end{array}$ $\begin{array}{lllllllll}0173 & 0 & 0.41213 & 0.68954 & 0.39785 & 0.00000 & \text { Uiso } & 1.00\end{array}$ $\begin{array}{llllllll}0174 & \mathrm{O} & 0.45123 & 0.32184 & 0.78803 & 0.00000 & \text { Uiso } & 1.00\end{array}$ $\begin{array}{llllllll}0175 & \mathrm{O} & 0.44977 & 0.66124 & 0.70631 & 0.00000 & \text { Uiso } & 1.00\end{array}$ $\begin{array}{llllllll}0176 & \mathrm{O} & 0.40113 & 0.61744 & 0.85289 & 0.00000 & \text { Uiso } & 1.00\end{array}$ $\begin{array}{llllllll}0177 & \mathrm{O} & 0.43607 & 0.47676 & 0.45173 & 0.00000 & \text { Uiso } & 1.00\end{array}$ $\begin{array}{llllllll}0178 & \mathrm{O} & 0.37031 & 0.16572 & 0.52944 & 0.00000 & \text { Uiso } & 1.00\end{array}$

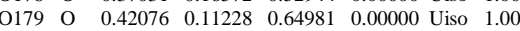
$\begin{array}{llllllll}\mathrm{O} 180 & \mathrm{O} & 0.40912 & 0.93876 & 0.64705 & 0.00000 & \text { Uiso } & 1.00\end{array}$ $\begin{array}{llllllll}0181 & \mathrm{O} & 0.39766 & 0.86797 & 0.43122 & 0.00000 & \text { Uiso } & 1.00\end{array}$ $\begin{array}{llllllll}0182 & \mathrm{O} & 0.42666 & 0.16887 & 0.41720 & 0.00000 & \text { Uiso } & 1.00\end{array}$ $\begin{array}{llllllll}0183 & \mathrm{O} & 0.39824 & 0.03570 & 0.45588 & 0.00000 & \text { Uiso } & 1.00\end{array}$ $\begin{array}{lllllllll}0184 & 0 & 0.45078 & 0.94156 & 0.48805 & 0.00000 & \text { Uiso } & 1.00\end{array}$ $\begin{array}{lllllllll}0185 & \mathrm{O} & 0.40957 & 0.90319 & 0.09422 & 0.00000 & \text { Uiso } & 1.00\end{array}$ $\begin{array}{llllllll}0186 & \mathrm{O} & 0.42964 & 0.98601 & 0.26870 & 0.00000 & \text { Uiso } & 1.00\end{array}$ $\begin{array}{llllllllll}0187 & 0 & 0.38862 & 0.68428 & 0.17297 & 0.00000 & \text { Uiso } & 1.00\end{array}$

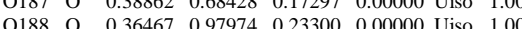
$\begin{array}{lllllllllll}0189 & 0 & 0.36467 & 0.97974 & 0.23300 & 0.00000 & \text { Uiso } & 1.00 \\ 0189 & 0 & 0.44229 & 0.78579 & 0.55841 & 0.00000 & \text { Uis } & 1.00\end{array}$ $\begin{array}{lllllllll}0189 & 0 & 0.44229 & 0.78579 & 0.55841 & 0.00000 & \text { Uiso } & 1.00\end{array}$ $\begin{array}{lllllllll}0190 & \mathrm{O} & 0.41755 & 0.75151 & 0.98913 & 0.00000 & \text { Uiso } & 1.00\end{array}$ $\begin{array}{llllllll} & \text { Sil91 Si } & 0.39178 & 0.75851 & 0.98913 & 0.00000 & \text { Uiso } & 1.00\end{array}$

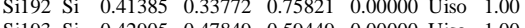
$\begin{array}{lllllllll}\text { Sil93 Si } & 0.42905 & 0.47849 & 0.59449 & 0.00000 & \text { Uiso } & 1.00\end{array}$ $\begin{array}{llllllll}\mathrm{Si194} & \mathrm{Si} & 0.38650 & 0.48929 & 0.15566 & 0.00000 & \text { Uiso } & 1.00\end{array}$ $\begin{array}{llllllll}\mathrm{Si} 195 & \mathrm{Si} & 0.39616 & 0.15552 & 0.04687 & 0.00000 & \text { Uiso } & 1.00\end{array}$ $\begin{array}{llllllll}\text { Si196 Si } & 0.39130 & 0.63957 & 0.30501 & 0.00000 & \text { Uiso } & 1.00\end{array}$ $\begin{array}{llllllll}\mathrm{Si} 197 & \mathrm{Si} & 0.37513 & 0.30939 & 0.14745 & 0.00000 & \text { Uiso } & 1.00\end{array}$ $\begin{array}{llllllll}\text { Si198 Si } & 0.41228 & 0.63449 & 0.71688 & 0.00000 & \text { Uiso } & 1.00\end{array}$

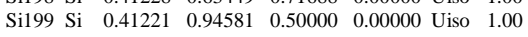


$\begin{array}{lllllll}\mathrm{Si} 200 \mathrm{Si} & 0.40539 & 0.12473 & 0.52048 & 0.00000 & \text { Uiso } & 1.00\end{array}$ $\begin{array}{llllllll}\mathrm{Si} 201 \mathrm{Si} & 0.40087 & 0.98337 & 0.16979 & 0.00000 & \text { Uiso } & 1.00\end{array}$ $\begin{array}{llllllll}\mathrm{Si} 202 & \mathrm{Si} & 0.39959 & 0.83472 & 0.99623 & 0.00000 & \text { Uiso } & 1.00\end{array}$ $\begin{array}{lllllllll}\mathrm{H} 203 & \mathrm{H} & 0.46922 & 0.44674 & 0.71462 & 0.00000 & \text { Uiso } & 1.00\end{array}$ $\begin{array}{llllllllll}\mathrm{H} 204 & \mathrm{H} & 0.45437 & 0.29192 & 0.86198 & 0.00000 & \text { Uiso } & 1.00\end{array}$ $\begin{array}{llllllll}\mathrm{H} 205 & \mathrm{H} & 0.46358 & 0.61492 & 0.69903 & 0.00000 & \text { Uiso } & 1.00\end{array}$ $\begin{array}{llllllllll} & \mathrm{H} 206 & \mathrm{H} & 0.42245 & 0.50191 & 0.39079 & 0.00000 & \text { Uiso } & 1.00\end{array}$ $\begin{array}{llllllllllll}4206 & \mathrm{H} & 0.4224 & 0.50191 & 0.39079 & 0.00000 & \text { Uiso } & 1.00\end{array}$ $\begin{array}{llllllllllllll} & 1.00\end{array}$ \begin{tabular}{lllllllll}
4208 & $\mathrm{H}$ & 0.45650 & 0.94141 & 0.40301 & 0.0000 & Uiso & 1.00 \\
\hline
\end{tabular} $\begin{array}{lllllllll}1209 & \mathrm{H} & 0.43375 & 0.04086 & 0.29348 & 0.00000 & \text { Uiso } & 1.00\end{array}$ \begin{tabular}{lllllllll}
$\mathrm{H} 210$ & $\mathrm{H}$ & 0.45368 & 0.83626 & 0.54658 & 0.00000 & Uiso & -1.00 \\
\hline 211 & $\mathrm{H}$ & 0.45140 & 0.74724 & 0.61711 & 0.0000 & Uiso & 1.00
\end{tabular} $\begin{array}{llllllllll}\mathrm{H} 211 & \mathrm{H} & 0.45140 & 0.74724 & 0.6171 & 0.00000 & \text { Uiso } & 1.00 \\ \mathrm{H} 212 & \mathrm{H} & 0.49980 & 0.75060 & 0.04062 & 0.0000 & \text { Uis } & 1.00\end{array}$ $\begin{array}{llllllllllllll}\mathrm{H} 212 & \mathrm{H} & 0.49580 & 0.75060 & 0.04062 & 0.00000 & \text { Usiso } & 1.00\end{array}$ $\begin{array}{llllllll}\mathrm{H} 213 & \mathrm{H} & 0.45821 & 0.61319 & 0.91840 & 0.00000 & \text { Uiso } & 1.00\end{array}$ $\begin{array}{lllllllll} & -1214 & \mathrm{H} & 0.44925 & 0.51143 & 0.88519 & 0.00000 & \text { Uiso } & 1.00\end{array}$ $\begin{array}{llllllll}\mathrm{H} 215 & \mathrm{H} & 0.48590 & 0.53784 & 0.95074 & 0.00000 & \text { Uiso } & 1.00\end{array}$ $\begin{array}{lllllllll}\mathrm{H} 216 & \mathrm{H} & 0.45168 & 0.66417 & 0.13291 & 0.00000 & \text { Uiso } & 1.00\end{array}$ $\begin{array}{lllllllll}\mathrm{H} 217 & \mathrm{H} & 0.48554 & 0.60567 & 0.14912 & 0.00000 & \text { Uiso } & 1.00\end{array}$ $\begin{array}{llllllll}\mathrm{H} 218 & \mathrm{H} & 0.45452 & 0.59308 & 0.25290 & 0.00000 & \text { Uiso } & 1.00\end{array}$ $\begin{array}{lllllllll}\mathrm{H} 219 & \mathrm{H} & 0.44929 & 0.44586 & 0.21290 & 0.00000 & \text { Uiso } & 1.00\end{array}$ $\begin{array}{llllllll}\mathrm{H} 220 & \mathrm{H} & 0.48272 & 0.45441 & 0.12056 & 0.00000 & \text { Uiso } & 1.00\end{array}$ $\begin{array}{llllllll}\mathrm{H} 221 & \mathrm{H} & 0.44818 & 0.40999 & 0.06102 & 0.00000 & \text { Uiso } & 1.00\end{array}$ $\begin{array}{lllllllll}\mathrm{H} 222 & \mathrm{H} & 0.47668 & 0.93550 & 0.14737 & 0.00000 & \text { Uiso } & 1.00\end{array}$ $\begin{array}{llllllll}\mathrm{H} 223 & \mathrm{H} & 0.51398 & 0.95536 & 0.08677 & 0.00000 & \text { Uiso } & 1.00\end{array}$ $\begin{array}{lllllllll}\mathrm{H} 224 & \mathrm{H} & 0.50732 & 0.86442 & 0.16679 & 0.00000 & \text { Uiso } & 1.00\end{array}$

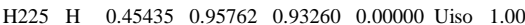
$\begin{array}{llllllllll}\mathrm{H} 226 & \mathrm{H} & 0.46531 & 0.88934 & 0.81616 & 0.00000 & \text { Uiso } & 1.00\end{array}$

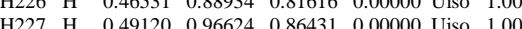
$\begin{array}{llllllllll}1227 & \mathrm{H} & 0.49120 & 0.9624 & 0.86431 & 0.00000 & \text { Uiso } & 1.00 \\ \mathrm{H} & \mathrm{H} 228 & \mathrm{H} & 0.48197 & 0.76073 & 0.88852 & 0.00000 & \text { Uiso } & 1.00\end{array}$ $\begin{array}{lllllllll}1.228 & \mathrm{H} & 0.48197 & 0.76073 & 0.88852 & 0.00000 & \text { Uiso } & 1.00\end{array}$ $\begin{array}{lllllllll}\mathrm{C} 229 & \mathrm{C} & 0.44792 & 0.53898 & 0.07496 & 0.00000 & \text { Uiso } & 1.00 \\ \mathrm{C} 230 & \mathrm{C} & 0.46086 & 0.55126 & 0.94894 & 0.00000 & \text { Uis } & 1.00\end{array}$ $\begin{array}{lllllllllll}C 230 & \mathrm{C} & 0.46086 & 0.55126 & 0.94894 & 0.00000 & \text { Uiso } & 1.00\end{array}$ $\begin{array}{lllllllll}C 231 & \mathrm{C} & 0.46006 & 0.60440 & 0.15842 & 0.00000 & \text { Uiso } & 1.00\end{array}$ $\begin{array}{lllllllll}\mathrm{C} 232 & \mathrm{C} & 0.45716 & 0.45678 & 0.12057 & 0.00000 & \text { Uiso } & 1.00\end{array}$ $\begin{array}{lllllllll}\mathrm{C} 233 & \mathrm{C} & 0.49006 & 0.78918 & 0.97102 & 0.00000 & \text { Uiso } & 1.00\end{array}$ \begin{tabular}{llllllll}
$C 234$ & C & 0.48727 & 0.86788 & 0.98852 & 0.00000 & Uiso & 1.00 \\
\hline & C & 0.49697 & 0.90742 & 0.10384 & 0.00000 & Uiso & 1.00
\end{tabular} $\begin{array}{llllllll}\mathrm{C} 235 & \mathrm{C} & 0.49697 & 0.90742 & 0.10384 & 0.00000 & \text { Uiso } & 1.00 \\ \mathrm{C} 236 & \mathrm{C} & 0.47373 & 0.92252 & 0.89486 & 0.00000 & \text { Uiso } & 1.00\end{array}$

\section{File $\mathbf{n}^{\circ} 15$}

$\begin{array}{llllllll}\text { Al2 } & \mathrm{Al} & 0.35125 & 0.05679 & 0.89452 & 0.00000 & \text { Uiso } & 1.00\end{array}$ $\begin{array}{llllllll}\mathrm{Al} 3 & \mathrm{Al} & 0.35008 & 0.03694 & 0.38063 & 0.00000 & \text { Uiso } & 1.00\end{array}$ $\begin{array}{llllllll}\mathrm{Al} 4 & \mathrm{Al} & 0.34413 & 0.56591 & 0.89966 & 0.00000 & \text { Uiso } & 1.00\end{array}$ $\begin{array}{llllllll}\mathrm{Al} 5 & \mathrm{Al} & 0.34386 & 0.53271 & 0.38260 & 0.00000 & \text { Uiso } & 1.00\end{array}$ $\begin{array}{lllllllll}\text { Al6 } & \text { Al } & 0.34447 & 0.87433 & 0.89712 & 0.00000 & \text { Uiso } & 1.00\end{array}$ $\begin{array}{llllllllll}\mathrm{A} 17 & \mathrm{Al} & 0.35000 & 0.85122 & 0.41037 & 0.00000 & \text { Uiso } & 1.00\end{array}$ $\begin{array}{llllllll}\mathrm{Al} 8 & \mathrm{Al} & 0.35336 & 0.40130 & 0.88417 & 0.00000 & \text { Uiso } & 1.00\end{array}$ $\begin{array}{llllllll}\text { Al9 } & \text { Al } & 0.35336 & 0.40130 & 0.88417 & 0.00000 & \text { Uiso } & 1.00 \\ \text { Al } & \text { Al } & 0.32883 & 0.38648 & 0.51923 & 0.00000 & \text { Uiso } & 1.00\end{array}$

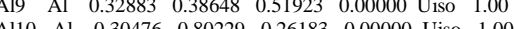

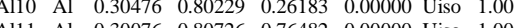
$\begin{array}{llllllll}\text { Al11 Al } & 0.30076 & 0.80726 & 0.76482 & 0.0000 & \text { Uiso } & 1.00\end{array}$ $\begin{array}{lllllllll}\text { Al12 Al } & 0.30453 & 0.30668 & 0.26029 & 0.00000 & \text { Uiso } & 1.00\end{array}$ $\begin{array}{llllllll}\text { Al13 Al } & 0.30163 & 0.31414 & 0.77259 & 0.00000 & \text { Uiso } & 1.00\end{array}$ $\begin{array}{llllllll}\text { Al14 Al } & 0.30632 & 0.13413 & 0.26128 & 0.00000 & \text { Uiso } & 1.00\end{array}$ $\begin{array}{llllllll}\text { Al15 } & \text { Al } & 0.30317 & 0.13599 & 0.75866 & 0.00000 & \text { Uiso } & 1.00\end{array}$ $\begin{array}{llllllll}\text { Al16 } & \mathrm{Al} & 0.29754 & 0.63492 & 0.26782 & 0.00000 & \text { Uiso } & 1.00\end{array}$ $\begin{array}{llllllll}\text { Al17 } & \mathrm{Al} & 0.29901 & 0.64121 & 0.76050 & 0.00000 & \text { Uiso } & 1.00\end{array}$ $\begin{array}{llllllll}\text { Al18 } & \mathrm{Al} & 0.21191 & 0.97110 & 0.25381 & 0.00000 & \text { Uiso } & 1.00\end{array}$ $\begin{array}{llllllll}\text { Al19 } & \mathrm{Al} & 0.21176 & 0.97245 & 0.75450 & 0.00000 & \text { Uiso } & 1.00\end{array}$ $\begin{array}{llllllll}\mathrm{Al} 20 & \mathrm{Al} & 0.20935 & 0.47056 & 0.25063 & 0.00000 & \text { Uiso } & 1.00\end{array}$ $\begin{array}{lllllllll}\mathrm{Al} 21 & \mathrm{Al} & 0.21015 & 0.47068 & 0.75172 & 0.00000 & \text { Uiso } & 1.00\end{array}$ $\begin{array}{lllllllll}\text { Al22 } & \text { Al } & 0.32933 & 0.97014 & 0.13018 & 0.00000 & \text { Uiso } & 1.00\end{array}$

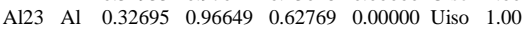
$\begin{array}{llllllllll}\mathrm{Al} 24 & \mathrm{Al} & 0.32458 & 0.47531 & 0.12735 & 0.00000 & \text { Uiso } & 1.00\end{array}$ $\begin{array}{llllllll}\mathrm{Al} 25 & \mathrm{Al} & 0.33978 & 0.53519 & 0.65873 & 0.00000 & \text { Uiso } & 1.00\end{array}$ $\begin{array}{llllllllll}\mathrm{Al} 26 & \mathrm{Al} & 0.28079 & 0.22011 & 0.02367 & 0.00000 & \text { Uiso } & 1.00\end{array}$ $\begin{array}{llllllll}\mathrm{Al} 27 & \mathrm{Al} & 0.28186 & 0.22138 & 0.51694 & 0.00000 & \text { Uiso } & 1.00\end{array}$ $\begin{array}{llllllllll}\mathrm{Al} 28 & \mathrm{Al} & 0.28100 & 0.71860 & 0.00725 & 0.00000 & \text { Uiso } & 1.00\end{array}$ \begin{tabular}{lllllllll}
\hline Al29 & Al & 0.28101 & 0.718098 & 0.50697 & 0.00000 & Uiso & 1.00
\end{tabular} $\begin{array}{llllllll}\text { Al29 Al } & 0.28101 & 0.72098 & 0.50697 & 0.00000 & \text { Uiso } & 1.00\end{array}$ $\begin{array}{llllllll}A 131 & \mathrm{Al} & 0.34586 & 0.23225 & 0.91703 & 0.0000 & \text { Uiso } & 1.00\end{array}$ $\begin{array}{lllllllllll}A 131 & \text { Al } & 0.35625 & 0.24023 & 0.65632 & 0.00000 & \text { Uiso } & 1.00\end{array}$ $\begin{array}{llllllllll} & \text { Al32 Al } & 0.35264 & 0.73144 & 0.12540 & 0.00000 & \text { Uiso } & 1.00\end{array}$ $\begin{array}{lllllllll}\text { Al33 Al } & 0.35163 & 0.73136 & 0.66579 & 0.00000 & \text { Uiso } & 1.00\end{array}$ $\begin{array}{lllllllll}\text { Al34 Al } & 0.21198 & 0.97156 & 0.00634 & 0.00000 & \text { Uiso } & 1.00\end{array}$ $\begin{array}{lllllllll}\text { Al35 Al } & 0.21143 & 0.97204 & 0.50418 & 0.00000 & \text { Uiso } & 1.00\end{array}$ $\begin{array}{llllllll}\text { Al36 Al } & 0.21023 & 0.46983 & 0.00295 & 0.00000 & \text { Uiso } & 1.00\end{array}$ $\begin{array}{llllllll}\text { Al37 } & \text { Al } & 0.20960 & 0.47001 & 0.50306 & 0.00000 & \text { Uiso } & 1.00\end{array}$ $\begin{array}{llllllll}\text { Al38 Al } & 0.28284 & 0.96122 & 0.37886 & 0.00000 & \text { Uiso } & 1.00\end{array}$ $\begin{array}{llllllll}\mathrm{Al} 39 & \mathrm{Al} & 0.28193 & 0.97594 & 0.87932 & 0.00000 & \text { Uiso } & 1.00\end{array}$ $\begin{array}{lllllllll}\mathrm{Al} 40 & \mathrm{Al} & 0.27839 & 0.46739 & 0.37680 & 0.00000 & \text { Uiso } & 1.00\end{array}$ $\begin{array}{llllllll}\mathrm{Al} 41 & \mathrm{Al} & 0.28078 & 0.47449 & 0.87721 & 0.00000 & \text { Uiso } & 1.00\end{array}$ $\begin{array}{llllllll}\mathrm{Al} 42 & \mathrm{Al} & 0.23655 & 0.21902 & 0.25537 & 0.00000 & \text { Uiso } & 1.00\end{array}$ $\begin{array}{llllllll}\mathrm{Al} 43 & \mathrm{Al} & 0.23539 & 0.22002 & 0.75396 & 0.00000 & \text { Uiso } & 1.00\end{array}$ $\begin{array}{llllllll}\mathrm{Al} 44 & \mathrm{Al} & 0.23490 & 0.72406 & 0.25056 & 0.00000 & \text { Uiso } & 1.00\end{array}$ $\begin{array}{llllllll}\mathrm{Al} 45 & \mathrm{Al} & 0.23417 & 0.72320 & 0.74999 & 0.00000 & \text { Uiso } & 1.00\end{array}$ $\begin{array}{lllllllll}\mathrm{Al} 46 & \mathrm{Al} & 0.26186 & 0.05810 & 0.13329 & 0.00000 & \text { Uiso } & 1.00\end{array}$ $\begin{array}{llllllll}\mathrm{Al} 47 & \mathrm{Al} & 0.26127 & 0.05827 & 0.62615 & 0.00000 & \text { Uiso } & 1.00\end{array}$ $\begin{array}{lllllllll}\text { Al48 } & \text { Al } & 0.25841 & 0.55813 & 0.12481 & 0.00000 & \text { Uiso } & 1.00\end{array}$ $\begin{array}{llllllllllll}\text { Al49 Al } & 0.25901 & 0.55861 & 0.62456 & 0.00000 & \text { Uiso } & 1.00\end{array}$ $\begin{array}{llllllll}\text { Al49 Al } & 0.25901 & 0.55861 & 0.62456 & 0.00000 & \text { Uiso } & 1.00 \\ \text { Al5 } & \text { Al } & 0.26183 & 0.88315 & 0.12666 & 0.00000 & \text { Uiso } & 1.00\end{array}$ $\begin{array}{lllllllll}A 151 & \mathrm{Al} & 0.25962 & 0.88322 & 0.62709 & 0.00000 & \text { Uiso } & 1.00\end{array}$ \begin{tabular}{lllllllll}
\hline 151 & Al & 0.25962 & 0.88322 & 0.62709 & 0.00000 & Uiso & 1.00
\end{tabular} $\begin{array}{llllllll}A 152 & \text { Al } & 0.25952 & 0.38232 & 0.13199 & 0.00000 & \text { Uiso } & 1.00\end{array}$ $\begin{array}{llllllll} & \text { Al53 Al } & 0.25925 & 0.38332 & 0.63006 & 0.0000 & \text { Uiso } & 1.00\end{array}$ $\begin{array}{lllllllll}\text { Al54 Al } & 0.21396 & 0.80723 & 0.00144 & 0.00000 & \text { Uiso } & 1.00\end{array}$ $\begin{array}{llllllll}\text { Al55 Al } & 0.21308 & 0.80675 & 0.50080 & 0.00000 & \text { Uiso } & 1.00\end{array}$ $\begin{array}{llllllll}\text { Al56 Al } & 0.21299 & 0.30593 & 0.00341 & 0.00000 & \text { Uiso } & 1.00\end{array}$ $\begin{array}{llllllll}\mathrm{Al} 57 & \mathrm{Al} & 0.21299 & 0.30600 & 0.50084 & 0.00000 & \text { Uiso } & 1.00\end{array}$ $\begin{array}{llllllll}\text { Al58 } & \text { Al } & 0.21369 & 0.13512 & 0.00596 & 0.00000 & \text { Uiso } & 1.00\end{array}$ $\begin{array}{llllllll}\text { Al59 } & \text { Al } & 0.21368 & 0.13572 & 0.50169 & 0.00000 & \text { Uiso } & 1.00\end{array}$ $\begin{array}{lllllll}\text { Al60 Al } & 0.21270 & 0.63517 & 0.99915 & 0.00000 & \text { Uiso } & 1.00\end{array}$

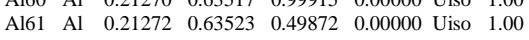


$\begin{array}{lllllll}\text { Al62 Al } & 0.40250 & 0.15132 & 0.78416 & 0.00000 & \text { Uiso } & 1.00\end{array}$ $\begin{array}{lllllllll}\text { Al63 } & \mathrm{Al} & 0.35104 & 0.22612 & 0.39951 & 0.00000 & \text { Uiso } & 1.00\end{array}$ $\begin{array}{llllllll}\mathrm{Al} 64 & \mathrm{Al} & 0.39693 & 0.62818 & 0.01967 & 0.00000 & \text { Uiso } & 1.00\end{array}$ $\begin{array}{lllllllll}\text { Al65 } & \mathrm{Al} & 0.40229 & 0.76557 & 0.50797 & 0.00000 & \text { Uiso } & 1.00\end{array}$ $\begin{array}{lllllllll}066 & \mathrm{O} & 0.30865 & 0.87752 & 0.38122 & 0.00000 & \text { Uiso } & 1.00\end{array}$ $\begin{array}{lllllllll}067 & \mathrm{O} & 0.30403 & 0.88771 & 0.88234 & 0.00000 & \text { Uiso } & 1.00\end{array}$ $\begin{array}{llllllllll}068 & 0 & 0.30656 & 0.38909 & 0.38513 & 0.00000 & \text { Uiso } & 1.00\end{array}$ $\begin{array}{llllllll}068 & 0 & 0.30656 & 0.3890 & 0.38513 & 0.00000 & \text { Uiso } & 1.00\end{array}$

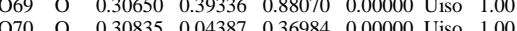
$\begin{array}{lllllllllll}071 & 0 & 0.30835 & 0.04387 & 0.36984 & 0.00000 & \text { Uiso } & 1.00 \\ 071 & 0 & 0.30941 & 0.05794 & 0.87208 & 0.00000 & \text { Uiso } & 1.00\end{array}$ $\begin{array}{llllllllll}071 & 0 & 0.30941 & 0.05794 & 0.87208 & 0.0000 & \text { Uiso } & 1.00\end{array}$ $\begin{array}{llllllllll}072 & 0 & 0.30386 & 0.55062 & 0.37161 & 0.00000 & \text { Uiso } & 1.00\end{array}$ $\begin{array}{lllllllll}073 & \mathrm{O} & 0.30289 & 0.56330 & 0.87872 & 0.00000 & \text { Uiso } & 1.00\end{array}$ $\begin{array}{lllllllll}074 & \mathrm{O} & 0.30735 & 0.22375 & 0.38769 & 0.00000 & \text { Uiso } & 1.00\end{array}$ $\begin{array}{llllllll}\mathrm{O} 75 & \mathrm{O} & 0.30452 & 0.21989 & 0.88858 & 0.00000 & \text { Uiso } & 1.00 \\ \mathrm{O} 76 & \mathrm{O} & 0.30182 & 0.71602 & 0.37045 & 0.00000 & \text { Uiso } & 1.00\end{array}$ $\begin{array}{llllllll}076 & \mathrm{O} & 0.30182 & 0.71602 & 0.37045 & 0.00000 & \text { Uiso } & 1.00 \\ 077 & \mathrm{O} & 0.30063 & 0.72210 & 0.87089 & 0.00000 & \text { Uiso } & 1.00\end{array}$ $\begin{array}{llllllll}\mathrm{O} 77 & \mathrm{O} & 0.30063 & 0.72210 & 0.87089 & 0.00000 & \text { Uiso } & 1.00 \\ \mathrm{O} 78 & \mathrm{O} & 0.35150 & 0.13452 & 0.31376 & 0.00000 & \text { Uiso } & 1.00\end{array}$ $\begin{array}{llllllll}079 & \mathrm{O} & 0.35598 & 0.15364 & 0.78233 & 0.00000 & \text { Uiso } & 1.00\end{array}$ $\begin{array}{llllllll}080 & \mathrm{O} & 0.35967 & 0.62672 & 0.36902 & 0.00000 & \text { Uiso } & 1.00\end{array}$ $\begin{array}{llllllll}081 & \mathrm{O} & 0.34595 & 0.63179 & 0.73638 & 0.00000 & \text { Uiso } & 1.00\end{array}$ $\begin{array}{lllllllll}\mathrm{O} 82 & \mathrm{O} & 0.34950 & 0.79814 & 0.26258 & 0.00000 & \text { Uiso } & 1.00\end{array}$ $\begin{array}{llllllll}083 & \mathrm{O} & 0.34565 & 0.80629 & 0.77472 & 0.00000 & \text { Uiso } & 1.00\end{array}$ $\begin{array}{llllllll}084 & \mathrm{O} & 0.35598 & 0.31059 & 0.02383 & 0.00000 & \text { Uiso } & 1.00\end{array}$ $\begin{array}{llllllll}085 & \mathrm{O} & 0.41676 & 0.39773 & 0.64515 & 0.00000 & \text { Uiso } & 1.00\end{array}$ $\begin{array}{lllllllll}086 & \mathrm{O} & 0.35384 & 0.96507 & 0.75641 & 0.00000 & \text { Uiso } & 1.00\end{array}$ $\begin{array}{lllllllll}087 & \mathrm{O} & 0.35541 & 0.48817 & 0.23975 & 0.00000 & \text { Uiso } & 1.00\end{array}$ $\begin{array}{lllllllll}088 & \mathrm{O} & 0.35414 & 0.48723 & 0.78387 & 0.00000 & \text { Uiso } & 1.00\end{array}$ $\begin{array}{llllllllllll}0889 & \mathrm{O} & 0.35414 & 0.48723 & 0.78387 & 0.00000 & \text { Uiso } & 1.00 \\ 0.30491 & 0.88672 & 0.14312 & 0.00000 & \text { Uiso } & 1.00\end{array}$ $\begin{array}{lllllllllll}090 & 0 & 0.30158 & 0.88681 & 0.64349 & 0.00000 & \text { Uiso } & 1.00\end{array}$ $\begin{array}{llllllllll}090 & \mathrm{O} & 0.30158 & 0.88681 & 0.64349 & 0.00000 & \text { Uiso } & 1.00\end{array}$ \begin{tabular}{lllllllll}
091 & $\mathrm{O}$ & 0.30235 & 0.38941 & 0.15037 & 0.00000 & Uiso & 1.00 \\
\hline 92 & $\mathrm{O}$ & 0.30228 & 0.38165 & 0.63789 & 0.00000 & Uiso & 1.00
\end{tabular} $\begin{array}{llllllllllll}092 & 0 & 0.30228 & 0.38165 & 0.63789 & 0.0000 & \text { Uiso } & 1.00\end{array}$ $\begin{array}{llllllllll}093 & 0 & 0.30546 & 0.05544 & 0.13939 & 0.00000 & \text { Uiso } & 1.00\end{array}$ $\begin{array}{lllllllll}094 & \mathrm{O} & 0.30487 & 0.05585 & 0.63921 & 0.00000 & \text { Uiso } & 1.00\end{array}$ $\begin{array}{lllllllll}095 & \mathrm{O} & 0.30108 & 0.55974 & 0.14377 & 0.00000 & \text { Uiso } & 1.00\end{array}$ $\begin{array}{lllllllll}096 & \mathrm{O} & 0.30062 & 0.55961 & 0.63907 & 0.00000 & \text { Uiso } & 1.00\end{array}$ $\begin{array}{llllllll}097 & \mathrm{O} & 0.30654 & 0.22067 & 0.15022 & 0.00000 & \text { Uiso } & 1.00\end{array}$ $\begin{array}{lllllllll}098 & \mathrm{O} & 0.31016 & 0.21881 & 0.64668 & 0.00000 & \text { Uiso } & 1.00\end{array}$ $\begin{array}{lllllllll}099 & \mathrm{O} & 0.30983 & 0.71787 & 0.13647 & 0.00000 & \text { Uiso } & 1.00\end{array}$ $\begin{array}{lllllllll}0100 & \mathrm{O} & 0.30661 & 0.72441 & 0.63817 & 0.00000 & \text { Uiso } & 1.00\end{array}$ $\begin{array}{llllllll}0101 & \mathrm{O} & 0.35916 & 0.14325 & 0.00338 & 0.00000 & \text { Uiso } & 1.00\end{array}$ $\begin{array}{llllllll}0102 & \mathrm{O} & 0.35643 & 0.64526 & 0.00029 & 0.00000 & \text { Uiso } & 1.00\end{array}$ $\begin{array}{lllllllll}0103 & \mathrm{O} & 0.36505 & 0.81045 & 0.01572 & 0.00000 & \text { Uiso } & 1.00\end{array}$ $\begin{array}{lllllllll}0104 & \mathrm{O} & 0.35985 & 0.77793 & 0.52178 & 0.00000 & \text { Uiso } & 1.00\end{array}$ $\begin{array}{llllllll}0105 & \mathrm{O} & 0.34500 & 0.30472 & 0.78774 & 0.00000 & \text { Uiso } & 1.00\end{array}$ $\begin{array}{lllllllll}0106 & \mathrm{O} & 0.35334 & 0.30352 & 0.52033 & 0.00000 & \text { Uiso } & 1.00\end{array}$ $\begin{array}{llllllll}0107 & \mathrm{O} & 0.35080 & 0.96761 & 0.99206 & 0.00000 & \text { Uiso } & 1.00\end{array}$ $\begin{array}{llllllll}0108 & 0 & 0.34976 & 0.95694 & 0.49401 & 0.00000 & \text { Uiso } & 1.00\end{array}$ $\begin{array}{llllllllllll}0109 & 0 & 0.34845 & 0.47851 & 0.00210 & 0.00000 & \text { Uiso } & 1.00\end{array}$ $\begin{array}{lllllllllllll}0110 & 0 & 0.34940 & 0.48178 & 0.52104 & 0.00000 & \text { Uiso } & 1.00\end{array}$

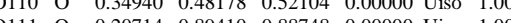
$\begin{array}{llllllllll}0111 & 0 & 0.20714 & 0.89410 & 0.88748 & 0.00000 & \text { Uiso } & 1.00\end{array}$ $\begin{array}{lllllllllllll}0112 & \mathrm{O} & 0.20694 & 0.89349 & 0.38613 & 0.00000 & \text { Uiso } & 1.00\end{array}$ $\begin{array}{lllllllll}0113 & 0 & 0.20580 & 0.39218 & 0.88554 & 0.00000 & \text { Uiso } & 1.00\end{array}$

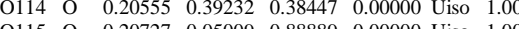
$\begin{array}{llllllllll}0115 & \mathrm{O} & 0.20727 & 0.02009 & 0.88889 & 0.00000 & \text { Uiso } & 1.00 \\ 0116 & 0 & 0.20705 & 0.0486 & 0.3862 & 0.0000 & \text { Uno } & 1.00\end{array}$ $\begin{array}{lllllllll}0116 & \mathrm{O} & 0.20705 & 0.04986 & 0.38642 & 0.00000 & \text { Uiso } & 1.00\end{array}$ $\begin{array}{lllllllll}0117 & 0 & 0.20574 & 0.54782 & 0.88445 & 0.00000 & \text { Uiso } & 1.00\end{array}$ $\begin{array}{lllllllll}0118 & \mathrm{O} & 0.20531 & 0.54773 & 0.38375 & 0.00000 & \text { Uiso } & 1.00\end{array}$ $\begin{array}{llllllll}0119 & \mathrm{O} & 0.21095 & 0.22046 & 0.88651 & 0.00000 & \text { Uiso } & 1.00\end{array}$ $\begin{array}{llllllll}0120 & \mathrm{O} & 0.21090 & 0.22066 & 0.38494 & 0.00000 & \text { Uiso } & 1.00\end{array}$ $\begin{array}{llllllll}0121 & \mathrm{O} & 0.21040 & 0.72146 & 0.88361 & 0.00000 & \text { Uiso } & 1.00\end{array}$ $\begin{array}{lllllllll}0122 & \mathrm{O} & 0.21029 & 0.72184 & 0.38227 & 0.00000 & \text { Uiso } & 1.00\end{array}$ $\begin{array}{lllllllll}0123 & \mathrm{O} & 0.26215 & 0.13521 & 0.25359 & 0.00000 & \text { Uiso } & 1.00\end{array}$ $\begin{array}{llllllllll}0124 & \mathrm{O} & 0.26141 & 0.13781 & 0.74601 & 0.00000 & \text { Uiso } & 1.00\end{array}$ $\begin{array}{lllllllll}0125 & \mathrm{O} & 0.25707 & 0.63595 & 0.24359 & 0.00000 & \text { Uiso } & 1.00\end{array}$

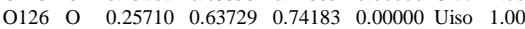
$\begin{array}{lllllllll}0127 & 0 & 0.26170 & 0.80403 & 0.24292 & 0.00000 & \text { Uiso } & 1.00\end{array}$ $\begin{array}{lllllllll}0128 & \mathrm{O} & 0.25846 & 0.80743 & 0.74601 & 0.00000 & \text { Uiso } & 1.00\end{array}$ $\begin{array}{lllllllll}0129 & 0 & 0.26074 & 0.30527 & 0.25416 & 0.00000 & \text { Uiso } & 1.00\end{array}$ $\begin{array}{llllllllllll}0130 & 0 & 0.25984 & 0.30508 & 0.75079 & 0.00000 & \text { Uiso } & 1.00\end{array}$ $\begin{array}{lllllllllll}0131 & 0 & 0.25642 & 0.96842 & 0.25014 & 0.00000 & \text { Uiso } & 1.00\end{array}$ $\begin{array}{llllllllll}0131 & 0 & 0.25642 & 0.96842 & 0.25014 & 0.00000 & \text { Uiso } & 1.00\end{array}$ $\begin{array}{llllllllllll}0132 & \mathrm{O} & 0.25571 & 0.97390 & 0.75027 & 0.00000 & \text { Uiso } & 1.00 \\ 0133 & \mathrm{O} & 0.25338 & 0.47022 & 0.24795 & 0.00000 & \text { Uiso } & 1.00\end{array}$ $\begin{array}{lllllllll}0133 & 0 & 0.25338 & 0.47022 & 0.24795 & 0.00000 & \text { Uiso } & 1.00\end{array}$ $\begin{array}{llllllllll}0134 & 0 & 0.25437 & 0.47040 & 0.74746 & 0.00000 & \text { Uiso } & 1.00 \\ 0135 & 0 & 0.21592 & 0.89300 & 0.12150 & 0.00000 & \text { Uiso } & 1.00\end{array}$ $\begin{array}{lllllllll}0135 & 0 & 0.21592 & 0.89300 & 0.12150 & 0.00000 & \text { Uiso } & 1.00\end{array}$ $\begin{array}{llllllll}0136 & \mathrm{O} & 0.21497 & 0.89461 & 0.62121 & 0.00000 & \text { Uiso } & 1.00\end{array}$ $\begin{array}{llllllll}0137 & \mathrm{O} & 0.21420 & 0.39217 & 0.12082 & 0.00000 & \text { Uiso } & 1.00\end{array}$

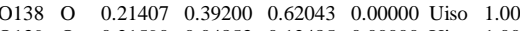
$\begin{array}{llllllll}0139 & \mathrm{O} & 0.21590 & 0.04853 & 0.12485 & 0.00000 & \text { Uiso } & 1.00\end{array}$ $\begin{array}{lllllllll}0140 & \mathrm{O} & 0.21583 & 0.04998 & 0.62089 & 0.00000 & \text { Uiso } & 1.00\end{array}$

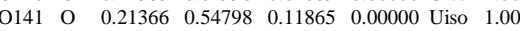
$\begin{array}{llllllll}0142 & \mathrm{O} & 0.21380 & 0.54805 & 0.61841 & 0.00000 & \text { Uiso } & 1.00\end{array}$ $\begin{array}{lllllllll}0143 & \mathrm{O} & 0.20848 & 0.21991 & 0.12777 & 0.00000 & \text { Uiso } & 1.00\end{array}$ $\begin{array}{lllllllll}0144 & \mathrm{O} & 0.20818 & 0.22101 & 0.62578 & 0.00000 & \text { Uiso } & 1.00\end{array}$

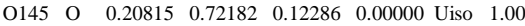
$\begin{array}{llllllll}0146 & \mathrm{O} & 0.20752 & 0.72170 & 0.62177 & 0.00000 & \text { Uiso } & 1.00\end{array}$ $\begin{array}{lllllllll}0147 & \mathrm{O} & 0.25600 & 0.13646 & 0.01719 & 0.00000 & \text { Uiso } & 1.00\end{array}$

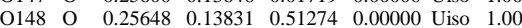
$\begin{array}{lllllllll}0149 & \mathrm{O} & 0.25471 & 0.63737 & 0.01157 & 0.00000 & \text { Uiso } & 1.00\end{array}$ $\begin{array}{lllllllll}0150 & 0 & 0.25487 & 0.63733 & 0.50809 & 0.00000 & \text { Uiso } & 1.00\end{array}$ $\begin{array}{llllllllllll}0151 & 0 & 0.25632 & 0.8037 & 0.5080 & 0.00000 & \text { Uiso } & 1.00\end{array}$ $\begin{array}{llllllll}0152 & 0 & 0.25525 & 0.80440 & 0.51081 & 0.00000 & \text { Uiso } & 1.00\end{array}$ $\begin{array}{llllllllll}0152 & 0 & 0.25525 & 0.80440 & 0.51081 & 0.00000 & \text { Uiso } & 1.00 \\ 0153 & 0 & 0.25541 & 0.30358 & 0.01769 & 0.00000 & \text { Uiso } & 1.00\end{array}$ $\begin{array}{llllllllll}0153 & \mathrm{O} & 0.25541 & 0.30358 & 0.01769 & 0.00000 & \text { Uiso } & 1.00 \\ 0154 & \mathrm{O} & 0.25553 & 0.30338 & 0.51503 & 0.00000 & \text { Uiso } & 1.00\end{array}$ $\begin{array}{llllllllllllllll}0154 & 0 & 0.25553 & 0.30338 & 0.51503 & 0.00000 & \text { Uiso } & 1.00\end{array}$ $\begin{array}{lllllllll}0155 & \mathrm{O} & 0.25884 & 0.97248 & 0.01255 & 0.00000 & \text { Uiso } & 1.00\end{array}$ $\begin{array}{lllllllll}0156 & \mathrm{O} & 0.25840 & 0.97017 & 0.50951 & 0.0000 & \text { Uiso } & 1.00\end{array}$ $\begin{array}{lllllllll}0157 & \mathrm{O} & 0.25710 & 0.47055 & 0.01117 & 0.00000 & \text { Uiso } & 1.00\end{array}$ $\begin{array}{lllllllll}0158 & \mathrm{O} & 0.25616 & 0.46930 & 0.51001 & 0.00000 & \text { Uiso } & 1.00\end{array}$ $\begin{array}{lllllllll}0159 & \mathrm{O} & 0.35219 & 0.29831 & 0.27093 & 0.00000 & \text { Uiso } & 1.00\end{array}$ $\begin{array}{lllllllll}0160 & \mathrm{O} & 0.39609 & 0.71281 & 0.65828 & 0.00000 & \text { Uiso } & 1.00\end{array}$ $\begin{array}{lllllllll}0161 & \mathrm{O} & 0.41526 & 0.18274 & 0.92864 & 0.00000 & \text { Uiso } & 1.00\end{array}$ $\begin{array}{llllllll}0162 & \mathrm{O} & 0.39940 & 0.88623 & 0.86705 & 0.00000 & \text { Uiso } & 1.00\end{array}$ 
$\begin{array}{llllllll}0163 & \mathrm{O} & 0.39756 & 0.38913 & 0.17670 & 0.00000 & \text { Uiso } & 1.00\end{array}$ $\begin{array}{lllllllll}0164 & \mathrm{O} & 0.39455 & 0.37896 & 0.87136 & 0.00000 & \text { Uiso } & 1.00\end{array}$ $\begin{array}{lllllllll}0165 & \mathrm{O} & 0.39807 & 0.25388 & 0.71337 & 0.00000 & \text { Uiso } & 1.00\end{array}$ $\begin{array}{llllllll}0166 & 0 & 0.41026 & 0.55878 & 0.65322 & 0.00000 & \text { Uiso } & 1.00\end{array}$ $\begin{array}{lllllllll}0167 & \mathrm{O} & 0.46470 & 0.49371 & 0.70771 & 0.00000 & \text { Uiso } & 1.00\end{array}$ $\begin{array}{llllllllll}0168 & \mathrm{O} & 0.40848 & 0.54461 & 0.29817 & 0.00000 & \text { Uiso } & 1.00\end{array}$ $\begin{array}{llllllllllllll}0168 & 0 & 0.40558 & 0.53200 & 0.07046 & 0.00000 & \text { Uiso } & 1.00\end{array}$

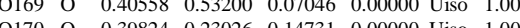
$\begin{array}{llllllllllll}017.00 & 0.000\end{array}$ $\begin{array}{llllllll}0171 & \mathrm{O} & 0.39554 & 0.04499 & 0.84435 & 0.00000 & \text { Uiso } & 1.00\end{array}$ $\begin{array}{llllllllll}0172 & 0 & 0.40547 & 0.07358 & 0.10876 & 0.00000 & \text { Uiso } & 1.00 \\ 0173 & 0 & 0.41428 & 0.6985 & 0.39705 & 0.00000 & \text { Uiso } & 1.00\end{array}$ $\begin{array}{lllllllll}0173 & \mathrm{O} & 0.41428 & 0.69685 & 0.39705 & 0.00000 & \text { Uiso } & 1.00 \\ 0174 & \mathrm{O} & 0.45047 & 0.32078 & 0.80215 & 0.00000 & \text { Uso } & 1.00\end{array}$

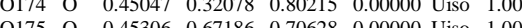
$\begin{array}{llllllllll}0175 & \mathrm{O} & 0.45306 & 0.67186 & 0.70628 & 0.00000 & \text { Uiso } & 1.00\end{array}$ $\begin{array}{llllllll}0176 & \mathrm{O} & 0.40978 & 0.63291 & 0.87000 & 0.00000 & \text { Uiso } & 1.00 \\ 0177 & \mathrm{O} & 0.4452 & 0.48960 & 0.48230 & 0.00000 & \text { Uis } & 1.00\end{array}$ $\begin{array}{llllllllll}0177 & 0 & 0.44552 & 0.48960 & 0.48230 & 0.00000 & \text { Uiso } & 1.00\end{array}$ $\begin{array}{lllllllll}0178 & \mathrm{O} & 0.37030 & 0.16732 & 0.53209 & 0.00000 & \text { Uiso } & 1.00\end{array}$ $\begin{array}{lllllllll}0179 & \mathrm{O} & 0.42077 & 0.11391 & 0.65281 & 0.00000 & \text { Uiso } & 1.00\end{array}$ $\begin{array}{llllllll}0180 & \mathrm{O} & 0.40932 & 0.94080 & 0.64896 & 0.00000 & \text { Uiso } & 1.00\end{array}$ $\begin{array}{llllllll}0181 & \mathrm{O} & 0.39800 & 0.87031 & 0.43286 & 0.00000 & \text { Uiso } & 1.00\end{array}$ $\begin{array}{llllllll}0182 & \mathrm{O} & 0.42692 & 0.16953 & 0.42003 & 0.00000 & \text { Uiso } & 1.00\end{array}$ $\begin{array}{lllllllll}0183 & \mathrm{O} & 0.39759 & 0.03782 & 0.45967 & 0.00000 & \text { Uiso } & 1.00\end{array}$ $\begin{array}{llllllll}0184 & \mathrm{O} & 0.45080 & 0.94566 & 0.49087 & 0.00000 & \text { Uiso } & 1.00\end{array}$

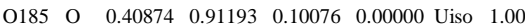
$\begin{array}{llllllll}0186 & \mathrm{O} & 0.42974 & 0.99206 & 0.27545 & 0.00000 & \text { Uiso } & 1.00\end{array}$ $\begin{array}{llllllll}0187 & \mathrm{O} & 0.39009 & 0.67795 & 0.17319 & 0.00000 & \text { Uiso } & 1.00\end{array}$ $\begin{array}{lllllllll}0188 & \mathrm{O} & 0.36434 & 0.98240 & 0.23792 & 0.00000 & \text { Uiso } & 1.00\end{array}$ $\begin{array}{llllllllllll}0189 & \mathrm{O} & 0.44340 & 0.79120 & 0.56047 & 0.00000 & \text { Uiso } & 1.00\end{array}$ $\begin{array}{lllllllllllllll}0189 & 0 & 0.44340 & 0.79120 & 0.5647 & 0.0000 & \text { Uiso } & 1.00\end{array}$

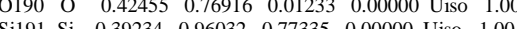
$\begin{array}{llllllll}\text { Sil91 Si } & 0.39234 & 0.96032 & 0.77335 & 0.00000 & \text { Uiso } & 1.00\end{array}$ $\begin{array}{llllllll}\mathrm{Si192} & \mathrm{Si} & 0.41377 & 0.33973 & 0.76444 & 0.00000 & \text { Uiso } & 1.00 \\ \mathrm{Si193} & \mathrm{Si} & 0.43368 & 0.48335 & 0.62045 & 0.00000 & \text { Uiso } & 1.00\end{array}$ $\begin{array}{llllllll}\text { Si193 Si } & 0.43368 & 0.48335 & 0.62045 & 0.00000 & \text { Uiso } & 1.00\end{array}$ $\begin{array}{llllllll}\text { Si194 Si } & 0.39305 & 0.48645 & 0.18746 & 0.00000 & \text { Uiso } & 1.00\end{array}$ $\begin{array}{lllllll}\text { Sil95 Si } & 0.39612 & 0.15811 & 0.04881 & 0.00000 & \text { Uiso } & 1.00\end{array}$ $\begin{array}{llllllll}\mathrm{Si} 196 \mathrm{Si} & 0.39418 & 0.63955 & 0.31235 & 0.00000 & \text { Uiso } & 1.00\end{array}$ $\begin{array}{llllllll}\mathrm{Si} 197 & \mathrm{Si} & 0.37541 & 0.30916 & 0.15305 & 0.00000 & \text { Uiso } & 1.00\end{array}$ $\begin{array}{llllllll}\text { Si198 Si } & 0.41596 & 0.64199 & 0.72885 & 0.00000 & \text { Uiso } & 1.00\end{array}$ $\begin{array}{llllllll}\mathrm{Si} 199 & \mathrm{Si} & 0.41212 & 0.94859 & 0.50194 & 0.00000 & \text { Uiso } & 1.00\end{array}$ $\begin{array}{lllllll}\mathrm{Si} 200 \mathrm{Si} & 0.40534 & 0.12665 & 0.52360 & 0.00000 & \text { Uiso } & 1.00\end{array}$ $\begin{array}{lllllll}\mathrm{Si2} 201 \mathrm{Si} & 0.40055 & 0.99050 & 0.17862 & 0.00000 & \text { Uiso } & 1.00\end{array}$

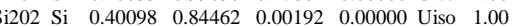
$\begin{array}{llllllll}\mathrm{H} 203 & \mathrm{H} & 0.46879 & 0.44819 & 0.76003 & 0.00000 & \text { Uiso } & 1.00\end{array}$ $\begin{array}{llllllll}\mathrm{H} 204 & \mathrm{H} & 0.45200 & 0.28966 & 0.87580 & 0.00000 & \text { Uiso } & 1.00\end{array}$ $\begin{array}{lllllllll}\mathrm{H} 205 & \mathrm{H} & 0.46785 & 0.62759 & 0.70682 & 0.00000 & \text { Uiso } & 1.00\end{array}$ $\begin{array}{llllllll}\mathrm{H} 206 & \mathrm{H} & 0.43195 & 0.51075 & 0.41864 & 0.00000 & \text { Uiso } & 1.00\end{array}$ $\begin{array}{lllllllll}\mathrm{H} 207 & \mathrm{H} & 0.43783 & 0.21822 & 0.44145 & 0.00000 & \text { Uiso } & 1.00\end{array}$ $\begin{array}{llllllll}\mathrm{H} 208 & \mathrm{H} & 0.45659 & 0.94672 & 0.40598 & 0.00000 & \text { Uiso } & 1.00\end{array}$ $\begin{array}{lllllllll}\mathrm{H} 209 & \mathrm{H} & 0.43466 & 0.04688 & 0.29848 & 0.00000 & \text { Uiso } & 1.00\end{array}$ $\begin{array}{llllllllll} & \mathrm{H} 210 & \mathrm{H} & 0.45421 & 0.84250 & 0.54799 & 0.00000 & \text { Uiso } & 1.00\end{array}$ $\begin{array}{lllllllll}\mathrm{H} 210 & \mathrm{H} & 0.45421 & 0.84250 & 0.54799 & 0.00000 & \text { Uiso } & 1.00 \\ \mathrm{H} 211 & \mathrm{H} & 0.45319 & 0.75225 & 0.61779 & 0.00000 & \text { Uiso } & 1.00\end{array}$ $\begin{array}{llllllllll}1211 & \mathrm{H} & 0.45319 & 0.75225 & 0.61779 & 0.00000 & \text { Uiso } & 1.00\end{array}$ $\begin{array}{llllllll}1212 & \mathrm{H} & 0.47000 & 0.87063 & 0.94402 & 0.00000 & \text { Uiso } & 1.00\end{array}$ $\begin{array}{lllllllll}\mathrm{H} 213 & \mathrm{H} & 0.45282 & 0.64596 & 0.12359 & 0.00000 & \text { Uiso } & 1.00\end{array}$ $\begin{array}{lllllllll}\mathrm{H} 214 & \mathrm{H} & 0.46134 & 0.64791 & 0.96494 & 0.00000 & \text { Uiso } & 1.00 \\ \mathrm{H} 215 & \mathrm{H} & 0.4906 & 0.66950 & 0.07357 & 0.00000 & \text { Uiso } & 1.00\end{array}$ $\begin{array}{llllllllll}\mathrm{H} 215 & \mathrm{H} & 0.49066 & 0.66950 & 0.07357 & 0.00000 & \text { Uiso } & 1.00\end{array}$ $\begin{array}{llllllll}H 216 & \mathrm{H} & 0.49066 & 0.66950 & 0.07357 & 0.00000 & \text { Uiso } & 1.00\end{array}$ $\begin{array}{lllllllll}\mathrm{H} 217 & \mathrm{H} & 0.45884 & 0.86684 & 0.17079 & 0.00000 & \text { Uiso } & 1.00\end{array}$ $\begin{array}{llllllllll}\mathrm{H} 218 & \mathrm{H} & 0.48647 & 0.79013 & 0.20112 & 0.00000 & \text { Uiso } & 1.00\end{array}$ $\begin{array}{lllllllll}\mathrm{H} 219 & \mathrm{H} & 0.47272 & 0.77817 & 0.86328 & 0.00000 & \text { Uiso } & 1.00\end{array}$ $\begin{array}{llllllll}\mathrm{H} 220 & \mathrm{H} & 0.50148 & 0.80479 & 0.97240 & 0.00000 & \text { Uiso } & 1.00\end{array}$ $\begin{array}{llllllll}\mathrm{H} 221 & \mathrm{H} & 0.48120 & 0.21835 & 0.98654 & 0.00000 & \text { Uiso } & 1.00\end{array}$ $\begin{array}{lllllllll}\mathrm{H} 222 & \mathrm{H} & 0.48392 & 0.15177 & 0.86135 & 0.00000 & \text { Uiso } & 1.00\end{array}$ $\begin{array}{llllllll}\mathrm{H} 223 & \mathrm{H} & 0.51765 & 0.17737 & 0.94724 & 0.00000 & \text { Uiso } & 1.00\end{array}$ $\begin{array}{lllllllll}\mathrm{H} 224 & \mathrm{H} & 0.46720 & 0.01763 & 0.90628 & 0.00000 & \text { Uiso } & 1.00\end{array}$

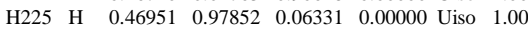
$\begin{array}{llllllll}\mathrm{H} 226 & \mathrm{H} & 0.48147 & 0.15973 & 0.20301 & 0.00000 & \text { Uiso } & 1.00\end{array}$ $\begin{array}{lllllllll}\mathrm{H} 227 & \mathrm{H} & 0.51983 & 0.12715 & 0.17400 & 0.00000 & \text { Uiso } & 1.00\end{array}$ $\begin{array}{llllllll}\mathrm{H} 228 & \mathrm{H} & 0.49117 & 0.05659 & 0.22114 & 0.00000 & \text { Uiso } & 1.00\end{array}$ $\begin{array}{lllllllll}\mathrm{C} 229 & \mathrm{C} & 0.46246 & 0.80212 & 0.17201 & 0.00000 & \text { Uiso } & 1.00\end{array}$ $\begin{array}{lllllllll}\mathrm{C} 230 & \mathrm{C} & 0.45788 & 0.76514 & 0.04779 & 0.00000 & \text { Uiso } & 1.00\end{array}$ $\begin{array}{llllllllllllllll}\mathrm{C} 231 & \mathrm{C} & 0.46589 & 0.67657 & 0.05186 & 0.00000 & \text { Uiso } & 1.00\end{array}$

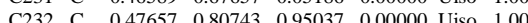
$\begin{array}{lllllllllll}\mathrm{C} 232 & \mathrm{C} & 0.47537 & 0.80743 & 0.95037 & 0.00000 & \text { Uiso } & 1.00\end{array}$

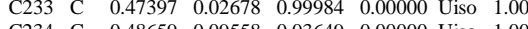

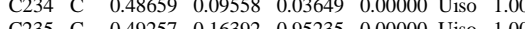
$\begin{array}{llllllll}\mathrm{C} 235 & \mathrm{C} & 0.49257 & 0.16392 & 0.95235 & 0.00000 & \text { Uiso } & 1.00 \\ \mathrm{C} 236 & \mathrm{C} & 0.49517 & 0.10986 & 0.16570 & 0.00000 & \text { Uiso } & 1.00\end{array}$

\section{File $\mathbf{n}^{\circ} 16$}

$\begin{array}{llllllll}\mathrm{A} 2 & \mathrm{Al} & 0.35085 & 0.05566 & 0.89374 & 0.00000 & \text { Uiso } & 1.00\end{array}$ $\begin{array}{llllllll}\mathrm{Al} 3 & \mathrm{Al} & 0.35015 & 0.03670 & 0.37607 & 0.00000 & \text { Uiso } & 1.00\end{array}$ $\begin{array}{llllllll}\mathrm{Al} 4 & \mathrm{Al} & 0.34663 & 0.56923 & 0.89875 & 0.00000 & \text { Uiso } & 1.00\end{array}$ $\begin{array}{lllllllll}\text { Al5 } & \mathrm{Al} & 0.34413 & 0.53279 & 0.38443 & 0.00000 & \text { Uiso } & 1.00\end{array}$ $\begin{array}{llllllll}\text { Al6 } & \text { Al } & 0.34424 & 0.87356 & 0.89786 & 0.00000 & \text { Uiso } & 1.00\end{array}$ $\begin{array}{llllllll}\mathrm{Al} 7 & \mathrm{Al} & 0.35033 & 0.85180 & 0.40972 & 0.00000 & \text { Uiso } & 1.00\end{array}$ $\begin{array}{llllllll}\text { Al8 } & \text { Al } & 0.35363 & 0.40239 & 0.88336 & 0.00000 & \text { Uiso } & 1.00\end{array}$ $\begin{array}{lllllllll}\mathrm{A} 19 & \mathrm{Al} & 0.32908 & 0.38487 & 0.51877 & 0.00000 & \text { Uiso } & 1.00\end{array}$ $\begin{array}{llllllll}\text { Al10 } & \text { Al } & 0.30479 & 0.80291 & 0.26326 & 0.00000 & \text { Uiso } & 1.00\end{array}$ $\begin{array}{llllllll}\text { Al1 } & \text { Al } & 0.30085 & 0.80713 & 0.76413 & 0.00000 & \text { Uiso } & 1.00\end{array}$ $\begin{array}{lllllllll}\text { Al12 } & \text { Al } & 0.30436 & 0.30716 & 0.26036 & 0.00000 & \text { Uiso } & 1.00\end{array}$ $\begin{array}{lllllllll}\text { Al12 } & \text { Al } & 0.30436 & 0.30716 & 0.26036 & 0.00000 & \text { Uiso } & 1.00 \\ \text { Al1 } & \text { Al } & 0.30155 & 0.31470 & 0.77372 & 0.00000 & \text { Uiso } & 1.00\end{array}$ $\begin{array}{llllllllll}\text { Al1 } & \text { Al } 0.301524 & 0.31411 & 0.2632 & 0.0000 & \text { Uiso } & 1.00\end{array}$

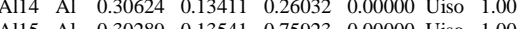
\begin{tabular}{llllllllll}
\hline Al15 Al & 0.30289 & 0.13541 & 0.75923 & 0.00000 & Uiso & 1.00
\end{tabular} $\begin{array}{lllllllll}\text { Al16 Al } & 0.29752 & 0.63541 & 0.26697 & 0.00000 & \text { Uiso } & 1.00\end{array}$ $\begin{array}{lllllllll}\text { Al17 } & \text { Al } & 0.29922 & 0.64076 & 0.76146 & 0.00000 & \text { Uiso } & 1.00\end{array}$ $\begin{array}{lllllllll}\text { Al18 Al } & 0.21191 & 0.97110 & 0.25381 & 0.00000 & \text { Uiso } & 1.00\end{array}$ $\begin{array}{llllllll}\text { Al19 Al } & 0.21176 & 0.97245 & 0.75450 & 0.00000 & \text { Uiso } & 1.00\end{array}$ $\begin{array}{llllllll}\text { Al20 Al } & 0.20935 & 0.47056 & 0.25063 & 0.00000 & \text { Uiso } & 1.00\end{array}$ $\begin{array}{llllllll}\mathrm{Al} 21 & \mathrm{Al} & 0.21015 & 0.47068 & 0.75172 & 0.00000 & \text { Uiso } & 1.00\end{array}$ $\begin{array}{llllllll}\text { Al22 Al } & 0.32925 & 0.96948 & 0.12902 & 0.00000 & \text { Uiso } & 1.00\end{array}$ $\begin{array}{llllllll}\mathrm{Al} 23 & \mathrm{Al} & 0.32707 & 0.96719 & 0.62619 & 0.00000 & \text { Uiso } & 1.00\end{array}$ $\begin{array}{lllllll}\text { Al24 Al } & 0.32455 & 0.47619 & 0.12492 & 0.00000 & \text { Uiso } & 1.00\end{array}$ 
$\begin{array}{llllllll}\text { Al25 } & \text { Al } & 0.33977 & 0.53444 & 0.65858 & 0.00000 & \text { Uiso } & 1.00\end{array}$ $\begin{array}{llllllll}\text { Al26 } & \text { Al } & 0.28072 & 0.22012 & 0.02404 & 0.00000 & \text { Uiso } & 1.00\end{array}$ $\begin{array}{llllllll}\mathrm{Al} 27 & \mathrm{Al} & 0.28183 & 0.22123 & 0.51690 & 0.00000 & \text { Uiso } & 1.00\end{array}$ $\begin{array}{lllllllll}\mathrm{Al} 28 & \mathrm{Al} & 0.28106 & 0.71861 & 0.00738 & 0.00000 & \text { Uiso } & 1.00\end{array}$ $\begin{array}{lllllllll}\text { Al29 } & \text { Al } & 0.28104 & 0.72090 & 0.50710 & 0.00000 & \text { Uiso } & 1.00\end{array}$ $\begin{array}{lllllllll}\mathrm{Al} 30 & \mathrm{Al} & 0.34570 & 0.23210 & 0.91779 & 0.00000 & \text { Uiso } & 1.00\end{array}$ $\begin{array}{llllllll}\mathrm{A} 131 & \mathrm{Al} & 0.35512 & 0.23210 & 0.91779 & 0.00000 & \text { Uiso } & 1.00\end{array}$ $\begin{array}{llllllll}\text { Al31 Al } & 0.35512 & 0.23790 & 0.65700 & 0.00000 & \text { Uiso } & 1.00\end{array}$ $\begin{array}{lllllllll}A 132 & \mathrm{Al} & 0.35216 & 0.73070 & 0.12552 & 0.00000 & \text { Uiso } & 1.00\end{array}$ $\begin{array}{lllllllll}A 133 & \mathrm{Al} & 0.35 & 160 & 0.73091 & 0.66493 & 0.00000 & \text { Uiso } & 1.00\end{array}$ $\begin{array}{lllllllll}\text { Al34 Al } & 0.21198 & 0.97156 & 0.00634 & 0.00000 & \text { Uiso } & 1.00\end{array}$ $\begin{array}{llllllll}\mathrm{Al35} & \mathrm{Al} & 0.21143 & 0.97204 & 0.50418 & 0.00000 & \text { Uiso } & 1.00\end{array}$ $\begin{array}{llllllll}\text { Al36 Al } & 0.21023 & 0.46983 & 0.00295 & 0.00000 & \text { Uiso } & 1.00\end{array}$ $\begin{array}{lllllllll}\text { Al37 } & \text { Al } & 0.20960 & 0.47001 & 0.50306 & 0.00000 & \text { Uiso } & 1.00\end{array}$ $\begin{array}{lllllllll}\text { Al38 } & \text { Al } & 0.28282 & 0.96168 & 0.37881 & 0.00000 & \text { Uiso } & 1.00\end{array}$ $\begin{array}{lllllllll}\mathrm{A} 139 & \mathrm{Al} & 0.28183 & 0.97554 & 0.87925 & 0.00000 & \text { Uiso } & 1.00\end{array}$ $\begin{array}{lllllllll}\mathrm{Al} 40 & \mathrm{Al} & 0.27856 & 0.46767 & 0.37676 & 0.00000 & \text { Uiso } & 1.00\end{array}$ $\begin{array}{llllllll}\mathrm{Al} 41 & \mathrm{Al} & 0.28120 & 0.47615 & 0.87746 & 0.00000 & \text { Uiso } & 1.00\end{array}$ $\begin{array}{llllllll}\mathrm{Al} 42 & \mathrm{Al} & 0.23655 & 0.21902 & 0.25537 & 0.00000 & \text { Uiso } & 1.00\end{array}$ $\begin{array}{llllllll}\mathrm{Al} 43 & \mathrm{Al} & 0.23539 & 0.22002 & 0.75396 & 0.00000 & \text { Uiso } & 1.00\end{array}$ $\begin{array}{llllllll}\mathrm{Al} 44 & \mathrm{Al} & 0.23490 & 0.72406 & 0.25056 & 0.00000 & \text { Uiso } & 1.00\end{array}$ $\begin{array}{llllllll}\mathrm{Al} 45 & \mathrm{Al} & 0.23417 & 0.72320 & 0.74999 & 0.00000 & \text { Uiso } & 1.00\end{array}$

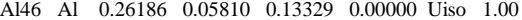
$\begin{array}{llllllll}\mathrm{Al} 47 & \mathrm{Al} & 0.26127 & 0.05827 & 0.62615 & 0.00000 & \text { Uiso } & 1.00\end{array}$ $\begin{array}{lllllllll}\mathrm{Al} 48 & \mathrm{Al} & 0.25841 & 0.55813 & 0.12481 & 0.00000 & \text { Uiso } & 1.00\end{array}$ $\begin{array}{lllllllll}\mathrm{A} 149 & \mathrm{Al} & 0.25901 & 0.55861 & 0.62456 & 0.00000 & \text { Uiso } & 1.00\end{array}$ $\begin{array}{lllllllll}\mathrm{Al} 50 & \mathrm{Al} & 0.26183 & 0.88315 & 0.12666 & 0.00000 & \text { Uiso } & 1.00\end{array}$ $\begin{array}{llllllllll}\mathrm{Al} 51 & \mathrm{Al} & 0.25962 & 0.88322 & 0.62709 & 0.00000 & \text { Uiso } & 1.00\end{array}$ $\begin{array}{lllllllllll}\text { Al51 } & \text { Al } & 0.2562 & 0.88322 & 0.62719 & 0.00000 & \text { Uiso } & 1.00\end{array}$ $\begin{array}{llllllll}\mathrm{A} 152 & \mathrm{Al} & 0.25952 & 0.38232 & 0.13199 & 0.00000 & \text { Uiso } & 1.00\end{array}$

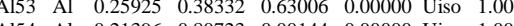
$\begin{array}{llllllll}\text { Al54 Al } & 0.21396 & 0.80723 & 0.00144 & 0.0000 & \text { Uiso } & 1.00\end{array}$ $\begin{array}{lllllllll}4 & \text { Al55 } & \text { Al } & 0.21308 & 0.80675 & 0.50080 & 0.00000 & \text { Uiso } & 1.00\end{array}$ $\begin{array}{llllllll}\text { Al56 } & \text { Al } & 0.21299 & 0.30593 & 0.00341 & 0.00000 & \text { Uiso } & 1.00\end{array}$ $\begin{array}{lllllll}\text { Al57 Al } & 0.2129 & 0.30600 & 0.50084 & 0.00000 & \text { Uiso } & 1.00\end{array}$ $\begin{array}{lllllllll}\text { Al58 Al } & 0.21369 & 0.13512 & 0.00596 & 0.00000 & \text { Uiso } & 1.00\end{array}$ $\begin{array}{llllllll}\mathrm{Al59} & \mathrm{Al} & 0.21368 & 0.13572 & 0.50169 & 0.00000 & \text { Uiso } & 1.00\end{array}$ $\begin{array}{llllllll}\mathrm{Al} 60 & \mathrm{Al} & 0.21270 & 0.63517 & 0.99915 & 0.00000 & \text { Uiso } & 1.00\end{array}$ $\begin{array}{llllllll}\text { Al61 } & \text { Al } & 0.21272 & 0.63523 & 0.49872 & 0.00000 & \text { Uiso } & 1.00\end{array}$ $\begin{array}{llllllll}\mathrm{Al} 62 & \mathrm{Al} & 0.40183 & 0.14785 & 0.78620 & 0.00000 & \text { Uiso } & 1.00\end{array}$ $\begin{array}{llllllll}\mathrm{Al} 63 & \mathrm{Al} & 0.35103 & 0.22481 & 0.39932 & 0.00000 & \text { Uiso } & 1.00\end{array}$ $\begin{array}{lllllllll}\mathrm{A} 164 & \mathrm{Al} & 0.40012 & 0.64450 & 0.01226 & 0.00000 & \text { Uiso } & 1.00\end{array}$ $\begin{array}{llllllll}\mathrm{Al} 65 & \mathrm{Al} & 0.40224 & 0.76563 & 0.50903 & 0.00000 & \text { Uiso } & 1.00\end{array}$ $\begin{array}{llllllllll}066 & \mathrm{O} & 0.30876 & 0.87883 & 0.38304 & 0.00000 & \text { Uiso } & 1.00\end{array}$ $\begin{array}{lllllllll}\mathrm{O} 67 & \mathrm{O} & 0.30387 & 0.88780 & 0.88167 & 0.00000 & \text { Uiso } & 1.00\end{array}$ $\begin{array}{lllllllll}\mathrm{O} 68 & \mathrm{O} & 0.30658 & 0.38821 & 0.38493 & 0.00000 & \text { Uiso } & 1.00\end{array}$ $\begin{array}{llllllll}069 & \mathrm{O} & 0.30686 & 0.39436 & 0.88085 & 0.00000 & \text { Uiso } & 1.00\end{array}$

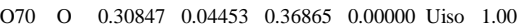
$\begin{array}{llllllll}071 & \mathrm{O} & 0.30902 & 0.05820 & 0.87274 & 0.00000 & \text { Uiso } & 1.00\end{array}$ $\begin{array}{lllllllll}072 & 0 & 0.30409 & 0.55058 & 0.37072 & 0.00000 & \text { Uiso } & 1.00\end{array}$ $\begin{array}{lllllllll}072 & 0 & 0.30409 & 0.55058 & 0.37072 & 0.00000 & \text { Uiso } & 1.00 \\ 073 & \mathrm{O} & 0.30437 & 0.56326 & 0.88003 & 0.00000 & \text { Uiso } & 1.00\end{array}$

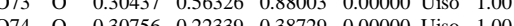
$\begin{array}{llllllll}074 & \mathrm{O} & 0.30756 & 0.22339 & 0.38729 & 0.00000 & \text { Uiso } & 1.00 \\ 075 & 0 & 0.30447 & 0.22013 & 0.88921 & 0.00000 & \text { Uiso } & 1.00\end{array}$ $\begin{array}{lllllllll}075 & \mathrm{O} & 0.30447 & 0.22013 & 0.88921 & 0.00000 & \text { Uiso } & 1.00 \\ 076 & 0 & 0.30183 & 0.71595 & 0.37080 & 0.00000 & \text { Uiso } & 1.00\end{array}$ $\begin{array}{lllllllll}076 & \mathrm{O} & 0.30183 & 0.71595 & 0.37080 & 0.00000 & \text { Uiso } & 1.00\end{array}$ $\begin{array}{llllllllll}077 & 0 & 0.30046 & 0.72213 & 0.87070 & 0.0000 & \text { Uiso } & 1.00\end{array}$ $\begin{array}{lllllllll}078 & \mathrm{O} & 0.35222 & 0.13459 & 0.31101 & 0.00000 & \text { Uiso } & 1.00 \\ 079 & \mathrm{O} & 0.3581 & 0.15243 & 0.78554 & 0.0000 & \text { Uiso } & 1.00\end{array}$ $\begin{array}{lllllllll}079 & \mathrm{O} & 0.35581 & 0.15243 & 0.78554 & 0.00000 & \text { Uiso } & 1.00\end{array}$ $\begin{array}{llllllll}080 & \mathrm{O} & 0.36067 & 0.62570 & 0.38610 & 0.00000 & \text { Uiso } & 1.00\end{array}$ $\begin{array}{llllllll}\mathrm{O} 81 & \mathrm{O} & 0.34578 & 0.63129 & 0.73550 & 0.00000 & \text { Uiso } & 1.00\end{array}$ $\begin{array}{llllllll}082 & \mathrm{O} & 0.34889 & 0.79925 & 0.26436 & 0.00000 & \text { Uiso } & 1.00\end{array}$ $\begin{array}{llllllll}\mathrm{O} 83 & \mathrm{O} & 0.34532 & 0.80612 & 0.77371 & 0.00000 & \text { Uiso } & 1.00\end{array}$ $\begin{array}{llllllll}084 & \mathrm{O} & 0.35611 & 0.31061 & 0.02348 & 0.00000 & \text { Uiso } & 1.00\end{array}$ $\begin{array}{llllllll}\mathrm{O} 85 & \mathrm{O} & 0.41520 & 0.39368 & 0.63975 & 0.00000 & \text { Uiso } & 1.00\end{array}$ $\begin{array}{llllllllll}086 & \mathrm{O} & 0.35353 & 0.96658 & 0.75534 & 0.00000 & \text { Uiso } & 1.00\end{array}$ $\begin{array}{llllllll}087 & \mathrm{O} & 0.35503 & 0.49108 & 0.23786 & 0.00000 & \text { Uiso } & 1.00\end{array}$ $\begin{array}{lllllllll}088 & \mathrm{O} & 0.35503 & 0.48701 & 0.78282 & 0.00000 & \text { Uiso } & 1.00\end{array}$ $\begin{array}{lllllllll}089 & \mathrm{O} & 0.30456 & 0.88710 & 0.14403 & 0.00000 & \text { Uiso } & 1.00\end{array}$ $\begin{array}{llllllllll}090 & \mathrm{O} & 0.30166 & 0.88735 & 0.64250 & 0.00000 & \text { Uiso } & 1.00\end{array}$ $\begin{array}{lllllllll}091 & \mathrm{O} & 0.30254 & 0.38960 & 0.14986 & 0.00000 & \text { Uiso } & 1.00\end{array}$ $\begin{array}{lllllllll}092 & \mathrm{O} & 0.30242 & 0.38046 & 0.63747 & 0.00000 & \text { Uiso } & 1.00\end{array}$ $\begin{array}{llllllllll}093 & 0 & 0.30554 & 0.385562 & 0.13896 & 0.00000 & \text { Uiso } & 1.00\end{array}$ $\begin{array}{lllllllll}093 & 0 & 0.30554 & 0.05562 & 0.13896 & 0.00000 & \text { Uiso } & 1.00\end{array}$ $\begin{array}{lllllllll}094 & \mathrm{O} & 0.30472 & 0.05609 & 0.63920 & 0.00000 & \text { Uiso } & 1.00\end{array}$ $\begin{array}{lllllllll}095 & \mathrm{O} & 0.30089 & 0.55976 & 0.14286 & 0.00000 & \text { Uiso } & 1.00\end{array}$ $\begin{array}{lllllllll}096 & \mathrm{O} & 0.30054 & 0.55849 & 0.63986 & 0.00000 & \text { Uiso } & 1.00\end{array}$ $\begin{array}{lllllllll}097 & 0 & 0.30672 & 0.22115 & 0.15034 & 0.00000 & \text { Uiso } & 1.00\end{array}$ $\begin{array}{llllllll}098 & 0 & 0.31003 & 0.21837 & 0.64744 & 0.00000 & \text { Uiso } & 1.00 \\ 099 & 0 & 0.30941 & 0.71702 & 0.13793 & 0.00000 & \text { Uiso } & 1.00\end{array}$ $\begin{array}{llllllll}099 & \mathrm{O} & 0.30941 & 0.71702 & 0.13793 & 0.00000 & \text { Uiso } & 1.00\end{array}$ $\begin{array}{llllllll}0100 & \mathrm{O} & 0.30654 & 0.72393 & 0.63797 & 0.00000 & \text { Uiso } & 1.00\end{array}$ $\begin{array}{llllllll}0101 & \mathrm{O} & 0.35953 & 0.14353 & 0.00505 & 0.00000 & \text { Uiso } & 1.00\end{array}$ $\begin{array}{lllllllll}0102 & \mathrm{O} & 0.35791 & 0.65001 & 0.99735 & 0.00000 & \text { Uiso } & 1.00\end{array}$ $\begin{array}{lllllllll}0103 & \mathrm{O} & 0.36150 & 0.81379 & 0.02012 & 0.00000 & \text { Uiso } & 1.00\end{array}$ $\begin{array}{llllllll}0104 & \mathrm{O} & 0.35989 & 0.78023 & 0.52320 & 0.00000 & \text { Uiso } & 1.00\end{array}$ $\begin{array}{lllllllll}0105 & \mathrm{O} & 0.34503 & 0.30411 & 0.78797 & 0.00000 & \text { Uiso } & 1.00\end{array}$ $\begin{array}{llllllll}\mathrm{O} 106 & \mathrm{O} & 0.35380 & 0.30134 & 0.52011 & 0.00000 & \text { Uiso } & 1.00\end{array}$ $\begin{array}{lllllllll}0107 & \mathrm{O} & 0.35056 & 0.96867 & 0.99146 & 0.00000 & \text { Uiso } & 1.00\end{array}$ $\begin{array}{lllllllll}0108 & \mathrm{O} & 0.35084 & 0.96027 & 0.49321 & 0.00000 & \text { Uiso } & 1.00\end{array}$ $\begin{array}{lllllllll}0109 & \mathrm{O} & 0.34985 & 0.47908 & 0.00287 & 0.00000 & \text { Uiso } & 1.00\end{array}$ $\begin{array}{lllllllll}0110 & \mathrm{O} & 0.34924 & 0.47955 & 0.52138 & 0.00000 & \text { Uiso } & 1.00\end{array}$

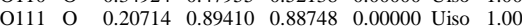
$\begin{array}{llllllll}0112 & 0 & 0.20694 & 0.89349 & 0.38613 & 0.00000 & \text { Uiso } & 1.00\end{array}$ $\begin{array}{llllllllll}0113 & 0 & 0.20580 & 0.39218 & 0.88554 & 0.00000 & \text { Uiso } & 1.00\end{array}$ $\begin{array}{lllllllllll}0114 & 0 & 0.20555 & 0.39232 & 0.38447 & 0.00000 & \text { Uiso } & 1.00\end{array}$

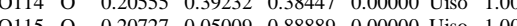
$\begin{array}{llllllll}0115 & 0 & 0.20727 & 0.05009 & 0.88889 & 0.00000 & \text { Uiso } & 1.00\end{array}$ $\begin{array}{lllllllll}0116 & 0 & 0.20705 & 0.04986 & 0.38642 & 0.00000 & \text { Uiso } & 1.00\end{array}$ $\begin{array}{lllllllll}0117 & 0 & 0.20574 & 0.54782 & 0.88445 & 0.00000 & \text { Uiso } & 1.00 \\ 0118 & 0 & 0.20531 & 0.54773 & 0.38375 & 0.00000 & \text { Uiso } & 1.00\end{array}$ $\begin{array}{lllllllll}0118 & \mathrm{O} & 0.20531 & 0.54773 & 0.38375 & 0.00000 & \text { Uiso } & 1.00\end{array}$ $\begin{array}{llllllllllll}0119 & 0 & 0.21095 & 0.22046 & 0.88651 & 0.00000 & \text { Uiso } & 1.00\end{array}$ $\begin{array}{lllllllll}0120 & \mathrm{O} & 0.21090 & 0.22066 & 0.38494 & 0.00000 & \text { Uiso } & 1.00\end{array}$ $\begin{array}{lllllllll}0121 & \mathrm{O} & 0.21040 & 0.72146 & 0.88361 & 0.00000 & \text { Uiso } & 1.00\end{array}$ $\begin{array}{lllllllll}0122 & \mathrm{O} & 0.21029 & 0.72184 & 0.38227 & 0.00000 & \text { Uiso } & 1.00\end{array}$ $\begin{array}{llllllll}\mathrm{O} 123 & \mathrm{O} & 0.26215 & 0.13521 & 0.25359 & 0.00000 & \text { Uiso } & 1.00 \\ \mathrm{O} 124 & \mathrm{O} & 0.26141 & 0.13781 & 0.74601 & 0.00000 & \text { Uiso } & 1.00\end{array}$ $\begin{array}{llllllll}0125 & \mathrm{O} & 0.25707 & 0.63595 & 0.24359 & 0.00000 & \text { Uiso } & 1.00\end{array}$ 
$\begin{array}{llllllll}0126 & \mathrm{O} & 0.25710 & 0.63729 & 0.74183 & 0.00000 & \text { Uiso } & 1.00\end{array}$ $\begin{array}{llllllll}0127 & \mathrm{O} & 0.26170 & 0.80403 & 0.24292 & 0.00000 & \text { Uiso } & 1.00\end{array}$ $\begin{array}{llllllll}0128 & \mathrm{O} & 0.25846 & 0.80743 & 0.74601 & 0.00000 & \text { Uiso } & 1.00\end{array}$ $\begin{array}{llllllll}0129 & 0 & 0.26074 & 0.30527 & 0.25416 & 0.00000 & \text { Uiso } & 1.00\end{array}$ $\begin{array}{llllllll}0130 & \mathrm{O} & 0.25984 & 0.30508 & 0.75079 & 0.00000 & \text { Uiso } & 1.00\end{array}$ $\begin{array}{lllllllll}0131 & \mathrm{O} & 0.25642 & 0.96842 & 0.25014 & 0.00000 & \text { Uiso } & 1.00\end{array}$ $\begin{array}{lllllllllllll}0132 & 0 & 0.25571 & 0.97390 & 0.75027 & 0.00000 & \text { Uiso } & 1.00\end{array}$ $\begin{array}{lllllllllll}0133 & 0 & 0.25738 & 0.4702 & 0.7527 & 0.00000 & \text { Uiso } & 1.00\end{array}$ $\begin{array}{llllllllll}0134 & 0 & 0.25338 & 0.47022 & 0.24795 & 0.00000 & 0.74740 & 1.00\end{array}$ $\begin{array}{lllllllll}0134 & \mathrm{O} & 0.25437 & 0.47040 & 0.74746 & 0.00000 & \text { Uiso } & 1.00\end{array}$ $\begin{array}{llllllllll}0135 & \mathrm{O} & 0.21592 & 0.89300 & 0.12150 & 0.00000 & \text { Uiso } & 1.00 \\ 0136 & \mathrm{O} & 0.21497 & 0.8961 & 0.62121 & 0.0000 & \text { Uiso } & 1.00\end{array}$ $\begin{array}{lllllllll}0136 & \mathrm{O} & 0.21497 & 0.89461 & 0.62121 & 0.00000 & \text { Uiso } & 1.00 \\ 0137 & \mathrm{O} & 0.21420 & 0.39217 & 0.12082 & 0.0000 & \text { Uiso } & 1.00\end{array}$ $\begin{array}{lllllllll}0137 & 0 & 0.21420 & 0.39217 & 0.12082 & 0.00000 & \text { Uiso } & 1.00 \\ 0138 & 0 & 0.21407 & 0.39200 & 0.62043 & 0.00000 & \text { Uis } & 1.00\end{array}$ $\begin{array}{lllllllll}0138 & 0 & 0.21407 & 0.39200 & 0.62043 & 0.00000 & \text { Uiso } & 1.00\end{array}$ $\begin{array}{llllllll}0139 & \mathrm{O} & 0.21590 & 0.04853 & 0.12485 & 0.00000 & \text { Uiso } & 1.00\end{array}$ $\begin{array}{lllllllll}0140 & 0 & 0.21583 & 0.04998 & 0.62089 & 0.00000 & \text { Uiso } & 1.00\end{array}$ $\begin{array}{lllllllll}0141 & \mathrm{O} & 0.21366 & 0.54798 & 0.11865 & 0.00000 & \text { Uiso } & 1.00\end{array}$ $\begin{array}{llllllll}0142 & \mathrm{O} & 0.21380 & 0.54805 & 0.61841 & 0.00000 & \text { Uiso } & 1.00\end{array}$ $\begin{array}{lllllllll}0143 & \mathrm{O} & 0.20848 & 0.21991 & 0.12777 & 0.00000 & \text { Uiso } & 1.00\end{array}$ $\begin{array}{llllllll}0144 & \mathrm{O} & 0.20818 & 0.22101 & 0.62578 & 0.00000 & \text { Uiso } & 1.00\end{array}$ $\begin{array}{lllllllll}0145 & \mathrm{O} & 0.20815 & 0.72182 & 0.12286 & 0.00000 & \text { Uiso } & 1.00\end{array}$ $\begin{array}{lllllllll}0146 & \mathrm{O} & 0.20752 & 0.72170 & 0.62177 & 0.00000 & \text { Uiso } & 1.00\end{array}$ $\begin{array}{llllllll}0147 & \mathrm{O} & 0.25600 & 0.13646 & 0.01719 & 0.00000 & \text { Uiso } & 1.00\end{array}$ $\begin{array}{llllllllll}0148 & \mathrm{O} & 0.25648 & 0.13831 & 0.51274 & 0.00000 & \text { Uiso } & 1.00\end{array}$ $\begin{array}{lllllllll}0149 & \mathrm{O} & 0.25471 & 0.63737 & 0.01157 & 0.00000 & \text { Uiso } & 1.00\end{array}$ $\begin{array}{llllllll}0150 & \mathrm{O} & 0.25487 & 0.63733 & 0.50809 & 0.00000 & \text { Uiso } & 1.00\end{array}$ $\begin{array}{llllllll}0151 & \mathrm{O} & 0.25632 & 0.80362 & 0.01316 & 0.00000 & \text { Uiso } & 1.00\end{array}$ $\begin{array}{llllllllll}0152 & 0 & 0.25525 & 0.80440 & 0.51081 & 0.00000 & \text { Uiso } & 1.00\end{array}$ $\begin{array}{lllllllllll}0152 & 0 & 0.25525 & 0.80440 & 0.51081 & 0.00000 & \text { Uiso } & 1.00\end{array}$ $\begin{array}{lllllllll}0154 & 0 & 0.25541 & 0.30358 & 0.01769 & 0.00000 & \text { Uiso } & 1.00\end{array}$ $\begin{array}{lllllllllllll}0154 & 0 & 0.25553 & 0.30338 & 0.51503 & 0.00000 & \text { Uiso } & 1.00\end{array}$ $\begin{array}{lllllllll}0155 & \mathrm{O} & 0.25884 & 0.97248 & 0.01255 & 0.00000 & \text { Uiso } & 1.00 \\ 0156 & \mathrm{O} & 0.25840 & 0.97017 & 0.50951 & 0.00000 & \text { Uis } & 1.00\end{array}$ $\begin{array}{llllllllll}0156 & \mathrm{O} & 0.25840 & 0.97017 & 0.50951 & 0.00000 & \text { Uiso } & 1.00 \\ 0157 & \mathrm{O} & 0.25710 & 0.47055 & 0.01117 & 0.00000 & \text { Uis } & 1.00\end{array}$ $\begin{array}{lllllllll}0157 & 0 & 0.25710 & 0.47055 & 0.01117 & 0.00000 & \text { Uiso } & 1.00\end{array}$ $\begin{array}{llllllllll}0158 & 0 & 0.25616 & 0.46930 & 0.51001 & 0.00000 & \text { Uiso } & 1.00\end{array}$ $\begin{array}{lllllllll}0159 & \mathrm{O} & 0.35326 & 0.29855 & 0.27096 & 0.00000 & \text { Uiso } & 1.00\end{array}$ $\begin{array}{lllllllll}0160 & \mathrm{O} & 0.39552 & 0.70930 & 0.65500 & 0.00000 & \text { Uiso } & 1.00\end{array}$ $\begin{array}{llllllll}0161 & \mathrm{O} & 0.41620 & 0.17988 & 0.93011 & 0.00000 & \text { Uiso } & 1.00\end{array}$ $\begin{array}{lllllllll}0162 & \mathrm{O} & 0.39732 & 0.88175 & 0.86591 & 0.00000 & \text { Uiso } & 1.00\end{array}$ $\begin{array}{llllllll}0163 & \mathrm{O} & 0.39738 & 0.39372 & 0.16999 & 0.00000 & \text { Uiso } & 1.00\end{array}$ $\begin{array}{llllllll}0164 & \mathrm{O} & 0.39502 & 0.37657 & 0.87039 & 0.00000 & \text { Uiso } & 1.00\end{array}$ $\begin{array}{lllllllll}0165 & \mathrm{O} & 0.39746 & 0.25055 & 0.71258 & 0.00000 & \text { Uiso } & 1.00\end{array}$ $\begin{array}{llllllll}\mathrm{O} 166 & \mathrm{O} & 0.41014 & 0.55572 & 0.63526 & 0.00000 & \text { Uiso } & 1.00\end{array}$ $\begin{array}{llllllllll}0167 & \mathrm{O} & 0.46435 & 0.48744 & 0.68949 & 0.00000 & \text { Uiso } & 1.00\end{array}$ $\begin{array}{llllllll}0168 & \mathrm{O} & 0.40770 & 0.54510 & 0.29754 & 0.00000 & \text { Uiso } & 1.00\end{array}$ $\begin{array}{lllllllll}0169 & \mathrm{O} & 0.40594 & 0.54244 & 0.06803 & 0.00000 & \text { Uiso } & 1.00\end{array}$ $\begin{array}{llllllll}0170 & \mathrm{O} & 0.39960 & 0.23218 & 0.14445 & 0.00000 & \text { Uiso } & 1.00\end{array}$ $\begin{array}{llllllll}0171 & \mathrm{O} & 0.39550 & 0.04268 & 0.84696 & 0.00000 & \text { Uiso } & 1.00\end{array}$ $\begin{array}{llllllll}0172 & 0 & 0.40619 & 0.07088 & 0.10939 & 0.00000 & \text { Uiso } & 1.00\end{array}$ $\begin{array}{lllllllll}0173 & 0 & 0.41515 & 0.69702 & 0.40025 & 0.00000 & \text { Uiso } & 1.00\end{array}$ $\begin{array}{lllllllll}0174 & 0 & 0.41515 & 0.69702 & 0.40216 & 0.00000 & \text { Uiso } & 1.00\end{array}$ $\begin{array}{llllllllllllll}0174 & 0 & 0.45016 & 0.3168 & 0.79216 & 0.00000 & \text { Uiso } & 1.00\end{array}$ $\begin{array}{lllllllll}0175 & 0 & 0.45219 & 0.66721 & 0.69849 & 0.00000 & \text { Uiso } & 1.00\end{array}$ $\begin{array}{llllllllll}0176 & \mathrm{O} & 0.40802 & 0.61851 & 0.85776 & 0.00000 & \text { Uiso } & 1.00 \\ 0177 & \mathrm{O} & 0.44258 & 0.47562 & 0.46602 & 0.0000 & \text { Uiso } & 1.00\end{array}$ $\begin{array}{llllllllll}0177 & \mathrm{O} & 0.44258 & 0.47562 & 0.46602 & 0.00000 & \text { Uiso } & 1.00 \\ 0178 & \mathrm{O} & 0.36726 & 0.16056 & 0.53700 & 0.0000 & \text { Uiso } & 1.00\end{array}$ $\begin{array}{lllllllll}0178 & \mathrm{O} & 0.36726 & 0.16056 & 0.53700 & 0.00000 & \text { Uiso } & 1.00 \\ 0179 & \mathrm{O} & 0.41993 & 0.1191 & 0.6522 & 0.0000 & \text { Uiso } & 1.00\end{array}$ $\begin{array}{llllllllll}018 & 0 & 0.41993 & 0.11191 & 0.65422 & 0.00000 & \text { Uiso } & 1.00\end{array}$ $\begin{array}{lllllllll}0180 & \mathrm{O} & 0.40922 & 0.94303 & 0.64928 & 0.00000 & \text { Uiso } & 1.00\end{array}$

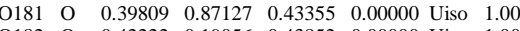
$\begin{array}{llllllll}0182 & \mathrm{O} & 0.42222 & 0.19056 & 0.43852 & 0.00000 & \text { Uiso } & 1.00\end{array}$ $\begin{array}{llllllll}0183 & \mathrm{O} & 0.40053 & 0.04059 & 0.45352 & 0.00000 & \text { Uiso } & 1.00\end{array}$ $\begin{array}{llllllll}\mathrm{O} 184 & \mathrm{O} & 0.45117 & 0.94445 & 0.48927 & 0.00000 & \text { Uiso } & 1.00\end{array}$ $\begin{array}{llllllll}0185 & \mathrm{O} & 0.40912 & 0.90354 & 0.09322 & 0.00000 & \text { Uiso } & 1.00\end{array}$ $\begin{array}{llllllll}\mathrm{O} 186 & \mathrm{O} & 0.41716 & 0.74931 & 0.00123 & 0.00000 & \text { Uiso } & 1.00\end{array}$ $\begin{array}{llllllll}0187 & \mathrm{O} & 0.43016 & 0.98251 & 0.26745 & 0.00000 & \text { Uiso } & 1.00\end{array}$ $\begin{array}{llllllll}0188 & \mathrm{O} & 0.38713 & 0.67811 & 0.18291 & 0.00000 & \text { Uiso } & 1.00\end{array}$ $\begin{array}{lllllllll}0189 & \mathrm{O} & 0.36469 & 0.98041 & 0.23420 & 0.00000 & \text { Uiso } & 1.00\end{array}$ $\begin{array}{llllllll}\mathrm{O} 190 & \mathrm{O} & 0.44231 & 0.79300 & 0.56949 & 0.00000 & \text { Uiso } & 1.00\end{array}$ $\begin{array}{llllllll}\text { Si191 Si } & 0.39185 & 0.95782 & 0.77483 & 0.00000 & \text { Uiso } & 1.00\end{array}$

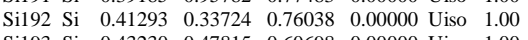

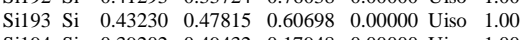
$\begin{array}{lllllll}\mathrm{Si} 194 \mathrm{Si} & 0.39202 & 0.49432 & 0.17948 & 0.00000 & \text { Uiso } & 1.00\end{array}$

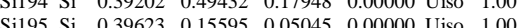
$\begin{array}{llllllll} & \text { Si196 Si } & 0.39375 & 0.64090 & 0.31789 & 0.00000 & \text { Uiso } & 1.00\end{array}$ $\begin{array}{llllllll}\text { Sil96 Si } & 0.39375 & 0.64090 & 0.31789 & 0.00000 & \text { Uiso } & 1.00\end{array}$ $\begin{array}{lllllll}\text { Silg7 Si } & 0.37557 & 0.31048 & 0.15151 & 0.00000 & \text { Uiso } & 1.00\end{array}$ $\begin{array}{llllllll}\text { Sil98 Si } & 0.41526 & 0.63605 & 0.72022 & 0.00000 & \text { Uiso } & 1.00\end{array}$ $\begin{array}{lllllllll}\text { Sily9 Si } & 0.41247 & 0.94917 & 0.50249 & 0.00000 & \text { Uiso } & 1.00\end{array}$ $\begin{array}{lllllllll}\text { Si200 Si } & 0.40339 & 0.12701 & 0.52774 & 0.00000 & \text { Uiso } & 1.00\end{array}$ $\begin{array}{llllllll}\mathrm{Si} 201 \mathrm{Si} & 0.40084 & 0.98269 & 0.17044 & 0.00000 & \text { Uiso } & 1.00\end{array}$ $\begin{array}{llllllll}\mathrm{Si} 202 & \mathrm{Si} & 0.39932 & 0.83235 & 0.99857 & 0.00000 & \text { Uiso } & 1.00\end{array}$ $\begin{array}{llllllll}\mathrm{H} 203 & \mathrm{H} & 0.46841 & 0.44233 & 0.74236 & 0.00000 & \text { Uiso } & 1.00\end{array}$ $\begin{array}{llllllll}\mathrm{H} 204 & \mathrm{H} & 0.45140 & 0.27368 & 0.85022 & 0.00000 & \text { Uiso } & 1.00\end{array}$ $\begin{array}{llllllll}\mathrm{H} 205 & \mathrm{H} & 0.46760 & 0.62484 & 0.70569 & 0.00000 & \text { Uiso } & 1.00\end{array}$ $\begin{array}{llllllll}\mathrm{H} 206 & \mathrm{H} & 0.42975 & 0.50502 & 0.40631 & 0.00000 & \text { Uiso } & 1.00\end{array}$ $\begin{array}{llllllll}\mathrm{H} 207 & \mathrm{H} & 0.42604 & 0.24046 & 0.48141 & 0.00000 & \text { Uiso } & 1.00\end{array}$ $\begin{array}{llllllllll}\mathrm{H} 208 & \mathrm{H} & 0.45611 & 0.93955 & 0.40374 & 0.00000 & \text { Uiso } & 1.00\end{array}$ $\begin{array}{lllllllll}\mathrm{H} 209 & \mathrm{H} & 0.43319 & 0.03599 & 0.29925 & 0.00000 & \text { Uiso } & 1.00\end{array}$ $\begin{array}{llllllll}\mathrm{H} 210 & \mathrm{H} & 0.45428 & 0.84164 & 0.55129 & 0.00000 & \text { Uiso } & 1.00\end{array}$ $\begin{array}{lllllllll}\mathrm{H} 211 & \mathrm{H} & 0.45253 & 0.75109 & 0.62087 & 0.00000 & \text { Uiso } & 1.00\end{array}$ $\begin{array}{llllllllllllllll}\mathrm{H} 212 & \mathrm{H} & 0.51251 & 0.75782 & 0.11084 & 0.00000 & \text { Uiso } & 1.00\end{array}$ $\begin{array}{llllllll}\mathrm{H} 213 & \mathrm{H} & 0.46924 & 0.68422 & 0.91883 & 0.00000 & \text { Uiso } & 1.00\end{array}$ $\begin{array}{llllllllll} & \mathrm{H} 214 & \mathrm{H} & 0.47445 & 0.58088 & 0.95002 & 0.00000 & \text { Uiso } & 1.00\end{array}$ $\begin{array}{lllllllll}\mathrm{H} 214 & \mathrm{H} & 0.4748 & 0.58088 & 0.9502 & 0.0000 & \text { Unso } & 1.00 \\ \mathrm{H} 215 & \mathrm{H} & 0.46820 & 0.61881 & 0.27849 & 0.00000 & \text { Uiso } & 1.00\end{array}$ $\begin{array}{lllllllllll}14215 & \mathrm{H} & 0.47820 & 0.6188 & 0.27849 & 0.00000 & \text { Uiso } & 1.00\end{array}$ $\begin{array}{lllllllll}\mathrm{H} 216 & \mathrm{H} & 0.48669 & 0.75119 & 0.23712 & 0.00000 & \text { Uiso } & 1.00\end{array}$ $\begin{array}{llllllll}\mathrm{H} 217 & \mathrm{H} & 0.47279 & 0.78507 & 0.09459 & 0.00000 & \text { Uiso } & 1.00 \\ \mathrm{H} 218 & \mathrm{H} & 0.46311 & 0.5460 & 0.15316 & 0.00000 & \text { Uis } & 1.00\end{array}$ $\begin{array}{llllllll}\mathrm{H} 218 & \mathrm{H} & 0.46311 & 0.54604 & 0.15316 & 0.00000 & \text { Uiso } & 1.00\end{array}$ $\begin{array}{lllllllll}\mathrm{H} 219 & \mathrm{H} & 0.50732 & 0.64646 & 0.94116 & 0.00000 & \text { Uiso } & 1.00\end{array}$ $\begin{array}{lllllllll}\mathrm{H} 220 \mathrm{H} & 0.45566 & 0.24040 & 0.03003 & 0.00000 & \text { Uiso } & 1.00\end{array}$ $\begin{array}{llllllll}\mathrm{H} 221 & \mathrm{H} & 0.49643 & 0.24273 & 0.03674 & 0.00000 & \text { Uiso } & 1.00\end{array}$ $\begin{array}{llllllll}\mathrm{H} 222 & \mathrm{H} & 0.47498 & 0.33323 & 0.00025 & 0.00000 & \text { Uiso } & 1.00\end{array}$ $\begin{array}{llllllll}\mathrm{H} 223 & \mathrm{H} & 0.47232 & 0.42041 & 0.17241 & 0.00000 & \text { Uiso } & 1.00\end{array}$ $\begin{array}{llllllll}\mathrm{H} 224 & \mathrm{H} & 0.48937 & 0.24422 & 0.34771 & 0.00000 & \text { Uiso } & 1.00\end{array}$ $\begin{array}{llllllll}\mathrm{H} 225 & \mathrm{H} & 0.46435 & 0.38187 & 0.32420 & 0.00000 & \text { Uiso } & 1.00\end{array}$ $\begin{array}{llllllll}\mathrm{H} 226 & \mathrm{H} & 0.43744 & 0.37200 & 0.20051 & 0.00000 & \text { Uiso } & 1.00\end{array}$ 
$\begin{array}{llllllll}\mathrm{H} 227 & \mathrm{H} & 0.45246 & 0.21261 & 0.30393 & 0.00000 & \text { Uiso } & 1.00\end{array}$ $\begin{array}{llllllll}\mathrm{H} 228 & \mathrm{H} & 0.48553 & 0.17289 & 0.22529 & 0.00000 & \text { Uiso } & 1.00\end{array}$ $\begin{array}{lllllllll}\mathrm{C} 229 & \mathrm{C} & 0.48294 & 0.64110 & 0.97139 & 0.00000 & \text { Uiso } & 1.00\end{array}$ $\begin{array}{lllllllll}\mathrm{C} 230 & \mathrm{C} & 0.48036 & 0.65923 & 0.10323 & 0.00000 & \text { Uiso } & 1.00\end{array}$ $\begin{array}{lllllllll}\mathrm{C} 231 & \mathrm{C} & 0.48864 & 0.74285 & 0.13950 & 0.00000 & \text { Uiso } & 1.00\end{array}$ $\begin{array}{llllllllll}\mathrm{C} 232 & \mathrm{C} & 0.47052 & 0.60496 & 0.18311 & 0.00000 & \text { Uiso } & 1.00\end{array}$ $\begin{array}{lllllllllll}C 233 & C & 0.47570 & 0.27924 & 0.05554 & 0.00000 & \text { Uiso } & 1.00\end{array}$ $\begin{array}{lllllllllll}C 234 & C & 0.47255 & 0.29383 & 0.18463 & 0.00000 & \text { Uiso } & 1.00\end{array}$ $\begin{array}{lllllllllll}C 234 & C & 0.47255 & 0.29383 & 0.18463 & 0.00000 & \text { Uiso } & 1.00\end{array}$ $\begin{array}{llllllll}\mathrm{C} 235 & \mathrm{C} & 0.46333 & 0.37079 & 0.22710 & 0.00000 & \text { Uiso } & 1.00 \\ \mathrm{C} 236 & \mathrm{C} & 0.47603 & 0.22672 & 0.26749 & 0.00000 & \text { Uiso } & 1.00\end{array}$

\section{File n ${ }^{\circ} 17$ :}

$\begin{array}{lllllllll}\text { Al2 } & \text { Al } & 0.35094 & 0.05667 & 0.89357 & 0.00000 & \text { Uiso } & 1.00\end{array}$ $\begin{array}{llllllll}\mathrm{Al} 3 & \mathrm{Al} & 0.35052 & 0.03596 & 0.37806 & 0.00000 & \text { Uiso } & 1.00\end{array}$ $\begin{array}{llllllll}\mathrm{Al} 4 & \mathrm{Al} & 0.34715 & 0.57026 & 0.89732 & 0.00000 & \text { Uiso } & 1.00\end{array}$ $\begin{array}{llllllll}\mathrm{Al} 5 & \mathrm{Al} & 0.34343 & 0.53329 & 0.38568 & 0.00000 & \text { Uiso } & 1.00\end{array}$ $\begin{array}{llllllll}\mathrm{Al} 6 & \mathrm{Al} & 0.34424 & 0.87514 & 0.89701 & 0.00000 & \text { Uiso } & 1.00\end{array}$ $\begin{array}{llllllll}\text { Al7 } & \text { Al } & 0.34961 & 0.85159 & 0.41216 & 0.00000 & \text { Uiso } & 1.00\end{array}$ $\begin{array}{llllllll}\mathrm{Al} 8 & \mathrm{Al} & 0.35443 & 0.40138 & 0.88620 & 0.00000 & \text { Uiso } & 1.00\end{array}$ $\begin{array}{llllllll}\mathrm{Al} 9 & \mathrm{Al} & 0.32873 & 0.38567 & 0.51934 & 0.00000 & \text { Uiso } & 1.00\end{array}$ $\begin{array}{lllllllll}\text { Al10 } & \text { Al } & 0.30443 & 0.80285 & 0.26387 & 0.00000 & \text { Uiso } & 1.00\end{array}$ $\begin{array}{lllllllll}\text { Al11 } & \text { Al } & 0.30073 & 0.80721 & 0.76526 & 0.00000 & \text { Uiso } & 1.00\end{array}$ $\begin{array}{lllllllll}\mathrm{Al} 12 & \mathrm{Al} & 0.30455 & 0.30672 & 0.25997 & 0.00000 & \text { Uiso } & 1.00\end{array}$ $\begin{array}{lllllllll}\mathrm{Al13} & \mathrm{Al} & 0.30176 & 0.31538 & 0.77462 & 0.00000 & \text { Uiso } & 1.00\end{array}$ $\begin{array}{llllllllll}\mathrm{Al} 14 & \mathrm{Al} & 0.30640 & 0.13384 & 0.26077 & 0.00000 & \text { Uiso } & 1.00\end{array}$ \begin{tabular}{llllllllll}
\hline 115 & Al & 0.30314 & 0.13618 & 0.75725 & 0.00000 & Uiso & 1.00
\end{tabular}

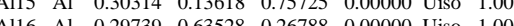

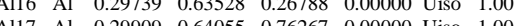

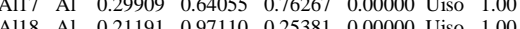
$\begin{array}{llllllll} & \text { Al1 } & \text { Al } 0.21191 & 0.97110 & 0.25381 & 0.00000 & \text { Uiso } & 1.00\end{array}$ $\begin{array}{llllllll}\text { Al19 Al } & 0.21176 & 0.97245 & 0.75450 & 0.00000 & \text { Uiso } & 1.00\end{array}$ $\begin{array}{llllllll}\text { Al20 Al } & 0.20935 & 0.47056 & 0.25063 & 0.00000 & \text { Uiso } & 1.00\end{array}$ $\begin{array}{lllllllll}\text { Al21 } & \text { Al } & 0.21015 & 0.47068 & 0.75172 & 0.00000 & \text { Uiso } & 1.00\end{array}$ $\begin{array}{llllllll}\text { Al22 } & \text { Al } & 0.32929 & 0.96979 & 0.12958 & 0.00000 & \text { Uiso } & 1.00\end{array}$ $\begin{array}{llllllll}\mathrm{Al} 23 & \mathrm{Al} & 0.32682 & 0.96649 & 0.62676 & 0.00000 & \text { Uiso } & 1.00\end{array}$ $\begin{array}{lllllllll}\text { Al24 } & \text { Al } & 0.32435 & 0.47636 & 0.12377 & 0.00000 & \text { Uiso } & 1.00\end{array}$ $\begin{array}{llllllll}\mathrm{A} 125 & \mathrm{Al} & 0.33956 & 0.53347 & 0.66102 & 0.00000 & \text { Uiso } & 1.00\end{array}$

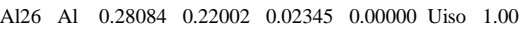
$\begin{array}{lllllllll}\mathrm{A} 127 & \mathrm{Al} & 0.28194 & 0.22121 & 0.51658 & 0.00000 & \text { Uiso } & 1.00\end{array}$ $\begin{array}{llllllll}\text { Al28 } & \text { Al } & 0.28094 & 0.71874 & 0.00797 & 0.00000 & \text { Uiso } & 1.00\end{array}$ $\begin{array}{llllllll}\mathrm{A} 229 & \mathrm{Al} & 0.28099 & 0.72101 & 0.50780 & 0.00000 & \text { Uiso } & 1.00\end{array}$ $\begin{array}{llllllll}\mathrm{Al} 30 & \mathrm{Al} & 0.34594 & 0.23159 & 0.91453 & 0.00000 & \text { Uiso } & 1.00\end{array}$ $\begin{array}{llllllll}\mathrm{Al} 31 & \mathrm{Al} & 0.35637 & 0.23992 & 0.65562 & 0.00000 & \text { Uiso } & 1.00\end{array}$ $\begin{array}{llllllll}\mathrm{Al} 32 & \mathrm{Al} & 0.35161 & 0.73319 & 0.12776 & 0.00000 & \text { Uiso } & 1.00\end{array}$ $\begin{array}{llllllll}\mathrm{Al} 33 & \mathrm{Al} & 0.35132 & 0.73085 & 0.66581 & 0.00000 & \text { Uiso } & 1.00\end{array}$ $\begin{array}{llllllllll}\text { Al34 } & \text { Al } & 0.21198 & 0.97156 & 0.00634 & 0.00000 & \text { Uiso } & 1.00\end{array}$ $\begin{array}{llllllll} & \text { Al35 Al } & 0.21143 & 0.97204 & 0.50418 & 0.00000 & \text { Uiso } & 1.00\end{array}$

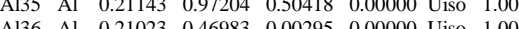
$\begin{array}{lllllllll}\text { A136 Al } & 0.21023 & 0.4683 & 0.00295 & 0.00000 & \text { Uiso } & 1.00\end{array}$

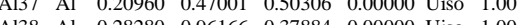
$\begin{array}{lllllllll}A & \text { Al38 Al } & 0.28280 & 0.96166 & 0.37884 & 0.00000 & \text { Uiso } & 1.00\end{array}$ $\begin{array}{lllllllll} & \text { Al39 Al } & 0.28186 & 0.97642 & 0.87924 & 0.00000 & \text { Uiso } & 1.00\end{array}$ $\begin{array}{llllllll}\text { A140 Al } & 0.27834 & 0.46722 & 0.37667 & 0.00000 & \text { Uiso } & 1.00\end{array}$ $\begin{array}{lllllllll}\text { Al41 Al } & 0.28118 & 0.47618 & 0.87756 & 0.00000 & \text { Uiso } & 1.00\end{array}$ $\begin{array}{lllllllll}\text { Al42 } & \text { Al } & 0.23655 & 0.21902 & 0.25537 & 0.00000 & \text { Uiso } & 1.00\end{array}$ $\begin{array}{llllllll}\text { Al43 Al } & 0.23539 & 0.22002 & 0.75396 & 0.00000 & \text { Uiso } & 1.00\end{array}$

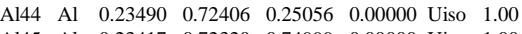
$\begin{array}{llllllll}\mathrm{A} 145 & \mathrm{Al} & 0.23417 & 0.72320 & 0.74999 & 0.00000 & \text { Uiso } & 1.00\end{array}$ $\begin{array}{llllllll}\mathrm{Al} 46 & \mathrm{Al} & 0.26186 & 0.05810 & 0.13329 & 0.00000 & \text { Uiso } & 1.00\end{array}$ $\begin{array}{llllllll}\mathrm{Al} 47 & \mathrm{Al} & 0.26127 & 0.05827 & 0.62615 & 0.00000 & \text { Uiso } & 1.00\end{array}$ $\begin{array}{llllllll}\mathrm{Al} 48 & \mathrm{Al} & 0.25841 & 0.55813 & 0.12481 & 0.00000 & \text { Uiso } & 1.00\end{array}$ $\begin{array}{llllllllll}\mathrm{A} 149 & \mathrm{Al} & 0.25901 & 0.55861 & 0.62456 & 0.00000 & \text { Uiso } & 1.00\end{array}$ $\begin{array}{llllllll}\mathrm{Al} 50 & \mathrm{Al} & 0.26183 & 0.88315 & 0.12666 & 0.00000 & \text { Uiso } & 1.00\end{array}$ $\begin{array}{lllllllll}\mathrm{Al} 151 & \mathrm{Al} & 0.25962 & 0.88322 & 0.62709 & 0.00000 & \text { Uiso } & 1.00\end{array}$ $\begin{array}{llllllll}\mathrm{Al} 52 & \mathrm{Al} & 0.25952 & 0.38232 & 0.13199 & 0.00000 & \text { Uiso } & 1.00\end{array}$ $\begin{array}{lllllllll}\mathrm{Al} 53 & \mathrm{Al} & 0.25925 & 0.38332 & 0.63006 & 0.00000 & \text { Uiso } & 1.00\end{array}$ $\begin{array}{lllllllll}\mathrm{Al} 154 & \mathrm{Al} & 0.21396 & 0.80723 & 0.00144 & 0.00000 & \text { Uiso } & 1.00\end{array}$ $\begin{array}{lllllllll}\mathrm{Al} 55 & \mathrm{Al} & 0.21308 & 0.80675 & 0.50080 & 0.00000 & \text { Uiso } & 1.00\end{array}$ $\begin{array}{lllllllll}4 & 156 & \mathrm{Al} & 0.21299 & 0.30593 & 0.00341 & 0.00000 & \text { Uiso } & 1.00\end{array}$ $\begin{array}{llllllllll}A 157 & \text { Al } & 0.21299 & 0.30600 & 0.50084 & 0.00000 & \text { Uiso } & 1.00\end{array}$

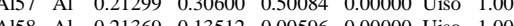
\begin{tabular}{lllllllll} 
Al58 Al & 0.21369 & 0.13512 & 0.0084 & 0.00000 & Uiso & 1.00 \\
\hline & 0.0000 & Uiso & 1.00
\end{tabular}

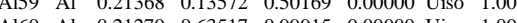
$\begin{array}{llllllll}\text { Al60 Al } & 0.21270 & 0.63517 & 0.99915 & 0.00000 & \text { Uiso } & 1.00\end{array}$ $\begin{array}{lllllll}\text { Al61 Al } & 0.21272 & 0.63523 & 0.49872 & 0.00000 & \text { Uiso } & 1.00\end{array}$ $\begin{array}{llllllll}\mathrm{Al62} & \mathrm{Al} & 0.40268 & 0.14918 & 0.78406 & 0.00000 & \text { Uiso } & 1.00\end{array}$ $\begin{array}{llllllll}\text { Al63 } & \text { Al } & 0.35125 & 0.22549 & 0.39876 & 0.00000 & \text { Uiso } & 1.00\end{array}$ $\begin{array}{llllllll}\text { Al64 Al } & 0.39927 & 0.65336 & 0.00941 & 0.00000 & \text { Uiso } & 1.00\end{array}$ $\begin{array}{llllllll}\text { Al65 } & \mathrm{Al} & 0.40198 & 0.76505 & 0.50824 & 0.00000 & \text { Uiso } & 1.00\end{array}$ $\begin{array}{lllllllll}\mathrm{O} 66 & \mathrm{O} & 0.30818 & 0.87767 & 0.38332 & 0.00000 & \text { Uiso } & 1.00\end{array}$ $\begin{array}{llllllll}\mathrm{O} 67 & \mathrm{O} & 0.30377 & 0.88815 & 0.88185 & 0.00000 & \text { Uiso } & 1.00\end{array}$ $\begin{array}{llllllll}068 & \mathrm{O} & 0.30655 & 0.38889 & 0.38490 & 0.00000 & \text { Uiso } & 1.00\end{array}$ $\begin{array}{llllllllll}069 & \mathrm{O} & 0.30750 & 0.39566 & 0.88338 & 0.00000 & \text { Uiso } & 1.00\end{array}$ $\begin{array}{lllllllll}\mathrm{O} 70 & \mathrm{O} & 0.30864 & 0.04372 & 0.36887 & 0.00000 & \text { Uiso } & 1.00\end{array}$ $\begin{array}{lllllllll}071 & \mathrm{O} & 0.30912 & 0.05885 & 0.87141 & 0.00000 & \text { Uiso } & 1.00\end{array}$ $\begin{array}{llllllll}072 & \mathrm{O} & 0.30352 & 0.55073 & 0.37170 & 0.00000 & \text { Uiso } & 1.00\end{array}$ $\begin{array}{lllllllll}073 & \mathrm{O} & 0.30479 & 0.56278 & 0.88216 & 0.00000 & \text { Uiso } & 1.00\end{array}$ $\begin{array}{lllllllll}074 & \mathrm{O} & 0.30753 & 0.22372 & 0.38715 & 0.00000 & \text { Uiso } & 1.00\end{array}$ $\begin{array}{lllllllll}075 & \mathrm{O} & 0.30449 & 0.22103 & 0.88792 & 0.00000 & \text { Uiso } & 1.00\end{array}$ $\begin{array}{lllllllll}076 & \mathrm{O} & 0.30160 & 0.71589 & 0.37125 & 0.00000 & \text { Uiso } & 1.00\end{array}$ $\begin{array}{llllllll}077 & 0 & 0.30160 & 0.71589 & 0.37725 & 0.00000 & \text { Uiso } & 1.00\end{array}$ $\begin{array}{lllllllllll}078 & 0 & 0.30042 & 0.72186 & 0.87150 & 0.0000 & \text { Uiso } & 1.00\end{array}$ $\begin{array}{llllllll}078 & 0 & 0.35175 & 0.13417 & 0.31235 & 0.00000 & \text { Uiso } & 1.00 \\ 079 & 0 & 0.35582 & 0.15350 & 0.78183 & 0.00000 & \text { Uiso } & 1.00\end{array}$ $\begin{array}{lllllllll}079 & 0 & 0.35582 & 0.15350 & 0.78183 & 0.0000 & \text { Uiso } & 1.00 \\ 080 & \mathrm{O} & 0.35931 & 0.62712 & 0.38108 & 0.0000 & \text { Uiso } & 1.00\end{array}$ $\begin{array}{llllllllll}080 & 0 & 0.35931 & 0.62712 & 0.38108 & 0.00000 & \text { Uiso } & 1.00\end{array}$ $\begin{array}{lllllllll}081 & 0 & 0.34581 & 0.63085 & 0.73721 & 0.00000 & \text { Uiso } & 1.00\end{array}$ $\begin{array}{lllllllll}082 & 0 & 0.34878 & 0.79849 & 0.26578 & 0.0000 & \text { Uiso } & 1.00\end{array}$ $\begin{array}{lllllllll}083 & \mathrm{O} & 0.34530 & 0.80558 & 0.77518 & 0.00000 & \text { Uiso } & 1.00\end{array}$ $\begin{array}{lllllllll}084 & \mathrm{O} & 0.35547 & 0.31119 & 0.02047 & 0.00000 & \text { Uiso } & 1.00\end{array}$ $\begin{array}{lllllllll}085 & \mathrm{O} & 0.41853 & 0.39431 & 0.64787 & 0.00000 & \text { Uiso } & 1.00\end{array}$ $\begin{array}{llllllll}086 & \mathrm{O} & 0.35332 & 0.96529 & 0.75572 & 0.00000 & \text { Uiso } & 1.00\end{array}$

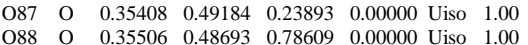


$\begin{array}{llllllll}\text { O89 } & \mathrm{O} & 0.30466 & 0.88696 & 0.14442 & 0.00000 & \text { Uiso } & 1.00\end{array}$ $\begin{array}{lllllllll}\mathrm{O} 90 & \mathrm{O} & 0.30156 & 0.88654 & 0.64310 & 0.00000 & \text { Uiso } & 1.00\end{array}$ $\begin{array}{lllllllll}091 & \mathrm{O} & 0.30256 & 0.38979 & 0.14902 & 0.00000 & \text { Uiso } & 1.00\end{array}$ $\begin{array}{llllllll}092 & \mathrm{O} & 0.30232 & 0.38181 & 0.63893 & 0.00000 & \text { Uiso } & 1.00\end{array}$ $\begin{array}{lllllllll}093 & \mathrm{O} & 0.30543 & 0.05553 & 0.13896 & 0.00000 & \text { Uiso } & 1.00\end{array}$ $\begin{array}{llllllllll}094 & \mathrm{O} & 0.30477 & 0.05591 & 0.63834 & 0.00000 & \text { Uiso } & 1.00\end{array}$ $\begin{array}{llllllllll}095 & 0 & 0.30089 & 0.55996 & 0.14270 & 0.00000 & \text { Uiso } & 1.00\end{array}$ $\begin{array}{lllllllll}095 & 0 & 0.3008 & 0.55996 & 0.1427 & 0.00000 & \text { Uiso } & 1.00 \\ 096 & 0 & 0.30050 & 0.55837 & 0.64169 & 0.00000 & \text { Uiso } & 1.00\end{array}$ $\begin{array}{llllllllll}0967 & \mathrm{O} & 0.30050 & 0.52837 & 0.64169 & 0.0000 & \text { Uiso } & 1.00\end{array}$ $\begin{array}{llllllllll}097 & \mathrm{O} & 0.30660 & 0.22087 & 0.1494 & 0.00000 & \text { Uiso } & 1.00\end{array}$ $\begin{array}{lllllllllll}098 & 0 & 0.31032 & 0.21876 & 0.64574 & 0.00000 & \text { Uiso } & 1.00\end{array}$ $\begin{array}{llllllllll}099 & 0 & 0.30927 & 0.71722 & 0.13831 & 0.0000 & \text { Uiso } & 1.00\end{array}$ $\begin{array}{lllllllll}0100 & \mathrm{O} & 0.30654 & 0.72338 & 0.63888 & 0.00000 & \text { Uiso } & 1.00 \\ 0101 & \mathrm{O} & 0.35915 & 0.14337 & 0.0255 & 0.0000 & \text { Uis } & 1.00\end{array}$ $\begin{array}{lllllllll}0101 & \mathrm{O} & 0.35915 & 0.14337 & 0.00255 & 0.00000 & \text { Uiso } & 1.00\end{array}$ $\begin{array}{lllllllll}0102 & \mathrm{O} & 0.35768 & 0.64963 & 0.99984 & 0.00000 & \text { Uiso } & 1.00\end{array}$ $\begin{array}{lllllllll}0103 & 0 & 0.36150 & 0.81463 & 0.02101 & 0.00000 & \text { Uiso } & 1.00\end{array}$ $\begin{array}{lllllllll}\mathrm{O} 104 & \mathrm{O} & 0.35956 & 0.77864 & 0.52336 & 0.00000 & \text { Uiso } & 1.00\end{array}$ $\begin{array}{llllllll}0105 & \mathrm{O} & 0.34526 & 0.30543 & 0.78685 & 0.00000 & \text { Uiso } & 1.00\end{array}$

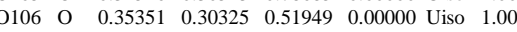
$\begin{array}{llllllll}0107 & \mathrm{O} & 0.35039 & 0.96922 & 0.99134 & 0.00000 & \text { Uiso } & 1.00\end{array}$ $\begin{array}{llllllll}0108 & \mathrm{O} & 0.34977 & 0.95700 & 0.49326 & 0.00000 & \text { Uiso } & 1.00\end{array}$ $\begin{array}{lllllllll}0109 & \mathrm{O} & 0.35312 & 0.48199 & 0.00992 & 0.00000 & \text { Uiso } & 1.00\end{array}$ $\begin{array}{lllllllll}0110 & \mathrm{O} & 0.34909 & 0.48071 & 0.52260 & 0.00000 & \text { Uiso } & 1.00\end{array}$ $\begin{array}{lllllllll}0111 & \mathrm{O} & 0.20714 & 0.89410 & 0.88748 & 0.00000 & \text { Uiso } & 1.00\end{array}$ $\begin{array}{llllllll}0112 & \mathrm{O} & 0.20694 & 0.89349 & 0.38613 & 0.00000 & \text { Uiso } & 1.00\end{array}$ $\begin{array}{llllllll}0113 & \mathrm{O} & 0.20580 & 0.39218 & 0.88554 & 0.00000 & \text { Uiso } & 1.00\end{array}$ $\begin{array}{llllllll}0114 & \mathrm{O} & 0.20555 & 0.39232 & 0.38447 & 0.00000 & \text { Uiso } & 1.00\end{array}$ $\begin{array}{llllllllll}0115 & 0 & 0.20727 & 0.05009 & 0.88889 & 0.00000 & \text { Uiso } & 1.00\end{array}$ $\begin{array}{llllllllll}0116 & 0 & 0.20727 & 0.050705 & 0.04986 & 0.38642 & 0.00000 & \text { Uiso } & 1.00\end{array}$ $\begin{array}{lllllllllll}0117 & 0 & 0.207574 & 0.54782 & 0.88445 & 0.00000 & \text { Uiso } & 1.00\end{array}$ $\begin{array}{llllllllll}0117 & 0 & 0.20574 & 0.54782 & 0.8845 & 0.00000 & \text { Ulso } & 1.00 \\ 0118 & \mathrm{O} & 0.20531 & 0.54773 & 0.38375 & 0.00000 & \text { Uiso } & 1.00\end{array}$ $\begin{array}{lllllllll}0118 & \mathrm{O} & 0.20531 & 0.54773 & 0.38375 & 0.00000 & \text { Uiso } & 1.00 \\ 0119 & \mathrm{O} & 0.21095 & 0.22046 & 0.88651 & 0.00000 & \text { Uiso } & 1.00\end{array}$ $\begin{array}{llllllllllll}0119 & 0 & 0.21095 & 0.22046 & 0.88651 & 0.00000 & \text { Uiso } & 1.00\end{array}$ $\begin{array}{llllllllll}0120 & \mathrm{O} & 0.21090 & 0.22066 & 0.38494 & 0.00000 & \text { Uiso } & 1.00\end{array}$ $\begin{array}{lllllllll}0121 & \mathrm{O} & 0.21040 & 0.72146 & 0.88361 & 0.00000 & \text { Uiso } & 1.00 \\ 0122 & 0 & 0.21029 & 0.72184 & 0.38227 & 0.0000 & \text { Uiso } & 1.00\end{array}$ $\begin{array}{lllllllll}0122 & \mathrm{O} & 0.21029 & 0.72184 & 0.38227 & 0.00000 & \text { Uiso } & 1.00\end{array}$ $\begin{array}{lllllllll}0123 & \mathrm{O} & 0.26215 & 0.13521 & 0.25359 & 0.00000 & \text { Uiso } & 1.00\end{array}$ $\begin{array}{llllllll}0124 & \mathrm{O} & 0.26141 & 0.13781 & 0.74601 & 0.00000 & \text { Uiso } & 1.00\end{array}$ $\begin{array}{llllllll}0125 & 0 & 0.25707 & 0.63595 & 0.24359 & 0.00000 & \text { Uiso } & 1.00\end{array}$ $\begin{array}{llllllll}0126 & \mathrm{O} & 0.25710 & 0.63729 & 0.74183 & 0.00000 & \text { Uiso } & 1.00\end{array}$ $\begin{array}{llllllll}0127 & \mathrm{O} & 0.26170 & 0.80403 & 0.24292 & 0.00000 & \text { Uiso } & 1.00\end{array}$ $\begin{array}{llllllllll}0128 & \mathrm{O} & 0.25846 & 0.80743 & 0.74601 & 0.00000 & \text { Uiso } & 1.00\end{array}$ $\begin{array}{llllllll}0129 & 0 & 0.26074 & 0.30527 & 0.25416 & 0.00000 & \text { Uiso } & 1.00\end{array}$ $\begin{array}{lllllllll}0130 & \mathrm{O} & 0.25984 & 0.30508 & 0.75079 & 0.00000 & \text { Uiso } & 1.00\end{array}$ $\begin{array}{llllllll}\mathrm{O} 131 & \mathrm{O} & 0.25642 & 0.96842 & 0.25014 & 0.00000 & \text { Uiso } & 1.00\end{array}$ $\begin{array}{lllllllll}0132 & \mathrm{O} & 0.25571 & 0.97390 & 0.75027 & 0.00000 & \text { Uiso } & 1.00\end{array}$ $\begin{array}{llllllll}0133 & \mathrm{O} & 0.25338 & 0.47022 & 0.24795 & 0.00000 & \text { Uiso } & 1.00\end{array}$ $\begin{array}{llllllllll}0134 & \mathrm{O} & 0.25437 & 0.47040 & 0.74746 & 0.00000 & \text { Uiso } & 1.00\end{array}$ $\begin{array}{llllllll}0135 & 0 & 0.21592 & 0.89300 & 0.12150 & 0.00000 & \text { Uiso } & 1.00\end{array}$ $\begin{array}{llllllllll}0136 & 0 & 0.21497 & 0.89461 & 0.62121 & 0.00000 & \text { Uiso } & 1.00\end{array}$ $\begin{array}{llllllllll}0136 & 0 & 0.21497 & 0.89461 & 0.62121 & 0.0000 & \text { Uiso } & 1.00\end{array}$

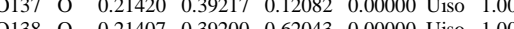
$\begin{array}{llllllll}0138 & \mathrm{O} & 0.21407 & 0.39200 & 0.62043 & 0.00000 & \text { Uiso } & 1.00\end{array}$ $\begin{array}{lllllllll}0139 & 0 & 0.21590 & 0.04853 & 0.12485 & 0.00000 & \text { Uiso } & 1.00\end{array}$ $\begin{array}{llllllll}0140 & 0 & 0.21583 & 0.0498 & 0.62089 & 0.00000 & \text { Uiso } & 1.00 \\ 0141 & 0 & 0.21366 & 0.54798 & 0.11865 & 0.00000 & \text { Uiso } & 1.00\end{array}$ $\begin{array}{lllllllll}0141 & \mathrm{O} & 0.21366 & 0.54798 & 0.11865 & 0.00000 & \text { Uiso } & 1.00 \\ 0142 & \mathrm{O} & 0.21380 & 0.5805 & 0.61841 & 0.00000 & \text { Uis } & 1.00\end{array}$ $\begin{array}{llllllllll}0142 & \mathrm{O} & 0.21380 & 0.54805 & 0.61841 & 0.0000 & \text { Uiso } & 1.00\end{array}$ $\begin{array}{lllllllll}0143 & \mathrm{O} & 0.20848 & 0.21991 & 0.12777 & 0.00000 & \text { Uiso } & 1.00\end{array}$ $\begin{array}{llllllll}0144 & \mathrm{O} & 0.20818 & 0.22101 & 0.62578 & 0.00000 & \text { Uiso } & 1.00\end{array}$ $\begin{array}{llllllll}\mathrm{O} 145 & \mathrm{O} & 0.20815 & 0.72182 & 0.12286 & 0.00000 & \text { Uiso } & 1.00\end{array}$ $\begin{array}{lllllllll}0146 & \mathrm{O} & 0.20752 & 0.72170 & 0.62177 & 0.00000 & \text { Uiso } & 1.00\end{array}$ $\begin{array}{llllllll}0147 & \mathrm{O} & 0.25600 & 0.13646 & 0.01719 & 0.00000 & \text { Uiso } & 1.00\end{array}$ $\begin{array}{llllllll}0148 & \mathrm{O} & 0.25648 & 0.13831 & 0.51274 & 0.00000 & \text { Uiso } & 1.00\end{array}$ $\begin{array}{llllllll}\mathrm{O} 149 & \mathrm{O} & 0.25471 & 0.63737 & 0.01157 & 0.00000 & \text { Uiso } & 1.00\end{array}$ $\begin{array}{lllllllll}0150 & \mathrm{O} & 0.25487 & 0.63733 & 0.50809 & 0.00000 & \text { Uiso } & 1.00\end{array}$ $\begin{array}{llllllll}0151 & \mathrm{O} & 0.25632 & 0.80362 & 0.01316 & 0.00000 & \text { Uiso } & 1.00\end{array}$ $\begin{array}{lllllllll}0152 & \mathrm{O} & 0.25525 & 0.80440 & 0.51081 & 0.00000 & \text { Uiso } & 1.00\end{array}$ $\begin{array}{lllllllll}0153 & \mathrm{O} & 0.25541 & 0.30358 & 0.01769 & 0.00000 & \text { Uiso } & 1.00\end{array}$ $\begin{array}{lllllllll}0154 & 0 & 0.25553 & 0.30338 & 0.51503 & 0.00000 & \text { Uiso } & 1.00\end{array}$ $\begin{array}{llllllll}0155 & \mathrm{O} & 0.25884 & 0.97248 & 0.01255 & 0.00000 & \text { Uiso } & 1.00\end{array}$ $\begin{array}{lllllllll}0156 & 0 & 0.25840 & 0.97017 & 0.50951 & 0.00000 & \text { Uiso } & 1.00\end{array}$ $\begin{array}{lllllllllll}0157 & 0 & 0.25840 & 0.97017 & 0.5091 & 0.00000 & \text { Uiso } & 1.00\end{array}$ $\begin{array}{lllllllllllllllll}0158 & 0 & 0.25710 & 0.47055 & 0.01117 & 0.00000 & \text { Uiso } & 1.00\end{array}$ $\begin{array}{lllllllllll}0158 & 0 & 0.25616 & 0.46930 & 0.51001 & 0.00000 & \text { Uiso } & 1.00\end{array}$ $\begin{array}{lllllllll}0159 & \mathrm{O} & 0.35237 & 0.29851 & 0.26962 & 0.00000 & \text { Uiso } & 1.00 \\ 0160 & \mathrm{O} & 0.39537 & 0.70908 & 0.65529 & 0.00000 & \text { Uiso } & 1.00\end{array}$

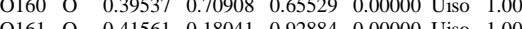
$\begin{array}{lllllllll}0161 & \mathrm{O} & 0.41561 & 0.18041 & 0.92884 & 0.00000 & \text { Uiso } & 1.00 \\ 0162 & \mathrm{O} & 0.39714 & 0.88359 & 0.86808 & 0.00000 & \text { Uis } & 1.00\end{array}$ $\begin{array}{lllllllll}0162 & 0 & 0.39714 & 0.88359 & 0.86808 & 0.00000 & \text { Uiso } & 1.00\end{array}$ $\begin{array}{lllllllll}0163 & \mathrm{O} & 0.39540 & 0.39302 & 0.16680 & 0.00000 & \text { Uiso } & 1.00\end{array}$ $\begin{array}{lllllllll}0164 & \mathrm{O} & 0.39567 & 0.37873 & 0.87321 & 0.00000 & \text { Uiso } & 1.00\end{array}$ $\begin{array}{llllllll}0165 & \mathrm{O} & 0.39814 & 0.25264 & 0.71549 & 0.00000 & \text { Uiso } & 1.00\end{array}$ $\begin{array}{lllllllll}0166 & \mathrm{O} & 0.41048 & 0.55477 & 0.64030 & 0.00000 & \text { Uiso } & 1.00\end{array}$ $\begin{array}{llllllll}0167 & \mathrm{O} & 0.46712 & 0.49435 & 0.67637 & 0.00000 & \text { Uiso } & 1.00\end{array}$ $\begin{array}{llllllll}0168 & \mathrm{O} & 0.40587 & 0.54519 & 0.29335 & 0.00000 & \text { Uiso } & 1.00\end{array}$

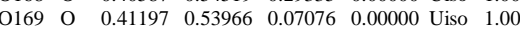
$\begin{array}{llllllll}0170 & \mathrm{O} & 0.39797 & 0.23085 & 0.14409 & 0.00000 & \text { Uiso } & 1.00\end{array}$ $\begin{array}{llllllll}0171 & \mathrm{O} & 0.39528 & 0.04369 & 0.84359 & 0.00000 & \text { Uiso } & 1.00\end{array}$ $\begin{array}{lllllllll}0172 & 0 & 0.40544 & 0.07025 & 0.10627 & 0.00000 & \text { Uiso } & 1.00\end{array}$ $\begin{array}{lllllllll}0173 & \mathrm{O} & 0.41431 & 0.69537 & 0.39916 & 0.00000 & \text { Uiso } & 1.00\end{array}$ $\begin{array}{lllllllll}0174 & \mathrm{O} & 0.45085 & 0.31573 & 0.80775 & 0.00000 & \text { Uiso } & 1.00\end{array}$ $\begin{array}{llllllllll}0175 & \mathrm{O} & 0.45204 & 0.66654 & 0.70682 & 0.00000 & \text { Uiso } & 1.00\end{array}$ $\begin{array}{llllllll}0176 & \mathrm{O} & 0.40592 & 0.61991 & 0.85944 & 0.00000 & \text { Uiso } & 1.00\end{array}$ $\begin{array}{llllllllll}0177 & 0 & 0.44087 & 0.47747 & 0.46349 & 0.00000 & \text { Uiso } & 1.00\end{array}$ $\begin{array}{llllllllllll}0177 & 0 & 0.44087 & 0.4747 & 0.46349 & 0.00000 & \text { Uiso } & 1.00\end{array}$ $\begin{array}{llllllllll}0179 & 0 & 0.37047 & 0.16710 & 0.53135 & 0.0000 & \text { Uiso } & 1.00\end{array}$ $\begin{array}{lllllllll}0179 & \mathrm{O} & 0.42077 & 0.11311 & 0.65184 & 0.00000 & \text { Uiso } & 1.00\end{array}$ $\begin{array}{lllllllll}0180 & \mathrm{O} & 0.40892 & 0.93999 & 0.64962 & 0.00000 & \text { Uiso } & 1.00 \\ 0181 & 0 & 0.39790 & 0.8691 & 0.43373 & 0.00000 & \text { Uiso } & 1.00\end{array}$ $\begin{array}{lllllllll}0181 & \mathrm{O} & 0.39790 & 0.86941 & 0.4337 & 0.00000 & \text { Uiso } & 1.00\end{array}$ $\begin{array}{lllllllll}0182 & \mathrm{O} & 0.42693 & 0.16931 & 0.41937 & 0.00000 & \text { Uiso } & 1.00\end{array}$ $\begin{array}{llllllllll}0183 & 0 & 0.39777 & 0.03697 & 0.45824 & 0.00000 & \text { Unso } & 1.00\end{array}$ $\begin{array}{lllllllll}0184 & \mathrm{O} & 0.45068 & 0.94485 & 0.49135 & 0.00000 & \text { Uiso } & 1.00\end{array}$ $\begin{array}{lllllllll}0185 & \mathrm{O} & 0.40914 & 0.90444 & 0.09798 & 0.00000 & \text { Uiso } & 1.00\end{array}$ $\begin{array}{lllllllll}0186 & \mathrm{O} & 0.42958 & 0.98764 & 0.27205 & 0.00000 & \text { Uiso } & 1.00\end{array}$ $\begin{array}{lllllllll}0187 & \mathrm{O} & 0.38762 & 0.67797 & 0.17896 & 0.00000 & \text { Uiso } & 1.00\end{array}$ $\begin{array}{llllllll}0188 & \mathrm{O} & 0.36451 & 0.98029 & 0.23548 & 0.00000 & \text { Uiso } & 1.00\end{array}$ $\begin{array}{llllllll}0189 & \mathrm{O} & 0.44262 & 0.79053 & 0.56597 & 0.00000 & \text { Uiso } & 1.00\end{array}$ 
$\begin{array}{llllllll}\mathrm{O} 190 & \mathrm{O} & 0.41735 & 0.75281 & 0.00000 & 0.00000 & \text { Uiso } & 1.00\end{array}$ $\begin{array}{llllllll}\mathrm{Si} 191 & \mathrm{Si} & 0.39175 & 0.95831 & 0.77417 & 0.00000 & \text { Uiso } & 1.00\end{array}$ $\begin{array}{llllllll}\mathrm{Si} 192 & \mathrm{Si} & 0.41464 & 0.33710 & 0.76712 & 0.00000 & \text { Uiso } & 1.00\end{array}$ $\begin{array}{lllllllll}\text { Sil93 Si } & 0.43345 & 0.47905 & 0.60642 & 0.00000 & \text { Uiso } & 1.00\end{array}$ $\begin{array}{llllllll}\mathrm{Si} 194 \mathrm{Si} & 0.39049 & 0.49007 & 0.17869 & 0.00000 & \text { Uiso } & 1.00\end{array}$ $\begin{array}{llllllll}\mathrm{Si} 195 & \mathrm{Si} & 0.39609 & 0.15610 & 0.04866 & 0.00000 & \text { Uiso } & 1.00\end{array}$ $\begin{array}{llllllllll}\mathrm{Si} 196 & \mathrm{Si} & 0.39270 & 0.64091 & 0.31578 & 0.00000 & \text { Uiso } & 1.00\end{array}$ $\begin{array}{lllllllllll}\text { Si196 Si } & 0.39270 & 0.64091 & 0.31578 & 0.00000 & \text { Uiso } & 1.00\end{array}$ $\begin{array}{llllllll}\text { Sil198 Si } & 0.41489 & 0.63627 & 0.72180 & 0.00000 & \text { Uiso } & 1.00\end{array}$ $\begin{array}{lllllllll}\text { Silis Si } & 0.41489 & 0.63627 & 0.72180 & 0.00000 & \text { Uiso } & 1.00\end{array}$ $\begin{array}{llllllll} & \text { Sily Si } & 0.41206 & 0.94757 & 0.50263 & 0.00000 & \text { Uiso } & 1.00\end{array}$ $\begin{array}{llllllll}\text { Si200 Si } & 0.40543 & 0.12575 & 0.52255 & 0.00000 & \text { Uiso } & 1.00\end{array}$ $\begin{array}{lllllllll}\mathrm{Si} 201 & \mathrm{Si} & 0.40079 & 0.98464 & 0.17349 & 0.00000 & \text { Uiso } & 1.00 \\ \mathrm{Si2} 202 & \mathrm{Si} & 0.39918 & 0.83587 & 0.00071 & 0.00000 & \text { Us } & 1.00\end{array}$ $\begin{array}{lllllll} & \text { Si202 Si } 0.39918 & 0.83587 & 0.00071 & 0.00000 & \text { Uiso } & 1.00\end{array}$ $\begin{array}{lllllllll}\mathrm{H} 203 & \mathrm{H} & 0.47250 & 0.45378 & 0.73532 & 0.00000 & \text { Uiso } & 1.00\end{array}$ $\begin{array}{llllllll}\mathrm{H} 204 & \mathrm{H} & 0.45138 & 0.28049 & 0.87738 & 0.00000 & \text { Uiso } & 1.00\end{array}$ $\begin{array}{lllllllll}\mathrm{H} 205 & \mathrm{H} & 0.46655 & 0.62191 & 0.69806 & 0.00000 & \text { Uiso } & 1.00\end{array}$ $\begin{array}{llllllll}\mathrm{H} 206 & \mathrm{H} & 0.42703 & 0.50551 & 0.40671 & 0.00000 & \text { Uiso } & 1.00\end{array}$ $\begin{array}{llllllll}\mathrm{H} 207 & \mathrm{H} & 0.43841 & 0.21678 & 0.44247 & 0.00000 & \text { Uiso } & 1.00\end{array}$

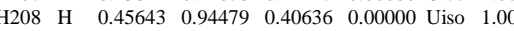
$\begin{array}{llllllll}\mathrm{H} 209 & \mathrm{H} & 0.43382 & 0.04265 & 0.29571 & 0.00000 & \text { Uiso } & 1.00\end{array}$ $\begin{array}{lllllllll}\mathrm{H} 210 & \mathrm{H} & 0.45404 & 0.84068 & 0.55175 & 0.00000 & \text { Uiso } & 1.00\end{array}$ $\begin{array}{lllllllll}\mathrm{H} 211 & \mathrm{H} & 0.45228 & 0.75116 & 0.62270 & 0.00000 & \text { Uiso } & 1.00\end{array}$ $\begin{array}{lllllllll}\mathrm{H} 212 & \mathrm{H} & 0.48265 & 0.49616 & 0.94861 & 0.00000 & \text { Uiso } & 1.00\end{array}$ $\begin{array}{lllllllll}\mathrm{H} 213 & \mathrm{H} & 0.48340 & 0.45598 & 0.14967 & 0.00000 & \text { Uiso } & 1.00\end{array}$ $\begin{array}{lllllllll}\mathrm{H} 214 & \mathrm{H} & 0.45103 & 0.45952 & 0.24624 & 0.00000 & \text { Uiso } 1.00\end{array}$ $\begin{array}{llllllll}\mathrm{H} 215 & \mathrm{H} & 0.44949 & 0.40091 & 0.11096 & 0.00000 & \text { Uiso } & 1.00\end{array}$ $\begin{array}{lllllllll}\mathrm{H} 216 & \mathrm{H} & 0.48710 & 0.60053 & 0.12622 & 0.00000 & \text { Uiso } & 1.00\end{array}$

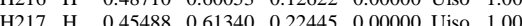
$\begin{array}{lllllllllll}1217 & H & 0.45488 & 0.61340 & 0.22445 & 0.00000 & \text { Ulso } & 1.00\end{array}$ $\begin{array}{lllllllllll}1.218 & \mathrm{H} & 0.45488 & 0.61340 & 0.22445 & 0.00000 & 0.6585 & 1.00\end{array}$ $\begin{array}{llllllllll}\mathrm{H} 219 & \mathrm{H} & 0.45650 & 0.57040 & 0.89491 & 0.0000 & \text { Uiso } & 1.00\end{array}$ $\begin{array}{llllllllllllll}14220 & \mathrm{H} & 0.44374 & 0.46910 & 0.90755 & 0.00000 & \text { Uiso } & 1.00\end{array}$

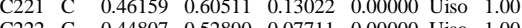
$\begin{array}{llllllllll}\mathrm{C} 222 & \mathrm{C} & 0.44807 & 0.52890 & 0.0771 & 0.00000 & \text { Uiso } & 1.00\end{array}$ $\begin{array}{llllllll}\mathrm{C} 223 & \mathrm{C} & 0.45783 & 0.45651 & 0.15138 & 0.00000 & \text { Uiso } & 1.00 \\ \mathrm{C} 224 & \mathrm{C} & 0.45823 & 0.51580 & 0.94803 & 0.00000 & \text { Uiso } & 1.00\end{array}$

\section{File ${ }^{\circ} 18$}

$\begin{array}{llllllll}\mathrm{Al} 2 & \mathrm{Al} & 0.35124 & 0.05687 & 0.89424 & 0.00000 & \text { Uiso } & 1.00\end{array}$ $\begin{array}{llllllll}\mathrm{Al} 3 & \mathrm{Al} & 0.35004 & 0.03692 & 0.38075 & 0.00000 & \text { Uiso } & 1.00\end{array}$ $\begin{array}{lllllllll}\mathrm{Al} 4 & \mathrm{Al} & 0.34401 & 0.56579 & 0.90006 & 0.00000 & \text { Uiso } & 1.00\end{array}$ $\begin{array}{llllllll}\text { Al5 } & \text { Al } & 0.34387 & 0.53294 & 0.38217 & 0.00000 & \text { Uiso } & 1.00\end{array}$ $\begin{array}{llllllll}\text { Al6 } & \text { Al } & 0.34458 & 0.87426 & 0.89724 & 0.00000 & \text { Uiso } & 1.00\end{array}$ $\begin{array}{lllllllll}\mathrm{Al} 7 & \mathrm{Al} & 0.34997 & 0.85104 & 0.41093 & 0.00000 & \text { Uiso } & 1.00\end{array}$ $\begin{array}{llllllll}\mathrm{Al} 8 & \mathrm{Al} & 0.35328 & 0.40136 & 0.88386 & 0.00000 & \text { Uiso } & 1.00\end{array}$ $\begin{array}{llllllll}\mathrm{Al} 9 & \mathrm{Al} & 0.32880 & 0.38683 & 0.51911 & 0.00000 & \text { Uiso } & 1.00\end{array}$ $\begin{array}{llllllllll} & \mathrm{Al} 10 & \mathrm{Al} & 0.30477 & 0.80229 & 0.26183 & 0.00000 & \text { Uiso } & 1.00\end{array}$ $\begin{array}{lllllllll}\text { Al11 Al } & 0.30477 & 0.80229 & 0.26183 & 0.00000 & \text { Uiso } & 1.00\end{array}$

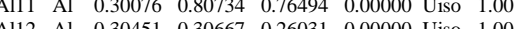
$\begin{array}{lllllllll}112 & \text { Al } & 0.30451 & 0.30667 & 0.26031 & 0.00000 & \text { Uiso } & 1.00\end{array}$ $\begin{array}{llllllll}\text { Al13 Al } & 0.30162 & 0.31414 & 0.77250 & 0.0000 & \text { Uiso } & 1.00\end{array}$ $\begin{array}{lllllllll} & \text { Al14 Al } & 0.30632 & 0.13415 & 0.26132 & 0.00000 & \text { Uiso } & 1.00\end{array}$ $\begin{array}{llllllll}\text { Al15 Al } & 0.30315 & 0.13612 & 0.75850 & 0.0000 & \text { Uiso } & 1.00\end{array}$ $\begin{array}{llllllll}\text { Al16 } & \text { Al } & 0.29759 & 0.63498 & 0.26762 & 0.00000 & \text { Uiso } & 1.00\end{array}$ $\begin{array}{llllllll}\text { Al17 } & \text { Al } & 0.29898 & 0.64125 & 0.76058 & 0.00000 & \text { Uiso } & 1.00\end{array}$ $\begin{array}{llllllll}\text { Al18 } & \mathrm{Al} & 0.21191 & 0.97110 & 0.25381 & 0.00000 & \text { Uiso } & 1.00\end{array}$ $\begin{array}{llllllll}\text { Al19 Al } & 0.21176 & 0.97245 & 0.75450 & 0.00000 & \text { Uiso } & 1.00\end{array}$ $\begin{array}{llllllll}\mathrm{Al} 20 & \mathrm{Al} & 0.20935 & 0.47056 & 0.25063 & 0.00000 & \text { Uiso } & 1.00\end{array}$ $\begin{array}{lllllllll}\mathrm{Al} 21 & \mathrm{Al} & 0.21015 & 0.47068 & 0.75172 & 0.00000 & \text { Uiso } & 1.00\end{array}$ $\begin{array}{llllllll}\mathrm{Al} 22 & \mathrm{Al} & 0.32928 & 0.97028 & 0.12998 & 0.00000 & \text { Uiso } & 1.00\end{array}$ $\begin{array}{llllllll}\mathrm{Al} 23 & \mathrm{Al} & 0.32688 & 0.96663 & 0.62749 & 0.00000 & \text { Uiso } & 1.00\end{array}$ $\begin{array}{llllllllll}\text { Al24 } & \text { Al } & 0.32460 & 0.47511 & 0.12741 & 0.00000 & \text { Uiso } & 1.00\end{array}$ $\begin{array}{llllllll}\mathrm{Al} 25 & \mathrm{Al} & 0.33977 & 0.53536 & 0.65881 & 0.00000 & \text { Uiso } & 1.00\end{array}$ $\begin{array}{llllllllll}\mathrm{Al} 26 & \mathrm{Al} & 0.28081 & 0.22011 & 0.02367 & 0.00000 & \text { Uiso } & 1.00\end{array}$ $\begin{array}{llllllll}\mathrm{Al} 27 & \mathrm{Al} & 0.28185 & 0.22140 & 0.51692 & 0.00000 & \text { Uiso } & 1.00\end{array}$ $\begin{array}{llllllllll}\mathrm{A} 228 & \mathrm{Al} & 0.28101 & 0.71861 & 0.00731 & 0.00000 & \text { Uiso } & 1.00\end{array}$ $\begin{array}{llllllll}\mathrm{Al} 29 & \mathrm{Al} & 0.28101 & 0.72099 & 0.50703 & 0.00000 & \text { Uiso } & 1.00\end{array}$ $\begin{array}{lllllllll}\mathrm{Al} 30 & \mathrm{Al} & 0.34592 & 0.23208 & 0.91659 & 0.00000 & \text { Uiso } & 1.00\end{array}$ $\begin{array}{lllllllll}A 131 & \mathrm{Al} & 0.34562 & 0.23208 & 0.91659 & 0.00000 & \text { Uiso } & 1.00\end{array}$ $\begin{array}{lllllllll}A 132 & \mathrm{Al} & 0.35632 & 0.24061 & 0.6515 & 0.0000 & \text { Uiso } & 1.00\end{array}$

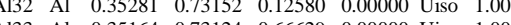
$\begin{array}{lllllllll} & \text { Al33 Al } & 0.35164 & 0.73124 & 0.66629 & 0.00000 & \text { Uiso } & 1.00\end{array}$ $\begin{array}{llllllll} & \text { Al34 Al } & 0.21198 & 0.97156 & 0.00634 & 0.00000 & \text { Uiso } & 1.00\end{array}$ $\begin{array}{llllllll}\text { Al35 Al } & 0.21143 & 0.97204 & 0.50418 & 0.00000 & \text { Uiso } & 1.00\end{array}$ $\begin{array}{lllllllll}\text { Al36 Al } & 0.21023 & 0.46983 & 0.00295 & 0.00000 & \text { Uiso } & 1.00\end{array}$ $\begin{array}{lllllllll}\text { Al37 } & \text { Al } & 0.20960 & 0.47001 & 0.50306 & 0.00000 & \text { Uiso } & 1.00\end{array}$ $\begin{array}{lllllllll}\mathrm{Al} 38 & \mathrm{Al} & 0.28283 & 0.96115 & 0.37888 & 0.00000 & \text { Uiso } & 1.00\end{array}$ $\begin{array}{llllllll}\text { Al39 } & \text { Al } & 0.28195 & 0.97601 & 0.87928 & 0.00000 & \text { Uiso } & 1.00\end{array}$ $\begin{array}{llllllll}\mathrm{Al} 40 & \mathrm{Al} & 0.27839 & 0.46754 & 0.37680 & 0.00000 & \text { Uiso } & 1.00\end{array}$ $\begin{array}{lllllllll}\mathrm{A} 141 & \mathrm{Al} & 0.28074 & 0.47443 & 0.87723 & 0.00000 & \text { Uiso } & 1.00\end{array}$ $\begin{array}{llllllll}\mathrm{Al} 42 & \mathrm{Al} & 0.23655 & 0.21902 & 0.25537 & 0.00000 & \text { Uiso } & 1.00\end{array}$ $\begin{array}{llllllll}\mathrm{Al} 43 & \mathrm{Al} & 0.23539 & 0.22002 & 0.75396 & 0.00000 & \text { Uiso } & 1.00\end{array}$ $\begin{array}{llllllll}\mathrm{Al} 44 & \mathrm{Al} & 0.23490 & 0.72406 & 0.25056 & 0.00000 & \text { Uiso } & 1.00\end{array}$ $\begin{array}{llllllll}\text { Al45 } & \text { Al } & 0.23417 & 0.72320 & 0.74999 & 0.00000 & \text { Uiso } & 1.00\end{array}$ $\begin{array}{llllllll}\mathrm{Al} 46 & \mathrm{Al} & 0.26186 & 0.05810 & 0.13329 & 0.00000 & \text { Uiso } & 1.00\end{array}$ $\begin{array}{llllllll}\mathrm{A} 147 & \mathrm{Al} & 0.26127 & 0.05827 & 0.62615 & 0.00000 & \text { Uiso } & 1.00\end{array}$ $\begin{array}{llllllll}\mathrm{Al} 48 & \mathrm{Al} & 0.25841 & 0.55813 & 0.12481 & 0.00000 & \text { Uiso } & 1.00\end{array}$ $\begin{array}{llllllll}\text { Al49 } & \text { Al } & 0.25901 & 0.55861 & 0.62456 & 0.00000 & \text { Uiso } & 1.00\end{array}$ $\begin{array}{lllllllll}\mathrm{Al} 50 & \mathrm{Al} & 0.26183 & 0.88315 & 0.12666 & 0.00000 & \text { Uiso } & 1.00\end{array}$ $\begin{array}{lllllllll}\mathrm{Al} 51 & \mathrm{Al} & 0.25962 & 0.88322 & 0.62709 & 0.00000 & \text { Uiso } & 1.00\end{array}$ $\begin{array}{llllllll}\mathrm{Al} 1 & \mathrm{Al} & 0.25962 & 0.88322 & 0.62709 & 0.00000 & \text { Uiso } & 1.00 \\ \mathrm{Al} 2 & \mathrm{Al} & 0.25952 & 0.38232 & 0.13199 & 0.00000 & \text { Uis } & 1.00\end{array}$ $\begin{array}{llllllll}4153 & \text { Al } & 0.25925 & 0.38332 & 0.63006 & 0.00000 & \text { Uiso } & 1.00\end{array}$

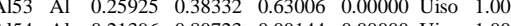
$\begin{array}{llllllll}\text { Al54 Al } & 0.21396 & 0.80723 & 0.00144 & 0.00000 & \text { Uiso } & 1.00\end{array}$

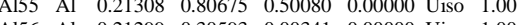

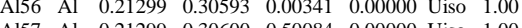
$\begin{array}{lllllllll}\text { Al57 Al } & 0.21299 & 0.30600 & 0.50084 & 0.00000 & \text { Uiso } & 1.00\end{array}$ $\begin{array}{llllllll}\text { Al58 Al } & 0.21369 & 0.13512 & 0.00596 & 0.00000 & \text { Uiso } & 1.00\end{array}$ $\begin{array}{llllllll}\mathrm{Al59} & \mathrm{Al} & 0.21368 & 0.13572 & 0.50169 & 0.00000 & \text { Uiso } & 1.00\end{array}$ $\begin{array}{llllllll}\mathrm{Al} 60 & \mathrm{Al} & 0.21270 & 0.63517 & 0.99915 & 0.00000 & \text { Uiso } & 1.00\end{array}$ $\begin{array}{llllllll}\text { Al61 } & \text { Al } & 0.21272 & 0.63523 & 0.49872 & 0.00000 & \text { Uiso } & 1.00\end{array}$ $\begin{array}{lllllll}\text { Al62 Al } & 0.40258 & 0.15166 & 0.78382 & 0.00000 & \text { Uiso } & 1.00\end{array}$

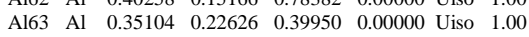


$\begin{array}{lllllll}\text { Al64 Al } & 0.39710 & 0.62750 & 0.01995 & 0.00000 & \text { Uiso } & 1.00\end{array}$ $\begin{array}{lllllllll}\mathrm{Al} 65 & \mathrm{Al} & 0.40224 & 0.76533 & 0.50846 & 0.00000 & \text { Uiso } & 1.00\end{array}$ $\begin{array}{lllllllll}\mathrm{O} 66 & \mathrm{O} & 0.30863 & 0.87741 & 0.38125 & 0.00000 & \text { Uiso } & 1.00\end{array}$ $\begin{array}{llllllll}067 & \mathrm{O} & 0.30410 & 0.88782 & 0.88239 & 0.00000 & \text { Uiso } & 1.00\end{array}$ $\begin{array}{llllllllll}068 & \mathrm{O} & 0.30654 & 0.38922 & 0.38502 & 0.00000 & \text { Uiso } & 1.00\end{array}$ $\begin{array}{lllllllll}\text { O69 } & \mathrm{O} & 0.30648 & 0.39337 & 0.88074 & 0.00000 & \text { Uiso } & 1.00\end{array}$ $\begin{array}{llllllllll}070 & 0 & 0.30832 & 0.04383 & 0.36978 & 0.00000 & \text { Uiso } & 1.00\end{array}$ $\begin{array}{lllllllll}071 & \mathrm{O} & 0.30832 & 0.04383 & 0.36978 & 0.00000 & \text { Uiso } & 1.00\end{array}$ $\begin{array}{llllllllll}072 & 0 & 0.30385 & 0.55069 & 0.37164 & 0.00000 & \text { Uiso } & 1.00\end{array}$ $\begin{array}{lllllllllll}072 & 0 & 0.30385 & 0.55069 & 0.37164 & 0.00000 & \text { Uiso } & 1.00 \\ 073 & 0 & 0.30282 & 0.56331 & 0.87893 & 0.00000 & \text { Uiso } & 1.00\end{array}$ $\begin{array}{llllllllll}073 & 0 & 0.30282 & 0.56331 & 0.87893 & 0.00000 & \text { Uiso } & 1.00\end{array}$ $\begin{array}{lllllllllllllll}074 & 0 & 0.30735 & 0.22382 & 0.38769 & 0.00000 & \text { Uiso } & 1.00\end{array}$ $\begin{array}{llllllllll}075 & \mathrm{O} & 0.30454 & 0.21994 & 0.88853 & 0.00000 & \text { Uiso } & 1.00\end{array}$ $\begin{array}{lllllllll}076 & \mathrm{O} & 0.30178 & 0.71607 & 0.37042 & 0.00000 & \text { Uiso } & 1.00\end{array}$ $\begin{array}{llllllll}\mathrm{O} 77 & \mathrm{O} & 0.30065 & 0.72213 & 0.87100 & 0.00000 & \text { Uiso } & 1.00 \\ 078 & \mathrm{O} & 0.35147 & 0.13455 & 0.31396 & 0.00000 & \text { Uiso } & 1.00\end{array}$ $\begin{array}{llllllllll}078 & \mathrm{O} & 0.35147 & 0.13455 & 0.31396 & 0.00000 & \text { Uiso } & 1.00\end{array}$ $\begin{array}{llllllll}\mathrm{O} 79 & \mathrm{O} & 0.35602 & 0.15402 & 0.78227 & 0.00000 & \text { Uiso } & 1.00\end{array}$

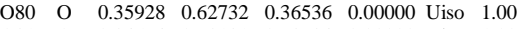
$\begin{array}{lllllllll}\mathrm{O} 81 & \mathrm{O} & 0.34594 & 0.63181 & 0.73684 & 0.00000 & \text { Uiso } & 1.00\end{array}$ $\begin{array}{lllllllll}082 & \mathrm{O} & 0.34955 & 0.79809 & 0.26285 & 0.00000 & \text { Uiso } & 1.00\end{array}$ $\begin{array}{llllllll}083 & \mathrm{O} & 0.34563 & 0.80645 & 0.77471 & 0.00000 & \text { Uiso } & 1.00\end{array}$ $\begin{array}{llllllll}084 & \mathrm{O} & 0.35617 & 0.30999 & 0.02365 & 0.00000 & \text { Uiso } & 1.00\end{array}$ $\begin{array}{llllllll}085 & \mathrm{O} & 0.41713 & 0.39792 & 0.64477 & 0.00000 & \text { Uiso } & 1.00\end{array}$ $\begin{array}{llllllll}\mathrm{O} 86 & \mathrm{O} & 0.35372 & 0.96605 & 0.75561 & 0.00000 & \text { Uiso } & 1.00\end{array}$ $\begin{array}{llllllll}087 & \mathrm{O} & 0.35542 & 0.48761 & 0.23982 & 0.00000 & \text { Uiso } & 1.00\end{array}$ $\begin{array}{lllllllll}088 & \mathrm{O} & 0.35402 & 0.48734 & 0.78396 & 0.00000 & \text { Uiso } & 1.00\end{array}$ $\begin{array}{lllllllll}\mathrm{O} 89 & \mathrm{O} & 0.30493 & 0.88674 & 0.14317 & 0.00000 & \text { Uiso } & 1.00\end{array}$ $\begin{array}{lllllllll}090 & \mathrm{O} & 0.30157 & 0.88684 & 0.64352 & 0.00000 & \text { Uiso } & 1.00\end{array}$ $\begin{array}{lllllllllll}091 & \mathrm{O} & 0.30234 & 0.38926 & 0.15020 & 0.00000 & \text { Uiso } & 1.00\end{array}$ $\begin{array}{lllllllllll}091 & 0 & 0.30234 & 0.38926 & 0.1352 & 0.00000 & \text { Uiso } & 1.00\end{array}$ $\begin{array}{lllllllll}092 & \mathrm{O} & 0.30228 & 0.38182 & 0.63788 & 0.00000 & \text { Uiso } & 1.00 \\ 093 & \mathrm{O} & 0.30544 & 0.05553 & 0.13935 & 0.00000 & \text { Uis } & 1.00\end{array}$ $\begin{array}{lllllllll}093 & \mathrm{O} & 0.30544 & 0.05553 & 0.13935 & 0.00000 & \text { Uiso } & 1.00\end{array}$ $\begin{array}{lllllllll}094 & 0 & 0.30483 & 0.05600 & 0.63902 & 0.0000 & \text { Uiso } & 1.00\end{array}$ $\begin{array}{lllllllllll}095 & 0 & 0.30111 & 0.55960 & 0.14377 & 0.00000 & \text { Uiso } & 1.00\end{array}$ $\begin{array}{lllllllll}096 & 0 & 0.30062 & 0.55969 & 0.63918 & 0.00000 & \text { Uiso } & 1.00\end{array}$ $\begin{array}{llllllll}097 & \mathrm{O} & 0.30655 & 0.22066 & 0.15024 & 0.00000 & \text { Uiso } & 1.00 \\ 098 & \mathrm{O} & 0.31016 & 0.21895 & 0.61663 & 0.00000 & \text { Uso } & 1.00\end{array}$ $\begin{array}{llllllll}098 & \mathrm{O} & 0.31016 & 0.21895 & 0.64663 & 0.00000 & \text { Uiso } & 1.00\end{array}$ $\begin{array}{llllllll}099 & \mathrm{O} & 0.30993 & 0.71788 & 0.13640 & 0.00000 & \text { Uiso } & 1.00\end{array}$ $\begin{array}{lllllllll}0100 & \mathrm{O} & 0.30661 & 0.72438 & 0.63832 & 0.00000 & \text { Uiso } & 1.00\end{array}$ $\begin{array}{llllllll}0101 & \mathrm{O} & 0.35925 & 0.14308 & 0.00331 & 0.00000 & \text { Uiso } & 1.00\end{array}$ $\begin{array}{llllllll}0102 & \mathrm{O} & 0.35659 & 0.64479 & 0.00084 & 0.00000 & \text { Uiso } & 1.00\end{array}$ $\begin{array}{llllllll}0103 & 0 & 0.36522 & 0.81055 & 0.01627 & 0.00000 & \text { Uiso } & 1.00\end{array}$ $\begin{array}{llllllll}\mathrm{O} 104 & \mathrm{O} & 0.35982 & 0.77755 & 0.52211 & 0.00000 & \text { Uiso } & 1.00\end{array}$ $\begin{array}{lllllllll}0105 & \mathrm{O} & 0.34499 & 0.30488 & 0.78761 & 0.00000 & \text { Uiso } & 1.00\end{array}$ $\begin{array}{lllllllll}0106 & \mathrm{O} & 0.35331 & 0.30386 & 0.52022 & 0.00000 & \text { Uiso } & 1.00\end{array}$ $\begin{array}{llllllllll}0107 & \mathrm{O} & 0.35077 & 0.96774 & 0.99193 & 0.00000 & \text { Uiso } & 1.00\end{array}$ $\begin{array}{lllllllll}0108 & \mathrm{O} & 0.34964 & 0.95670 & 0.49381 & 0.00000 & \text { Uiso } & 1.00\end{array}$ $\begin{array}{llllllll}0109 & \mathrm{O} & 0.34851 & 0.47837 & 0.00222 & 0.00000 & \text { Uiso } & 1.00\end{array}$ $\begin{array}{lllllllll}0110 & \mathrm{O} & 0.34942 & 0.48226 & 0.52085 & 0.00000 & \text { Uiso } & 1.00\end{array}$

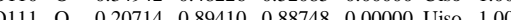
$\begin{array}{llllllllllll}0111 & 0 & 0.20714 & 0.89410 & 0.88748 & 0.00000 & \text { Uiso } & 1.00\end{array}$ $\begin{array}{llllllllll}0113 & 0 & 0.20580 & 0.393418 & 0.88554 & 0.00000 & \text { U1so } & 1.00\end{array}$ $\begin{array}{lllllllll}0114 & \mathrm{O} & 0.20580 & 0.39218 & 0.88554 & 0.00000 & \text { Uiso } & 1.00\end{array}$ $\begin{array}{lllllllll}0114 & \mathrm{O} & 0.20555 & 0.39232 & 0.38447 & 0.00000 & \text { Uiso } & 1.00 \\ 0115 & \mathrm{O} & 0.20727 & 0.05009 & 0.88889 & 0.00000 & \text { Uiso } & 1.00\end{array}$ $\begin{array}{llllllllll}0115 & 0 & 0.20727 & 0.05009 & 0.88889 & 0.00000 & \text { Uiso } & 1.00 \\ 0116 & \mathrm{O} & 0.20705 & 0.04986 & 0.38642 & 0.00000 & \text { Uss } & 1.00\end{array}$ $\begin{array}{lllllllll}0116 & 0 & 0.20705 & 0.04986 & 0.38642 & 0.00000 & \text { Uiso } & 1.00\end{array}$ $\begin{array}{lllllllllll}0117 & 0 & 0.20574 & 0.54782 & 0.88445 & 0.00000 & \text { Uiso } & 1.00 \\ 0118 & 0 & 0.20531 & 0.5773 & 0.38375 & 0.00000 & \text { Uis } & 1.00\end{array}$ $\begin{array}{lllllllll}0118 & \mathrm{O} & 0.20531 & 0.54773 & 0.38375 & 0.00000 & \text { Uiso } & 1.00\end{array}$ $\begin{array}{llllllll}0119 & \mathrm{O} & 0.21095 & 0.22046 & 0.88651 & 0.00000 & \text { Uiso } & 1.00\end{array}$ $\begin{array}{llllllll}0120 & \mathrm{O} & 0.21090 & 0.22066 & 0.38494 & 0.00000 & \text { Uiso } & 1.00\end{array}$

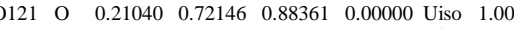
$\begin{array}{llllllll}0122 & \mathrm{O} & 0.21029 & 0.72184 & 0.38227 & 0.00000 & \text { Uiso } & 1.00\end{array}$ $\begin{array}{llllllll}0123 & \mathrm{O} & 0.26215 & 0.13521 & 0.25359 & 0.00000 & \text { Uiso } & 1.00\end{array}$ $\begin{array}{llllllll}0124 & \mathrm{O} & 0.26141 & 0.13781 & 0.74601 & 0.00000 & \text { Uiso } & 1.00\end{array}$ $\begin{array}{lllllllll}0125 & \mathrm{O} & 0.25707 & 0.63595 & 0.24359 & 0.00000 & \text { Uiso } & 1.00\end{array}$ $\begin{array}{lllllllll}0126 & \mathrm{O} & 0.25710 & 0.63729 & 0.74183 & 0.00000 & \text { Uiso } & 1.00\end{array}$ $\begin{array}{lllllllll}0127 & \mathrm{O} & 0.26170 & 0.80403 & 0.24292 & 0.00000 & \text { Uiso } & 1.00\end{array}$ $\begin{array}{llllllll}0128 & \mathrm{O} & 0.25846 & 0.80743 & 0.74601 & 0.00000 & \text { Uiso } & 1.00\end{array}$ $\begin{array}{lllllllll}0129 & \mathrm{O} & 0.26074 & 0.30527 & 0.25416 & 0.00000 & \text { Uiso } & 1.00\end{array}$ $\begin{array}{lllllllll}\mathrm{O} 130 & \mathrm{O} & 0.25984 & 0.30508 & 0.75079 & 0.00000 & \text { Uiso } & 1.00\end{array}$ $\begin{array}{llllllllll}0131 & \mathrm{O} & 0.25642 & 0.96842 & 0.25014 & 0.00000 & \text { Uiso } & 1.00\end{array}$ $\begin{array}{lllllllllll}0132 & 0 & 0.25571 & 0.97390 & 0.75027 & 0.00000 & \text { Uiso } & 1.00\end{array}$ $\begin{array}{lllllllllll}0133 & 0 & 0.25338 & 0.47022 & 0.24795 & 0.00000 & \text { Uiso } & 1.00\end{array}$ $\begin{array}{llllllllll}0134 & 0 & 0.25338 & 0.47022 & 0.24795 & 0.00000 & \text { Ulso } & 1.00\end{array}$ $\begin{array}{llllllllll}0134 & \mathrm{O} & 0.25437 & 0.47040 & 0.74746 & 0.00000 & \text { Uiso } & 1.00 \\ 0135 & \mathrm{O} & 0.21592 & 0.89300 & 0.12150 & 0.00000 & \text { Uiso } & 1.00\end{array}$ $\begin{array}{lllllllllll}0135 & 0 & 0.21592 & 0.89300 & 0.12150 & 0.00000 & \text { Uiso } & 1.00\end{array}$ $\begin{array}{lllllllll}0136 & \mathrm{O} & 0.21497 & 0.89461 & 0.62121 & 0.00000 & \text { Uiso } & 1.00 \\ 0137 & \mathrm{O} & 0.21420 & 0.39217 & 0.12082 & 0.0000 & \text { Uiso } & 1.00\end{array}$ $\begin{array}{llllllllll}0137 & 0 & 0.21420 & 0.39217 & 0.12082 & 0.00000 & \text { Uiso } & 1.00 \\ 0138 & 0 & 0.21407 & 0.39200 & 0.62043 & 0.0000 & \text { Uis } & 1.00\end{array}$ $\begin{array}{lllllllll}0138 & 0 & 0.21407 & 0.39200 & 0.62043 & 0.00000 & \text { Uiso } & 1.00\end{array}$

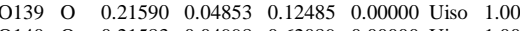

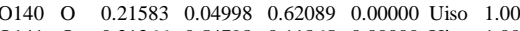
$\begin{array}{llllllll}0141 & \mathrm{O} & 0.21366 & 0.54798 & 0.11865 & 0.00000 & \text { Uiso } & 1.00\end{array}$ $\begin{array}{lllllllll}0142 & \mathrm{O} & 0.21380 & 0.54805 & 0.61841 & 0.00000 & \text { Uiso } & 1.00\end{array}$ $\begin{array}{llllllll}0143 & \mathrm{O} & 0.20848 & 0.21991 & 0.12777 & 0.00000 & \text { Uiso } & 1.00\end{array}$ $\begin{array}{llllllll}0144 & \mathrm{O} & 0.20818 & 0.22101 & 0.62578 & 0.00000 & \text { Uiso } & 1.00\end{array}$ $\begin{array}{lllllllll}0145 & \mathrm{O} & 0.20815 & 0.72182 & 0.12286 & 0.00000 & \text { Uiso } & 1.00\end{array}$ $\begin{array}{llllllll}0146 & \mathrm{O} & 0.20752 & 0.72170 & 0.62177 & 0.00000 & \text { Uiso } & 1.00\end{array}$ $\begin{array}{llllllll}0147 & \mathrm{O} & 0.25600 & 0.13646 & 0.01719 & 0.00000 & \text { Uiso } & 1.00\end{array}$ $\begin{array}{lllllllll}0148 & \mathrm{O} & 0.25648 & 0.13831 & 0.51274 & 0.00000 & \text { Uiso } & 1.00\end{array}$ $\begin{array}{lllllllll}0149 & \mathrm{O} & 0.25471 & 0.63737 & 0.01157 & 0.00000 & \text { Uiso } & 1.00\end{array}$ $\begin{array}{llllllll}0150 & \mathrm{O} & 0.25487 & 0.63733 & 0.50809 & 0.00000 & \text { Uiso } & 1.00\end{array}$

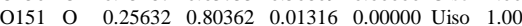
$\begin{array}{lllllllll}0152 & 0 & 0.25525 & 0.80440 & 0.51081 & 0.00000 & \text { Uiso } & 1.00\end{array}$ $\begin{array}{lllllllll}0153 & 0 & 0.25541 & 0.30358 & 0.01769 & 0.00000 & \text { Uiso } & 1.00\end{array}$ $\begin{array}{lllllllllll}0153 & 0 & 0.25541 & 0.30358 & 0.01769 & 0.00000 & \text { Uiso } & 1.00\end{array}$ $\begin{array}{llllllll}0154 & 0 & 0.25553 & 0.30338 & 0.51503 & 0.00000 & \text { Uiso } & 1.00 \\ 0155 & 0 & 0.25884 & 0.97248 & 0.01255 & 0.00000 & \text { Uiso } & 1.00\end{array}$ $\begin{array}{lllllllll}0155 & 0 & 0.25884 & 0.97248 & 0.01255 & 0.00000 & \text { Uiso } & 1.00\end{array}$ $\begin{array}{llllllllll}0156 & 0 & 0.25840 & 0.97017 & 0.50951 & 0.00000 & \text { Uiso } & 1.00\end{array}$ $\begin{array}{llllllll}0157 & \mathrm{O} & 0.25710 & 0.47055 & 0.01117 & 0.00000 & \text { Uiso } & 1.00\end{array}$ $\begin{array}{llllllllll}0158 & \mathrm{O} & 0.25616 & 0.46930 & 0.51001 & 0.00000 & \text { Uiso } & 1.00 \\ 0159 & \mathrm{O} & 0.35218 & 0.2933 & 0.27088 & 0.0000 & \text { Uiso } & 1.00\end{array}$ $\begin{array}{lllllllll}0159 & \mathrm{O} & 0.35218 & 0.29833 & 0.27088 & 0.00000 & \text { Uiso } & 1.00\end{array}$ $\begin{array}{llllllll}0160 & \mathrm{O} & 0.39610 & 0.71268 & 0.65905 & 0.00000 & \text { Uiso } & 1.00\end{array}$ $\begin{array}{lllllllll}\mathrm{O} 161 & \mathrm{O} & 0.41534 & 0.18307 & 0.92836 & 0.00000 & \text { Uiso } & 1.00\end{array}$ $\begin{array}{lllllllll}0162 & \mathrm{O} & 0.39867 & 0.88706 & 0.86682 & 0.00000 & \text { Uiso } & 1.00\end{array}$ $\begin{array}{llllllll}0163 & \mathrm{O} & 0.39762 & 0.38889 & 0.17675 & 0.00000 & \text { Uiso } & 1.00\end{array}$ $\begin{array}{llllllll}\mathrm{O} 164 & \mathrm{O} & 0.39447 & 0.37952 & 0.87075 & 0.00000 & \text { Uiso } & 1.00\end{array}$ 
$\begin{array}{llllllll}0165 & \mathrm{O} & 0.39815 & 0.25433 & 0.71322 & 0.00000 & \text { Uiso } & 1.00\end{array}$ $\begin{array}{lllllllll}0166 & \mathrm{O} & 0.41031 & 0.55854 & 0.65496 & 0.00000 & \text { Uiso } & 1.00\end{array}$ $\begin{array}{llllllll}0167 & \mathrm{O} & 0.46507 & 0.49401 & 0.70750 & 0.00000 & \text { Uiso } & 1.00\end{array}$ $\begin{array}{llllllllll}0168 & \mathrm{O} & 0.40824 & 0.54453 & 0.29888 & 0.00000 & \text { Uiso } & 1.00\end{array}$ $\begin{array}{llllllll}0169 & 0 & 0.40592 & 0.53171 & 0.07133 & 0.00000 & \text { Uiso } & 1.00\end{array}$ $\begin{array}{lllllllll}0170 & \mathrm{O} & 0.39826 & 0.23007 & 0.14771 & 0.00000 & \text { Uiso } & 1.00\end{array}$ $\begin{array}{lllllllllll}0171 & 0 & 0.39555 & 0.04534 & 0.84387 & 0.00000 & \text { Uiso } & 1.00\end{array}$ $\begin{array}{llllllllllllll}0172 & 0 & 0.40608 & 0.07380 & 0.10715 & 0.00000 & \text { Uiso } & 1.00\end{array}$ $\begin{array}{lllllllllll}0173 & 0 & 0.4068 & 0.07380 & 0.10715 & 0.00000 & \text { Uiso } & 1.00\end{array}$ $\begin{array}{llllllllll}0173 & \mathrm{O} & 0.41405 & 0.69624 & 0.39758 & 0.00000 & \text { Uiso } & 1.00\end{array}$ $\begin{array}{lllllllllll}0174 & \mathrm{O} & 0.45043 & 0.32147 & 0.80401 & 0.00000 & \text { Uiso } & 1.00 \\ 0175 & \mathrm{O} & 0.45314 & 0.67174 & 0.70498 & 0.00000 & \text { Uiso } & 1.00\end{array}$ $\begin{array}{llllllllll}0175 & \mathrm{O} & 0.45314 & 0.67174 & 0.70498 & 0.00000 & \text { Uiso } & 1.00 \\ 0176 & \mathrm{O} & 0.41030 & 0.63377 & 0.87123 & 0.0000 & \text { Uiso } & 1.00\end{array}$ $\begin{array}{lllllllll}0176 & 0 & 0.41030 & 0.63377 & 0.87123 & 0.00000 & \text { Uiso } & 1.00\end{array}$ $\begin{array}{lllllllll}0177 & \mathrm{O} & 0.44572 & 0.49102 & 0.48270 & 0.00000 & \text { Uiso } & 1.00\end{array}$ $\begin{array}{lllllllll}0178 & \mathrm{O} & 0.37034 & 0.16778 & 0.53186 & 0.00000 & \text { Uiso } & 1.00 \\ 0179 & \mathrm{O} & 0.42072 & 0.11405 & 0.65243 & 0.00000 & \text { Uis } & 1.00\end{array}$ $\begin{array}{lllllllll}0179 & 0 & 0.42072 & 0.11405 & 0.65243 & 0.00000 & \text { Uiso } & 1.00\end{array}$ $\begin{array}{lllllllll}0180 & \mathrm{O} & 0.40931 & 0.94055 & 0.64873 & 0.00000 & \text { Uiso } & 1.00\end{array}$ $\begin{array}{lllllllll}0181 & \mathrm{O} & 0.39789 & 0.87011 & 0.43301 & 0.00000 & \text { Uiso } & 1.00\end{array}$ $\begin{array}{lllllllll}0182 & \mathrm{O} & 0.42687 & 0.16824 & 0.41874 & 0.00000 & \text { Uiso } & 1.00\end{array}$ $\begin{array}{llllllll}0183 & \mathrm{O} & 0.39730 & 0.03761 & 0.46023 & 0.00000 & \text { Uiso } & 1.00\end{array}$ $\begin{array}{lllllllll}0184 & \mathrm{O} & 0.45073 & 0.94562 & 0.49072 & 0.00000 & \text { Uiso } & 1.00\end{array}$ $\begin{array}{lllllllll}0185 & \mathrm{O} & 0.40885 & 0.91302 & 0.10104 & 0.00000 & \text { Uiso } & 1.00\end{array}$ $\begin{array}{llllllllll}0186 & \mathrm{O} & 0.42952 & 0.99319 & 0.27635 & 0.00000 & \text { Uiso } & 1.00\end{array}$ $\begin{array}{llllllll}0187 & \mathrm{O} & 0.39073 & 0.67864 & 0.17219 & 0.00000 & \text { Uiso } & 1.00\end{array}$ $\begin{array}{llllllll}0188 & \mathrm{O} & 0.36430 & 0.98319 & 0.23745 & 0.00000 & \text { Uiso } & 1.00\end{array}$ $\begin{array}{llllllll}0189 & \mathrm{O} & 0.44331 & 0.79116 & 0.56053 & 0.00000 & \text { Uiso } & 1.00\end{array}$ $\begin{array}{lllllllll}0190 & \mathrm{O} & 0.42537 & 0.77256 & 0.00979 & 0.00000 & \text { Uiso } & 1.00\end{array}$ \begin{tabular}{lllllllll}
\hline & Si191 & Si & 0.39217 & 0.96089 & 0.77235 & 0.00000 & Uiso & 1.00
\end{tabular} $\begin{array}{llllllll}\mathrm{Si191} & \mathrm{Si} & 0.39217 & 0.96089 & 0.77235 & 0.00000 & \text { Uiso } & 1.00 \\ \mathrm{Si1} & \mathrm{Si} & 0.41387 & 0.34024 & 0.76432 & 0.00000 & \text { Uiso } & 1.00\end{array}$ $\begin{array}{llllllll}\mathrm{Si192} & \mathrm{Si} & 0.41387 & 0.34024 & 0.76432 & 0.00000 & \text { Uiso } & 1.00 \\ \mathrm{Si1} & \mathrm{Si} & 0.43396 & 0.48376 & 0.62076 & 0.00000 & \text { Us } & 1.00\end{array}$ $\begin{array}{lllllllll}\text { Silg4 Si } & 0.39310 & 0.48613 & 0.62076 & 0.00000 & \text { Uiso } & 1.00\end{array}$ $\begin{array}{llllllll}\text { Si194 Si } & 0.39310 & 0.48613 & 0.18798 & 0.00000 & \text { Uiso } & 1.00\end{array}$ $\begin{array}{llllllll}\text { Si195 Si } & 0.39620 & 0.15848 & 0.04840 & 0.00000 & \text { Uiso } & 1.00\end{array}$ $\begin{array}{llllllll}\text { Si196 Si } & 0.39414 & 0.63980 & 0.31150 & 0.00000 & \text { Uiso } & 1.00\end{array}$ $\begin{array}{lllllllll} & \text { Sil97 Si } & 0.37545 & 0.30901 & 0.15308 & 0.0000 & \text { Uiso } & 1.00\end{array}$ $\begin{array}{lllllllll}\mathrm{Si} 198 & \mathrm{Si} & 0.41611 & 0.64204 & 0.72969 & 0.00000 & \text { Uiso } & 1.00\end{array}$ $\begin{array}{llllllll}\text { Si199 Si } & 0.41207 & 0.94844 & 0.50176 & 0.00000 & \text { Uiso } & 1.00\end{array}$ $\begin{array}{lllllll}\mathrm{Si} 200 \mathrm{Si} & 0.40529 & 0.12660 & 0.52335 & 0.00000 & \text { Uiso } & 1.00\end{array}$ $\begin{array}{llllllll}\mathrm{Si} 201 \mathrm{Si} & 0.40051 & 0.99145 & 0.17879 & 0.00000 & \text { Uiso } & 1.00\end{array}$ $\begin{array}{lllllll}\mathrm{Si} 202 \mathrm{Si} & 0.40093 & 0.84611 & 0.00183 & 0.00000 & \text { Uiso } & 1.00\end{array}$ $\begin{array}{llllllll}\mathrm{H} 203 & \mathrm{H} & 0.46891 & 0.44902 & 0.76112 & 0.00000 & \text { Uiso } & 1.00\end{array}$ $\begin{array}{llllllll}\mathrm{H} 204 & \mathrm{H} & 0.45153 & 0.29286 & 0.88004 & 0.00000 & \text { Uiso } & 1.00\end{array}$

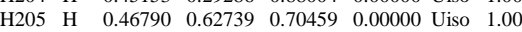
$\begin{array}{llllllll}\mathrm{H} 206 & \mathrm{H} & 0.43202 & 0.51170 & 0.41912 & 0.00000 & \text { Uiso } & 1.00\end{array}$ $\begin{array}{llllllll}\mathrm{H} 207 & \mathrm{H} & 0.43810 & 0.21676 & 0.43839 & 0.00000 & \text { Uiso } & 1.00\end{array}$ $\begin{array}{lllllllll}\mathrm{H} 208 & \mathrm{H} & 0.45666 & 0.94718 & 0.40601 & 0.00000 & \text { Uiso } & 1.00\end{array}$ $\begin{array}{lllllllll}\mathrm{H} 209 & \mathrm{H} & 0.43402 & 0.04788 & 0.30142 & 0.00000 & \text { Uiso } & 1.00\end{array}$ $\begin{array}{llllllll}\mathrm{H} 210 & \mathrm{H} & 0.45421 & 0.84221 & 0.54703 & 0.00000 & \text { Uiso } & 1.00\end{array}$ $\begin{array}{llllllll}\mathrm{H} 211 & \mathrm{H} & 0.45324 & 0.75188 & 0.61708 & 0.00000 & \text { Uiso } & 1.00\end{array}$ \begin{tabular}{llllllllll}
\hline & $\mathrm{H} 212$ & $\mathrm{H}$ & 0.46507 & 0.89749 & 0.97557 & 0.00000 & Uiso & 1.00
\end{tabular} $\begin{array}{llllllllllll}1212 & \mathrm{H} & 0.46507 & 0.89749 & 0.97557 & 0.00000 & \text { Uiso } & 1.00\end{array}$

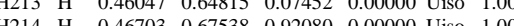
$\begin{array}{lllllllll}4214 & \mathrm{H} & 0.46703 & 0.67538 & 0.92080 & 0.0000 & \text { Uiso } & 1.00\end{array}$ $\begin{array}{llllllll}\mathrm{H} 215 & \mathrm{H} & 0.49652 & 0.6997 & 0.03204 & 0.00000 & \text { Uiso } & 1.00\end{array}$

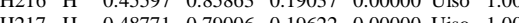
$\begin{array}{llllllll}\mathrm{H} 217 & \mathrm{H} & 0.48771 & 0.79006 & 0.19622 & 0.00000 & \text { Uiso } & 1.00\end{array}$ \begin{tabular}{lllllllllll}
$\mathrm{H} 218$ & $\mathrm{H}$ & 0.48771 & 0.79006 & 0.19622 & 0.00000 & Uiso & 1.00 \\
\hline & 0.75678 & 0.22952 & 0.00000 & Uiso & 1.00
\end{tabular} $\begin{array}{lllllllll}\mathrm{H} 219 & \mathrm{H} & 0.47012 & 0.82205 & 0.86278 & 0.00000 & \text { Uiso } & 1.00\end{array}$ $\begin{array}{lllllllll}\mathrm{H} 220 & \mathrm{H} & 0.49945 & 0.83873 & 0.97371 & 0.00000 & \text { Uiso } & 1.00\end{array}$ $\begin{array}{llllllll}\mathrm{C} 221 & \mathrm{C} & 0.46301 & 0.79688 & 0.17236 & 0.00000 & \text { Uiso } & 1.00\end{array}$ $\begin{array}{llllllll}\mathrm{C} 222 & \mathrm{C} & 0.45899 & 0.77577 & 0.03972 & 0.00000 & \text { Uiso } & 1.00\end{array}$ $\begin{array}{llllllll}\mathrm{C} 223 & \mathrm{C} & 0.47137 & 0.69232 & 0.01439 & 0.00000 & \text { Uiso } & 1.00\end{array}$ $\begin{array}{llllllll}\mathrm{C} 224 & \mathrm{C} & 0.47424 & 0.83736 & 0.95747 & 0.00000 & \text { Uiso } & 1.00\end{array}$

\section{File n ${ }^{\circ} 19$}

$\begin{array}{llllllll}\mathrm{Al} 2 & \mathrm{Al} & 0.35106 & 0.05704 & 0.89485 & 0.00000 & \text { Uiso } & 1.00\end{array}$ $\begin{array}{lllllllll}\mathrm{Al} 3 & \mathrm{Al} & 0.35054 & 0.03695 & 0.37821 & 0.00000 & \text { Uiso } & 1.00\end{array}$ $\begin{array}{lllllllll}\text { Al4 } & \text { Al } & 0.34547 & 0.56788 & 0.89902 & 0.00000 & \text { Uiso } & 1.00\end{array}$ $\begin{array}{llllllll}\text { Al5 } & \text { Al } & 0.34398 & 0.53289 & 0.38506 & 0.00000 & \text { Uiso } & 1.00\end{array}$ $\begin{array}{llllllllll}\mathrm{Al} 6 & \mathrm{Al} & 0.34407 & 0.87503 & 0.89630 & 0.00000 & \text { Uiso } & 1.00\end{array}$ $\begin{array}{lllllllll}\mathrm{Al} & \mathrm{Al} & 0.34407 & 0.8752 & 0.89630 & 0.00000 & \text { Uiso } & 1.00 \\ \mathrm{Al} & \mathrm{Al} & 0.34964 & 0.85227 & 0.41145 & 0.0000 & \text { Uiso } & 1.00\end{array}$

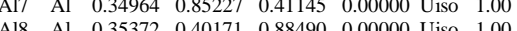
$\begin{array}{lllllllll}A 18 & \text { Al } & 0.35372 & 0.4017 & 0.88490 & 0.00000 & \text { Uiso } & 1.00\end{array}$ $\begin{array}{llllllllll}\text { Al9 Al } & 0.32881 & 0.38561 & 0.51921 & 0.00000 & \text { Uiso } & 1.00\end{array}$ $\begin{array}{lllllllll}\text { Al10 Al } & 0.30448 & 0.80312 & 0.26359 & 0.00000 & \text { Uiso } & 1.00\end{array}$ $\begin{array}{lllllllll}\text { Al11 Al } & 0.30076 & 0.80704 & 0.76466 & 0.00000 & \text { Uiso } & 1.00\end{array}$ $\begin{array}{lllllllll}\text { Al12 Al } & 0.30462 & 0.30714 & 0.25973 & 0.00000 & \text { Uiso } & 1.00\end{array}$ $\begin{array}{llllllllll}\text { Al13 } & \text { Al } & 0.30169 & 0.31470 & 0.77347 & 0.00000 & \text { Uiso } & 1.00\end{array}$ $\begin{array}{llllllll}\text { Al14 } & \mathrm{Al} & 0.30645 & 0.13425 & 0.26047 & 0.00000 & \text { Uiso } & 1.00\end{array}$ $\begin{array}{llllllll}\text { Al15 } & \text { Al } & 0.30314 & 0.13599 & 0.75825 & 0.00000 & \text { Uiso } & 1.00\end{array}$ $\begin{array}{llllllll}\mathrm{Al16} & \mathrm{Al} & 0.29741 & 0.63529 & 0.26723 & 0.00000 & \text { Uiso } & 1.00\end{array}$ $\begin{array}{llllllll}\text { Al17 } & \mathrm{Al} & 0.29911 & 0.64085 & 0.76108 & 0.00000 & \text { Uiso } & 1.00\end{array}$ $\begin{array}{lllllllll}\mathrm{Al18} & \mathrm{Al} & 0.21191 & 0.97110 & 0.25381 & 0.00000 & \text { Uiso } & 1.00\end{array}$ $\begin{array}{llllllll}\text { Al19 } & \text { Al } & 0.21176 & 0.97245 & 0.75450 & 0.00000 & \text { Uiso } & 1.00\end{array}$ $\begin{array}{llllllll}\mathrm{Al} 20 & \mathrm{Al} & 0.20935 & 0.47056 & 0.25063 & 0.00000 & \text { Uiso } & 1.00\end{array}$ $\begin{array}{llllllll}\mathrm{Al} 21 & \mathrm{Al} & 0.21015 & 0.47068 & 0.75172 & 0.00000 & \text { Uiso } & 1.00\end{array}$ $\begin{array}{lllllllll}\mathrm{A} 22 & \mathrm{Al} & 0.32933 & 0.96982 & 0.12964 & 0.00000 & \text { Uiso } & 1.00\end{array}$ $\begin{array}{lllllllll}\mathrm{Al} 23 & \mathrm{Al} & 0.32691 & 0.96650 & 0.62730 & 0.00000 & \text { Uiso } & 1.00\end{array}$ $\begin{array}{llllllll}\mathrm{Al} 24 & \mathrm{Al} & 0.32455 & 0.47629 & 0.12602 & 0.00000 & \text { Uiso } & 1.00\end{array}$ $\begin{array}{lllllllll}\mathrm{Al} 25 & \mathrm{Al} & 0.33985 & 0.53460 & 0.65890 & 0.00000 & \text { Uiso } & 1.00\end{array}$ $\begin{array}{lllllllll}\mathrm{A} 126 & \mathrm{Al} & 0.28082 & 0.22011 & 0.02345 & 0.00000 & \text { Uiso } & 1.00\end{array}$ $\begin{array}{llllllll}\text { Al2 } & \text { Al } & 0.28082 & 0.22011 & 0.02345 & 0.00000 & \text { Uiso } & 1.00 \\ \text { Al2 } & \text { Al } & 0.28198 & 0.22130 & 0.51625 & 0.00000 & \text { Uiso } & 1.00\end{array}$

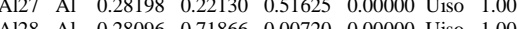

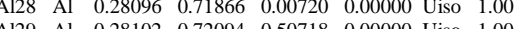
$\begin{array}{lllllllll}\text { A129 Al } & 0.28102 & 0.72094 & 0.50718 & 0.00000 & \text { Uiso } & 1.00\end{array}$ $\begin{array}{lllllllll} & \text { Al30 Al } & 0.34597 & 0.23236 & 0.91631 & 0.00000 & \text { Uiso } & -1.00\end{array}$ $\begin{array}{llllllll}\text { Al31 } & \text { Al } & 0.35605 & 0.23937 & 0.65561 & 0.00000 & \text { Uiso } & 1.00\end{array}$

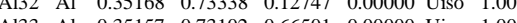
$\begin{array}{lllllllll}\mathrm{Al} 33 & \mathrm{Al} & 0.35157 & 0.73102 & 0.66501 & 0.00000 & \text { Uiso } & -1.00\end{array}$ $\begin{array}{lllllllll}\mathrm{Al} 34 & \mathrm{Al} & 0.21198 & 0.97156 & 0.00634 & 0.00000 & \text { Uiso } & 1.00\end{array}$ $\begin{array}{llllllll}\text { Al35 Al } & 0.21143 & 0.97204 & 0.50418 & 0.00000 & \text { Uiso } & 1.00\end{array}$ $\begin{array}{llllllll}\text { Al36 } & \text { Al } & 0.21023 & 0.46983 & 0.00295 & 0.00000 & \text { Uiso } & 1.00\end{array}$ $\begin{array}{llllllll}\mathrm{Al} 37 & \mathrm{Al} & 0.20960 & 0.47001 & 0.50306 & 0.00000 & \text { Uiso } & 1.00\end{array}$

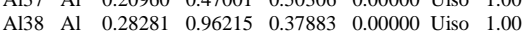


$\begin{array}{llllllll}\text { Al39 } & \text { Al } & 0.28186 & 0.97623 & 0.87925 & 0.00000 & \text { Uiso } & 1.00\end{array}$ $\begin{array}{llllllll}\mathrm{Al} 40 & \mathrm{Al} & 0.27849 & 0.46766 & 0.37672 & 0.00000 & \text { Uiso } & 1.00\end{array}$ $\begin{array}{llllllll}\mathrm{Al} 41 & \mathrm{Al} & 0.28099 & 0.47534 & 0.87730 & 0.00000 & \text { Uiso } & 1.00\end{array}$ $\begin{array}{llllllll}\mathrm{Al} 42 & \mathrm{Al} & 0.23655 & 0.21902 & 0.25537 & 0.00000 & \text { Uiso } & 1.00\end{array}$ $\begin{array}{lllllllll}\mathrm{Al} 43 & \mathrm{Al} & 0.23539 & 0.22002 & 0.75396 & 0.00000 & \text { Uiso } & 1.00\end{array}$ $\begin{array}{llllllllll}\mathrm{Al} 44 & \mathrm{Al} & 0.23490 & 0.72406 & 0.25056 & 0.00000 & \text { Uiso } & 1.00\end{array}$

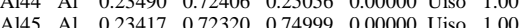
$\begin{array}{lllllllll}A 146 & \text { Al } & 0.26186 & 0.05810 & 0.13329 & 0.00000 & \text { Uiso } & 1.00\end{array}$ $\begin{array}{lllllllll}\text { Al46 } & \mathrm{Al} & 0.26186 & 0.05810 & 0.13329 & 0.0000 & \text { Uiso } & 1.00\end{array}$

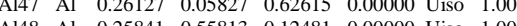

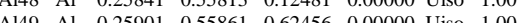
$\begin{array}{lllllllll}\text { Al49 Al } & 0.25901 & 0.55861 & 0.62456 & 0.00000 & \text { Uiso } & 1.00\end{array}$ $\begin{array}{llllllll}\text { Al50 Al } & 0.26183 & 0.88315 & 0.12666 & 0.00000 & \text { Uiso } & 1.00\end{array}$ $\begin{array}{llllllll}\text { Al51 Al } & 0.25962 & 0.88322 & 0.62709 & 0.00000 & \text { Uiso } & 1.00\end{array}$ $\begin{array}{llllllll}\text { Al52 Al } & 0.25952 & 0.38232 & 0.13199 & 0.00000 & \text { Uiso } & 1.00\end{array}$ \begin{tabular}{llllllll}
\hline Al53 Al & 0.25925 & 0.38332 & 0.63006 & 0.00000 & Uiso & -1.00
\end{tabular} $\begin{array}{llllllll}\mathrm{Al} 54 & \mathrm{Al} & 0.21396 & 0.80723 & 0.00144 & 0.00000 & \text { Uiso } & 1.00\end{array}$ $\begin{array}{llllllll}\text { Al55 } & \mathrm{Al} & 0.21308 & 0.80675 & 0.50080 & 0.00000 & \text { Uiso } & 1.00\end{array}$ $\begin{array}{llllllll}\text { Al56 } & \text { Al } & 0.21299 & 0.30593 & 0.00341 & 0.00000 & \text { Uiso } & 1.00\end{array}$ $\begin{array}{lllllllll}\mathrm{A} 157 & \mathrm{Al} & 0.21299 & 0.30600 & 0.50084 & 0.00000 & \text { Uiso } & 1.00\end{array}$ $\begin{array}{llllllll}\mathrm{Al} 58 & \mathrm{Al} & 0.21369 & 0.13512 & 0.00596 & 0.00000 & \text { Uiso } & 1.00\end{array}$ $\begin{array}{llllllll}\text { Al59 } & \text { Al } & 0.21368 & 0.13572 & 0.50169 & 0.00000 & \text { Uiso } & 1.00\end{array}$ $\begin{array}{llllllll}\mathrm{Al} 60 & \mathrm{Al} & 0.21270 & 0.63517 & 0.99915 & 0.00000 & \text { Uiso } & 1.00\end{array}$ $\begin{array}{llllllll}\mathrm{Al} 61 & \mathrm{Al} & 0.21272 & 0.63523 & 0.49872 & 0.00000 & \text { Uiso } & 1.00\end{array}$ $\begin{array}{llllllll}\text { Al62 } & \mathrm{Al} & 0.40248 & 0.14924 & 0.78414 & 0.00000 & \text { Uiso } & 1.00\end{array}$ $\begin{array}{lllllllll}\mathrm{Al} 63 & \mathrm{Al} & 0.35143 & 0.22625 & 0.39786 & 0.00000 & \text { Uiso } & 1.00\end{array}$ $\begin{array}{lllllllll}\mathrm{Al} 64 & \mathrm{Al} & 0.39864 & 0.64582 & 0.01025 & 0.00000 & \text { Uiso } & 1.00\end{array}$ $\begin{array}{lllllllll}\mathrm{Al} 165 & \mathrm{Al} & 0.40211 & 0.76637 & 0.50642 & 0.00000 & \text { Uiso } & 1.00\end{array}$ \begin{tabular}{lllllllll}
\hline 165 & Al & 0.40211 & 0.76637 & 0.50642 & 0.00000 & Uiso & 1.00 \\
\hline & 0.66 & 0 & 0.30820 & 0.87818 & 0.38325 & 0.00000 & Uiso & 1.00
\end{tabular} $\begin{array}{llllllll}066 & \mathrm{O} & 0.30820 & 0.87818 & 0.38325 & 0.00000 & \text { Uiso } & 1.00 \\ 067 & \mathrm{O} & 0.30368 & 0.88787 & 0.88183 & 0.00000 & \text { Uiso } & 1.00\end{array}$ \begin{tabular}{llllllll}
067 & $\mathrm{O}$ & 0.30368 & 0.88787 & 0.88183 & 0.00000 & Uiso & 1.00 \\
\hline & $\mathrm{O}$ & 0.30659 & 0.38898 & 0.38474 & 0.00000 & Uis & 1.00
\end{tabular} $\begin{array}{lllllllll}068 & \mathrm{O} & 0.30659 & 0.38898 & 0.38474 & 0.00000 & \text { Uiso } & 1.00\end{array}$ $\begin{array}{llllllll}069 & \mathrm{O} & 0.30692 & 0.39436 & 0.88160 & 0.00000 & \text { Uiso } & 1.00\end{array}$ $\begin{array}{lllllllll}071 & 0 & 0.30867 & 0.04425 & 0.36903 & 0.00000 & \text { Uiso } & 1.00\end{array}$ $\begin{array}{llllllllll}071 & \mathrm{O} & 0.30924 & 0.05839 & 0.87206 & 0.0000 & \text { Uiso } & 1.00\end{array}$ $\begin{array}{lllllllll}072 & \mathrm{O} & 0.30400 & 0.55081 & 0.37088 & 0.00000 & \text { Uiso } & 1.00 \\ 073 & \mathrm{O} & 0.3037 & 0.56322 & 0.87996 & 0.00000 & \text { Uso } & 1.00\end{array}$ $\begin{array}{llllllll}\mathrm{O} 73 & \mathrm{O} & 0.30376 & 0.56322 & 0.87996 & 0.00000 & \text { Uiso } & 1.00\end{array}$ $\begin{array}{lllllllll}074 & \mathrm{O} & 0.30765 & 0.22405 & 0.38672 & 0.00000 & \text { Uiso } & 1.00\end{array}$ $\begin{array}{llllllll}075 & \mathrm{O} & 0.30457 & 0.22042 & 0.88834 & 0.00000 & \text { Uiso } & 1.00\end{array}$ $\begin{array}{lllllllll}076 & \mathrm{O} & 0.30186 & 0.71585 & 0.37074 & 0.00000 & \text { Uiso } & 1.00\end{array}$ $\begin{array}{lllllllll}077 & \mathrm{O} & 0.30045 & 0.72192 & 0.87073 & 0.00000 & \text { Uiso } & 1.00\end{array}$ $\begin{array}{lllllllll}078 & \mathrm{O} & 0.35184 & 0.13474 & 0.31158 & 0.00000 & \text { Uiso } & 1.00\end{array}$ $\begin{array}{llllllll}079 & \mathrm{O} & 0.35588 & 0.15297 & 0.78258 & 0.00000 & \text { Uiso } & 1.00\end{array}$ $\begin{array}{lllllllll}080 & \mathrm{O} & 0.36075 & 0.62565 & 0.38754 & 0.00000 & \text { Uiso } & 1.00\end{array}$ $\begin{array}{lllllllll}\mathrm{O} 81 & \mathrm{O} & 0.34619 & 0.63137 & 0.73521 & 0.00000 & \text { Uiso } & 1.00\end{array}$ $\begin{array}{llllllllll}082 & \mathrm{O} & 0.34873 & 0.79974 & 0.26495 & 0.00000 & \text { Uiso } & 1.00\end{array}$ $\begin{array}{llllllll}083 & \mathrm{O} & 0.34543 & 0.80563 & 0.77462 & 0.00000 & \text { Uiso } & 1.00\end{array}$ $\begin{array}{lllllllll}084 & \mathrm{O} & 0.35600 & 0.31170 & 0.02169 & 0.00000 & \text { Uiso } & 1.00\end{array}$ $\begin{array}{llllllll}085 & \mathrm{O} & 0.41561 & 0.39459 & 0.64045 & 0.00000 & \text { Uiso } & 1.00\end{array}$ $\begin{array}{lllllllll}086 & \mathrm{O} & 0.35341 & 0.96393 & 0.75681 & 0.00000 & \text { Uiso } & 1.00\end{array}$ $\begin{array}{lllllllll}086 & 0 & 0.35341 & 0.96393 & 0.75681 & 0.00000 & \text { Uiso } & 1.00 \\ 087 & 0 & 0.35512 & 0.49328 & 0.23794 & 0.00000 & \text { Uiso } & 1.00\end{array}$ \begin{tabular}{lllllllll}
087 & $\mathrm{O}$ & 0.35512 & 0.49328 & 0.23794 & 0.00000 & Uiso & 1.00 \\
\hline 088 & $\mathrm{O}$ & 0.35456 & 0.48736 & 0.78446 & 0.00000 & Uiso & 1.00
\end{tabular} $\begin{array}{llllllll}088 & \mathrm{O} & 0.35456 & 0.48736 & 0.78446 & 0.00000 & \text { Uiso } & 1.00 \\ 089 & \mathrm{O} & 0.30465 & 0.88706 & 0.14401 & 0.00000 & \text { Uiso } & 1.00\end{array}$ $\begin{array}{llllllll}089 & \mathrm{O} & 0.30465 & 0.88706 & 0.14401 & 0.00000 & \text { Uiso } & 1.00 \\ 090 & \mathrm{O} & 0.30159 & 0.88666 & 0.64303 & 0.00000 & \text { Uiso } & 1.00\end{array}$ $\begin{array}{lllllllllll}090 & 0 & 0.30159 & 0.88666 & 0.64303 & 0.0000 & \text { Uiso } & 1.00\end{array}$ $\begin{array}{lllllllll}091 & \mathrm{O} & 0.30251 & 0.38997 & 0.14981 & 0.00000 & \text { Uiso } & 1.00\end{array}$ $\begin{array}{llllllll}092 & \mathrm{O} & 0.30227 & 0.38135 & 0.63812 & 0.00000 & \text { Uiso } & 1.00\end{array}$ $\begin{array}{lllllllll}093 & \mathrm{O} & 0.30549 & 0.05555 & 0.13900 & 0.00000 & \text { Uiso } & 1.00\end{array}$ $\begin{array}{llllllll}094 & \mathrm{O} & 0.30482 & 0.05578 & 0.63905 & 0.00000 & \text { Uiso } & 1.00\end{array}$ $\begin{array}{llllllll}095 & \mathrm{O} & 0.30093 & 0.56012 & 0.14273 & 0.00000 & \text { Uiso } & 1.00\end{array}$ $\begin{array}{llllllll}096 & \mathrm{O} & 0.30061 & 0.55891 & 0.64001 & 0.00000 & \text { Uiso } & 1.00\end{array}$ $\begin{array}{llllllll}\mathrm{O} 97 & \mathrm{O} & 0.30668 & 0.22116 & 0.14985 & 0.00000 & \text { Uiso } & 1.00\end{array}$ $\begin{array}{llllllll}098 & \mathrm{O} & 0.31021 & 0.21850 & 0.64608 & 0.00000 & \text { Uiso } & 1.00\end{array}$ $\begin{array}{lllllllll}099 & \mathrm{O} & 0.30939 & 0.71730 & 0.13757 & 0.00000 & \text { Uiso } & 1.00\end{array}$ $\begin{array}{lllllllll}0100 & \mathrm{O} & 0.30657 & 0.72383 & 0.63814 & 0.00000 & \text { Uiso } & 1.00\end{array}$ $\begin{array}{llllllll}0101 & \mathrm{O} & 0.35931 & 0.14384 & 0.00358 & 0.00000 & \text { Uiso } & 1.00\end{array}$ $\begin{array}{llllllllll}0102 & \mathrm{O} & 0.35754 & 0.65028 & 0.99276 & 0.00000 & \text { Uiso } & 1.00\end{array}$ $\begin{array}{lllllllll}\mathrm{O} 103 & \mathrm{O} & 0.36173 & 0.81422 & 0.01961 & 0.00000 & \text { Uiso } & 1.00\end{array}$ $\begin{array}{lllllllll}0104 & \mathrm{O} & 0.35976 & 0.77935 & 0.52266 & 0.00000 & \text { Uiso } & 1.00\end{array}$ $\begin{array}{lllllllll}0105 & \mathrm{O} & 0.34512 & 0.30476 & 0.78691 & 0.00000 & \text { Uiso } & 1.00\end{array}$ $\begin{array}{llllllllll}0106 & 0 & 0.35350 & 0.30297 & 0.51961 & 0.00000 & \text { Uiso } & 1.00\end{array}$ $\begin{array}{lllllllllll}0107 & 0 & 0.35064 & 0.96883 & 0.99163 & 0.00000 & \text { Uiso } & 1.00\end{array}$ $\begin{array}{lllllllllll}0108 & 0 & 0.35064 & 0.9683 & 0.99163 & 0.00000 & \text { Uiso } & 1.00\end{array}$ $\begin{array}{llllllllllllll}0108 & 0 & 0.3498 & 0.95783 & 0.49368 & 0.0000 & 0.000\end{array}$ $\begin{array}{lllllllllll}0110 & 0 & 0.35020 & 0.48024 & 0.00456 & 0.00000 & \text { Uiso } & 1.00\end{array}$ $\begin{array}{llllllllll}0110 & \mathrm{O} & 0.34927 & 0.48011 & 0.52196 & 0.00000 & \text { Uiso } & 1.00\end{array}$ $\begin{array}{llllllllll}0111 & \mathrm{O} & 0.20714 & 0.89410 & 0.88748 & 0.00000 & \text { Uiso } & 1.00 \\ 0112 & \mathrm{O} & 0.20694 & 0.8349 & 0.38613 & 0.0000 & \text { Uiso } & 1.00\end{array}$ $\begin{array}{lllllllll}0112 & \mathrm{O} & 0.20694 & 0.89349 & 0.38613 & 0.00000 & \text { Uiso } & 1.00\end{array}$ $\begin{array}{llllllll}0113 & 0 & 0.20580 & 0.39218 & 0.88554 & 0.00000 & \text { Unso } & 1.00\end{array}$ $\begin{array}{lllllllll}0114 & \mathrm{O} & 0.20555 & 0.39232 & 0.38447 & 0.00000 & \text { Uiso } & 1.00\end{array}$ $\begin{array}{llllllll}0115 & \mathrm{O} & 0.20727 & 0.05009 & 0.88889 & 0.00000 & \text { Uiso } & 1.00\end{array}$ $\begin{array}{lllllllll}0116 & \mathrm{O} & 0.20705 & 0.04986 & 0.38642 & 0.00000 & \text { Uiso } & 1.00\end{array}$ $\begin{array}{lllllllll}0117 & \mathrm{O} & 0.20574 & 0.54782 & 0.88445 & 0.00000 & \text { Uiso } & 1.00\end{array}$ $\begin{array}{llllllll}0118 & \mathrm{O} & 0.20531 & 0.54773 & 0.38375 & 0.00000 & \text { Uiso } & 1.00\end{array}$ $\begin{array}{llllllll}0119 & \mathrm{O} & 0.21095 & 0.22046 & 0.88651 & 0.00000 & \text { Uiso } & 1.00\end{array}$ $\begin{array}{lllllllll}0120 & \mathrm{O} & 0.21090 & 0.22066 & 0.38494 & 0.00000 & \text { Uiso } & 1.00\end{array}$ $\begin{array}{lllllllll}0121 & \mathrm{O} & 0.21040 & 0.72146 & 0.88361 & 0.00000 & \text { Uiso } & 1.00\end{array}$ $\begin{array}{llllllll}0122 & \mathrm{O} & 0.21029 & 0.72184 & 0.38227 & 0.00000 & \text { Uiso } & 1.00\end{array}$ $\begin{array}{llllllll}0123 & \mathrm{O} & 0.26215 & 0.13521 & 0.25359 & 0.00000 & \text { Uiso } & 1.00\end{array}$ $\begin{array}{lllllllll}0124 & \mathrm{O} & 0.26141 & 0.13781 & 0.74601 & 0.00000 & \text { Uiso } & 1.00\end{array}$ $\begin{array}{lllllllll}0125 & \mathrm{O} & 0.25707 & 0.63595 & 0.24359 & 0.00000 & \text { Uiso } & 1.00\end{array}$ $\begin{array}{llllllll}0126 & \mathrm{O} & 0.25710 & 0.63729 & 0.74183 & 0.00000 & \text { Uiso } & 1.00\end{array}$ $\begin{array}{llllllllll}0127 & 0 & 0.26170 & 0.80403 & 0.24292 & 0.00000 & \text { Uiso } & 1.00\end{array}$ $\begin{array}{lllllllllll}0128 & 0 & 0.25846 & 0.80743 & 0.74601 & 0.00000 & \text { Uiso } & 1.00\end{array}$ $\begin{array}{lllllllllll}0129 & 0 & 0.2584 & 0.80743 & 0.74601 & 0.00000 & \text { Uiso } & 1.00\end{array}$ $\begin{array}{lllllllll}0130 & 0 & 0.26074 & 0.30527 & 0.25416 & 0.00000 & \text { Uiso } & 1.00\end{array}$

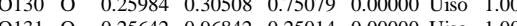
$\begin{array}{llllllllll}0131 & 0 & 0.25642 & 0.96842 & 0.25014 & 0.00000 & \text { Uiso } & 1.00\end{array}$ $\begin{array}{lllllllll}0132 & 0 & 0.25571 & 0.97390 & 0.75027 & 0.00000 & \text { Uiso } & 1.00\end{array}$ $\begin{array}{llllllllll}0133 & 0 & 0.25338 & 0.47022 & 0.24795 & 0.00000 & \text { Uiso } & 1.00\end{array}$ $\begin{array}{llllllllll}0134 & \mathrm{O} & 0.25437 & 0.47040 & 0.74746 & 0.00000 & \text { Uiso } & 1.00\end{array}$ $\begin{array}{lllllllll}0135 & \mathrm{O} & 0.21592 & 0.89300 & 0.12150 & 0.00000 & \text { Uiso } & 1.00\end{array}$ $\begin{array}{llllllll}0136 & \mathrm{O} & 0.21497 & 0.89461 & 0.62121 & 0.00000 & \text { Uiso } & 1.00\end{array}$ $\begin{array}{lllllllll}0137 & \mathrm{O} & 0.21420 & 0.39217 & 0.12082 & 0.00000 & \text { Uiso } & 1.00\end{array}$ $\begin{array}{llllllll}0138 & 0 & 0.21407 & 0.39200 & 0.62043 & 0.00000 & \text { Uiso } & 1.00\end{array}$ $\begin{array}{llllllll}0139 & \mathrm{O} & 0.21590 & 0.04853 & 0.12485 & 0.00000 & \text { Uiso } & 1.00\end{array}$ 
$\begin{array}{llllllll}\mathrm{O} 140 & \mathrm{O} & 0.21583 & 0.04998 & 0.62089 & 0.00000 & \text { Uiso } & 1.00\end{array}$ $\begin{array}{lllllllll}0141 & \mathrm{O} & 0.21366 & 0.54798 & 0.11865 & 0.00000 & \text { Uiso } & 1.00\end{array}$ $\begin{array}{lllllllll}0142 & \mathrm{O} & 0.21380 & 0.54805 & 0.61841 & 0.00000 & \text { Uiso } & 1.00\end{array}$ $\begin{array}{lllllllll}0143 & \mathrm{O} & 0.20848 & 0.21991 & 0.12777 & 0.00000 & \text { Uiso } & 1.00\end{array}$ $\begin{array}{lllllllll}0144 & \mathrm{O} & 0.20818 & 0.22101 & 0.62578 & 0.00000 & \text { Uiso } & 1.00\end{array}$ $\begin{array}{llllllllll}0145 & 0 & 0.20815 & 0.72182 & 0.12286 & 0.00000 & \text { Uiso } & 1.00\end{array}$ $\begin{array}{lllllllll}0146 & 0 & 0.20752 & 0.72170 & 0.62177 & 0.00000 & \text { Uiso } & 1.00\end{array}$ $\begin{array}{llllllllll}0146 & 0 & 0.20752 & 0.72170 & 0.62177 & 0.00000 & \text { Uiso } & 1.00\end{array}$ $\begin{array}{lllllllllll}0148 & 0 & 0.25648 & 0.1368 & 0.01719 & 0.00000 & 0.51274 & 0.00000 & \text { Uiso } & 1.00\end{array}$ $\begin{array}{lllllllll}0148 & 0 & 0.25648 & 0.13831 & 0.51274 & 0.00000 & \text { Uiso } & 1.00\end{array}$ $\begin{array}{lllllllll}0149 & 0 & 0.25471 & 0.63737 & 0.01157 & 0.00000 & \text { Uiso } & 1.00 \\ 0150 & 0 & 0.25487 & 0.63733 & 0.50809 & 0.00000 & \text { Uiso } & 1.00\end{array}$ $\begin{array}{lllllllll}0150 & \mathrm{O} & 0.25487 & 0.63733 & 0.5080 & 0.00000 & \text { Uiso } & 1.00 \\ 0151 & \mathrm{O} & 0.25632 & 0.80362 & 0.01316 & 0.00000 & \text { Uiso } & 1.00\end{array}$ $\begin{array}{llllllllll}0151 & 0 & 0.25632 & 0.80362 & 0.01316 & 0.00000 & \text { Uiso } & 1.00 \\ 0152 & 0 & 0.25525 & 0.80440 & 0.51081 & 0.0000 & \text { Uis } & 1.00\end{array}$ $\begin{array}{lllllllll}0152 & 0 & 0.25525 & 0.80440 & 0.51081 & 0.00000 & \text { Uiso } & 1.00\end{array}$ $\begin{array}{llllllll}0153 & \mathrm{O} & 0.25541 & 0.30358 & 0.01769 & 0.00000 & \text { Uiso } & 1.00 \\ 0154 & 0 & 0.25553 & 0.3038 & 0.51503 & 0.00000 & \text { Us } & 1.00\end{array}$ $\begin{array}{llllllllll}0154 & 0 & 0.25553 & 0.30338 & 0.51503 & 0.00000 & \text { Uiso } & 1.00\end{array}$ $\begin{array}{lllllllll}0155 & \mathrm{O} & 0.25884 & 0.97248 & 0.01255 & 0.00000 & \text { Uiso } & 1.00\end{array}$ $\begin{array}{lllllllll}0156 & \mathrm{O} & 0.25840 & 0.97017 & 0.50951 & 0.00000 & \text { Uiso } & 1.00\end{array}$ $\begin{array}{lllllllll}0157 & \mathrm{O} & 0.25710 & 0.47055 & 0.01117 & 0.00000 & \text { Uiso } & 1.00\end{array}$ $\begin{array}{llllllll}0158 & \mathrm{O} & 0.25616 & 0.46930 & 0.51001 & 0.00000 & \text { Uiso } & 1.00\end{array}$

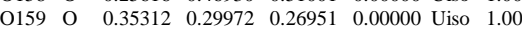
$\begin{array}{llllllll}0160 & \mathrm{O} & 0.39613 & 0.71123 & 0.65493 & 0.00000 & \text { Uiso } & 1.00\end{array}$ $\begin{array}{lllllllll}0161 & \mathrm{O} & 0.41563 & 0.18074 & 0.92827 & 0.00000 & \text { Uiso } & 1.00\end{array}$

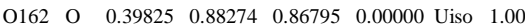
$\begin{array}{lllllllll}0163 & \mathrm{O} & 0.39689 & 0.39511 & 0.16662 & 0.00000 & \text { Uiso } & 1.00\end{array}$ $\begin{array}{lllllllll}0164 & \mathrm{O} & 0.39495 & 0.37896 & 0.87018 & 0.00000 & \text { Uiso } & 1.00\end{array}$ $\begin{array}{llllllll}0165 & \mathrm{O} & 0.39798 & 0.25221 & 0.71381 & 0.00000 & \text { Uiso } & 1.00\end{array}$ $\begin{array}{lllllllll}0166 & 0 & 0.40975 & 0.55629 & 0.64065 & 0.00000 & \text { Uiso } & 1.00\end{array}$ $\begin{array}{lllllllll}0166 & 0 & 0.40975 & 0.55629 & 0.64065 & 0.0000 & \text { Uiso } & 1.00\end{array}$

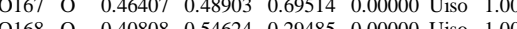
$\begin{array}{lllllllll}0168 & 0 & 0.40808 & 0.54624 & 0.29485 & 0.00000 & \text { Uiso } & 1.00\end{array}$ $\begin{array}{lllllllll}0169 & \mathrm{O} & 0.40708 & 0.54476 & 0.06865 & 0.00000 & \text { Uiso } & 1.00\end{array}$ $\begin{array}{lllllllllll}0170 & \mathrm{O} & 0.39868 & 0.23187 & 0.14421 & 0.00000 & \text { Uiso } & 1.00 \\ 0171 & \mathrm{O} & 0.39533 & 0.04323 & 0.84411 & 0.00000 & \text { Uis } & 1.00\end{array}$ $\begin{array}{lllllllll}0171 & 0 & 0.39533 & 0.04323 & 0.84411 & 0.00000 & \text { Uiso } & 1.00 \\ 0172 & 0 & 0.40557 & 0.07081 & 0.10610 & 0.00000 & \text { Uiso } & 1.00\end{array}$ $\begin{array}{llllllllll}0172 & \mathrm{O} & 0.40557 & 0.07081 & 0.10610 & 0.00000 & \text { Uiso } & 1.00\end{array}$ $\begin{array}{lllllllll}0173 & \mathrm{O} & 0.41508 & 0.69778 & 0.39666 & 0.00000 & \text { Uiso } & 1.00\end{array}$ $\begin{array}{llllllllll}0174 & \mathrm{O} & 0.45050 & 0.32167 & 0.79843 & 0.00000 & \text { Uiso } & 1.00\end{array}$ $\begin{array}{lllllllll}0175 & \mathrm{O} & 0.45344 & 0.66641 & 0.69054 & 0.00000 & \text { Uiso } & 1.00\end{array}$ $\begin{array}{llllllll}0176 & \mathrm{O} & 0.41181 & 0.62520 & 0.85993 & 0.00000 & \text { Uiso } & 1.00\end{array}$ $\begin{array}{llllllll}0177 & \mathrm{O} & 0.44366 & 0.48282 & 0.47067 & 0.00000 & \text { Uiso } & 1.00\end{array}$ $\begin{array}{llllllll}0178 & \mathrm{O} & 0.36990 & 0.16632 & 0.53182 & 0.00000 & \text { Uiso } & 1.00\end{array}$ $\begin{array}{lllllllll}0179 & \mathrm{O} & 0.42055 & 0.11279 & 0.65187 & 0.00000 & \text { Uiso } & 1.00\end{array}$ $\begin{array}{llllllll}\mathrm{O} 180 & \mathrm{O} & 0.40877 & 0.94027 & 0.64934 & 0.00000 & \text { Uiso } & 1.00\end{array}$ $\begin{array}{lllllllll}0181 & \mathrm{O} & 0.39776 & 0.87078 & 0.43219 & 0.00000 & \text { Uiso } & 1.00\end{array}$ $\begin{array}{llllllll}0182 & \mathrm{O} & 0.42636 & 0.17196 & 0.42070 & 0.00000 & \text { Uiso } & 1.00\end{array}$ $\begin{array}{llllllll}0183 & \mathrm{O} & 0.39783 & 0.03822 & 0.45676 & 0.00000 & \text { Uiso } & 1.00\end{array}$ $\begin{array}{lllllllll}0184 & \mathrm{O} & 0.45029 & 0.94636 & 0.49002 & 0.00000 & \text { Uiso } & 1.00\end{array}$ $\begin{array}{llllllll}0185 & \mathrm{O} & 0.40932 & 0.90455 & 0.09827 & 0.00000 & \text { Uiso } & 1.00\end{array}$ $\begin{array}{lllllllll}0186 & \mathrm{O} & 0.41756 & 0.75207 & 0.00716 & 0.00000 & \text { Uiso } & 1.00\end{array}$ $\begin{array}{lllllllll}0186 & 0 & 0.41756 & 0.75207 & 0.00716 & 0.00000 & \text { Uiso } & 1.00\end{array}$ $\begin{array}{lllllllllll}0187 & 0 & 0.4296 & 0.9836 & 0.27056 & 0.0000 & \text { Uiso } & 1.00 \\ 0188 & 0 & 0.38641 & 0.67785 & 0.18123 & 0.0000 & \text { Uis } & 1.00\end{array}$ $\begin{array}{llllllllll}0189 & 0 & 0.38641 & 0.6785 & 0.18123 & 0.0000 & 0.000 & \text { Uiso } & 1.00\end{array}$ $\begin{array}{lllllllll}0189 & 0 & 0.36463 & 0.98073 & 0.23547 & 0.00000 & \text { Uiso } & 1.00\end{array}$ $\begin{array}{lllllllll}0190 & \mathrm{O} & 0.44298 & 0.79326 & 0.56105 & 0.00000 & \text { Uiso } & 1.00\end{array}$ $\begin{array}{lllllllllll}\text { Sil91 Si } & 0.39186 & 0.95783 & 0.77484 & 0.00000 & \text { Uiso } & 1.00\end{array}$ $\begin{array}{llllllll}\text { Si192 Si } & 0.41341 & 0.33861 & 0.76232 & 0.00000 & \text { Uiso } & 1.00\end{array}$ $\begin{array}{llllllll}\text { Sil93 Si } & 0.43238 & 0.47933 & 0.60997 & 0.00000 & \text { Utso } & -1.00\end{array}$ $\begin{array}{llllllll}\mathrm{Si} 194 & \mathrm{Si} & 0.39205 & 0.49316 & 0.17862 & 0.00000 & \text { Uiso } & 1.00\end{array}$ $\begin{array}{llllllll}\mathrm{Si} 195 \mathrm{Si} & 0.39633 & 0.15717 & 0.04899 & 0.00000 & \text { Uiso } & 1.00\end{array}$ $\begin{array}{lllllll}\text { Si196 Si } & 0.39335 & 0.64066 & 0.31714 & 0.00000 & \text { Uiso } & 1.00\end{array}$ $\begin{array}{llllllll}\text { Si197 Si } & 0.37592 & 0.31155 & 0.15007 & 0.00000 & \text { Uiso } & 1.00\end{array}$ $\begin{array}{llllllll}\text { Si198 Si } & 0.41619 & 0.63838 & 0.71865 & 0.00000 & \text { Uiso } & 1.00\end{array}$ $\begin{array}{llllllll}\text { Si199 Si } & 0.41156 & 0.94883 & 0.50256 & 0.00000 & \text { Uiso } & 1.00\end{array}$ $\begin{array}{lllllll}\mathrm{Si} 200 \mathrm{Si} & 0.40512 & 0.12670 & 0.52301 & 0.00000 & \text { Uiso } & 1.00\end{array}$ $\begin{array}{lllllllll}\mathrm{Si} 201 \mathrm{Si} & 0.40082 & 0.98521 & 0.17312 & 0.00000 & \text { Uiso } & 1.00\end{array}$ $\begin{array}{llllllll}\mathrm{Si} 202 & \mathrm{Si} & 0.39923 & 0.83586 & 0.00128 & 0.00000 & \text { Uiso } & 1.00\end{array}$ $\begin{array}{llllllll}\mathrm{H} 203 & \mathrm{H} & 0.46801 & 0.44184 & 0.74450 & 0.00000 & \text { Uiso } & 1.00\end{array}$ $\begin{array}{llllllll}\mathrm{H} 204 & \mathrm{H} & 0.45218 & 0.29179 & 0.87323 & 0.00000 & \text { Uiso } & 1.00\end{array}$ $\begin{array}{lllllllll}\mathrm{H} 205 & \mathrm{H} & 0.46732 & 0.62090 & 0.67985 & 0.00000 & \text { Uiso } & 1.00\end{array}$ $\begin{array}{llllllll}\mathrm{H} 206 & \mathrm{H} & 0.42986 & 0.50638 & 0.40898 & 0.00000 & \text { Uiso } & 1.00\end{array}$ $\begin{array}{llllllllll}\mathrm{H} 207 & \mathrm{H} & 0.43667 & 0.22119 & 0.44366 & 0.00000 & \text { Uiso } & 1.00\end{array}$

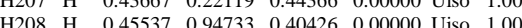
$\begin{array}{lllllllll}\mathrm{H} 208 & \mathrm{H} & 0.45537 & 0.94733 & 0.40426 & 0.00000 & \text { Uiso } & 1.00\end{array}$ $\begin{array}{llllllllll} & 1208 & \text { H } & 0.43387 & 0.04350 & 0.29444 & 0.00000 & \text { Uiso } & 1.00\end{array}$ $\begin{array}{lllllllll}\mathrm{H} 210 & \mathrm{H} & 0.45392 & 0.84424 & 0.54688 & 0.00000 & \text { Uiso } & 1.00\end{array}$ $\begin{array}{lllllllll}\mathrm{H} 211 & \mathrm{H} & 0.45340 & 0.75210 & 0.61251 & 0.00000 & \text { Uiso } & 1.00\end{array}$ $\begin{array}{llllllll}\mathrm{H} 212 & \mathrm{H} & 0.49448 & 0.75625 & 0.03787 & 0.00000 & \text { Uiso } & 1.00\end{array}$ $\begin{array}{llllllll}\mathrm{H} 213 & \mathrm{H} & 0.46225 & 0.60740 & 0.8975 & 0.00000 & \text { Uiso } & 1.00\end{array}$ $\begin{array}{lllllllll}\mathrm{H} 214 & \mathrm{H} & 0.49603 & 0.55049 & 0.92852 & 0.00000 & \text { Uiso } & 1.00\end{array}$ $\begin{array}{llllllll}\mathrm{H} 215 & \mathrm{H} & 0.47189 & 0.59308 & 0.24322 & 0.00000 & \text { Uiso } & 1.00\end{array}$ $\begin{array}{llllllll}\mathrm{H} 216 & \mathrm{H} & 0.48000 & 0.73164 & 0.18904 & 0.00000 & \text { Uiso } & 1.00\end{array}$ $\begin{array}{llllllll}\mathrm{H} 217 & \mathrm{H} & 0.45456 & 0.73555 & 0.06002 & 0.00000 & \text { Uiso } & 1.00\end{array}$ $\begin{array}{llllllll}\mathrm{H} 218 & \mathrm{H} & 0.48363 & 0.51643 & 0.13471 & 0.00000 & \text { Uiso } & 1.00\end{array}$ $\begin{array}{llllllll}\mathrm{H} 219 & \mathrm{H} & 0.44695 & 0.56102 & 0.12162 & 0.00000 & \text { Uiso } & 1.00\end{array}$ $\begin{array}{lllllllll}\mathrm{H} 220 & \mathrm{H} & 0.49884 & 0.65503 & 0.88452 & 0.00000 & \text { Uiso } & 1.00\end{array}$ $\begin{array}{llllllll}\mathrm{C} 221 & \mathrm{C} & 0.48629 & 0.61062 & 0.93809 & 0.00000 & \text { Uiso } & 1.00\end{array}$ $\begin{array}{lllllllll}\mathrm{C} 222 & \mathrm{C} & 0.48135 & 0.63458 & 0.06342 & 0.00000 & \text { Uiso } & 1.00\end{array}$ $\begin{array}{lllllllll}\mathrm{C} 223 & \mathrm{C} & 0.47932 & 0.71816 & 0.09275 & 0.00000 & \text { Uiso } & 1.00\end{array}$ $\begin{array}{llllllll}\mathrm{C} 224 & \mathrm{C} & 0.47224 & 0.57429 & 0.14904 & 0.00000 & \text { Uiso } & 1.00\end{array}$

\section{File ${ }^{\circ} 20$ :}

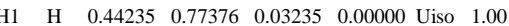
$\begin{array}{llllllll}\mathrm{H} 2 & \mathrm{H} & 0.46867 & 0.44769 & 0.37987 & 0.00000 & \text { Uiso } & 1.00\end{array}$ $\begin{array}{llllllllll} & \mathrm{H} 2 & \mathrm{H} & 0.46867 & 0.44769 & 0.37987 & 0.00000 & \text { Uiso } & 1.00\end{array}$ $\begin{array}{lllllllll} & \mathrm{H} 3 & \mathrm{H} & 0.45171 & 0.29409 & 0.43981 & 0.00000 & \text { Uiso } & 1.00\end{array}$ $\begin{array}{llllllll}145 & \mathrm{H} & 0.46832 & 0.62850 & 0.35344 & 0.00000 & \text { Uiso } & 1.00\end{array}$

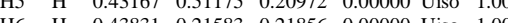

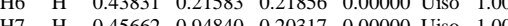
$\begin{array}{llllllll}\mathrm{H} 7 & \mathrm{H} & 0.45662 & 0.94840 & 0.20317 & 0.00000 & \text { Uiso } & 1.00\end{array}$ $\begin{array}{llllllll}\mathrm{H} 8 & \mathrm{H} & 0.43379 & 0.04890 & 0.15085 & 0.00000 & \text { Uiso } & 1.00\end{array}$ $\begin{array}{lllllllll}\mathrm{H} 9 & \mathrm{H} & 0.45424 & 0.84226 & 0.27356 & 0.00000 & \text { Uiso } & 1.00\end{array}$ $\begin{array}{lllllllll}\mathrm{H} 10 & \mathrm{H} & 0.45348 & 0.75202 & 0.30830 & 0.00000 & \text { Uiso } & 1.00\end{array}$ $\begin{array}{llllllll}\text { Al11 Al } & 0.35125 & 0.05697 & 0.44702 & 0.00000 & \text { Uiso } & 1.00\end{array}$ $\begin{array}{lllllll}\text { Al12 Al } & 0.35014 & 0.03644 & 0.19026 & 0.00000 & \text { Uiso } & 1.00\end{array}$ 
$\begin{array}{llllllll}\text { Al13 } & \text { Al } & 0.34387 & 0.56554 & 0.45015 & 0.00000 & \text { Uiso } & 1.00\end{array}$ $\begin{array}{llllllll}\text { Al14 } & \mathrm{Al} & 0.34381 & 0.53288 & 0.19082 & 0.00000 & \text { Uiso } & 1.00\end{array}$ $\begin{array}{lllllllll}\text { Al15 } & \text { Al } & 0.34466 & 0.87468 & 0.44839 & 0.00000 & \text { Uiso } & 1.00\end{array}$ $\begin{array}{llllllll}\text { Al16 } & \mathrm{Al} & 0.34995 & 0.85057 & 0.20542 & 0.00000 & \text { Uiso } & 1.00\end{array}$ $\begin{array}{lllllllll}\text { Al17 } & \text { Al } & 0.35317 & 0.40132 & 0.44196 & 0.00000 & \text { Uiso } & 1.00\end{array}$ $\begin{array}{lllllllll}\text { Al18 } & \text { Al } & 0.32886 & 0.38701 & 0.25958 & 0.00000 & \text { Uiso } & 1.00\end{array}$

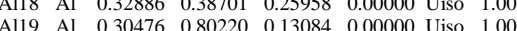
$\begin{array}{llllllllll}\text { Al19 } & \text { Al } & 0.30476 & 0.80220 & 0.13084 & 0.00000 & \text { Uiso } & 1.00\end{array}$

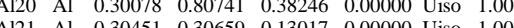

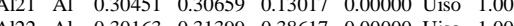

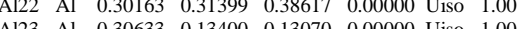
$\begin{array}{lllllllll}\text { Al22 Al } & 0.30633 & 0.13400 & 0.13070 & 0.00000 & \text { Uiso } & 1.00\end{array}$ $\begin{array}{llllllll}\text { Al24 } & \text { Al } & 0.30315 & 0.13613 & 0.37925 & 0.0000 & \text { Uiso } & 1.00\end{array}$ $\begin{array}{llllllll}\text { Al25 Al } & 0.29763 & 0.63498 & 0.13379 & 0.00000 & \text { Uiso } & 1.00\end{array}$ $\begin{array}{llllllll}\text { Al26 Al } & 0.29900 & 0.64138 & 0.38019 & 0.00000 & \text { Uiso } & 1.00\end{array}$ $\begin{array}{lllllllll}\mathrm{A} 227 & \mathrm{Al} & 0.21191 & 0.97110 & 0.12691 & 0.00000 & \text { Uiso } & 1.00\end{array}$ $\begin{array}{llllllll}\mathrm{Al} 28 & \mathrm{Al} & 0.21176 & 0.97245 & 0.37725 & 0.00000 & \text { Uiso } & 1.00\end{array}$ $\begin{array}{llllllll}\text { Al29 } & \text { Al } & 0.20935 & 0.47056 & 0.12532 & 0.00000 & \text { Uiso } & 1.00\end{array}$ $\begin{array}{lllllllll}\mathrm{Al} 30 & \mathrm{Al} & 0.21015 & 0.47068 & 0.37586 & 0.00000 & \text { Uiso } & 1.00\end{array}$ $\begin{array}{llllllll}\mathrm{Al} 31 & \mathrm{Al} & 0.32937 & 0.97028 & 0.06496 & 0.00000 & \text { Uiso } & 1.00\end{array}$ $\begin{array}{llllllll}\mathrm{Al} 32 & \mathrm{Al} & 0.32684 & 0.96669 & 0.31355 & 0.00000 & \text { Uiso } & 1.00\end{array}$ $\begin{array}{lllllllll}\mathrm{Al} 33 & \mathrm{Al} & 0.32451 & 0.47491 & 0.06382 & 0.00000 & \text { Uiso } & 1.00\end{array}$ $\begin{array}{llllllll}\mathrm{Al} 34 & \mathrm{Al} & 0.33980 & 0.53552 & 0.32944 & 0.00000 & \text { Uiso } & 1.00\end{array}$ $\begin{array}{llllllll}\mathrm{Al} 35 & \mathrm{Al} & 0.28083 & 0.22009 & 0.01185 & 0.00000 & \text { Uiso } & 1.00\end{array}$ $\begin{array}{llllllll}\text { Al36 } & \mathrm{Al} & 0.28185 & 0.22139 & 0.25848 & 0.00000 & \text { Uiso } & 1.00\end{array}$ $\begin{array}{lllllllll}\mathrm{Al} 37 & \mathrm{Al} & 0.28101 & 0.71862 & 0.00362 & 0.00000 & \text { Uiso } & 1.00\end{array}$ $\begin{array}{lllllllll}\mathrm{Al} 38 & \mathrm{Al} & 0.28101 & 0.72101 & 0.25350 & 0.00000 & \text { Uiso } & 1.00\end{array}$ $\begin{array}{lllllllll}\mathrm{Al} 39 & \mathrm{Al} & 0.34596 & 0.23201 & 0.45830 & 0.00000 & \text { Uiso } & 1.00\end{array}$ $\begin{array}{llllllll}\text { Al40 Al } & 0.35641 & 0.232076 & 0.45830 & 0.00000 & \text { Uiso } & 1.00\end{array}$ $\begin{array}{llllllllll}A 140 & A 1 & 0.35641 & 0.24076 & 0.32813 & 0.0000 & \text { Uiso } & 1.00\end{array}$

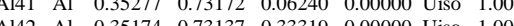
$\begin{array}{lllllll}\text { A142 Al } & 0.35174 & 0.73137 & 0.33319 & 0.0000 & \text { Uiso } & 1.00\end{array}$

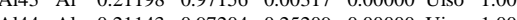

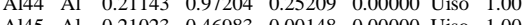
$\begin{array}{lllllllll}\text { Al45 Al } & 0.21023 & 0.46983 & 0.00148 & 0.00000 & \text { Uiso } & 1.00\end{array}$ $\begin{array}{llllllll}\text { Al46 Al } & 0.20960 & 0.47001 & 0.25153 & 0.00000 & \text { Uiso } & 1.00\end{array}$ $\begin{array}{lllllllll}\mathrm{A} 147 & \text { Al } & 0.28282 & 0.96087 & 0.18939 & 0.00000 & \text { Uiso } & 1.00\end{array}$ $\begin{array}{llllllll}\mathrm{Al} 48 & \mathrm{Al} & 0.28195 & 0.97604 & 0.43965 & 0.00000 & \text { Uiso } & 1.00\end{array}$ $\begin{array}{llllllll}\mathrm{Al} 49 & \mathrm{Al} & 0.27837 & 0.46745 & 0.18842 & 0.00000 & \text { Uiso } & 1.00\end{array}$ $\begin{array}{llllllll}\mathrm{Al} 50 & \mathrm{Al} & 0.28071 & 0.47437 & 0.43859 & 0.00000 & \text { Uiso } & 1.00\end{array}$

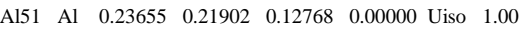
$\begin{array}{lllllllll}\mathrm{Al} 152 & \mathrm{Al} & 0.23539 & 0.22002 & 0.37698 & 0.00000 & \text { Uiso } & 1.00\end{array}$ $\begin{array}{llllllll}\mathrm{A} 153 & \mathrm{Al} & 0.23490 & 0.72406 & 0.12528 & 0.00000 & \text { Uiso } & 1.00\end{array}$ $\begin{array}{lllllllll}\mathrm{Al} 54 & \mathrm{Al} & 0.23417 & 0.72320 & 0.37500 & 0.00000 & \text { Uiso } & 1.00\end{array}$ $\begin{array}{llllllll}\mathrm{Al} 55 & \mathrm{Al} & 0.26186 & 0.05810 & 0.06665 & 0.00000 & \text { Uiso } & 1.00\end{array}$ $\begin{array}{llllllll}\text { Al56 } & \mathrm{Al} & 0.26127 & 0.05827 & 0.31307 & 0.00000 & \text { Uiso } & 1.00\end{array}$ $\begin{array}{lllllllll}\text { Al57 } & \mathrm{Al} & 0.25841 & 0.55813 & 0.06240 & 0.00000 & \text { Uiso } & 1.00\end{array}$ $\begin{array}{lllllllll}\mathrm{Al} 58 & \mathrm{Al} & 0.25901 & 0.55861 & 0.31228 & 0.00000 & \text { Uiso } & 1.00\end{array}$ $\begin{array}{lllllllll}\text { Al59 } & \text { Al } & 0.26183 & 0.88315 & 0.06333 & 0.00000 & \text { Uiso } & 1.00\end{array}$ $\begin{array}{lllllllll}\mathrm{Al} 60 & \mathrm{Al} & 0.25962 & 0.88322 & 0.31355 & 0.00000 & \text { Uiso } & 1.00\end{array}$ $\begin{array}{llllllll}\mathrm{A} 161 & \mathrm{Al} & 0.25952 & 0.88322 & 0.31355 & 0.00000 & \text { Uiso } & 1.00\end{array}$ $\begin{array}{llllllll}A 161 & \text { Al } & 0.25925 & 0.38232 & 0.06600 & 0.00000 & \text { Uiso } & 1.00\end{array}$ $\begin{array}{llllllll}162 & \mathrm{~A} & 0.25925 & 0.38332 & 0.31503 & 0.00000 & \text { Uiso } & 1.00\end{array}$

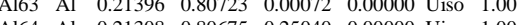
$\begin{array}{llllllll}\text { Al64 Al } & 0.21308 & 0.80675 & 0.25040 & 0.00000 & \text { Uiso } & -1.00\end{array}$ $\begin{array}{llllllll}\mathrm{A} 165 & \mathrm{Al} & 0.21299 & 0.30593 & 0.00170 & 0.00000 & \text { Uiso } & 1.00\end{array}$ $\begin{array}{llllllll}\text { Al66 Al } & 0.21299 & 0.30600 & 0.25042 & 0.00000 & \text { Uiso } & 1.00\end{array}$ $\begin{array}{lllllllll}\text { Al67 } & \text { Al } & 0.21369 & 0.13512 & 0.00298 & 0.00000 & \text { Uiso } & 1.00\end{array}$ $\begin{array}{llllllll}\mathrm{Al} 68 & \mathrm{Al} & 0.21368 & 0.13572 & 0.25084 & 0.00000 & \text { Uiso } & 1.00\end{array}$ $\begin{array}{llllllll}\text { Al69 Al } & 0.21270 & 0.63517 & 0.49958 & 0.00000 & \text { Uiso } & 1.00\end{array}$ $\begin{array}{llllllll}\mathrm{Al} 70 & \mathrm{Al} & 0.21272 & 0.63523 & 0.24936 & 0.00000 & \text { Uiso } & 1.00\end{array}$ $\begin{array}{lllllllll}\mathrm{Al} 71 & \mathrm{Al} & 0.40273 & 0.15173 & 0.39193 & 0.00000 & \text { Uiso } & 1.00\end{array}$ $\begin{array}{llllllll}\mathrm{Al} 172 & \mathrm{Al} & 0.35104 & 0.22616 & 0.19981 & 0.00000 & \text { Uiso } & 1.00\end{array}$ $\begin{array}{llllllll}\mathrm{Al} 73 & \mathrm{Al} & 0.39708 & 0.62691 & 0.00979 & 0.00000 & \text { Uiso } & 1.00\end{array}$ $\begin{array}{lllllllll}\text { Al74 Al } & 0.40242 & 0.76507 & 0.25422 & 0.00000 & \text { Uiso } & 1.00\end{array}$ $\begin{array}{llllllll}\mathrm{O} 75 & \mathrm{O} & 0.30862 & 0.87706 & 0.19065 & 0.00000 & \text { Uiso } & 1.00\end{array}$ $\begin{array}{llllllllll}076 & \mathrm{O} & 0.30414 & 0.88795 & 0.44114 & 0.00000 & \text { Uiso } & 1.00\end{array}$ $\begin{array}{llllllll}077 & \mathrm{O} & 0.30659 & 0.38926 & 0.19252 & 0.00000 & \text { Uiso } & 1.00\end{array}$ $\begin{array}{llllllll}\mathrm{O} 78 & \mathrm{O} & 0.30644 & 0.39326 & 0.44030 & 0.00000 & \text { Uiso } & 1.00\end{array}$ $\begin{array}{llllllll}079 & \mathrm{O} & 0.30839 & 0.04341 & 0.18480 & 0.00000 & \text { Uiso } & 1.00\end{array}$ $\begin{array}{llllllll}080 & \mathrm{O} & 0.30939 & 0.05815 & 0.43595 & 0.00000 & \text { Uiso } & 1.00\end{array}$ $\begin{array}{llllllll}\mathrm{O} 81 & \mathrm{O} & 0.30380 & 0.55065 & 0.18583 & 0.00000 & \text { Uiso } & 1.00\end{array}$ $\begin{array}{lllllllllll}082 & 0 & 0.30274 & 0.56335 & 0.43838 & 0.00000 & \text { Uiso } & 1.00\end{array}$ $\begin{array}{llllllllll}082 & 0 & 0.30274 & 0.56335 & 0.43938 & 0.0000 & \text { Uiso } & 1.00 \\ 083 & 0 & 0.30736 & 0.22377 & 0.19389 & 0.00000 & \text { Uiso } & 1.00\end{array}$ $\begin{array}{llllllll}083 & \mathrm{O} & 0.30736 & 0.22377 & 0.19389 & 0.00000 & \text { Uiso } & 1.00\end{array}$ $\begin{array}{lllllllllll}085 & 0 & 0.30457 & 0.21993 & 0.44428 & 0.00000 & \text { Uiso } & -1.00\end{array}$ $\begin{array}{llllllllll}085 & 0 & 0.30178 & 0.71602 & 0.18520 & 0.0000 & \text { Uiso } & 1.00\end{array}$ $\begin{array}{lllllllll}086 & 0 & 0.30068 & 0.72217 & 0.43548 & 0.0000 & \text { Uiso } & 1.00\end{array}$ $\begin{array}{llllllll}087 & \mathrm{O} & 0.35148 & 0.13431 & 0.15713 & 0.00000 & \text { Uiso } & 1.00\end{array}$ $\begin{array}{llllllll}088 & \mathrm{O} & 0.35607 & 0.15416 & 0.39112 & 0.00000 & \text { Uiso } & 1.00\end{array}$ $\begin{array}{lllllllll}089 & \mathrm{O} & 0.35910 & 0.62717 & 0.18058 & 0.00000 & \text { Uiso } & 1.00\end{array}$ $\begin{array}{lllllllll}\mathrm{O} 90 & \mathrm{O} & 0.34601 & 0.63194 & 0.36837 & 0.00000 & \text { Uiso } & 1.00\end{array}$ $\begin{array}{lllllllll}\mathrm{O} 91 & \mathrm{O} & 0.34958 & 0.79774 & 0.13132 & 0.00000 & \text { Uiso } & 1.00\end{array}$ $\begin{array}{llllllll}092 & \mathrm{O} & 0.34563 & 0.80667 & 0.38720 & 0.00000 & \text { Uiso } & 1.00\end{array}$ $\begin{array}{lllllllll}093 & \mathrm{O} & 0.35619 & 0.30976 & 0.01187 & 0.00000 & \text { Uiso } & 1.00\end{array}$ $\begin{array}{lllllllll}094 & \mathrm{O} & 0.41655 & 0.39866 & 0.32266 & 0.00000 & \text { Uiso } & 1.00\end{array}$ $\begin{array}{llllllllll}\mathrm{O} 95 & \mathrm{O} & 0.35367 & 0.96649 & 0.37762 & 0.00000 & \text { Uiso } & 1.00\end{array}$ $\begin{array}{lllllllll}096 & \mathrm{O} & 0.35539 & 0.48658 & 0.12002 & 0.00000 & \text { Uiso } & 1.00\end{array}$ $\begin{array}{llllllll}097 & \mathrm{O} & 0.35400 & 0.48738 & 0.39202 & 0.00000 & \text { Uiso } & 1.00\end{array}$ $\begin{array}{llllllllll}098 & 0 & 0.30494 & 0.88681 & 0.07164 & 0.00000 & \text { Uiso } & 1.00\end{array}$ $\begin{array}{lllllllll}099 & \mathrm{O} & 0.30155 & 0.88690 & 0.32173 & 0.00000 & \text { Uiso } & 1.00\end{array}$ $\begin{array}{llllllll}0100 & \mathrm{O} & 0.30230 & 0.38909 & 0.07511 & 0.00000 & \text { Uiso } & 1.00\end{array}$ $\begin{array}{lllllllllllll}0101 & 0 & 0.30239 & 0.38189 & 0.31891 & 0.00000 & \text { Uiso } & 1.00\end{array}$ $\begin{array}{llllllllll}0102 & 0 & 0.30229 & 0.38189 & 0.31891 & 0.0000 & \text { Uiso } & 1.00\end{array}$ $\begin{array}{lllllllll}0103 & 0 & 0.30546 & 0.0554 & 0.0695 & 0.0000 & \text { Uiso } & 1.00\end{array}$ $\begin{array}{lllllllll}0104 & 0 & 0.30483 & 0.05611 & 0.31940 & 0.00000 & \text { Uiso } & 1.00\end{array}$ $\begin{array}{lllllllll}0104 & \mathrm{O} & 0.30112 & 0.55957 & 0.07192 & 0.00000 & \text { Uiso } & 1.00\end{array}$ $\begin{array}{llllllllll}0105 & \mathrm{O} & 0.30064 & 0.55976 & 0.31956 & 0.00000 & \text { Uiso } & 1.00\end{array}$ $\begin{array}{lllllllll}0106 & \mathrm{O} & 0.30655 & 0.22059 & 0.07516 & 0.00000 & \text { Uiso } & 1.00\end{array}$ $\begin{array}{llllllllll}0107 & \mathrm{O} & 0.31015 & 0.21905 & 0.32337 & 0.00000 & \text { Uiso } & 1.00\end{array}$ $\begin{array}{lllllllll}0108 & \mathrm{O} & 0.30991 & 0.71783 & 0.06809 & 0.00000 & \text { Uiso } & 1.00\end{array}$ $\begin{array}{lllllllll}0109 & \mathrm{O} & 0.30663 & 0.72444 & 0.31913 & 0.00000 & \text { Uiso } & 1.00\end{array}$ $\begin{array}{llllllll}\mathrm{O} 110 & \mathrm{O} & 0.35924 & 0.14289 & 0.00165 & 0.00000 & \text { Uiso } & 1.00\end{array}$ $\begin{array}{lllllllll}0111 & \mathrm{O} & 0.35660 & 0.64474 & 0.00034 & 0.00000 & \text { Uiso } & 1.00\end{array}$ $\begin{array}{llllllll}0112 & \mathrm{O} & 0.36490 & 0.81138 & 0.00819 & 0.00000 & \text { Uiso } & 1.00\end{array}$ $\begin{array}{llllllll}0113 & \mathrm{O} & 0.35994 & 0.77690 & 0.26087 & 0.00000 & \text { Uiso } & 1.00\end{array}$ 
$\begin{array}{llllllll}0114 & \mathrm{O} & 0.34501 & 0.30495 & 0.39382 & 0.00000 & \text { Uiso } & 1.00\end{array}$ $\begin{array}{lllllllll}0115 & \mathrm{O} & 0.35333 & 0.30397 & 0.26015 & 0.00000 & \text { Uiso } & 1.00\end{array}$ $\begin{array}{llllllll}0116 & \mathrm{O} & 0.35086 & 0.96802 & 0.49593 & 0.00000 & \text { Uiso } & 1.00\end{array}$ $\begin{array}{lllllllll}0117 & 0 & 0.34963 & 0.95617 & 0.24681 & 0.00000 & \text { Uiso } & 1.00\end{array}$ $\begin{array}{llllllll}0118 & \mathrm{O} & 0.34826 & 0.47821 & 0.00110 & 0.00000 & \text { Uiso } & 1.00\end{array}$ $\begin{array}{lllllllll}0119 & \mathrm{O} & 0.34948 & 0.48263 & 0.26033 & 0.00000 & \text { Uiso } & 1.00\end{array}$ $\begin{array}{lllllllllllll}0120 & 0 & 0.20714 & 0.89410 & 0.44374 & 0.00000 & \text { Uiso } & 1.00\end{array}$ $\begin{array}{lllllllllll}0121 & 0 & 0.20714 & 0.89410 & 0.43374 & 0.00000 & \text { Uiso } & 1.00\end{array}$

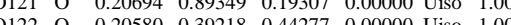
$\begin{array}{lllllllll}0122 & \mathrm{O} & 0.20580 & 0.39218 & 0.44277 & 0.00000 & \text { Uiso } & 1.00\end{array}$ $\begin{array}{lllllllll}0123 & \mathrm{O} & 0.20555 & 0.3232 & 0.19223 & 0.00000 & \text { Uiso } & 1.00 \\ 0124 & 0 & 0.20727 & 0.05009 & 0.4444 & 0.00000 & \text { Uiso } & 1.00\end{array}$ $\begin{array}{llllllllll}0124 & \mathrm{O} & 0.20727 & 0.05009 & 0.44444 & 0.00000 & \text { Uiso } & 1.00 \\ 0125 & \mathrm{O} & 0.20705 & 0.0488 & 0.19321 & 0.0000 & \text { Uiso } & 1.00\end{array}$ $\begin{array}{llllllllll}0125 & 0 & 0.2075 & 0.04986 & 0.19321 & 0.00000 & \text { Uiso } & 1.00 \\ 0126 & 0 & 0.20574 & 0.54782 & 0.4223 & 0.0000 & \text { Uis } & 1.00\end{array}$ $\begin{array}{lllllllll}0126 & \mathrm{O} & 0.20574 & 0.54782 & 0.44223 & 0.00000 & \text { Uiso } & 1.00\end{array}$ $\begin{array}{lllllllll}\mathrm{O} 127 & \mathrm{O} & 0.20531 & 0.54773 & 0.19188 & 0.00000 & \text { Uiso } & 1.00\end{array}$ $\begin{array}{lllllllllll}0128 & 0 & 0.21095 & 0.22046 & 0.44326 & 0.00000 & \text { Uiso } & 1.00\end{array}$ $\begin{array}{lllllllll}0129 & \mathrm{O} & 0.21090 & 0.22066 & 0.19247 & 0.00000 & \text { Uiso } & 1.00\end{array}$ $\begin{array}{llllllll}0130 & \mathrm{O} & 0.21040 & 0.72146 & 0.44180 & 0.00000 & \text { Uiso } & 1.00\end{array}$ $\begin{array}{lllllllll}0131 & \mathrm{O} & 0.21029 & 0.72184 & 0.19113 & 0.00000 & \text { Uiso } & 1.00\end{array}$

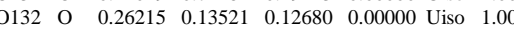
$\begin{array}{lllllllll}0133 & \mathrm{O} & 0.26141 & 0.13781 & 0.37300 & 0.00000 & \text { Uiso } & 1.00\end{array}$ $\begin{array}{llllllll}0134 & \mathrm{O} & 0.25707 & 0.63595 & 0.12179 & 0.00000 & \text { Uiso } & 1.00\end{array}$ $\begin{array}{llllllll}0135 & \mathrm{O} & 0.25710 & 0.63729 & 0.37091 & 0.00000 & \text { Uiso } & 1.00\end{array}$ $\begin{array}{llllllll}0136 & \mathrm{O} & 0.26170 & 0.80403 & 0.12146 & 0.00000 & \text { Uiso } & 1.00\end{array}$ $\begin{array}{llllllll}0137 & \mathrm{O} & 0.25846 & 0.80743 & 0.37301 & 0.00000 & \text { Uiso } & 1.00\end{array}$ $\begin{array}{llllllll}0138 & \mathrm{O} & 0.26074 & 0.30527 & 0.12708 & 0.00000 & \text { Uiso } & 1.00\end{array}$ $\begin{array}{llllllll}0139 & \mathrm{O} & 0.25984 & 0.30508 & 0.37539 & 0.00000 & \text { Uiso } & 1.00\end{array}$ $\begin{array}{llllllllll}0140 & 0 & 0.25642 & 0.96842 & 0.12507 & 0.00000 & \text { Uiso } & 1.00\end{array}$ $\begin{array}{lllllllllll}0141 & 0 & 0.25642 & 0.96842 & 0.12507 & 0.00000 & 0.07590 & 1.00\end{array}$ $\begin{array}{llllllllll}0142 & 0 & 0.25338 & 0.4702 & 0.12398 & 0.00000 & \text { Uiso } & 1.00\end{array}$ $\begin{array}{lllllllll}0142 & 0 & 0.25338 & 0.47022 & 0.12398 & 0.00000 & \text { Uiso } & 1.00\end{array}$ $\begin{array}{lllllllll}0143 & \mathrm{O} & 0.25437 & 0.47040 & 0.37373 & 0.00000 & \text { Uiso } & 1.00\end{array}$ $\begin{array}{lllllllllll}0144 & \mathrm{O} & 0.21592 & 0.89300 & 0.06075 & 0.00000 & \text { Uiso } & 1.00 \\ 0145 & \mathrm{O} & 0.21497 & 0.89461 & 0.31060 & 0.00000 & \text { Uis } & 1.00\end{array}$ $\begin{array}{llllllllll}0145 & \mathrm{O} & 0.21497 & 0.89461 & 0.31060 & 0.00000 & \text { Uiso } & 1.00\end{array}$ $\begin{array}{lllllllll}0146 & \mathrm{O} & 0.21420 & 0.39217 & 0.06041 & 0.00000 & \text { Uiso } & 1.00\end{array}$ $\begin{array}{lllllllll}0147 & \mathrm{O} & 0.21407 & 0.39200 & 0.31022 & 0.00000 & \text { Uiso } & 1.00\end{array}$ $\begin{array}{lllllllll}0148 & \mathrm{O} & 0.21590 & 0.04853 & 0.06243 & 0.00000 & \text { Uiso } & 1.00\end{array}$ $\begin{array}{llllllll}0149 & \mathrm{O} & 0.21583 & 0.04998 & 0.31045 & 0.00000 & \text { Uiso } & 1.00\end{array}$ $\begin{array}{llllllll}0150 & \mathrm{O} & 0.21366 & 0.54798 & 0.05933 & 0.00000 & \text { Uiso } & 1.00\end{array}$ $\begin{array}{llllllll}0151 & \mathrm{O} & 0.21380 & 0.54805 & 0.30921 & 0.00000 & \text { Uiso } & 1.00\end{array}$ $\begin{array}{llllllll}0152 & \mathrm{O} & 0.20848 & 0.21991 & 0.06388 & 0.00000 & \text { Uiso } & 1.00\end{array}$ $\begin{array}{llllllll}0153 & \mathrm{O} & 0.20818 & 0.22101 & 0.31289 & 0.00000 & \text { Uiso } & 1.00\end{array}$ $\begin{array}{llllllll}0154 & \mathrm{O} & 0.20815 & 0.72182 & 0.06143 & 0.00000 & \text { Uiso } & 1.00\end{array}$ $\begin{array}{lllllllll}0155 & \mathrm{O} & 0.20752 & 0.72170 & 0.31089 & 0.00000 & \text { Uiso } & 1.00\end{array}$ $\begin{array}{llllllll}0156 & \mathrm{O} & 0.25600 & 0.13646 & 0.00859 & 0.00000 & \text { Uiso } & 1.00\end{array}$ $\begin{array}{lllllllll}0157 & \mathrm{O} & 0.25648 & 0.13831 & 0.25637 & 0.00000 & \text { Uiso } & 1.00\end{array}$ $\begin{array}{lllllllll}0158 & \mathrm{O} & 0.25471 & 0.63737 & 0.00579 & 0.00000 & \text { Uiso } & 1.00\end{array}$ $\begin{array}{llllllll}0159 & 0 & 0.25487 & 0.63733 & 0.25404 & 0.00000 & \text { Uiso } & 1.00\end{array}$ $\begin{array}{lllllllll}0160 & \mathrm{O} & 0.25632 & 0.80362 & 0.00658 & 0.00000 & \text { Uiso } & 1.00\end{array}$ $\begin{array}{llllllllllll}0161 & 0 & 0.25525 & 0.80440 & 0.25541 & 0.00000 & \text { Uiso } & 1.00\end{array}$ $\begin{array}{lllllllll}0161 & 0 & 0.25525 & 0.80440 & 0.2541 & 0.0000 & \text { Uiso } & 1.00\end{array}$ $\begin{array}{llllllllll}0162 & 0 & 0.25541 & 0.30358 & 0.00884 & 0.0000 & \text { Uiso } & 1.00\end{array}$ $\begin{array}{lllllllll}0163 & \mathrm{O} & 0.25553 & 0.30338 & 0.25751 & 0.00000 & \text { Uiso } & 1.00\end{array}$ $\begin{array}{llllllllll}0164 & \mathrm{O} & 0.25884 & 0.97248 & 0.00628 & 0.00000 & \text { Uiso } & 1.00 \\ 0165 & \mathrm{O} & 0.25840 & 0.97017 & 0.25476 & 0.00000 & \text { Uis } & 1.00\end{array}$ \begin{tabular}{llllllllll}
0165 & $\mathrm{O}$ & 0.25840 & 0.97017 & 0.25476 & 0.00000 & Uiso & 1.00 \\
\hline 166 & $\mathrm{O}$ & 0.25710 & 0.47055 & 0.00558 & 0.0000 & Uiso & 1.00
\end{tabular} $\begin{array}{lllllllll}0166 & \mathrm{O} & 0.25710 & 0.47055 & 0.00558 & 0.00000 & \text { Uiso } & 1.00 \\ 0167 & \mathrm{O} & 0.25616 & 0.4630 & 0.2550 & 0.0000 & \text { Uiso } & 1.00\end{array}$ $\begin{array}{llllllllll}0167 & \mathrm{O} & 0.25616 & 0.46930 & 0.25501 & 0.0000 & \text { Uiso } & 1.00\end{array}$ $\begin{array}{lllllllll}0168 & \mathrm{O} & 0.35221 & 0.29808 & 0.13546 & 0.00000 & \text { Uiso } & 1.00\end{array}$ $\begin{array}{llllllll}0169 & \mathrm{O} & 0.39636 & 0.71351 & 0.33001 & 0.00000 & \text { Uiso } & 1.00\end{array}$ $\begin{array}{llllllll}\mathrm{O} 170 & \mathrm{O} & 0.41534 & 0.18300 & 0.46432 & 0.00000 & \text { Uiso } & 1.00\end{array}$ $\begin{array}{llllllll}0171 & \mathrm{O} & 0.39821 & 0.88752 & 0.43360 & 0.00000 & \text { Uiso } & 1.00\end{array}$ $\begin{array}{llllllll}0172 & \mathrm{O} & 0.39775 & 0.38819 & 0.08848 & 0.00000 & \text { Uiso } & 1.00\end{array}$ $\begin{array}{llllllll}0173 & \mathrm{O} & 0.39437 & 0.37958 & 0.43585 & 0.00000 & \text { Uiso } & 1.00\end{array}$ $\begin{array}{llllllll}0174 & \mathrm{O} & 0.39820 & 0.25452 & 0.35681 & 0.00000 & \text { Uiso } & 1.00\end{array}$ $\begin{array}{lllllllll}0175 & \mathrm{O} & 0.41027 & 0.55966 & 0.32768 & 0.00000 & \text { Uiso } & 1.00\end{array}$ $\begin{array}{llllllll}0176 & \mathrm{O} & 0.46470 & 0.49370 & 0.35410 & 0.00000 & \text { Uiso } & 1.00\end{array}$ $\begin{array}{lllllllll}0177 & \mathrm{O} & 0.40835 & 0.54445 & 0.14866 & 0.00000 & \text { Uiso } & 1.00\end{array}$ $\begin{array}{lllllllll}0178 & \mathrm{O} & 0.40535 & 0.53068 & 0.03513 & 0.00000 & \text { Uiso } & 1.00\end{array}$ $\begin{array}{lllllllll}0179 & 0 & 0.39823 & 0.22926 & 0.07406 & 0.00000 & \text { Uiso } & 1.00\end{array}$ $\begin{array}{lllllllll}\mathrm{O} 180 & \mathrm{O} & 0.39560 & 0.04554 & 0.42170 & 0.00000 & \text { Uiso } & 1.00\end{array}$ $\begin{array}{llllllllll}0181 & 0 & 0.40608 & 0.07263 & 0.05296 & 0.00000 & \text { Uiso } & 1.00\end{array}$ $\begin{array}{llllllllll}0181 & 0 & 0.40608 & 0.07263 & 0.05296 & 0.00000 & \text { Uiso } & 1.00\end{array}$ $\begin{array}{lllllllll}0182 & 0 & 0.41387 & 0.69542 & 0.1981 & 0.00000 & \text { Uiso } & 1.00\end{array}$ $\begin{array}{llllllllll}0182 & 0 & 0.45036 & 0.32257 & 0.40178 & 0.00000 & \text { Uiso } & 1.00\end{array}$ $\begin{array}{llllllll}0184 & \mathrm{O} & 0.45343 & 0.67249 & 0.35267 & 0.00000 & \text { Uiso } & 1.00\end{array}$ $\begin{array}{llllllllll}0185 & 0 & 0.41090 & 0.63368 & 0.43607 & 0.00000 & \text { Uiso } & 1.00 \\ 0186 & 0 & 0.4533 & 0.49103 & 0.24153 & 0.00000 & \text { Uiso } & 1.00\end{array}$ $\begin{array}{lllllllll}0186 & \mathrm{O} & 0.44533 & 0.49103 & 0.24153 & 0.00000 & \text { Uiso } & 1.00 \\ 0187 & \mathrm{O} & 0.37047 & 0.1813 & 0.26594 & 0.0000 & \text { Uiso } & 1.00\end{array}$ $\begin{array}{lllllllll}0187 & 0 & 0.37047 & 0.16813 & 0.26594 & 0.00000 & \text { Uiso } & 1.00\end{array}$ $\begin{array}{lllllllll}0188 & 0 & 0.42082 & 0.11432 & 0.32609 & 0.00000 & \text { Uiso } & 1.00\end{array}$ $\begin{array}{lllllllll}0189 & \mathrm{O} & 0.40927 & 0.94017 & 0.32440 & 0.00000 & \text { Uiso } & 1.00\end{array}$ $\begin{array}{llllllll}\mathrm{O} 190 & \mathrm{O} & 0.39800 & 0.86985 & 0.21658 & 0.00000 & \text { Uiso } & 1.00\end{array}$ $\begin{array}{llllllll}0191 & \mathrm{O} & 0.42690 & 0.16749 & 0.20899 & 0.00000 & \text { Uiso } & 1.00\end{array}$ $\begin{array}{llllllll}0192 & \mathrm{O} & 0.39710 & 0.03746 & 0.23037 & 0.00000 & \text { Uiso } & 1.00\end{array}$ $\begin{array}{llllllll}0193 & \mathrm{O} & 0.45071 & 0.94614 & 0.24552 & 0.00000 & \text { Uiso } & 1.00\end{array}$ $\begin{array}{llllllll}0194 & \mathrm{O} & 0.40939 & 0.91105 & 0.05180 & 0.00000 & \text { Uiso } & 1.00\end{array}$ $\begin{array}{llllllll}0195 & \mathrm{O} & 0.42572 & 0.77391 & 0.00122 & 0.00000 & \text { Uiso } & 1.00\end{array}$ $\begin{array}{llllllllll}0196 & \mathrm{O} & 0.42960 & 0.99392 & 0.13861 & 0.00000 & \text { Uiso } & 1.00\end{array}$ $\begin{array}{lllllllll}0197 & \mathrm{O} & 0.39134 & 0.67940 & 0.08524 & 0.00000 & \text { Uiso } & 1.00\end{array}$ $\begin{array}{llllllll}\mathrm{O} 198 & \mathrm{O} & 0.36447 & 0.98277 & 0.11866 & 0.00000 & \text { Uiso } & 1.00\end{array}$ $\begin{array}{lllllllll}0199 & \mathrm{O} & 0.44383 & 0.79024 & 0.27878 & 0.00000 & \text { Uiso } & 1.00\end{array}$ $\begin{array}{lllllll}\mathrm{Si} 200 \mathrm{Si} & 0.39211 & 0.96113 & 0.38606 & 0.00000 & \text { Uiso } & 1.00\end{array}$ $\begin{array}{llllllll}\mathrm{Si} 201 & \mathrm{Si} & 0.41367 & 0.34069 & 0.38236 & 0.00000 & \text { Uiso } & 1.00\end{array}$ $\begin{array}{llllllll}\mathrm{Si} 202 & \mathrm{Si} & 0.43358 & 0.48430 & 0.31065 & 0.00000 & \text { Uiso } & 1.00\end{array}$ $\begin{array}{llllllll}\mathrm{Si2} 22 & \mathrm{Si} & 0.43358 & 0.48430 & 0.31065 & 0.00000 & \text { Uiso } & 1.00 \\ \mathrm{Si2} 23 & \mathrm{Si} & 0.39296 & 0.48532 & 0.09379 & 0.00000 & \text { Uiso } & 1.00\end{array}$ $\begin{array}{llllllllll}\mathrm{Si} 203 & \mathrm{Si} & 0.39296 & 0.48532 & 0.09379 & 0.00000 & \text { Uiso } & 1.00 \\ \mathrm{Si} 204 & \mathrm{Si} & 0.39620 & 0.15780 & 0.02426 & 0.00000 & \text { Uis } & 1.00\end{array}$ $\begin{array}{llllllll}\text { Si204 Si } & 0.39620 & 0.15780 & 0.02426 & 0.00000 & \text { Uiso } & 1.00\end{array}$ $\begin{array}{llllllll}\mathrm{Si} 205 & \mathrm{Si} & 0.39419 & 0.63960 & 0.15482 & 0.00000 & \text { Uiso } & 1.00 \\ \mathrm{Si} 206 & \mathrm{Si} & 0.37551 & 0.30841 & 0.07658 & 0.00000 & \text { Us } & 1.00\end{array}$

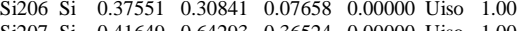
$\begin{array}{lllllll}\mathrm{Si2} 27 \mathrm{Si} & 0.41649 & 0.64293 & 0.36524 & 0.00000 & \text { Uiso } & 1.00 \\ \mathrm{Si} 208 \mathrm{Si} & 0.41207 & 0.94837 & 0.25092 & 0.00000 & \text { Uiso } & 1.00\end{array}$ $\begin{array}{lllllllll}\text { Si208 Si } & 0.41207 & 0.94837 & 0.25092 & 0.00000 & \text { Uiso } & 1.00\end{array}$ $\begin{array}{llllllll}\mathrm{Si} 209 & \mathrm{Si} & 0.40530 & 0.12653 & 0.26160 & 0.00000 & \text { Uiso } & 1.00\end{array}$ $\begin{array}{lllllll}\mathrm{Si} 210 \mathrm{Si} & 0.40080 & 0.99058 & 0.08950 & 0.00000 & \text { Uiso } & 1.00\end{array}$ $\begin{array}{llllllll}\mathrm{Si} 211 & \mathrm{Si} & 0.40079 & 0.84635 & 0.00082 & 0.00000 & \text { Uiso } & 1.00\end{array}$ $\begin{array}{lllllllll}\mathrm{C} 212 & \mathrm{C} & 0.47330 & 0.63277 & 0.54782 & 0.00000 & \text { Uiso } & 1.00\end{array}$ $\begin{array}{llllllll}\mathrm{C} 213 & \mathrm{C} & 0.48466 & 0.71647 & 0.55759 & 0.00000 & \text { Uiso } & 1.00\end{array}$ $\begin{array}{llllllll}\mathrm{C} 214 & \mathrm{C} & 0.47327 & 0.76342 & 0.60154 & 0.00000 & \text { Uiso } & 1.00\end{array}$ 
$\begin{array}{llllllll}\text { C215 } & \text { C } & 0.50870 & 0.74529 & 0.51367 & 0.00000 & \text { Uiso } & 1.00\end{array}$ $\begin{array}{lllllllll}\mathrm{H} 216 & \mathrm{H} & 0.45595 & 0.74179 & 0.63358 & 0.00000 & \text { Uiso } & 1.00\end{array}$ $\begin{array}{llllllll}\mathrm{H} 217 & \mathrm{H} & 0.48182 & 0.82438 & 0.60706 & 0.00000 & \text { Uiso } & 1.00\end{array}$ $\begin{array}{llllllll}\mathrm{H} 218 & \mathrm{H} & 0.44235 & 0.77376 & 0.53235 & 0.00000 & \text { Uiso } & 1.00\end{array}$ $\begin{array}{llllllll}\mathrm{H} 219 & \mathrm{H} & 0.45605 & 0.61429 & 0.58143 & 0.00000 & \text { Uiso } & 1.00\end{array}$ $\begin{array}{llllllllll}\mathrm{H} 220 & \mathrm{H} & 0.49280 & 0.59015 & 0.54964 & 0.00000 & \text { Uiso } & 1.00\end{array}$ $\begin{array}{lllllllll} & \mathrm{H} 221 & \mathrm{H} & 0.46326 & 0.62668 & 0.50236 & 0.00000 & \text { Uiso } & 1.00\end{array}$

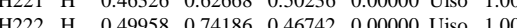
$\begin{array}{llllllll} & 0.523 & 0.4958 & 0.74186 & 0.4672 & 0.0000\end{array}$ $\begin{array}{llllllllll}\text { H223 } & \mathrm{H} & 0.52938 & 0.70644 & 0.51519 & 0.00000 & \text { Uiso } & 1.00\end{array}$ \begin{tabular}{lllllllll}
$\mathrm{H} 224$ & $\mathrm{H}$ & 0.46867 & 0.44769 & 0.87987 & 0.00000 & Uiso & 1.00 \\
\hline & +225 & $\mathrm{H}$ & 0.45171 & 0.29409 & 0.93981 & 0.00000 & Uiso & 1.00
\end{tabular} $\begin{array}{lllllllll}\mathrm{H} 225 & \mathrm{H} & 0.45171 & 0.2909 & 0.93881 & 0.00000 & \text { Uiso } & 1.00 \\ \mathrm{H} 226 & \mathrm{H} & 0.4632 & 0.62850 & 0.8534 & 0.0000 & \text { Uiso } & 1.00\end{array}$ $\begin{array}{lllllllll}\mathrm{H} 226 & \mathrm{H} & 0.46832 & 0.62850 & 0.85344 & 0.00000 & \text { Uiso } & 1.00 \\ \mathrm{H} 227 & \mathrm{H} & 0.43167 & 0.51175 & 0.70972 & 0.00000 & \text { Uis } & 1.00\end{array}$ $\begin{array}{llllllll}\mathrm{H} 227 & \mathrm{H} & 0.43167 & 0.51175 & 0.70972 & 0.00000 & \text { Uiso } & 1.00\end{array}$ $\begin{array}{llllllll}\mathrm{H} 228 & \mathrm{H} & 0.43831 & 0.21583 & 0.71856 & 0.00000 & \text { Uiso } & 1.00\end{array}$ $\begin{array}{lllllllll}\mathrm{H} 229 & \mathrm{H} & 0.45662 & 0.94840 & 0.70317 & 0.00000 & \text { Uiso } & -1.00\end{array}$ $\begin{array}{lllllllll}\mathrm{H} 230 & \mathrm{H} & 0.51594 & 0.80689 & 0.52295 & 0.00000 & \text { Uiso } & 1.00\end{array}$ $\begin{array}{llllllll}\mathrm{H} 231 & \mathrm{H} & 0.43379 & 0.04890 & 0.65085 & 0.00000 & \text { Uiso } & 1.00\end{array}$ $\begin{array}{lllllllll}\mathrm{H} 232 & \mathrm{H} & 0.45424 & 0.84226 & 0.77356 & 0.00000 & \text { Uiso } & 1.00\end{array}$ $\begin{array}{lllllllll}\mathrm{H} 233 & \mathrm{H} & 0.45348 & 0.75202 & 0.80830 & 0.00000 & \text { Uiso } & 1.00\end{array}$ $\begin{array}{llllllll}\mathrm{Al} 234 & \mathrm{Al} & 0.35125 & 0.05697 & 0.94702 & 0.00000 & \text { Uiso } & 1.00\end{array}$ $\begin{array}{llllllll}\mathrm{Al} 235 \mathrm{Al} & 0.35014 & 0.03644 & 0.69026 & 0.00000 & \text { Uiso } & 1.00\end{array}$ $\begin{array}{llllllll}\mathrm{Al} 236 & \mathrm{Al} & 0.34387 & 0.56554 & 0.95015 & 0.00000 & \text { Uiso } & 1.00\end{array}$ $\begin{array}{llllllll}\mathrm{Al} 237 & \mathrm{Al} & 0.34381 & 0.53288 & 0.69082 & 0.00000 & \text { Uiso } & 1.00\end{array}$ $\begin{array}{llllllll}\text { Al238 Al } & 0.34466 & 0.87468 & 0.94839 & 0.00000 & \text { Uiso } & 1.00\end{array}$

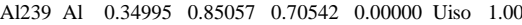
$\begin{array}{llllllll}\mathrm{Al} 240 & \mathrm{Al} & 0.35317 & 0.40132 & 0.94196 & 0.00000 & \text { Uiso } & 1.00\end{array}$ $\begin{array}{llllllll}\mathrm{A} 2241 & \mathrm{Al} & 0.32886 & 0.38701 & 0.75958 & 0.00000 & \text { Uiso } & 1.00\end{array}$

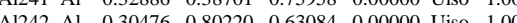

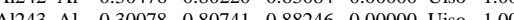
$\begin{array}{llllllllll}\text { Al243 Al } & 0.30078 & 0.80741 & 0.88246 & 0.00000 & \text { Uiso } & 1.00\end{array}$ $\begin{array}{lllllll}\text { Al244 Al } & 0.30451 & 0.30659 & 0.63017 & 0.00000 & \text { Uiso } & 1.00\end{array}$

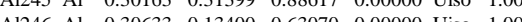
$\begin{array}{llllllll}\text { Al246 Al } & 0.30633 & 0.13400 & 0.63070 & 0.00000 & \text { Uiso } & 1.00\end{array}$ $\begin{array}{lllllllll} & \text { Al247 Al } & 0.30315 & 0.13613 & 0.87925 & 0.00000 & \text { Uiso } & 1.00\end{array}$ $\begin{array}{llllllll}\text { Al248 Al } & 0.29763 & 0.63498 & 0.63379 & 0.00000 & \text { Uiso } & 1.00\end{array}$

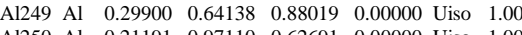
$\begin{array}{lllllll}\mathrm{Al} 250 \mathrm{Al} & 0.21191 & 0.97110 & 0.62691 & 0.00000 & \text { Uiso } & 1.00\end{array}$ $\begin{array}{llllllll}\mathrm{Al} 251 & \mathrm{Al} & 0.21176 & 0.97245 & 0.87725 & 0.00000 & \text { Uiso } & 1.00\end{array}$ $\begin{array}{llllllll}\mathrm{A} 1252 \mathrm{Al} & 0.20935 & 0.47056 & 0.62532 & 0.00000 & \text { Uiso } & 1.00\end{array}$ $\begin{array}{llllllll}\mathrm{Al} 253 & \mathrm{Al} & 0.21015 & 0.47068 & 0.87586 & 0.00000 & \text { Uiso } & 1.00\end{array}$ $\begin{array}{llllllll}\mathrm{A} 1254 \mathrm{Al} & 0.32937 & 0.97028 & 0.56496 & 0.00000 & \text { Uiso } & 1.00\end{array}$ $\begin{array}{lllllll}\mathrm{A} 1255 \mathrm{Al} & 0.32684 & 0.96669 & 0.81355 & 0.00000 & \text { Uiso } & 1.00\end{array}$ $\begin{array}{llllllll}\mathrm{Al} 256 & \mathrm{Al} & 0.32451 & 0.47491 & 0.56382 & 0.00000 & \text { Uiso } & 1.00\end{array}$ $\begin{array}{llllllll}\mathrm{Al} 257 & \mathrm{Al} & 0.33980 & 0.53552 & 0.82944 & 0.00000 & \text { Uiso } & 1.00\end{array}$ $\begin{array}{llllllll}\mathrm{Al} 258 \mathrm{Al} & 0.28083 & 0.22009 & 0.51185 & 0.00000 & \text { Uiso } & 1.00\end{array}$

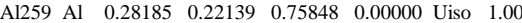

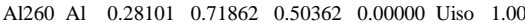
$\begin{array}{lllllllll}\mathrm{Al} 261 & \mathrm{Al} & 0.28101 & 0.72101 & 0.75350 & 0.00000 & \text { Uiso } & 1.00\end{array}$ $\begin{array}{llllllll}4262 & \mathrm{Al} & 0.34596 & 0.23201 & 0.95830 & 0.00000 & \text { Uiso } & 1.00\end{array}$ $\begin{array}{llllllllllll}A 1262 & \text { Al } & 0.34596 & 0.23201 & 0.95830 & 0.0000 & \text { Uiso } & 1.00\end{array}$

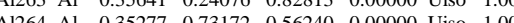
$\begin{array}{llllllll}\text { Al264 Al } & 0.35277 & 0.73172 & 0.56240 & 0.00000 & \text { Uiso } & 1.00\end{array}$ \begin{tabular}{lllllllll}
\hline & $\mathrm{A} 1266$ & $\mathrm{Al}$ & 0.35174 & 0.73137 & 0.83319 & 0.00000 & Uiso & 1.00
\end{tabular} $\begin{array}{llllllll}\mathrm{A} 266 & \mathrm{Al} & 0.21198 & 0.97156 & 0.50317 & 0.00000 & \text { Uiso } & 1.00\end{array}$ $\begin{array}{llllllllll}\text { Al267 Al } & 0.21143 & 0.97204 & 0.75209 & 0.00000 & \text { Uiso } & 1.00\end{array}$ $\begin{array}{llllllllllllllll}\text { Al268 Al } & 0.21023 & 0.46983 & 0.50148 & 0.00000 & \text { Uiso } & 1.00\end{array}$ $\begin{array}{llllllll}\mathrm{Al} 269 & \mathrm{Al} & 0.20960 & 0.47001 & 0.75153 & 0.00000 & \text { Uiso } & 1.00\end{array}$ $\begin{array}{llllllll}\mathrm{Al} 270 \mathrm{Al} & 0.28282 & 0.96087 & 0.68939 & 0.00000 & \text { Uiso } & 1.00\end{array}$

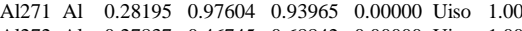
$\begin{array}{llllllll}\mathrm{A} 2272 \mathrm{Al} & 0.27837 & 0.46745 & 0.68842 & 0.00000 & \text { Uiso } & 1.00\end{array}$ $\begin{array}{llllllll}\mathrm{Al} 273 & \mathrm{Al} & 0.28071 & 0.47437 & 0.93859 & 0.00000 & \text { Uiso } & 1.00\end{array}$ $\begin{array}{lllllll}\mathrm{Al} 274 \mathrm{Al} & 0.23655 & 0.21902 & 0.62768 & 0.00000 & \text { Uiso } & 1.00\end{array}$

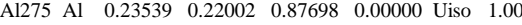
$\begin{array}{llllllll}\mathrm{A} 1276 \mathrm{Al} & 0.23490 & 0.72406 & 0.62528 & 0.00000 & \text { Uiso } & 1.00\end{array}$ $\begin{array}{lllllll}\mathrm{Al} 277 \mathrm{Al} & 0.23417 & 0.72320 & 0.87500 & 0.00000 & \text { Uiso } & 1.00\end{array}$ $\begin{array}{llllllll}\mathrm{Al} 278 & \mathrm{Al} & 0.26186 & 0.05810 & 0.56665 & 0.00000 & \text { Uiso } & 1.00\end{array}$ $\begin{array}{lllllll}\mathrm{Al} 279 \mathrm{Al} & 0.26127 & 0.05827 & 0.81307 & 0.00000 & \text { Uiso } & 1.00\end{array}$ $\begin{array}{llllllll}\mathrm{Al} 280 & \mathrm{Al} & 0.25841 & 0.55813 & 0.56240 & 0.00000 & \text { Uiso } & 1.00\end{array}$ $\begin{array}{lllllll}\mathrm{Al} 281 \mathrm{Al} & 0.25901 & 0.55861 & 0.81228 & 0.00000 & \text { Uiso } & 1.00\end{array}$ $\begin{array}{lllllllll} & \mathrm{Al} 282 & \mathrm{Al} & 0.26183 & 0.88315 & 0.56333 & 0.00000 & \text { Uiso } & 1.00\end{array}$ $\begin{array}{llllllllllll} & \mathrm{A} 282 & \mathrm{Al} & 0.26183 & 0.88315 & 0.56333 & 0.0000 & \text { Uiso } & 1.00\end{array}$ $\begin{array}{lllllllll}\mathrm{A} & \mathrm{A} 283 & \mathrm{Al} & 0.25962 & 0.88322 & 0.81355 & 0.00000 & \text { Uiso } & 1.00\end{array}$ $\begin{array}{lllllllll}A 1284 & \text { Al } & 0.25952 & 0.38232 & 0.56600 & 0.00000 & \text { Ulso } & 1.00\end{array}$ $\begin{array}{llllllll}\text { Al285 Al } & 0.25925 & 0.38332 & 0.81503 & 0.00000 & \text { Uiso } & 1.00\end{array}$ $\begin{array}{lllllllll}\text { Al286 Al } & 0.21396 & 0.80723 & 0.50072 & 0.00000 & \text { Uiso } & 1.00\end{array}$

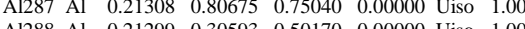
$\begin{array}{llllllll}\text { Al288 Al } & 0.21299 & 0.30593 & 0.50170 & 0.00000 & \text { Uiso } & 1.00\end{array}$ $\begin{array}{lllllll}\text { Al289 Al } & 0.21299 & 0.30600 & 0.75042 & 0.00000 & \text { Uiso } & 1.00\end{array}$

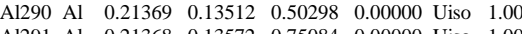
$\begin{array}{llllllll}\mathrm{Al} 291 & \mathrm{Al} & 0.21368 & 0.13572 & 0.75084 & 0.00000 & \text { Uiso } & 1.00\end{array}$ $\begin{array}{lllllll}\mathrm{Al} 292 \mathrm{Al} & 0.21270 & 0.63517 & 0.99958 & 0.00000 & \text { Uiso } & 1.00\end{array}$ $\begin{array}{llllllll}\mathrm{Al} 293 & \mathrm{Al} & 0.21272 & 0.63523 & 0.74936 & 0.00000 & \text { Uiso } & 1.00\end{array}$ $\begin{array}{llllllll}\mathrm{Al} 294 \mathrm{Al} & 0.40273 & 0.15173 & 0.89193 & 0.00000 & \text { Uiso } & 1.00\end{array}$ $\begin{array}{lllllll}\mathrm{Al} 295 \mathrm{Al} & 0.35104 & 0.22616 & 0.69981 & 0.00000 & \text { Uiso } & 1.00\end{array}$ $\begin{array}{llllllll}\mathrm{Al} 296 \mathrm{Al} & 0.39708 & 0.62691 & 0.50979 & 0.00000 & \text { Uiso } & 1.00\end{array}$ $\begin{array}{llllllll}\mathrm{Al} 297 \mathrm{Al} & 0.40242 & 0.76507 & 0.75422 & 0.00000 & \text { Uiso } & 1.00\end{array}$ $\begin{array}{lllllllll}\mathrm{O} 298 & \mathrm{O} & 0.30862 & 0.87706 & 0.69065 & 0.00000 & \text { Uiso } & 1.00\end{array}$ $\begin{array}{llllllll}\mathrm{O} 299 & \mathrm{O} & 0.30414 & 0.88795 & 0.94114 & 0.00000 & \text { Uiso } & 1.00\end{array}$ $\begin{array}{lllllllll}0300 & 0 & 0.30659 & 0.38926 & 0.69252 & 0.00000 & \text { Uiso } & 1.00\end{array}$ $\begin{array}{lllllllll}0301 & \mathrm{O} & 0.30644 & 0.39326 & 0.94030 & 0.00000 & \text { Uiso } & 1.00\end{array}$ $\begin{array}{lllllllll}0302 & \mathrm{O} & 0.30839 & 0.04341 & 0.68480 & 0.00000 & \text { Uiso } & 1.00\end{array}$ $\begin{array}{llllllllll}0303 & 0 & 0.30939 & 0.05815 & 0.93595 & 0.00000 & \text { Uiso } & 1.00\end{array}$ $\begin{array}{lllllllllllllllll}0304 & 0 & 0.30380 & 0.55065 & 0.68583 & 0.00000 & \text { Uiso } & 1.00\end{array}$

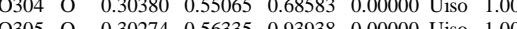
$\begin{array}{llllllllll}0306 & 0 & 0.30274 & 0.56335 & 0.93938 & 0.00000 & \text { Uiso } & 1.00\end{array}$ $\begin{array}{lllllllll}0306 & 0 & 0.30736 & 0.22377 & 0.69389 & 0.00000 & \text { Uiso } & 1.00\end{array}$ $\begin{array}{lllllllll}0307 & \mathrm{O} & 0.30457 & 0.21993 & 0.94428 & 0.00000 & \text { Uiso } & 1.00\end{array}$ $\begin{array}{lllllllll}0308 & \mathrm{O} & 0.30178 & 0.71602 & 0.68520 & 0.00000 & \text { Uiso } & 1.00\end{array}$ $\begin{array}{lllllllll}0309 & \mathrm{O} & 0.30068 & 0.72217 & 0.93548 & 0.0000 & \text { Uiso } & 1.00\end{array}$ $\begin{array}{lllllllll}0310 & \mathrm{O} & 0.35148 & 0.13431 & 0.65713 & 0.00000 & \text { Uiso } & 1.00\end{array}$ $\begin{array}{lllllllll}0311 & \mathrm{O} & 0.35607 & 0.15416 & 0.89112 & 0.00000 & \text { Uiso } & 1.00\end{array}$ $\begin{array}{llllllll}\mathrm{O} 312 & \mathrm{O} & 0.35910 & 0.62717 & 0.68058 & 0.00000 & \text { Uiso } & 1.00\end{array}$ $\begin{array}{lllllllll}\mathrm{O} 313 & \mathrm{O} & 0.34601 & 0.63194 & 0.86837 & 0.00000 & \text { Uiso } & 1.00\end{array}$ $\begin{array}{lllllllll}0314 & \mathrm{O} & 0.34958 & 0.79774 & 0.63132 & 0.00000 & \text { Uiso } & 1.00\end{array}$ $\begin{array}{llllllll}0315 & \text { O } & 0.34563 & 0.80667 & 0.88720 & 0.00000 & \text { Uiso } & 1.00\end{array}$ 
$\begin{array}{llllllll}0316 & \mathrm{O} & 0.35619 & 0.30976 & 0.51187 & 0.00000 & \text { Uiso } & 1.00\end{array}$ $\begin{array}{llllllll}0317 & \mathrm{O} & 0.41655 & 0.39866 & 0.82266 & 0.00000 & \text { Uiso } & 1.00\end{array}$ $\begin{array}{lllllllll}0318 & \mathrm{O} & 0.35367 & 0.96649 & 0.87762 & 0.00000 & \text { Uiso } & 1.00\end{array}$ $\begin{array}{llllllll}0319 & \mathrm{O} & 0.35539 & 0.48658 & 0.62002 & 0.00000 & \text { Uiso } & 1.00\end{array}$

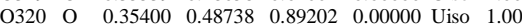
$\begin{array}{lllllllll}0321 & \mathrm{O} & 0.30494 & 0.88681 & 0.57164 & 0.00000 & \text { Uiso } & 1.00\end{array}$ $\begin{array}{lllllllllll}0322 & 0 & 0.30155 & 0.88690 & 0.82173 & 0.00000 & \text { Uiso } & 1.00\end{array}$

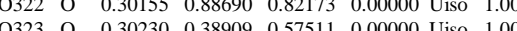

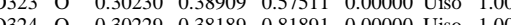
$\begin{array}{lllllllll}0324 & \mathrm{O} & 0.30229 & 0.38189 & 0.81891 & 0.00000 & \text { Uiso } & 1.00\end{array}$ $\begin{array}{llllllll}0325 & \mathrm{O} & 0.30546 & 0.0549 & 0.56965 & 0.00000 & \text { Uiso } & 1.00 \\ 0326 & 0 & 0.30483 & 0.05611 & 0.81940 & 0.000000 & \text { Uiso } & 1.00\end{array}$

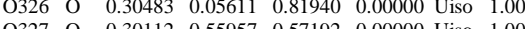
$\begin{array}{lllllllll}0327 & 0 & 0.30112 & 0.55957 & 0.57192 & 0.00000 & \text { Uiso } & 1.00\end{array}$ $\begin{array}{lllllllllll}0328 & 0 & 0.30064 & 0.55976 & 0.81956 & 0.00000 & \text { Uiso } & 1.00\end{array}$ $\begin{array}{llllllll}\mathrm{O} 329 & \mathrm{O} & 0.30655 & 0.22059 & 0.57516 & 0.00000 & \text { Uiso } & 1.00\end{array}$ $\begin{array}{lllllllll}0330 & 0 & 0.31015 & 0.21905 & 0.82337 & 0.00000 & \text { Uiso } & 1.00\end{array}$ $\begin{array}{lllllllll}\mathrm{O} 331 & \mathrm{O} & 0.30991 & 0.71783 & 0.56809 & 0.00000 & \text { Uiso } & 1.00\end{array}$ $\begin{array}{lllllllll}0332 & \mathrm{O} & 0.30663 & 0.72444 & 0.81913 & 0.00000 & \text { Uiso } & 1.00\end{array}$ $\begin{array}{lllllllll}0333 & \mathrm{O} & 0.35924 & 0.14289 & 0.50165 & 0.00000 & \text { Uiso } & 1.00\end{array}$ $\begin{array}{llllllllll}0334 & \mathrm{O} & 0.35660 & 0.64474 & 0.50034 & 0.00000 & \text { Uiso } & 1.00\end{array}$

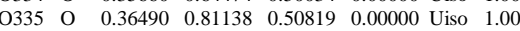
$\begin{array}{lllllllll}0336 & \mathrm{O} & 0.35994 & 0.77690 & 0.76087 & 0.00000 & \text { Uiso } & 1.00\end{array}$ $\begin{array}{llllllll}0337 & \mathrm{O} & 0.34501 & 0.30495 & 0.89382 & 0.00000 & \text { Uiso } & 1.00\end{array}$ $\begin{array}{lllllllll}0338 & \mathrm{O} & 0.35333 & 0.30397 & 0.76015 & 0.00000 & \text { Uiso } & 1.00\end{array}$ $\begin{array}{llllllllll}0339 & \mathrm{O} & 0.35086 & 0.96802 & 0.99593 & 0.00000 & \text { Uiso } & 1.00\end{array}$ $\begin{array}{llllllll}0340 & \mathrm{O} & 0.34963 & 0.95617 & 0.74681 & 0.00000 & \text { Uiso } & 1.00\end{array}$ $\begin{array}{llllllll}\mathrm{O} 341 & \mathrm{O} & 0.34826 & 0.47821 & 0.50110 & 0.00000 & \text { Uiso } & 1.00\end{array}$ $\begin{array}{lllllllllll}0342 & 0 & 0.34948 & 0.48263 & 0.76033 & 0.00000 & \text { Uiso } & 1.00\end{array}$ $\begin{array}{lllllllllllll}0343 & 0 & 0.349714 & 0.89410 & 0.94374 & 0.00000 & \text { Uiso } & 1.00\end{array}$

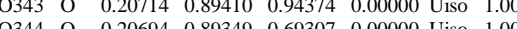
$\begin{array}{llllllllll}0344 & 0 & 0.20694 & 0.8340 & 0.69307 & 0.00000 & \text { Unso } & 1.00\end{array}$ $\begin{array}{llllllll}0345 & \mathrm{O} & 0.20580 & 0.39218 & 0.94277 & 0.00000 & \text { Uiso } & 1.00\end{array}$ $\begin{array}{lllllllll}0346 & \mathrm{O} & 0.20555 & 0.39232 & 0.69223 & 0.00000 & \text { Uiso } & 1.00\end{array}$ $\begin{array}{lllllllll}0347 & \mathrm{O} & 0.20727 & 0.05009 & 0.94444 & 0.00000 & \text { Uiso } & 1.00\end{array}$ $\begin{array}{llllllllll}0348 & 0 & 0.20705 & 0.04986 & 0.69321 & 0.00000 & \text { Uiso } & 1.00\end{array}$ $\begin{array}{lllllllll}0349 & \mathrm{O} & 0.20574 & 0.54782 & 0.94223 & 0.00000 & \text { Uiso } & 1.00\end{array}$ $\begin{array}{lllllllll}0350 & \mathrm{O} & 0.20531 & 0.54773 & 0.69188 & 0.00000 & \text { Uiso } & 1.00\end{array}$ $\begin{array}{llllllll}0351 & \mathrm{O} & 0.21095 & 0.22046 & 0.94326 & 0.00000 & \text { Uiso } & 1.00\end{array}$ $\begin{array}{lllllllll}0352 & \mathrm{O} & 0.21090 & 0.22066 & 0.69247 & 0.00000 & \text { Uiso } & 1.00\end{array}$ $\begin{array}{llllllll}0353 & \mathrm{O} & 0.21040 & 0.72146 & 0.94180 & 0.00000 & \text { Uiso } & 1.00\end{array}$

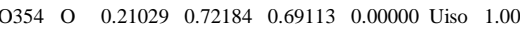
$\begin{array}{lllllllll}0355 & \mathrm{O} & 0.26215 & 0.13521 & 0.62680 & 0.00000 & \text { Uiso } & 1.00\end{array}$ $\begin{array}{llllllll}0356 & \mathrm{O} & 0.26141 & 0.13781 & 0.87300 & 0.00000 & \text { Uiso } & 1.00\end{array}$ $\begin{array}{llllllll}0357 & \mathrm{O} & 0.25707 & 0.63595 & 0.62179 & 0.00000 & \text { Uiso } & 1.00\end{array}$ $\begin{array}{llllllll}0358 & \mathrm{O} & 0.25710 & 0.63729 & 0.87091 & 0.00000 & \text { Uiso } & 1.00\end{array}$ $\begin{array}{lllllllll}0359 & \mathrm{O} & 0.26170 & 0.80403 & 0.62146 & 0.00000 & \text { Uiso } & 1.00\end{array}$ $\begin{array}{llllllll}0360 & \mathrm{O} & 0.25846 & 0.80743 & 0.87301 & 0.00000 & \text { Uiso } & 1.00\end{array}$ $\begin{array}{llllllll}0361 & \mathrm{O} & 0.26074 & 0.30527 & 0.62708 & 0.00000 & \text { Uiso } & 1.00\end{array}$ $\begin{array}{lllllllll}0362 & \mathrm{O} & 0.25984 & 0.30508 & 0.87539 & 0.00000 & \text { Uiso } & 1.00\end{array}$ $\begin{array}{lllllllll}0363 & 0 & 0.25642 & 0.96842 & 0.62507 & 0.00000 & \text { Uiso } & 1.00\end{array}$ $\begin{array}{llllllllll}0363 & \mathrm{O} & 0.25642 & 0.96842 & 0.62507 & 0.0000 & \text { Uiso } & 1.00\end{array}$ $\begin{array}{lllllllll}0364 & 0 & 0.25571 & 0.97390 & 0.87513 & 0.00000 & \text { Uiso } & 1.00 \\ 0365 & 0 & 0.25338 & 0.47022 & 0.62398 & 0.00000 & \text { Uiso } & 1.00\end{array}$ $\begin{array}{lllllllll}0365 & 0 & 0.25338 & 0.47022 & 0.62398 & 0.00000 & \text { Uiso } & 1.00\end{array}$ $\begin{array}{lllllllll}0366 & 0 & 0.25437 & 0.47040 & 0.87373 & 0.00000 & \text { Uiso } & 1.00 \\ 0367 & 0 & 0.21592 & 0.89300 & 0.56075 & 0.00000 & \text { Uis } & 1.00\end{array}$ \begin{tabular}{lllllllll}
0367 & $\mathrm{O}$ & 0.21592 & 0.8300 & 0.56075 & 0.00000 & Uiso & 1.00 \\
\hline 368 & $\mathrm{O}$ & 0.21497 & 0.89461 & 0.81060 & 0.0000 & Uiso & 1.00
\end{tabular} $\begin{array}{lllllllll}0368 & \mathrm{O} & 0.21497 & 0.89461 & 0.81060 & 0.00000 & \text { Uiso } & 1.00\end{array}$ $\begin{array}{lllllllll}0369 & \mathrm{O} & 0.21420 & 0.39217 & 0.56041 & 0.00000 & \text { Uiso } & 1.00\end{array}$ $\begin{array}{lllllllll}0370 & \mathrm{O} & 0.21407 & 0.39200 & 0.81022 & 0.00000 & \text { Uiso } & 1.00\end{array}$ $\begin{array}{llllllll}0371 & \mathrm{O} & 0.21590 & 0.04853 & 0.56243 & 0.00000 & \text { Uiso } & 1.00\end{array}$ $\begin{array}{llllllll}0372 & \mathrm{O} & 0.21583 & 0.04998 & 0.81045 & 0.00000 & \text { Uiso } & 1.00\end{array}$ $\begin{array}{llllllll}0373 & \mathrm{O} & 0.21366 & 0.54798 & 0.55933 & 0.00000 & \text { Uiso } & 1.00\end{array}$ $\begin{array}{llllllll}0374 & \mathrm{O} & 0.21380 & 0.54805 & 0.80921 & 0.00000 & \text { Uiso } & 1.00\end{array}$ $\begin{array}{lllllllll}\mathrm{O} 375 & \mathrm{O} & 0.20848 & 0.21991 & 0.56388 & 0.00000 & \text { Uiso } & 1.00\end{array}$ $\begin{array}{llllllll}\mathrm{O} 376 & \mathrm{O} & 0.20818 & 0.22101 & 0.81289 & 0.00000 & \text { Uiso } & 1.00\end{array}$ $\begin{array}{llllllllll}0377 & \mathrm{O} & 0.20815 & 0.72182 & 0.56143 & 0.00000 & \text { Uiso } & 1.00\end{array}$ $\begin{array}{llllllll}0378 & \mathrm{O} & 0.20752 & 0.72170 & 0.81089 & 0.00000 & \text { Uiso } & 1.00\end{array}$ $\begin{array}{lllllllll}0 & 0 & 0 & 0.25600 & 0.13646 & 0.50859 & 0.00000 & \text { Uiso } & 1.00\end{array}$ $\begin{array}{llllllll}\mathrm{O} 380 & \mathrm{O} & 0.25648 & 0.13831 & 0.75637 & 0.00000 & \text { Uiso } & 1.00\end{array}$ $\begin{array}{llllllll}0381 & \mathrm{O} & 0.25471 & 0.63737 & 0.50579 & 0.00000 & \text { Uiso } & 1.00\end{array}$ $\begin{array}{lllllllll}0382 & \mathrm{O} & 0.25487 & 0.63733 & 0.75404 & 0.00000 & \text { Uiso } & 1.00\end{array}$ $\begin{array}{llllllllll}0383 & 0 & 0.25632 & 0.80362 & 0.50658 & 0.00000 & \text { Uiso } & 1.00\end{array}$

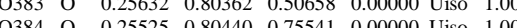
$\begin{array}{lllllllll}0384 & 0 & 0.25525 & 0.80440 & 0.75541 & 0.00000 & \text { Uiso } & 1.00\end{array}$ $\begin{array}{llllllllll}0385 & 0 & 0.25541 & 0.30358 & 0.50884 & 0.00000 & \text { Uiso } & 1.00\end{array}$ $\begin{array}{lllllllll}0386 & \mathrm{O} & 0.25553 & 0.30338 & 0.75751 & 0.00000 & \text { Uiso } & 1.00 \\ 0387 & \mathrm{O} & 0.25884 & 0.97248 & 0.50628 & 0.0000 & \text { Uiso } & 1.00\end{array}$ $\begin{array}{lllllllll}0387 & \mathrm{O} & 0.25884 & 0.97248 & 0.50628 & 0.00000 & \text { Uiso } & 1.00 \\ 0388 & \mathrm{O} & 0.25840 & 0.97017 & 0.75476 & 0.00000 & \text { Uis } & 1.00\end{array}$ $\begin{array}{lllllllll}0388 & \mathrm{O} & 0.25840 & 0.97017 & 0.75476 & 0.00000 & \text { Uiso } & 1.00\end{array}$ $\begin{array}{lllllllll}0389 & 0 & 0.25710 & 0.47055 & 0.50558 & 0.00000 & \text { Uiso } & 1.00 \\ 0390 & 0 & 0.25616 & 0.4693 & 0.75501 & 0.0000 & \text { Uis } & 1.00\end{array}$ $\begin{array}{lllllllll}0390 & \mathrm{O} & 0.25610 & 0.46930 & 0.75501 & 0.00000 & \text { Uiso } & 1.00\end{array}$ $\begin{array}{llllllll}0391 & \mathrm{O} & 0.35221 & 0.29808 & 0.63546 & 0.00000 & \text { Uiso } & 1.00\end{array}$ $\begin{array}{lllllllll}0392 & \mathrm{O} & 0.39636 & 0.71351 & 0.83001 & 0.00000 & \text { Uiso } & 1.00\end{array}$ $\begin{array}{llllllll}0393 & \mathrm{O} & 0.41534 & 0.18300 & 0.96432 & 0.00000 & \text { Uiso } & 1.00\end{array}$ $\begin{array}{lllllllll}0394 & \mathrm{O} & 0.39821 & 0.88752 & 0.93360 & 0.00000 & \text { Uiso } & 1.00\end{array}$ $\begin{array}{llllllll}\mathrm{O} 395 & \mathrm{O} & 0.39775 & 0.38819 & 0.58848 & 0.00000 & \text { Uiso } & 1.00\end{array}$ $\begin{array}{lllllllll}0396 & \mathrm{O} & 0.39437 & 0.37958 & 0.93585 & 0.00000 & \text { Uiso } & 1.00\end{array}$ $\begin{array}{llllllll}0397 & \mathrm{O} & 0.39820 & 0.25452 & 0.85681 & 0.00000 & \text { Uiso } & 1.00\end{array}$ $\begin{array}{lllllllll}0398 & \mathrm{O} & 0.41027 & 0.55966 & 0.82768 & 0.00000 & \text { Uiso } & 1.00\end{array}$ $\begin{array}{lllllllll}0399 & \mathrm{O} & 0.46470 & 0.49370 & 0.85410 & 0.00000 & \text { Uiso } & 1.00\end{array}$ $\begin{array}{llllllll}\mathrm{O} 400 & \mathrm{O} & 0.40835 & 0.54445 & 0.64866 & 0.00000 & \text { Uiso } & 1.00\end{array}$ $\begin{array}{lllllllll}0401 & \mathrm{O} & 0.40535 & 0.53068 & 0.53513 & 0.00000 & \text { Uiso } & 1.00\end{array}$ $\begin{array}{llllllllll}0402 & \mathrm{O} & 0.39823 & 0.22926 & 0.57406 & 0.00000 & \text { Uiso } & 1.00\end{array}$ $\begin{array}{lllllllll}0403 & \mathrm{O} & 0.39560 & 0.04554 & 0.92170 & 0.00000 & \text { Uiso } & 1.00\end{array}$ $\begin{array}{lllllllllll}0404 & 0 & 0.40608 & 0.07263 & 0.55296 & 0.00000 & \text { Uiso } & 1.00\end{array}$ $\begin{array}{lllllllll}0404 & 0 & 0.40608 & 0.07263 & 0.55296 & 0.00000 & \text { Uiso } & 1.00\end{array}$ $\begin{array}{lllllllll}040.40 & 0 & 0.41387 & 0.69542 & 0.6981 & 0.0000 & \text { Uiso } & 1.00\end{array}$ $\begin{array}{lllllllll}0406 & \mathrm{O} & 0.45036 & 0.32257 & 0.90178 & 0.00000 & \text { Uiso } & 1.00\end{array}$ $\begin{array}{lllllllll}0407 & \mathrm{O} & 0.45343 & 0.67249 & 0.85267 & 0.00000 & \text { Uiso } & 1.00\end{array}$ $\begin{array}{lllllllll}0408 & \mathrm{O} & 0.41090 & 0.63368 & 0.93607 & 0.00000 & \text { Uiso } & 1.00\end{array}$ $\begin{array}{llllllll}0409 & 0 & 0.44533 & 0.49103 & 0.74153 & 0.00000 & \text { Uiso } & 1.00\end{array}$ $\begin{array}{lllllllll}0410 & 0 & 0.37047 & 0.16813 & 0.76594 & 0.00000 & \text { Uiso } & 1.00\end{array}$ $\begin{array}{llllllll}0411 & \mathrm{O} & 0.42082 & 0.11432 & 0.82609 & 0.00000 & \text { Uiso } & 1.00\end{array}$ $\begin{array}{llllllll}0412 & \mathrm{O} & 0.40927 & 0.94017 & 0.82440 & 0.00000 & \text { Uiso } & 1.00\end{array}$ $\begin{array}{llllllll}\mathrm{O} 413 & \mathrm{O} & 0.39800 & 0.86985 & 0.71658 & 0.00000 & \text { Uiso } & 1.00\end{array}$ $\begin{array}{llllllll}\mathrm{O} 414 & \mathrm{O} & 0.42690 & 0.16749 & 0.70899 & 0.00000 & \text { Uiso } & 1.00 \\ \mathrm{O} 415 & \mathrm{O} & 0.39710 & 0.03746 & 0.73037 & 0.00000 & \text { Uiso } & 1.00\end{array}$ $\begin{array}{lllllllll}0416 & \mathrm{O} & 0.45071 & 0.94614 & 0.74552 & 0.00000 & \text { Uiso } & 1.00\end{array}$ 
$\begin{array}{llllllll}0417 & \mathrm{O} & 0.40939 & 0.91105 & 0.55180 & 0.00000 & \text { Uiso } & 1.00\end{array}$ $\begin{array}{lllllllll}0418 & \mathrm{O} & 0.42572 & 0.77391 & 0.50122 & 0.00000 & \text { Uiso } & 1.00\end{array}$ $\begin{array}{llllllll}\mathrm{O} 419 & \mathrm{O} & 0.42960 & 0.99392 & 0.63861 & 0.00000 & \text { Uiso } & 1.00\end{array}$ $\begin{array}{lllllllll}0420 & \mathrm{O} & 0.39134 & 0.67940 & 0.58524 & 0.00000 & \text { Uiso } & 1.00\end{array}$ $\begin{array}{llllllllll}0421 & 0 & 0.36447 & 0.98277 & 0.61866 & 0.00000 & \text { Uiso } & 1.00\end{array}$ $\begin{array}{lllllllllllll}0422 & 0 & 0.44383 & 0.79024 & 0.77878 & 0.00000 & \text { Uiso } & 1.00\end{array}$

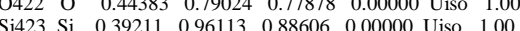
$\begin{array}{llllllll}\mathrm{Si423} & \mathrm{Si} & 0.39211 & 0.96113 & 0.88606 & 0.00000 & \text { Uiso } & 1.00 \\ \mathrm{Si424} & \mathrm{Si} & 0.41367 & 0.34069 & 0.88236 & 0.00000 & \text { Uiso } & 1.00\end{array}$

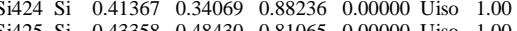

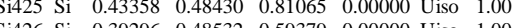
$\begin{array}{llllllll}\text { Si426 Si } & 0.39296 & 0.48532 & 0.59379 & 0.00000 & \text { Uiso } & 1.00\end{array}$

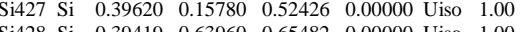
$\begin{array}{llllllll}\mathrm{Si} 428 & \mathrm{Si} & 0.39419 & 0.63960 & 0.65482 & 0.00000 & \text { Uiso } & 1.00\end{array}$ $\begin{array}{llllllll}\mathrm{Si} 429 & \mathrm{Si} & 0.37551 & 0.30841 & 0.57658 & 0.00000 & \text { Uiso } & 1.00\end{array}$ $\begin{array}{llllllll}\mathrm{Si} 430 & \mathrm{Si} & 0.41649 & 0.64293 & 0.86524 & 0.00000 & \text { Uiso } & 1.00 \\ \mathrm{Si} 431 & \mathrm{Si} & 0.41207 & 0.94837 & 0.75092 & 0.00000 & \text { Us } & 1.00\end{array}$ $\begin{array}{llllllll}\mathrm{Si} 431 & \mathrm{Si} & 0.41207 & 0.94837 & 0.75092 & 0.00000 & \text { Uiso } & 1.00 \\ \mathrm{Si} 432 & \mathrm{Si} & 0.40530 & 0.12653 & 0.76160 & 0.00000 & \text { Uiso } & 1.00\end{array}$ $\begin{array}{llllllll}\mathrm{Si} 432 & \mathrm{Si} & 0.40530 & 0.12653 & 0.76160 & 0.00000 & \text { Uiso } & 1.00 \\ \mathrm{Si} 433 & \mathrm{Si} & 0.40080 & 0.99058 & 0.58950 & 0.00000 & \text { Uiso } & 1.00\end{array}$ $\begin{array}{llllllll}\mathrm{Si} 433 & \mathrm{Si} & 0.40080 & 0.99058 & 0.58950 & 0.00000 & \text { Uiso } & 1.00 \\ \mathrm{Si} 434 & \mathrm{Si} & 0.40079 & 0.84635 & 0.50082 & 0.00000 & \text { Uiso } & 1.00\end{array}$ 


\section{References}

(1) Tetrode, H. Ann. Phys. (Berlin) 1912, 38, 434.

(2) Tetrode, H. Ann. Phys. (Berlin) 1912, 39, 255.

(3) Sackur, O. Ann. Phys. (Berlin) 1913, 40, 67.

(4) Leydier, F.; Chizallet, C.; Chaumonnot, A.; Digne, M.; Soyer, E.; Quoineaud, A. A.; Costa, D.; Raybaud, P. J. Catal. 2011, 284, 215.

(5) Leydier, F.; Chizallet, C.; Costa, D.; Raybaud, P. Chem. Commun. 2012, 48, 4076. 